Supporting Information

\title{
Anionic Diels-Alder Chemistry of Cyclic Sodium Dien-1-olates Delivering Highly Stereoselective and Functionalized Polycyclic Adducts
}

Jing-Kai Huang and Kak-Shan Shia*

Institute of Biotechnology and Pharmaceutical Research, National Health Research Institutes, Miaoli County 35053, Taiwan, R.O.C.

E-mail: ksshia@nhri.edu.tw 


\section{Table of Contents}

Proposed reaction-energy profiles for dienolates $(Z)-7 \mathbf{a}$ and $(E)-7 \mathbf{a}$ ion pairs and products 9 and $\mathbf{1 0}$

Materials and Methods

Synthetic procedures and characterization of various cross-conjugated enones and precursors

X-ray crystal structure and crystal parameters of anionic Diels-Alder products 9, 10, 15, 22,

24-27, 29, 30, 32, 36, 41, 43 .

References .568

${ }^{1} \mathrm{H},{ }^{13} \mathrm{C}$ NMR, DEPT, ${ }^{1} \mathrm{H}-{ }^{1} \mathrm{H}$ COSY and NOESY spectra for all new compounds S69

2D NOESY spectrum for compound 31a. S154

1D NOESY spectra for compound 35a. 


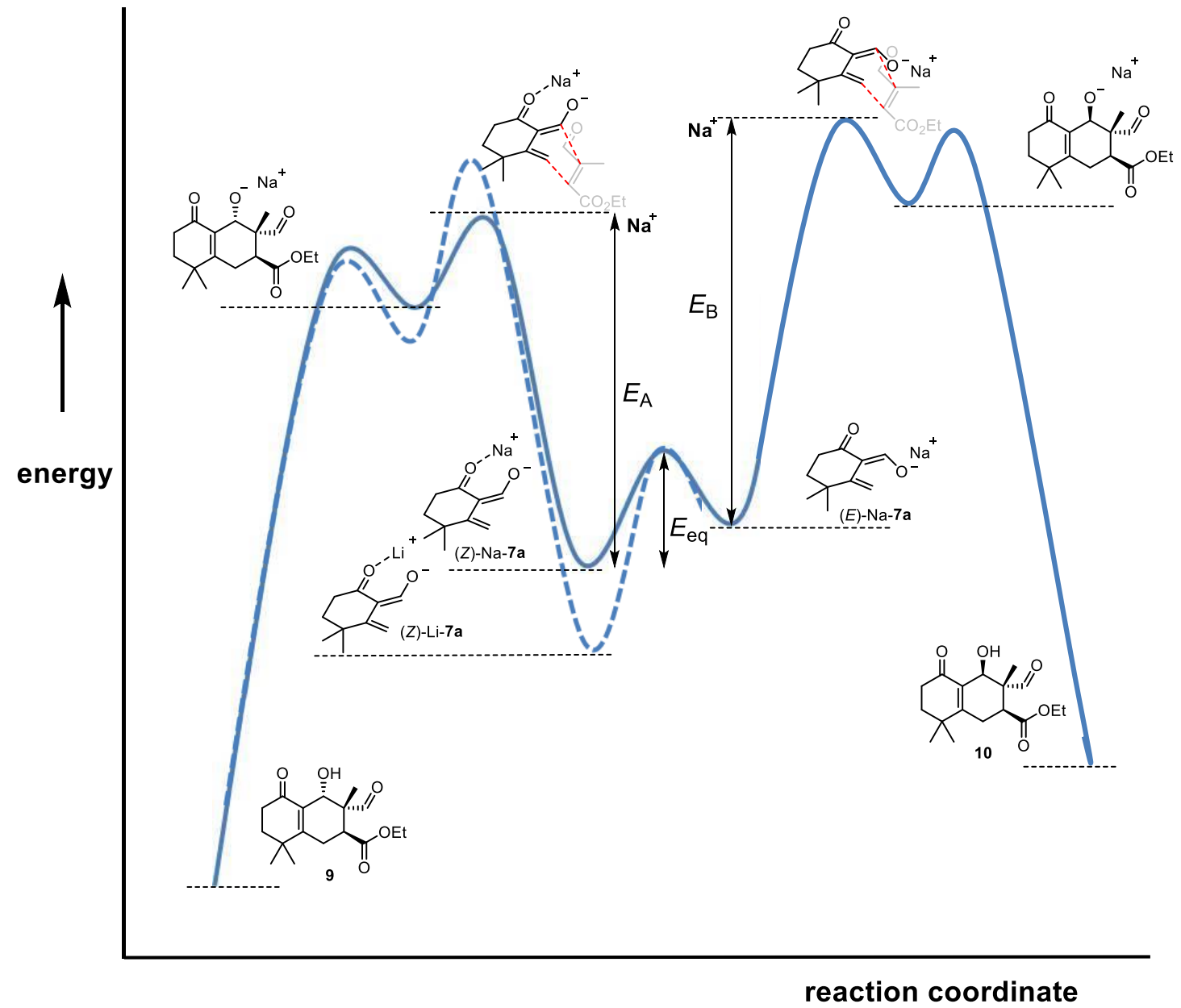

Figure S1. Proposed reaction-energy profiles for dienolates $(Z)-7 \mathbf{a}$ and $(E)-\mathbf{7 a}$ ion pairs and products 9 and 10. The vertical axis represents the total energy. The transition state is the highest point on the graph, and the activation energy, $E_{\mathrm{A}}$ and $E_{\mathrm{B}}$, is the energy difference between the reactants and the transition state. $E_{\text {eq }}$ is the equilibrium energy barrier between conformers $(Z)-7 \mathbf{a}$ and $(E)-7 \mathbf{a}$. 


\section{Materials and Methods}

All reactions were performed under nitrogen using flame-dried glassware and stir bars unless otherwise stated. Room temperature refers to $23-26{ }^{\circ} \mathrm{C}$. A pre-heated oil bath was used to maintain reaction temperature when it was higher than room temperature. All reagents were employed as received without further purification. All solvents were dried and distilled by standard techniques. Tetrahydrofuran was distilled from potassium under $\mathrm{N}_{2}$. Dichloromethane and toluene were distilled from calcium hydride under $\mathrm{N}_{2}$. Analytical thin layer chromatography was performed on $\mathrm{SiO}_{2} 60$ F-254 plates and flash column chromatography was carried out using $\mathrm{SiO}_{2} 60$ (particle size 0.040-0.055 mm, 230-400 mesh). Visualization was performed under UV irradiation at $254 \mathrm{~nm}$ followed by staining with aqueous potassium permanganate and charring by heat gun. Infrared spectra (IR) were recorded on a FT-IR spectrometer and expressed in $\mathrm{cm}^{-1} .{ }^{1} \mathrm{H}$ and ${ }^{13} \mathrm{C}-\mathrm{NMR}$ spectra were recorded by VNMR-300, VNMR-400, Bruker-400 or Bruker-600. Chemical shifts are expressed in ppm using TMS in $\mathrm{CDCl}_{3}(\delta=0.00)$ as internal standard in ${ }^{1} \mathrm{H}-\mathrm{NMR}$ spectra. ${ }^{13} \mathrm{C}$-NMR spectra were recorded in $\mathrm{CDCl}_{3}$, using the central resonances of $\mathrm{CDCl}_{3}(\delta=77.00)$ as the internal references. Multiplicities are recorded as s (singlet), $d$ (doublet), $t$ (triplet), $q$ (quartet), quint (quintet), dd (doublet of doublets), dt (doublet of triplets), tt (triplet of triplets), ddd (doublet of doublet of doublets), m (multiplet), br (broad). Coupling constants $(J)$ are expressed in Hz. HRMS was obtained on a triple quadrupole mass analysis using electrospray ionization (ESI) source, and spectral data were recorded as $\mathrm{m} / \mathrm{z}$ values. The single-crystal X-ray diffraction data of crystals were individually collected on a Bruker D8 Venture diffractometer equipped with a Cu-target $(\mathrm{K} \alpha=1.54178 \AA)$ or Mo-target $(\mathrm{K} \alpha=0.71073 \AA)$ microfocus X-ray generators and a PHOTON-II CMOS detector. The temperature was adjusted with a nitrogen flow (Oxford Cryosystems). After collection, the data were integrated with the Bruker SAINT software package using a narrow-frame algorithm and were corrected for absorption effects using the Multi-Scan method (SADABS). Then, the molecular structure was solved and refined by the Bruker SHELXTL Software Package and the final anisotropic full-matrix least-squares method was used to refine on F2 with variables parameters to determine crystal structure. Melting points were measured using an Electrothermal instrument. 
Synthetic procedures and characterization of various cross-conjugated enones and their precursors

General procedure for preparation of 2-(hydroxymethyl)-3-alkyl (aryl or alkynyl) cyclic enones

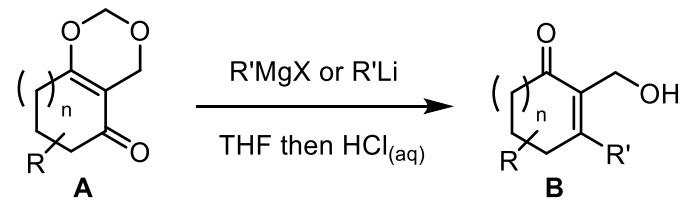

Condition A:

To a stirred solution of 1,3-dioxin $\mathbf{A}(5.0 \mathrm{mmol})$ in dry THF $(15 \mathrm{~mL})$ at $0{ }^{\circ} \mathrm{C}$ was added Grignard reagent $(7.5 \mathrm{mmol})$ dropwise under $\mathrm{N}_{2}$. The mixture was then allowed to react at 0 ${ }^{\circ} \mathrm{C}$ to room temperature for $2 \mathrm{~h}$ under $\mathrm{N}_{2}$. After the reaction was complete, $5 \% \mathrm{HCl}_{(\mathrm{aq})}(20 \mathrm{~mL})$ was added at $0{ }^{\circ} \mathrm{C}$ to acidify the reaction. The reaction mixture then allowed to react at room temperature for $30 \mathrm{~min}$. After the reaction was complete, the organic layer was separated, and the aqueous layer was extracted with EtOAc $(30 \mathrm{~mL} \times 2)$. The organic portions were combined, washed with sat. $\mathrm{NaHCO}_{3(\mathrm{aq})}$, dried over $\mathrm{MgSO}_{4}$, filtered and concentrated to give the crude residue, which was purified by chromatography on silical gel to afford the 2-(hydroxymethyl) cyclic enones B, including compounds: 6, S1-S3, S5, S9 and S14-S16 as indicated below.

Condition B:

To a stirred solution of 1,3-dioxin $\mathbf{A}(5.0 \mathrm{mmol})$ in dry THF $(15 \mathrm{~mL})$ at $-78{ }^{\circ} \mathrm{C}$ was added organic lithium reagent $(7.5 \mathrm{mmol})$ dropwise under $\mathrm{N}_{2}$. The mixture was then allowed to react at -78 to $0{ }^{\circ} \mathrm{C}$ for $2 \mathrm{~h}$ under $\mathrm{N}_{2}$. After the reaction was complete, $5 \% \mathrm{HCl}_{(\mathrm{aq})}(20 \mathrm{~mL})$ was added at $0{ }^{\circ} \mathrm{C}$ to acidify the reaction. The reaction mixture then allowed to react at room temperature for $30 \mathrm{~min}$. After the reaction was complete, the organic layer was separated, and the aqueous layer was extracted with EtOAc $(30 \mathrm{~mL} \times 2)$. The organic portions were combined, washed with sat. $\mathrm{NaHCO}_{3(\mathrm{aq})}$, dried over $\mathrm{MgSO}_{4}$, filtered and concentrated to give the crude residue, which was purified by chromatography on silical gel to afford the 2-(hydroxymethyl) cyclic enones B, including compounds: S4, S6, S8, S10, S12, S17 and S19 as indicated below.

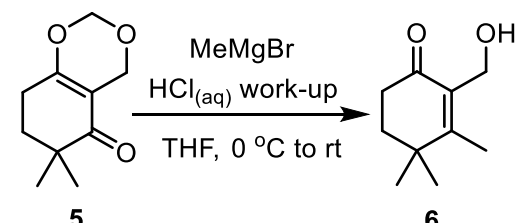

2-(Hydroxymethyl)-3,4,4-trimethylcyclohex-2-en-1-one (6). Following the general procedure of condition A, 2-(hydroxymethyl) enone 6 (740 $\mathrm{mg}, 88 \%$ yield) was prepared from 1,3-dioxin 5 (911 mg, $5.0 \mathrm{mmol})$ and $\mathrm{MeMgBr}(2.5 \mathrm{~mL}, 3.0 \mathrm{M}$ in diethyl ether) as a 
colorless oil: IR $\left(\mathrm{CH}_{2} \mathrm{Cl}_{2}\right.$ cast, $\left.\mathrm{cm}^{-1}\right) v_{\max } 3432$ (br), 2963, 2927, 2869, 1660, 1609; ${ }^{1} \mathrm{H}$ NMR $\left(\mathrm{CDCl}_{3}, 400 \mathrm{MHz}\right): \delta 1.18$ (s, 6H), 1.84 (t, $\left.J=7.6 \mathrm{~Hz}, 2 \mathrm{H}\right), 1.97$ (s, 3H), 2.48 (t, $J=7.6 \mathrm{~Hz}$, $2 \mathrm{H}), 2.80(\mathrm{t}, J=6.8 \mathrm{~Hz}, 1 \mathrm{H}), 4.35(\mathrm{~d}, J=6.8 \mathrm{~Hz}, 2 \mathrm{H}) ;{ }^{13} \mathrm{C} \mathrm{NMR}\left(\mathrm{CDCl}_{3}, 150 \mathrm{MHz}\right): \delta 15.5$ $\left(\mathrm{CH}_{3}\right), 26.3\left(\mathrm{CH}_{3}\right), 34.2\left(\mathrm{CH}_{2}\right), 36.1(\mathrm{C}), 36.7\left(\mathrm{CH}_{2}\right), 58.0\left(\mathrm{CH}_{2}\right), 133.2(\mathrm{C}), 165.3(\mathrm{C}), 200.1$ (CO); HRMS (ESI) m/z: [M + Na $]^{+}$calcd. for $\mathrm{C}_{10} \mathrm{H}_{16} \mathrm{O}_{2} \mathrm{Na}$ 191.1043, found 191.1035.

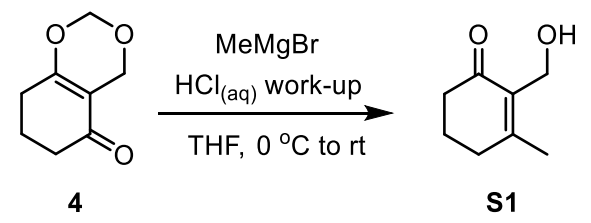

2-(Hydroxymethyl)-3-methylcyclohex-2-en-1-one (S1). Following the general procedure of condition A, 2-(hydroxymethyl) enone $\mathbf{S 1}$ (568 mg, 81\% yield) was prepared from 1,3-dioxin 4 (771 mg, $5.0 \mathrm{mmol})$ and $\mathrm{MeMgBr}(2.5 \mathrm{~mL}, 3.0 \mathrm{M}$ in diethyl ether) as a colorless oil: IR $\left(\mathrm{CH}_{2} \mathrm{Cl}_{2}\right.$ cast, $\left.\mathrm{cm}^{-1}\right) v_{\max } 3420$ (br), 2927, 2890, 1654, 1627; ${ }^{1} \mathrm{H} \mathrm{NMR}\left(\mathrm{CDCl}_{3}, 400 \mathrm{MHz}\right): \delta$ 1.94-2.00 (m, 2H), $2.03(\mathrm{~s}, 3 \mathrm{H}), 2.39(\mathrm{t}, J=6.0 \mathrm{~Hz}, 2 \mathrm{H}), 2.42(\mathrm{t}, J=6.4 \mathrm{~Hz}, 2 \mathrm{H}), 2.88$ (br s, $1 \mathrm{H}), 4.37(\mathrm{~s}, 2 \mathrm{H}) ;{ }^{13} \mathrm{C} \mathrm{NMR}\left(\mathrm{CDCl}_{3}, 150 \mathrm{MHz}\right): \delta 20.8\left(\mathrm{CH}_{3}\right), 21.9\left(\mathrm{CH}_{2}\right), 32.8\left(\mathrm{CH}_{2}\right), 37.5$ $\left(\mathrm{CH}_{2}\right), 57.3\left(\mathrm{CH}_{2}\right), 134.1(\mathrm{C}), 158.9(\mathrm{C}), 200.6(\mathrm{CO})$; HRMS (ESI) m/z: [M + Na] ${ }^{+}$calcd. for $\mathrm{C}_{8} \mathrm{H}_{12} \mathrm{O}_{2} \mathrm{Na}$ 163.0730, found 163.0726.

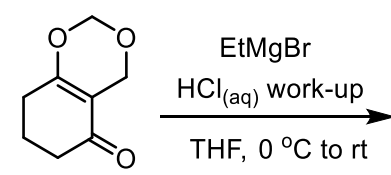

4<smiles>CCC1=C(CO)C(=O)CCC1</smiles>

S2

3-Ethyl-2-(hydroxymethyl)cyclohex-2-en-1-one (S2). Following the general procedure of condition A, 2-(hydroxymethyl) enone S2 (702 mg, 91\% yield) was prepared from 1,3-dioxin $4(771 \mathrm{mg}, 5.0 \mathrm{mmol})$ and $\mathrm{EtMgBr}(7.5 \mathrm{~mL}, 1.0 \mathrm{M}$ in diethyl ether) as a colorless oil: IR $\left(\mathrm{CH}_{2} \mathrm{Cl}_{2}\right.$ cast, $\left.\mathrm{cm}^{-1}\right) v_{\max } 3436$ (br), 2965, 2937, 2878, 1656, 1622; ${ }^{1} \mathrm{H} \mathrm{NMR}\left(\mathrm{CDCl}_{3}, 400\right.$ MHz): $\delta 1.12(\mathrm{t}, J=7.6 \mathrm{~Hz}, 3 \mathrm{H}), 1.94-2.01(\mathrm{~m}, 2 \mathrm{H}), 2.35(\mathrm{q}, J=7.6 \mathrm{~Hz}, 2 \mathrm{H}), 2.41(\mathrm{t}, J=6.8$ $\mathrm{Hz}, 2 \mathrm{H}), 2.42$ (t, $J=6.0 \mathrm{~Hz}, 2 \mathrm{H}), 2.87$ (br s, 1H), $4.36(\mathrm{~s}, 2 \mathrm{H}) ;{ }^{13} \mathrm{C} \mathrm{NMR}\left(\mathrm{CDCl}_{3}, 150 \mathrm{MHz}\right)$ : $\delta 12.8\left(\mathrm{CH}_{3}\right), 22.2\left(\mathrm{CH}_{2}\right), 27.7\left(\mathrm{CH}_{2}\right), 30.4\left(\mathrm{CH}_{2}\right), 37.7\left(\mathrm{CH}_{2}\right), 57.0\left(\mathrm{CH}_{2}\right), 133.5(\mathrm{C}), 164.3$ (C), 201.1 (CO); HRMS (ESI) m/z: $[\mathrm{M}+\mathrm{Na}]^{+}$calcd. for $\mathrm{C}_{9} \mathrm{H}_{14} \mathrm{O}_{2} \mathrm{Na} 177.0886$, found 177.0875 .

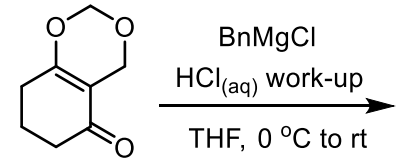

4<smiles>O=C1CCCC(Cc2ccccc2)=C1CO</smiles>

S3 
3-Benzyl-2-(hydroxymethyl)cyclohex-2-en-1-one (S3). Following the general procedure of condition A, 2-(hydroxymethyl) enone $\mathbf{S 3}$ (876 $\mathrm{mg}$, 81\% yield) was prepared from 1,3-dioxin $4(771 \mathrm{mg}, 5.0 \mathrm{mmol})$ and benzylmagnesium chloride $(7.5 \mathrm{~mL}, 1.0 \mathrm{M}$ in diethyl ether) as a white solid: $\mathrm{mp}=72-74{ }^{\circ} \mathrm{C}$; IR $\left(\mathrm{KBr}, \mathrm{cm}^{-1}\right) v_{\max } 3429$ (br), 3084, 3060, 3027, 2940, 2888, 1660, 1624, 1602, 1494, 1453, 1426; ${ }^{1} \mathrm{H} \mathrm{NMR}\left(\mathrm{CDCl}_{3}, 400 \mathrm{MHz}\right): \delta 1.91$ (quint, $J=6.4 \mathrm{~Hz}$, $2 \mathrm{H}), 2.31(\mathrm{t}, J=6.4 \mathrm{~Hz}, 2 \mathrm{H}), 2.43(\mathrm{t}, J=6.4 \mathrm{~Hz}, 2 \mathrm{H}), 2.88(\mathrm{br} \mathrm{s}, 1 \mathrm{H}), 3.69(\mathrm{~s}, 2 \mathrm{H}), 4.51(\mathrm{~s}$, $2 \mathrm{H}), 7.17(\mathrm{~d}, J=7.2 \mathrm{~Hz}, 2 \mathrm{H}), 7.22-7.33(\mathrm{~m}, 3 \mathrm{H}) ;{ }^{13} \mathrm{C} \mathrm{NMR}\left(\mathrm{CDCl}_{3}, 100 \mathrm{MHz}\right): \delta 22.0\left(\mathrm{CH}_{2}\right)$, $30.4\left(\mathrm{CH}_{2}\right), 37.7\left(\mathrm{CH}_{2}\right), 40.1\left(\mathrm{CH}_{2}\right), 57.1\left(\mathrm{CH}_{2}\right), 126.7(\mathrm{CH}), 128.6(\mathrm{CH}), 128.7(\mathrm{CH}), 134.7$ (C), 137.3 (C), 160.1 (C), 200.9 (CO); HRMS (ESI) m/z: [M + Na $]^{+}$calcd. for $\mathrm{C}_{14} \mathrm{H}_{16} \mathrm{O}_{2} \mathrm{Na}$ 239.1043, found 239.1040.

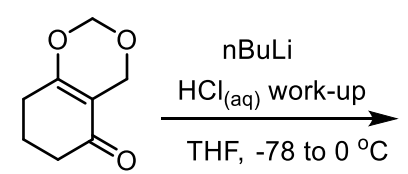

4

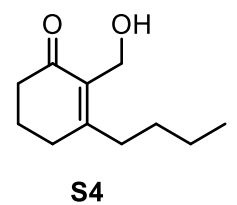

S4

3-Butyl-2-(hydroxymethyl)cyclohex-2-en-1-one (S4). Following the general procedure of condition B, 2-(hydroxymethyl) enone $\mathbf{S 4}$ (747 mg, 82\% yield) was prepared from 1,3-dioxin 4 (771 mg, $5.0 \mathrm{mmol})$ and $n$-butyllithium ( $3 \mathrm{~mL}, 2.5 \mathrm{M}$ in $n$-hexane) as a colorless oil: IR $\left(\mathrm{CH}_{2} \mathrm{Cl}_{2}\right.$ cast, $\left.\mathrm{cm}^{-1}\right) v_{\max } 3436$ (br), 2956, 2931, 2871, 1657, 1622; ${ }^{1} \mathrm{H}$ NMR $\left(\mathrm{CDCl}_{3}, 400\right.$ MHz): $\delta 0.93(\mathrm{t}, J=7.2 \mathrm{~Hz}, 3 \mathrm{H}), 1.32-1.41(\mathrm{~m}, 2 \mathrm{H}), 1.43-1.51(\mathrm{~m}, 2 \mathrm{H}), 1.93-2.00(\mathrm{~m}, 2 \mathrm{H})$, $2.32(\mathrm{t}, J=7.6 \mathrm{~Hz}, 2 \mathrm{H}), 2.40(\mathrm{t}, J=6.0 \mathrm{~Hz}, 2 \mathrm{H}), 2.42(\mathrm{t}, J=6.8 \mathrm{~Hz}, 2 \mathrm{H}), 2.94(\mathrm{t}, J=6.8 \mathrm{~Hz}$, $1 \mathrm{H}), 4.35(\mathrm{~d}, J=6.8 \mathrm{~Hz}, 2 \mathrm{H}) ;{ }^{13} \mathrm{C} \mathrm{NMR}\left(\mathrm{CDCl}_{3}, 150 \mathrm{MHz}\right): \delta 13.8\left(\mathrm{CH}_{3}\right), 22.2\left(\mathrm{CH}_{2}\right), 22.8$ $\left(\mathrm{CH}_{2}\right), 30.5\left(\mathrm{CH}_{2}\right), 30.9\left(\mathrm{CH}_{2}\right), 34.4\left(\mathrm{CH}_{2}\right), 37.7\left(\mathrm{CH}_{2}\right), 57.1\left(\mathrm{CH}_{2}\right), 133.9(\mathrm{C}), 163.0(\mathrm{C})$, 201.1 (CO); HRMS (ESI) m/z: [M + Na $]^{+}$calcd. for $\mathrm{C}_{11} \mathrm{H}_{18} \mathrm{O}_{2} \mathrm{Na} 205.1199$, found 205.1187.

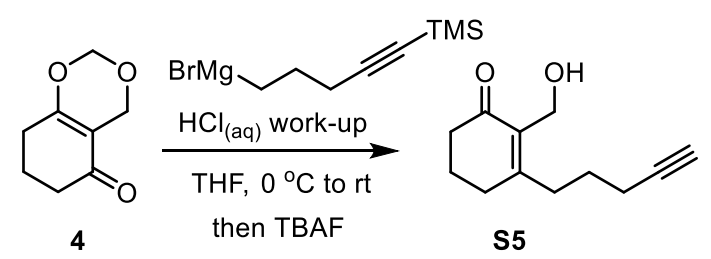

2-(Hydroxymethyl)-3-(pent-4-yn-1-yl)cyclohex-2-en-1-one (S5). Following the general procedure of condition A, 2-(hydroxymethyl) enone $\mathbf{S 5}$ (682 $\mathrm{mg}, 71 \%$ yield over 2 steps) was prepared from 1,3-dioxin 4 (771 $\mathrm{mg}, 5.0 \mathrm{mmol})$ and (5-(trimethylsilyl)pent-4-yn-1-yl) magnesium bromide $(7.5 \mathrm{~mL}, 1 \mathrm{M}$ in THF) and TBAF (10 mL, $1.0 \mathrm{M}$ in THF) as a light yellow oil: IR $\left(\mathrm{CH}_{2} \mathrm{Cl}_{2}\right.$ cast, $\left.\mathrm{cm}^{-1}\right) v_{\max } 3436$ (br), 3290, 2937, 2870, 2215, 1656, 1622; ${ }^{1} \mathrm{H}$ NMR ( $\left.\mathrm{CDCl}_{3}, 400 \mathrm{MHz}\right): \delta 1.68-1.76(\mathrm{~m}, 2 \mathrm{H}), 1.98$ (quint, $\left.J=6.0 \mathrm{~Hz}, 2 \mathrm{H}\right), 2.02(\mathrm{t}, J=2.8$ $\mathrm{Hz}, 1 \mathrm{H}), 2.25(\mathrm{td}, J=6.8,2.8 \mathrm{~Hz}, 2 \mathrm{H}), 2.40-2.48(\mathrm{~m}, 6 \mathrm{H}), 2.82(\mathrm{t}, J=6.8 \mathrm{~Hz}, 1 \mathrm{H}), 4.37(\mathrm{~d}, J$ $=6.8 \mathrm{~Hz}, 2 \mathrm{H}) ;{ }^{13} \mathrm{C} \mathrm{NMR}\left(\mathrm{CDCl}_{3}, 100 \mathrm{MHz}\right): \delta 18.3\left(\mathrm{CH}_{2}\right), 22.1\left(\mathrm{CH}_{2}\right), 27.0\left(\mathrm{CH}_{2}\right), 30.8$ 
$\left(\mathrm{CH}_{2}\right), 33.4\left(\mathrm{CH}_{2}\right), 37.6\left(\mathrm{CH}_{2}\right), 56.9\left(\mathrm{CH}_{2}\right), 69.4(\mathrm{C}), 83.2(\mathrm{CH}), 134.6(\mathrm{C}), 161.5(\mathrm{C}), 200.8$ (CO); HRMS (ESI) m/z: [M + Na $]^{+}$calcd. for $\mathrm{C}_{12} \mathrm{H}_{16} \mathrm{O}_{2} \mathrm{Na} 215.1043$, found 215.1047.

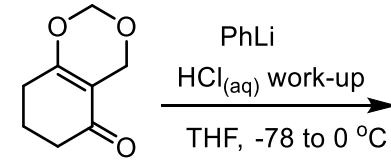

4<smiles>O=C1CCCC(c2ccccc2)=C1CO</smiles>

s6

2-(Hydroxymethyl)-5,6-dihydro-[1,1'-biphenyl]-3(4H)-one (S6). Following the general procedure of condition B, 2-(hydroxymethyl) enone S6 (849 $\mathrm{mg}, 84 \%$ yield) was prepared from 1,3-dioxin 4 (771 mg, $5.0 \mathrm{mmol}$ ) and phenyllithium (7.5 mL, 1.0 M in THF) as a white solid: $\mathrm{mp}=86-88^{\circ} \mathrm{C}$; IR $\left(\mathrm{KBr}, \mathrm{cm}^{-1}\right) v_{\max } 3450$ (br), 3057, 2921, 2850, 1660, 1614, 1572, 1424, 1361; ${ }^{1} \mathrm{H}$ NMR $\left(\mathrm{CDCl}_{3}, 400 \mathrm{MHz}\right): \delta 2.10-2.17(\mathrm{~m}, 2 \mathrm{H}), 2.55(\mathrm{t}, J=7.2 \mathrm{~Hz}, 2 \mathrm{H}), 2.71$ (t, $J=6.0 \mathrm{~Hz}, 2 \mathrm{H}), 2.92$ (t, $J=7.2 \mathrm{~Hz}, 1 \mathrm{H}), 4.21(\mathrm{~d}, J=7.2 \mathrm{~Hz}, 2 \mathrm{H}), 7.27-7.31(\mathrm{~m}, 2 \mathrm{H})$, 7.35-7.44 (m, 3H); ${ }^{13} \mathrm{C}$ NMR $\left(\mathrm{CDCl}_{3}, 150 \mathrm{MHz}\right): \delta 22.4\left(\mathrm{CH}_{2}\right), 32.7\left(\mathrm{CH}_{2}\right), 37.7\left(\mathrm{CH}_{2}\right), 58.7$ $\left(\mathrm{CH}_{2}\right), 127.3(\mathrm{CH}), 128.5(\mathrm{CH}), 128.8(\mathrm{CH}), 134.5(\mathrm{C}), 139.4(\mathrm{C}), 159.8(\mathrm{C}), 201.5(\mathrm{CO})$; HRMS (ESI) m/z: [M + Na $]^{+}$calcd. for $\mathrm{C}_{13} \mathrm{H}_{14} \mathrm{O}_{2} \mathrm{Na} 225.0886$, found 225.0888 .

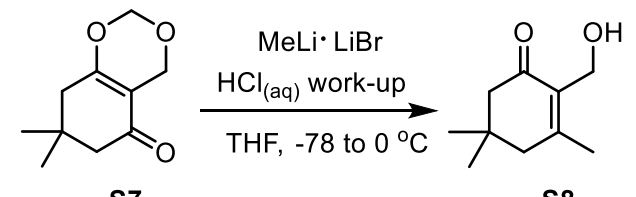

s7

S8

2-(Hydroxymethyl)-3,5,5-trimethylcyclohex-2-en-1-one (S8). Following the general procedure of condition B, 2-(hydroxymethyl) enone $\mathbf{S 8}$ (732 $\mathrm{mg}$, 87\% yield) was prepared from 1,3-dioxin $\mathbf{S} 7$ (911 mg, $5.0 \mathrm{mmol}$ ) and $\mathrm{MeLi} \cdot \mathrm{LiBr}(7.5 \mathrm{~mL}, 1.0 \mathrm{M}$ in diethyl ether) as a white solid: $\mathrm{mp}=51-52{ }^{\circ} \mathrm{C}$; IR $\left(\mathrm{CH}_{2} \mathrm{Cl}_{2}\right.$ cast, $\left.\mathrm{cm}^{-1}\right) v_{\max } 3420,2957,2893,2887,1661,1635$; ${ }^{1} \mathrm{H}$ NMR (CDCl $\left.3,400 \mathrm{MHz}\right): \delta 1.03(\mathrm{~s}, 6 \mathrm{H}), 1.61(\mathrm{~s}, 2 \mathrm{H}), 2.00(\mathrm{~s}, 2 \mathrm{H}), 2.28(\mathrm{~s}, 3 \mathrm{H}), 2.79(\mathrm{t}, J$ $=6.0 \mathrm{~Hz}, 1 \mathrm{H}), 4.38(\mathrm{~d}, J=6.0 \mathrm{~Hz}, 2 \mathrm{H}) ;{ }^{13} \mathrm{C} \mathrm{NMR}\left(\mathrm{CDCl}_{3}, 150 \mathrm{MHz}\right): \delta 20.9\left(\mathrm{CH}_{3}\right), 28.2$ $\left(\mathrm{CH}_{3}\right), 32.7(\mathrm{C}), 46.9\left(\mathrm{CH}_{2}\right), 51.1\left(\mathrm{CH}_{2}\right), 57.0\left(\mathrm{CH}_{2}\right), 132.9(\mathrm{C}), 156.3(\mathrm{C}), 200.8(\mathrm{CO})$; HRMS (ESI) m/z: [M + Na] $]^{+}$calcd. for $\mathrm{C}_{10} \mathrm{H}_{16} \mathrm{O}_{2} \mathrm{Na}$ 191.1043, found 191.1043.

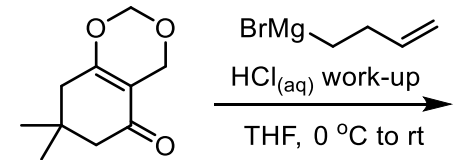

S7

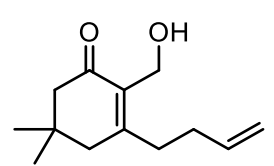

S9

3-(But-3-en-1-yl)-2-(hydroxymethyl)-5,5-dimethylcyclohex-2-en-1-one (S9). Following the general procedure of condition A, 2-(hydroxymethyl) enone $\mathbf{S 9}$ (781 mg, 75\% yield) was prepared from 1,3-dioxin $\mathbf{S 7}$ (911 $\mathrm{mg}, 5.0 \mathrm{mmol}$ ) and but-3-en-1-ylmagnesium bromide (7.5 $\mathrm{mL}, 1.0 \mathrm{M}$ in THF) as a colorless oil: IR $\left(\mathrm{CH}_{2} \mathrm{Cl}_{2}\right.$ cast, $\left.\mathrm{cm}^{-1}\right) v_{\max } 3445$ (br), 3078, 2957, 2891, 
2869, 1662, 1628, 1412, 1368; ${ }^{1} \mathrm{H}$ NMR ( $\left.\mathrm{CDCl}_{3}, 400 \mathrm{MHz}\right): \delta 1.03(\mathrm{~s}, 6 \mathrm{H}), 2.21-2.27(\mathrm{~m}, 2 \mathrm{H})$, $2.28(\mathrm{~s}, 2 \mathrm{H}), 2.29(\mathrm{~s}, 2 \mathrm{H}), 2.40(\mathrm{t}, J=7.6 \mathrm{~Hz}, 2 \mathrm{H}), 2.77(\mathrm{t}, J=6.8 \mathrm{~Hz}, 1 \mathrm{H}), 4.36(\mathrm{~d}, J=6.8 \mathrm{~Hz}$, 2H), 5.02 (dd, $J=7.6,0.8 \mathrm{~Hz}, 1 \mathrm{H}$ ), 5.06 (dd, $J=17.6,1.6 \mathrm{~Hz}, 1 \mathrm{H}$ ), 5.79 (ddt, $J=17.6,7.6$, $6.0 \mathrm{~Hz}, 1 \mathrm{H}) ;{ }^{13} \mathrm{C} \mathrm{NMR}\left(\mathrm{CDCl}_{3}, 100 \mathrm{MHz}\right): \delta 28.1\left(\mathrm{CH}_{3}\right), 31.9\left(\mathrm{CH}_{2}\right), 32.9(\mathrm{C}), 33.8\left(\mathrm{CH}_{2}\right)$, $44.9\left(\mathrm{CH}_{2}\right), 51.1\left(\mathrm{CH}_{2}\right), 56.8\left(\mathrm{CH}_{2}\right), 115.8\left(\mathrm{CH}_{2}\right), 133.3(\mathrm{C}), 136.7(\mathrm{CH}), 159.0(\mathrm{C}), 201.1$ (CO); HRMS (ESI) m/z: [M + Na $]^{+}$calcd. for $\mathrm{C}_{13} \mathrm{H}_{20} \mathrm{O}_{2} \mathrm{Na} 231.1356$, found 231.1359.

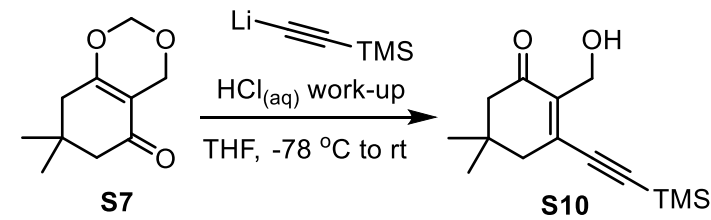

2-(Hydroxymethyl)-5,5-dimethyl-3-((trimethylsilyl)ethynyl)cyclohex-2-en-1-one (S10). Following the general procedure of condition A, 2-(hydroxymethyl) enone S10 (776 mg, 62\% yield) was prepared from 1,3-dioxin $\quad \mathbf{S 7}$ (911 $\mathrm{mg}, \quad 5.0 \mathrm{mmol})$ and Lithium (trimethylsilyl)acetylide (15 mL, $0.5 \mathrm{M}$ in THF) as a light yellow oil: $\mathrm{IR}\left(\mathrm{CH}_{2} \mathrm{Cl}_{2}\right.$ cast, $\left.\mathrm{cm}^{-1}\right)$ $v_{\max } 3469$ (br), 2959, 2898, 2871, 2140, 1663, 1598, 1412, 1389, 1359; ${ }^{1} \mathrm{H} \mathrm{NMR}\left(\mathrm{CDCl}_{3}, 400\right.$ MHz): $\delta 0.23(\mathrm{~s}, 9 \mathrm{H}), 1.06(\mathrm{~s}, 6 \mathrm{H}), 2.32(\mathrm{~s}, 2 \mathrm{H}), 2.41(\mathrm{~s}, 2 \mathrm{H}), 2.92(\mathrm{t}, J=7.2 \mathrm{~Hz}, 1 \mathrm{H}), 4.54(\mathrm{~d}$, $J=7.2 \mathrm{~Hz}, 2 \mathrm{H}) ;{ }^{13} \mathrm{C} \mathrm{NMR}\left(\mathrm{CDCl}_{3}, 150 \mathrm{MHz}\right): \delta 0.42\left(\mathrm{CH}_{3}\right), 27.9\left(\mathrm{CH}_{3}\right), 33.4(\mathrm{C}), 44.7\left(\mathrm{CH}_{2}\right)$, $51.5\left(\mathrm{CH}_{2}\right), 60.0\left(\mathrm{CH}_{2}\right), 101.7(\mathrm{C}), 110.9(\mathrm{C}), 137.2(\mathrm{C}), 139.8(\mathrm{C}), 200.4(\mathrm{CO})$; HRMS (ESI) $\mathrm{m} / \mathrm{z}:[\mathrm{M}+\mathrm{Na}]^{+}$calcd. for $\mathrm{C}_{14} \mathrm{H}_{22} \mathrm{O}_{2} \mathrm{SiNa} 273.1281$, found 273.1272 .

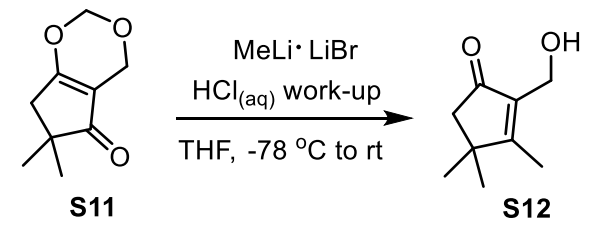

2-(Hydroxymethyl)-3,4,4-trimethylcyclopent-2-en-1-one (S12). Following the general procedure of condition B, 2-(hydroxymethyl) enone $\mathbf{S 1 2}$ (663 $\mathrm{mg}, 86 \%$ yield) was prepared from 1,3-dioxin $\mathbf{S 1 1}(841 \mathrm{mg}, 5.0 \mathrm{mmol}$ ) and $\mathrm{MeLi} \cdot \mathrm{LiBr}$ ( $7.5 \mathrm{~mL}, 1.0 \mathrm{M}$ in diethyl ether) as a colorless oil: IR $\left(\mathrm{CH}_{2} \mathrm{Cl}_{2}\right.$ cast, $\left.\mathrm{cm}^{-1}\right) v_{\max } 3412$ (br), 2962, 2928, 2871, 1693, 1642; ${ }^{1} \mathrm{H}$ NMR $\left(\mathrm{CDCl}_{3}, 400 \mathrm{MHz}\right): \delta 1.22$ (s, 6H), $2.01(\mathrm{~s}, 3 \mathrm{H}), 2.32(\mathrm{~s}, 2 \mathrm{H}), 2.87$ (br s, $\left.1 \mathrm{H}\right), 4.33(\mathrm{~s}, 2 \mathrm{H}) ;{ }^{13} \mathrm{C}$ NMR $\left(\mathrm{CDCl}_{3}, 150 \mathrm{MHz}\right): \delta 11.6\left(\mathrm{CH}_{3}\right), 26.6\left(\mathrm{CH}_{3}\right), 41.7(\mathrm{C}), 50.7\left(\mathrm{CH}_{2}\right), 56.0\left(\mathrm{CH}_{2}\right), 136.7$ (C), $179.4(\mathrm{C}), 208.7(\mathrm{CO})$; HRMS (ESI) m/z: $[\mathrm{M}+\mathrm{Na}]^{+}$calcd. for $\mathrm{C}_{9} \mathrm{H}_{14} \mathrm{O}_{2} \mathrm{Na}$ 177.0886, found 177.1882 .

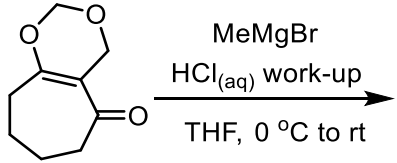

S13

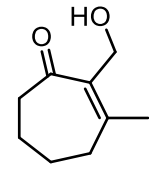

S14 
2-(Hydroxymethyl)-3-methylcyclohept-2-en-1-one (S14). Following the general procedure of condition A, 2-(hydroxymethyl) enone $\mathbf{S 1 4}$ (709 mg, 92\% yield) was prepared from 1,3-dioxin $\mathbf{S 1 3}(841 \mathrm{mg}, 5.0 \mathrm{mmol}$ ) and $\mathrm{MeMgBr}(2.5 \mathrm{~mL}, 3.0 \mathrm{M}$ in diethyl ether) as a colorless oil: IR $\left(\mathrm{CH}_{2} \mathrm{Cl}_{2}\right.$ cast, $\left.\mathrm{cm}^{-1}\right) v_{\max } 3424$ (br), 2939, 2867, 1712, 1655, 1458, 1424, 1375; ${ }^{1} \mathrm{H}$ NMR $\left(\mathrm{CDCl}_{3}, 400 \mathrm{MHz}\right): \delta 1.72-1.80(\mathrm{~m}, 4 \mathrm{H}), 2.06(\mathrm{~s}, 3 \mathrm{H}), 2.44(\mathrm{dd}, J=6.4,4.4$ $\mathrm{Hz}, 2 \mathrm{H}), 2.58(\mathrm{dd}, J=6.8,6.4 \mathrm{~Hz}, 2 \mathrm{H}), 2.81(\mathrm{br} \mathrm{s}, 1 \mathrm{H}), 4.31(\mathrm{~s}, 2 \mathrm{H}) ;{ }^{13} \mathrm{C} \mathrm{NMR}_{\left(\mathrm{CDCl}_{3}, 150\right.}$ MHz): $\delta 20.8\left(\mathrm{CH}_{2}\right), 23.0\left(\mathrm{CH}_{3}\right), 23.7\left(\mathrm{CH}_{2}\right), 34.7\left(\mathrm{CH}_{2}\right), 41.8\left(\mathrm{CH}_{2}\right), 59.8\left(\mathrm{CH}_{2}\right), 137.1(\mathrm{C})$, 155.1 (C), 207.5 (CO); HRMS (ESI) m/z: [M + Na $]^{+}$calcd. for $\mathrm{C}_{9} \mathrm{H}_{14} \mathrm{O}_{2} \mathrm{Na} 177.0886$, found 177.0882.

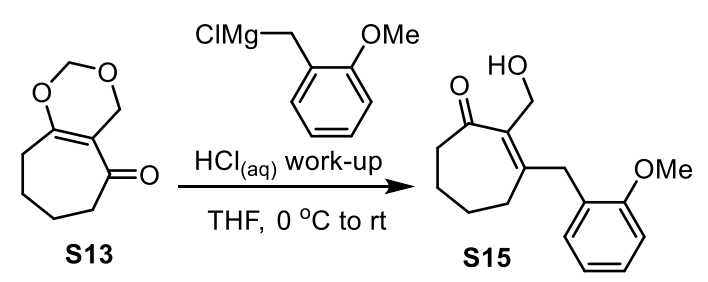

2-(Hydroxymethyl)-3-(2-methoxybenzyl)cyclohept-2-en-1-one (S15). Following the general procedure of condition A, 2-(hydroxymethyl) enone $\mathbf{S 1 5}$ (729 mg, 56\% yield) was prepared from 1,3-dioxin $\mathbf{S 1 3}$ (841 $\mathrm{mg}, 5.0 \mathrm{mmol}$ ) and 2-methoxybenzylmagnesium chloride (20 mL, $0.25 \mathrm{M}$ in THF) as a light yellow oil: IR $\left(\mathrm{CH}_{2} \mathrm{Cl}_{2}\right.$ cast, $\left.\mathrm{cm}^{-1}\right) v_{\max } 3447$ (br), 3060, 2937, 2864, 1654, 1599, 1556, 1492, 1458, 1439; ${ }^{1} \mathrm{H}$ NMR $\left(\mathrm{CDCl}_{3}, 400 \mathrm{MHz}\right): \delta 1.44-1.51$ (m, 2H), 1.68-1.75 (m, 2H), $2.34(\mathrm{t}, J=6.4 \mathrm{~Hz}, 2 \mathrm{H}), 2.55(\mathrm{t}, J=6.4 \mathrm{~Hz}, 2 \mathrm{H}), 2.81$ (br s, $1 \mathrm{H})$, $3.68(\mathrm{~s}, 2 \mathrm{H}), 3.83(\mathrm{~s}, 3 \mathrm{H}), 4.46(\mathrm{~s}, 2 \mathrm{H}), 6.87(\mathrm{dd}, J=8.2,0.8 \mathrm{~Hz}, 1 \mathrm{H}), 6.91(\mathrm{td}, J=7.4,1.2 \mathrm{~Hz}$, $1 \mathrm{H}), 7.09(\mathrm{dd}, J=7.4,1.6 \mathrm{~Hz}, 1 \mathrm{H}), 7.24(\mathrm{td}, J=8.2,1.6 \mathrm{~Hz}, 1 \mathrm{H}) ;{ }^{13} \mathrm{C} \mathrm{NMR}\left(\mathrm{CDCl}_{3}, 150\right.$ MHz): $\delta 20.7\left(\mathrm{CH}_{2}\right), 24.1\left(\mathrm{CH}_{2}\right), 31.7\left(\mathrm{CH}_{2}\right), 36.0\left(\mathrm{CH}_{2}\right), 41.7\left(\mathrm{CH}_{2}\right), 55.3\left(\mathrm{CH}_{3}\right), 59.7\left(\mathrm{CH}_{2}\right)$, $110.4(\mathrm{CH}), 120.7(\mathrm{CH}), 125.8(\mathrm{C}), 128.0(\mathrm{CH}), 130.3(\mathrm{CH}), 138.0(\mathrm{C}), 154.7(\mathrm{C}), 157.6(\mathrm{C})$, 208.4 (CO); HRMS (ESI) m/z: [M + Na $]^{+}$calcd. for $\mathrm{C}_{16} \mathrm{H}_{20} \mathrm{O}_{3} \mathrm{Na} 283.1305$, found 283.1308.

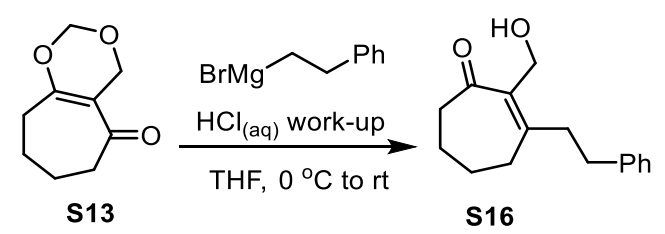

2-(Hydroxymethyl)-3-phenethylcyclohept-2-en-1-one (S16). Following the general procedure of condition A, 2-(hydroxymethyl) enone S16 (941 mg, 77\% yield) was prepared from 1,3-dioxin $\mathbf{S 1 3}$ (841 mg, $5.0 \mathrm{mmol}$ ) and (7.5 mL, 1.0 M in THF) as a colorless oil: IR $\left(\mathrm{CH}_{2} \mathrm{Cl}_{2}\right.$ cast, $\left.\mathrm{cm}^{-1}\right) v_{\max } 3435$ (br), 3084, 3061, 3026, 2937, 2865, 1654, 1496, 1454; ${ }^{1} \mathrm{H} \mathrm{NMR}$ $\left(\mathrm{CDCl}_{3}, 400 \mathrm{MHz}\right): \delta 1.70-1.81(\mathrm{~m}, 4 \mathrm{H}), 2.45(\mathrm{dd}, J=6.8,4.4 \mathrm{~Hz}, 2 \mathrm{H}), 2.52(\mathrm{t}, J=6.4 \mathrm{~Hz}$, $1 \mathrm{H}), 2.56(\mathrm{dd}, J=6.8,4.8 \mathrm{~Hz}, 2 \mathrm{H}), 2.63(\mathrm{dd}, J=8.2,7.2 \mathrm{~Hz}, 2 \mathrm{H}), 2.80(\mathrm{dd}, J=8.2,7.6 \mathrm{~Hz}$, $2 \mathrm{H}), 4.16(\mathrm{~d}, J=6.4 \mathrm{~Hz}, 2 \mathrm{H}), 7.17-7.24(\mathrm{~m}, 3 \mathrm{H}), 7.28-7.32(\mathrm{~m}, 2 \mathrm{H}) ;{ }^{13} \mathrm{C} \mathrm{NMR}\left(\mathrm{CDCl}_{3}, 100\right.$ 
MHz): $\delta 20.7\left(\mathrm{CH}_{2}\right), 24.2\left(\mathrm{CH}_{2}\right), 33.0\left(\mathrm{CH}_{2}\right), 34.6\left(\mathrm{CH}_{2}\right), 38.8\left(\mathrm{CH}_{2}\right), 41.7\left(\mathrm{CH}_{2}\right), 59.5\left(\mathrm{CH}_{2}\right)$, $126.3(\mathrm{CH}), 128.3(\mathrm{CH}), 128.5(\mathrm{CH}), 137.9(\mathrm{C}), 140.6(\mathrm{C}), 156.5(\mathrm{C}), 207.9(\mathrm{CO})$; HRMS (ESI) $\mathrm{m} / \mathrm{z}:[\mathrm{M}+\mathrm{Na}]^{+}$calcd. for $\mathrm{C}_{16} \mathrm{H}_{20} \mathrm{O}_{2} \mathrm{Na} 267.1356$, found 267.1356 .

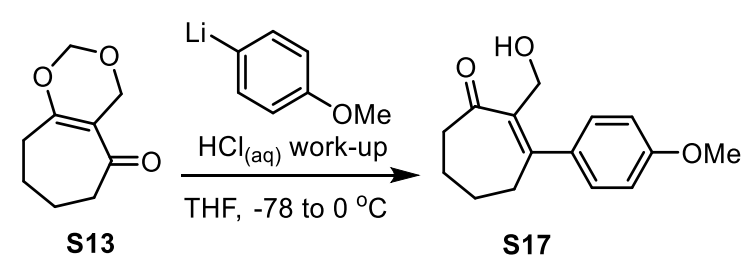

2-(Hydroxymethyl)-3-(4-methoxyphenyl)cyclohept-2-en-1-one (S17). Following the general procedure of condition B, 2-(hydroxymethyl) enone $\mathbf{S 1 7}$ (985 mg, 80\% yield) was prepared from 1,3-dioxin $\mathbf{S 1 3}$ (841 mg, $5.0 \mathrm{mmol}$ ) and 4-methoxyphenyllithium (7.5 mL, 1.0 $\mathrm{M}$ in THF) as a light yellow oil: IR $\left(\mathrm{CH}_{2} \mathrm{Cl}_{2}\right.$ cast, $\left.\mathrm{cm}^{-1}\right) v_{\max } 3446$ (br), 3062, 2936, 2864, 1654, 1606, 1570, 1509, 1458; ${ }^{1} \mathrm{H}$ NMR ( $\left.\mathrm{CDCl}_{3}, 400 \mathrm{MHz}\right): \delta 1.84-1.94(\mathrm{~m}, 4 \mathrm{H}), 2.68(\mathrm{t}, J=$ $6.0 \mathrm{~Hz}, 2 \mathrm{H}), 2.75(\mathrm{t}, J=6.0 \mathrm{~Hz}, 2 \mathrm{H}), 2.86(\mathrm{td}, J=6.4,4.0,1 \mathrm{H}), 3.83(\mathrm{~s}, 3 \mathrm{H}), 4.15(\mathrm{~d}, J=6.4$ $\mathrm{Hz}, 2 \mathrm{H}), 6.91(\mathrm{~d}, J=8.8 \mathrm{~Hz}, 2 \mathrm{H}), 7.22(\mathrm{~d}, J=8.8 \mathrm{~Hz}, 2 \mathrm{H}) ;{ }^{13} \mathrm{C} \mathrm{NMR}\left(\mathrm{CDCl}_{3}, 100 \mathrm{MHz}\right): \delta$ $20.8\left(\mathrm{CH}_{2}\right), 24.2\left(\mathrm{CH}_{2}\right), 34.8\left(\mathrm{CH}_{2}\right), 41.8\left(\mathrm{CH}_{2}\right), 55.2\left(\mathrm{CH}_{3}\right), 61.3\left(\mathrm{CH}_{2}\right), 113.6(\mathrm{CH}), 128.9$ (CH), 134.2 (C), $137.3(\mathrm{C}), 156.0(\mathrm{C}), 159.5$ (C), 208.4 (CO); HRMS (ESI) m/z: [M + Na $]^{+}$ calcd. for $\mathrm{C}_{15} \mathrm{H}_{18} \mathrm{O}_{3} \mathrm{Na} 269.1148$, found 269.1146 .
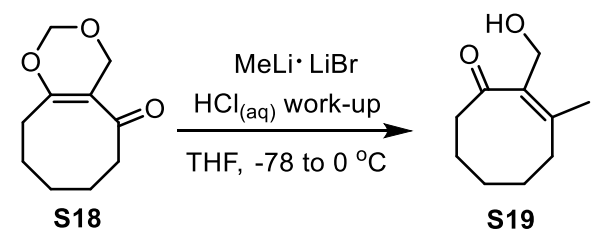

(Z)-2-(Hydroxymethyl)-3-methylcyclooct-2-en-1-one (S19). Following the general procedure of condition B, 2-(hydroxymethyl) enone $\mathbf{S 1 9}$ (740 mg, 88\% yield) was prepared from 1,3-dioxin $\mathbf{S 1 8}(911 \mathrm{mg}, 5.0 \mathrm{mmol})$ and $\mathrm{MeLi} \cdot \mathrm{LiBr}(7.5 \mathrm{~mL}, 1.0 \mathrm{M}$ in diethyl ether) as a colorless oil: IR $\left(\mathrm{CH}_{2} \mathrm{Cl}_{2}\right.$ cast, $\left.\mathrm{cm}^{-1}\right) v_{\max } 3429$ (br), 2930, 2870, 1677, 1454, 1420, 1407; ${ }^{1} \mathrm{H}$ NMR (CDCl $3,600 \mathrm{MHz}): \delta 1.57-1.61(\mathrm{~m}, 2 \mathrm{H}), 1.63-1.67$ (m, 2H), 1.80-1.84 (m, 2H), 1.92 (s, $3 \mathrm{H}), 2.38(\mathrm{t}, J=6.0 \mathrm{~Hz}, 2 \mathrm{H}), 2.41(\mathrm{t}, J=4.2 \mathrm{~Hz}, 1 \mathrm{H}), 2.65(\mathrm{t}, J=6.6 \mathrm{~Hz}, 2 \mathrm{H}), 4.29$ (d, $J=4.2$ $\mathrm{Hz}, 2 \mathrm{H}) ;{ }^{13} \mathrm{C} \mathrm{NMR}\left(\mathrm{CDCl}_{3}, 150 \mathrm{MHz}\right): \delta 21.2\left(\mathrm{CH}_{3}\right), 22.5\left(\mathrm{CH}_{2}\right), 23.4\left(\mathrm{CH}_{2}\right), 26.8\left(\mathrm{CH}_{2}\right)$, $35.9\left(\mathrm{CH}_{2}\right), 45.1\left(\mathrm{CH}_{2}\right), 60.9\left(\mathrm{CH}_{2}\right), 135.3(\mathrm{C}), 144.9(\mathrm{C}), 211.5(\mathrm{CO})$; HRMS (ESI) m/z: [M $+\mathrm{Na}]^{+}$calcd. for $\mathrm{C}_{10} \mathrm{H}_{16} \mathrm{O}_{2} \mathrm{Na}$ 191.1043, found 191.1037. 
The following known precursors were prepared by reported procedures: compounds $\mathbf{4},{ }^{[1]} \mathbf{5}^{[2]}$ S7, ${ }^{[3]} \mathbf{S 1 3},{ }^{[1]} \mathbf{S 2 0},{ }^{[4]} \mathbf{S 2 6},{ }^{[5]} \mathbf{S 2 8},{ }^{[6]} \mathbf{S 3 6},{ }^{[7]} \mathbf{S 3 7}{ }^{[8]}$ and $\mathbf{S 3 8},{ }^{[9]}$ The remaining precursors $\mathbf{7}, \mathbf{S 1 8}$, S22, S25, S27, S29, S31, S33 and S35 were prepared by the following procedures.

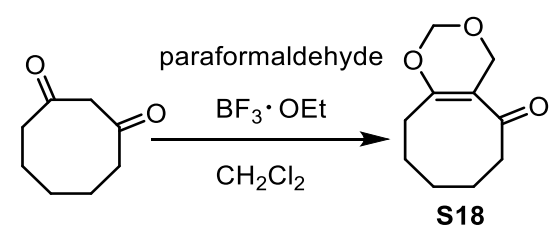

4,6,7,8,9,10-Hexahydro-5H-cycloocta[ $d][1,3]$ dioxin-5-one (S18). ${ }^{[1]}$ To a stirred solution of cycloctane-1,3-dione (2.80 g, $20.0 \mathrm{mmol})$ and paraformaldehyde $(3.03 \mathrm{~g}, 100.0 \mathrm{mmol})$ in dry $\mathrm{CH}_{2} \mathrm{Cl}_{2}(200 \mathrm{~mL})$ at room temperature was added $\mathrm{BF}_{3} \cdot \mathrm{OEt}_{2}(6.28 \mathrm{~mL}, 50.0 \mathrm{mmol})$ under $\mathrm{N}_{2}$. The mixture was then allowed to react at room temperature for $3 \mathrm{~h}$ under $\mathrm{N}_{2}$. After the reaction was complete, sat. $\mathrm{NaHCO}_{3(\mathrm{aq})}(200 \mathrm{~mL})$ was added to quench the reaction at $0{ }^{\circ} \mathrm{C}$, and the resulting mixture was filtrated with celite. The organic portions were washed with sat. $\mathrm{NaHCO}_{3(\text { aq) }}$ and brine, dried over $\mathrm{MgSO}_{4}$, filtered and concentrated to give the crude residue, which was purified by chromatography on silical gel to afford the 1,3-dioxin S18 (2.48 g, $68 \%$ yield $)$ as a colorless oil: IR $\left(\mathrm{CH}_{2} \mathrm{Cl}_{2}\right.$ cast, $\left.\mathrm{cm}^{-1}\right) v_{\max } 2929,2859,2795,1613,1479,1411$, 1385; ${ }^{1} \mathrm{H}$ NMR $\left(\mathrm{CDCl}_{3}, 400 \mathrm{MHz}\right): \delta 1.57-1.63(\mathrm{~m}, 2 \mathrm{H}), 7.72$ (quint, $J=6.8 \mathrm{~Hz}, 2 \mathrm{H}$ ), 1.74 (quint, $J=7.2 \mathrm{~Hz}, 2 \mathrm{H}), 2.79(\mathrm{t}, J=7.2 \mathrm{~Hz}, 2 \mathrm{H}), 2.81(\mathrm{t}, J=6.8 \mathrm{~Hz}, 2 \mathrm{H}), 4.48(\mathrm{~s}, 2 \mathrm{H}), 5.07(\mathrm{~s}$, $2 \mathrm{H}) ;{ }^{13} \mathrm{C} \mathrm{NMR}\left(\mathrm{CDCl}_{3}, 150 \mathrm{MHz}\right): \delta 23.1\left(\mathrm{CH}_{2}\right), 23.2\left(\mathrm{CH}_{2}\right), 23.5\left(\mathrm{CH}_{2}\right), 32.9\left(\mathrm{CH}_{2}\right), 41.9$ $\left(\mathrm{CH}_{2}\right), 64.4\left(\mathrm{CH}_{2}\right), 91.0\left(\mathrm{CH}_{2}\right), 116.6(\mathrm{C}), 166.2(\mathrm{C}), 198.7(\mathrm{CO})$; HRMS (ESI) m/z: [M + $\mathrm{Na}]^{+}$calcd. for $\mathrm{C}_{10} \mathrm{H}_{14} \mathrm{O}_{3} \mathrm{Na} 205.0835$, found 205.0834.

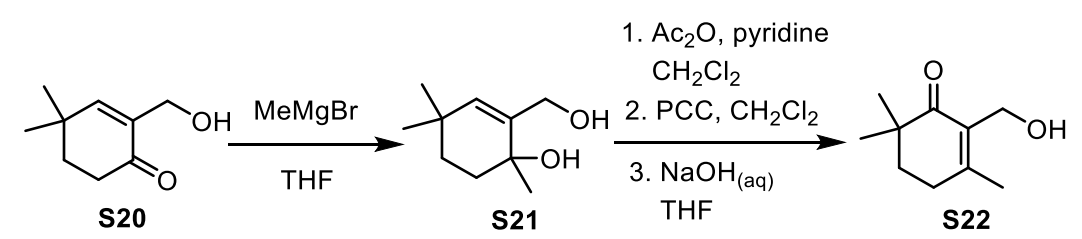

2-(Hydroxymethyl)-1,4,4-trimethylcyclohex-2-en-1-ol (S21). ${ }^{[10]}$ To a stirred solution of 2-(hydroxymethyl) enone $\mathbf{S 2 0}(1.54 \mathrm{~g}, 10.0 \mathrm{mmol})$ in dry THF $(50 \mathrm{~mL})$ at $0{ }^{\circ} \mathrm{C}$ was added $\mathrm{MeMgBr}$ solution ( $8 \mathrm{~mL}, 3.0 \mathrm{M}$ in $\mathrm{Et}_{2} \mathrm{O}$, $\mathrm{xx} \mathrm{mmol}$ ) dropwise under $\mathrm{N}_{2}$. The mixture was then allowed to react at $0{ }^{\circ} \mathrm{C}$ to room temperature for $1 \mathrm{~h}$ under $\mathrm{N}_{2}$. After the reaction was complete, $5 \% \mathrm{HCl}_{(\mathrm{aq})}(20 \mathrm{~mL})$ was added to quench the reaction at $0{ }^{\circ} \mathrm{C}$, and the resulting mixture was extracted with EtOAc $(50 \mathrm{~mL} \times 2)$. The organic portions were combined, washed with sat. $\mathrm{NaHCO}_{3(\mathrm{aq})}$ and brine, dried over $\mathrm{MgSO}_{4}$, filtered and concentrated to give the crude residue, which was recrystallized from EtOAc/ $n$-hexane to afford diol $\mathbf{S 2 1}(1.41 \mathrm{~g}, 83 \%$ yield) as a white solid: $\mathrm{mp}=124-126^{\circ} \mathrm{C}$; IR $\left(\mathrm{KBr}, \mathrm{cm}^{-1}\right) v_{\max } 3254$ (br), 2960, 2933, 2920, 2852, 1458, 1359; ${ }^{1} \mathrm{H}$ NMR $\left(\mathrm{CDCl}_{3}, 400 \mathrm{MHz}\right): \delta 0.95$ (s, 3H), 1.05 (s, 3H), $1.40(\mathrm{~s}, 3 \mathrm{H}), 1.41-1.48(\mathrm{~m}$, 1H), 1.58-1.65 (m, 1H), 1.71-1.83 (m, 2H), 2.41 (br s, 2H), 3.99 (d, $J=12.0 \mathrm{~Hz}, 1 \mathrm{H}), 4.46$ (d, 
$J=12.0 \mathrm{~Hz}, 1 \mathrm{H}), 5.43(\mathrm{~s}, 1 \mathrm{H}) ;{ }^{13} \mathrm{C} \mathrm{NMR}\left(\mathrm{CDCl}_{3}, 150 \mathrm{MHz}\right): \delta 27.5\left(\mathrm{CH}_{3}\right), 28.5\left(\mathrm{CH}_{3}\right), 29.5$ $\left(\mathrm{CH}_{3}\right), 32.2(\mathrm{C}), 33.8\left(\mathrm{CH}_{2}\right), 36.4\left(\mathrm{CH}_{2}\right), 65.5\left(\mathrm{CH}_{2}\right), 70.9(\mathrm{C}), 137.6(\mathrm{C}), 138.1(\mathrm{CH})$; HRMS (ESI) $\mathrm{m} / \mathrm{z}:[\mathrm{M}+\mathrm{Na}]^{+}$calcd. for $\mathrm{C}_{10} \mathrm{H}_{18} \mathrm{O}_{2} \mathrm{Na}$ 193.1199, found 193.1196.

2-(Hydroxymethyl)-3,6,6-trimethylcyclohex-2-en-1-one (S22). ${ }^{[10]}$ To a stirred solution of the diol $\mathbf{S 2 1}(851 \mathrm{mg}, 5.0 \mathrm{mmol})$ and pyridine $(4 \mathrm{~mL})$ in dry $\mathrm{CH}_{2} \mathrm{Cl}_{2}(6 \mathrm{~mL})$ was added $\mathrm{Ac}_{2} \mathrm{O}$ $\left(613 \mathrm{mg}, 6.0 \mathrm{mmol}\right.$ ) in one portion at $0{ }^{\circ} \mathrm{C}$. The resulting mixture was then stirred at $40{ }^{\circ} \mathrm{C}$ under $\mathrm{N}_{2}$ for $3 \mathrm{~h}$. After reaction was complete, the reaction mixture was quenched with $5 \%$ $\mathrm{HCl}_{(\text {aq) }}(20 \mathrm{~mL})$ and extracted with $\mathrm{CH}_{2} \mathrm{Cl}_{2}(20 \mathrm{~mL} \times 2)$. The combined organic extract was washed with saturated $\mathrm{NaHCO}_{3(\mathrm{aq})}$ and brine, dried over $\mathrm{MgSO}_{4}$, filtered and concentrated to give the crude acetyl intermediate. To a stirred solution of the acetyl intermediate and celite (3 g) in dry $\mathrm{CH}_{2} \mathrm{Cl}_{2}(30 \mathrm{~mL})$ was added PCC $(1.62 \mathrm{~g}, 7.5 \mathrm{mmol})$ in one portion at $0{ }^{\circ} \mathrm{C}$. The resulting mixture was then stirred at room temperature under $\mathrm{N}_{2}$ for $6 \mathrm{~h}$. After reaction was complete, the reaction mixture was filtered and concentrated to give the crude enone acetate intermediate. To a stirred solution of the crude enone acetate intermediate in THF $(20 \mathrm{~mL})$ was added $4 \mathrm{~N} \mathrm{NaOH}_{(\mathrm{aq})}(5 \mathrm{~mL}, 20.0 \mathrm{mmol})$ in one portion at $0{ }^{\circ} \mathrm{C}$. The resulting mixture was then stirred at room temperature under $\mathrm{N}_{2}$ for $10 \mathrm{~h}$. After reaction was complete, the reaction mixture was extracted with EtOAc $(20 \mathrm{~mL} \times 3)$. The organic portions were combined, washed with water and brine, dried over $\mathrm{MgSO}_{4}$, filtered and concentrated to give the crude residue, which was purified by chromatography on silical gel to afford the 2-(hydroxymethyl) enone S22 (639 mg, 76\% yield over 3 steps) as a colorless oil: IR $\left(\mathrm{CH}_{2} \mathrm{Cl}_{2}\right.$ cast, $\left.\mathrm{cm}^{-1}\right) v_{\max } 3448$ (br), 2963, 2922, 2869, 1660, 1636, 1472, 1452, 1425, 1385; ${ }^{1} \mathrm{H}$ NMR $\left(\mathrm{CDCl}_{3}, 400 \mathrm{MHz}\right): \delta 1.11$ (s, 6H), $1.80(\mathrm{t}, J=6.2 \mathrm{~Hz}, 2 \mathrm{H}), 2.00(\mathrm{~s}, 3 \mathrm{H}), 2.39(\mathrm{t}, J=6.2 \mathrm{~Hz}, 2 \mathrm{H}), 2.84(\mathrm{br} \mathrm{s}, 1 \mathrm{H}), 4.34$ (s, $2 \mathrm{H}) ;{ }^{13} \mathrm{C} \mathrm{NMR}\left(\mathrm{CDCl}_{3}, 100 \mathrm{MHz}\right): \delta 20.6\left(\mathrm{CH}_{3}\right), 24.2\left(\mathrm{CH}_{3}\right), 29.6\left(\mathrm{CH}_{2}\right), 35.3\left(\mathrm{CH}_{2}\right), 40.1$ (C), $57.5\left(\mathrm{CH}_{2}\right), 132.2(\mathrm{C}), 156.6(\mathrm{C}), 205.4(\mathrm{CO})$; HRMS (ESI) m/z: [M + Na $]^{+}$calcd. for $\mathrm{C}_{10} \mathrm{H}_{16} \mathrm{O}_{2} \mathrm{Na}$ 191.1043, found 191.1025.

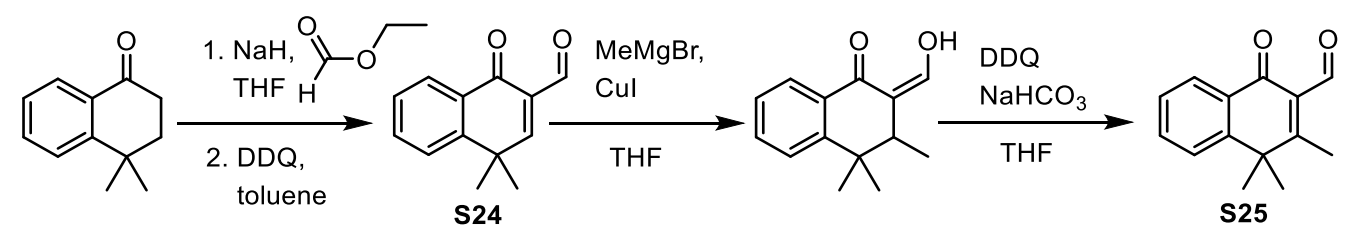

4,4-Dimethyl-1-oxo-1,4-dihydronaphthalene-2-carbaldehyde (S24). To a stirred solution of 4,4-dimethyl-3,4-dihydronaphthalen-1(2H)-one (3.48 g, $20.0 \mathrm{mmol})$ and ethyl formate $(7.41 \mathrm{~g}, 100.0 \mathrm{mmol})$ in dry THF $(60 \mathrm{~mL})$ at room temperature was added $60 \% \mathrm{NaH}(1.20 \mathrm{~g}$, $30.0 \mathrm{mmol}$ ) portionwise under $\mathrm{N}_{2}$. The mixture was then allowed to react at reflux for $6 \mathrm{~h}$ under $\mathrm{N}_{2}$. After the reaction was complete, $5 \% \mathrm{HCl}_{(\mathrm{aq})}(40 \mathrm{~mL})$ was added to quench the reaction at $0{ }^{\circ} \mathrm{C}$, and the resulting mixture was extracted with EtOAc $(60 \mathrm{~mL} \times 2)$. The organic portions were combined, washed with sat. $\mathrm{NaHCO}_{3(\mathrm{aq})}$ and brine, dried over $\mathrm{MgSO}_{4}$, filtered and concentrated to give the 2-formylketone intermediate. To a stirred solution of the 
2-formylketone intermediate in dry toluene $(60 \mathrm{~mL})$ was added DDQ $(4.99 \mathrm{~g}, 22.0 \mathrm{mmol})$ in one portion. The resulting mixture was then stirred at $60{ }^{\circ} \mathrm{C}$ under $\mathrm{N}_{2}$ for $2 \mathrm{~h}$. After reaction was complete, the reaction mixture was filtrated with celite, and quenched with saturated $\mathrm{NaHCO}_{3(\text { aq })}(60 \mathrm{~mL})$ and extracted with EtOAc $(50 \mathrm{~mL} \times 3)$. The combined organic extract was washed with saturated $\mathrm{NaHCO}_{3(\mathrm{aq})}$ and brine, dried over $\mathrm{MgSO}_{4}$, filtered and concentrated to give the crude residue, which was purified by chromatography on silical gel to afford 2-formylenone $\mathbf{S 2 4}$ (2.92 g, 73\% yield over 2 steps) as a white solid: $\mathrm{mp}=102$ $105{ }^{\circ} \mathrm{C}$; IR $\left(\mathrm{KBr}, \mathrm{cm}^{-1}\right) v_{\max } 3198,2973,2919,2874,2850,1706,1663,1620,1600 ;{ }^{1} \mathrm{H}$ NMR $\left(\mathrm{CDCl}_{3}, 400 \mathrm{MHz}\right): \delta 1.58(\mathrm{~s}, 6 \mathrm{H}), 7.46(\mathrm{td}, J=7.2,1.2 \mathrm{~Hz}, 1 \mathrm{H}), 7.57(\mathrm{dd}, J=8.0,1.2 \mathrm{~Hz}$, $1 \mathrm{H}), 7.65$ (td, $J=7.2,1.2 \mathrm{~Hz}, 1 \mathrm{H}), 7.72(\mathrm{~s}, 1 \mathrm{H}), 8.26$ (dd, $J=8.0,1.2 \mathrm{~Hz}, 1 \mathrm{H}), 10.40(\mathrm{~s}, 1 \mathrm{H})$; ${ }^{13} \mathrm{C} \mathrm{NMR}\left(\mathrm{CDCl}_{3}, 100 \mathrm{MHz}\right): \delta 29.1\left(\mathrm{CH}_{3}\right), 37.7(\mathrm{C}), 126.2(\mathrm{CH}), 126.9(\mathrm{CH}), 127.2(\mathrm{CH})$, $130.1(\mathrm{C}), 130.6(\mathrm{C}), 133.5(\mathrm{CH}), 162.1(\mathrm{CH}), 183.5$ (C), 190.1 (CHO); HRMS (ESI) m/z: [M $+\mathrm{Na}]^{+}$calcd. for $\mathrm{C}_{13} \mathrm{H}_{12} \mathrm{O}_{2} \mathrm{Na}$ 223.0730, found 223.0735.

3,4,4-Trimethyl-1-oxo-1,4-dihydronaphthalene-2-carbaldehyde (S25). To a stirred solution of $\mathrm{CuI}(2.29 \mathrm{~g}, 12.0 \mathrm{mmol})$ in dry THF $(20 \mathrm{~mL})$ at $0{ }^{\circ} \mathrm{C}$ was added $\mathrm{MeMgBr}$ solution (4 $\mathrm{mL}, 3.0 \mathrm{M}$ in $\mathrm{Et}_{2} \mathrm{O}, 12.0 \mathrm{mmol}$ ) dropwise under $\mathrm{N}_{2}$. The resulting mixture was then allowed to react at $0{ }^{\circ} \mathrm{C}$ for $30 \mathrm{~min}$ under $\mathrm{N}_{2}$. To this stirred mixture at $0{ }^{\circ} \mathrm{C}$ was then added the 2-formylenone $\mathbf{S 2 4}$ solution (2.00 $\mathrm{g}$ in $10 \mathrm{~mL}$ dry THF, $10.0 \mathrm{mmol}$ ) dropwise under $\mathrm{N}_{2}$ for $30 \mathrm{~min}$. After the reaction was complete, sat. $\mathrm{NH}_{4} \mathrm{Cl}_{(\mathrm{aq})}(20 \mathrm{~mL})$ was added to quench the reaction at $0{ }^{\circ} \mathrm{C}$, and the resulting mixture was diluted with EtOAc $(100 \mathrm{~mL})$ and filtrated with celite. The organic layer was separated, and the aqueous layer was extracted with EtOAc $(25 \mathrm{~mL} \times 2)$. The organic portions were combined, washed with water and brine, dried over $\mathrm{MgSO}_{4}$, filtered and concentrated to give the crude residue, which was purified by chromatography on silical gel to afford 3-methyl-2-ketone intermediate. To a stirred solution of the 3-methyl-2-ketone intermediate and $\mathrm{NaHCO}_{3}(840 \mathrm{mg}, 10.0 \mathrm{mmol})$ in dry THF (50 $\mathrm{mL})$, which was previously degassed with argon, was added DDQ $(2.27 \mathrm{~g}, 10.0 \mathrm{mmol})$ in one portion at $0{ }^{\circ} \mathrm{C}$. The resulting mixture was then stirred at room temperature under argon for 6 h. After reaction was complete, the reaction mixture was quenched with saturated $\mathrm{NaHCO}_{3(\mathrm{aq})}$ $(50 \mathrm{~mL})$ and extracted with EtOAc $(50 \mathrm{~mL} \times 3)$. The combined organic extract was washed with saturated $\mathrm{NaHCO}_{3(\text { aq) }}(50 \mathrm{~mL} \times 3)$ and brine, dried over $\mathrm{MgSO}_{4}$, filtered and concentrated to give the crude residue, which was purified by chromatography on neutralized silica gel to afford 3-methyl-2-formylenone S25 (1.09 g, 51\% yield over 2 steps) as a white solid: $\mathrm{mp}=131-133{ }^{\circ} \mathrm{C}$; IR $\left(\mathrm{KBr}, \mathrm{cm}^{-1}\right) v_{\max } 3054,2989,2936,2870,1699,1648,1602,1571$, 1472, 1454; ${ }^{1} \mathrm{H}$ NMR $\left(\mathrm{CDCl}_{3}, 400 \mathrm{MHz}\right): \delta 1.60$ (s, 6H), 2.50 (s, 3H), 7.41-7.45 (m, 1H), 7.59-7.66 (m, 2H), 8.22-8.24 (m, 1H), $10.61(\mathrm{~s}, 1 \mathrm{H}) ;{ }^{13} \mathrm{C} \mathrm{NMR}\left(\mathrm{CDCl}_{3}, 75 \mathrm{MHz}\right): \delta 17.7$ $\left(\mathrm{CH}_{3}\right), 28.4(\mathrm{CH}), 41.5(\mathrm{C}), 126.4(\mathrm{CH}), 127.0(\mathrm{CH}), 129.2(\mathrm{C}), 129.3(\mathrm{C}), 133.3(\mathrm{CH}), 149.8$ (C), 173.8 (C), 184.0 (C), 194.5 (CHO); HRMS (ESI) m/z: [M + Na] $]^{+}$calcd. for $\mathrm{C}_{14} \mathrm{H}_{14} \mathrm{O}_{2} \mathrm{Na}$ 237.0886, found 237.0881 . 


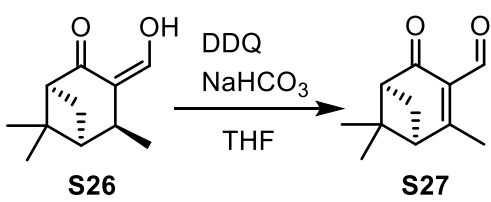

(1R,5S)-2,6,6-Trimethyl-4-oxobicyclo[3.1.1]hept-2-ene-3-carbaldehyde (S27). To a stirred solution of the 3-methyl-2-formylketone $\mathbf{S 2 6}$ (1.80 g, $10.0 \mathrm{mmol}$ ) and $\mathrm{NaHCO}_{3}(840 \mathrm{mg}, 10.0$ mmol) in dry THF $(50 \mathrm{~mL})$, which was previously degassed with argon, was added DDQ $(2.27 \mathrm{~g}, 10.0 \mathrm{mmol})$ in one portion at $0{ }^{\circ} \mathrm{C}$. The resulting mixture was then stirred at room temperature under argon for $10 \mathrm{~min}$. After reaction was complete, the reaction mixture was quenched with saturated $\mathrm{NaHCO}_{3(\mathrm{aq})}(50 \mathrm{~mL})$ and extracted with EtOAc $(50 \mathrm{~mL} \times 3)$. The combined organic extract was washed with saturated $\mathrm{NaHCO}_{3(\mathrm{aq})}(50 \mathrm{~mL} \times 3)$ and brine, dried over $\mathrm{MgSO}_{4}$, filtered and concentrated to give the crude residue, which was purified by chromatography on neutralized silica gel to afford 3-methyl-2-formylenone S27 (1.34 g, 75\% yield) as a light yellow oil: IR $\left(\mathrm{CH}_{2} \mathrm{Cl}_{2}\right.$ cast, $\left.\mathrm{cm}^{-1}\right) v_{\max } 2957,2871,1683,1648,1587 ;{ }^{1} \mathrm{H}$ NMR (CDCl $3,400 \mathrm{MHz}): \delta 1.01(\mathrm{~s}, 3 \mathrm{H}), 1.53(\mathrm{~s}, 3 \mathrm{H}), 2.10(\mathrm{~d}, J=9.6 \mathrm{~Hz}, 1 \mathrm{H}), 2.51(\mathrm{~s}, 3 \mathrm{H})$, $2.65(\mathrm{dt}, J=6.0,5.6 \mathrm{~Hz}, 1 \mathrm{H}), 2.79(\mathrm{t}, J=6.0 \mathrm{~Hz}, 1 \mathrm{H}), 2.84(\mathrm{dt}, J=9.6,5.6 \mathrm{~Hz}, 1 \mathrm{H}), 10.25(\mathrm{~s}$, $1 \mathrm{H}) ;{ }^{13} \mathrm{C} \mathrm{NMR}\left(\mathrm{CDCl}_{3}, 75 \mathrm{MHz}\right): \delta 21.9\left(\mathrm{CH}_{3}\right), 22.2\left(\mathrm{CH}_{3}\right), 26.4\left(\mathrm{CH}_{3}\right), 38.5\left(\mathrm{CH}_{2}\right), 52.7(\mathrm{C})$, $53.4(\mathrm{CH}), 57.3(\mathrm{CH}), 126.4(\mathrm{C}), 181.7(\mathrm{C}), 191.2(\mathrm{C}), 201.5(\mathrm{CHO}) ;$ HRMS (ESI) m/z: [M + $\mathrm{Na}]^{+}$calcd. for $\mathrm{C}_{11} \mathrm{H}_{14} \mathrm{O}_{2} \mathrm{Na}$ 201.0886, found 201.0886.

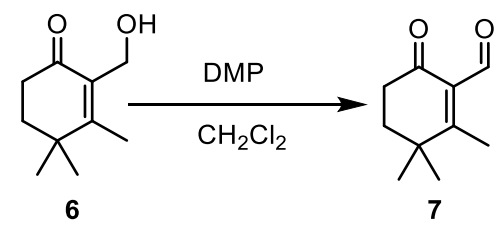

2,3,3-Trimethyl-6-oxocyclohex-1-ene-1-carbaldehyde (7). To a stirred solution of the 2-(hydroxymethyl) enone $6(168 \mathrm{mg}, 1.0 \mathrm{mmol})$ in dry $\mathrm{CH}_{2} \mathrm{Cl}_{2}(10 \mathrm{~mL})$ was added DessMartin periodinane $(551 \mathrm{mg}, 1.3 \mathrm{mmol})$ in one portion at $0{ }^{\circ} \mathrm{C}$. The resulting mixture was then stirred at room temperature under $\mathrm{N}_{2}$ for $30 \mathrm{~min}$. After reaction was complete, the reaction mixture was quenched with $5 \% \mathrm{Na}_{2} \mathrm{~S}_{2} \mathrm{O}_{3 \text { (aq) }}(20 \mathrm{~mL})$. The resulting solution was then stirred at room temperature for $20 \mathrm{~min}$. The aquous layer was extracted with $\mathrm{CH}_{2} \mathrm{Cl}_{2}(10 \mathrm{~mL} \times 2)$. The organic portions were combined, washed with sat. $\mathrm{NaHCO}_{3(\text { aq) }}$ and brine, dried over $\mathrm{MgSO}_{4}$, filtered and concentrated to give the 3-methyl-2-formylenone 7 (158 $\mathrm{mg}, 95 \%$ yield) as a light yellow solid: $\mathrm{mp}=63-65^{\circ} \mathrm{C}$; IR $\left(\mathrm{KBr}, \mathrm{cm}^{-1}\right) v_{\max } 2959,2932,2863,2774,1694,1665,1567$; ${ }^{1} \mathrm{H}$ NMR $\left(\mathrm{CDCl}_{3}, 400 \mathrm{MHz}\right): \delta 1.25(\mathrm{~s}, 6 \mathrm{H}), 1.89(\mathrm{t}, J=6.8 \mathrm{~Hz}, 2 \mathrm{H}), 2.28(\mathrm{~s}, 3 \mathrm{H}), 2.54(\mathrm{t}, J=$ $6.8 \mathrm{~Hz}, 2 \mathrm{H}), 10.25(\mathrm{~s}, 1 \mathrm{H}) ;{ }^{13} \mathrm{C} \mathrm{NMR}\left(\mathrm{CDCl}_{3}, 100 \mathrm{MHz}\right): \delta 17.1\left(\mathrm{CH}_{3}\right), 26.2\left(\mathrm{CH}_{3}\right), 34.3$ $\left(\mathrm{CH}_{2}\right), 36.0\left(\mathrm{CH}_{2}\right), 37.7(\mathrm{C}), 130.3(\mathrm{C}), 178.4(\mathrm{C}), 193.9$ (CHO), 198.2 (CO); HRMS (ESI) $\mathrm{m} / \mathrm{z}:[\mathrm{M}+\mathrm{Na}]^{+}$calcd. for $\mathrm{C}_{10} \mathrm{H}_{14} \mathrm{O}_{2} \mathrm{Na}$ 189.0886, found 189.0887 . 


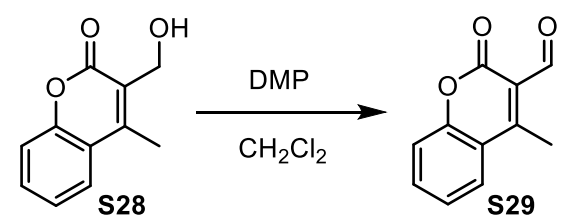

4-Methyl-2-oxo-2H-chromene-3-carbaldehyde (S29). To a stirred solution of the 2-(hydroxymethyl) enone $\mathbf{S 2 8}$ (190 mg, $1.0 \mathrm{mmol})$ in dry $\mathrm{CH}_{2} \mathrm{Cl}_{2}(10 \mathrm{~mL})$ was added DessMartin periodinane $(551 \mathrm{mg}, 1.3 \mathrm{mmol})$ in one portion at $0{ }^{\circ} \mathrm{C}$. The resulting mixture was then stirred at room temperature under $\mathrm{N}_{2}$ for $15 \mathrm{~h}$. After reaction was complete, the reaction mixture was quenched with $5 \% \mathrm{Na}_{2} \mathrm{~S}_{2} \mathrm{O}_{3(\mathrm{aq})}(50 \mathrm{~mL})$. The resulting solution was then stirred at room temperature for $20 \mathrm{~min}$. The aquous layer was extracted with $\mathrm{CH}_{2} \mathrm{Cl}_{2}(10 \mathrm{~mL} \times 2)$. The organic portions were combined, washed with sat. $\mathrm{NaHCO}_{3(\text { aq) }}$ and brine, dried over $\mathrm{MgSO}_{4}$, filtered and concentrated to give the 3-methyl-2-formylenone $\mathbf{S 2 9}$ (175 mg, 93\% yield) as a light yellow solid: $\mathrm{mp}=136-139{ }^{\circ} \mathrm{C}$; IR $\left(\mathrm{CH}_{2} \mathrm{Cl}_{2}\right.$ cast, $\left.\mathrm{cm}^{-1}\right) v_{\max } 3113,3078,3056,2918$, 2873, 2850, 1719, 1695, 1608, 1596, 1555, 1452; ${ }^{1} \mathrm{H} \mathrm{NMR}\left(\mathrm{CDCl}_{3}, 400 \mathrm{MHz}\right): \delta 2.88$ (s, 3H), 7.37-7.41 (m, 2H), 7.64-7.69 (m, 1H), 7.88-7.91 (m, 1H), $10.47(\mathrm{~s}, 1 \mathrm{H}) ;{ }^{13} \mathrm{C} \mathrm{NMR}\left(\mathrm{CDCl}_{3}\right.$, $150 \mathrm{MHz}): \delta 14.7\left(\mathrm{CH}_{3}\right), 117.4(\mathrm{CH}), 119.0(\mathrm{C}), 120.2(\mathrm{C}), 125.0(\mathrm{CH}), 126.9(\mathrm{CH}), 134.3$ (CH), 153.9 (C), 158.7 (C), 160.9 (C), 191.5 (CHO); HRMS (ESI) m/z: [M + Na] $]^{+}$calcd. for $\mathrm{C}_{11} \mathrm{H}_{8} \mathrm{O}_{3} \mathrm{Na} 211.0366$, found 211.0371.

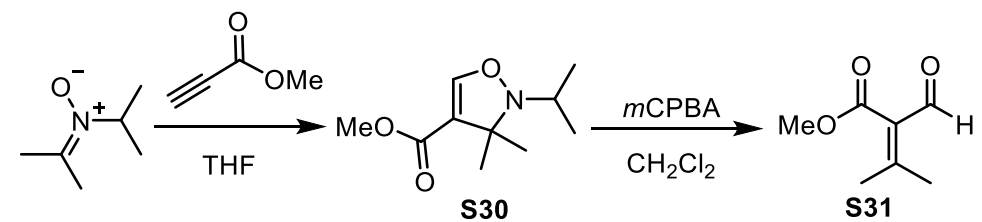

Methyl 2-isopropyl-3,3-dimethyl-2,3-dihydroisoxazole-4-carboxylate (S30). ${ }^{[1]}$ To a stirred solution of the $N$-(propan-2-yl)propanimine oxide $(1.15 \mathrm{~g}, 10.0 \mathrm{mmol})$ in dry THF $(30 \mathrm{~mL})$ was added methyl propilote $(1.68 \mathrm{~g}, 20.0 \mathrm{mmol})$ in one portion. The resulting mixture was then stirred at $40{ }^{\circ} \mathrm{C}$ under $\mathrm{N}_{2}$ for $3 \mathrm{~h}$. After reaction was complete, the reaction mixture was concentrated to give the crude residue, which was purified by chromatography on silical gel to afford isoxazoline $\mathbf{S 3 0}$ (1.65 g, 83\% yield) as a colorless oil: IR $\left(\mathrm{CH}_{2} \mathrm{Cl}_{2}\right.$ cast, $\left.\mathrm{cm}^{-1}\right) v_{\max }$ 3093, 2978, 2951, 2876, 1711, 1626, 1458, 1438; ${ }^{1} \mathrm{H}$ NMR $\left(\mathrm{CDCl}_{3}, 400 \mathrm{MHz}\right): \delta 1.19$ (d, $J=$ $6.4 \mathrm{~Hz}, 6 \mathrm{H}), 1.49$ (s, 6H), 3.38 (septet, $J=6.4 \mathrm{~Hz}, 1 \mathrm{H}), 3.70(\mathrm{~s}, 3 \mathrm{H}), 7.26(\mathrm{~s}, 1 \mathrm{H}) ;{ }^{13} \mathrm{C}$ NMR $\left(\mathrm{CDCl}_{3}, 100 \mathrm{MHz}\right): \delta 21.2\left(\mathrm{CH}_{3}\right), 24.1\left(\mathrm{CH}_{3}\right), 50.8\left(\mathrm{CH}_{3}\right), 52.7(\mathrm{CH}), 67.2(\mathrm{C}), 115.0(\mathrm{C})$, $152.9(\mathrm{CH}), 164.1(\mathrm{C})$; HRMS (ESI) m/z: $[\mathrm{M}+\mathrm{Na}]^{+}$calcd. for $\mathrm{C}_{10} \mathrm{H}_{17} \mathrm{NO}_{3} \mathrm{Na} 222.1101$, found 222.1105

Methyl 2-formyl-3-methylbut-2-enoate (S31) ${ }^{[12]}$ To a stirred solution of the isoxazoline S30 (996 mg, $5.0 \mathrm{mmol})$ in dry $\mathrm{CH}_{2} \mathrm{Cl}_{2}(50 \mathrm{~mL})$ was added $70 \% \mathrm{mCPBA}(1.12 \mathrm{~g}, 6.5 \mathrm{mmol})$ in one portion at $0{ }^{\circ} \mathrm{C}$. The resulting mixture was then stirred at room temperature under $\mathrm{N}_{2}$ 
for $30 \mathrm{~min}$. After reaction was complete, the reaction mixture was quenched with 5\% $\mathrm{Na}_{2} \mathrm{~S}_{2} \mathrm{O}_{3(\text { aq })}(50 \mathrm{~mL})$. The resulting solution was then stirred at room temperature for $30 \mathrm{~min}$. The aquous layer was extracted with $\mathrm{CH}_{2} \mathrm{Cl}_{2}(20 \mathrm{~mL})$. The organic portions were combined, washed with sat. $\mathrm{NaHCO}_{3(\mathrm{aq})}$ and brine, dried over $\mathrm{MgSO}_{4}$, filtered and concentrated to give the cross-conjugated enone $\mathbf{S 3 1}$ (654 mg, $92 \%$ yield) as a colorless oil: $\mathrm{IR}\left(\mathrm{CH}_{2} \mathrm{Cl}_{2}\right.$ cast, $\left.\mathrm{cm}^{-1}\right)$ $v_{\max } 2999,2954,2919,2850,1736,1666,1632,1601,1436,1375,1317 ;{ }^{1} \mathrm{H}$ NMR $\left(\mathrm{CDCl}_{3}\right.$, $400 \mathrm{MHz}): \delta 2.10$ (s, 3H), 2.27 (s, 3H), $3.84(\mathrm{~s}, 3 \mathrm{H}), 9.96$ (s, 3H); ${ }^{13} \mathrm{C} \mathrm{NMR}\left(\mathrm{CDCl}_{3}, 150\right.$ MHz): $\delta 20.9\left(\mathrm{CH}_{3}\right), 24.9\left(\mathrm{CH}_{3}\right), 52.1\left(\mathrm{CH}_{3}\right), 132.4(\mathrm{C}), 161.7(\mathrm{C}), 166.9(\mathrm{CO}), 188.0(\mathrm{CHO})$; HRMS (ESI) m/z: [M + Na $]^{+}$calcd. for $\mathrm{C}_{7} \mathrm{H}_{10} \mathrm{O}_{3} \mathrm{Na}$ 165.0522, found 165.0522.

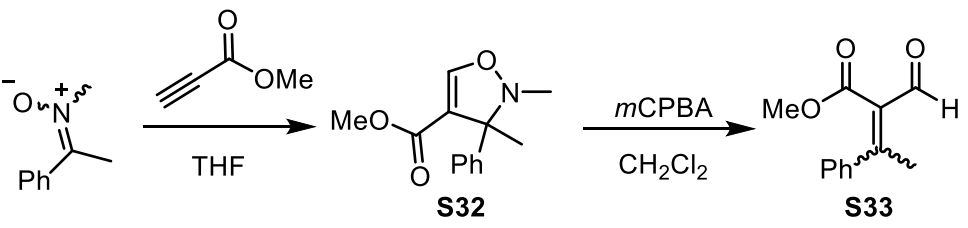

Methyl 2,3-dimethyl-3-phenyl-2,3-dihydroisoxazole-4-carboxylate (S32). According to the synthetic procedures similar to compound S30, isoxazoline $\mathbf{S 3 2}$ (1.77 g, 76\% yield) was prepared from the corresponding nitrone $(1.49 \mathrm{~g}, 10.0 \mathrm{mmol})$ and methyl propilote $(1.68 \mathrm{~g}$, $20.0 \mathrm{mmol})$ as a colorless oil: IR $\left(\mathrm{CH}_{2} \mathrm{Cl}_{2}\right.$ cast, $\left.\mathrm{cm}^{-1}\right) v_{\max } 3089,3059,3024,2986,2951,2920$, 2888, 2850, 1712, 1619, 1437, 1343; ${ }^{1} \mathrm{H}$ NMR $\left(\mathrm{CDCl}_{3}, 400 \mathrm{MHz}\right): \delta 1.83$ (s, 3H), 2.60 (s, 3H), $3.65(\mathrm{~s}, 3 \mathrm{H}), 7.25-7.30(\mathrm{~m}, 1 \mathrm{H}), 7.33-7.37(\mathrm{~m}, 2 \mathrm{H}), 7.46(\mathrm{~s}, 1 \mathrm{H}), 7.48-7.50(\mathrm{~m}, 2 \mathrm{H}) ;{ }^{13} \mathrm{C} \mathrm{NMR}$ $\left(\mathrm{CDCl}_{3}, 100 \mathrm{MHz}\right): \delta 21.1\left(\mathrm{CH}_{3}\right), 39.5\left(\mathrm{CH}_{3}\right), 51.0\left(\mathrm{CH}_{3}\right), 72.0(\mathrm{C}), 114.1(\mathrm{C}), 126.9(\mathrm{CH})$, $127.6(\mathrm{CH}), 128.1(\mathrm{CH}), 141.5(\mathrm{C}), 153.8(\mathrm{CH}), 164.0(\mathrm{CO})$; HRMS (ESI) m/z: $[\mathrm{M}+\mathrm{Na}]^{+}$ calcd. for $\mathrm{C}_{13} \mathrm{H}_{15} \mathrm{NO}_{3} 256.0944$, found 256.1953 .

Methyl (E)/(Z)-2-formyl-3-phenylbut-2-enoate (S33). According to the synthetic procedures similar to compound $\mathbf{S 3 1}$, a mixture of cross-conjugated enones $(E)$ and $(Z)-\mathbf{S 3 3}$ ( $950 \mathrm{mg}$, 93\% yield) was prepared from the corresponding isoxazoline $\mathbf{S 3 2}$ (1.17 g, $5.0 \mathrm{mmol}$ ) as a light yellow oil: IR $\left(\mathrm{CH}_{2} \mathrm{Cl}_{2}\right.$ cast, $\left.\mathrm{cm}^{-1}\right) v_{\max } 3058,3000,2953,2919,2851,2741,1736$, $1673,1616,1573,1491,1435 ;{ }^{1} \mathrm{H} \mathrm{NMR}\left(\mathrm{CDCl}_{3}, 400 \mathrm{MHz}\right)$ mixture of $E / Z=10 / 1: \delta 2.36$ (s, $3 \mathrm{H}), 2.58(\mathrm{~s}, 0.3 \mathrm{H}), 3.54(\mathrm{~s}, 0.3 \mathrm{H}), 3.90(\mathrm{~s}, 3 \mathrm{H}), 7.29-7.46(\mathrm{~m}, 5.5 \mathrm{H}), 9.36(\mathrm{~s}, 1 \mathrm{H}), 10.10(\mathrm{~s}$, $0.1 \mathrm{H}) ;{ }^{13} \mathrm{C} \mathrm{NMR}\left(\mathrm{CDCl}_{3}, 150 \mathrm{MHz}\right)$ major $E$-form: $\delta 24.2\left(\mathrm{CH}_{3}\right), 52.4\left(\mathrm{CH}_{3}\right), 128.4(\mathrm{CH})$, $128.6(\mathrm{CH}), 129.7(\mathrm{CH}), 134.5(\mathrm{C}), 137.4(\mathrm{C}), 161.4(\mathrm{C}), 166.8$ (CO), 189.2 (CHO); HRMS (ESI) $\mathrm{m} / \mathrm{z}:[\mathrm{M}+\mathrm{Na}]^{+}$calcd. for $\mathrm{C}_{12} \mathrm{H}_{12} \mathrm{O}_{3} \mathrm{Na} 227.0679$, found 227.0671 .

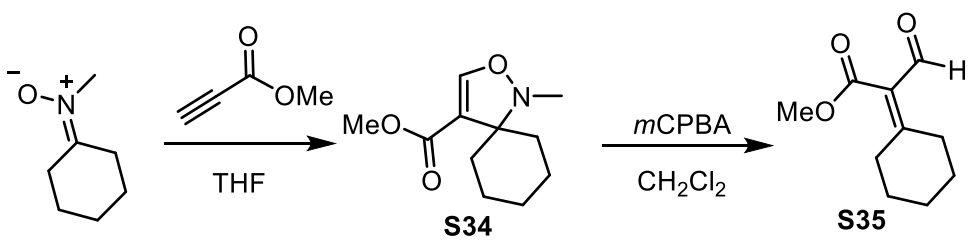


Methyl 1-methyl-2-oxa-1-azaspiro[4.5]dec-3-ene-4-carboxylate (S34). According to the synthetic procedures similar to compound S30, isoxazoline $\mathbf{S 3 4}$ (1.52 g, 72\% yield) was prepared from the corresponding nitrone $(1.27 \mathrm{~g}, 10.0 \mathrm{mmol})$ and methyl propilote $(1.68 \mathrm{~g}$, $20.0 \mathrm{mmol})$ as a colorless oil: IR $\left(\mathrm{CH}_{2} \mathrm{Cl}_{2}\right.$ cast, $\left.\mathrm{cm}^{-1}\right) v_{\max } 3091,2931,2858,1713,1616$, 1436; ${ }^{1} \mathrm{H}$ NMR $\left(\mathrm{CDCl}_{3}, 400 \mathrm{MHz}\right): \delta 1.22-1.35$ (m, 2H), 1.58-1.69 (m, 8H), 2.72 (d, $J=0.8$ $\mathrm{Hz}, 3 \mathrm{H}), 3.71(\mathrm{~s}, 3 \mathrm{H}), 7.33(\mathrm{q}, J=0.8 \mathrm{~Hz}, 1 \mathrm{H}) ;{ }^{13} \mathrm{C} \mathrm{NMR}\left(\mathrm{CDCl}_{3}, 150 \mathrm{MHz}\right): \delta 22.8\left(\mathrm{br}, \mathrm{CH}_{2}\right)$, $25.1\left(\mathrm{CH}_{2}\right), 35.7$ (br, $\left.\mathrm{CH}_{2}\right), 39.9\left(\mathrm{CH}_{3}\right), 50.9\left(\mathrm{CH}_{3}\right), 69.6(\mathrm{C}), 112.7(\mathrm{C}), 154.2(\mathrm{CH}), 164.6$ (CO); HRMS (ESI) m/z: [M + Na $]^{+}$calcd. for $\mathrm{C}_{11} \mathrm{H}_{17} \mathrm{NO}_{3} \mathrm{Na} 234.1101$, found 234.1103.

Methyl 2-cyclohexylidene-3-oxopropanoate (S35). According to the synthetic procedures similar to compound S31, cross-conjugated enone S35 (866 mg, 95\% yield) was prepared from the corresponding isoxazoline $\mathbf{S 3 4}(1.06 \mathrm{~g}, 5.0 \mathrm{mmol})$ as a colorless oil: $\mathrm{IR}\left(\mathrm{CH}_{2} \mathrm{Cl}_{2}\right.$ cast, $\left.\mathrm{cm}^{-1}\right) v_{\max } 2936,2859,1737,1668,1624,1446,1436 ;{ }^{1} \mathrm{H} \mathrm{NMR}\left(\mathrm{CDCl}_{3}, 400 \mathrm{MHz}\right): \delta$ 1.64-1.70 (m, 2H), 1.74-1.82 (m, 4H), 2.37 (t, $J=6.4 \mathrm{~Hz}, 2 \mathrm{H}), 2.75(\mathrm{t}, J=6.4 \mathrm{~Hz}, 2 \mathrm{H}), 3.82$ (s, 3H), $10.01(\mathrm{~s}, 1 \mathrm{H}) ;{ }^{13} \mathrm{C} \mathrm{NMR}\left(\mathrm{CDCl}_{3}, 100 \mathrm{MHz}\right): \delta 25.9\left(\mathrm{CH}_{2}\right), 28.2\left(\mathrm{CH}_{2}\right), 28.3\left(\mathrm{CH}_{2}\right)$, $29.7\left(\mathrm{CH}_{2}\right), 52.2\left(\mathrm{CH}_{3}\right), 131.0(\mathrm{C}), 167.0(\mathrm{C}), 167.2(\mathrm{CO}), 187.0(\mathrm{CHO}) ; \mathrm{HRMS}(\mathrm{ESI}) \mathrm{m} / \mathrm{z}$ : $[\mathrm{M}+\mathrm{H}]^{+}$calcd. for $\mathrm{C}_{10} \mathrm{H}_{15} \mathrm{O}_{3}$ 183.1016, found 183.1017. 


\section{Synthetic procedures and characterization of new anionic Diels-Alder compounds}

General procedure for aldehyde-type anionic Diels-Alder reaction:

Condition A (one step):

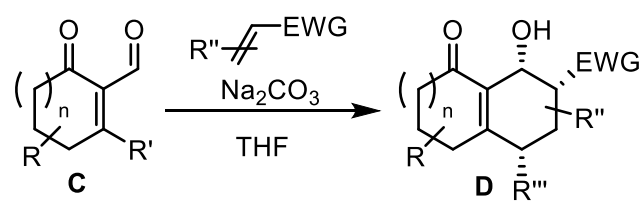

To a stirred solution of the cross-conjugated enone $\mathbf{C}(1.0 \mathrm{mmol})$ and $\mathrm{Na}_{2} \mathrm{CO}_{3}(127 \mathrm{mg}, 1.2$ mmol) in dry THF (5 mL), which was previously degassed with argon, was added dienophile $(1.2 \mathrm{mmol})$ in one portion at room temperature. The resulting mixture was then stirred under individual temperature and time as indicated in Table 2. After reaction was complete, the reaction mixture was quenched with sat. $\mathrm{NH}_{4} \mathrm{Cl}_{(\mathrm{aq})}$ and extracted with EtOAc $(10 \mathrm{~mL} \times 2)$. The combined organic extract was washed with water and brine, dried over $\mathrm{MgSO}_{4}$, filtered and concentrated to give the crude residue, which was purified by chromatography on silica gel to afford anionic Diels-Alder adduct $D$, including compounds 9, 11, 15, 19-22, 24, 25, 28 and $\mathbf{3 4}$ as indicated below.

Condition B (two steps):

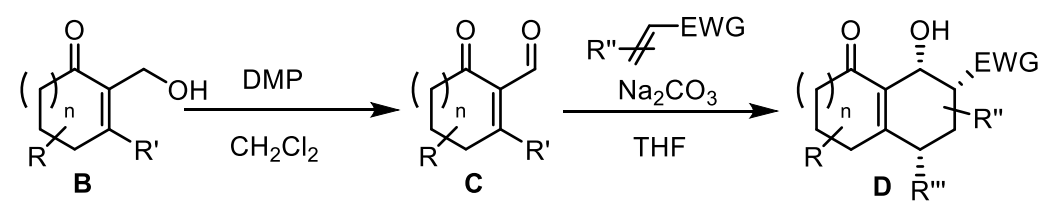

To a stirred solution of the 2-(hydroxymethyl) enone $\mathbf{B}(1.0 \mathrm{mmol})$ in dry $\mathrm{CH}_{2} \mathrm{Cl}_{2}(10 \mathrm{~mL})$ was added Dess-Martin periodinane $(551 \mathrm{mg}, 1.3 \mathrm{mmol})$ in one portion at $0{ }^{\circ} \mathrm{C}$. The resulting mixture was then stirred at room temperature under $\mathrm{N}_{2}$ for $30 \mathrm{~min}$. After reaction was complete, the reaction mixture was quenched with $5 \% \mathrm{Na}_{2} \mathrm{~S}_{2} \mathrm{O}_{3(\text { aq })}(20 \mathrm{~mL})$. The resulting solution was then stirred at room temperature for $20 \mathrm{~min}$. The aquous layer was extracted with $\mathrm{CH}_{2} \mathrm{Cl}_{2}(10 \mathrm{~mL} \times 2)$. The organic portions were combined, washed with sat. $\mathrm{NaHCO}_{3(\text { aq })}$ and brine, dried over $\mathrm{MgSO}_{4}$, filtered and concentrated to give the cross-conjugated enone $\mathbf{C}$ individually. To a stirred solution of the crude cross-conjugated enone $\mathbf{C}$ and $\mathrm{Na}_{2} \mathrm{CO}_{3}$ (127 $\mathrm{mg}, 1.2 \mathrm{mmol})$ in dry THF $(5 \mathrm{~mL})$, which was previously degassed with argon, was added dienophile $(1.2 \mathrm{mmol})$ in one portion at room temperature. The resulting mixture was then stirred under individual temperature and time as indicated in Table 2. After reaction was complete, the reaction mixture was quenched with sat. $\mathrm{NH}_{4} \mathrm{Cl}_{(\mathrm{aq})}$ and extracted with EtOAc $(10 \mathrm{~mL} \times 2)$. The combined organic extract was washed with water and brine, dried over $\mathrm{MgSO}_{4}$, filtered and concentrated to give the crude residue, which was purified by chromatography on silica gel to afford anionic Diels-Alder adduct $\mathbf{D}$, including compounds 12-14, 16-18, 23, 26, 27, 29-33 and 35-37 as indicated below. 
<smiles>[3H]C1(C)CCC(=O)C1C</smiles><smiles>CCOC(=O)C=C(C)C=O</smiles><smiles>CCOC(=O)C1CC2=C(C(=O)CCC2(C)C)[C@@H](O)C1C=O</smiles><smiles>CCOC(=O)C1CC2=C(C(=O)CCC2(C)C)C(O)C1C=O</smiles>

Ethyl

$\left(2 S^{*}, 3 R^{*}, 4 R^{*}\right)$-3-formyl-4-hydroxy-3,8,8-trimethyl-5-oxo-1,2,3,4,5,6,7,8-octahydronapht halene-2-carboxylate (9). Following the general procedure of condition A, compound 9 (299 $\mathrm{mg}, 97 \%$ yield) was prepared from enone 7 (166 mg, $1.0 \mathrm{mmol})$ and dienophile 8 (171 $\mathrm{mg}$, $1.2 \mathrm{mmol})$ as a white solid: $\mathrm{mp}=97-98{ }^{\circ} \mathrm{C}$; IR $\left(\mathrm{KBr}, \mathrm{cm}^{-1}\right) v_{\max } 3454$ (br), 2967, 2934, 2872, 1729, 1667, 1621; ${ }^{1} \mathrm{H}$ NMR $\left(\mathrm{CDCl}_{3}, 400 \mathrm{MHz}\right): \delta 0.99$ (s, 3H), 1.21 (s, 3H), $1.24(\mathrm{~s}, 3 \mathrm{H})$, $1.27(\mathrm{t}, J=7.2 \mathrm{~Hz}, 3 \mathrm{H}), 1.85-1.91(\mathrm{~m}, 2 \mathrm{H}), 2.46-2.59(\mathrm{~m}, 3 \mathrm{H}), 2.71(\mathrm{dd}, J=20.0,5.6 \mathrm{~Hz}, 1 \mathrm{H})$, $2.83(\mathrm{~d}, J=3.2 \mathrm{~Hz}, 1 \mathrm{H}), 3.39(\mathrm{dd}, J=11.2,5.6 \mathrm{~Hz}, 1 \mathrm{H}), 4.13-4.23(\mathrm{~m}, 2 \mathrm{H}), 4.56(\mathrm{~d}, J=3.2$ $\mathrm{Hz}, 1 \mathrm{H}), 9.81(\mathrm{~s}, 1 \mathrm{H}) ;{ }^{13} \mathrm{C} \mathrm{NMR}\left(\mathrm{CDCl}_{3}, 100 \mathrm{MHz}\right): \delta 12.5\left(\mathrm{CH}_{3}\right), 14.0\left(\mathrm{CH}_{3}\right), 25.7\left(\mathrm{CH}_{3}\right)$, $26.1\left(\mathrm{CH}_{2}\right), 27.2\left(\mathrm{CH}_{3}\right), 34.1\left(\mathrm{CH}_{2}\right), 35.3(\mathrm{C}), 36.8\left(\mathrm{CH}_{2}\right), 38.1(\mathrm{CH}), 49.2(\mathrm{C}), 61.1\left(\mathrm{CH}_{2}\right)$, $69.1(\mathrm{CH}), 130.8(\mathrm{C}), 164.2$ (C), 173.2 (CO), 198.9 (CO), 204.4 (CHO); HRMS (ESI) m/z: $[\mathrm{M}+\mathrm{Na}]^{+}$calcd. for $\mathrm{C}_{17} \mathrm{H}_{24} \mathrm{O}_{5} \mathrm{Na} 331.1516$, found 331.1514 .

\section{Ethyl}

$\left(2 S^{*}, 3 R^{*}, 4 S^{*}\right)$-3-formyl-4-hydroxy-3,8,8-trimethyl-5-oxo-1,2,3,4,5,6,7,8-octahydronapht halene-2-carboxylate (10). As listed in Table 1, isomer $\mathbf{1 0}$ was also isolated as a white solid: $\mathrm{mp}=65-67{ }^{\circ} \mathrm{C}$; IR $\left(\mathrm{KBr}, \mathrm{cm}^{-1}\right) v_{\max } 3474(\mathrm{br}), 2966,2932,2852,1731,1648,1613 ;{ }^{1} \mathrm{H}$ NMR $\left(\mathrm{CDCl}_{3}, 400 \mathrm{MHz}\right): \delta 1.19(\mathrm{~s}, 3 \mathrm{H}), 1.20(\mathrm{~s}, 3 \mathrm{H}), 1.23(\mathrm{~s}, 3 \mathrm{H}), 1.25(\mathrm{t}, J=7.2 \mathrm{~Hz}, 3 \mathrm{H}), 1.80(\mathrm{dt}$, $J=13.6,5.2 \mathrm{~Hz}, 1 \mathrm{H}), 1.93(\mathrm{ddd}, J=13.6,10.8,6.0 \mathrm{~Hz}, 1 \mathrm{H}), 2.45-2.54(\mathrm{~m}, 2 \mathrm{H}), 2.55-2.67(\mathrm{~m}$, 2H), $2.89(\mathrm{dd}, J=10.0,6.0 \mathrm{~Hz}, 1 \mathrm{H}), 4.08-4.21(\mathrm{~m}, 2 \mathrm{H}), 4.37$ (d, $J=2.4 \mathrm{~Hz}, 1 \mathrm{H}), 4.83(\mathrm{~d}, J=$ $2.4 \mathrm{~Hz}, 1 \mathrm{H}), 9.66(\mathrm{~s}, 1 \mathrm{H}) ;{ }^{13} \mathrm{C} \mathrm{NMR}\left(\mathrm{CDCl}_{3}, 100 \mathrm{MHz}\right): \delta 9.3\left(\mathrm{CH}_{3}\right), 14.0\left(\mathrm{CH}_{3}\right), 25.2\left(\mathrm{CH}_{3}\right)$, $25.8\left(\mathrm{CH}_{2}\right), 27.3\left(\mathrm{CH}_{3}\right), 34.3\left(\mathrm{CH}_{2}\right), 35.3(\mathrm{C}), 36.3\left(\mathrm{CH}_{2}\right), 42.3(\mathrm{CH}), 50.2(\mathrm{C}), 61.3\left(\mathrm{CH}_{2}\right)$, $68.6(\mathrm{CH}), 130.4(\mathrm{C}), 163.7(\mathrm{C}), 171.6(\mathrm{CO}), 200.6(\mathrm{CO}), 203.3(\mathrm{CHO})$; HRMS (ESI) m/z: $[\mathrm{M}+\mathrm{Na}]^{+}$calcd. for $\mathrm{C}_{17} \mathrm{H}_{24} \mathrm{O}_{5} \mathrm{Na} 331.1516$, found 331.1513 .<smiles>C/C(C=O)=C/C([13CH2])=O</smiles>

Ethyl

$\left(2 S^{*}, 3 R^{*}, 4 R^{*}\right)$-3-formyl-4-hydroxy-3,9,9-trimethyl-10-oxo-1,2,3,4,9,10-hexahydroanthra cene-2-carboxylate (11). Following the general procedure of condition A, compound 11 (342 $\mathrm{mg}, 96 \%$ yield) was prepared from enone $\mathbf{S 2 5}$ (214 $\mathrm{mg}, 1.0 \mathrm{mmol}$ ) and dienophile $\mathbf{8}$ (171 $\mathrm{mg}$, $1.2 \mathrm{mmol})$ as a white solid: $\mathrm{mp}=152-154{ }^{\circ} \mathrm{C}$; IR $\left(\mathrm{KBr}, \mathrm{cm}^{-1}\right) v_{\max } 3462(\mathrm{br}), 3065,2980$, 2935, 1726, 1649, 1600, 1575, 1467; ${ }^{1} \mathrm{H}$ NMR ( $\left.\mathrm{CDCl}_{3}, 400 \mathrm{MHz}\right): \delta 1.06$ (s, 3H), 1.29 (t, $J=$ 
$7.2 \mathrm{~Hz}, 3 \mathrm{H}), 1.56$ (s, 3H), 1.59 (s, 3H), 2.69 (dd, $J=19.6,11.6 \mathrm{~Hz}, 1 \mathrm{H}), 2.94$ (dd, $J=19.6$, $5.2 \mathrm{~Hz}, 1 \mathrm{H}), 3.17$ (d, $J=2.8 \mathrm{~Hz}, 1 \mathrm{H}), 3.57$ (dd, $J=11.6,5.2 \mathrm{~Hz}, 1 \mathrm{H}), 4.14-4.29$ (m, 2H), 4.83 $(\mathrm{d}, J=2.8 \mathrm{~Hz}, 1 \mathrm{H}), 7.40-7.44(\mathrm{~m}, 1 \mathrm{H}), 7.62-7.63(\mathrm{~m}, 2 \mathrm{H}), 8.18(\mathrm{~d}, 8.0 \mathrm{~Hz}, 1 \mathrm{H}), 9.91(\mathrm{~s}, 1 \mathrm{H})$; ${ }^{13} \mathrm{C} \mathrm{NMR}\left(\mathrm{CDCl}_{3}, 100 \mathrm{MHz}\right): \delta 12.5\left(\mathrm{CH}_{3}\right), 14.1\left(\mathrm{CH}_{3}\right), 26.0\left(\mathrm{CH}_{2}\right), 27.7\left(\mathrm{CH}_{3}\right), 30.2\left(\mathrm{CH}_{3}\right)$, $38.4(\mathrm{CH}), 39.8(\mathrm{C}), 49.3(\mathrm{C}), 61.2\left(\mathrm{CH}_{2}\right), 69.6(\mathrm{CH}), 126.1(\mathrm{CH}), 126.6(\mathrm{CH}), 126.9(\mathrm{CH})$, 129.5 (C), 130.7 (C), $133.1(\mathrm{CH}), 150.6$ (C), 160.3 (C), 173.2 (CO), 184.4 (CO), 204.3 (CHO); HRMS (ESI) m/z: [M + Na $]^{+}$calcd. for $\mathrm{C}_{21} \mathrm{H}_{24} \mathrm{O}_{5} \mathrm{Na} 379.1516$, found 379.1509.

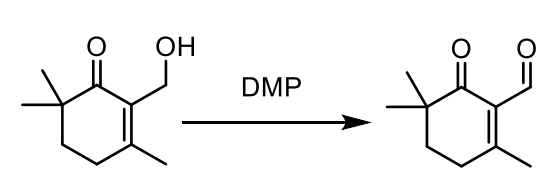

S22
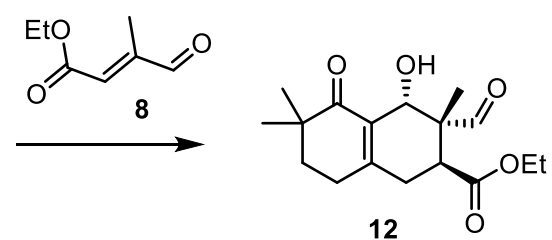

\section{Ethyl}

$\left(2 S^{*}, 3 R^{*}, 4 R^{*}\right)$-3-formyl-4-hydroxy-3,6,6-trimethyl-5-oxo-1,2,3,4,5,6,7,8-octahydronapht halene-2-carboxylate (12). Following the general procedure of condition B, compound $\mathbf{1 2}$ (281 mg, 91\% yield over 2 steps) was prepared from 2-(hydroxymethyl) enone S22 (168 mg, $1.0 \mathrm{mmol})$ and dienophile $\mathbf{8}(171 \mathrm{mg}, 1.2 \mathrm{mmol})$ as a white solid: $\mathrm{mp}=138-140{ }^{\circ} \mathrm{C}$; $\mathrm{IR}(\mathrm{KBr}$, $\left.\mathrm{cm}^{-1}\right) v_{\max } 3430(\mathrm{br}), 2977,2920,2861,1729,1640 ;{ }^{1} \mathrm{H} \mathrm{NMR}\left(\mathrm{CDCl}_{3}, 400 \mathrm{MHz}\right): \delta 1.00$ (s, $3 \mathrm{H}), 1.12(\mathrm{~s}, 3 \mathrm{H}), 1.13(\mathrm{~s}, 3 \mathrm{H}), 1.26(\mathrm{t}, J=7.2 \mathrm{~Hz}, 3 \mathrm{H}), 1.84(\mathrm{t}, J=6.4 \mathrm{~Hz}, 2 \mathrm{H}), 2.38(\mathrm{t}, J=$ $6.4 \mathrm{~Hz}, 2 \mathrm{H}), 2.52(\mathrm{dd}, J=20.0,10.8 \mathrm{~Hz}, 1 \mathrm{H}), 2.61(\mathrm{dd}, J=20.0,6.4 \mathrm{~Hz}, 1 \mathrm{H}), 2.72(\mathrm{~d}, J=3.2$ $\mathrm{Hz}, 1 \mathrm{H}), 3.47(\mathrm{dd}, J=10.8,6.4 \mathrm{~Hz}, 1 \mathrm{H}), 4.12-4.21(\mathrm{~m}, 2 \mathrm{H}), 4.53(\mathrm{~d}, J=3.2 \mathrm{~Hz}, 1 \mathrm{H}), 9.83(\mathrm{~s}$, $1 \mathrm{H}) ;{ }^{13} \mathrm{C} \mathrm{NMR}\left(\mathrm{CDCl}_{3}, 100 \mathrm{MHz}\right): \delta 12.8\left(\mathrm{CH}_{3}\right), 14.1\left(\mathrm{CH}_{3}\right), 24.0\left(\mathrm{CH}_{3}\right), 24.4\left(\mathrm{CH}_{3}\right), 27.4$ $\left(\mathrm{CH}_{2}\right), 30.8\left(\mathrm{CH}_{2}\right), 35.3\left(\mathrm{CH}_{2}\right), 38.1(\mathrm{CH}), 40.7(\mathrm{C}), 49.8(\mathrm{C}), 61.1\left(\mathrm{CH}_{2}\right), 69.1(\mathrm{CH}), 130.3$ (C), 155.5 (C), 173.0 (CO), $204.1(\mathrm{CO}), 204.5$ (CHO); HRMS (ESI) m/z: [M + Na] ${ }^{+}$calcd. for $\mathrm{C}_{17} \mathrm{H}_{24} \mathrm{O}_{5} \mathrm{Na} 331.1516$, found 331.1511.

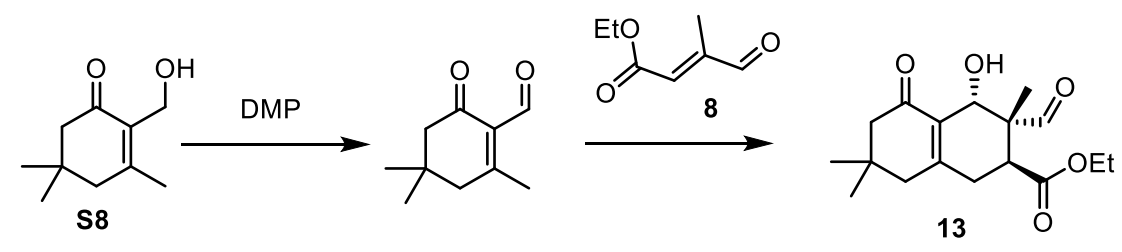

\section{Ethyl}

$\left(2 S^{*}, 3 R^{*}, 4 R^{*}\right)$-3-formyl-4-hydroxy-3,7,7-trimethyl-5-oxo-1,2,3,4,5,6,7,8-octahydronapht halene-2-carboxylate (13). Following the general procedure of condition $\mathrm{B}$, compound adduct 13 (296 mg, 96\% yield over 2 steps) was prepared from 2-(hydroxymethyl) enone S8 (168 $\mathrm{mg}, 1.0 \mathrm{mmol})$ and dienophile $\mathbf{8}(171 \mathrm{mg}, 1.2 \mathrm{mmol})$ as a colorless oil: $\mathrm{IR}\left(\mathrm{CH}_{2} \mathrm{Cl}_{2}\right.$ cast, $\left.\mathrm{cm}^{-1}\right) v_{\max } 3442$ (br), 2958, 2903, 2871, 1728, 1666; ${ }^{1} \mathrm{H}$ NMR $\left(\mathrm{CDCl}_{3}, 400 \mathrm{MHz}\right): \delta 1.00$ (s, 
3H), $1.01(\mathrm{~s}, 3 \mathrm{H}), 1.08(\mathrm{~s}, 3 \mathrm{H}), 1.26(\mathrm{t}, J=7.2 \mathrm{~Hz}, 3 \mathrm{H}), 2.22(\mathrm{~d}, J=18.0 \mathrm{~Hz}, 1 \mathrm{H}), 2.31(\mathrm{~d}, J=$ $18.0 \mathrm{~Hz}, 1 \mathrm{H}), 2.32(\mathrm{~s}, 2 \mathrm{H}), 2.50(\mathrm{dd}, J=20.0,10.8 \mathrm{~Hz}, 1 \mathrm{H}), 2.60(\mathrm{dd}, J=20.0,6.4 \mathrm{~Hz}, 1 \mathrm{H})$, 2.82 (br s, 1H), 3.49 (dd, $J=10.8,6.4 \mathrm{~Hz}, 1 \mathrm{H}), 4.13-4.22(\mathrm{~m}, 2 \mathrm{H}), 4.59$ (s, 1H), 9.84 (s, 1H); ${ }^{13} \mathrm{C} \mathrm{NMR}\left(\mathrm{CDCl}_{3}, 100 \mathrm{MHz}\right): \delta 12.6\left(\mathrm{CH}_{3}\right), 14.0\left(\mathrm{CH}_{3}\right), 27.2\left(\mathrm{CH}_{3}\right), 29.1\left(\mathrm{CH}_{3}\right), 31.2\left(\mathrm{CH}_{2}\right)$, $33.2(\mathrm{C}), 38.1(\mathrm{CH}), 44.4\left(\mathrm{CH}_{2}\right), 49.7(\mathrm{C}), 51.1\left(\mathrm{CH}_{2}\right), 61.1\left(\mathrm{CH}_{2}\right), 68.2(\mathrm{CH}), 131.0(\mathrm{C})$, 155.6 (C), 172.9 (CO), 199.1 (CO), 204.6 (CHO); HRMS (ESI) m/z: [M + Na $]^{+}$calcd. for $\mathrm{C}_{17} \mathrm{H}_{24} \mathrm{O}_{5} \mathrm{Na} 331.1516$, found 331.1512 .

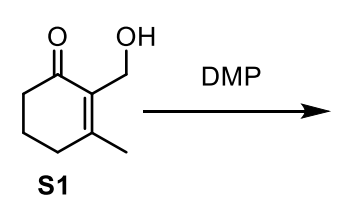

Ethyl<smiles>CC1=C(C=O)C(=O)CCC1</smiles><smiles>C1CCC1</smiles>

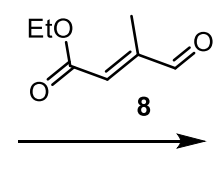

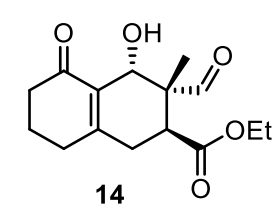

$\left(2 S^{*}, 3 R^{*}, 4 R^{*}\right)$-3-formyl-4-hydroxy-3-methyl-5-oxo-1,2,3,4,5,6,7,8-octahydronaphthalene

-2-carboxylate (14). Following the general procedure of condition B, compound 14 (247 mg, $88 \%$ yield over 2 steps) was prepared from 2-(hydroxymethyl) enone $\mathbf{S 1}$ (140 mg, $1.0 \mathrm{mmol}$ ) and dienophile 8 (171 mg, $1.2 \mathrm{mmol})$ as a white solid: $\mathrm{mp}=98-99{ }^{\circ} \mathrm{C}$; $\mathrm{IR}\left(\mathrm{KBr}, \mathrm{cm}^{-1}\right) v_{\max }$ 3448 (br), 2980, 2921, 2850, 1727, 1663, 1621; ${ }^{1} \mathrm{H}$ NMR $\left(\mathrm{CDCl}_{3}, 400 \mathrm{MHz}\right): \delta 1.01$ (s, 3H), $1.26(\mathrm{t}, J=7.2 \mathrm{~Hz}, 3 \mathrm{H}), 1.94-2.09(\mathrm{~m}, 2 \mathrm{H}), 2.39(\mathrm{t}, J=6.0 \mathrm{~Hz}, 2 \mathrm{H}), 2.40-2.55(\mathrm{~m}, 2 \mathrm{H}), 2.55$ $(\mathrm{dd}, J=20.4,10.8 \mathrm{~Hz}, 1 \mathrm{H}), 2.61(\mathrm{dd}, J=10.8,6.4 \mathrm{~Hz}, 1 \mathrm{H}), 2.86(\mathrm{~d}, J=3.2 \mathrm{~Hz}, 1 \mathrm{H}), 3.48(\mathrm{dd}$, $J=10.8,6.4 \mathrm{~Hz}, 1 \mathrm{H}), 4.12-4.21(\mathrm{~m}, 2 \mathrm{H}), 4.58(\mathrm{~d}, J=3.2 \mathrm{~Hz}, 1 \mathrm{H}), 9.83(\mathrm{~s}, 1 \mathrm{H}) ;{ }^{13} \mathrm{C} \mathrm{NMR}$ $\left(\mathrm{CDCl}_{3}, 100 \mathrm{MHz}\right): \delta 12.6\left(\mathrm{CH}_{3}\right), 14.0\left(\mathrm{CH}_{3}\right), 21.9\left(\mathrm{CH}_{2}\right), 30.3\left(\mathrm{CH}_{2}\right), 31.0\left(\mathrm{CH}_{2}\right), 37.5\left(\mathrm{CH}_{2}\right)$, $38.0(\mathrm{CH}), 49.7(\mathrm{C}), 61.1\left(\mathrm{CH}_{2}\right), 68.5(\mathrm{CH}), 132.0(\mathrm{C}), 157.9(\mathrm{C}), 172.9(\mathrm{CO}), 198.9(\mathrm{CO})$, 204.5 (CHO); HRMS (ESI) m/z: [M + Na $]^{+}$calcd. for $\mathrm{C}_{15} \mathrm{H}_{20} \mathrm{O}_{5} \mathrm{Na} 303.1203$, found 303.1206.

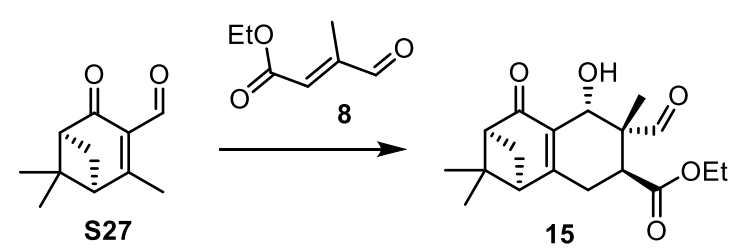

\section{Ethyl}

$\left(1 R^{*}, 3 S^{*}, 5 R^{*}, 6 R^{*}, 7 S^{*}\right)$-6-formyl-5-hydroxy-2,2,6-trimethyl-4-oxo-1,2,3,4,5,6,7,8-octahyd ro-1,3-methanonaphthalene-7-carboxylate (15). Following the general procedure of condition A, compound $\mathbf{1 5}$ (285 mg, 89\% yield) was prepared from enone S27 (178 mg, 1.0 mmol) and dienophile 8 (171 mg, $1.2 \mathrm{mmol})$ as a white solid: $\mathrm{mp}=121-123{ }^{\circ} \mathrm{C}$; IR (KBr, $\left.\mathrm{cm}^{-1}\right) v_{\max } 3447$ (br), 2980, 2941, 2873, 1729, 1676, 1623; ${ }^{1} \mathrm{H}$ NMR $\left(\mathrm{CDCl}_{3}, 400 \mathrm{MHz}\right): \delta$ 0.99 (s, 3H), 1.09 (s, 3H), $1.26(\mathrm{t}, J=7.2 \mathrm{~Hz}, 3 \mathrm{H}), 1.52$ (s, 3H), 2.15 (d, $J=9.6 \mathrm{~Hz}, 1 \mathrm{H}), 2.45$ $(\mathrm{t}, J=5.6 \mathrm{~Hz}, 1 \mathrm{H}), 2.51(\mathrm{dd}, J=20.4,9.6 \mathrm{~Hz}, 1 \mathrm{H}), 2.72-2.79(\mathrm{~m}, 3 \mathrm{H}), 2.88(\mathrm{dt}, J=9.6,5.6$ 
Hz, 1H), $3.46(\mathrm{dd}, J=9.6,6.2 \mathrm{~Hz}, 1 \mathrm{H}), 4.13-4.20(\mathrm{~m}, 2 \mathrm{H}), 4.65$ (d, $J=2.4 \mathrm{~Hz}, 1 \mathrm{H}), 9.85(\mathrm{~s}$, $1 \mathrm{H}) ;{ }^{13} \mathrm{C} \mathrm{NMR}\left(\mathrm{CDCl}_{3}, 75 \mathrm{MHz}\right): \delta 13.2\left(\mathrm{CH}_{3}\right), 14.1\left(\mathrm{CH}_{3}\right), 22.2\left(\mathrm{CH}_{3}\right), 26.6\left(\mathrm{CH}_{3}\right), 30.4$ $\left(\mathrm{CH}_{2}\right), 39.2(\mathrm{CH}), 41.0\left(\mathrm{CH}_{2}\right), 48.3\left(\mathrm{CH}_{3}\right), 49.7(\mathrm{C}), 54.6(\mathrm{C}), 57.6(\mathrm{CH}), 61.1\left(\mathrm{CH}_{2}\right), 67.3$ (CH), 127.5 (C), 165.0 (C), 172.9 (CO), 202.9 (CO), 204.6 (CHO); HRMS (ESI) m/z: [M + $\mathrm{Na}]^{+}$calcd. for $\mathrm{C}_{18} \mathrm{H}_{24} \mathrm{O}_{5} \mathrm{Na} 343.1516$, found 343.1514.
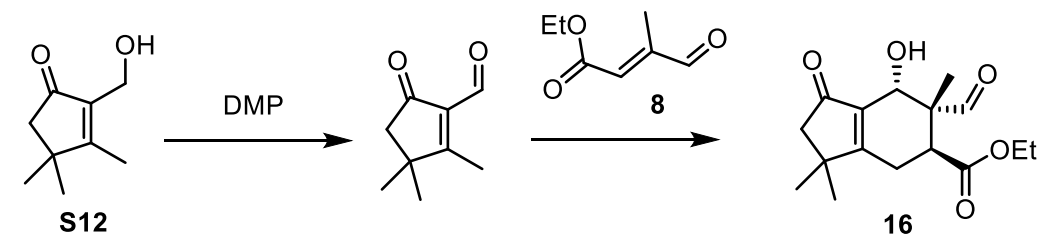

\section{Ethyl}

$\left(5 S^{*}, 6 R^{*}, 7 R^{*}\right)-6$-formyl-7-hydroxy-3,3,6-trimethyl-1-oxo-2,3,4,5,6,7-hexahydro-1H-inde ne-5-carboxylate (16). Following the general procedure of condition B, compound 16 (221 $\mathrm{mg}, 75 \%$ yield over 2 steps) was prepared from 2-(hydroxymethyl) enone $\mathbf{S 1 2}$ (154 mg, 1.0 mmol) and dienophile $8(171 \mathrm{mg}, 1.2 \mathrm{mmol})$ as a white solid: $\mathrm{mp}=129-131{ }^{\circ} \mathrm{C}$; IR $(\mathrm{KBr}$, $\left.\mathrm{cm}^{-1}\right) v_{\max } 3426$ (br), 2962, 2919, 2850, 1729, 1710, 1651; ${ }^{1} \mathrm{H}$ NMR $\left(\mathrm{CDCl}_{3}, 400 \mathrm{MHz}\right): \delta$ $1.04(\mathrm{~s}, 3 \mathrm{H}), 1.24$ (s, 3H), $1.27(\mathrm{~s}, 3 \mathrm{H}), 1.28$ (t, $J=7.2 \mathrm{~Hz}, 3 \mathrm{H}), 2.36(\mathrm{~d}, J=18.8 \mathrm{~Hz}, 1 \mathrm{H})$, $2.39(\mathrm{~d}, J=18.8 \mathrm{~Hz}, 1 \mathrm{H}), 2.53(\mathrm{dd}, J=20.0,9.6 \mathrm{~Hz}, 1 \mathrm{H}), 2.78(\mathrm{dd}, J=20.0,6.0 \mathrm{~Hz}, 1 \mathrm{H})$, $2.99(\mathrm{~d}, J=3.0 \mathrm{~Hz}, 1 \mathrm{H}), 3.48(\mathrm{dd}, J=9.6,6.0 \mathrm{~Hz}, 1 \mathrm{H}), 4.13-4.26(\mathrm{~m}, 2 \mathrm{H}), 4.48(\mathrm{~d}, J=3.0 \mathrm{~Hz}$, $1 \mathrm{H}), 9.84(\mathrm{~s}, 1 \mathrm{H}) ;{ }^{13} \mathrm{C} \mathrm{NMR}\left(\mathrm{CDCl}_{3}, 100 \mathrm{MHz}\right): \delta 13.1\left(\mathrm{CH}_{3}\right), 14.1\left(\mathrm{CH}_{3}\right), 23.4\left(\mathrm{CH}_{2}\right), 26.4$ $\left(\mathrm{CH}_{3}\right), 26.8\left(\mathrm{CH}_{3}\right), 39.6(\mathrm{CH}), 40.8(\mathrm{C}), 50.3(\mathrm{C}), 51.5\left(\mathrm{CH}_{2}\right), 61.3\left(\mathrm{CH}_{2}\right), 67.0(\mathrm{CH}), 135.8$ (C), 172.9 (C), 180.6 (CO), 204.5 (CHO), 206.3 (CO); HRMS (ESI) m/z: [M + Na] ${ }^{+}$calcd. for $\mathrm{C}_{16} \mathrm{H}_{22} \mathrm{O}_{5} \mathrm{Na} 317.1359$, found 317.1361.

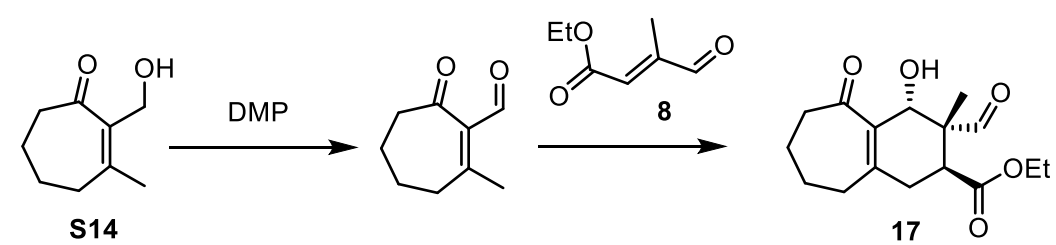

\section{Ethyl}

$\left(2 S^{*}, 3 R^{*}, 4 R^{*}\right)$-3-formyl-4-hydroxy-3-methyl-5-oxo-2,3,4,5,6,7,8,9-octahydro-1H-benzo[7 Jannulene-2-carboxylate (17). Following the general procedure of condition B, compound 17 (253 mg, 86\% yield over 2 steps) was prepared from 2-(hydroxymethyl) enone S14 (154 $\mathrm{mg}, 1.0 \mathrm{mmol})$ and dienophile $\mathbf{8}(171 \mathrm{mg}, 1.2 \mathrm{mmol})$ as a colorless oil: $\mathrm{IR}\left(\mathrm{CH}_{2} \mathrm{Cl}_{2}\right.$ cast, $\left.\mathrm{cm}^{-1}\right)$ $v_{\max } 3446$ (br), 2979, 2939, 2869, 1728, 1652; ${ }^{1} \mathrm{H} \mathrm{NMR}\left(\mathrm{CDCl}_{3}, 400 \mathrm{MHz}\right): \delta 0.98$ (s, 3H), $1.26(\mathrm{t}, J=7.2 \mathrm{~Hz}, 3 \mathrm{H}), 1.71-1.87(\mathrm{~m}, 4 \mathrm{H}), 2.37-2.72(\mathrm{~m}, 6 \mathrm{H}), 2.92($ br s, $1 \mathrm{H}), 3.52$ (dd, $J=$ $10.4,7.2 \mathrm{~Hz}, 1 \mathrm{H}), 4.10-4.23(\mathrm{~m}, 2 \mathrm{H}), 4.46(\mathrm{~s}, 1 \mathrm{H}), 9.83(\mathrm{~s}, 1 \mathrm{H}) ;{ }^{13} \mathrm{C} \mathrm{NMR}\left(\mathrm{CDCl}_{3}, 100\right.$ 
MHz): $\delta 12.4\left(\mathrm{CH}_{3}\right), 14.0\left(\mathrm{CH}_{3}\right), 20.6\left(\mathrm{CH}_{2}\right), 23.8\left(\mathrm{CH}_{3}\right), 32.9\left(\mathrm{CH}_{2}\right), 33.1\left(\mathrm{CH}_{2}\right), 38.2(\mathrm{CH})$, $41.7\left(\mathrm{CH}_{2}\right), 49.4(\mathrm{C}), 61.0\left(\mathrm{CH}_{2}\right), 71.1(\mathrm{CH}), 134.9(\mathrm{C}), 154.1(\mathrm{C}), 173.0(\mathrm{CO}), 204.9(\mathrm{CHO})$, 205.5 (CO); HRMS (ESI) m/z: [M + Na $]^{+}$calcd. for $\mathrm{C}_{16} \mathrm{H}_{22} \mathrm{O}_{5} \mathrm{Na} 317.1359$, found 317.1365.

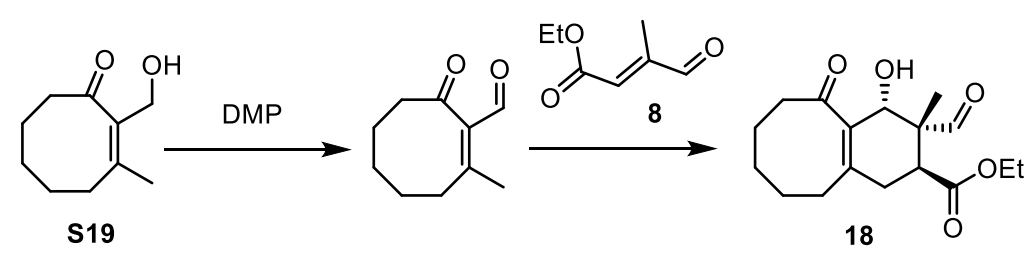

\section{Ethyl}

$\left(2 S^{*}, 3 R^{*}, 4 R^{*}\right)$-3-formyl-4-hydroxy-3-methyl-5-oxo-1,2,3,4,5,6,7,8,9,10-decahydrobenzo[ 8]annulene-2-carboxylate (18). Following the general procedure of condition B, compound 18 (163 mg, 53\% yield over 2 steps) was prepared from 2-(hydroxymethyl) enone S19 (168 $\mathrm{mg}, 1.0 \mathrm{mmol})$ and dienophile $8(171 \mathrm{mg}, 1.2 \mathrm{mmol})$ as a white solid: $\mathrm{mp}=113-115{ }^{\circ} \mathrm{C}$; IR $\left(\mathrm{KBr}, \mathrm{cm}^{-1}\right) v_{\max } 3456(\mathrm{br}), 2979,2931,2856,1727,1671,1648 ;{ }^{1} \mathrm{H} \mathrm{NMR}\left(\mathrm{CDCl}_{3}, 400 \mathrm{MHz}\right)$ : $\delta 1.01(\mathrm{~s}, 3 \mathrm{H}), 1.52-1.59(\mathrm{~m} 2 \mathrm{H}), 1.66-1.89(\mathrm{~m} 4 \mathrm{H}), 2.44-2.71(\mathrm{~m}, 5 \mathrm{H}), 2.84(\mathrm{ddd}, J=17.2$, 8.4, 6.0 Hz, 1H), 3.09 (br s, 1H), $3.53(\mathrm{dd}, J=10.8,7.2 \mathrm{~Hz}, 1 \mathrm{H}), 4.10-4.23(\mathrm{~m}, 2 \mathrm{H}), 4.34$ (s, $1 \mathrm{H}), 9.82(\mathrm{~s}, 1 \mathrm{H}) ;{ }^{13} \mathrm{C} \mathrm{NMR}\left(\mathrm{CDCl}_{3}, 100 \mathrm{MHz}\right): \delta 12.4\left(\mathrm{CH}_{3}\right), 14.0\left(\mathrm{CH}_{3}\right), 22.5\left(\mathrm{CH}_{2}\right), 23.5$ $\left(\mathrm{CH}_{2}\right), 25.0\left(\mathrm{CH}_{2}\right), 32.6\left(\mathrm{CH}_{2}\right), 34.2\left(\mathrm{CH}_{2}\right), 38.2(\mathrm{CH}), 43.4\left(\mathrm{CH}_{2}\right), 49.5(\mathrm{C}), 61.0\left(\mathrm{CH}_{2}\right), 72.3$ (CH), 134.5 (C), 147.7 (C), 173.1 (CO), 204.9 (CHO), 207.7 (CO); HRMS (ESI) m/z: [M + $\mathrm{Na}]^{+}$calcd. for $\mathrm{C}_{17} \mathrm{H}_{24} \mathrm{O}_{5} \mathrm{Na} 331.1516$, found 331.1513.

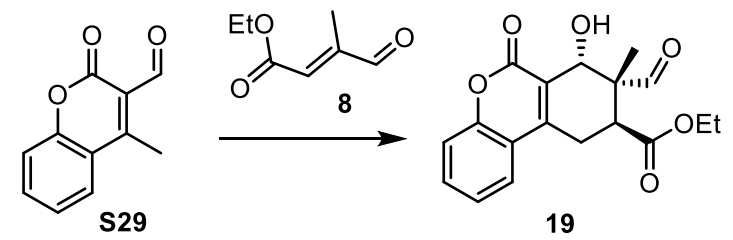

\section{Ethyl}

$\left(7 R^{*}, 8 R^{*}, 9 S^{*}\right)$-8-formyl-7-hydroxy-8-methyl-6-oxo-7,8,9,10-tetrahydro-6H-benzo[c]chro mene-9-carboxylate (19). Following the general procedure of condition A, compound 19 (294 mg, 89\% yield) was prepared from enone $\mathbf{S 2 9}$ (188 mg, $1.0 \mathrm{mmol}$ ) and dienophile $\mathbf{8}$ (171 $\mathrm{mg}, 1.2 \mathrm{mmol})$ as a white solid: $\mathrm{mp}=138-140{ }^{\circ} \mathrm{C}$; IR $\left(\mathrm{KBr}, \mathrm{cm}^{-1}\right) v_{\max } 3448$ (br), 3362, 3080, 2980, 2918, 2850, 1720, 1630, 1608, 1453; ${ }^{1} \mathrm{H}$ NMR $\left(\mathrm{CDCl}_{3}, 400 \mathrm{MHz}\right): \delta 1.10$ (s, 3H), 1.31 (t, $J=7.2 \mathrm{~Hz}, 3 \mathrm{H}), 3.04(\mathrm{dd}, J=19.6,11.2 \mathrm{~Hz}, 1 \mathrm{H}), 3.16(\mathrm{~d}, J=3.4 \mathrm{~Hz}, 1 \mathrm{H}), 3.37$ (dd, $J=$ 19.6, $6.0 \mathrm{~Hz},), 3.71(\mathrm{dd}, J=11.2,6.0 \mathrm{~Hz}, 1 \mathrm{H}), 4.18-4.31(\mathrm{~m}, 2 \mathrm{H}), 4.83(\mathrm{~d}, J=3.4 \mathrm{~Hz}, 1 \mathrm{H})$, 7.35-7.39 (m, 2H), 7.59 (td, $J=7.6,1.6 \mathrm{~Hz}, 1 \mathrm{H}), 7.70(\mathrm{dd}, J=8.0,1.2 \mathrm{~Hz}, 1 \mathrm{H}), 9.93(\mathrm{~s}, 1 \mathrm{H})$; ${ }^{13} \mathrm{C} \mathrm{NMR}\left(\mathrm{CDCl}_{3}, 100 \mathrm{MHz}\right): \delta 12.6\left(\mathrm{CH}_{3}\right), 14.0\left(\mathrm{CH}_{3}\right), 25.1\left(\mathrm{CH}_{2}\right), 37.7(\mathrm{CH}), 49.6(\mathrm{C}), 61.5$ $\left(\mathrm{CH}_{2}\right), 69.6(\mathrm{CH}), 117.1(\mathrm{CH}), 118.7(\mathrm{C}), 122.2(\mathrm{C}), 124.1(\mathrm{CH}), 124.7(\mathrm{CH}), 132.1(\mathrm{CH})$, 
147.2 (C), 152.5 (C), 161.2 (CO), 172.6 (CO), 203.7 (CHO); HRMS (ESI) m/z: [M + Na $]^{+}$ calcd. for $\mathrm{C}_{18} \mathrm{H}_{18} \mathrm{O}_{6} \mathrm{Na}$ 353.0996, found 353.0999.

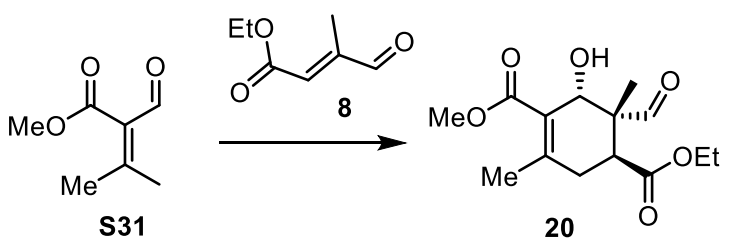

4-Ethyl 1-methyl $\left(4 S^{*}, 5 R^{*}, 6 R^{*}\right)$-5-formyl-6-hydroxy-2,5-dimethylcyclohex-1-ene-1,4dicarboxylate (20). Following the general procedure of condition A, compound $\mathbf{2 0}$ (230 mg, $81 \%$ yield) was prepared from enone $\mathbf{S 3 1}(142 \mathrm{mg}, 1.0 \mathrm{mmol})$ and dienophile $\mathbf{8}(171 \mathrm{mg}, 1.2$ mmol) as a colorless oil: IR $\left(\mathrm{CH}_{2} \mathrm{Cl}_{2}\right.$ cast, $\left.\mathrm{cm}^{-1}\right) v_{\max } 3483$ (br), 2983, 2951, 2851, 1724, 1645; ${ }^{1} \mathrm{H}$ NMR $\left(\mathrm{CDCl}_{3}, 400 \mathrm{MHz}\right): \delta 1.01(\mathrm{~s}, 3 \mathrm{H}), 1.26(\mathrm{t}, J=7.2 \mathrm{~Hz}, 3 \mathrm{H}), 2.15(\mathrm{~s}, 3 \mathrm{H}), 2.53(\mathrm{dd}, J$ $=20.0,11.2 \mathrm{~Hz}, 1 \mathrm{H}), 2.62(\mathrm{dd}, J=20.0,6.4 \mathrm{~Hz}, 1 \mathrm{H}), 3.52(\mathrm{dd}, J=11.2,6.4 \mathrm{~Hz}, 1 \mathrm{H}), 3.79(\mathrm{~s}$, $3 \mathrm{H}), 4.11-4.23(\mathrm{~m}, 2 \mathrm{H}), 4.41(\mathrm{~s}, 1 \mathrm{H}), 9.83(\mathrm{~s}, 1 \mathrm{H}) ;{ }^{13} \mathrm{C} \mathrm{NMR}\left(\mathrm{CDCl}_{3}, 150 \mathrm{MHz}\right): \delta 12.4\left(\mathrm{CH}_{3}\right)$, 14.1 $\left(\mathrm{CH}_{3}\right), 21.5\left(\mathrm{CH}_{3}\right), 33.4\left(\mathrm{CH}_{2}\right), 37.9(\mathrm{CH}), 49.6(\mathrm{C}), 51.7\left(\mathrm{CH}_{3}\right), 61.1\left(\mathrm{CH}_{2}\right), 71.9(\mathrm{CH})$, 124.5 (C), 150.4 (C), 167.7 (CO), 172.9 (CO), 204.9 (CHO); HRMS (ESI) m/z: [M + Na $]^{+}$ calcd. for $\mathrm{C}_{14} \mathrm{H}_{20} \mathrm{O}_{6} \mathrm{Na} 307.1152$, found 307.1151.

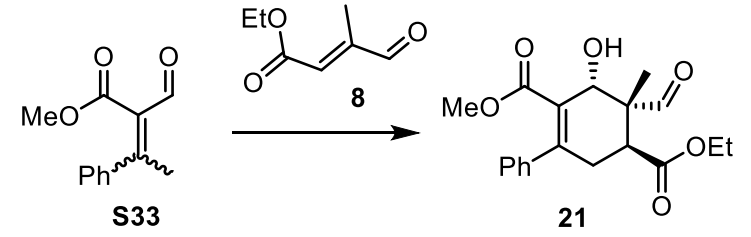

5-Ethyl

2-methyl $\left(3 R^{*}, 4 R^{*}, 5 S^{*}\right)$-4-formyl-3-hydroxy-4-methyl-3,4,5,6-tetrahydro-[1,1'-biphenyl]-2,5-dica rboxylate (21). Following the general procedure of condition A, compound 21 (291 mg, 84\% yield) was prepared from the mixture enones $(E) /(Z)-\mathbf{S 3 3}(204 \mathrm{mg}, 1.0 \mathrm{mmol})$ and dienophile 8 (171 mg, $1.2 \mathrm{mmol})$ as a colorless oil: IR $\left(\mathrm{CH}_{2} \mathrm{Cl}_{2}\right.$ cast, $\left.\mathrm{cm}^{-1}\right) v_{\max } 3473(\mathrm{br}), 3057,3022$, 2982, 2938, 2853, 1726, 1648, 1435; ${ }^{1} \mathrm{H} \mathrm{NMR}\left(\mathrm{CDCl}_{3}, 400 \mathrm{MHz}\right): \delta 1.17$ (s, 3H), 1.27 (t, $J=$ $7.2 \mathrm{~Hz}, 3 \mathrm{H}), 2.71(\mathrm{dd}, J=19.6,11.2 \mathrm{~Hz}, 1 \mathrm{H}), 2.87(\mathrm{dd}, J=19.6,6.0 \mathrm{~Hz}, 1 \mathrm{H}), 2.96(\mathrm{~d}, J=2.8$ $\mathrm{Hz}, 1 \mathrm{H}), 3.45$ (s, 3H), $3.64(\mathrm{dd}, J=11.2,6.0 \mathrm{~Hz}, 1 \mathrm{H}), 4.12-4.21(\mathrm{~m}, 2 \mathrm{H}), 4.52(\mathrm{~d}, J=2.8 \mathrm{~Hz}$, $1 \mathrm{H})$, 7.13-7.16 (m, 2H), 7.32-7.38 (m, 3H), $9.88(\mathrm{~s}, 1 \mathrm{H}) ;{ }^{13} \mathrm{C} \mathrm{NMR}\left(\mathrm{CDCl}_{3}, 100 \mathrm{MHz}\right): \delta 12.5$ $\left(\mathrm{CH}_{3}\right), 14.0\left(\mathrm{CH}_{3}\right), 32.6\left(\mathrm{CH}_{2}\right), 38.3(\mathrm{CH}), 49.6(\mathrm{C}), 51.7\left(\mathrm{CH}_{3}\right), 61.1\left(\mathrm{CH}_{2}\right), 72.1(\mathrm{CH}), 126.4$ $(\mathrm{CH}), 126.7(\mathrm{C}), 128.0(\mathrm{CH}), 128.2(\mathrm{CH}), 140.7(\mathrm{C}), 149.4(\mathrm{C}), 168.5(\mathrm{CO}), 172.7(\mathrm{CO})$, 204.6 (CHO); HRMS (ESI) m/z: [M + Na $]^{+}$calcd. for $\mathrm{C}_{19} \mathrm{H}_{22} \mathrm{O}_{6} \mathrm{Na} 369.1309$, found 369.1305. 


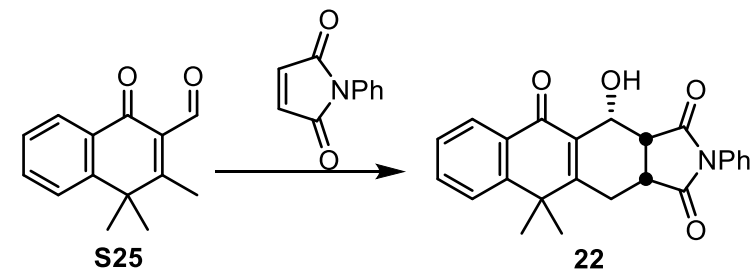

$\left(3 \mathrm{a} R *, 4 R^{*}, 11 \mathrm{a} R^{*}\right)-4-H y d r o x y-10,10$-dimethyl-2-phenyl-4,10,11,11a-tetrahydro-1H-naph tho[2,3-f]isoindole-1,3,5(2H,3aH)-trione (22). Following the general procedure of condition A, compound 22 (368 mg, 95\% yield) was prepared from enone $\mathbf{S 2 5}$ (214 mg, $1.0 \mathrm{mmol}$ ) and $N$-phenylmaleimide ( $208 \mathrm{mg}, 1.2 \mathrm{mmol})$ as a white solid: $\mathrm{mp}=236-239{ }^{\circ} \mathrm{C}$; IR $\left(\mathrm{KBr}, \mathrm{cm}^{-1}\right)$ $v_{\max } 3448$ (br), 3064, 2976, 2920, 2850, 1778, 1708, 1644, 1599, 1499, 1467, 1388; ${ }^{1} \mathrm{H}$ NMR $\left(\mathrm{CDCl}_{3}, 400 \mathrm{MHz}\right): \delta 1.56(\mathrm{~s}, 3 \mathrm{H}), 1.59(\mathrm{~s}, 3 \mathrm{H}), 2.59(\mathrm{~d}, J=4.4 \mathrm{~Hz}, 1 \mathrm{H}), 3.02(\mathrm{dt}, J=15.2$, $8.8 \mathrm{~Hz}, 1 \mathrm{H}), 3.18(\mathrm{dd}, J=8.8,4.4, \mathrm{~Hz}, 1 \mathrm{H}), 3.18-3.32(\mathrm{~m}, 2 \mathrm{H}), 5.98(\mathrm{t}, J=4.4 \mathrm{~Hz}, 1 \mathrm{H})$, 7.26-7.34 (m, 2H), 7.40-7.44 (m, 2H), 7.44-7.51 (m, 2H), 7.60-7.66 (m, 2H), $8.23(\mathrm{~d}, J=8.4$ $\mathrm{Hz}, 1 \mathrm{H}) ;{ }^{13} \mathrm{C} \mathrm{NMR}\left(\mathrm{CDCl}_{3}, 150 \mathrm{MHz}\right): \delta 24.7\left(\mathrm{CH}_{2}\right), 27.2\left(\mathrm{CH}_{3}\right), 27.9\left(\mathrm{CH}_{3}\right), 37.9(\mathrm{CH}), 39.8$ (C), $46.0(\mathrm{CH}), 60.7(\mathrm{CH}), 126.4(\mathrm{CH}), 126.6(\mathrm{CH}), 126.9(\mathrm{CH}), 127.0(\mathrm{CH}), 128.7(\mathrm{CH})$, 129.1 (CH), 129.5 (C), $131.9(\mathrm{C}), 132.9$ (CH), 133.1 (C), 150.1 (C), 164.1 (C), 175.8 (CO), 178.5 (CO), 181.4 (CO); HRMS (ESI) m/z: $[\mathrm{M}+\mathrm{Na}]^{+}$calcd. for $\mathrm{C}_{24} \mathrm{H}_{21} \mathrm{NO}_{4} \mathrm{Na} 410.1363$, found 410.1363 .

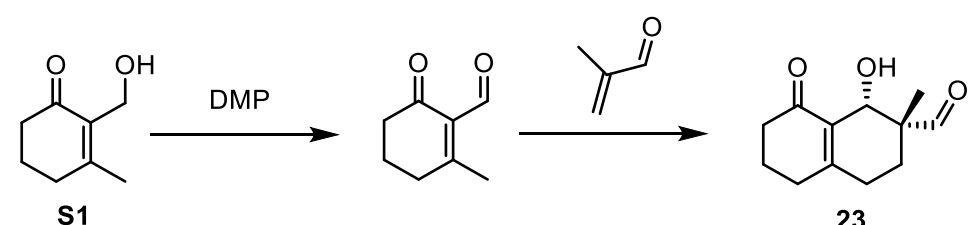

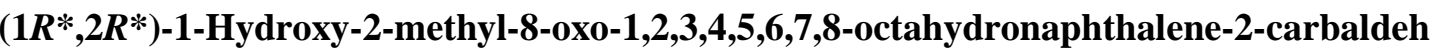
yde (23). Following the general procedure of condition B, compound 23 (196 mg, 94\% yield over 2 steps) was prepared from 2-(hydroxymethyl) enone S1 (140 mg, $1.0 \mathrm{mmol}$ ) and methacrolein (84 mg, $1.2 \mathrm{mmol})$ as a colorless oil: IR $\left(\mathrm{CH}_{2} \mathrm{Cl}_{2} \mathrm{cast}, \mathrm{cm}^{-1}\right) v_{\max } 3448$ (br), 2934, 2875, 2825, 2715, 1726, 1656, 1632; ${ }^{1} \mathrm{H}$ NMR $\left(\mathrm{CDCl}_{3}, 400 \mathrm{MHz}\right): \delta 1.01(\mathrm{~s}, 3 \mathrm{H}), 1.56-1.62$ (m, 1H), 1.91-2.08 (m, 4H), 2.22-2.52 (m, 5H), 3.28 (br s, 1H), $4.66(\mathrm{~s}, 1 \mathrm{H}), 9.75(\mathrm{~s}, 1 \mathrm{H}) ;{ }^{13} \mathrm{C}$ NMR $\left(\mathrm{CDCl}_{3}, 100 \mathrm{MHz}\right): \delta 17.2\left(\mathrm{CH}_{3}\right), 21.9\left(\mathrm{CH}_{2}\right), 22.9\left(\mathrm{CH}_{2}\right), 28.4\left(\mathrm{CH}_{2}\right), 30.8\left(\mathrm{CH}_{2}\right), 37.7$ $\left(\mathrm{CH}_{2}\right), 48.1(\mathrm{C}), 67.2$ (CH), 132.1 (C), 160.3 (C), 200.1 (CO), 205.0 (CHO); HRMS (ESI) $\mathrm{m} / \mathrm{z}:[\mathrm{M}+\mathrm{Na}]^{+}$calcd. for $\mathrm{C}_{12} \mathrm{H}_{16} \mathrm{O}_{3} \mathrm{Na} 231.0992$, found 231.0990 .<smiles>[Z4]C1(C)CCC(=O)C(C=O)=C1C</smiles> 
$\left(4 \mathrm{a} R^{*}, 9 \mathrm{a} R^{*}, 10 R^{*}\right)-10-H y d r o x y-3,4 \mathrm{a}, 8,8$-tetramethyl-6,7,8,9,9a,10-hexahydroanthracene1,4,5(4aH)-trione (24). Following the general procedure of condition A, anionic compound 24 (230 mg, 76\% yield) was prepared from enone 7 (166 mg, $1.0 \mathrm{mmol})$ and 2,6-dimethylbenzoquinone $(127 \mathrm{mg}, 1.2 \mathrm{mmol})$ as a white solid: $\mathrm{mp}=173-175^{\circ} \mathrm{C}$; $\mathrm{IR}(\mathrm{KBr}$, $\left.\mathrm{cm}^{-1}\right) v_{\max } 3480$ (br), 2965, 2928, 1677, 1622, 1468, 1377; ${ }^{1} \mathrm{H}$ NMR $\left(\mathrm{CDCl}_{3}, 400 \mathrm{MHz}\right): \delta$ $1.22(\mathrm{~s}, 3 \mathrm{H}), 1.23(\mathrm{~s}, 3 \mathrm{H}), 1.35(\mathrm{~s}, 3 \mathrm{H}), 1.81-1.95(\mathrm{~m}, 2 \mathrm{H}), 2.01(\mathrm{~d}, J=1.6 \mathrm{~Hz}, 3 \mathrm{H}), 2.24(\mathrm{dd}$, $J=20.0,7.2 \mathrm{~Hz}, 1 \mathrm{H}), 2.41-2.58(\mathrm{~m}, 2 \mathrm{H}), 2.66(\mathrm{~d}, J=2.8 \mathrm{~Hz}, 1 \mathrm{H}), 2.96(\mathrm{dd}, J=7.2,0.8 \mathrm{~Hz}$, $1 \mathrm{H}), 3.31(\mathrm{dd}, J=20.0,0.8 \mathrm{~Hz}, 1 \mathrm{H}), 4.42(\mathrm{~d}, J=2.8 \mathrm{~Hz}, 1 \mathrm{H}), 6.68(\mathrm{q}, \mathrm{J}=1.6 \mathrm{~Hz}, 1 \mathrm{H}) ;{ }^{13} \mathrm{C}$ NMR (CDCl $3,100 \mathrm{MHz}): \delta 16.4\left(\mathrm{CH}_{3}\right), 20.2\left(\mathrm{CH}_{3}\right), 21.7\left(\mathrm{CH}_{2}\right), 25.6\left(\mathrm{CH}_{3}\right), 27.2\left(\mathrm{CH}_{3}\right), 34.1$ $\left(\mathrm{CH}_{2}\right), 35.4(\mathrm{C}), 36.6\left(\mathrm{CH}_{2}\right), 48.1(\mathrm{CH}), 50.1(\mathrm{C}), 68.9(\mathrm{CH}), 130.3(\mathrm{C}), 138.1(\mathrm{CH}), 150.5(\mathrm{C})$, $163.5(\mathrm{C}), 196.8(\mathrm{CO}), 199.0(\mathrm{CO}), 201.4(\mathrm{CO})$; HRMS (ESI) m/z: [M + Na ${ }^{+}$calcd. for $\mathrm{C}_{18} \mathrm{H}_{22} \mathrm{O}_{4} \mathrm{Na} 325.1410$, found 325.1407 .

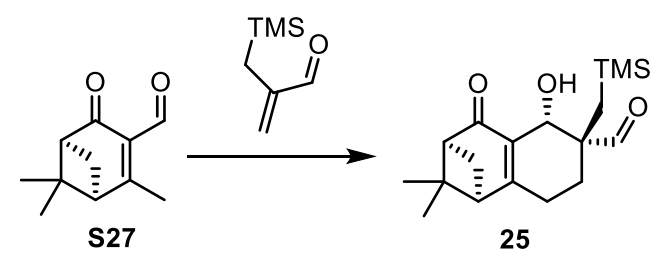

$\left(1 R^{*}, 3 S^{*}, 5 S^{*}, 6 S^{*}\right)-5$-Hydroxy-2,2-dimethyl-4-oxo-6-((trimethylsilyl)methyl)-1,2,3,4,5,6,7 ,8-octahydro-1,3-methanonaphthalene-6-carbaldehyde (25). Following the general procedure of condition A, compound $\mathbf{2 5}$ (288 $\mathrm{mg}$, 90\% yield) was prepared from enone S27 (178 mg, $1.0 \mathrm{mmol})$ and 2-((trimethylsilyl)methyl)acrolein (171 mg, $1.2 \mathrm{mmol})$ as a white solid: $\mathrm{mp}=164-166^{\circ} \mathrm{C}$; IR $\left(\mathrm{KBr}, \mathrm{cm}^{-1}\right) v_{\max } 3444$ (br), 2951, 2925, 2851, 1725, 1663, 1636; ${ }^{1} \mathrm{H}$ NMR $\left(\mathrm{CDCl}_{3}, 400 \mathrm{MHz}\right): \delta 0.05(\mathrm{~s}, 9 \mathrm{H}), 0.83(\mathrm{~d}, J=15.2 \mathrm{~Hz}, 1 \mathrm{H}), 0.90(\mathrm{~d}, J=15.2 \mathrm{~Hz}$, $1 \mathrm{H}), 0.98(\mathrm{~s}, 3 \mathrm{H}), 1.50(\mathrm{~s}, 3 \mathrm{H}), 1.62(\mathrm{dt}, J=13.6,5.2 \mathrm{~Hz}, 1 \mathrm{H}), 2.06-2.22(\mathrm{~m}, 4 \mathrm{H}), 2.38(\mathrm{t}, J=$ $5.6 \mathrm{~Hz}, 1 \mathrm{H}), 2.53(\mathrm{dtd}, J=18.8,5.2,1.2 \mathrm{~Hz}, 1 \mathrm{H}), 2.71(\mathrm{t}, J=5.6 \mathrm{~Hz}, 1 \mathrm{H}), 2.82(\mathrm{dt}, J=9.2$, $5.6 \mathrm{~Hz}, 1 \mathrm{H}), 3.37(\mathrm{~d}, J=2.8 \mathrm{~Hz}, 1 \mathrm{H}), 4.71(\mathrm{~d}, J=2.8 \mathrm{~Hz}, 1 \mathrm{H}), 9.79(\mathrm{~s}, 1 \mathrm{H}) ;{ }^{13} \mathrm{C} \mathrm{NMR}\left(\mathrm{CDCl}_{3}\right.$, $100 \mathrm{MHz}): \delta 0.51\left(\mathrm{CH}_{3}\right), 20.8\left(\mathrm{CH}_{2}\right), 22.2\left(\mathrm{CH}_{3}\right), 25.0\left(\mathrm{CH}_{2}\right), 26.6\left(\mathrm{CH}_{3}\right), 28.2\left(\mathrm{CH}_{2}\right), 40.8$ $\left(\mathrm{CH}_{2}\right), 48.5(\mathrm{CH}), 51.0(\mathrm{C}), 55.0(\mathrm{C}), 57.8(\mathrm{CH}), 68.4(\mathrm{CH}), 127.9(\mathrm{C}), 167.5(\mathrm{C}), 204.1(\mathrm{CO})$, $205.5(\mathrm{CHO})$; HRMS (ESI) m/z: $[\mathrm{M}+\mathrm{Na}]^{+}$calcd. for $\mathrm{C}_{18} \mathrm{H}_{28} \mathrm{O}_{3} \mathrm{SiNa} 343.1700$, found 343.1700 .

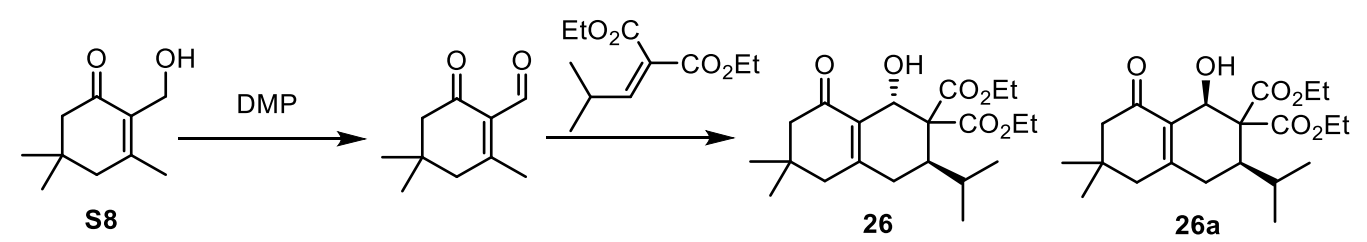

Diethyl

$\left(1 S^{*}, 3 R^{*}\right)$-1-hydroxy-3-isopropyl-6,6-dimethyl-8-oxo-3,4,5,6,7,8-hexahydronaphthalene- 
2,2(1H)-dicarboxylate (26). Following the general procedure of condition B, compound 26 (289 mg, 76\% yield over 2 steps) was prepared from 2-(hydroxymethyl) enone $\mathbf{S 8}$ (168 mg, $1.0 \mathrm{mmol})$ and diethyl isobutylidenemalonate $(257 \mathrm{mg}, 1.2 \mathrm{mmol})$ as a white solid: $\mathrm{mp}=112$ $114{ }^{\circ} \mathrm{C}$; IR (KBr, cm $\left.{ }^{-1}\right) v_{\max } 3450$ (br), 2960, 2928, 2870, 1752, 1726, 1657; ${ }^{1} \mathrm{H}$ NMR $\left(\mathrm{CDCl}_{3}\right.$, $400 \mathrm{MHz}): \delta 0.82(\mathrm{~d}, J=6.8 \mathrm{~Hz}, 3 \mathrm{H}), 0.99(\mathrm{~s}, 3 \mathrm{H}), 1.05(\mathrm{~d}, J=6.8 \mathrm{~Hz}, 3 \mathrm{H}), 1.06(\mathrm{~s}, 3 \mathrm{H})$, 1.20 (t, $J=7.2 \mathrm{~Hz}, 3 \mathrm{H}), 1.29$ (t, $J=7.2 \mathrm{~Hz}, 3 \mathrm{H}$ ), 2.11-2.37 (m, 7H), 2.47 (ddd, $J=10.8,6.0$, $2.4 \mathrm{~Hz}, 1 \mathrm{H}), 3.24(\mathrm{~d}, J=3.2 \mathrm{~Hz}, 1 \mathrm{H}), 4.00-4.27(\mathrm{~m}, 4 \mathrm{H}), 5.08(\mathrm{~d}, J=3.2 \mathrm{~Hz}, 1 \mathrm{H}) ;{ }^{13} \mathrm{C} \mathrm{NMR}$ $\left(\mathrm{CDCl}_{3}, 100 \mathrm{MHz}\right): \delta 13.8\left(\mathrm{CH}_{3}\right), 14.0\left(\mathrm{CH}_{3}\right), 18.6\left(\mathrm{CH}_{3}\right), 24.0\left(\mathrm{CH}_{3}\right), 26.7\left(\mathrm{CH}_{3}\right), 27.5(\mathrm{CH})$, $29.5\left(\mathrm{CH}_{3}\right), 30.9(\mathrm{CH}), 33.2(\mathrm{C}), 37.4\left(\mathrm{CH}_{3}\right), 44.7\left(\mathrm{CH}_{2}\right), 51.1\left(\mathrm{CH}_{2}\right), 60.9\left(\mathrm{CH}_{2}\right), 61.0(\mathrm{C})$, $61.2\left(\mathrm{CH}_{2}\right), 65.1(\mathrm{CH}), 130.8(\mathrm{C}), 157.5(\mathrm{C}), 169.0(\mathrm{CO}), 170.6(\mathrm{CO}), 198.6(\mathrm{CO})$; HRMS (ESI) $\mathrm{m} / \mathrm{z}:[\mathrm{M}+\mathrm{Na}]^{+}$calcd. for $\mathrm{C}_{21} \mathrm{H}_{32} \mathrm{O}_{6} \mathrm{Na}$ 403.2091, found 403.2091 .

\section{Diethyl}

$\left(1 R^{*}, 3 R^{*}\right)-1$-hydroxy-3-isopropyl-6,6-dimethyl-8-oxo-3,4,5,6,7,8-hexahydronaphthalene-

2,2(1H)-dicarboxylate (26a). cis-isomer $\mathbf{2 6 a}$ (15 mg, 4\% yield) was also isolated as a white solid: $\mathrm{mp}=78-80{ }^{\circ} \mathrm{C}$; IR $\left(\mathrm{KBr}, \mathrm{cm}^{-1}\right) v_{\max } 3488$ (br), 2958, 2928, 2870, 1732, 1661; ${ }^{1} \mathrm{H} \mathrm{NMR}$ $\left(\mathrm{CDCl}_{3}, 400 \mathrm{MHz}\right): \delta 0.87(\mathrm{~d}, J=9.2 \mathrm{~Hz}, 3 \mathrm{H}), 0.99$ (s, 3H), $1.01(\mathrm{~d}, J=9.2 \mathrm{~Hz}, 3 \mathrm{H}), 1.06$ (s, $3 \mathrm{H}), 1.22(\mathrm{t}, J=7.2 \mathrm{~Hz}, 3 \mathrm{H}), 1.28(\mathrm{t}, J=7.2 \mathrm{~Hz}, 3 \mathrm{H}), 2.12-2.35(\mathrm{~m}, 7 \mathrm{H}), 2.42-2.46(\mathrm{~m}, 1 \mathrm{H})$, $3.74(\mathrm{~d}, J=3.6 \mathrm{~Hz}, 1 \mathrm{H}), 4.13-4.18(\mathrm{~m}, 2 \mathrm{H}), 4.21-4.27(\mathrm{~m}, 2 \mathrm{H}), 5.27$ (br s, $1 \mathrm{H}) ;{ }^{13} \mathrm{C} \mathrm{NMR}$ $\left(\mathrm{CDCl}_{3}, 100 \mathrm{MHz}\right): \delta 13.9\left(\mathrm{CH}_{3}\right), 14.0\left(\mathrm{CH}_{3}\right), 19.2\left(\mathrm{CH}_{3}\right), 24.5\left(\mathrm{CH}_{3}\right), 27.7\left(\mathrm{CH}_{3}\right), 28.4\left(\mathrm{CH}_{3}\right)$, $28.7(\mathrm{CH}), 31.7\left(\mathrm{CH}_{2}\right), 33.0(\mathrm{C}), 40.8(\mathrm{CH}), 44.8\left(\mathrm{CH}_{2}\right), 51.3\left(\mathrm{CH}_{2}\right), 61.2\left(\mathrm{CH}_{2}\right), 61.6\left(\mathrm{CH}_{2}\right)$, $61.9(\mathrm{C}), 66.1(\mathrm{CH}), 130.6(\mathrm{C}), 156.7$ (C), 169.5 (CO), 169.8 (CO), 199.1 (CO); HRMS (ESI) $\mathrm{m} / \mathrm{z}:[\mathrm{M}+\mathrm{Na}]^{+}$calcd. for $\mathrm{C}_{21} \mathrm{H}_{32} \mathrm{O}_{6} \mathrm{Na}$ 403.2091, found 403.2093.

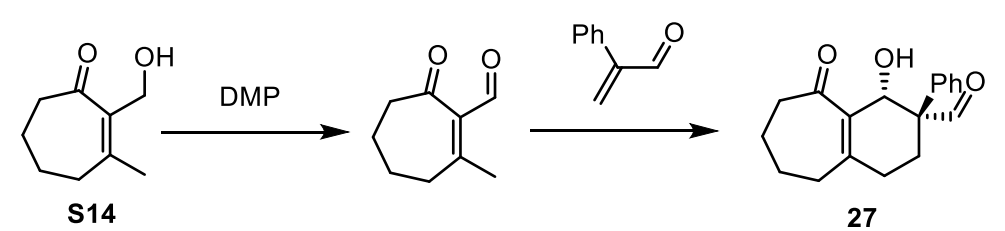

$\left(1 R^{*}, 2 S^{*}\right)$-1-Hydroxy-9-oxo-2-phenyl-2,3,4,5,6,7,8,9-octahydro-1H-benzo[7]annulene-2-c arbaldehyde (27). Following the general procedure of condition B, compound 27 (247 mg, 87\% yield over 2 steps) was prepared from 2-(hydroxymethyl) enone $\mathbf{S 1 4}$ (154 mg, $1.0 \mathrm{mmol}$ ) and 2-phenylpropenal $(159 \mathrm{mg}, 1.2 \mathrm{mmol})$ as a white solid: $\mathrm{mp}=175-177^{\circ} \mathrm{C}$; $\mathrm{IR}\left(\mathrm{KBr}, \mathrm{cm}^{-1}\right)$ $v_{\max } 3378$ (br), 3058, 2918, 2989, 2869, 1726, 1637, 1624, 1495, 1454; ${ }^{1} \mathrm{H}$ NMR $\left(\mathrm{CDCl}_{3}, 400\right.$ MHz): $\delta 1.50-1.62(\mathrm{~m}, 1 \mathrm{H}), 1.72-1.91(\mathrm{~m}, 4 \mathrm{H}), 2.18-2.43(\mathrm{~m}, 5 \mathrm{H}), 2.68(\mathrm{t}, J=6.4 \mathrm{~Hz}, 2 \mathrm{H})$, $2.96(\mathrm{~d}, J=3.6 \mathrm{~Hz}, 1 \mathrm{H}), 5.50(\mathrm{~d}, J=3.6 \mathrm{~Hz}, 1 \mathrm{H}), 7.11-7.14(\mathrm{~m}, 2 \mathrm{H}), 7.25-7.35(\mathrm{~m}, 3 \mathrm{H}), 9.55$ (s, $1 \mathrm{H}) ;{ }^{13} \mathrm{C} \mathrm{NMR}\left(\mathrm{CDCl}_{3}, 100 \mathrm{MHz}\right): \delta 20.8\left(\mathrm{CH}_{2}\right), 22.6\left(\mathrm{CH}_{2}\right), 23.8\left(\mathrm{CH}_{2}\right), 31.1\left(\mathrm{CH}_{2}\right), 33.3$ $\left(\mathrm{CH}_{2}\right), 41.8\left(\mathrm{CH}_{2}\right), 57.0(\mathrm{C}), 64.8(\mathrm{CH}), 127.5(\mathrm{CH}), 127.7(\mathrm{CH}), 128.8(\mathrm{CH}), 135.0(\mathrm{C})$, 135.3 (C), 158.7 (C), 199.2 (CO), 205.5 (CO); HRMS (ESI) m/z: [M + Na $]^{+}$calcd. for $\mathrm{C}_{18} \mathrm{H}_{20} \mathrm{O}_{3} \mathrm{Na} 307.1305$, found 307.1314. 


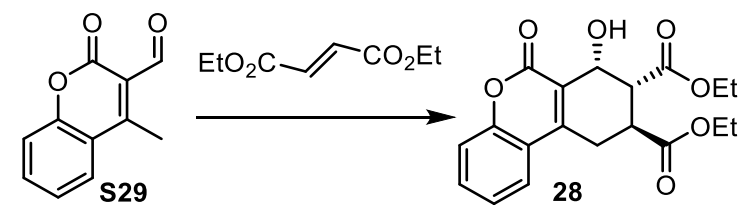

\section{Diethyl}

$\left(7 R^{*}, 8 R^{*}, 9 S^{*}\right)$-7-hydroxy-6-oxo-7,8,9,10-tetrahydro-6H-benzo[c]chromene-8,9-dicarbox ylate (28). Following the general procedure of condition A, compound 28 (317 mg, 88\% yield) was prepared from enone $\mathbf{S 2 9}$ (188 $\mathrm{mg}, 1.0 \mathrm{mmol})$ and diethyl fumarate $(207 \mathrm{mg}, 1.2 \mathrm{mmol})$ as a white solid: $\mathrm{mp}=129-130{ }^{\circ} \mathrm{C}$; IR $\left(\mathrm{KBr}, \mathrm{cm}^{-1}\right) v_{\max } 3482$ (br), 3067, 2981, 2917, 2848, 1734, 1719, 1628, 1608; ${ }^{1} \mathrm{H}$ NMR $\left(\mathrm{CDCl}_{3}, 400 \mathrm{MHz}\right): \delta 1.32(\mathrm{t}, J=7.2 \mathrm{~Hz}, 3 \mathrm{H}), 1.34(\mathrm{t}, J=$ $7.2 \mathrm{~Hz}, 3 \mathrm{H}), 2.78(\mathrm{dd}, J=16.0,3.6 \mathrm{~Hz}, 1 \mathrm{H}), 3.01(\mathrm{~d}, J=3.2 \mathrm{~Hz}, 1 \mathrm{H}), 3.05(\mathrm{dd}, J=12.4,3.6$ $\mathrm{Hz}, 1 \mathrm{H}), 3.33-3.42(\mathrm{~m}, 2 \mathrm{H}), 4.19-4.34(\mathrm{~m}, 4 \mathrm{H}), 5.30(\mathrm{t}, J=3.2 \mathrm{~Hz}, 1 \mathrm{H}), 7.34(\mathrm{t}, J=8.2 \mathrm{~Hz}$, $1 \mathrm{H}), 7.37(\mathrm{~d}, J=8.2 \mathrm{~Hz}, 1 \mathrm{H}), 7.57(\mathrm{t}, J=8.2 \mathrm{~Hz}, 1 \mathrm{H}), 7.60(\mathrm{~d}, J=8.2 \mathrm{~Hz}, 1 \mathrm{H}) ;{ }^{13} \mathrm{C} \mathrm{NMR}$ $\left(\mathrm{CDCl}_{3}, 100 \mathrm{MHz}\right): \delta 14.1\left(\mathrm{CH}_{3}\right) \times 2,28.9\left(\mathrm{CH}_{2}\right), 35.6(\mathrm{CH}), 46.8(\mathrm{CH}), 61.2\left(\mathrm{CH}_{2}\right) \times 2,62.8$ $(\mathrm{CH}), 117.1(\mathrm{CH}), 118.6(\mathrm{C}), 123.1(\mathrm{C}), 124.0(\mathrm{CH}), 124.7(\mathrm{CH}), 132.0(\mathrm{CH}), 146.7(\mathrm{C})$, $152.5(\mathrm{C}), 161.0(\mathrm{CO}), 170.9(\mathrm{CO}), 174.4(\mathrm{CO})$; HRMS (ESI) m/z: $[\mathrm{M}+\mathrm{Na}]^{+}$calcd. for $\mathrm{C}_{19} \mathrm{H}_{20} \mathrm{O}_{7} \mathrm{Na} 383.1101$, found 383.1100.

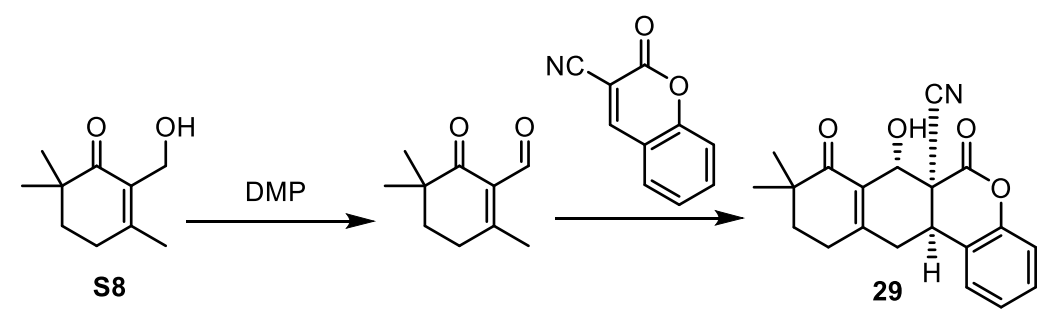

$\left(6 \mathrm{a} R^{*}, 7 S^{*}, 12 \mathrm{a} R^{*}\right)-7-H y d r o x y-9,9-d i m e t h y l-6,8-d i o x o-8,9,10,11,12,12 \mathrm{a}-h e x a h y d r o-6 H$-na phtho[2,3-c]chromene-6a(7H)-carbonitrile (29). Following the general procedure of condition B, compound 29 (310 mg, 92\% yield over 2 steps) was prepared from 2-(hydroxymethyl) enone $\mathbf{S 8}$ (168 $\mathrm{mg}, 1.0 \mathrm{mmol}$ ) and 3-cyanocoumarin (205 mg, $1.2 \mathrm{mmol})$ as a white solid: $\mathrm{mp}=194-197{ }^{\circ} \mathrm{C}$; IR $\left(\mathrm{KBr}, \mathrm{cm}^{-1}\right) v_{\max } 3357$ (br), 3051, 2924, 2851, 1773, 1654; ${ }^{1} \mathrm{H}$ NMR $\left(\mathrm{CDCl}_{3}, 400 \mathrm{MHz}\right): \delta 1.12(\mathrm{~s}, 3 \mathrm{H}), 1.15(\mathrm{~s}, 3 \mathrm{H}), 1.85(\mathrm{t}, J=6.0 \mathrm{~Hz}, 2 \mathrm{H})$, 2.24-2.39 (m, 3H), $2.75(\mathrm{dd}, J=20.4,6.4 \mathrm{~Hz}, 1 \mathrm{H}), 3.44(\mathrm{~d}, J=4.0 \mathrm{~Hz}, 1 \mathrm{H}), 3.73(\mathrm{dd}, J=10.0$, $6.4 \mathrm{~Hz}, 1 \mathrm{H}), 5.32(\mathrm{~d}, J=4.0 \mathrm{~Hz}, 1 \mathrm{H}), 7.17-7.22(\mathrm{~m}, 1 \mathrm{H}), 7.24(\mathrm{dd}, J=7.6,1.2 \mathrm{~Hz}, 1 \mathrm{H}), 7.30$ $(\mathrm{dd}, J=7.6,1.6 \mathrm{~Hz}, 1 \mathrm{H}), 7.39-7.43(\mathrm{~m}, 1 \mathrm{H}) ;{ }^{13} \mathrm{C} \mathrm{NMR}\left(\mathrm{CDCl}_{3}, 100 \mathrm{MHz}\right): \delta 24.0\left(\mathrm{CH}_{3}\right), 24.1$ $\left(\mathrm{CH}_{3}\right), 27.3\left(\mathrm{CH}_{2}\right), 34.1(\mathrm{CH}), 34.5\left(\mathrm{CH}_{2}\right), 34.9\left(\mathrm{CH}_{2}\right), 40.8(\mathrm{C}), 47.7(\mathrm{C}), 62.4(\mathrm{CH}), 114.8$ (C), $117.5(\mathrm{CH}), 123.9(\mathrm{C}), 125.8(\mathrm{CH}), 127.5(\mathrm{CH}), 129.1(\mathrm{C}), 130.1(\mathrm{CH}), 150.2(\mathrm{C}), 152.0$ (C), $160.4(\mathrm{CO}), 203.0(\mathrm{CO})$; HRMS (ESI) m/z: $[\mathrm{M}+\mathrm{Na}]^{+}$calcd. for $\mathrm{C}_{20} \mathrm{H}_{19} \mathrm{NO}_{4} \mathrm{Na} 360.1206$, found 360.1208 . 


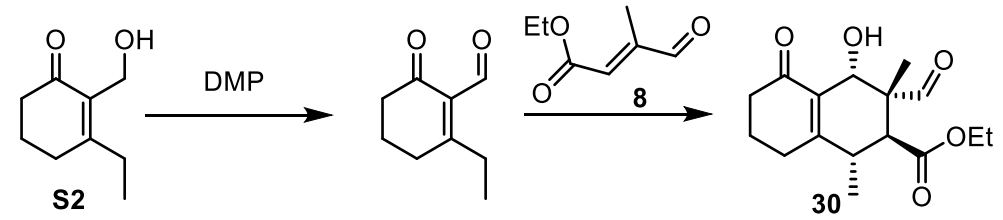

\section{Ethyl}

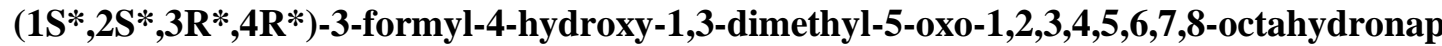
hthalene-2-carboxylate (30). Following the general procedure of condition $\mathrm{B}$, compound $\mathbf{3 0}$ (247 mg, 84\% yield over 2 steps) was prepared from 2-(hydroxymethyl) enone S2 (154 mg, $1.0 \mathrm{mmol})$ and dienophile $8(171 \mathrm{mg}, 1.2 \mathrm{mmol})$ as a white solid: $\mathrm{mp}=125-126^{\circ} \mathrm{C}$; $\mathrm{IR}(\mathrm{KBr}$, $\left.\mathrm{cm}^{-1}\right) v_{\max } 3448(\mathrm{br}), 2980,2938,2882,1726,1664,1630 ;{ }^{1} \mathrm{H} \mathrm{NMR}\left(\mathrm{CDCl}_{3}, 400 \mathrm{MHz}\right): \delta$ $0.96(\mathrm{~s}, 3 \mathrm{H}), 1.23$ (d, $J=7.2 \mathrm{~Hz}, 3 \mathrm{H}), 1.27$ (t, $J=7.2 \mathrm{~Hz}, 3 \mathrm{H}), 1.84-1.95(\mathrm{~m}, 1 \mathrm{H}), 2.05-2.13$ (m, 1H), 2.73-2.40 (m, 2H), 2.47-2.55 (m, 2H), 2.74-2.81 (m, 1H), $2.78(\mathrm{~d}, J=3.2 \mathrm{~Hz}, 1 \mathrm{H})$, $3.16(\mathrm{~d}, J=10.4 \mathrm{~Hz}, 1 \mathrm{H}), 4.13-4.24(\mathrm{~m}, 2 \mathrm{H}), 4.59(\mathrm{~d}, J=3.2 \mathrm{~Hz}, 1 \mathrm{H}), 9.83(\mathrm{~s}, 1 \mathrm{H}) ;{ }^{13} \mathrm{C}$ NMR $\left(\mathrm{CDCl}_{3}, 100 \mathrm{MHz}\right): \delta 12.8\left(\mathrm{CH}_{3}\right), 14.0\left(\mathrm{CH}_{3}\right), 17.3\left(\mathrm{CH}_{3}\right), 22.1\left(\mathrm{CH}_{2}\right), 28.6\left(\mathrm{CH}_{2}\right), 35.4(\mathrm{CH})$, $37.2\left(\mathrm{CH}_{2}\right), 45.7(\mathrm{CH}), 50.3(\mathrm{C}), 60.9\left(\mathrm{CH}_{2}\right), 67.5(\mathrm{CH}), 131.3(\mathrm{C}), 161.9(\mathrm{C}), 173.2(\mathrm{CO})$, $199.0(\mathrm{CO}), 204.8(\mathrm{CHO})$; HRMS (ESI) m/z: $[\mathrm{M}+\mathrm{Na}]^{+}$calcd. for $\mathrm{C}_{16} \mathrm{H}_{22} \mathrm{O}_{5} \mathrm{Na} 317.1359$, found 317.1360 .

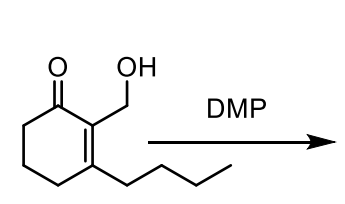

S4

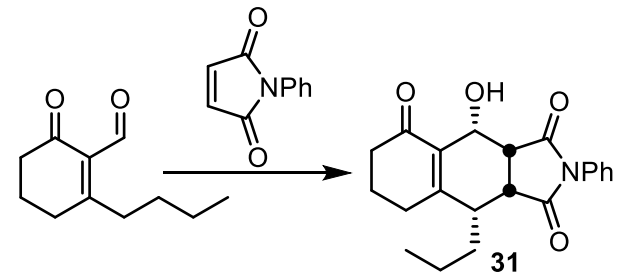

31

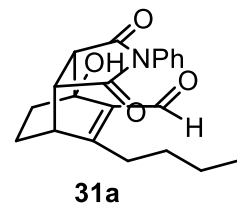

$31 \mathrm{a}$

$\left(3 \mathrm{a} R^{*}, 4 R^{*}, 9 S^{*}, 9 \mathrm{a} R^{*}\right)-4-H y d r o x y-2-p h e n y l-9-p r o p y l-4,6,7,8,9,9 \mathrm{a}-h e x a h y d r o-1 H$-benzo[f]i soindole-1,3,5(2H,3aH)-trione (31). Following the general procedure of condition $\mathrm{B}$, compound $\mathbf{3 1}$ (300 mg, 85\% yield over 2 steps) was prepared from 2-(hydroxymethyl) enone S4 (182 mg, $1.0 \mathrm{mmol})$ and $N$-phenylmaleimide $(208 \mathrm{mg}, 1.2 \mathrm{mmol})$ as a white solid: $\mathrm{mp}=$ 159-161 ${ }^{\circ} \mathrm{C}$; IR $\left(\mathrm{KBr}, \mathrm{cm}^{-1}\right) v_{\max } 3469,2957,2931,2872,1775,1709,1662,1628,1498$, 1387; ${ }^{1} \mathrm{H}$ NMR $\left(\mathrm{CDCl}_{3}, 400 \mathrm{MHz}\right): \delta 0.91(\mathrm{t}, J=7.2 \mathrm{~Hz}, 3 \mathrm{H}), 1.29-1.49(\mathrm{~m}, 2 \mathrm{H}), 1.78-1.87$ (m, $1 \mathrm{H}), 1.94-2.11(\mathrm{~m}, 3 \mathrm{H}), 2.43-2.62(\mathrm{~m}, 4 \mathrm{H}), 2.93(\mathrm{ddd}, J=12.8,10.0,7.2 \mathrm{~Hz}, 1 \mathrm{H}), 3.15(\mathrm{~d}$, $J=2.8 \mathrm{~Hz}, 1 \mathrm{H}), 3.27(\mathrm{dd}, J=10.2,6.8 \mathrm{~Hz}, 1 \mathrm{H}), 3.35(\mathrm{dd}, J=10.2,7.2 \mathrm{~Hz}, 1 \mathrm{H}), 5.54(\mathrm{dd}, J=$ 6.8, $2.8 \mathrm{~Hz}, 1 \mathrm{H}), 7.26-7.30(\mathrm{~m}, 2 \mathrm{H}), 7.41(\mathrm{tt}, J=7.2,1.2 \mathrm{~Hz}, 1 \mathrm{H}), 7.47-7.51(\mathrm{~m}, 2 \mathrm{H}) ;{ }^{13} \mathrm{C}$ NMR (CDCl $3,100 \mathrm{MHz}): \delta 14.3\left(\mathrm{CH}_{3}\right), 21.8\left(\mathrm{CH}_{2}\right), 22.1\left(\mathrm{CH}_{2}\right), 32.4\left(\mathrm{CH}_{2}\right), 34.0\left(\mathrm{CH}_{2}\right), 37.4$ $\left(\mathrm{CH}_{2}\right), 41.1(\mathrm{CH}), 43.2(\mathrm{CH}), 45.3(\mathrm{CH}), 58.4(\mathrm{CH}), 126.5(\mathrm{CH}), 128.8(\mathrm{CH}), 129.2(\mathrm{CH})$, 131.7 (C), 133.6 (C), 166.3 (C), 176.2 (CO), 176.7 (CO), 196.7 (CO); HRMS (ESI) m/z: [M + $\mathrm{Na}]^{+}$calcd. for $\mathrm{C}_{21} \mathrm{H}_{23} \mathrm{NO}_{4} \mathrm{Na} 376.1519$, found 376.1524 . 
$\left(3 \mathrm{a} R^{*}, 4 R^{*}, 7 R^{*}, 7 \mathrm{a} R^{*}\right)-6$-Butyl-4-hydroxy-1,3-dioxo-2-phenyl-2,3,3a,4,7,7a-hexahydro-1H -4,7-ethanoisoindole-5-carbaldehyde (31a). Ketone-type cyclic adduct 31a (28 mg, 8\% yield over 2 steps) was also isolated as a white solid: $\mathrm{mp}=102-104{ }^{\circ} \mathrm{C} ; \mathrm{IR}\left(\mathrm{KBr}, \mathrm{cm}^{-1}\right) v_{\max }$ 3465 (br), 2957, 2930, 2872, 1774, 1710, 1651, 1597, 1498, 1380; ${ }^{1} \mathrm{H}$ NMR $\left(\mathrm{CDCl}_{3}, 400\right.$ MHz): $\delta 0.88(\mathrm{t}, J=7.2 \mathrm{~Hz}, 3 \mathrm{H}), 1.25-1.39(\mathrm{~m}, 2 \mathrm{H}), 1.41-1.53(\mathrm{~m}, 2 \mathrm{H}), 1.58-1.66(\mathrm{~m}, 1 \mathrm{H})$, $1.70-1.78(\mathrm{~m}, 1 \mathrm{H}), 1.87-1.97(\mathrm{~m}, 2 \mathrm{H}), 2.36(\mathrm{ddd}, J=12.8,8.4,6.4 \mathrm{~Hz}, 1 \mathrm{H}), 2.81$ (ddd, $J=$ $12.8,8.8,7.2 \mathrm{~Hz}, 1 \mathrm{H}), 3.08$ (d, $J=8.4 \mathrm{~Hz}, 1 \mathrm{H}), 3.23-3.26(\mathrm{~m}, 2 \mathrm{H}), 5.85$ (br s, 1H), 7.08-7.11 $(\mathrm{m}, 2 \mathrm{H}), 7.37(\mathrm{tt}, J=7.2,1.2 \mathrm{~Hz}, 1 \mathrm{H}), 7.41-7.45(\mathrm{~m}, 2 \mathrm{H}), 10.02(\mathrm{~s}, 1 \mathrm{H}) ;{ }^{13} \mathrm{C} \mathrm{NMR}\left(\mathrm{CDCl}_{3}\right.$, $100 \mathrm{MHz}): \delta 13.6\left(\mathrm{CH}_{3}\right), 22.6\left(\mathrm{CH}_{2}\right), 23.7\left(\mathrm{CH}_{2}\right), 30.0\left(\mathrm{CH}_{2}\right), 30.6\left(\mathrm{CH}_{2}\right), 32.9\left(\mathrm{CH}_{2}\right), 38.6$ $(\mathrm{CH}), 44.4(\mathrm{CH}), 49.3(\mathrm{CH}), 75.6(\mathrm{C}), 126.0(\mathrm{CH}), 128.7(\mathrm{CH}), 129.1(\mathrm{CH}), 131.2(\mathrm{C}), 134.8$ (C), $163.5(\mathrm{C}), 175.5(\mathrm{CO}), 176.0(\mathrm{CO}), 189.1(\mathrm{CHO})$; HRMS (ESI) m/z: [M + Na ${ }^{+}$calcd. for $\mathrm{C}_{21} \mathrm{H}_{23} \mathrm{NO}_{4} \mathrm{Na} 376.1519$, found 376.1517 .

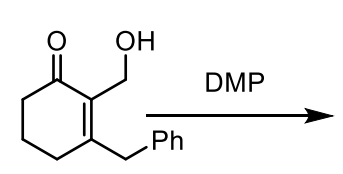

S3

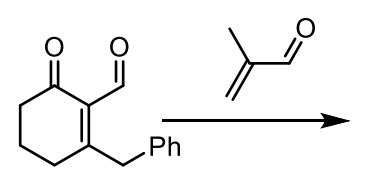

$\left(1 R^{*}, 2 R^{*}, 4 R^{*}\right)-1$-Hydroxy-2-methyl-8-oxo-4-phenyl-1,2,3,4,5,6,7,8-octahydronaphthalen e-2-carbaldehyde (32). Following the general procedure of condition B, compound 32 (264 $\mathrm{mg}, 93 \%$ yield over 2 steps) was prepared from 2-(hydroxymethyl) enone $\mathbf{S 3}$ (216 mg, 1.0 mmol) and methacrolein ( $84 \mathrm{mg}, 1.2 \mathrm{mmol})$ as a white solid: $\mathrm{mp}=158-160{ }^{\circ} \mathrm{C}$; IR $(\mathrm{KBr}$, $\left.\mathrm{cm}^{-1}\right) v_{\max } 3461$ (br), 3082, 3059, 3025, 2932, 2874, 2807, 2713, 1725, 1655, 1627, 1600, 1493, 1455; ${ }^{1} \mathrm{H}$ NMR $\left(\mathrm{CDCl}_{3}, 400 \mathrm{MHz}\right): \delta 1.04$ (s, 3H), 1.79-1.94 (m, 3H), 1.98-2.11 (m, 2H), $2.26(\mathrm{dd}, J=13.6,11.6 \mathrm{~Hz}, 1 \mathrm{H}), 2.39(\mathrm{ddd}, J=17.2,10.8,5.6 \mathrm{~Hz}, 1 \mathrm{H}), 2.49(\mathrm{dt}, J=$ 17.2, $5.2 \mathrm{~Hz}, 1 \mathrm{H}), 3.17$ (br s, 1H), 3.45 (dd, $J=11.6,6.4 \mathrm{~Hz}, 1 \mathrm{H}), 4.81(\mathrm{~s}, 1 \mathrm{H}), 7.19-7.21(\mathrm{~m}$, 2H), 7.24-7.28 (m, 1H), 7.31-7.34 (m, 2H), $9.68(\mathrm{~s}, 1 \mathrm{H}) ;{ }^{13} \mathrm{C} \mathrm{NMR}\left(\mathrm{CDCl}_{3}, 100 \mathrm{MHz}\right): \delta 16.4$ $\left(\mathrm{CH}_{3}\right), 22.1\left(\mathrm{CH}_{2}\right), 29.4\left(\mathrm{CH}_{2}\right), 31.9\left(\mathrm{CH}_{2}\right), 37.6\left(\mathrm{CH}_{2}\right), 45.6(\mathrm{CH}), 48.3(\mathrm{C}), 65.2(\mathrm{CH}), 127.0$ $(\mathrm{CH}), 128.5(\mathrm{CH}), 128.8(\mathrm{CH}), 133.0(\mathrm{C}), 142.1(\mathrm{C}), 161.3(\mathrm{C}), 199.8(\mathrm{CO}), 204.1(\mathrm{CHO})$; HRMS (ESI) m/z: [M + Na $]^{+}$calcd. for $\mathrm{C}_{18} \mathrm{H}_{20} \mathrm{O}_{3} \mathrm{Na}$ 307.1305, found 307.1307.

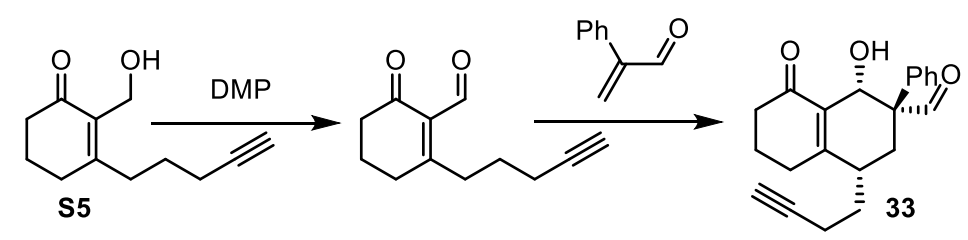

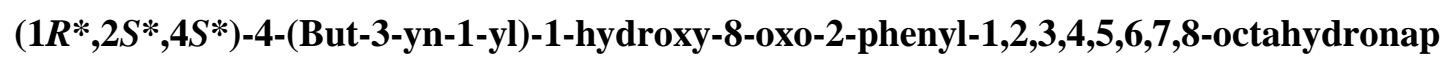
hthalene-2-carbaldehyde (33). Following the general procedure of condition B, compound 33 (261 mg, 81\% yield over 2 steps) was prepared from 2-(hydroxymethyl) enone $\mathbf{S 5}$ (192 mg, $1.0 \mathrm{mmol})$ and 2-phenylpropenal $(159 \mathrm{mg}, 1.2 \mathrm{mmol})$ as a white solid: $\mathrm{mp}=103-105{ }^{\circ} \mathrm{C}$; IR 
$\left(\mathrm{KBr}, \mathrm{cm}^{-1}\right) v_{\max } 3444,3289,3088,3058,2929,2869,2825,2719,2116,1724,1660,1622$, 1495, 1448; ${ }^{1} \mathrm{H}$ NMR $\left(\mathrm{CDCl}_{3}, 400 \mathrm{MHz}\right): \delta 1.50-1.59(\mathrm{~m}, 1 \mathrm{H}), 1.70-1.82(\mathrm{~m}, 1 \mathrm{H}), 1.92(\mathrm{t}, J=$ $2.8 \mathrm{~Hz}, 1 \mathrm{H}), 1.95-2.14$ (m, 5H), 2.33-2.58 (m, 6H), 2.98 (br s, 1H), 5.62 (s, 1H), 7.10-7.13 (m, 2H), 7.24-7.34 (m, 3H), $9.51(\mathrm{~s}, 1 \mathrm{H}) ;{ }^{13} \mathrm{C} \mathrm{NMR}\left(\mathrm{CDCl}_{3}, 100 \mathrm{MHz}\right): \delta 15.6\left(\mathrm{CH}_{2}\right), 22.2\left(\mathrm{CH}_{2}\right)$, $26.6\left(\mathrm{CH}_{2}\right), 28.6\left(\mathrm{CH}_{2}\right), 30.2\left(\mathrm{CH}_{2}\right), 36.6(\mathrm{CH}), 37.3\left(\mathrm{CH}_{2}\right), 56.8(\mathrm{C}), 62.2(\mathrm{CH}), 69.4(\mathrm{C})$, $82.8(\mathrm{C}), 127.5(\mathrm{CH}), 127.7(\mathrm{CH}), 128.9(\mathrm{CH}), 132.9(\mathrm{C}), 134.5(\mathrm{C}), 164.0(\mathrm{C}), 198.8(\mathrm{CHO})$, 199.6 (CO); HRMS (ESI) m/z: [M + Na $]^{+}$calcd. for $\mathrm{C}_{21} \mathrm{H}_{22} \mathrm{O}_{3} \mathrm{Na} 345.1461$, found 345.1461.

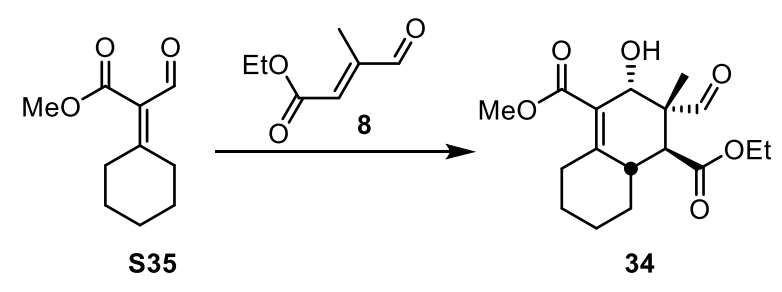

1-Ethyl

4-methyl

$\left(1 S^{*}, 2 R^{*}, 3 R^{*}, 8 \mathrm{a} S^{*}\right)$-2-formyl-3-hydroxy-2-methyl-1,2,3,5,6,7,8,8a-octahydronaphthalene -1,4-dicarboxylate (34). Following the general procedure of condition A, compound 34 (237 $\mathrm{mg}, 73 \%$ yield) was prepared from enone $\mathbf{S 3 5}(182 \mathrm{mg}, 1.0 \mathrm{mmol})$ and dienophile $\mathbf{8}$ (171 $\mathrm{mg}$, $1.2 \mathrm{mmol})$ as a colorless oil: IR (KBr, cm $\left.{ }^{-1}\right) v_{\max } 3484(\mathrm{br}), 2981,2933,2858,1722,1638 ;{ }^{1} \mathrm{H}$ NMR $\left(\mathrm{CDCl}_{3}, 400 \mathrm{MHz}\right): \delta 0.95(\mathrm{~s}, 3 \mathrm{H}), 1.20-1.31(\mathrm{~m}, 1 \mathrm{H}), 1.25(\mathrm{t}, J=7.2 \mathrm{~Hz}, 3 \mathrm{H})$, 1.36-1.60 (m, 2H), 1.76-1.94 (m, 3H), 2.07-2.12 (m, 1H), $2.55(\mathrm{ddd}, J=12.4,10.0,4.8 \mathrm{~Hz}$, $1 \mathrm{H}), 2.73$ (br s, 1H), 3.16 (d, $J=10.0 \mathrm{~Hz}, 1 \mathrm{H}), 3.48-3.53(\mathrm{~m}, 1 \mathrm{H}), 3.79(\mathrm{~s}, 3 \mathrm{H}), 4.11-4.23$ (m, 2H), $4.36(\mathrm{~s}, 1 \mathrm{H}), 9.82(\mathrm{~s}, 1 \mathrm{H}) ;{ }^{13} \mathrm{C} \mathrm{NMR}\left(\mathrm{CDCl}_{3}, 100 \mathrm{MHz}\right): \delta 13.0\left(\mathrm{CH}_{3}\right), 14.0\left(\mathrm{CH}_{3}\right), 25.5$ $\left(\mathrm{CH}_{2}\right), 27.2\left(\mathrm{CH}_{2}\right), 31.0\left(\mathrm{CH}_{2}\right), 34.5\left(\mathrm{CH}_{2}\right), 39.2(\mathrm{CH}), 44.9(\mathrm{CH}), 50.2(\mathrm{C}), 51.7\left(\mathrm{CH}_{3}\right), 60.9$ $\left(\mathrm{CH}_{2}\right), 71.6(\mathrm{CH}), 122.6(\mathrm{C}), 155.0(\mathrm{C}), 168.3(\mathrm{CO}), 173.2(\mathrm{CO}), 205.2(\mathrm{CHO})$; HRMS (ESI) $\mathrm{m} / \mathrm{z}:[\mathrm{M}+\mathrm{Na}]^{+}$calcd. for $\mathrm{C}_{17} \mathrm{H}_{24} \mathrm{O}_{6} \mathrm{Na} 347.1465$, found 347.1466 .

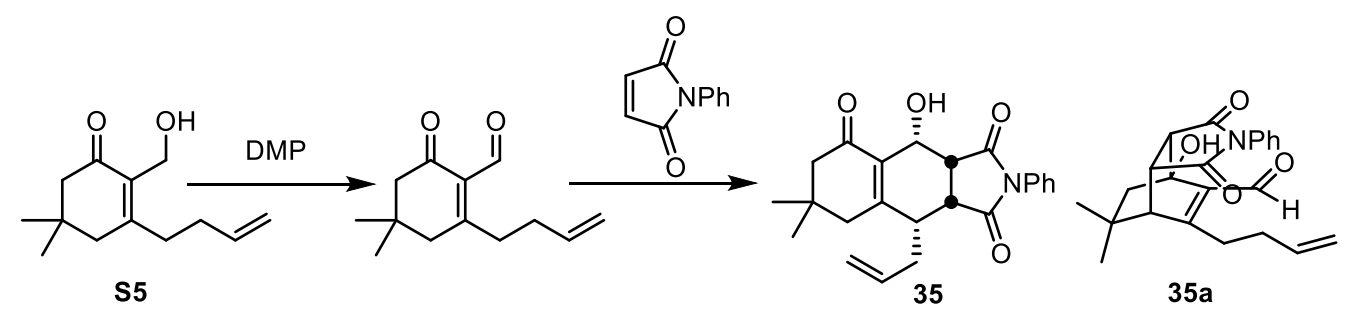

$\left(3 \mathrm{a} R^{*}, 4 R^{*}, 9 S^{*}, 9 \mathrm{a} R^{*}\right)-9-A l l y l-4-h y d r o x y-7,7-d i m e t h y l-2-p h e n y l-4,6,7,8,9,9 \mathrm{a}-h e x a h y d r o-1$ $\boldsymbol{H}$-benzo $[f]$ isoindole-1,3,5(2H,3aH)-trione (35). Following the general procedure of condition B, compound 35 (300 mg, 79\% yield over 2 steps) was prepared from 2-(hydroxymethyl) enone $\mathbf{S 5}$ (208 mg, $1.0 \mathrm{mmol}$ ) and $N$-phenylmaleimide (208 mg, $1.2 \mathrm{mmol}$ ) as a white solid: $\mathrm{mp}=204-206^{\circ} \mathrm{C}$; IR $\left(\mathrm{KBr}, \mathrm{cm}^{-1}\right) v_{\max } 3440(\mathrm{br}), 3071,2957,2923,2852$, 1778, 1708, 1665, 1630, 1498, 1386; ${ }^{1} \mathrm{H}$ NMR $\left(\mathrm{CDCl}_{3}, 400 \mathrm{MHz}\right): \delta 1.04$ (s, 3H), 1.06 (s, 3H), 
$2.32(\mathrm{~d}, J=16.0 \mathrm{~Hz}, 1 \mathrm{H}), 2.33(\mathrm{~d}, J=16.0 \mathrm{~Hz}, 1 \mathrm{H}), 2.34(\mathrm{~d}, J=18.8 \mathrm{~Hz}, 1 \mathrm{H}), 2.48(\mathrm{~d}, J=$ $18.8 \mathrm{~Hz}, 1 \mathrm{H}), 2.70(\mathrm{dt}, J=13.2,6.0 \mathrm{~Hz}, 1 \mathrm{H}), 2.79(\mathrm{dt}, J=13.2,8.4 \mathrm{~Hz}, 1 \mathrm{H}), 2.93$ (ddd, $J=$ 8.4, 6.8, $6.0 \mathrm{~Hz}, 1 \mathrm{H}), 3.19$ (d, $J=4.0 \mathrm{~Hz}, 1 \mathrm{H}), 3.29$ (dd, $J=10.4,6.4 \mathrm{~Hz}, 1 \mathrm{H}), 3.36$ (dd, $J=$ $10.4,6.8 \mathrm{~Hz}, 1 \mathrm{H}), 5.05(\mathrm{~d}, J=10.0 \mathrm{~Hz}, 1 \mathrm{H}), 5.11(\mathrm{~d}, J=17.2 \mathrm{~Hz}, 1 \mathrm{H}), 5.52(\mathrm{dd}, J=6.4,4.0$ $\mathrm{Hz}, 1 \mathrm{H})$, 5.75-5.86 (m, 1H), 7.26-7.30 (m, 2H), 7.39-7.43 (m, $1 \mathrm{H}), 7.46-7.51(\mathrm{~m}, 2 \mathrm{H}) ;{ }^{13} \mathrm{C}$ NMR ( $\left.\mathrm{CDCl}_{3}, 100 \mathrm{MHz}\right): \delta 27.7\left(\mathrm{CH}_{3}\right), 29.1\left(\mathrm{CH}_{3}\right), 33.5(\mathrm{C}), 35.9\left(\mathrm{CH}_{2}\right), 40.9(\mathrm{CH}), 42.7$ $(\mathrm{CH}), 45.5(\mathrm{CH}), 46.0\left(\mathrm{CH}_{2}\right), 51.0\left(\mathrm{CH}_{2}\right), 58.7(\mathrm{CH}), 118.0\left(\mathrm{CH}_{2}\right), 126.5(\mathrm{CH}), 128.8(\mathrm{CH})$, $129.2(\mathrm{CH}), 131.6(\mathrm{C}), 132.8(\mathrm{C}), 135.8(\mathrm{CH}), 162.9(\mathrm{C}), 175.9(\mathrm{CO}), 176.5(\mathrm{CO}), 197.1$ (CO); HRMS (ESI) m/z: [M + Na $]^{+}$calcd. for $\mathrm{C}_{23} \mathrm{H}_{25} \mathrm{NO}_{4} \mathrm{Na} 402.1676$, found 402.1676.

$\left(3 \mathrm{a} R^{*}, 4 R^{*}, 7 S^{*}, 7 \mathrm{a} R^{*}\right)-6$-(But-3-en-1-yl)-4-hydroxy-8,8-dimethyl-1,3-dioxo-2-phenyl-2,3,3 a,4,7,7a-hexahydro-1H-4,7-ethanoisoindole-5-carbaldehyde (35a). Ketone-type cyclic adduct 35a (38 mg, $10 \%$ yield over 2 steps) was also isolated as a white solid: $\mathrm{mp}=72$ $75^{\circ} \mathrm{C}$; IR $\left(\mathrm{KBr}, \mathrm{cm}^{-1}\right) v_{\max } 3470(\mathrm{br}), 3361,3068,2959,2920,2850,1774,1711,1654,1597$, 1498, 1385; ${ }^{1} \mathrm{H}$ NMR $\left(\mathrm{CDCl}_{3}, 400 \mathrm{MHz}\right): \delta 1.02$ (s, 3H), 1.25 (s, 3H), 1.59 (d, $J=12.8 \mathrm{~Hz}$, $1 \mathrm{H}), 1.66(\mathrm{~d}, J=12.8 \mathrm{~Hz}, 1 \mathrm{H}), 2.10-2.20(\mathrm{~m}, 1 \mathrm{H}), 2.22-2.32(\mathrm{~m}, 1 \mathrm{H}), 2.58-2.72(\mathrm{~m}, 2 \mathrm{H}), 2.87$ $(\mathrm{d}, J=3.6 \mathrm{~Hz}, 1 \mathrm{H}), 3.00(\mathrm{~d}, J=8.4 \mathrm{~Hz}, 1 \mathrm{H}), 3.55(\mathrm{dd}, J=8.4,3.6 \mathrm{~Hz}, 1 \mathrm{H}), 4.99(\mathrm{dq}, J=10.4$, $1.2 \mathrm{~Hz}, 1 \mathrm{H}$ ), 5.03 (dq, $J=17.2,1.6 \mathrm{~Hz}, 1 \mathrm{H}$ ), 5.63 (br s, $1 \mathrm{H}$ ), 5.75 (ddt, $J=17.2,10.4,6.4 \mathrm{~Hz}$, 1H), 7.07-7.10 (m, 2H), 7.37 (tt, $J=7.2,1.6 \mathrm{~Hz}, 1 \mathrm{H}), 7.41-7.46(\mathrm{~m}, 2 \mathrm{H}), 10.05(\mathrm{~s}, 1 \mathrm{H}) ;{ }^{13} \mathrm{C}$ NMR $\left(\mathrm{CDCl}_{3}, 100 \mathrm{MHz}\right): \delta 29.3\left(\mathrm{CH}_{3}\right), 31.2\left(\mathrm{CH}_{3}\right), 31.5\left(\mathrm{CH}_{2}\right), 31.8\left(\mathrm{CH}_{2}\right), 34.1(\mathrm{C}), 41.8$ $(\mathrm{CH}), 48.2(\mathrm{CH}), 48.2\left(\mathrm{CH}_{2}\right), 50.1(\mathrm{CH}), 76.4(\mathrm{C}), 116.1\left(\mathrm{CH}_{2}\right), 126.1(\mathrm{CH}), 128.8(\mathrm{CH})$, $129.2(\mathrm{CH}), 131.2(\mathrm{C}), 134.0(\mathrm{C}), 136.4(\mathrm{CH}), 162.9(\mathrm{C}), 175.5(\mathrm{CO}), 176.8(\mathrm{CO}), 189.5$ (CHO); HRMS (ESI) m/z: [M + Na $]^{+}$calcd. for $\mathrm{C}_{23} \mathrm{H}_{25} \mathrm{NO}_{4} \mathrm{Na} 402.1676$, found 402.1678.

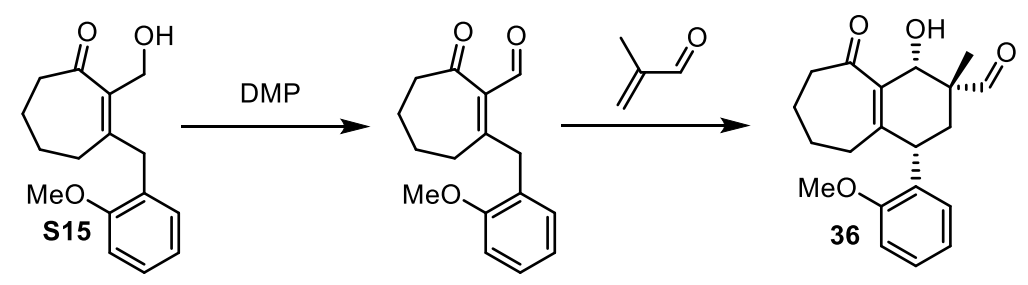

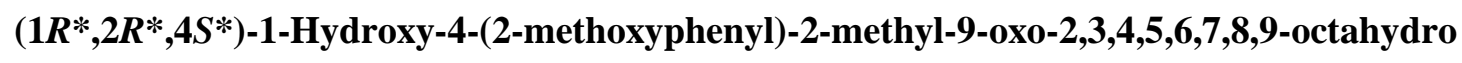
-1H-benzo[7] annulene-2-carbaldehyde (36). Following the general procedure of condition B, compound 36 ( $253 \mathrm{mg}, 77 \%$ yield over 2 steps) was prepared from 2-(hydroxymethyl) enone $\mathbf{S 1 5}$ (260 mg, $1.0 \mathrm{mmol}$ ) and 2-phenylpropenal (159 mg, $1.2 \mathrm{mmol}$ ) as a white solid: $\mathrm{mp}=120-123{ }^{\circ} \mathrm{C}$; IR $\left(\mathrm{KBr}, \mathrm{cm}^{-1}\right) v_{\max } 3449$ (br), 3061, 2937, 2867, 2837, 2713, 1726, 1654, 1624, 1598, 1493, 1460; ${ }^{1} \mathrm{H}$ NMR $\left(\mathrm{CDCl}_{3}, 400 \mathrm{MHz}\right): \delta 1.04$ (s, 3H), 1.40-1.58 (m, 2H), 1.75 (quint, $J=6.8 \mathrm{~Hz}, 2 \mathrm{H}), 1.80-1.89(\mathrm{~m}, 1 \mathrm{H}), 2.09-2.25(\mathrm{~m}, 3 \mathrm{H}), 2.57(\mathrm{dt}, J=15.2,6.0 \mathrm{~Hz}, 1 \mathrm{H})$, 2.65 (dt, $J=15.2,6.8 \mathrm{~Hz}, 1 \mathrm{H}), 2.72$ (br s, 1H), 3.83 (s, 3H), 3.98 (br s, 1H), 4.81 (d, $J=4.4$ $\mathrm{Hz}, 1 \mathrm{H}), 6.90(\mathrm{~d}, J=8.0 \mathrm{~Hz}, 1 \mathrm{H}), 6.94(\mathrm{t}, J=7.6 \mathrm{~Hz}, 1 \mathrm{H}), 7.12(\mathrm{~d}, J=6.4 \mathrm{~Hz}, 1 \mathrm{H}), 7.25$ (t, $J$ $=7.6 \mathrm{~Hz}, 1 \mathrm{H}) ;{ }^{13} \mathrm{C} \mathrm{NMR}\left(\mathrm{CDCl}_{3}, 100 \mathrm{MHz}\right): \delta 16.3\left(\mathrm{CH}_{3}\right), 20.7\left(\mathrm{CH}_{2}\right), 24.4\left(\mathrm{CH}_{2}\right), 30.9(\mathrm{br}$, 
$\left.\mathrm{CH}_{2}\right), 38.5$ (br, C), $41.5\left(\mathrm{CH}_{2}\right), 48.6(\mathrm{C}), 55.2\left(\mathrm{CH}_{3}\right), 67.5(\mathrm{CH}), 110.7(\mathrm{br}, \mathrm{CH}), 121.0(\mathrm{CH})$, 128.1 (br, CH), 157.0 (C), 204.7 (CHO), 205.8 (CO); HRMS (ESI) m/z: [M + Na $]^{+}$calcd. for $\mathrm{C}_{20} \mathrm{H}_{24} \mathrm{O}_{4} \mathrm{Na} 351.1567$, found 351.1564 .

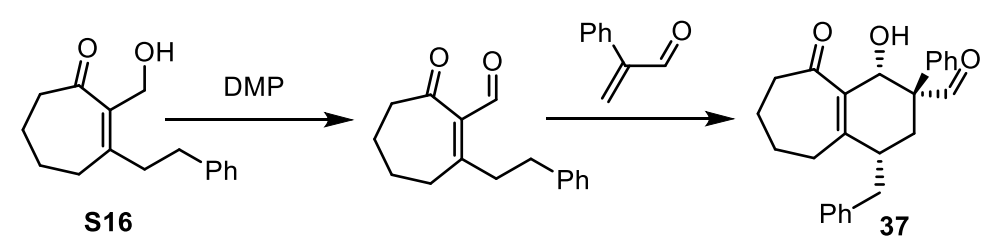

$\left(1 R^{*}, 2 S^{*}, 4 S^{*}\right)$-4-Benzyl-1-hydroxy-9-oxo-2-phenyl-2,3,4,5,6,7,8,9-octahydro-1 $H$-benzo[7

]annulene-2-carbaldehyde (37). Following the general procedure of condition B, compound 37 (333 mg, 89\% yield over 2 steps) was prepared from 2-(hydroxymethyl) enone S16 (244 $\mathrm{mg}, 1.0 \mathrm{mmol})$ and 2-phenylpropenal $(159 \mathrm{mg}, 1.2 \mathrm{mmol})$ as a colorless oil: $\mathrm{IR}\left(\mathrm{CH}_{2} \mathrm{Cl}_{2}\right.$ cast, $\left.\mathrm{cm}^{-1}\right) v_{\max } 3360$ (br), 3026, 2919, 2850, 1723, 1652, 1634, 1494, 1470, 1454; ${ }^{1} \mathrm{H} \mathrm{NMR}\left(\mathrm{CDCl}_{3}\right.$, $400 \mathrm{MHz}): \delta 1.37-1.47(\mathrm{~m}, 1 \mathrm{H}), 1.74-1.88(\mathrm{~m}, 3 \mathrm{H}), 2.01-2.19(\mathrm{~m}, 3 \mathrm{H}), 2.34-2.58(\mathrm{~m}, 4 \mathrm{H})$, $2.61(\mathrm{dt}, J=14.8,5.2 \mathrm{~Hz}, 1 \mathrm{H}), 2.75(\mathrm{dt}, J=14.8,6.0 \mathrm{~Hz}, 1 \mathrm{H}), 3.26(\mathrm{dd}, J=17.6,9.0 \mathrm{~Hz}, 1 \mathrm{H})$, $5.74(\mathrm{~d}, J=3.6 \mathrm{~Hz}, 1 \mathrm{H}), 7.02-7.05(\mathrm{~m}, 2 \mathrm{H}), 7.16-7.18(\mathrm{~m}, 2 \mathrm{H}), 7.21-7.35(\mathrm{~m}, 6 \mathrm{H}), 9.43(\mathrm{~s}$, $1 \mathrm{H}) ;{ }^{13} \mathrm{C} \mathrm{NMR}\left(\mathrm{CDCl}_{3}, 100 \mathrm{MHz}\right): \delta 21.1\left(\mathrm{CH}_{2}\right), 24.9\left(\mathrm{CH}_{2}\right), 27.6\left(\mathrm{CH}_{2}\right), 31.2\left(\mathrm{CH}_{2}\right), 39.2$ $\left(\mathrm{CH}_{2}\right), 41.5\left(\mathrm{CH}_{2}\right), 41.9(\mathrm{CH}), 57.2(\mathrm{C}), 64.2(\mathrm{CH}), 126.4(\mathrm{CH}), 127.4(\mathrm{CH}), 127.5(\mathrm{CH})$, $128.6(\mathrm{CH}), 128.8(\mathrm{CH}), 128.8(\mathrm{CH}), 134.8(\mathrm{C}), 136.6(\mathrm{C}), 139.2(\mathrm{C}), 160.6(\mathrm{C}), 199.4(\mathrm{CHO})$, 205.1 (CO); HRMS (ESI) m/z: [M + Na $]^{+}$calcd. for $\mathrm{C}_{25} \mathrm{H}_{26} \mathrm{O}_{3} \mathrm{Na} 397.1774$, found 397.1773. 
General procedure for ketone-type anionic Diels-Alder reaction:

Condition C:

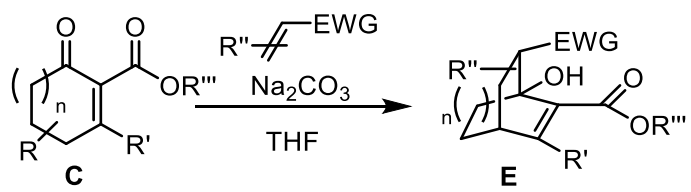

To a stirred solution of the cross-conjugated enone $\mathbf{C}(1.0 \mathrm{mmol})$ and $\mathrm{Na}_{2} \mathrm{CO}_{3}(127 \mathrm{mg}, 1.2$ mmol) in dry THF (5 mL), which was previously degassed with argon, was added dienophile $(1.2 \mathrm{mmol})$ in one portion at room temperature. The resulting mixture was then stirred under individual temperature and time as indicated in Table 3. After reaction was complete, the reaction mixture was quenched with sat. $\mathrm{NH}_{4} \mathrm{Cl}_{(\mathrm{aq})}$ and extracted with EtOAc $(10 \mathrm{~mL} \times 2)$. The combined organic extract was washed with water and brine, dried over $\mathrm{MgSO}_{4}$, filtered and concentrated to give the crude residue, which was purified by chromatography on silica gel to afford anionic Diels-Alder adduct $\mathbf{E}$, including compounds 38-41 as indicated below. Condition D:

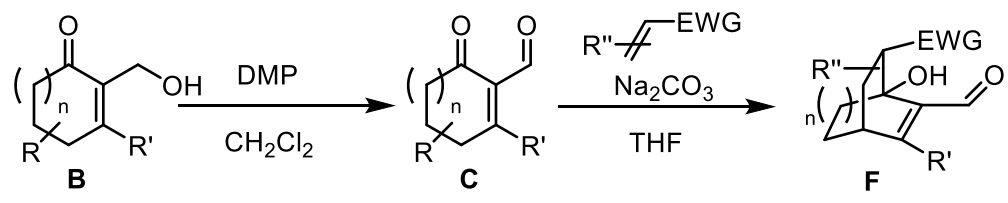

To a stirred solution of the 2-(hydroxymethyl) enone $\mathbf{B}(1.0 \mathrm{mmol})$ in $\operatorname{dry} \mathrm{CH}_{2} \mathrm{Cl}_{2}(10 \mathrm{~mL})$ was added Dess-Martin periodinane $(551 \mathrm{mg}, 1.3 \mathrm{mmol})$ in one portion at $0{ }^{\circ} \mathrm{C}$. The resulting mixture was then stirred at room temperature under $\mathrm{N}_{2}$ for 30 min. After reaction was complete, the reaction mixture was quenched with $5 \% \mathrm{Na}_{2} \mathrm{~S}_{2} \mathrm{O}_{3(\text { aq })}(20 \mathrm{~mL})$. The resulting solution was then stirred at room temperature for $20 \mathrm{~min}$. The aquous layer was extracted with $\mathrm{CH}_{2} \mathrm{Cl}_{2}(10 \mathrm{~mL} \times 2)$. The organic portions were combined, washed with sat. $\mathrm{NaHCO}_{3(\mathrm{aq})}$ and brine, dried over $\mathrm{MgSO}_{4}$, filtered and concentrated to give the cross-conjugated enone $\mathbf{C}$ individually. To a stirred solution of the crude cross-conjugated enone $\mathbf{C}$ and $\mathrm{Na}_{2} \mathrm{CO}_{3}$ (127 $\mathrm{mg}, 1.2 \mathrm{mmol})$ in dry THF $(5 \mathrm{~mL})$, which was previously degassed with argon, was added dienophile $(1.2 \mathrm{mmol})$ in one portion at room temperature. The resulting mixture was then stirred under individual temperature and time as indicated in Table 3. After reaction was complete, the reaction mixture was quenched with sat. $\mathrm{NH}_{4} \mathrm{Cl}_{(\mathrm{aq})}$ and extracted with EtOAc $(10 \mathrm{~mL} \times 2)$. The combined organic extract was washed with water and brine, dried over $\mathrm{MgSO}_{4}$, filtered and concentrated to give the crude residue, which was purified by chromatography on silica gel to afford anionic Diels-Alder adduct $\mathbf{F}$, including compounds 42-46 as indicated below. 


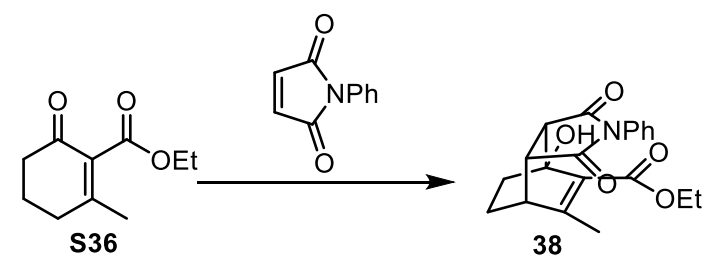

\section{Ethyl}

$\left(3 \mathrm{a} R^{*}, 4 R^{*}, 7 R^{*}, 7 \mathrm{a} R^{*}\right)-4$-hydroxy-6-methyl-1,3-dioxo-2-phenyl-2,3,3a,4,7,7a-hexahydro-1 $\boldsymbol{H - 4 , 7 - e t h a n o i s o i n d o l e - 5 - c a r b o x y l a t e ~ ( 3 8 ) . ~ F o l l o w i n g ~ t h e ~ g e n e r a l ~ p r o c e d u r e ~ o f ~ c o n d i t i o n ~ C , ~}$ compound 38 (270 mg, 76\% yield) was prepared from enone $\mathbf{S 3 6}$ (182 mg, $1.0 \mathrm{mmol}$ ) and $\mathrm{N}$-phenylmaleimide (208 mg, $1.2 \mathrm{mmol})$ as a white solid: $\mathrm{mp}=111-113{ }^{\circ} \mathrm{C}$; $\mathrm{IR}\left(\mathrm{KBr}, \mathrm{cm}^{-1}\right)$ $v_{\max } 3057$ (br), 3361, 3196, 3067, 2919, 2875, 2850, 1775, 1710, 1634, 1598, 1499, 1382; ${ }^{1} \mathrm{H}$ NMR $\left(\mathrm{CDCl}_{3}, 600 \mathrm{MHz}\right): \delta 1.29(\mathrm{t}, J=7.2 \mathrm{~Hz}, 3 \mathrm{H}), 1.65-1.74(\mathrm{~m}, 2 \mathrm{H}), 1.77-1.90(\mathrm{~m}, 2 \mathrm{H})$, $2.04(\mathrm{~s}, 3 \mathrm{H}), 3.02(\mathrm{~d}, J=8.4 \mathrm{~Hz}, 1 \mathrm{H}), 3.09-3.11(\mathrm{~m}, 1 \mathrm{H}), 3.22(\mathrm{dd}, J=8.4,3.6 \mathrm{~Hz}, 1 \mathrm{H})$, 4.22-4.32 (m, 2H), $4.88(\mathrm{~s}, 1 \mathrm{H}), 7.15(\mathrm{~d}, J=7.2 \mathrm{~Hz}, 2 \mathrm{H}), 7.37(\mathrm{t}, J=7.2 \mathrm{~Hz}, 1 \mathrm{H}), 7.44(\mathrm{t}, J=$ $7.2 \mathrm{~Hz}, 2 \mathrm{H}) ;{ }^{13} \mathrm{C} \mathrm{NMR}\left(\mathrm{CDCl}_{3}, 150 \mathrm{MHz}\right): \delta 14.2\left(\mathrm{CH}_{3}\right), 19.8\left(\mathrm{CH}_{3}\right), 22.5\left(\mathrm{CH}_{2}\right), 32.7\left(\mathrm{CH}_{2}\right)$, $39.0(\mathrm{CH}), 44.9(\mathrm{CH}), 49.3(\mathrm{CH}), 61.1\left(\mathrm{CH}_{2}\right), 74.4(\mathrm{C}), 126.4(\mathrm{CH}), 128.7(\mathrm{CH}), 129.1(\mathrm{CH})$, 130.5 (C), 131.5 (C), 147.3 (C), 165.2 (CO), 175.8 (CO), 176.4 (CO); HRMS (ESI) m/z: [M + $\mathrm{Na}]^{+}$calcd. for $\mathrm{C}_{20} \mathrm{H}_{21} \mathrm{NO}_{5} \mathrm{Na} 378.1312$, found 378.1307.

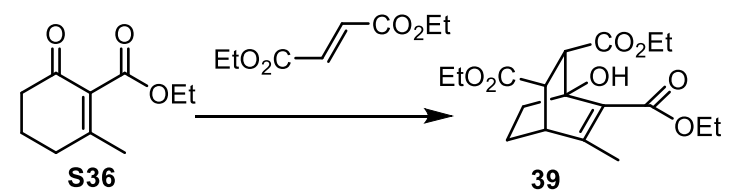

\section{Triethyl}

$\left(1 R^{*}, 2 S^{*}, 3 R^{*}, 4 R^{*}\right)$-4-hydroxy-6-methylbicyclo[2.2.2] ]oct-5-ene-2,3,5-tricarboxylate (39). Following the general procedure of condition C, compound 39 (252 mg, $71 \%$ yield) was prepared from enone $\mathbf{S 3 6}$ (182 $\mathrm{mg}, 1.0 \mathrm{mmol}$ ) and diethyl fumarate (207 $\mathrm{mg}, 1.2 \mathrm{mmol}$ ) as a colorless oil: IR $\left(\mathrm{CH}_{2} \mathrm{Cl}_{2}\right.$ cast, $\left.\mathrm{cm}^{-1}\right) v_{\max } 3362$ (br), 2981, 2960, 2938, 2919, 2876, 2850, 1731, 1675, 1633; ${ }^{1} \mathrm{H}$ NMR ( $\left.\mathrm{CDCl}_{3}, 400 \mathrm{MHz}\right): \delta 1.27$ (t, $\left.J=7.2 \mathrm{~Hz}, 3 \mathrm{H}\right), 1.28$ (t, $J=7.2 \mathrm{~Hz}$, $3 \mathrm{H}), 1.34(\mathrm{t}, J=7.2 \mathrm{~Hz}, 3 \mathrm{H}), 1.35-1.44(\mathrm{~m}, 1 \mathrm{H}), 1.56(\mathrm{td}, J=12.0,4.0 \mathrm{~Hz}, 1 \mathrm{H}), 1.66-1.74(\mathrm{~m}$, 1H), 1.82 (ddd, $J=12.0,10.0,4.4 \mathrm{~Hz}, 1 \mathrm{H}), 2.22(\mathrm{~s}, 3 \mathrm{H}), 2.79$ (q, $J=2.8 \mathrm{~Hz}, 1 \mathrm{H}), 2.90$ (ddd, $J$ $=5.6,2.8,2.0 \mathrm{~Hz}, 1 \mathrm{H}), 3.26(\mathrm{~d}, J=5.6 \mathrm{~Hz}, 1 \mathrm{H}), 4.12-4.31(\mathrm{~m}, 6 \mathrm{H}), 5.69(\mathrm{~s}, 1 \mathrm{H}) ;{ }^{13} \mathrm{C} \mathrm{NMR}$ $\left(\mathrm{CDCl}_{3}, 150 \mathrm{MHz}\right): \delta 14.2\left(\mathrm{CH}_{3}\right), 14.2\left(\mathrm{CH}_{3}\right), 14.2\left(\mathrm{CH}_{3}\right), 19.7\left(\mathrm{CH}_{3}\right), 20.3\left(\mathrm{CH}_{2}\right), 33.7\left(\mathrm{CH}_{2}\right)$, $40.7(\mathrm{CH}), 46.0(\mathrm{CH}), 51.8(\mathrm{CH}), 60.8\left(\mathrm{CH}_{2}\right), 61.1\left(\mathrm{CH}_{2}\right), 61.2\left(\mathrm{CH}_{2}\right), 74.3(\mathrm{C}), 128.5(\mathrm{C})$, 151.9 (C), 166.8 (CO), $172.3(\mathrm{CO}), 173.0(\mathrm{CO})$; HRMS (ESI) m/z: $[\mathrm{M}+\mathrm{Na}]^{+}$calcd. for $\mathrm{C}_{18} \mathrm{H}_{26} \mathrm{O}_{7} \mathrm{Na} 377.1571$, found 377.1564 . 


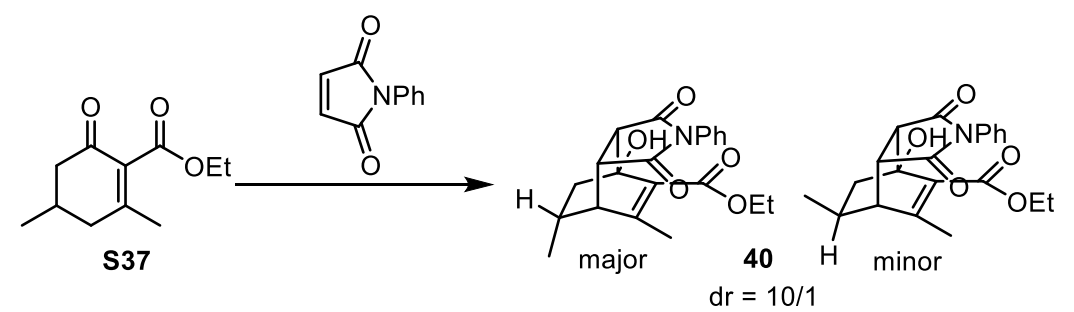

\section{Ethyl}

$\left(3 \mathrm{a} R^{*}, 4 R^{*}, 7 R^{*}, 7 \mathrm{a} R^{*}, 9 R^{*}\right)-7-h y d r o x y-5,9-d i m e t h y l-1,3-d i o x o-2-p h e n y l-2,3,3 \mathrm{a}, 4,7,7 \mathrm{a}-\mathrm{hexa}$ hydro-1H-4,7-ethanoisoindole-6-carboxylate (40). Following the general procedure of condition C, compound $\mathbf{4 0}$ (292 mg, 79\% yield) was prepared from enone S37 (196 mg, 1.0 mmol) and $N$-phenylmaleimide (208 mg, $1.2 \mathrm{mmol})$ as a white solid: $\mathrm{mp}=129-132{ }^{\circ} \mathrm{C}$; IR $\left(\mathrm{KBr}, \mathrm{cm}^{-1}\right) v_{\max } 3462$ (br), 3063, 2938, 2857, 1773, 1708, 1631, 1597, 1498, 1387; ${ }^{1} \mathrm{H}$ NMR $\left(\mathrm{CDCl}_{3}, 600 \mathrm{MHz}\right)$ mixture of diastereomers: $\delta 0.97(\mathrm{~d}, J=6.0 \mathrm{~Hz}, 3 \mathrm{H}), 1.17(\mathrm{~d}, J=6.0 \mathrm{~Hz}$, $0.3 \mathrm{H}), 1.24-1.27(\mathrm{~m}, 1.1 \mathrm{H}), 1.29(\mathrm{t}, J=7.2 \mathrm{~Hz}, 3.3 \mathrm{H}), 2.04(\mathrm{~s}, 0.3 \mathrm{H}), 2.05(\mathrm{~s}, 3 \mathrm{H}), 2.08-2.11$ $(\mathrm{m}, 2.2 \mathrm{H}), 2.83-2.85(\mathrm{~m}, 0.1 \mathrm{H}), 2.94-2.95(\mathrm{~m}, 1 \mathrm{H}), 2.97(\mathrm{~d}, J=8.4 \mathrm{~Hz}, 1 \mathrm{H}), 2.99(\mathrm{~d}, J=8.4$ $\mathrm{Hz}, 0.1 \mathrm{H}), 3.23(\mathrm{dd}, J=8.4,3.6 \mathrm{~Hz}, 1 \mathrm{H}), 3.42(\mathrm{dd}, J=8.4,3.0 \mathrm{~Hz}, 0.1 \mathrm{H}), 4.21-4.32(\mathrm{~m}$, $2.2 \mathrm{H}), 4.86$ (br s, 1H), 7.14 (d, $J=7.2 \mathrm{~Hz}, 2 \mathrm{H}), 7.37$ (t, $J=7.2 \mathrm{~Hz}, 1.1 \mathrm{H}), 7.43$ (t, $J=7.2 \mathrm{~Hz}$, 2.2H); ${ }^{13} \mathrm{C} \mathrm{NMR}\left(\mathrm{CDCl}_{3}, 150 \mathrm{MHz}\right)$ major: $\delta 14.2\left(\mathrm{CH}_{3}\right), 21.6\left(\mathrm{CH}_{3}\right), 21.6\left(\mathrm{CH}_{3}\right), 30.3(\mathrm{CH})$, $41.5\left(\mathrm{CH}_{2}\right), 45.3(\mathrm{CH}), 45.6(\mathrm{CH}), 48.5(\mathrm{CH}), 61.1\left(\mathrm{CH}_{2}\right), 74.6(\mathrm{C}), 126.3(\mathrm{CH}), 128.7(\mathrm{CH})$, $129.1(\mathrm{CH}), 130.0(\mathrm{C}), 131.6(\mathrm{C}), 146.3(\mathrm{C}), 165.2(\mathrm{CO}), 175.7$ (CO), $176.3(\mathrm{CO})$; HRMS (ESI) $\mathrm{m} / \mathrm{z}:[\mathrm{M}+\mathrm{Na}]^{+}$calcd. for $\mathrm{C}_{21} \mathrm{H}_{23} \mathrm{NO}_{5} \mathrm{Na} 392.1468$, found 392.1471 .

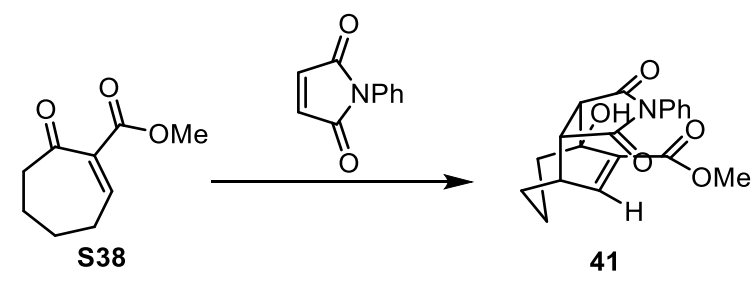

\section{Methyl}

$\left(3 \mathrm{a} R^{*}, 8 R^{*}, 8 \mathrm{a} R^{*}\right)-8$-hydroxy-1,3-dioxo-2-phenyl-1,2,3,3a,4,5,6,7,8,8a-decahydro-4,8-ethe nocyclohepta[ $[c]$ pyrrole-9-carboxylate (41). Following the general procedure of condition $\mathrm{C}$, compound 41 (321 mg, 94\% yield) was prepared from enone $\mathbf{S 3 8}$ (168 mg, $1.0 \mathrm{mmol}$ ) and $\mathrm{N}$-phenylmaleimide (208 mg, $1.2 \mathrm{mmol})$ as a white solid: $\mathrm{mp}=161-163{ }^{\circ} \mathrm{C}$; IR $\left(\mathrm{KBr}, \mathrm{cm}^{-1}\right)$ $v_{\max } 3462$ (br), 3063, 2938, 2857, 1773, 1708, 1631, 1597, 1498, 1387; ${ }^{1} \mathrm{H}$ NMR $\left(\mathrm{CDCl}_{3}, 400\right.$ MHz): $\delta 1.67-1.94(\mathrm{~m}, 5 \mathrm{H}), 2.06-2.13(\mathrm{~m}, 1 \mathrm{H}), 3.25-3.29(\mathrm{~m}, 1 \mathrm{H}), 3.28(\mathrm{dd}, J=8.8,1.6 \mathrm{~Hz}$, $1 \mathrm{H}), 3.41(\mathrm{~d}, J=8.8 \mathrm{~Hz}, 1 \mathrm{H}), 3.78(\mathrm{~s}, 3 \mathrm{H}), 5.20(\mathrm{~s}, 1 \mathrm{H}), 6.78(\mathrm{~d}, J=8.0 \mathrm{~Hz}, 1 \mathrm{H}), 7.19-7.22$ $(\mathrm{m}, 2 \mathrm{H}), 7.39(\mathrm{tt}, J=7.2,1.2 \mathrm{~Hz}, 1 \mathrm{H}), 7.43-7.48(\mathrm{~m}, 2 \mathrm{H}) ;{ }^{13} \mathrm{C} \mathrm{NMR}\left(\mathrm{CDCl}_{3}, 150 \mathrm{MHz}\right): \delta$ $21.7\left(\mathrm{CH}_{2}\right), 26.3\left(\mathrm{CH}_{2}\right), 35.0(\mathrm{CH}), 38.6\left(\mathrm{CH}_{2}\right), 44.2(\mathrm{CH}), 50.1(\mathrm{CH}), 52.2\left(\mathrm{CH}_{3}\right), 73.5(\mathrm{C})$, 
$126.4(\mathrm{C}), 128.8(\mathrm{CH}), 129.1(\mathrm{CH}), 131.4(\mathrm{C}), 137.5(\mathrm{C}), 139.6(\mathrm{CH}), 166.2(\mathrm{CO}), 176.9(\mathrm{CO})$, 177.4 (CO); HRMS (ESI) m/z: [M + Na $]^{+}$calcd. for $\mathrm{C}_{19} \mathrm{H}_{19} \mathrm{NO}_{5} \mathrm{Na} 364.1155$, found 364.1156.

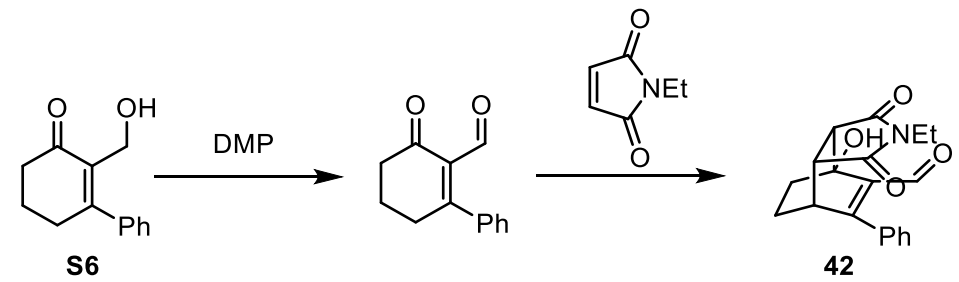

$\left(3 \mathrm{a} R^{*}, 4 R^{*}, 7 R^{*}, 7 \mathrm{a} S^{*}\right)$-2-Ethyl-4-hydroxy-1,3-dioxo-6-phenyl-2,3,3a,4,7,7a-hexahydro-1H -4,7-ethanoisoindole-5-carbaldehyde (42). Following the general procedure of condition D, compound adduct 42 (273 mg, 84\% yield over 2 steps) was prepared from 2-(hydroxymethyl) enone $\mathbf{S 6}$ (202 $\mathrm{mg}, 1.0 \mathrm{mmol})$ and $N$-ethylmaleimide $(150 \mathrm{mg}, 1.2 \mathrm{mmol})$ as a white solid: $\mathrm{mp}$ $=170-172{ }^{\circ} \mathrm{C}$; IR $\left(\mathrm{KBr}, \mathrm{cm}^{-1}\right) v_{\max } 3446$ (br), 2919, 2850, 1770, 1697, 1651, 1568; ${ }^{1} \mathrm{H}$ NMR $\left(\mathrm{CDCl}_{3}, 400 \mathrm{MHz}\right): \delta 0.94(\mathrm{t}, J=7.2 \mathrm{~Hz}, 3 \mathrm{H}), 1.68-1.84(\mathrm{~m}, 2 \mathrm{H}), 1.91-2.02(\mathrm{~m}, 2 \mathrm{H}), 3.03(\mathrm{~d}$, $J=8.4 \mathrm{~Hz}, 1 \mathrm{H}), 3.17(\mathrm{dd}, J=8.4,3.2 \mathrm{~Hz}, 1 \mathrm{H}), 3.42-3.51(\mathrm{~m}, 2 \mathrm{H}), 3.54(\mathrm{q}, J=3.2 \mathrm{~Hz}, 1 \mathrm{H})$, $6.22(\mathrm{~s}, 1 \mathrm{H}), 7.29-7.32(\mathrm{~m}, 2 \mathrm{H}), 7.41-7.48(\mathrm{~m}, 3 \mathrm{H}), 9.54(\mathrm{~s}, 1 \mathrm{H}) ;{ }^{13} \mathrm{C} \mathrm{NMR}\left(\mathrm{CDCl}_{3}, 150\right.$ MHz): $\delta 13.2\left(\mathrm{CH}_{3}\right), 23.3\left(\mathrm{CH}_{2}\right), 32.5\left(\mathrm{CH}_{2}\right), 33.5\left(\mathrm{CH}_{2}\right), 40.1(\mathrm{CH}), 44.8(\mathrm{CH}), 49.8(\mathrm{CH})$, $76.1(\mathrm{C}), 128.8(\mathrm{CH}), 129.4(\mathrm{CH}), 130.2(\mathrm{CH}), 133.7(\mathrm{C}), 135.1(\mathrm{C}), 161.4(\mathrm{C}), 175.7(\mathrm{CO})$, 177.2 (CO), 191.5 (CHO); HRMS (ESI) m/z: $[\mathrm{M}+\mathrm{Na}]^{+}$calcd. for $\mathrm{C}_{19} \mathrm{H}_{19} \mathrm{NO}_{4} \mathrm{Na} 348.1206$, found 348.1208 .

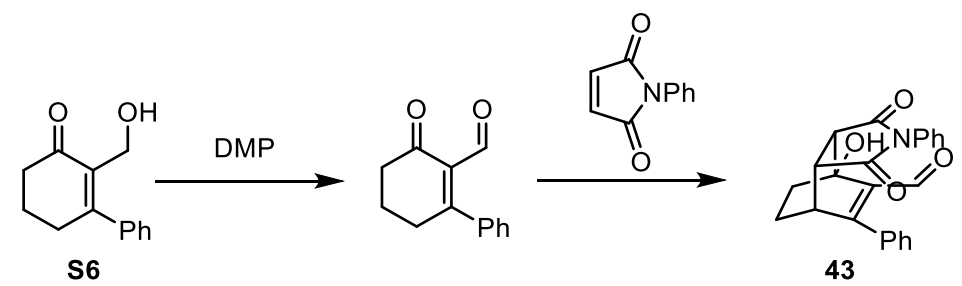

$\left(3 \mathrm{a} R^{*}, 4 R^{*}, 7 R^{*}, 7 \mathrm{a} R^{*}\right)-4$-hydroxy-1,3-dioxo-2,6-diphenyl-2,3,3a,4,7,7a-hexahydro-1H-4,7ethanoisoindole-5-carbaldehyde (43). Following the general procedure of condition D, compound 43 (347 mg, 93\% yield over 2 steps) was prepared from 2-(hydroxymethyl) enone S6 (202 mg, $1.0 \mathrm{mmol})$ and $N$-phenylmaleimide $(208 \mathrm{mg}, 1.2 \mathrm{mmol})$ as a white solid: $\mathrm{mp}=$ 178-180 ${ }^{\circ} \mathrm{C}$; IR (KBr, cm $\left.{ }^{-1}\right) v_{\max } 3464$ (br), 3061, 2917, 2874, 2850, 1774, 1711, 1648, 1597, 1498, 1383; ${ }^{1} \mathrm{H}$ NMR $\left(\mathrm{CDCl}_{3}, 400 \mathrm{MHz}\right): \delta 1.73-1.90$ (m, 2H), 1.96-2.10 (m, 2H), $3.22(\mathrm{~d}, J$ $=8.8 \mathrm{~Hz}, 1 \mathrm{H}), 3.37(\mathrm{dd}, J=8.8,3.2 \mathrm{~Hz}, 1 \mathrm{H}), 3.64(\mathrm{q}, J=3.2 \mathrm{~Hz}, 1 \mathrm{H}), 6.25(\mathrm{~s}, 1 \mathrm{H}), 7.05-7.08$ (m, 2H), 7.30-7.48 (m, 8H), $9.64(\mathrm{~s}, 1 \mathrm{H}) ;{ }^{13} \mathrm{C} \mathrm{NMR}\left(\mathrm{CDCl}_{3}, 100 \mathrm{MHz}\right): \delta 23.4\left(\mathrm{CH}_{2}\right), 32.6$ $\left(\mathrm{CH}_{2}\right), 40.3(\mathrm{CH}), 44.9(\mathrm{CH}), 49.9(\mathrm{CH}), 76.2(\mathrm{C}), 126.0(\mathrm{CH}), 128.7(\mathrm{CH}), 128.9(\mathrm{CH})$, $129.1(\mathrm{CH}), 129.4(\mathrm{CH}), 130.3(\mathrm{CH}), 131.2(\mathrm{C}), 133.7(\mathrm{C}), 135.4(\mathrm{C}), 161.4(\mathrm{C}), 174.9(\mathrm{CO})$, 176.3 (CO), 191.6 (CO); HRMS (ESI) m/z: [M + Na] calcd. for $\mathrm{C}_{23} \mathrm{H}_{19} \mathrm{NO}_{4}$ 396.1206, found 396.1207. 


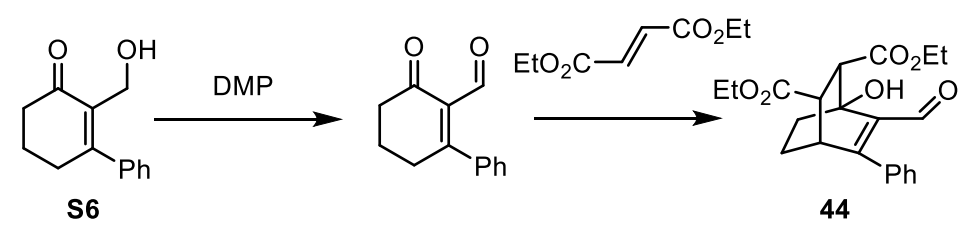

\section{Diethyl}

$\left(1 R^{*}, 2 R^{*}, 3 S^{*}, 4 R^{*}\right)$-6-formyl-1-hydroxy-5-phenylbicyclo[2.2.2] oct-5-ene-2,3-dicarboxylat e (44). Following the general procedure of condition D, compound 44 (272 mg, 73\% yield over 2 steps) was prepared from 2-(hydroxymethyl) enone $\mathbf{S 6}$ (202 mg, $1.0 \mathrm{mmol}$ ) and diethyl fumarate (207 mg, $1.2 \mathrm{mmol}$ ) as a colorless oil: IR $\left(\mathrm{CH}_{2} \mathrm{Cl}_{2}\right.$ cast, $\left.\mathrm{cm}^{-1}\right) v_{\max } 3401$ (br), 3058, 1980, 2940, 2875, 1732, 1650, 1594; ${ }^{1} \mathrm{H}$ NMR $\left(\mathrm{CDCl}_{3}, 400 \mathrm{MHz}\right): \delta 1.27$ (t, $\left.J=7.2 \mathrm{~Hz}, 3 \mathrm{H}\right)$, $1.28(\mathrm{t}, J=7.2 \mathrm{~Hz}, 3 \mathrm{H}), 1.59-1.74(\mathrm{~m}, 2 \mathrm{H}), 1.90-1.99(\mathrm{~m}, 2 \mathrm{H}), 2.93(\mathrm{dt}, J=5.6,2.8 \mathrm{~Hz}, 1 \mathrm{H})$, $3.31(\mathrm{q}, J=2.8 \mathrm{~Hz}, 1 \mathrm{H}), 3.37$ (d, $J=5.6 \mathrm{~Hz}, 1 \mathrm{H}), 4.09-4.24(\mathrm{~m}, 4 \mathrm{H}), 6.59$ (s, 1H), 7.36-7.39 (m, 2H), 7.45-7.48 (m, 3H), $9.62(\mathrm{~s}, 1 \mathrm{H}) ;{ }^{13} \mathrm{C} \mathrm{NMR}\left(\mathrm{CDCl}_{3}, 150 \mathrm{MHz}\right): \delta 14.2\left(\mathrm{CH}_{3}\right), 14.2$ $\left(\mathrm{CH}_{3}\right), 21.0\left(\mathrm{CH}_{2}\right), 33.6\left(\mathrm{CH}_{2}\right), 40.9(\mathrm{CH}), 46.8(\mathrm{CH}), 51.9(\mathrm{CH}), 61.1\left(\mathrm{CH}_{2}\right), 61.4\left(\mathrm{CH}_{2}\right)$, $76.5(\mathrm{C}), 128.6(\mathrm{CH}), 129.1(\mathrm{CH}), 129.8(\mathrm{CH}), 134.7(\mathrm{C}), 135.9(\mathrm{C}), 162.4(\mathrm{C}), 171.7(\mathrm{CO})$, 173.4 (CO), 193.0 (CO); HRMS (ESI) m/z: $[\mathrm{M}+\mathrm{Na}]^{+}$calcd. for $\mathrm{C}_{21} \mathrm{H}_{24} \mathrm{O}_{6} \mathrm{Na} 395.1465$, found 395.1464 .

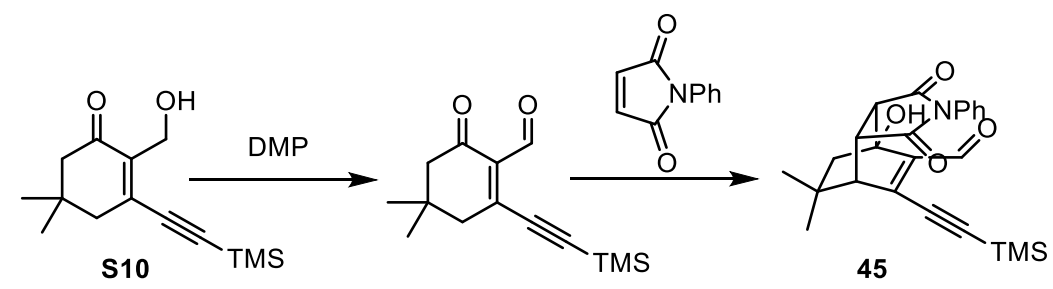

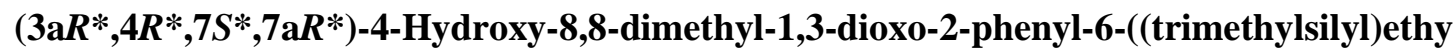
nyl)-2,3,3a,4,7,7a-hexahydro-1H-4,7-ethanoisoindole-5-carbaldehyde (45). Following the general procedure of condition D, compound 45 (400 mg, 95\% yield over 2 steps) was prepared from 2-(hydroxymethyl) enone $\mathbf{S 1 0}(250 \mathrm{mg}, 1.0 \mathrm{mmol})$ and $\mathrm{N}$-phenylmaleimide (208 mg, $1.2 \mathrm{mmol}$ ) as a white solid: $\mathrm{mp}=135-137^{\circ} \mathrm{C}$; IR $\left(\mathrm{KBr}, \mathrm{cm}^{-1}\right) v_{\max } 3478$ (br), 3065, 2962, 2925, 2871, 2851, 2139, 1778, 1714, 1662, 1598, 1570, 1500, 1385; ${ }^{1} \mathrm{H}$ NMR $\left(\mathrm{CDCl}_{3}\right.$, $400 \mathrm{MHz}): \delta 0.23(\mathrm{~s}, 9 \mathrm{H}), 1.09$ (s, 3H), $1.24(\mathrm{~s}, 3 \mathrm{H}), 1.63(\mathrm{~d}, J=12.8 \mathrm{~Hz}, 1 \mathrm{H}), 1.69(\mathrm{~d}, J=$ $12.8,1 \mathrm{H}), 3.05(\mathrm{~d}, J=3.2 \mathrm{~Hz}, 1 \mathrm{H}), 3.06(\mathrm{~d}, J=8.4 \mathrm{~Hz}, 1 \mathrm{H}), 3.53(\mathrm{dd}, J=8.4,3.2 \mathrm{~Hz}, 1 \mathrm{H})$, $5.73(\mathrm{~s}, 1 \mathrm{H}), 7.10-7.13(\mathrm{~m}, 2 \mathrm{H}), 7.35-7.45(\mathrm{~m}, 3 \mathrm{H}), 10.06(\mathrm{~s}, 1 \mathrm{H}) ;{ }^{13} \mathrm{C} \mathrm{NMR}\left(\mathrm{CDCl}_{3}, 150\right.$ MHz): $\delta 0.5\left(\mathrm{CH}_{3}\right), 28.9\left(\mathrm{CH}_{3}\right), 30.4\left(\mathrm{CH}_{3}\right), 34.2(\mathrm{C}), 41.3(\mathrm{CH}), 47.9\left(\mathrm{CH}_{2}\right), 48.9(\mathrm{CH}), 51.5$ $(\mathrm{CH}), 76.5(\mathrm{C}), 98.1(\mathrm{C}), 111.5(\mathrm{C}), 126.2(\mathrm{CH}), 128.8(\mathrm{CH}), 129.1(\mathrm{CH}), 131.4(\mathrm{C}), 141.5$ (C), $142.8(\mathrm{C}), 174.6(\mathrm{CO}), 175.8(\mathrm{CO}), 189.9(\mathrm{CO})$; HRMS (ESI) m/z: [M + Na] $]^{+}$calcd. for $\mathrm{C}_{24} \mathrm{H}_{27} \mathrm{NO}_{4} \mathrm{SiNa} 444.1602$, found 444.1604. 


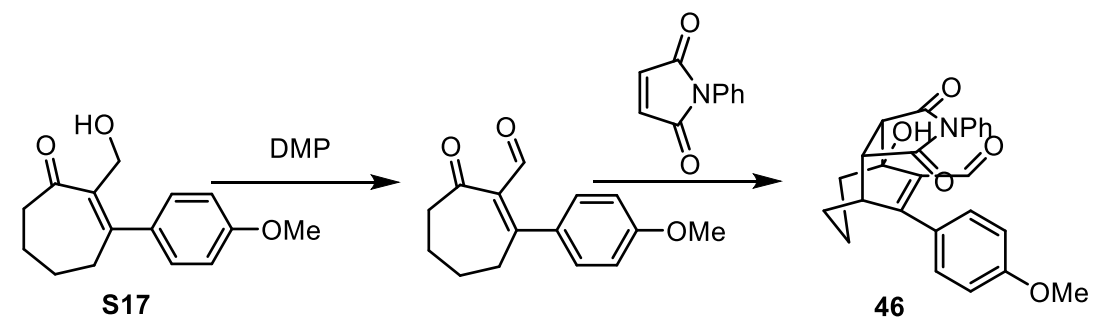

$\left(3 \mathrm{a} R^{*}, 8 R^{*}, 8 \mathrm{a} R^{*}\right)-8$-hydroxy-10-(4-methoxyphenyl)-1,3-dioxo-2-phenyl-1,2,3,3a,4,5,6,7,8, 8a-decahydro-4,8-ethenocyclohepta[c]pyrrole-9-carbaldehyde (46). Following the general procedure of condition D, product 46 (376 mg, 90\% yield over 2 steps) was prepared from 2-(hydroxymethyl) enone $\mathbf{S 1 7}$ (246 mg, $1.0 \mathrm{mmol}$ ) and $N$-phenylmaleimide (208 mg, 1.2 mmol) as a white solid: $\mathrm{mp}=167-169{ }^{\circ} \mathrm{C}$; IR $\left(\mathrm{KBr}, \mathrm{cm}^{-1}\right) v_{\max } 3502(\mathrm{br}), 3065,2922,2851$, 1774, 1709, 1647, 1602, 1509, 1386; ${ }^{1} \mathrm{H}$ NMR $\left(\mathrm{CDCl}_{3}, 400 \mathrm{MHz}\right): \delta 1.74-1.83(\mathrm{~m}, 3 \mathrm{H})$, $1.85-1.96(\mathrm{~m}, 1 \mathrm{H}), 2.02-2.09(\mathrm{~m}, 1 \mathrm{H}), 2.14-2.21(\mathrm{~m}, 1 \mathrm{H}), 3.40(\mathrm{dd}, J=8.8,1.6 \mathrm{~Hz}, 1 \mathrm{H}), 3.52$ $(\mathrm{d}, J=8.8 \mathrm{~Hz}, 1 \mathrm{H}), 3.57-3.59(\mathrm{~m}, 1 \mathrm{H}), 3.83(\mathrm{~s}, 3 \mathrm{H}), 6.91-6.94(\mathrm{~m}, 2 \mathrm{H}), 7.13-7.16(\mathrm{~m}, 2 \mathrm{H})$, 7.19-7.22 (m, 2H), 7.35-7.45 (m, 3H), $9.53(\mathrm{~s}, 1 \mathrm{H}) ;{ }^{13} \mathrm{C} \mathrm{NMR}\left(\mathrm{CDCl}_{3}, 100 \mathrm{MHz}\right): \delta 21.9$ $\left(\mathrm{CH}_{2}\right), 26.2\left(\mathrm{CH}_{2}\right), 40.8\left(\mathrm{CH}_{2}\right), 43.7(\mathrm{CH}), 43.9(\mathrm{CH}), 50.3(\mathrm{CH}), 55.3(\mathrm{CH}), 75.6(\mathrm{C}), 114.1$ $(\mathrm{CH}), 126.2(\mathrm{CH}), 127.6(\mathrm{C}), 128.7(\mathrm{CH}), 129.1(\mathrm{CH}), 130.7(\mathrm{CH}), 131.4(\mathrm{C}), 136.9(\mathrm{C})$, $161.1(\mathrm{C}), 161.3(\mathrm{C}), 175.4(\mathrm{CO}), 177.4(\mathrm{CO}), 193.0$ (CHO); HRMS (ESI) m/z: [M + Na $]^{+}$ calcd. for $\mathrm{C}_{25} \mathrm{H}_{23} \mathrm{NO}_{5} \mathrm{Na} 440.1468$, found 440.1473 . 


\section{X-ray crystal structure of anionic Diels-Alder compounds}

The recrystallization of $\mathbf{9}$ was carried out by the vapor diffusion method: A 4-mL vial containing a solution of $9(15 \mathrm{mg})$ in ehtyl acetate $(2 \mathrm{~mL})$ was placed into a $20 \mathrm{~mL}$ vial containing $n$-hexane $(10 \mathrm{~mL})$. The outer bottle was capped and allowed to stand at $4{ }^{\circ} \mathrm{C}$ for 3 days to afford the compound 9 crystals.

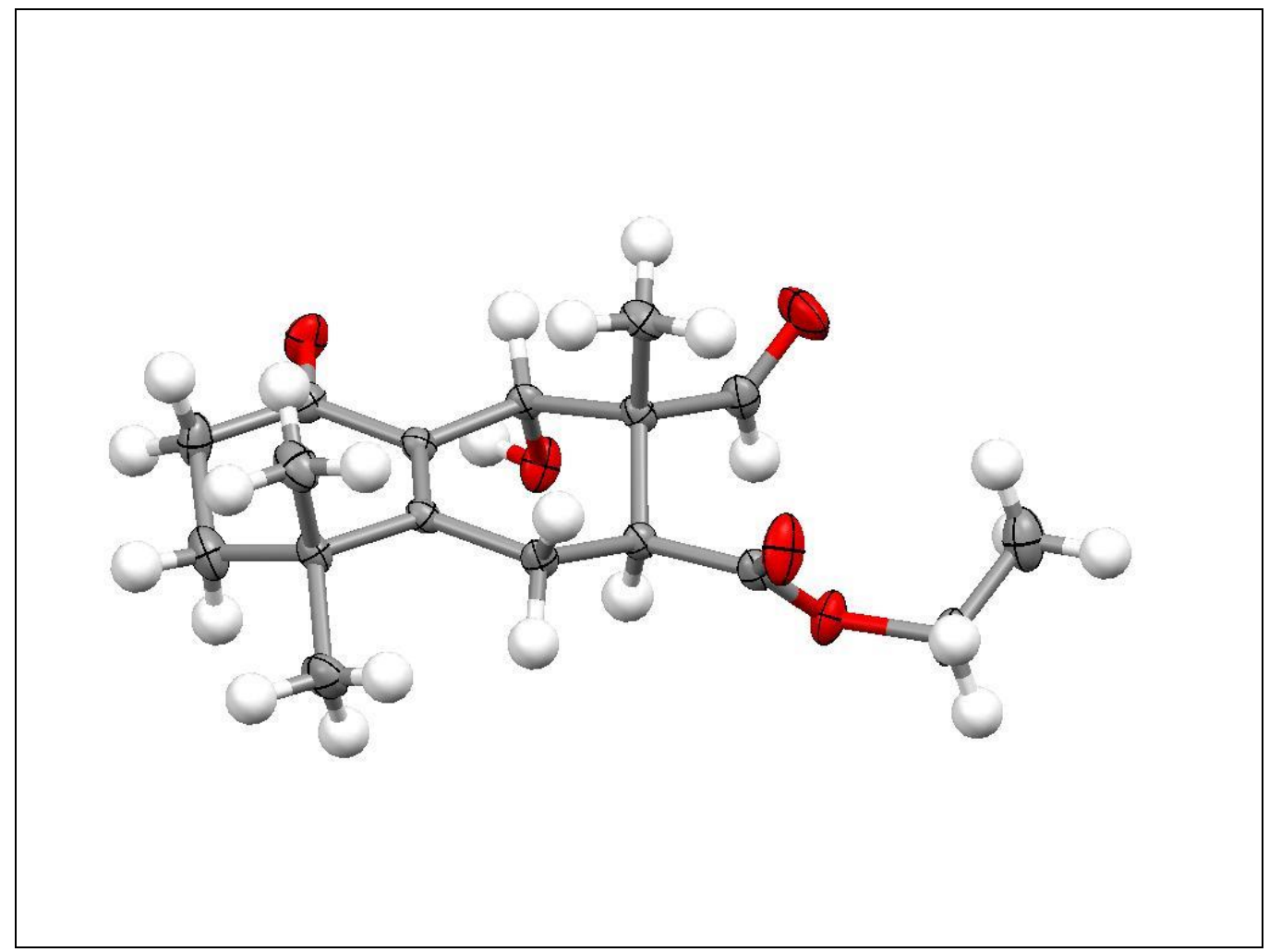

X-ray crystal structure of $\mathbf{9}$ (CCDC 2074048)

Thermal ellipsoid plot at 50\% probability level. 
Table S1. Crystal data and structure refinement for 9 (d22713).

Identification code

d22713

Empirical formula

$\mathrm{C}_{17} \mathrm{H}_{24} \mathrm{O}_{5}$

Formula weight

308.36

Temperature

200(2) K

Wavelength

$0.71073 \AA$

Crystal system

Monoclinic

Space group

Unit cell dimensions

P 21/c

$\begin{array}{ll}\mathrm{a}=7.7174(4) \AA & \mathrm{a}=90^{\circ} . \\ \mathrm{b}=17.1948(6) \AA & \mathrm{b}=\end{array}$

$102.4860(10)^{\circ}$.

Volume

$\mathrm{c}=12.3310(6) \AA$

$\mathrm{g}=90^{\circ}$.

1597.61(13) $\AA^{3}$

$\mathrm{Z}$

4

Density (calculated)

$1.282 \mathrm{Mg} / \mathrm{m}^{3}$

Absorption coefficient

$0.093 \mathrm{~mm}^{-1}$

$\mathrm{F}(000)$

664

Crystal size

$0.68 \times 0.55 \times 0.47 \mathrm{~mm}^{3}$

Theta range for data collection

2.37 to $25.06^{\circ}$.

Index ranges

$-8<=\mathrm{h}<=9,-19<=\mathrm{k}<=20,-13<=\mathrm{l}<=14$

Reflections collected

24255

Independent reflections

Completeness to theta $=25.06^{\circ}$

$2817[\mathrm{R}(\mathrm{int})=0.0836]$

$99.3 \%$

Absorption correction

multi-scan

Max. and min. transmission

0.9574 and 0.9392

Refinement method

Full-matrix least-squares on $\mathrm{F}^{2}$

Data / restraints / parameters

2817 / 0 / 203

Goodness-of-fit on $\mathrm{F}^{2}$

1.032

Final $\mathrm{R}$ indices [I>2sigma(I)]

$\mathrm{R} 1=0.0558, \mathrm{wR} 2=0.1520$

$\mathrm{R}$ indices (all data)

$\mathrm{R} 1=0.0642, \mathrm{wR} 2=0.1598$

Largest diff. peak and hole

0.607 and -0.281 e. $\AA^{-3}$ 
The recrystallization of $\mathbf{1 0}$ was carried out by the vapor diffusion method: A 4-mL vial containing a solution of $\mathbf{1 0}(15 \mathrm{mg})$ in diethyl ether $(2 \mathrm{~mL})$ was placed into a $20 \mathrm{~mL}$ vial containing $n$-hexane $(10 \mathrm{~mL})$. The outer bottle was capped and allowed to stand at $-20{ }^{\circ} \mathrm{C}$ for 10 days to afford the compound $\mathbf{1 0}$ crystals.

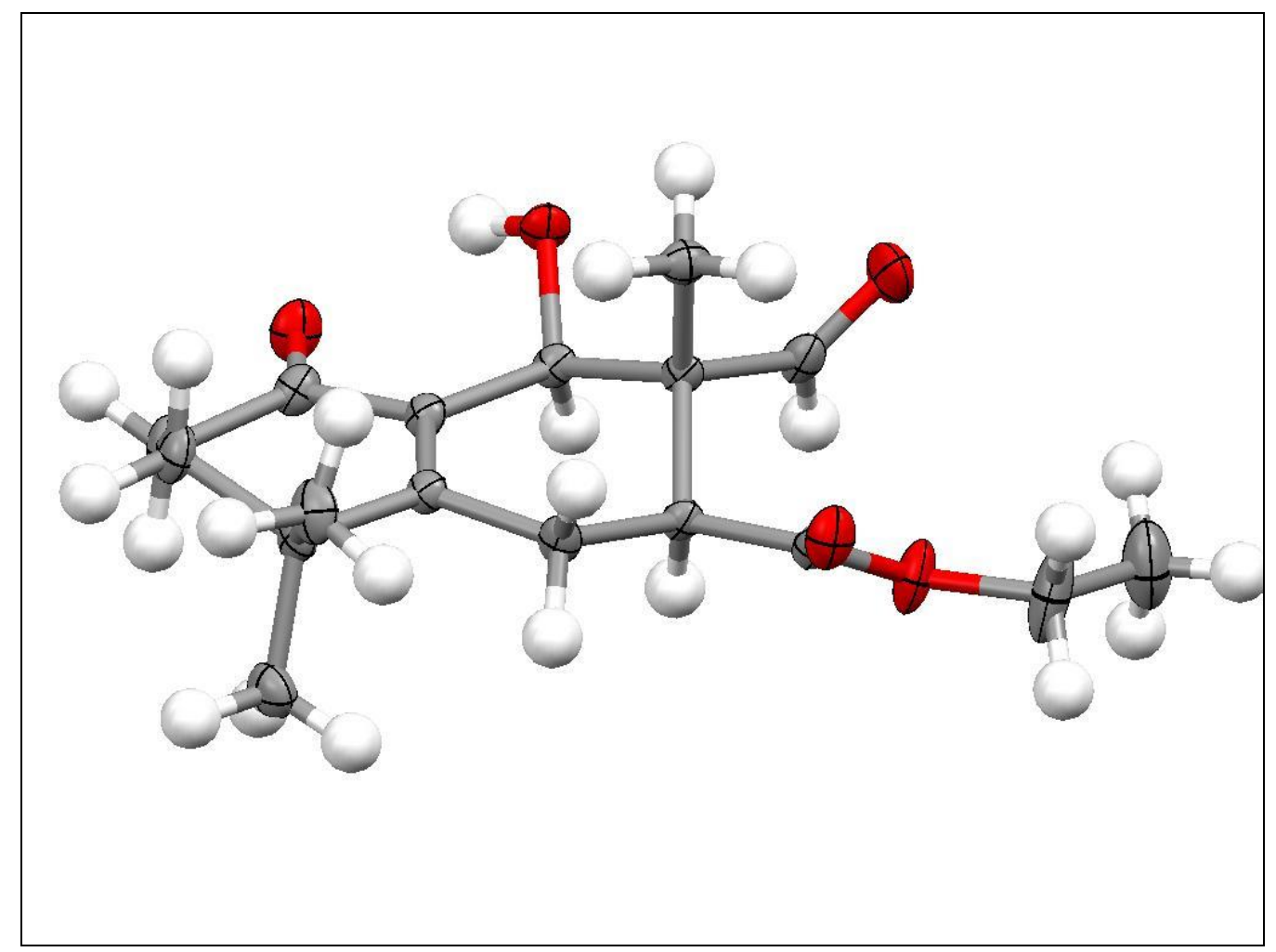

X-ray crystal structure of $\mathbf{1 0}$ (CCDC 2074049)

Thermal ellipsoid plot at 50\% probability level. 
Table S2. Crystal data and structure refinement for 10 (d22829).

Identification code

d22829

Empirical formula

$\mathrm{C}_{17} \mathrm{H}_{24} \mathrm{O}_{5}$

Formula weight

308.36

Temperature

200(2) K

Wavelength

$0.71073 \AA$

Crystal system

Triclinic

Space group

P -1

Unit cell dimensions

$\mathrm{a}=10.4190(6) \AA$

$a=113.236(2)^{\circ}$.

$\mathrm{b}=12.7565(7) \AA$

$\mathrm{b}=90.229(2)^{\circ}$.

$\mathrm{c}=14.1228(8) \AA$

$\mathrm{g}=106.772(2)^{\circ}$.

Volume

1636.55(16) $\AA^{3}$

$\mathrm{Z}$

4

Density (calculated)

$1.252 \mathrm{Mg} / \mathrm{m}^{3}$

Absorption coefficient

$0.091 \mathrm{~mm}^{-1}$

$\mathrm{F}(000)$

664

Crystal size

$0.65 \times 0.62 \times 0.07 \mathrm{~mm}^{3}$

Theta range for data collection

2.06 to $25.12^{\circ}$.

Index ranges

$-12<=\mathrm{h}<=12,-15<=\mathrm{k}<=15,-16<=1<=16$

Reflections collected

42139

Independent reflections

$5792[\mathrm{R}($ int $)=0.1064]$

Completeness to theta $=25.12^{\circ}$

$99.1 \%$

Absorption correction

multi-scan

Max. and min. transmission

0.9936 and 0.9431

Refinement method

Full-matrix least-squares on $\mathrm{F}^{2}$

Data / restraints / parameters

5792 / 0 / 405

Goodness-of-fit on $\mathrm{F}^{2}$

1.034

Final $\mathrm{R}$ indices [I>2sigma(I)]

$\mathrm{R} 1=0.0785, \mathrm{wR} 2=0.2149$

$\mathrm{R}$ indices (all data)

$\mathrm{R} 1=0.0995, \mathrm{wR} 2=0.2391$

Largest diff. peak and hole 0.489 and -0.578 e. $\AA^{-3}$ 
The recrystallization of $\mathbf{1 5}$ was carried out by the vapor diffusion method: A 4-mL vial containing a solution of $\mathbf{1 5}(15 \mathrm{mg})$ in diethyl ether $(2 \mathrm{~mL})$ was placed into a $20 \mathrm{~mL}$ vial containing $n$-hexane $(6 \mathrm{~mL})$. The outer bottle was capped and allowed to stand at $4{ }^{\circ} \mathrm{C}$ for 5 days to afford the compound $\mathbf{1 5}$ crystals.

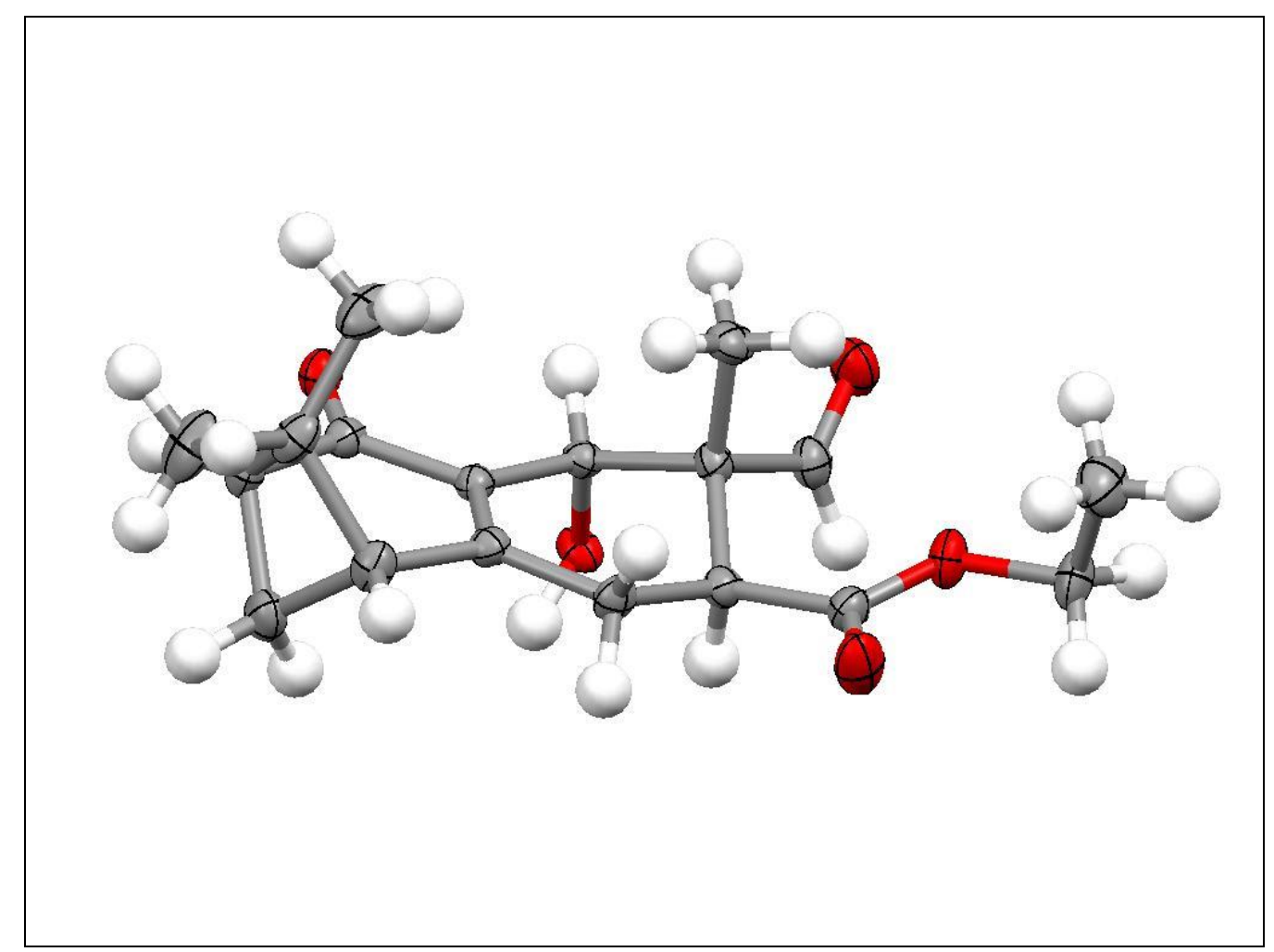

X-ray crystal structure of $\mathbf{1 5}$ (CCDC 2007692)

Thermal ellipsoid plot at 50\% probability level. 
Table S3. Crystal data and structure refinement for $\mathbf{1 5}$ (d22403).

Identification code

Empirical formula

Formula weight

Temperature

Wavelength

Crystal system

Space group

Unit cell dimensions

Volume

Z

Density (calculated)

Absorption coefficient

$\mathrm{F}(000)$

Crystal size

Theta range for data collection

Index ranges

Reflections collected

Independent reflections

Completeness to theta $=25.14^{\circ}$

Absorption correction

Max. and min. transmission

Refinement method

Data / restraints / parameters

Goodness-of-fit on $\mathrm{F}^{2}$

Final $\mathrm{R}$ indices [I $>2 \operatorname{sigma}(\mathrm{I})]$

$\mathrm{R}$ indices (all data)

Absolute structure parameter

Largest diff. peak and hole d22403

$\mathrm{C}_{18} \mathrm{H}_{24} \mathrm{O}_{5}$

320.37

200(2) K

$0.71073 \AA$

Monoclinic

P 21

$$
\begin{array}{ll}
\mathrm{a}=10.403(3) \AA & \mathrm{a}=90^{\circ} . \\
\mathrm{b}=7.4914(16) \AA & \mathrm{b}=90.839(7)^{\circ} . \\
\mathrm{c}=10.777(3) \AA & \mathrm{g}=90^{\circ} .
\end{array}
$$

$839.8(3) \AA^{3}$

2

$$
1.267 \mathrm{Mg} / \mathrm{m}^{3}
$$

$0.092 \mathrm{~mm}^{-1}$

344

$0.79 \times 0.13 \times 0.04 \mathrm{~mm}^{3}$

2.70 to $25.14^{\circ}$.

$-12<=\mathrm{h}<=12,-8<=\mathrm{k}<=8,-11<=\mathrm{l}<=12$

13459

$2978[\mathrm{R}(\mathrm{int})=0.0628]$

$99.6 \%$

multi-scan

0.9963 and 0.9312

Full-matrix least-squares on $\mathrm{F}^{2}$

2978 / 1 / 212

1.057

$\mathrm{R} 1=0.0416, \mathrm{wR} 2=0.0970$

$\mathrm{R} 1=0.0551, \mathrm{wR} 2=0.1035$

$-1.4(12)$

0.144 and -0.147 e. $\AA^{-3}$ 
The compound $22(30 \mathrm{mg})$ in a screw-capped vial (20 mL vial) was dissolved in $\mathrm{CH}_{2} \mathrm{Cl}_{2}(10$ $\mathrm{mL}$ ). The $20 \mathrm{~mL}$ vial was closed gently with a screw cap and stands it for 5 days until most of solvent was completed evaporate. The crystals of compound $\mathbf{2 2}$ was formed and subjected to singlecrystal X-ray analysis.

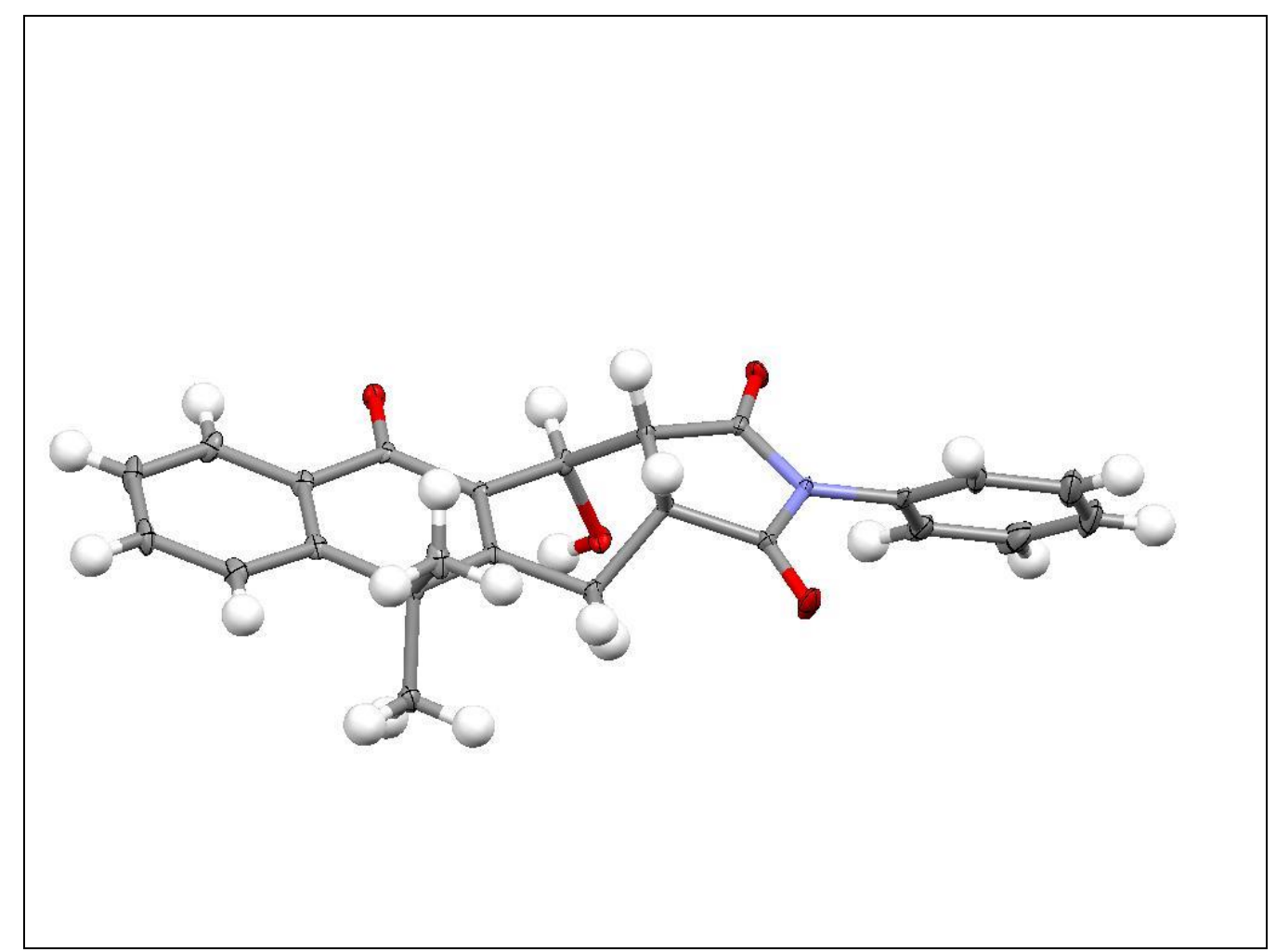

X-ray crystal structure of $\mathbf{2 2}$ (CCDC 2074040)

Thermal ellipsoid plot at $35 \%$ probability level. 
Table S4. Crystal data and structure refinement for 22 (cu_2002081t_cu_0m_a).

Identification code

Empirical formula

Formula weight

Temperature

Wavelength

Crystal system

Space group

Unit cell dimensions

Volume

$\mathrm{Z}$

Density (calculated)

Absorption coefficient

$\mathrm{F}(000)$

Crystal size

Theta range for data collection

Index ranges

Reflections collected

Independent reflections

Completeness to theta $=67.679^{\circ}$

Absorption correction

Max. and min. transmission

Refinement method

Data / restraints / parameters

Goodness-of-fit on $\mathrm{F}^{2}$

Final R indices [I $>2 \operatorname{sigma}(\mathrm{I})]$

$\mathrm{R}$ indices (all data)

Extinction coefficient

Largest diff. peak and hole cu_2002081t_cu_0m_a

$\mathrm{C}_{147} \mathrm{H}_{132} \mathrm{Cl}_{6} \mathrm{~N}_{6} \mathrm{O}_{24}$

2579.28

100(2) K

$1.54178 \AA$

Monoclinic

$\mathrm{P} 21 / \mathrm{n}$

$\mathrm{a}=30.0942(9) \AA \quad \mathrm{a}=90^{\circ}$.

$\mathrm{b}=12.1684(4) \AA$

$\mathrm{b}=97.433(2)^{\circ}$.

$\mathrm{c}=34.5883(11) \AA$

$\mathrm{g}=90^{\circ}$.
4

$1.364 \mathrm{Mg} / \mathrm{m}^{3}$

$1.881 \mathrm{~mm}^{-1}$

5400

$0.20 \times 0.06 \times 0.06 \mathrm{~mm}^{3}$

1.832 to $66.683^{\circ}$.

$-33<=\mathrm{h}<=34,-12<=\mathrm{k}<=14,-37<=1<=41$

118819

$21469[\mathrm{R}(\mathrm{int})=0.0474]$

$94.4 \%$

Semi-empirical from equivalents

0.7528 and 0.6322

Full-matrix least-squares on $\mathrm{F}^{2}$

21469 / 0 / 1666

1.051

$\mathrm{R} 1=0.0908, \mathrm{wR} 2=0.2511$

$\mathrm{R} 1=0.1007, \mathrm{wR} 2=0.2620$

$\mathrm{n} / \mathrm{a}$

1.783 and -1.235 e. $\AA^{-3}$ 
The recrystallization of $\mathbf{2 4}$ was carried out by the vapor diffusion method: A 4-mL vial containing a solution of $\mathbf{2 4}(15 \mathrm{mg})$ in ehtyl acetate $(2 \mathrm{~mL})$ was placed into a $20 \mathrm{~mL}$ vial containing $n$-hexane $(6 \mathrm{~mL})$. The outer bottle was capped and allowed to stand at $4{ }^{\circ} \mathrm{C}$ for 3 days to afford the compound $\mathbf{2 4}$ crystals.

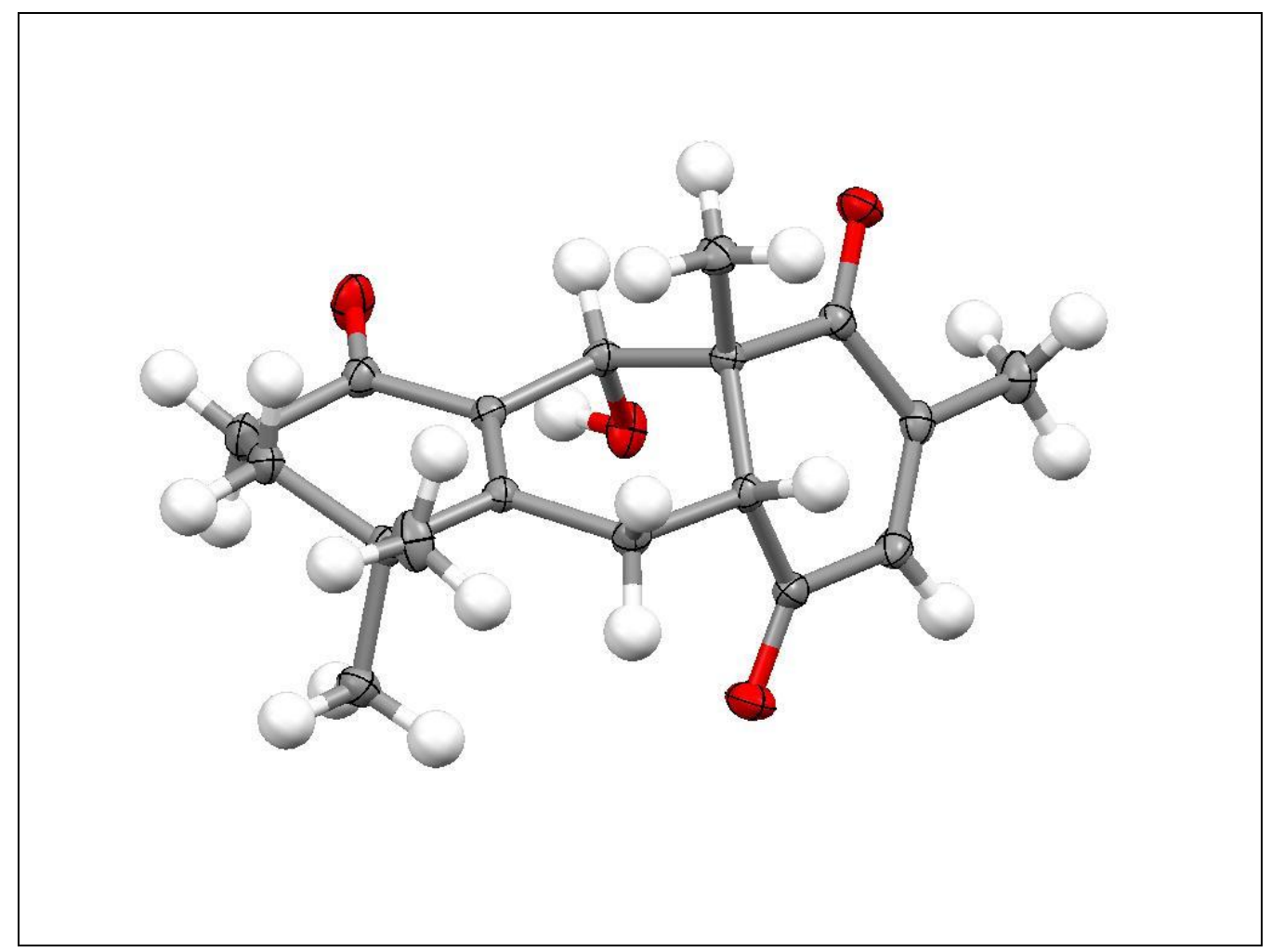

X-ray crystal structure of $\mathbf{2 4}$ (CCDC 2074071)

Thermal ellipsoid plot at 50\% probability level. 
Table S5. Crystal data and structure refinement for $\mathbf{2 4}$ (d22830).

Identification code

Empirical formula

Formula weight

Temperature

Wavelength

Crystal system

Space group

Unit cell dimensions

Volume

$\mathrm{Z}$

Density (calculated)

Absorption coefficient

$\mathrm{F}(000)$

Crystal size

Theta range for data collection

Index ranges

Reflections collected

Independent reflections

Completeness to theta $=25.08^{\circ}$

Absorption correction

Max. and min. transmission

Refinement method

Data / restraints / parameters

Goodness-of-fit on $\mathrm{F}^{2}$

Final R indices [I $>2 \operatorname{sigma}(\mathrm{I})]$

$\mathrm{R}$ indices (all data)

Largest diff. peak and hole d22830

$\mathrm{C}_{18} \mathrm{H}_{22} \mathrm{O}_{4}$

302.36

200(2) K

$0.71073 \AA$

Monoclinic

P 21/c

$$
\begin{array}{ll}
\mathrm{a}=8.1849(8) \AA & \mathrm{a}=90^{\circ} . \\
\mathrm{b}=10.1802(9) \AA & \mathrm{b}=99.363(3)^{\circ} . \\
\mathrm{c}=18.3743(18) \AA & \mathrm{g}=90^{\circ} .
\end{array}
$$

$1510.6(2) \AA^{3}$

4

$1.329 \mathrm{Mg} / \mathrm{m}^{3}$

$0.093 \mathrm{~mm}^{-1}$

648

$0.46 \times 0.42 \times 0.40 \mathrm{~mm}^{3}$

2.29 to $25.08^{\circ}$.

$-9<=\mathrm{h}<=9,-12<=\mathrm{k}<=11,-21<=1<=21$

30060

$2670[\mathrm{R}(\mathrm{int})=0.0509]$

$99.4 \%$

multi-scan

0.9638 and 0.9585

Full-matrix least-squares on $\mathrm{F}^{2}$

2670 / 0 / 203

1.020

$\mathrm{R} 1=0.0392, \mathrm{wR} 2=0.1136$

$\mathrm{R} 1=0.0456, \mathrm{wR} 2=0.1211$

0.231 and -0.246 e. $\AA^{-3}$ 
The recrystallization of $\mathbf{2 5}$ was carried out by the vapor diffusion method: A 4-mL vial containing a solution of $\mathbf{2 5}(15 \mathrm{mg})$ in diethyl ether $(2 \mathrm{~mL})$ was placed into a $20 \mathrm{~mL}$ vial containing $n$-hexane $(6 \mathrm{~mL})$. The outer bottle was capped and allowed to stand at $4{ }^{\circ} \mathrm{C}$ for 5 days to afford the compound $\mathbf{2 5}$ crystals.

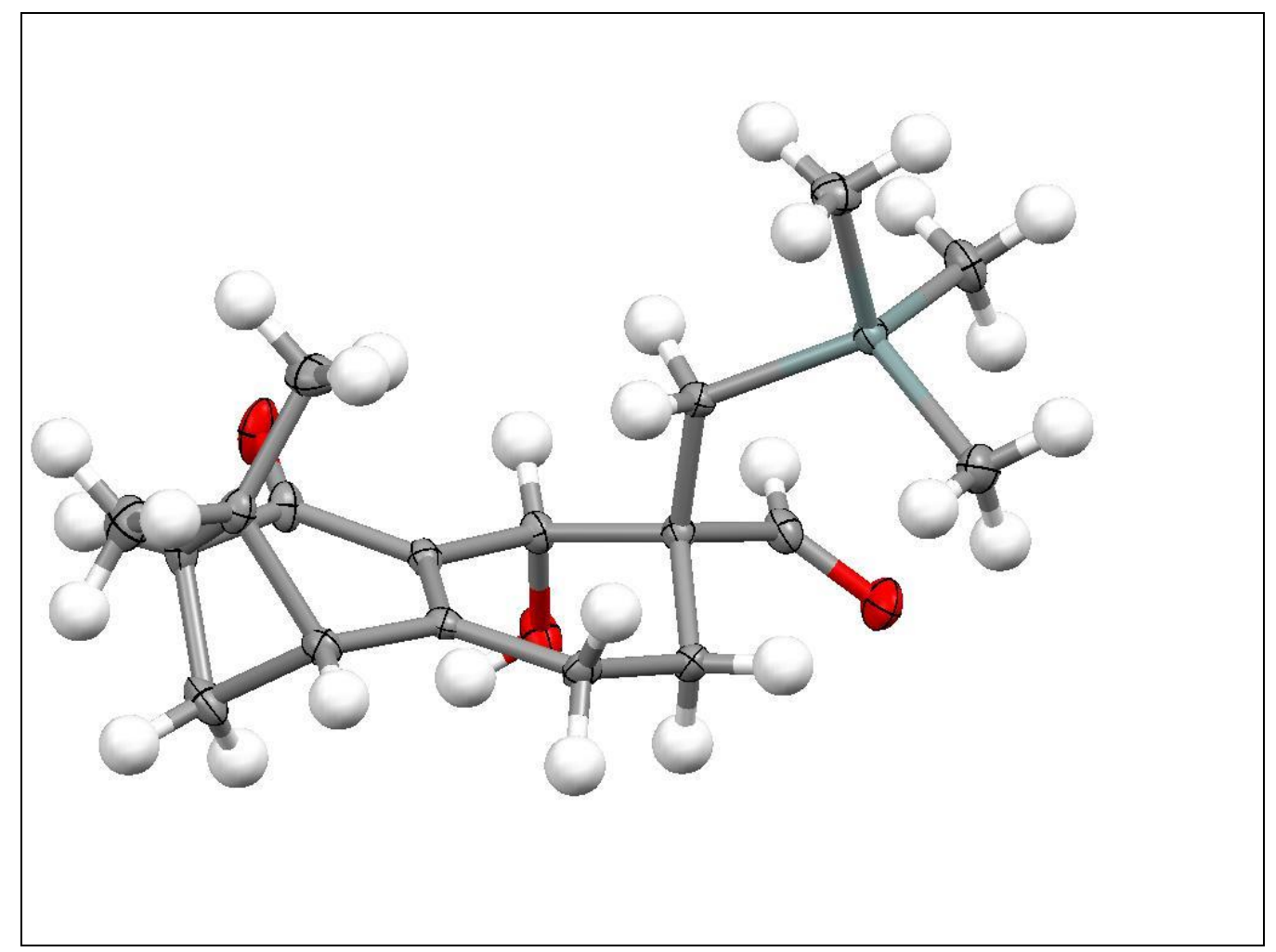

X-ray crystal structure of $\mathbf{2 5}$ (CCDC 2007693)

Thermal ellipsoid plot at 50\% probability level. 
Table S6. Crystal data and structure refinement for 25 (d22404).

Identification code

Empirical formula

Formula weight

Temperature

Wavelength

Crystal system

Space group

Unit cell dimensions

Volume

$\mathrm{Z}$

Density (calculated)

Absorption coefficient

$\mathrm{F}(000)$

Crystal size

Theta range for data collection

Index ranges

Reflections collected

Independent reflections

Completeness to theta $=25.04^{\circ}$

Absorption correction

Max. and min. transmission

Refinement method

Data / restraints / parameters

Goodness-of-fit on $\mathrm{F}^{2}$

Final R indices [I $>2 \operatorname{sigma}(\mathrm{I})]$

$\mathrm{R}$ indices (all data)

Absolute structure parameter

Largest diff. peak and hole d22404

$\mathrm{C}_{18} \mathrm{H}_{28} \mathrm{O}_{3} \mathrm{Si}$

320.49

200(2) K

$0.71073 \AA$

Orthorhombic

P 212121

$\begin{array}{ll}\mathrm{a}=6.4125(3) \AA & \mathrm{a}=90^{\circ} . \\ \mathrm{b}=12.7287(6) \AA & \mathrm{b}=90^{\circ} . \\ \mathrm{c}=21.8917(9) \AA & \mathrm{g}=90^{\circ} .\end{array}$

$1786.86(14) \AA^{3}$

4

$1.191 \mathrm{Mg} / \mathrm{m}^{3}$

$0.141 \mathrm{~mm}^{-1}$

696

$0.51 \times 0.39 \times 0.23 \mathrm{~mm}^{3}$

3.20 to $25.04^{\circ}$.

$-7<=\mathrm{h}<=7,-15<=\mathrm{k}<=15,-23<=\mathrm{l}<=26$

25393

$3123[\mathrm{R}(\mathrm{int})=0.0302]$

$98.6 \%$

multi-scan

0.9682 and 0.9313

Full-matrix least-squares on $\mathrm{F}^{2}$

3123 / 0 / 206

1.084

$\mathrm{R} 1=0.0251, \mathrm{wR} 2=0.0660$

$\mathrm{R} 1=0.0256, \mathrm{wR} 2=0.0663$

$0.02(10)$

0.194 and -0.155 e. $\AA^{-3}$ 
The recrystallization of $\mathbf{2 6}$ was carried out by the vapor diffusion method: A 4-mL vial containing a solution of $\mathbf{2 6}(15 \mathrm{mg})$ in $\mathrm{CH}_{2} \mathrm{Cl}_{2}(2 \mathrm{~mL})$ was placed into a $20 \mathrm{~mL}$ vial containing $n$-hexane $(6 \mathrm{~mL})$. The outer bottle was capped and allowed to stand at $4{ }^{\circ} \mathrm{C}$ for 5 days to afford the compound 26 crystals.

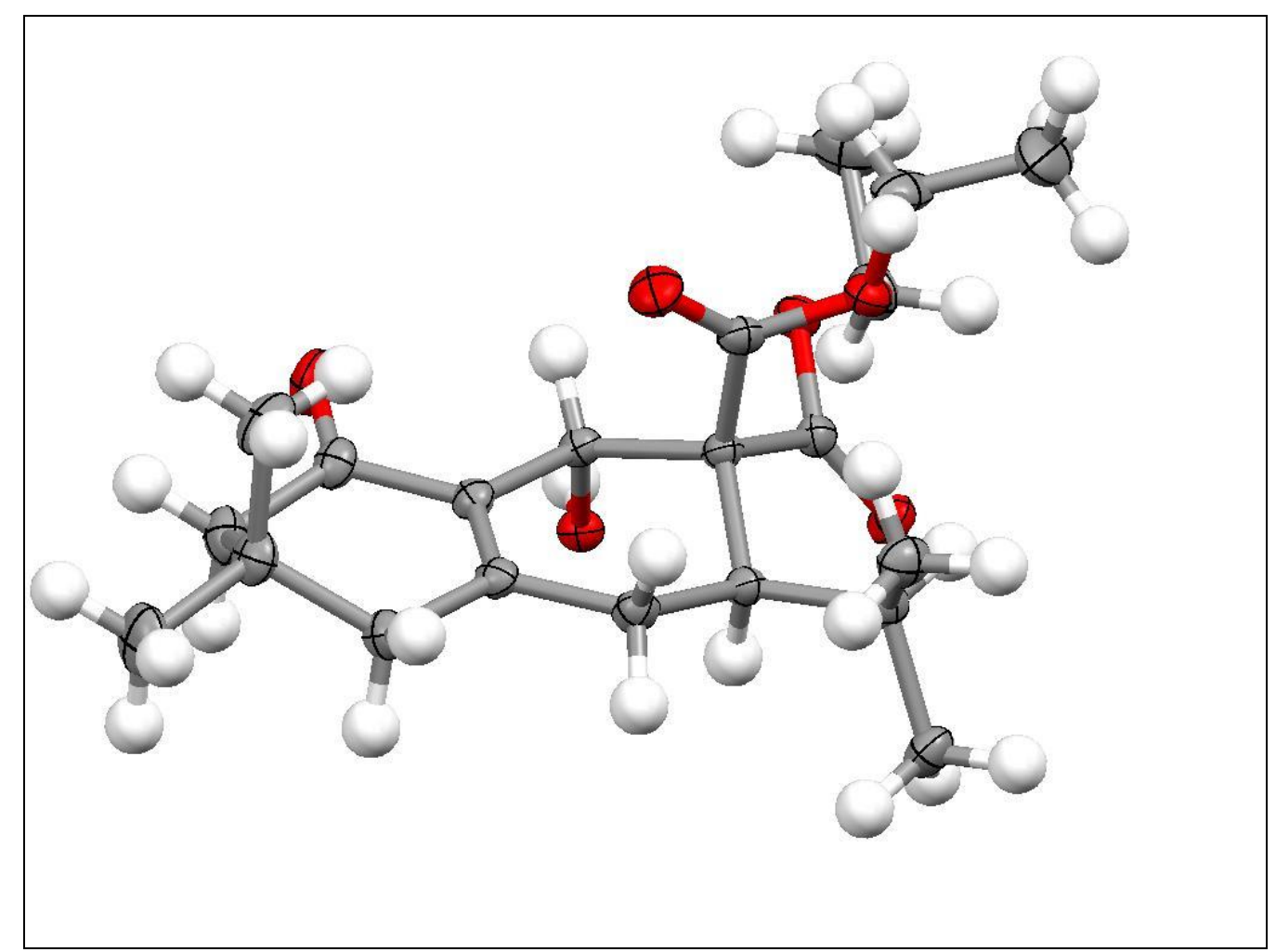

X-ray crystal structure of $\mathbf{2 6}$ (CCDC 2074072)

Thermal ellipsoid plot at 50\% probability level. 
Table S7. Crystal data and structure refinement for 26 (d22969).

Identification code

d22969

Empirical formula

$\mathrm{C}_{21} \mathrm{H}_{32} \mathrm{O}_{6}$

Formula weight

380.47

Temperature

200(2) K

Wavelength

$0.71073 \AA$

Crystal system

Monoclinic

Space group

Unit cell dimensions

C $2 / \mathrm{c}$

$a=26.5949(5) \AA$

$\mathrm{a}=90^{\circ}$.

$\mathrm{b}=11.8063(3) \AA$

$\mathrm{b}=$

$104.6430(10)^{\circ}$.

Volume

$\mathrm{c}=28.2854(6) \AA$

$\mathrm{g}=90^{\circ}$.

8592.8(3) $\AA^{3}$

Z

16

Density (calculated)

$1.176 \mathrm{Mg} / \mathrm{m}^{3}$

Absorption coefficient

$0.085 \mathrm{~mm}^{-1}$

$\mathrm{F}(000)$

3296

Crystal size

$0.50 \times 0.47 \times 0.44 \mathrm{~mm}^{3}$

Theta range for data collection

1.90 to $25.05^{\circ}$.

Index ranges

$-27<=\mathrm{h}<=31,-14<=\mathrm{k}<=14,-33<=1<=33$

Reflections collected

67960

Independent reflections

Completeness to theta $=25.05^{\circ}$

$7598[\mathrm{R}(\mathrm{int})=0.0458]$

$99.7 \%$

Absorption correction

multi-scan

Max. and min. transmission

0.9636 and 0.9587

Refinement method

Full-matrix least-squares on $\mathrm{F}^{2}$

Data / restraints / parameters

7598 / 0 / 503

Goodness-of-fit on $\mathrm{F}^{2}$

1.046

Final $\mathrm{R}$ indices [I>2sigma(I)]

$\mathrm{R} 1=0.0401, \mathrm{wR} 2=0.1082$

$\mathrm{R}$ indices (all data)

$\mathrm{R} 1=0.0518, \mathrm{wR} 2=0.1209$

Largest diff. peak and hole

0.266 and -0.225 e. $\AA^{-3}$ 
The recrystallization of $\mathbf{2 7}$ was carried out by the vapor diffusion method: A 4-mL vial containing a solution of $\mathbf{2 7}(15 \mathrm{mg})$ in ethyl acetate $(2 \mathrm{~mL})$ was placed into a $20 \mathrm{~mL}$ vial containing $n$-hexane $(6 \mathrm{~mL})$. The outer bottle was capped and allowed to stand at $4{ }^{\circ} \mathrm{C}$ for 10 days to afford the compound $\mathbf{2 7}$ crystals.

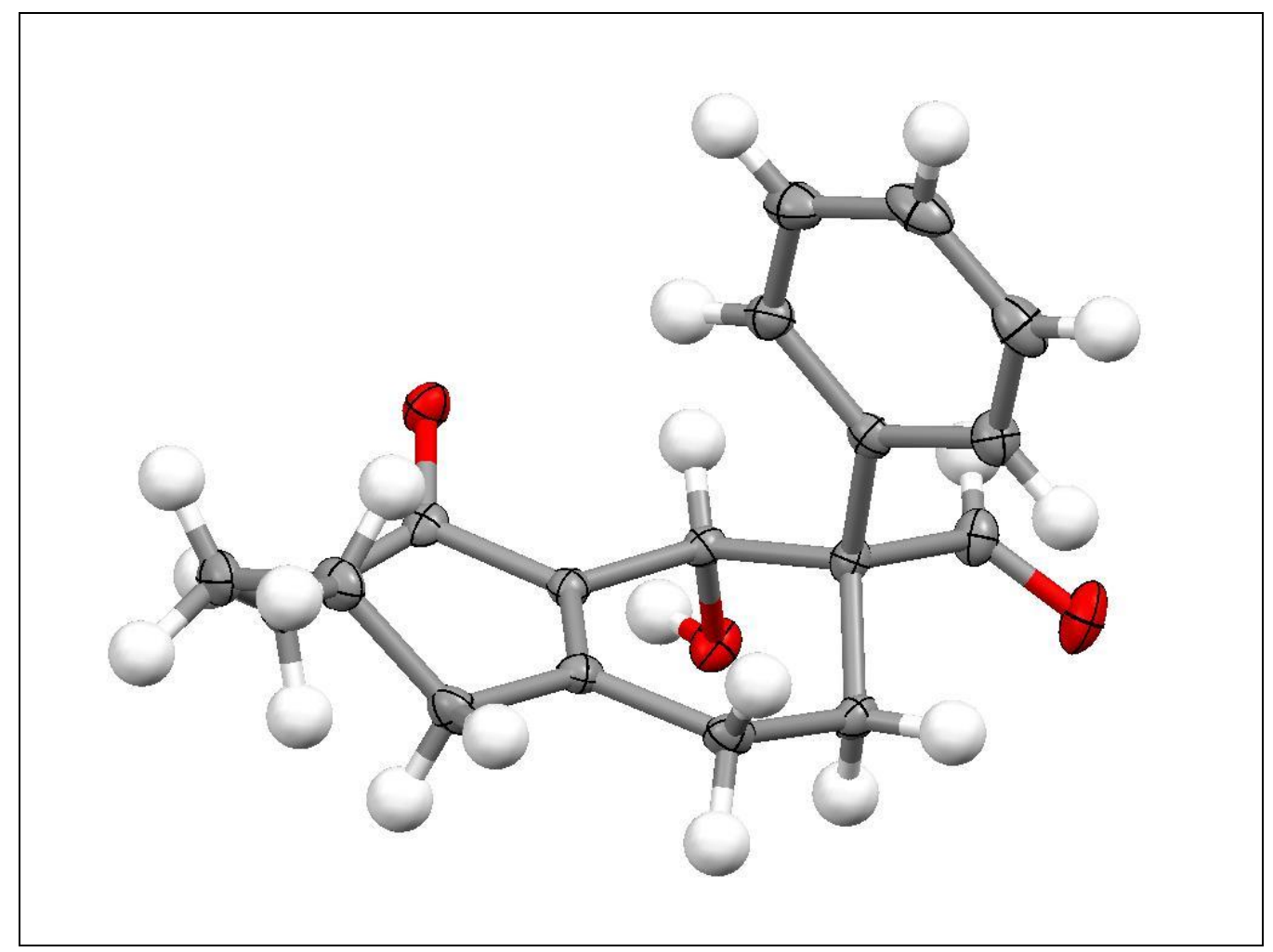

X-ray crystal structure of 27 (CCDC 2074073)

Thermal ellipsoid plot at 50\% probability level. 
Table S8. Crystal data and structure refinement for 27 (d22970).

Identification code

d22970

Empirical formula

$\mathrm{C}_{18} \mathrm{H}_{20} \mathrm{O}_{3}$

Formula weight

284.34

Temperature

200(2) K

Wavelength

$0.71073 \AA$

Crystal system

Triclinic

Space group

P -1

Unit cell dimensions

$\mathrm{a}=7.1806(3) \AA$

$\mathrm{a}=87.5350(10)^{\circ}$.

$\mathrm{b}=9.7298(3) \AA$

$\mathrm{b}=$

$86.4550(10)^{\circ}$.

$\mathrm{c}=10.3457(4) \AA \quad \mathrm{g}=$

$88.8730(10)^{\circ}$.

Volume

720.66(5) $\AA^{3}$

Z

2

Density (calculated)

$1.310 \mathrm{Mg} / \mathrm{m}^{3}$

Absorption coefficient

$0.088 \mathrm{~mm}^{-1}$

$\mathrm{F}(000)$

304

Crystal size

$0.39 \times 0.30 \times 0.06 \mathrm{~mm}^{3}$

Theta range for data collection

2.82 to $25.17^{\circ}$.

Index ranges

$-8<=\mathrm{h}<=8,-11<=\mathrm{k}<=11,-12<=\mathrm{l}<=12$

Reflections collected

25742

Independent reflections

$2580[\mathrm{R}(\mathrm{int})=0.0565]$

Completeness to theta $=25.17^{\circ}$

$99.2 \%$

Absorption correction

multi-scan

Max. and min. transmission

0.9947 and 0.9665

Refinement method

Full-matrix least-squares on $\mathrm{F}^{2}$

Data / restraints / parameters

2580 / 0 / 192

Goodness-of-fit on $\mathrm{F}^{2}$

1.002

Final $\mathrm{R}$ indices $[\mathrm{I}>2 \operatorname{sigma}(\mathrm{I})]$

$\mathrm{R} 1=0.0357, \mathrm{wR} 2=0.1071$

$\mathrm{R}$ indices (all data)

$\mathrm{R} 1=0.0406, \mathrm{wR} 2=0.1142$

Largest diff. peak and hole

0.265 and -0.199 e. $\AA^{-3}$ 
The recrystallization of $\mathbf{2 9}$ was carried out by the vapor diffusion method: A 4-mL vial containing a solution of $\mathbf{2 9}(15 \mathrm{mg})$ in $\mathrm{CH}_{2} \mathrm{Cl}_{2}(2 \mathrm{~mL})$ was placed into a $20 \mathrm{~mL}$ vial containing $n$-hexane $(10 \mathrm{~mL})$. The outer bottle was capped and allowed to stand at room temperature for 3 days to afford the compound $\mathbf{2 9}$ crystals.

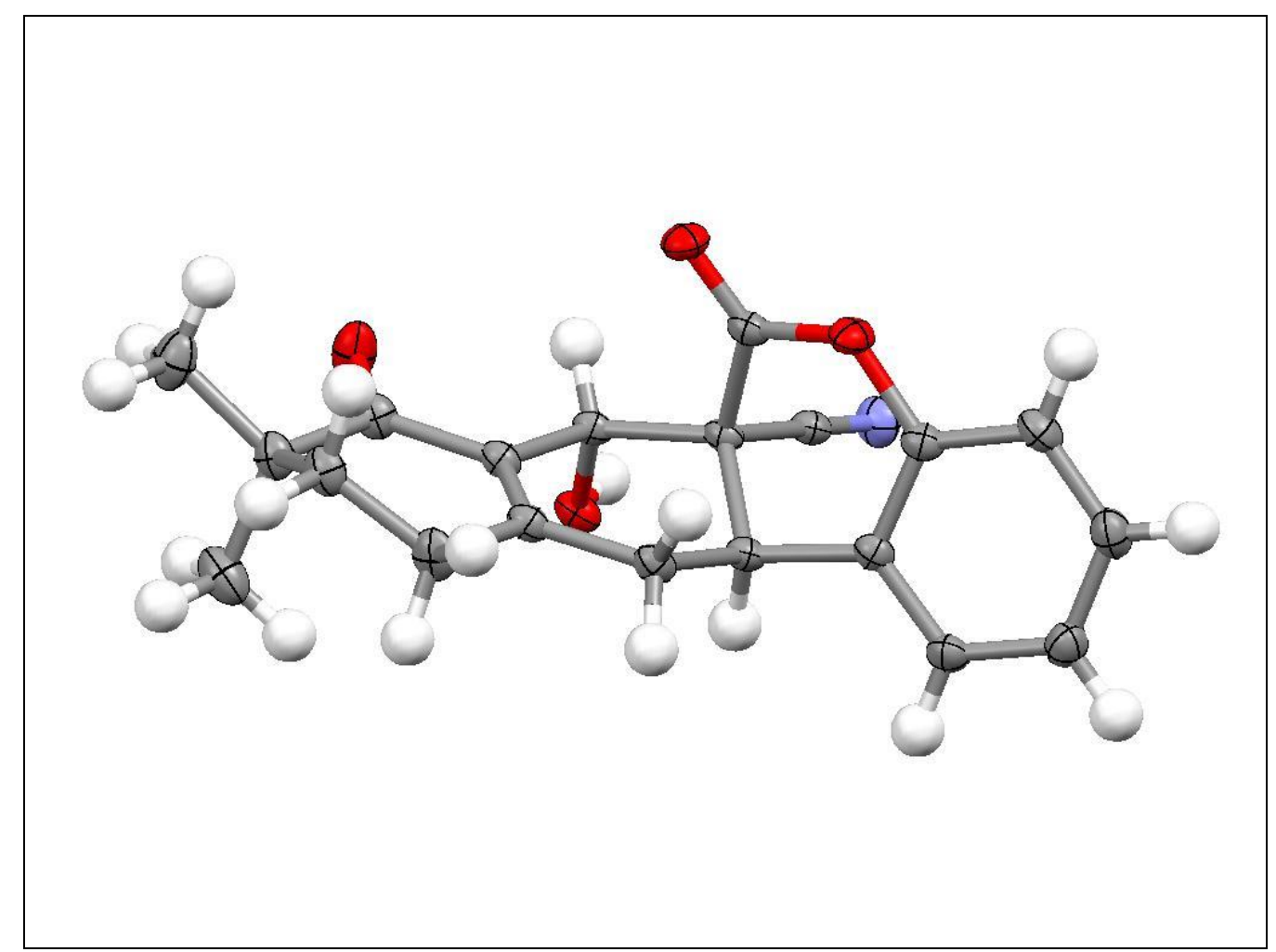

X-ray crystal structure of $\mathbf{2 9}$ (CCDC 2074074)

Thermal ellipsoid plot at $50 \%$ probability level. 
Table S9. Crystal data and structure refinement for $\mathbf{2 9}$ (d23038).

Identification code

Empirical formula

Formula weight

Temperature

Wavelength

Crystal system

Space group

Unit cell dimensions

Volume

$\mathrm{Z}$

Density (calculated)

Absorption coefficient

$\mathrm{F}(000)$

Crystal size

Theta range for data collection

Index ranges

Reflections collected

Independent reflections

Completeness to theta $=25.21^{\circ}$

Absorption correction

Max. and min. transmission

Refinement method

Data / restraints / parameters

Goodness-of-fit on $\mathrm{F}^{2}$

Final R indices [I $>2 \operatorname{sigma}(\mathrm{I})]$

$\mathrm{R}$ indices (all data)

Largest diff. peak and hole d23038

$\mathrm{C}_{20} \mathrm{H}_{19} \mathrm{NO}_{4}$

337.36

200(2) K

$0.71073 \AA$

Monoclinic

P 21/n

$$
\begin{array}{ll}
\mathrm{a}=7.2284(5) \AA & \mathrm{a}=90^{\circ} . \\
\mathrm{b}=20.5421(11) \AA & \mathrm{b}=95.141(2)^{\circ} . \\
\mathrm{c}=11.3844(7) \AA & \mathrm{g}=90^{\circ} .
\end{array}
$$$$
\text { 1683.63(18) } \AA^{3}
$$

4

$1.331 \mathrm{Mg} / \mathrm{m}^{3}$

$0.093 \mathrm{~mm}^{-1}$

712

$0.72 \times 0.05 \times 0.02 \mathrm{~mm}^{3}$

2.68 to $25.21^{\circ}$.

$-8<=\mathrm{h}<=8,-24<=\mathrm{k}<=24,-13<=\mathrm{l}<=12$

28885

$3004[\mathrm{R}(\mathrm{int})=0.0821]$

$98.9 \%$

multi-scan

0.9981 and 0.9361

Full-matrix least-squares on $\mathrm{F}^{2}$

3004 / 0 / 230

1.044

$\mathrm{R} 1=0.0388, \mathrm{wR} 2=0.0982$

$\mathrm{R} 1=0.0500, \mathrm{wR} 2=0.1089$

0.205 and -0.206 e. $\AA^{-3}$ 
The recrystallization of $\mathbf{3 0}$ was carried out by the vapor diffusion method: A 4-mL vial containing a solution of $\mathbf{3 0}(15 \mathrm{mg})$ in ehtyl acetate $(2 \mathrm{~mL})$ was placed into a $20 \mathrm{~mL}$ vial containing $n$-hexane $(6 \mathrm{~mL})$. The outer bottle was capped and allowed to stand at $4{ }^{\circ} \mathrm{C}$ for 3 days to afford the compound $\mathbf{3 0}$ crystals.

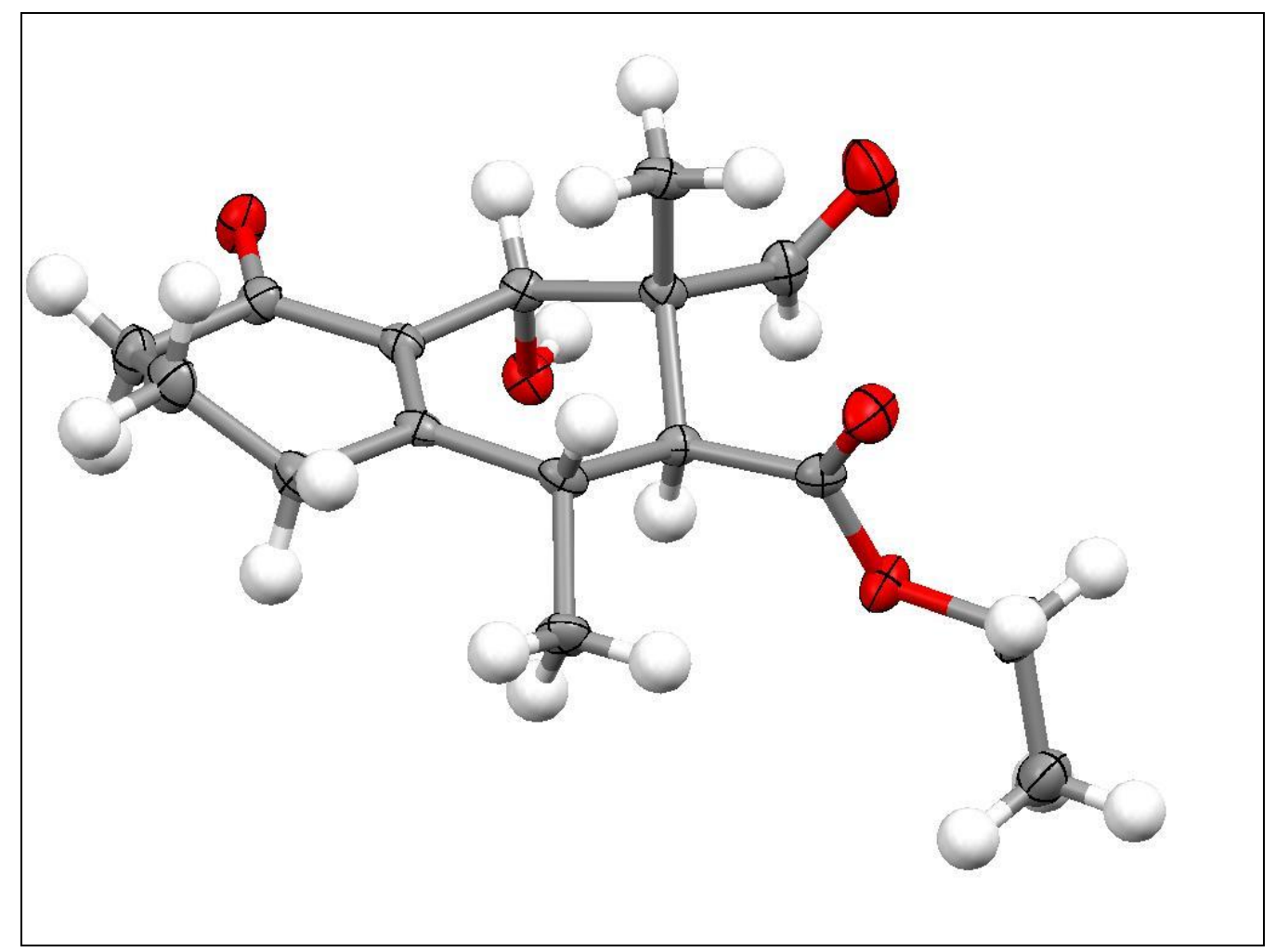

X-ray crystal structure of $\mathbf{3 0}$ (CCDC 2074075)

Thermal ellipsoid plot at $50 \%$ probability level. 
Table S10. Crystal data and structure refinement for $\mathbf{3 0}$ (d23039).

Identification code

d23039

Empirical formula

$\mathrm{C}_{16} \mathrm{H}_{22} \mathrm{O}_{5}$

Formula weight

294.34

Temperature

200(2) K

Wavelength

$0.71073 \AA$

Crystal system

Triclinic

Space group

Unit cell dimensions

Volume

P -1

$\mathrm{a}=7.9928(16) \AA$

$\mathrm{a}=91.834(5)^{\circ}$.

$\mathrm{b}=9.3677(18) \AA$

$\mathrm{b}=93.703(5)^{\circ}$.

$\mathrm{c}=10.3408(19) \AA$

$\mathrm{g}=103.042(5)^{\circ}$.

Z

$751.8(3) \AA^{3}$

Density (calculated)

Absorption coefficient

$1.300 \mathrm{Mg} / \mathrm{m}^{3}$

$0.096 \mathrm{~mm}^{-1}$

$\mathrm{F}(000)$

316

Crystal size

$0.47 \times 0.02 \times 0.01 \mathrm{~mm}^{3}$

Theta range for data collection

1.98 to $25.07^{\circ}$.

Index ranges

$-9<=\mathrm{h}<=9,-11<=\mathrm{k}<=11,-12<=\mathrm{l}<=12$

Reflections collected

19647

Independent reflections

Completeness to theta $=25.07^{\circ}$

Absorption correction

Max. and min. transmission

$2648[\mathrm{R}(\mathrm{int})=0.1523]$

$99.5 \%$

multi-scan

0.9990 and 0.9563

Refinement method

Full-matrix least-squares on $\mathrm{F}^{2}$

Data / restraints / parameters

Goodness-of-fit on $\mathrm{F}^{2}$

2648 / 0 / 195

Final R indices [I $>2 \operatorname{sigma}(\mathrm{I})]$

1.008

$\mathrm{R}$ indices (all data)

$\mathrm{R} 1=0.0665, \mathrm{wR} 2=0.1475$

$\mathrm{R} 1=0.1289, \mathrm{wR} 2=0.1783$

Largest diff. peak and hole

0.518 and -0.254 e. $\AA^{-3}$ 
The recrystallization of $\mathbf{3 2}$ was carried out by the vapor diffusion method: A 4-mL vial containing a solution of $\mathbf{3 2}(15 \mathrm{mg})$ in ehtyl acetate $(2 \mathrm{~mL})$ was placed into a $20 \mathrm{~mL}$ vial containing $n$-hexane $(10 \mathrm{~mL})$. The outer bottle was capped and allowed to stand at $4{ }^{\circ} \mathrm{C}$ for 5 days to afford the compound $\mathbf{3 2}$ crystals.

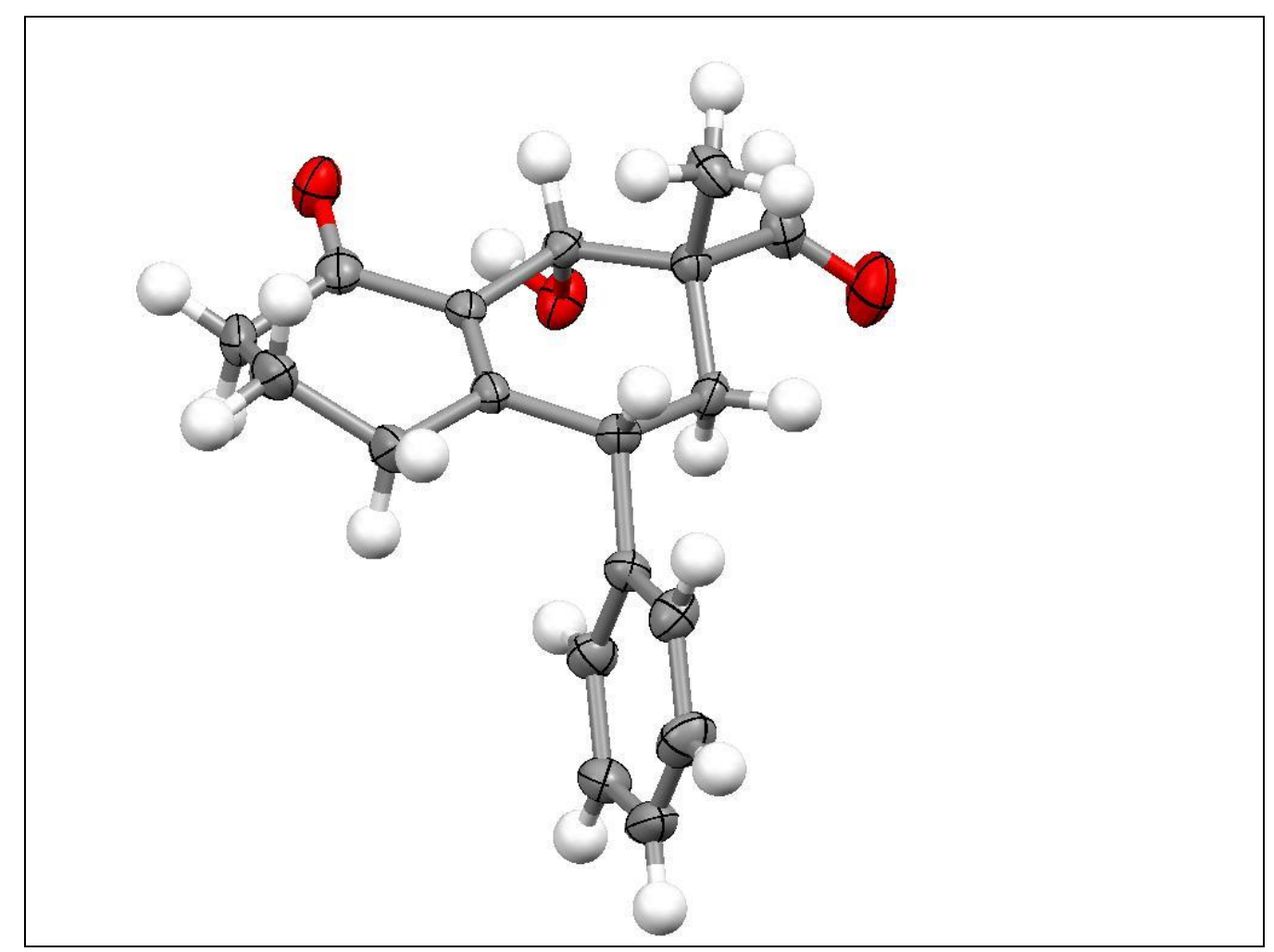

X-ray crystal structure of $\mathbf{3 2}$ (CCDC 2074055)

Thermal ellipsoid plot at 50\% probability level. 
Table S11. Crystal data and structure refinement for 32 (d22827).

Identification code

d22827

Empirical formula

$\mathrm{C}_{18} \mathrm{H}_{20} \mathrm{O}_{3}$

Formula weight

284.34

Temperature

200(2) K

Wavelength

$0.71073 \AA$

Crystal system

Orthorhombic

Space group

P 212121

Unit cell dimensions

$\begin{array}{ll}\mathrm{a}=5.8485(5) \AA & \mathrm{a}=90^{\circ} . \\ \mathrm{b}=15.7132(15) \AA & \mathrm{b}=90^{\circ} . \\ \mathrm{c}=16.2264(17) \AA & \mathrm{g}=90^{\circ} .\end{array}$

Volume

1491.2(2) $\AA^{3}$

$\mathrm{Z}$

4

Density (calculated)

$1.267 \mathrm{Mg} / \mathrm{m}^{3}$

Absorption coefficient

$0.085 \mathrm{~mm}^{-1}$

$\mathrm{F}(000)$

608

Crystal size

$0.79 \times 0.23 \times 0.02 \mathrm{~mm}^{3}$

Theta range for data collection

2.51 to $25.03^{\circ}$.

Index ranges

$-6<=\mathrm{h}<=6,-15<=\mathrm{k}<=18,-12<=\mathrm{l}<=19$

Reflections collected

9204

Independent reflections

Completeness to theta $=25.03^{\circ}$

Absorption correction

Max. and min. transmission

Refinement method

Data / restraints / parameters

Goodness-of-fit on $\mathrm{F}^{2}$

$2620[\mathrm{R}(\mathrm{int})=0.1024]$

$99.8 \%$

multi-scan

0.9983 and 0.9359

Full-matrix least-squares on $\mathrm{F}^{2}$

2620 / 0 / 191

0.986

Final R indices [I $>2 \operatorname{sigma}(\mathrm{I})]$

$\mathrm{R} 1=0.0542, \mathrm{wR} 2=0.1101$

$\mathrm{R}$ indices (all data)

$\mathrm{R} 1=0.0855, \mathrm{wR} 2=0.1233$

Absolute structure parameter

$0.2(19)$

Largest diff. peak and hole

0.222 and -0.197 e. $\AA^{-3}$ 
The recrystallization of $\mathbf{3 6}$ was carried out by the vapor diffusion method: A 4-mL vial containing a solution of $\mathbf{3 6}(15 \mathrm{mg})$ in ehtyl acetate $(2 \mathrm{~mL})$ was placed into a $20 \mathrm{~mL}$ vial containing $n$-hexane $(6 \mathrm{~mL})$. The outer bottle was capped and allowed to stand at $4{ }^{\circ} \mathrm{C}$ for 10 days to afford the compound $\mathbf{3 6}$ crystals.

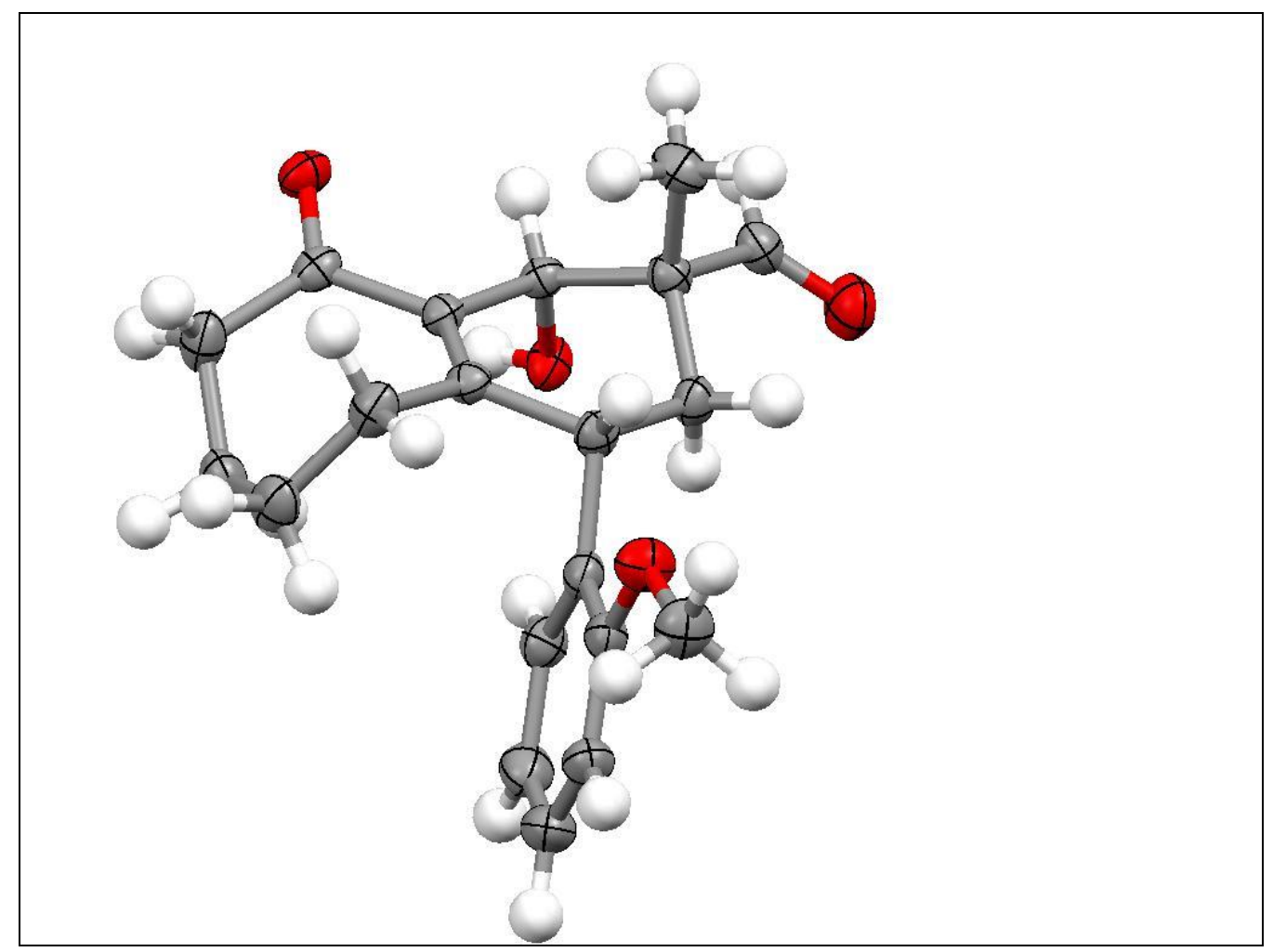

X-ray crystal structure of $\mathbf{3 6}$ (CCDC 2074053)

Thermal ellipsoid plot at 50\% probability level. 
Table S12. Crystal data and structure refinement for $\mathbf{3 6}$ (d22714).

Identification code

d22714

Empirical formula

$\mathrm{C}_{20} \mathrm{H}_{24} \mathrm{O}_{4}$

Formula weight

328.39

Temperature

200(2) K

Wavelength

$0.71073 \AA$

Crystal system

Orthorhombic

Space group

Unit cell dimensions

$\mathrm{Pbc} \mathrm{a}$

Volume

$a=7.4767(2) \AA$ $\mathrm{a}=90^{\circ}$.

$\mathrm{b}=14.1191(5) \AA$

$\mathrm{b}=90^{\circ}$.

$\mathrm{c}=32.2835(9) \AA$

$\mathrm{g}=90^{\circ}$.

$\mathrm{Z}$

3407.98(18) $\AA^{3}$

Density (calculated)

8

$1.280 \mathrm{Mg} / \mathrm{m}^{3}$

Absorption coefficient

$0.088 \mathrm{~mm}^{-1}$

$\mathrm{F}(000)$

1408

Crystal size

$0.18 \times 0.15 \times 0.03 \mathrm{~mm}^{3}$

Theta range for data collection

2.52 to $25.10^{\circ}$.

Index ranges

$-8<=\mathrm{h}<=6,-12<=\mathrm{k}<=16,-38<=\mathrm{l}<=29$

Reflections collected

16421

Independent reflections

$3007[\mathrm{R}(\mathrm{int})=0.0420]$

Completeness to theta $=25.10^{\circ}$

$99.2 \%$

Absorption correction

multi-scan

Max. and min. transmission

0.9974 and 0.9843

Refinement method

Full-matrix least-squares on $\mathrm{F}^{2}$

Data / restraints / parameters

3007 / 0 / 221

Goodness-of-fit on $\mathrm{F}^{2}$

1.070

Final $\mathrm{R}$ indices [I $>2 \operatorname{sigma}(\mathrm{I})]$

$\mathrm{R} 1=0.0413, \mathrm{wR} 2=0.0928$

$\mathrm{R}$ indices (all data)

$\mathrm{R} 1=0.0559, \mathrm{wR} 2=0.1021$

Largest diff. peak and hole

0.296 and -0.162 e. $\AA^{-3}$ 
The recrystallization of $\mathbf{4 1}$ was carried out by the vapor diffusion method: A 4-mL vial containing a solution of $\mathbf{4 1}(15 \mathrm{mg})$ in ehtyl acetate $(2 \mathrm{~mL})$ was placed into a $20 \mathrm{~mL}$ vial containing $n$-hexane $(10 \mathrm{~mL})$. The outer bottle was capped and allowed to stand at room temperature for 5 days to afford the compound $\mathbf{4 1}$ crystals.

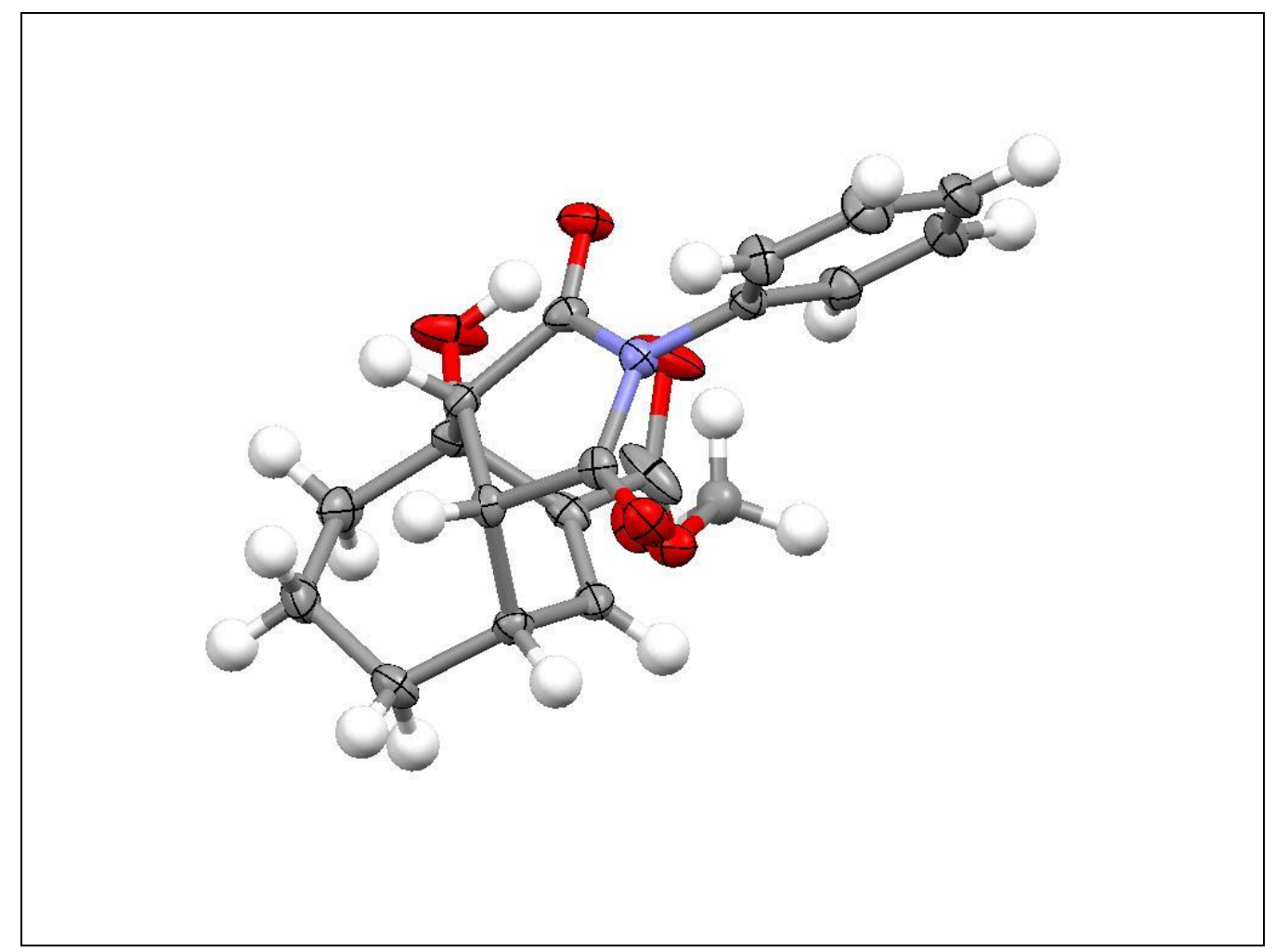

X-ray crystal structure of $\mathbf{4 1}$ (CCDC 2074076)

Thermal ellipsoid plot at 50\% probability level. 
Table S13. Crystal data and structure refinement for $\mathbf{4 1}$ (d23173).

Identification code

d23173

Empirical formula

$\mathrm{C}_{19} \mathrm{H}_{19} \mathrm{NO}_{5}$

Formula weight

341.35

Temperature

200(2) K

Wavelength

$0.71073 \AA$

Crystal system

Monoclinic

Space group

Unit cell dimensions

P 21/c

$\begin{array}{ll}\mathrm{a}=8.4700(4) \AA & \mathrm{a}=90^{\circ} . \\ \mathrm{b}=18.7943(8) \AA & \mathrm{b}=\end{array}$

$111.1640(10)^{\circ}$.

Volume

$\mathrm{c}=11.0135(5) \AA$

$\mathrm{g}=90^{\circ}$.

1634.96(13) $\AA^{3}$

Z

4

Density (calculated)

$1.387 \mathrm{Mg} / \mathrm{m}^{3}$

Absorption coefficient

$0.101 \mathrm{~mm}^{-1}$

$\mathrm{F}(000)$

720

Crystal size

$0.45 \times 0.25 \times 0.17 \mathrm{~mm}^{3}$

Theta range for data collection

2.80 to $25.11^{\circ}$.

Index ranges

$-10<=\mathrm{h}<=8,-22<=\mathrm{k}<=22,-13<=1<=13$

Reflections collected

23001

Independent reflections

Completeness to theta $=25.11^{\circ}$

$2906[\mathrm{R}(\mathrm{int})=0.0395]$

$99.8 \%$

Absorption correction

multi-scan

Max. and min. transmission

0.9830 and 0.9560

Refinement method

Full-matrix least-squares on $\mathrm{F}^{2}$

Data / restraints / parameters

2906 / 2 / 245

Goodness-of-fit on $\mathrm{F}^{2}$

1.045

Final $\mathrm{R}$ indices [I>2sigma(I)]

$\mathrm{R} 1=0.0433, \mathrm{wR} 2=0.1061$

$\mathrm{R}$ indices (all data)

$\mathrm{R} 1=0.0510, \mathrm{wR} 2=0.1132$

Largest diff. peak and hole

0.253 and -0.413 e. $\AA^{-3}$ 
The recrystallization of $\mathbf{4 3}$ was carried out by the vapor diffusion method: A 4-mL vial containing a solution of $\mathbf{4 3}(15 \mathrm{mg})$ in $\mathrm{CH}_{2} \mathrm{Cl}_{2}(2 \mathrm{~mL})$ was placed into a $20 \mathrm{~mL}$ vial containing $n$-hexane $(10 \mathrm{~mL})$. The outer bottle was capped and allowed to stand at room temperature for 3 days to afford the compound $\mathbf{4 3}$ crystals.

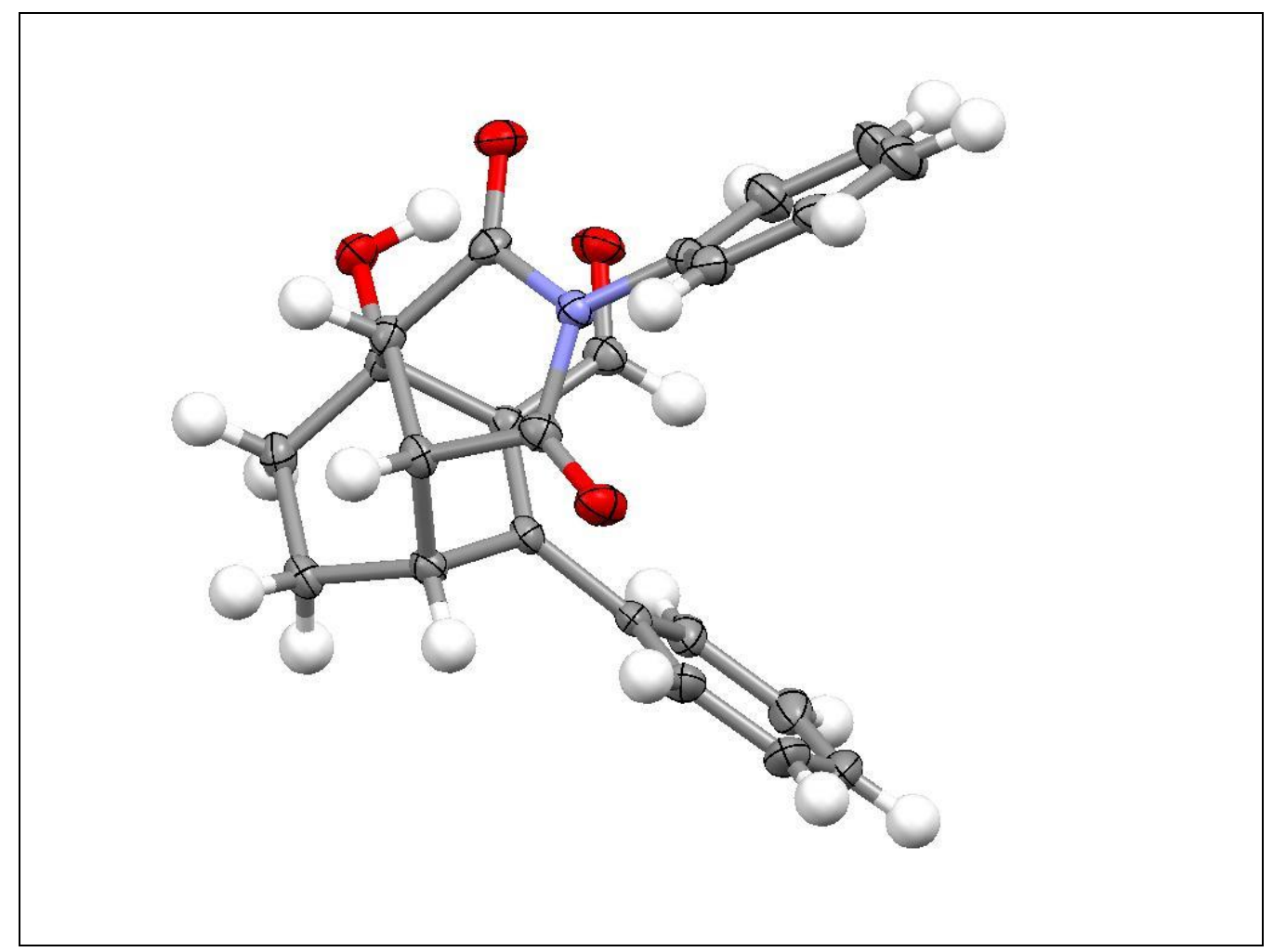

X-ray crystal structure of $\mathbf{4 3}$ (CCDC 2074078)

Thermal ellipsoid plot at $50 \%$ probability level. 
Table S14. Crystal data and structure refinement for $\mathbf{4 3}$ (d23119).

Identification code

d23119

Empirical formula

$\mathrm{C}_{23} \mathrm{H}_{19} \mathrm{NO}_{4}$

Formula weight

373.39

Temperature

200(2) K

Wavelength

$0.71073 \AA$

Crystal system

Monoclinic

Space group

P $21 / n$

Unit cell dimensions

$$
\begin{array}{ll}
\mathrm{a}=14.2305(13) \AA & \mathrm{a}=90^{\circ} . \\
\mathrm{b}=7.4723(5) \AA & \mathrm{b}=99.991(3)^{\circ} . \\
\mathrm{c}=17.0649(15) \AA & \mathrm{g}=90^{\circ} .
\end{array}
$$

Volume

1787.1(3) $\AA^{3}$

Z

4

Density (calculated)

$1.388 \mathrm{Mg} / \mathrm{m}^{3}$

Absorption coefficient

$0.095 \mathrm{~mm}^{-1}$

$\mathrm{F}(000)$

784

Crystal size

$0.20 \times 0.11 \times 0.07 \mathrm{~mm}^{3}$

Theta range for data collection

2.42 to $25.25^{\circ}$.

Index ranges

$-16<=\mathrm{h}<=17,-8<=\mathrm{k}<=8,-20<=\mathrm{l}<=20$

Reflections collected

33679

Independent reflections

Completeness to theta $=25.26^{\circ}$

Absorption correction

Max. and min. transmission

$3183[\mathrm{R}(\mathrm{int})=0.0495]$

$98.4 \%$

multi-scan

0.9934 and 0.9812

Refinement method

Full-matrix least-squares on $\mathrm{F}^{2}$

Data / restraints / parameters

3183 / 0 / 253

Goodness-of-fit on $\mathrm{F}^{2}$

1.080

Final R indices [I $>2 \operatorname{sigma}(\mathrm{I})]$

$\mathrm{R} 1=0.0374, \mathrm{wR} 2=0.0998$

$\mathrm{R}$ indices (all data)

$\mathrm{R} 1=0.0459, \mathrm{wR} 2=0.1063$

Largest diff. peak and hole

0.386 and -0.210 e. $\AA^{-3}$ 


\section{Reference}

[1] A. B. Smith, B. D. Dorsey, M. Ohba, A. T. Lupo, M. S. Malamas, J. Org. Chem. 1988, $53,4314-4325$.

[2] I. Kolodziej, J. R. Green, Org. Biomol. Chem. 2015, 13, 10852-10864.

[3] a) A. Parida, M. Sharique, B. N. Kakde, S. Ghosh, A. Bisai, Synthesis 2015, 47, 2965-2970; b) B. N. Kakde, S. Bhunia, A. Bisai, Tetrahedron Lett. 2013, 54, 1436-1439.

[4] a) F. Su, Y. Lu, L. Kong, J. Liu, T. Luo, 2018, 57, 760-764; b) C. Zheng, I. Dubovyk, K. E. Lazarski, R. J. Thomson, J. Am. Chem. Soc. 2014, 136, 17750-17756; c) A. Porzelle, C. M. Williams, B. D. Schwartz, I. R. Gentle, Synlett 2005, 2005, 2923-2926.

[5] A. Kmieciak, M. P. Krzemiński, Beilstein J. Org. Chem. 2019, 15, 2493-2499.

[6] T. Rajale, S. Sharma, D. A. Stroud, D. K. Unruh, E. Miaou, K. Lai, D. M. Birney, Tetrahedron Lett. 2014, 55, 6627-6630.

[7] a) Y.-T. Liu, J.-Q. Chen, L.-P. Li, X.-Y. Shao, J.-H. Xie, Q.-L. Zhou, Org. Lett. 2017, 19, 3231-3234; b) T. Nishizuka, S. Hirosawa, S. Kondo, D. Ikeda, T. Takeuchi, J. Antibiot. 1997, 50, 755-764.

[8] G. D. Brown, Q. Shi, G. V. Delucca, D. G. Batt, M. A. Galella, M.-E. Cvijic, R.-Q. Liu, F. Qiu, Q. Zhao, J. C. Barrish, P. H. Carter, Bioorg. Med. Chem. Lett. 2016, 26, 662-666.

[9] S. Ikeda, M. Shibuya, N. Kanoh, Y. Iwabuchi, Org. Lett. 2009, 11, 1833-1836.

[10] F. Rezgui, M. M. El Gaïed, J. Chem. Res. Synop. 1999, 576-577.

[11] U. Chiachio, A. Liguori, A. Rescifina, G. Romeo, F. Rossano, G. Sindona, N. Uccella, Tetrahedron 1992, 48, 123-132.

[12] a) D. P. Canterbury, I. R. Herrick, J. Um, K. N. Houk, A. J. Frontier, Tetrahedron 2009, 65, 3165-3179; b) A. Padwa, U. Chiacchio, D. N. Kline, J. Perumattam, J. Org. Chem. 1988, 53, 2238-2245. 
${ }^{1} \mathrm{H},{ }^{13} \mathrm{C}$ NMR, DEPT, ${ }^{1} \mathrm{H}-{ }^{1} \mathrm{H}$ COSY and NOESY spectra for all new compounds:

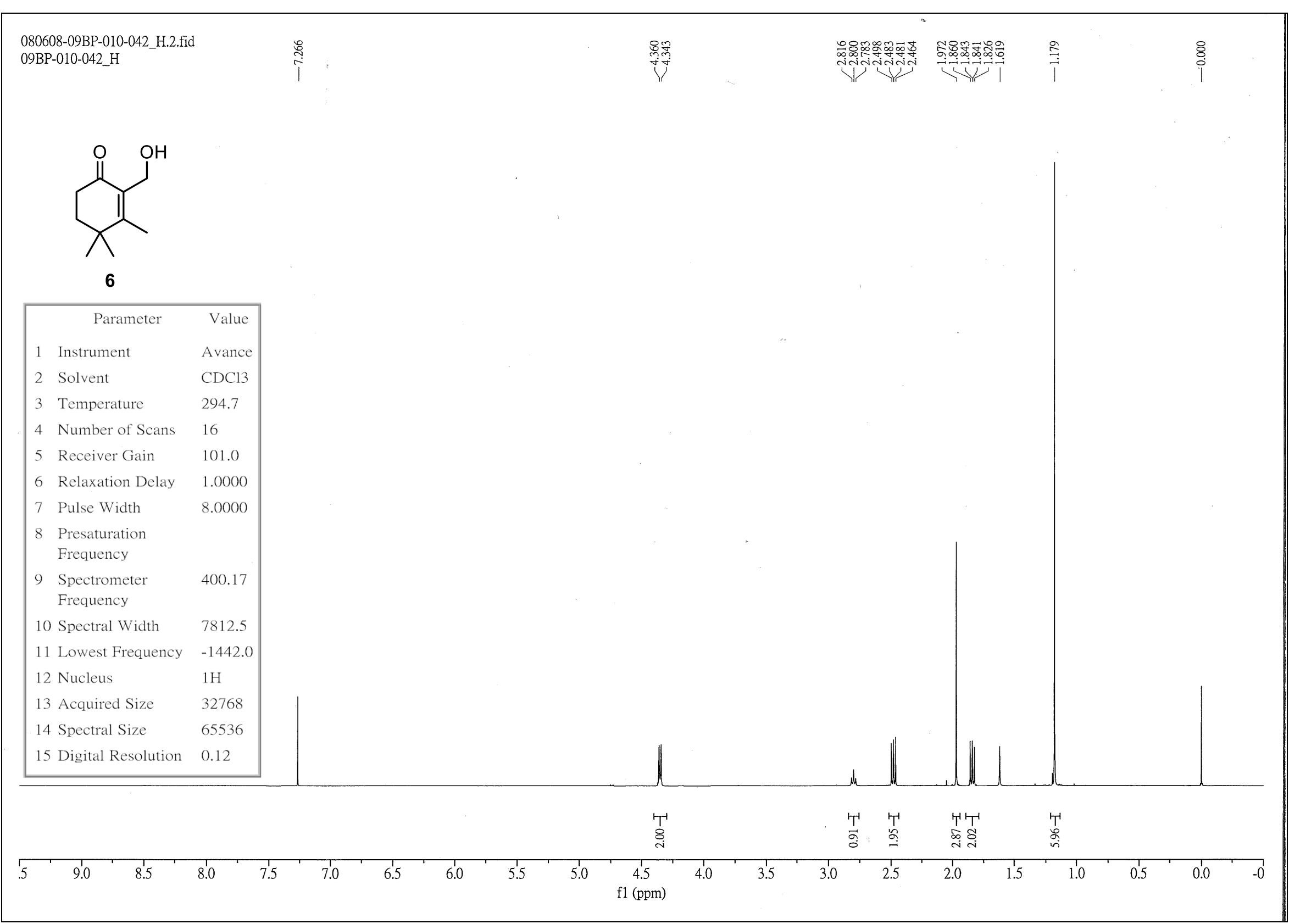

${ }^{1} \mathrm{H}$ NMR spectrum for compound 6 


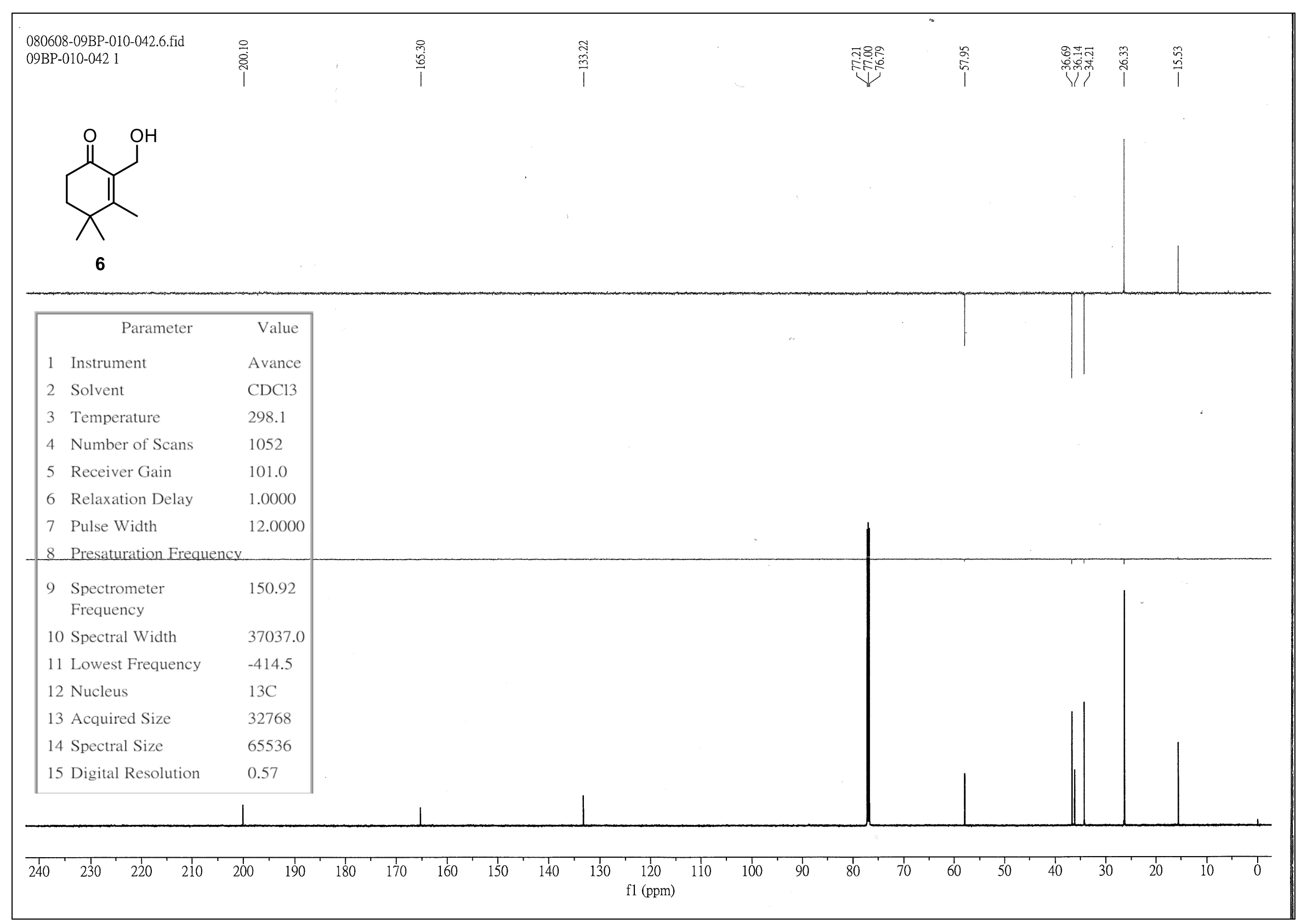

${ }^{13} \mathrm{C}$ NMR + DEPT spectra for compound $\mathbf{6}$ 


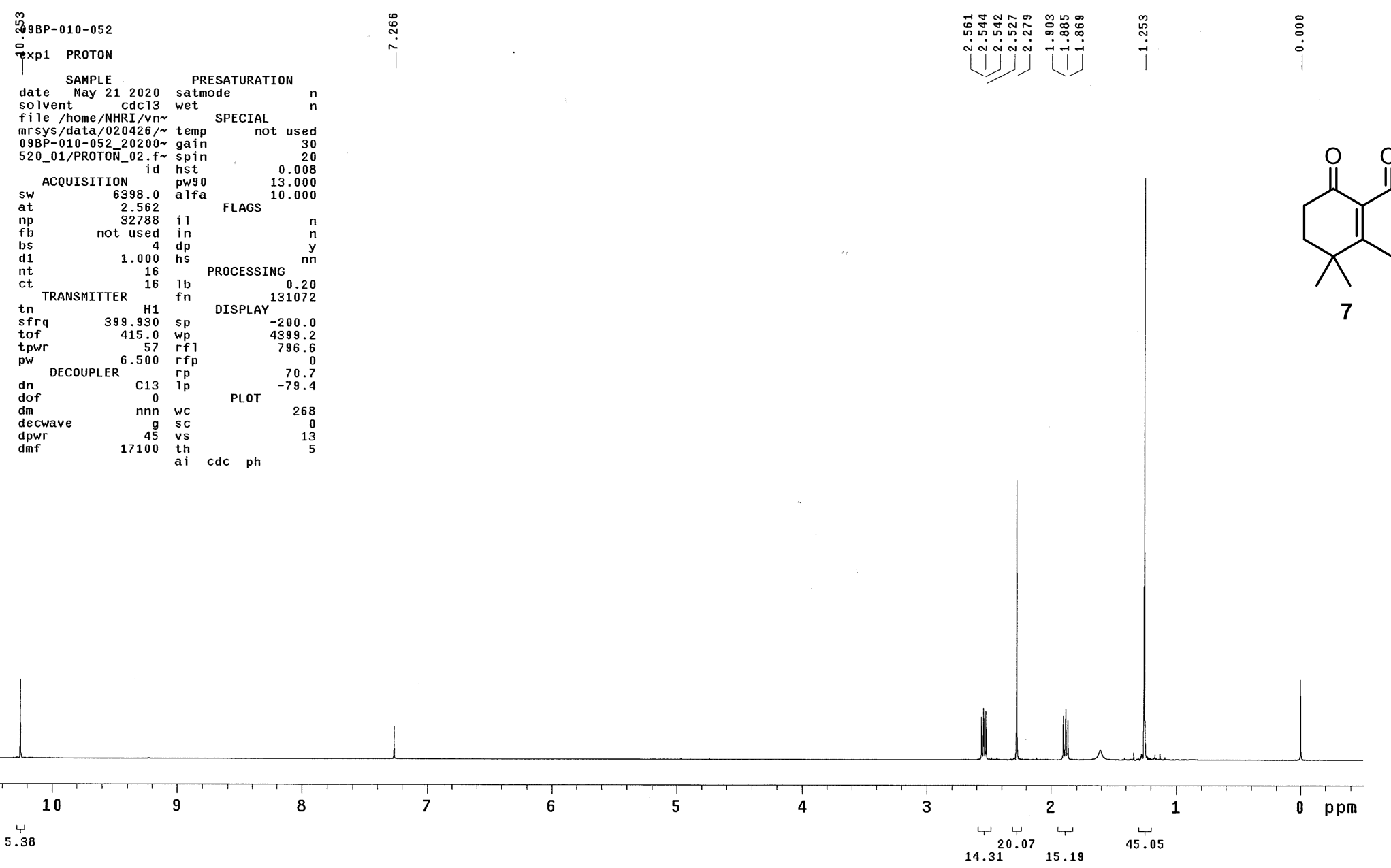

${ }^{1} \mathrm{H}$ NMR spectrum for compound 7 


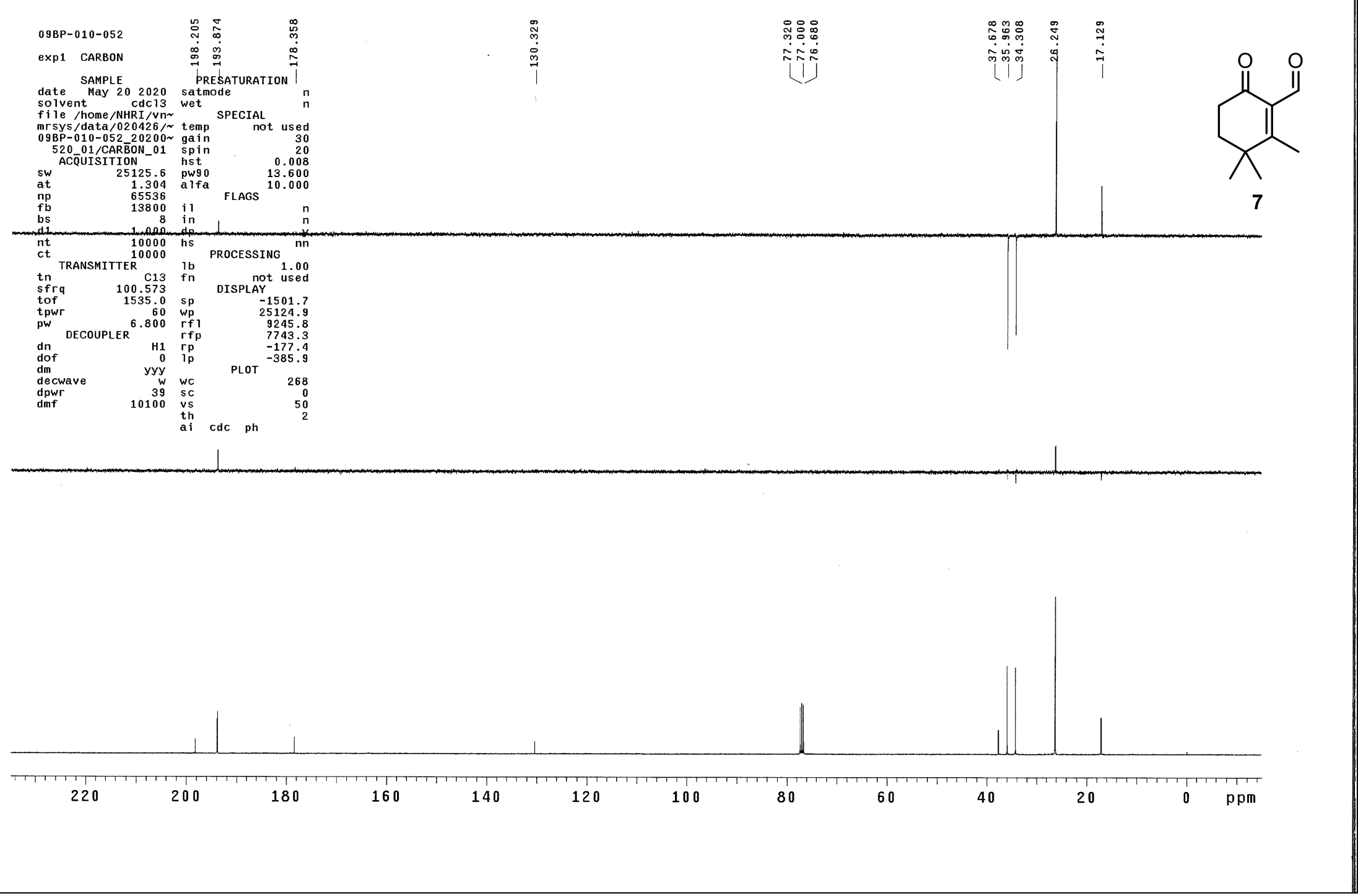

${ }^{13} \mathrm{C}$ NMR + DEPT spectra for compound 7 


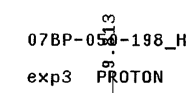

SAMPLE

SamPLE
dun 18 2020
solvent $\begin{gathered}\text { PRESATURATION } \\ \text { satmode }\end{gathered}$

file /home/NHRI/Vn NPECIAL

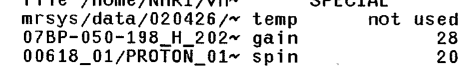

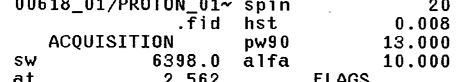

$\begin{array}{lr}\text { at } & 2.562 \\ \mathrm{np} & 32788 \text { i }\end{array}$

$\begin{array}{lrl}\text { np } & \text { not used in } \\ \text { fb } & \text { in } \\ \text { b1 } & \text { not } \\ \text { d1 } & 1.000 & \text { hp } \\ \text { nt } & & 16\end{array}$

16 hs 16 procest

TRANSMITTER $\begin{array}{rrr}16 & \mathrm{lb} & 0.20 \\ \mathrm{fn} & 131072\end{array}$

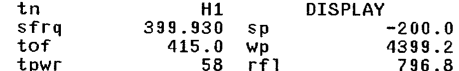

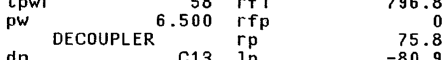

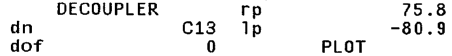

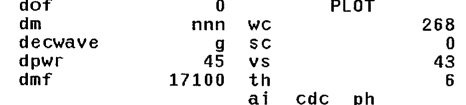

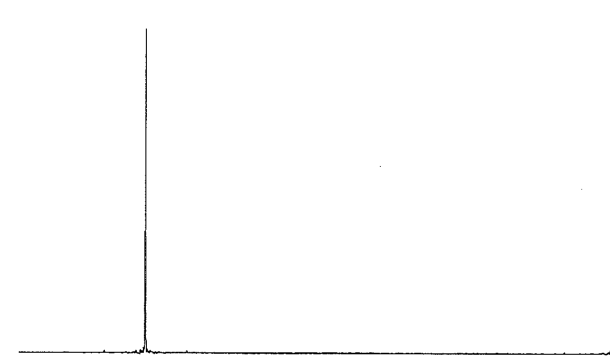

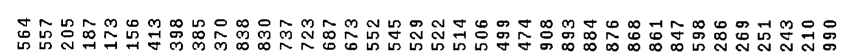

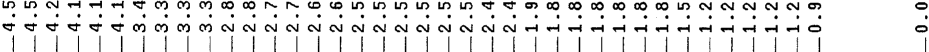

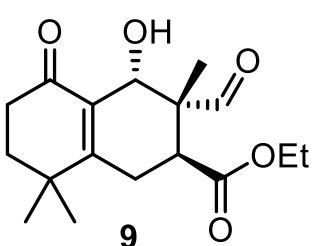

10

9

8

7

6

跴

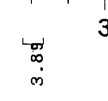

3 in

我 1

o ppm

${ }^{1} \mathrm{H}$ NMR spectrum for compound 9 


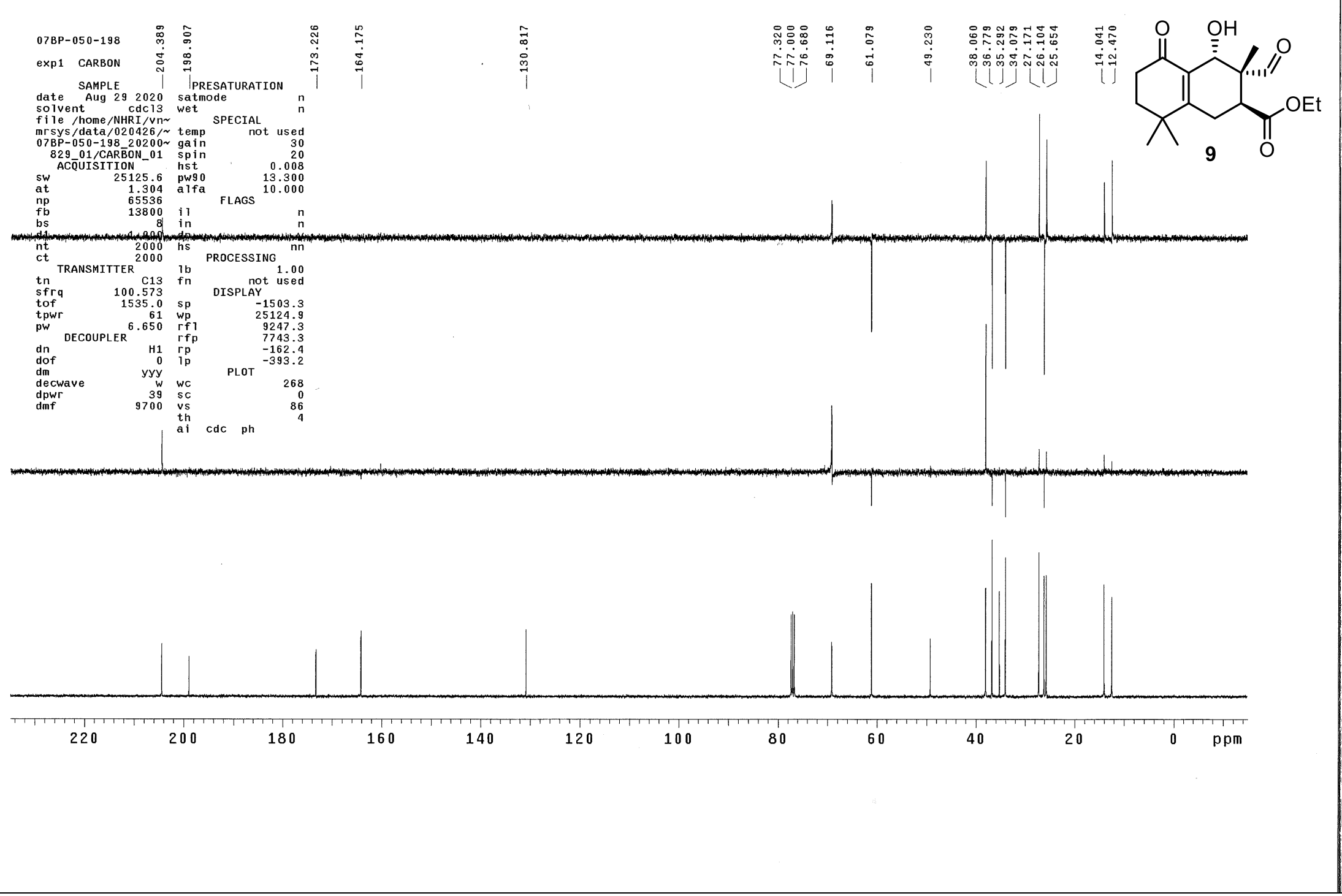

${ }^{13} \mathrm{C}$ NMR + DEPT spectra for compound 9 


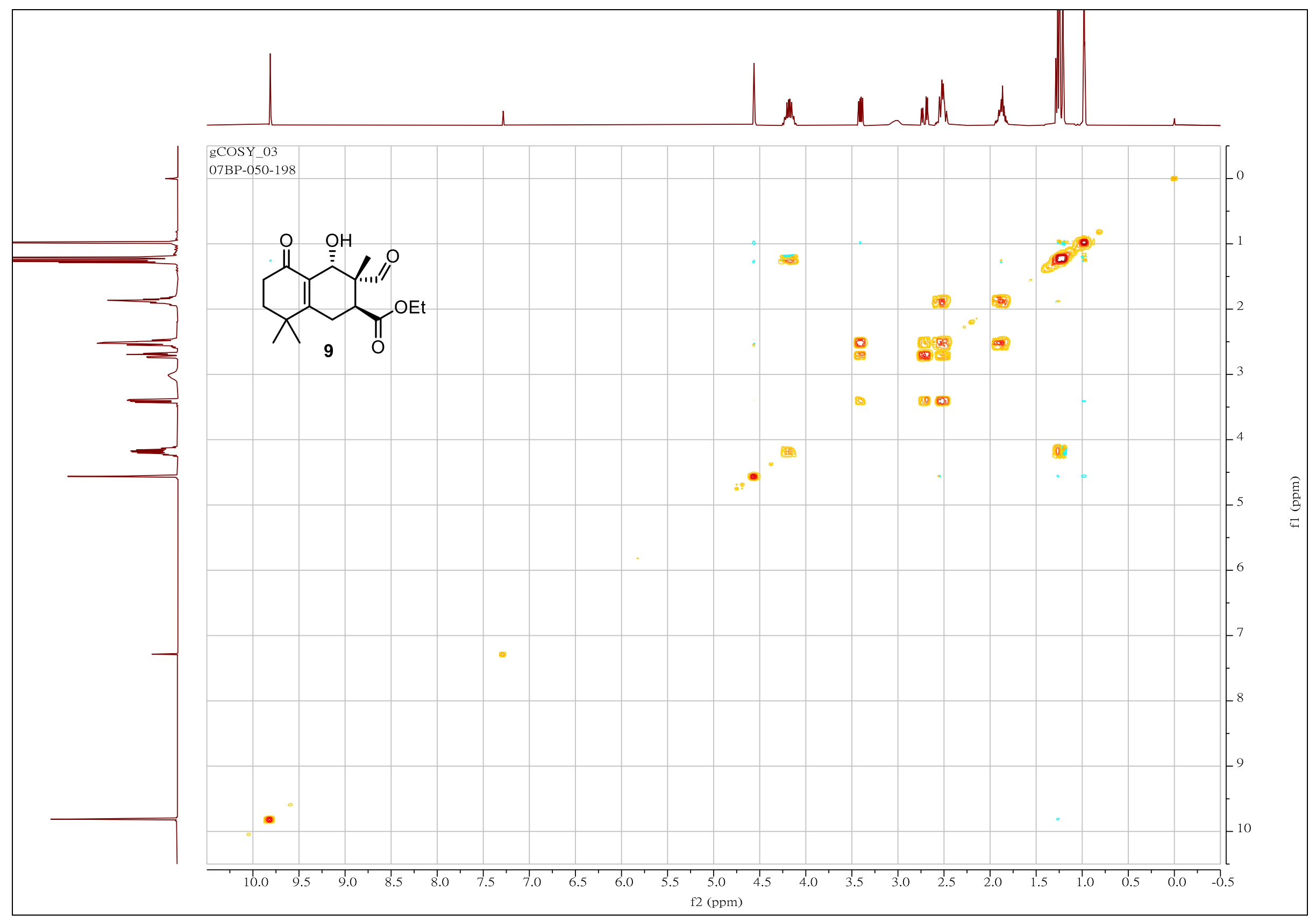

${ }^{1} \mathrm{H}-{ }^{1} \mathrm{H}$ COSY spectrum for compound 9 


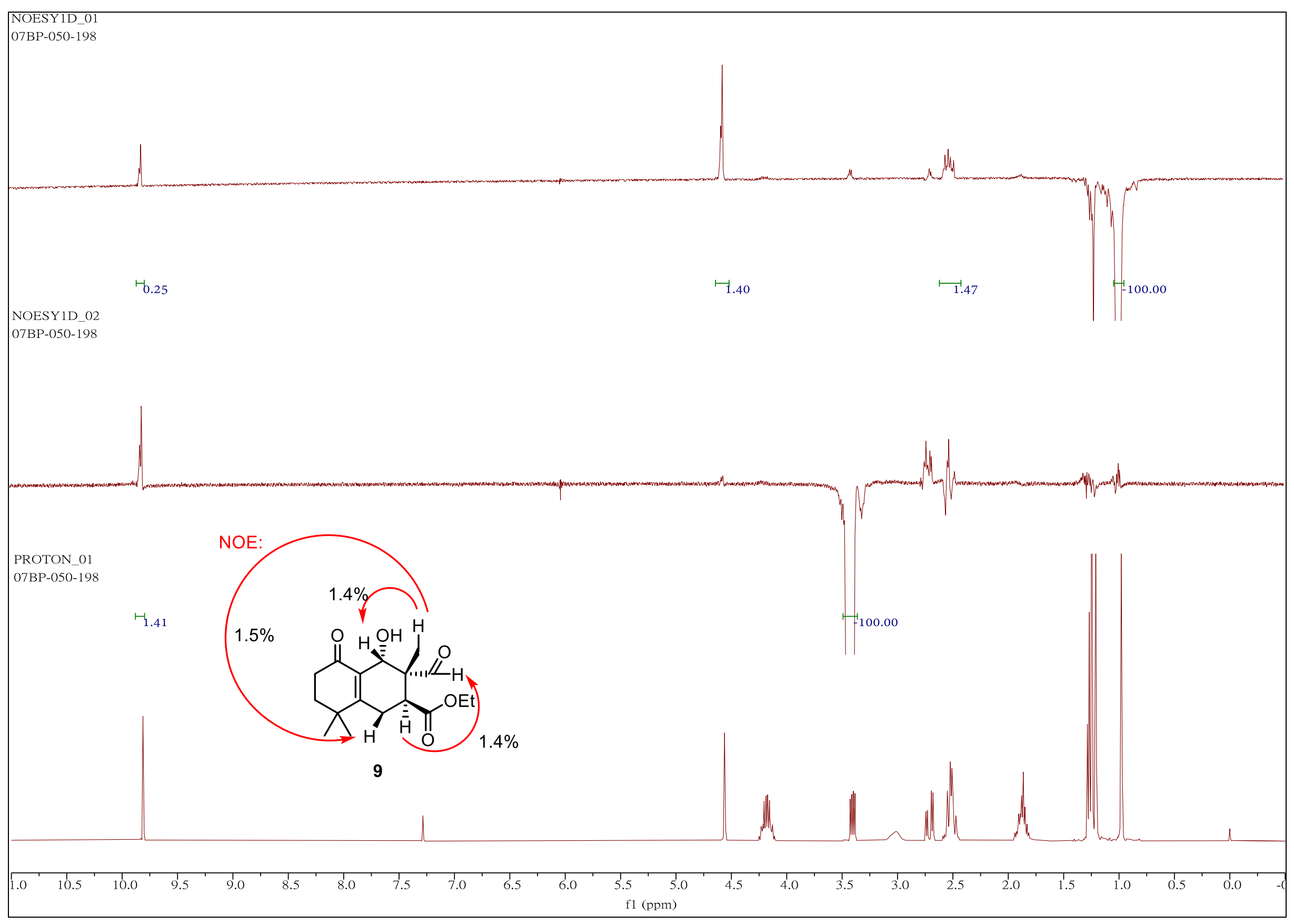

1D NOESY spectra for compound 9

S76 
$09 \mathrm{BP}-010-\stackrel{\infty}{\leftrightarrow 0}$

exp4 PROTंON

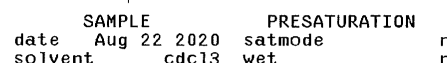

file /home NHRI/Vn N SPECAAL

09BP-010-099A_2020 gain not used

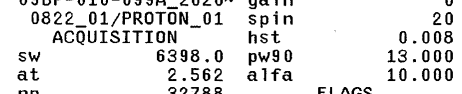

$\begin{array}{lrll}\text { at } & 2.562 & \text { alfa } & \\ \text { np } & 32788 & 10.000 \\ \text { f } & \text { not used } & \text { il } & \text { FLAGS }\end{array}$

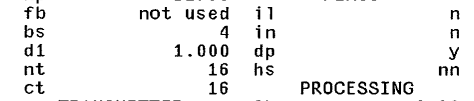

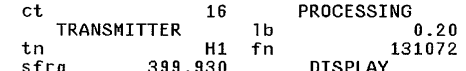

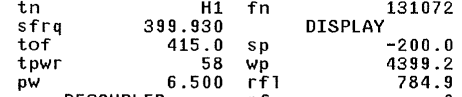

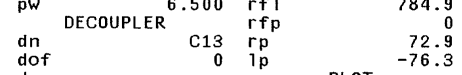

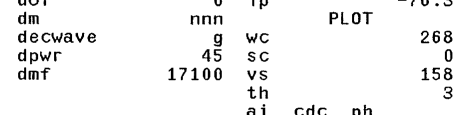

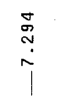

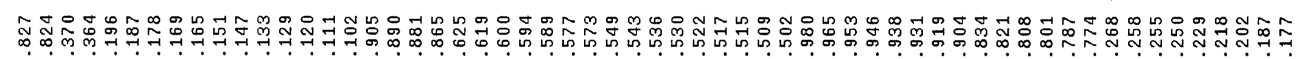
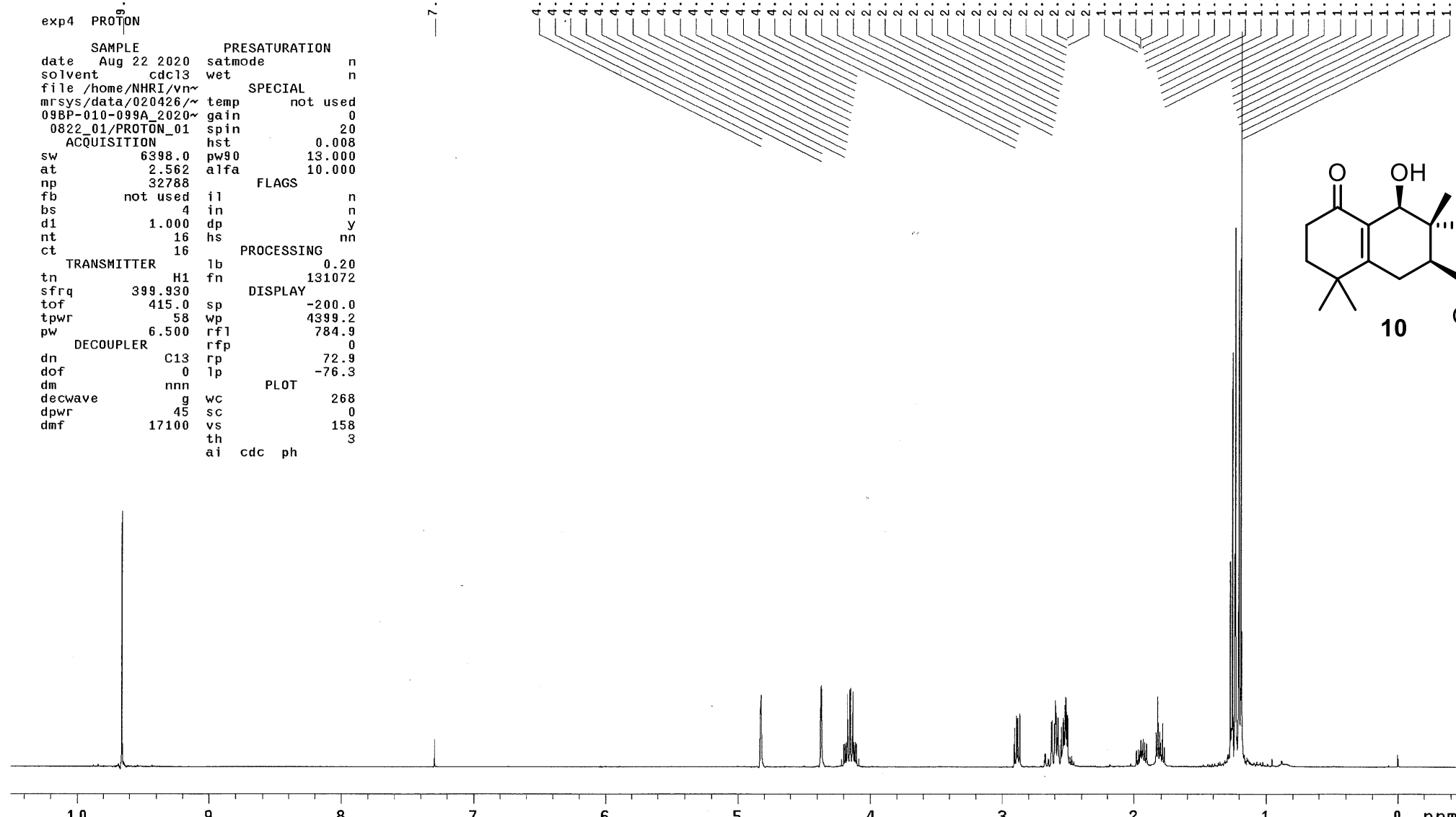

10 :

6

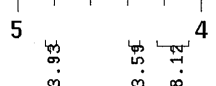

$3 \underset{\infty}{3}$

$\stackrel{\substack{2 \\ \dot{x}}}{2}$

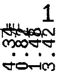

${ }^{1} \mathrm{H}$ NMR spectrum for compound $\mathbf{1 0}$ 


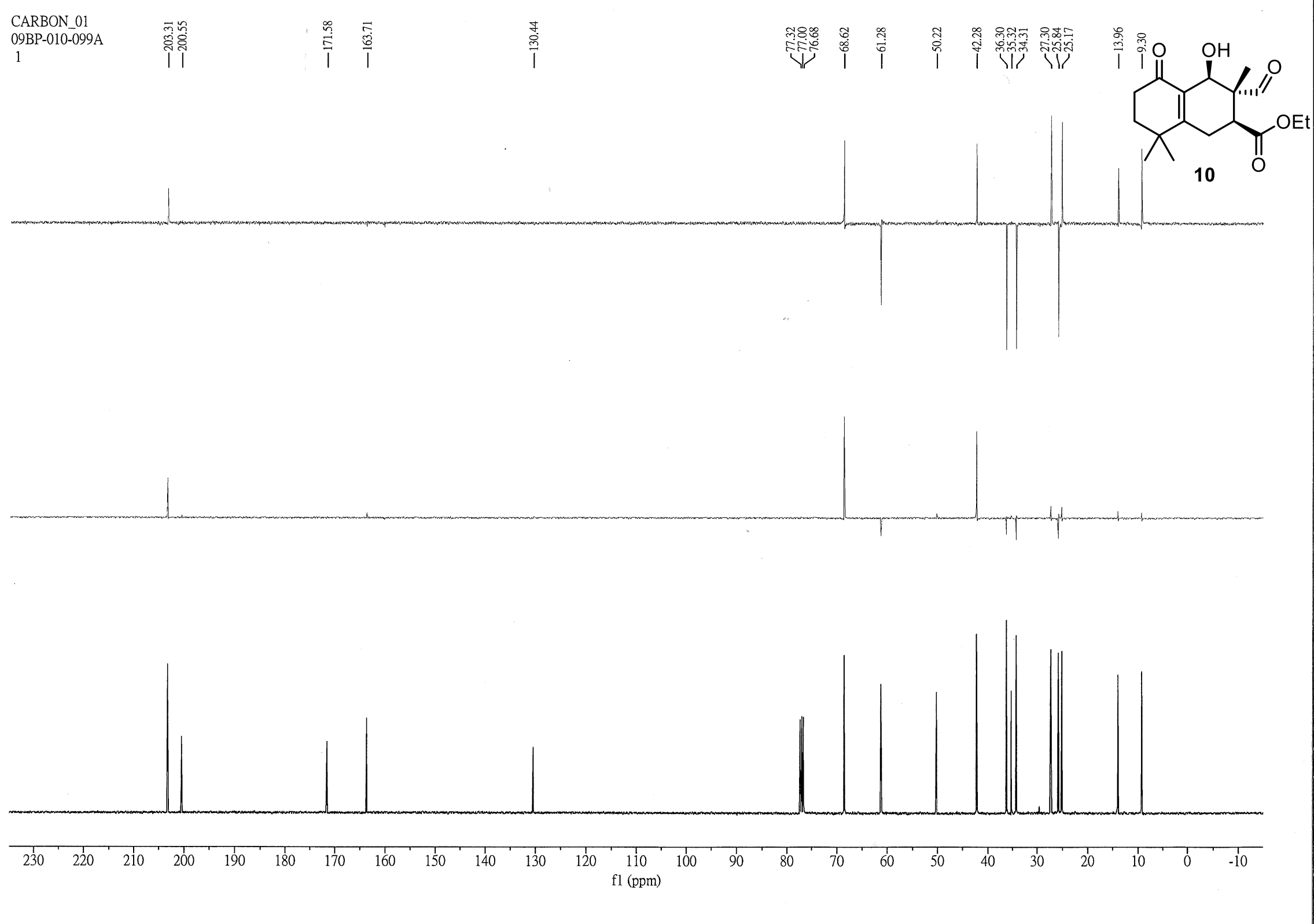

${ }^{13} \mathrm{C}$ NMR + DEPT spectra for compound $\mathbf{1 0}$ 


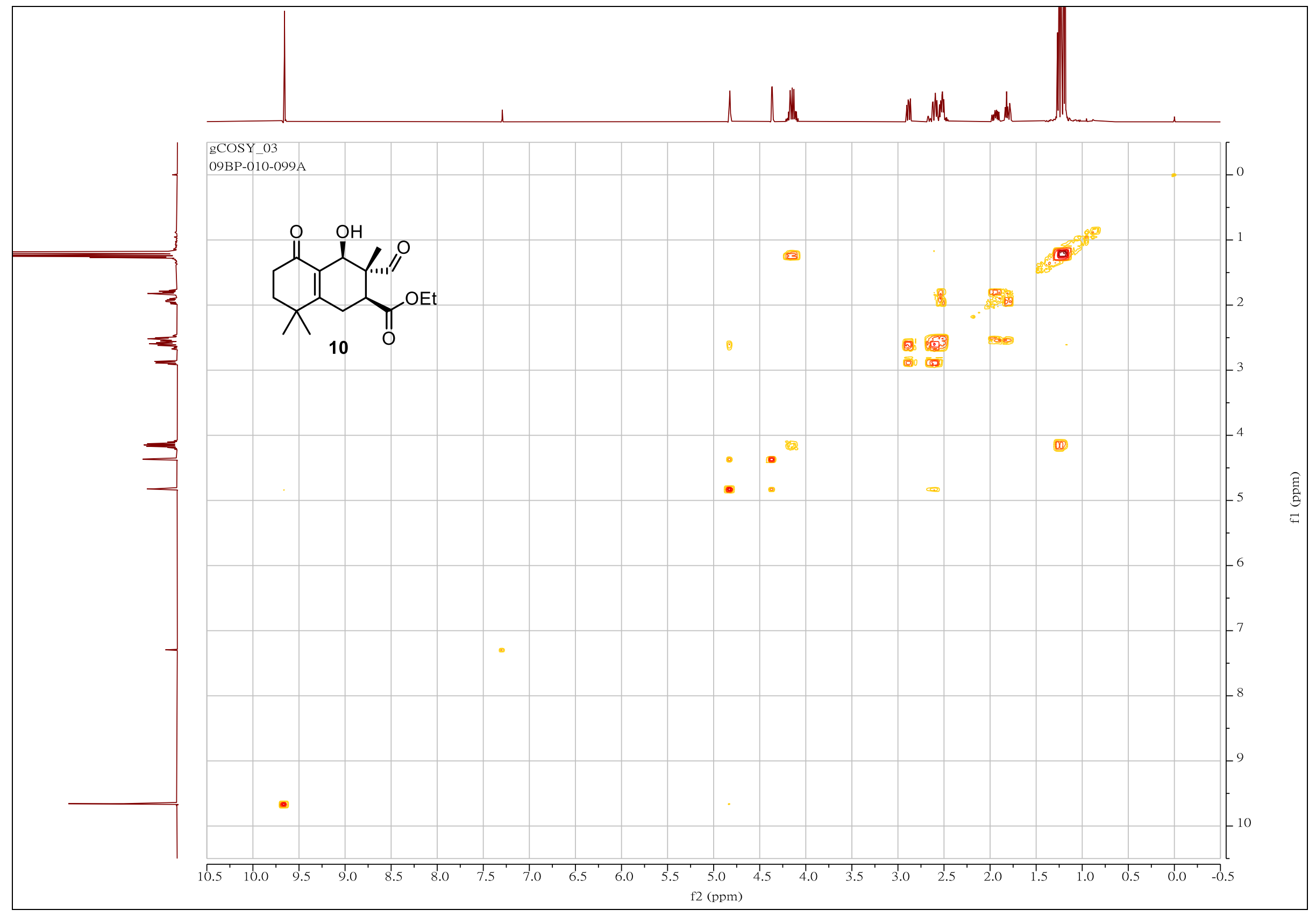

${ }^{1} \mathrm{H}-{ }^{1} \mathrm{H}$ COSY spectrum for compound $\mathbf{1 0}$ 


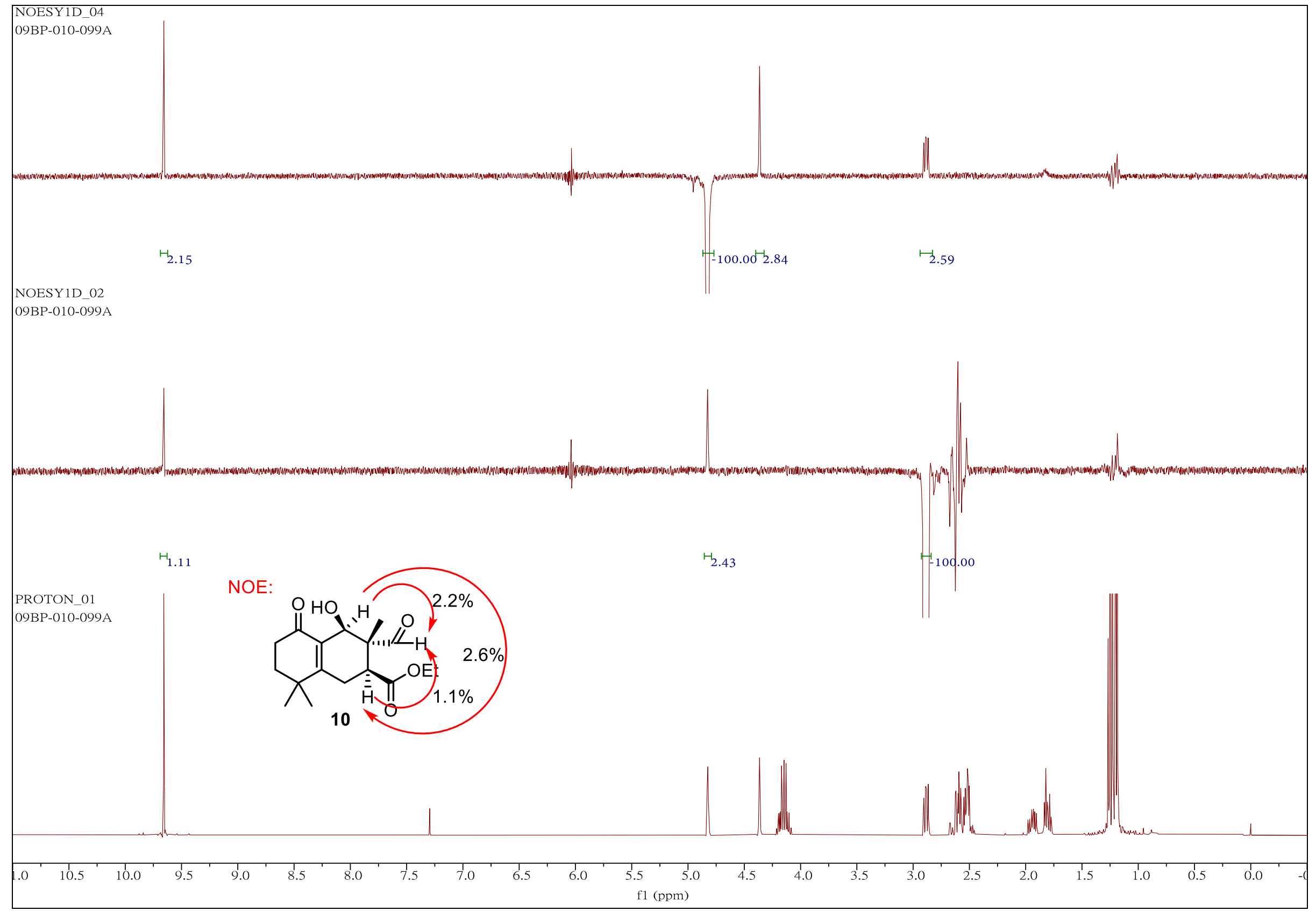

1D NOESY spectra for compound $\mathbf{1 0}$ 

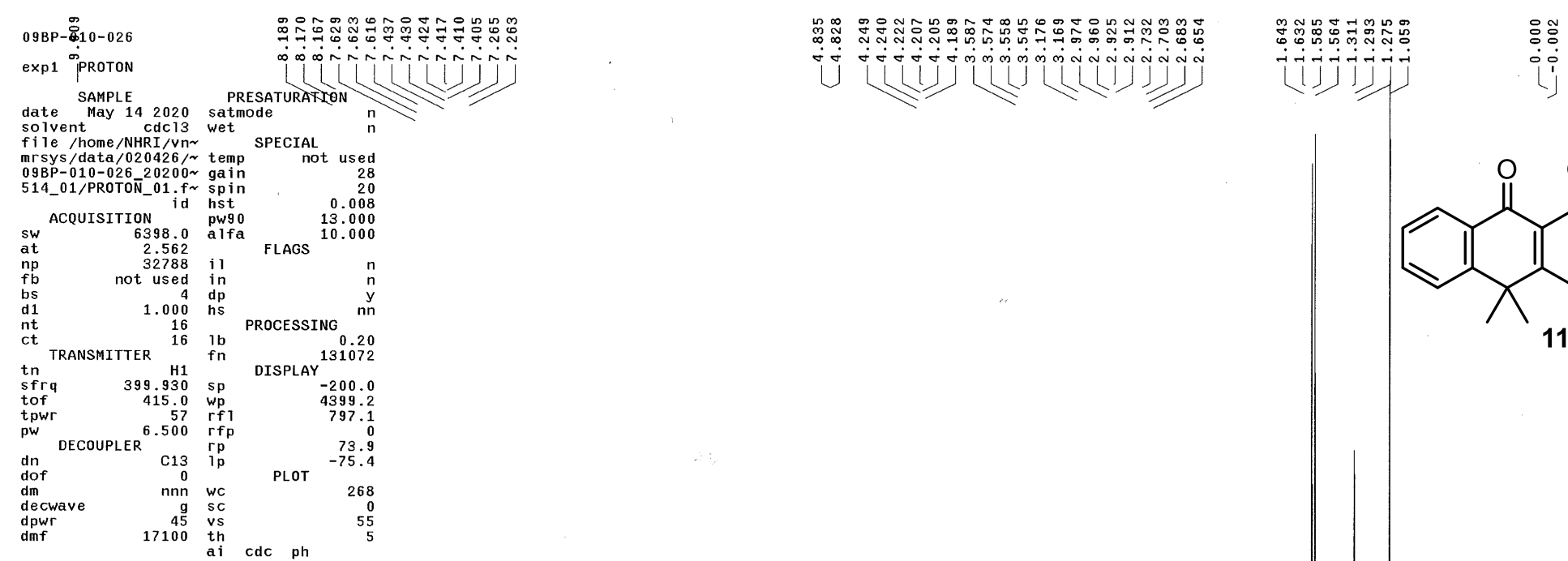

exp1 ${ }^{\circ}$ PROTO

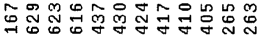

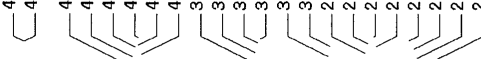

$99 \mathrm{BP}-010-026$ -

Ad hst

16 PROCESSING ${ }^{n n}$

TRANSMITTER $\begin{array}{ll}16 & 0.20 \\ & \end{array}$

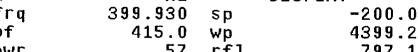

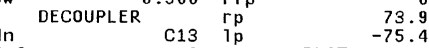

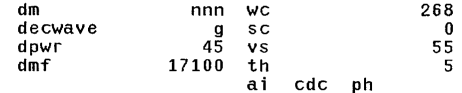

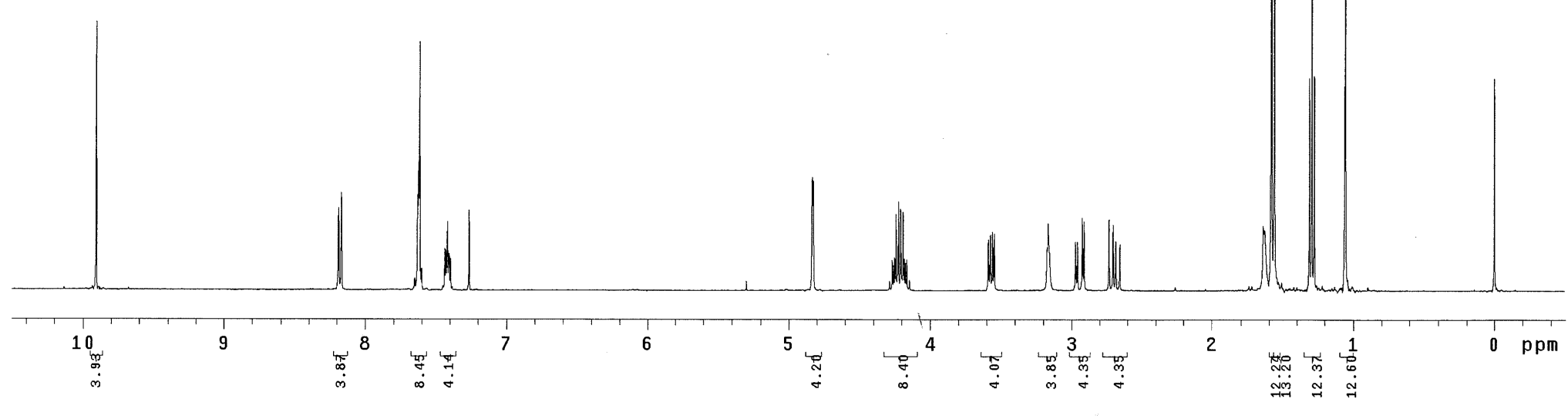

${ }^{1}$ H NMR spectrum for compound $\mathbf{1 1}$ 


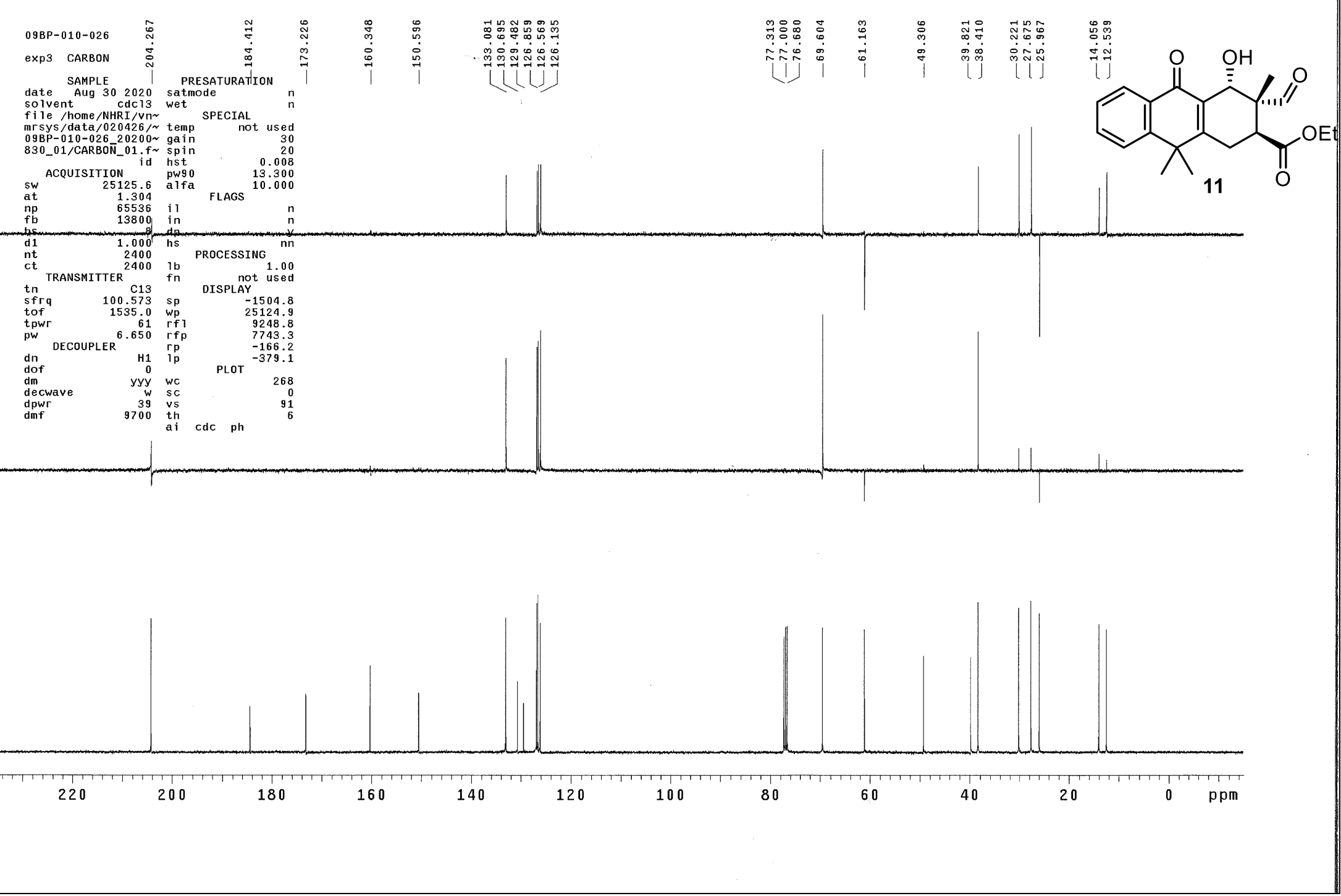

${ }^{13} \mathrm{C}$ NMR + DEPT spectra for compound $\mathbf{1 1}$ 


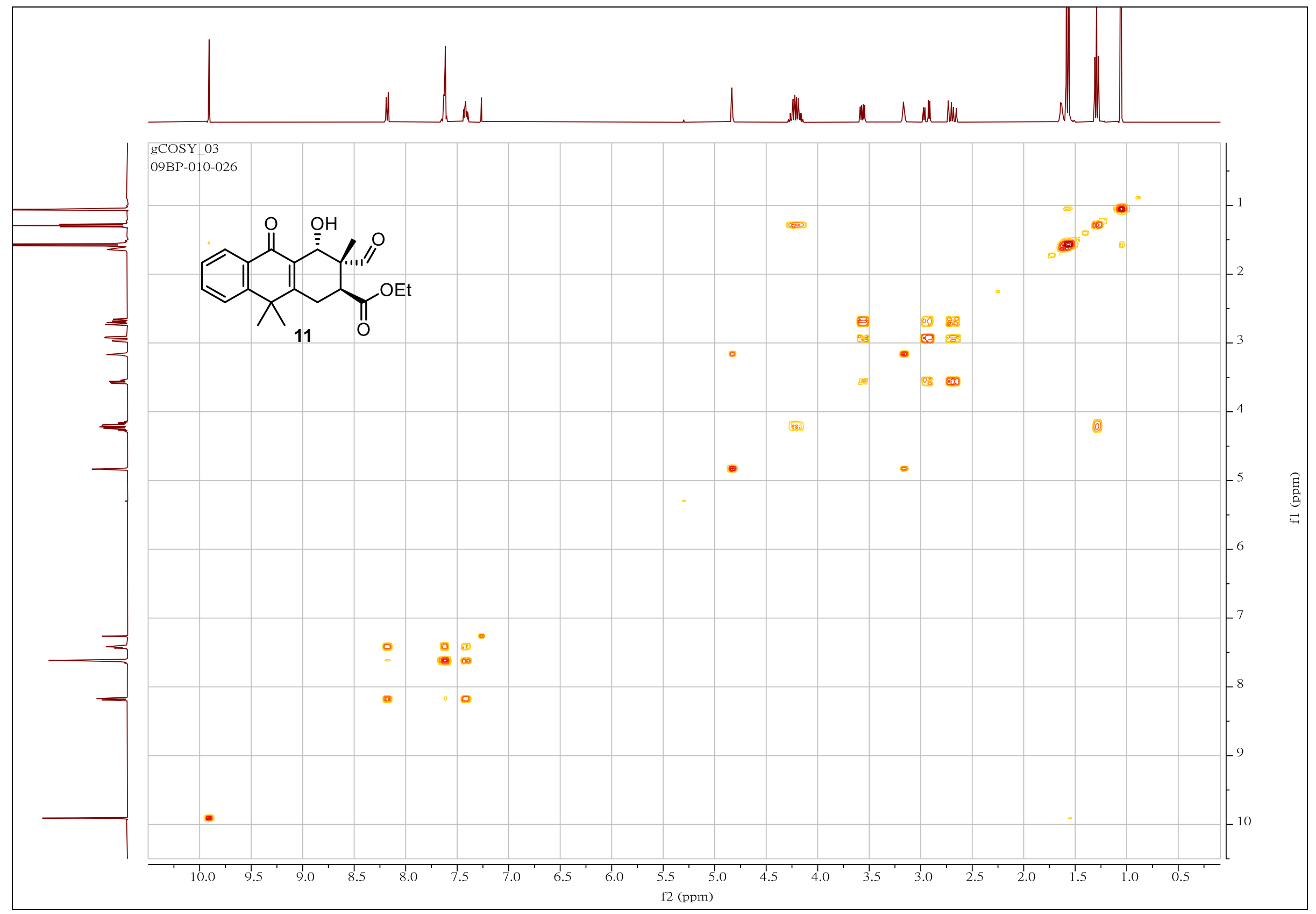

${ }^{1} \mathrm{H}-{ }^{1} \mathrm{H}$ COSY spectrum for compound $\mathbf{1 1}$ 


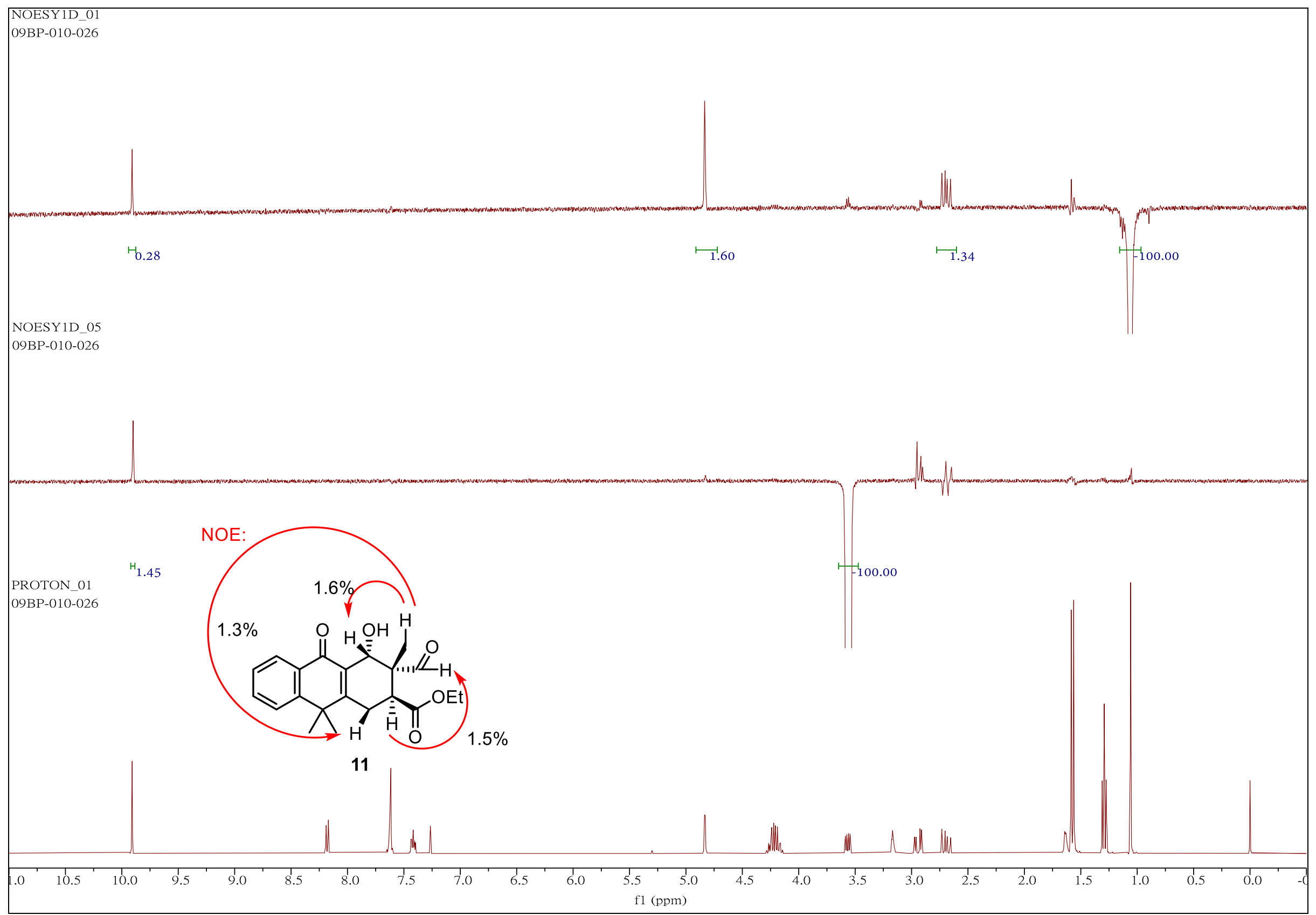

1D NOESY spectra for compound $\mathbf{1 1}$ 
09BP-0 0 迎- 025

exp1 PिRoton

SAMPLE
date
solvent 172020 $\begin{aligned} & \text { PRESATURATION } \\ & \text { satmode } \\ & \text { wetclion }\end{aligned}$

09BP-010-025-20200 gain not used

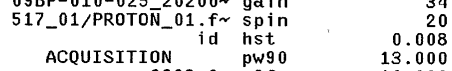

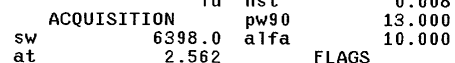

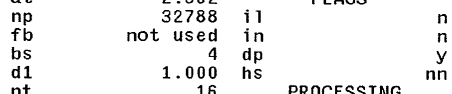

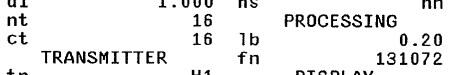

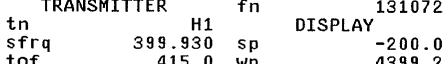

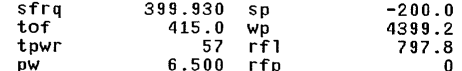

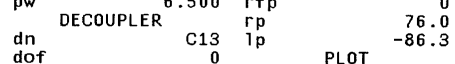

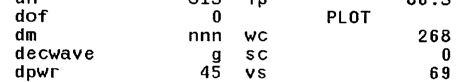

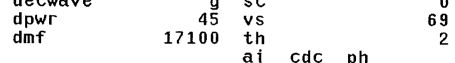
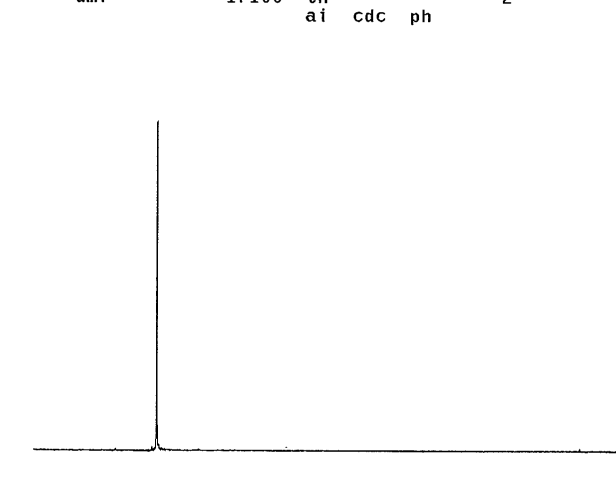

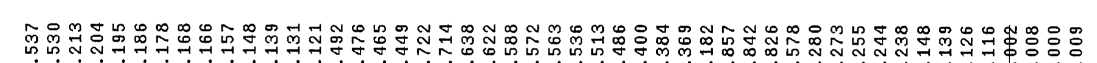

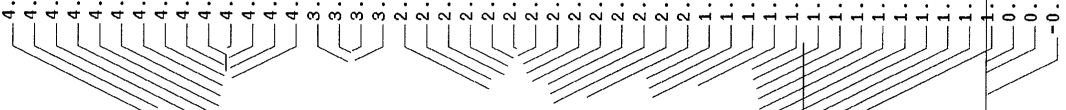

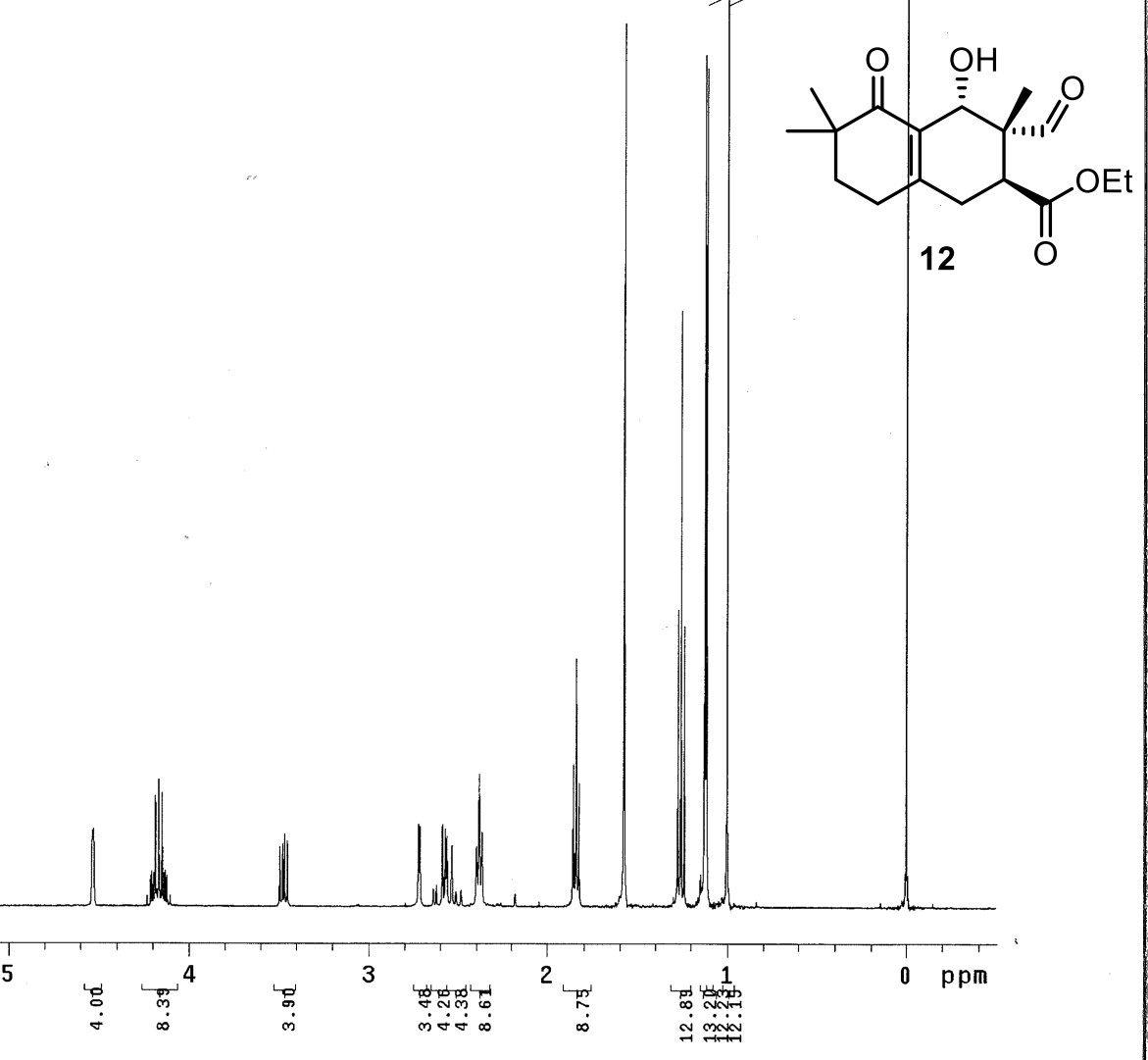

${ }^{1} \mathrm{H}$ NMR spectrum for compound $\mathbf{1 2}$ 


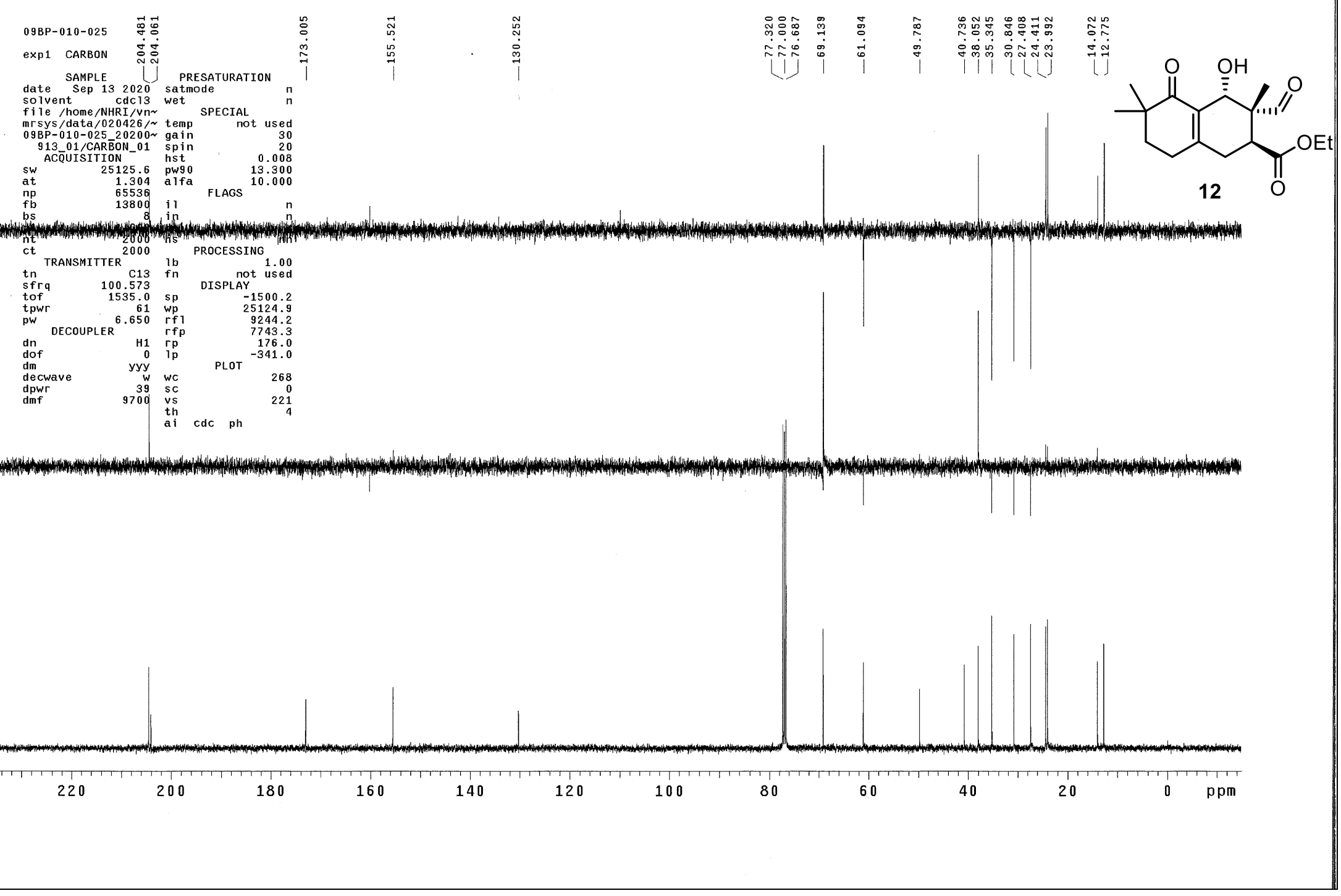

${ }^{13} \mathrm{C}$ NMR + DEPT spectra for compound $\mathbf{1 2}$ 


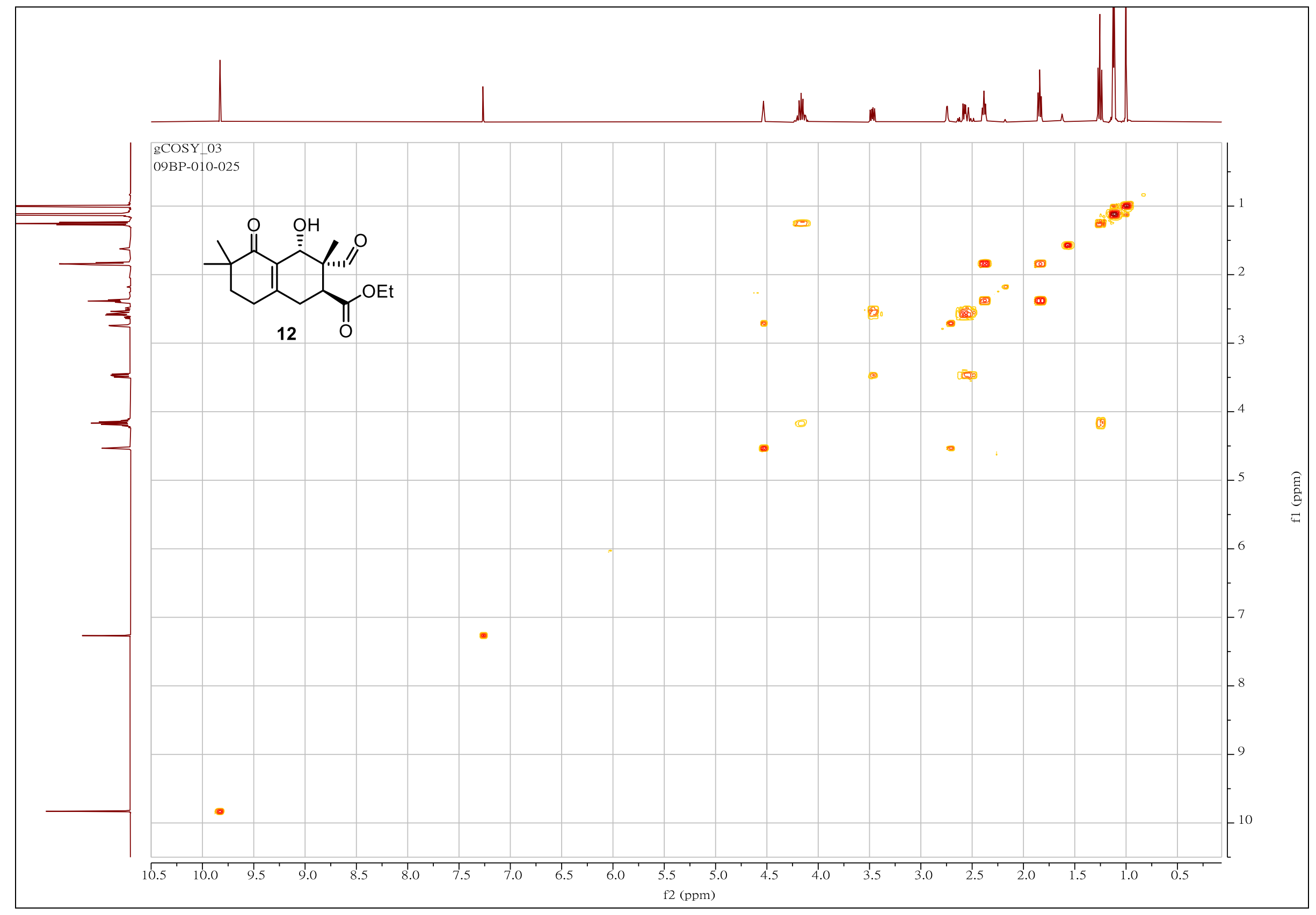

${ }^{1} \mathrm{H}-{ }^{1} \mathrm{H}$ COSY spectrum for compound $\mathbf{1 2}$ 


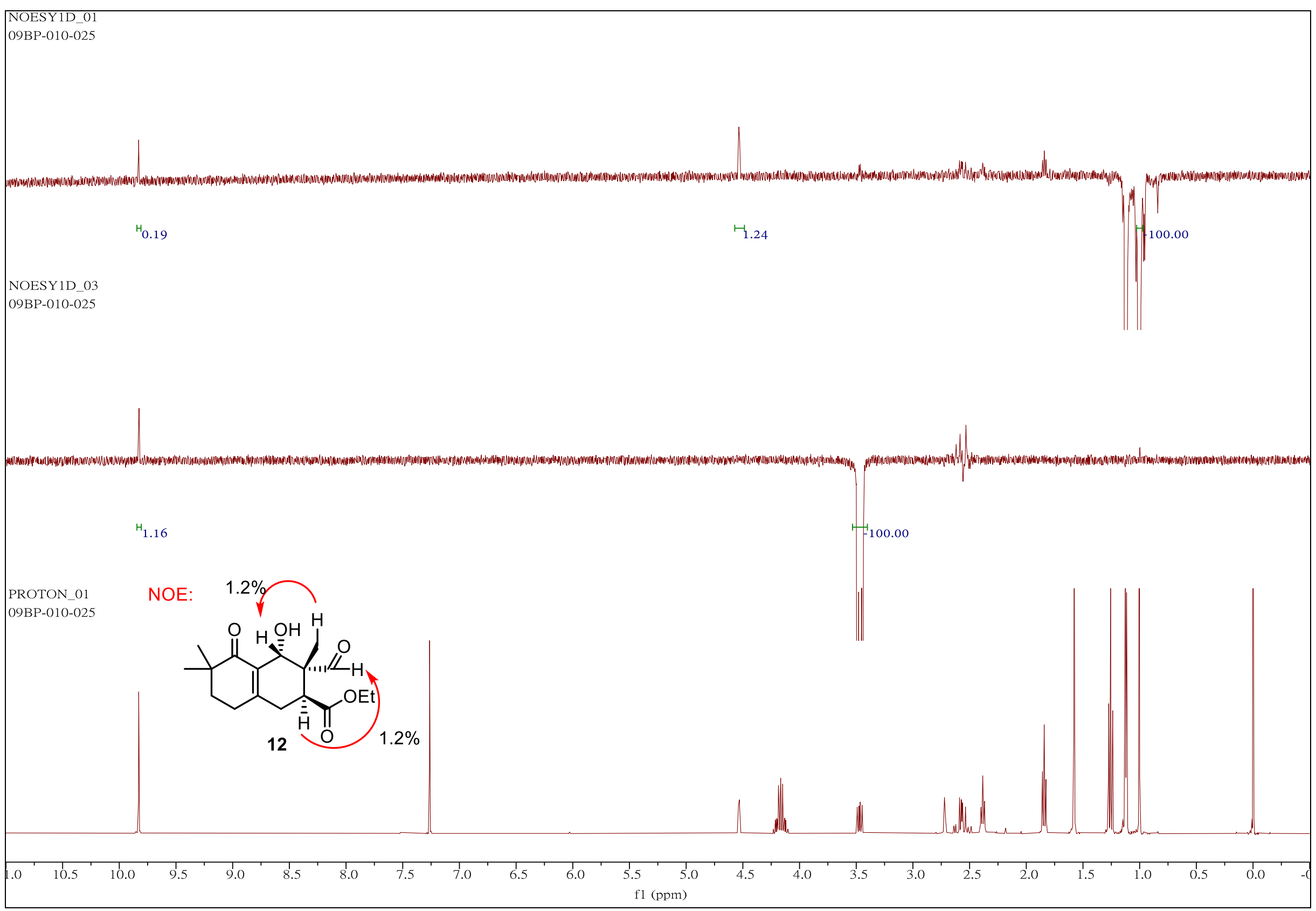

1D NOESY spectra for compound $\mathbf{1 2}$ 


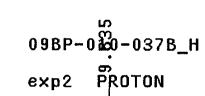

SAMPLE

date SAMPLE 102020 PRESATURATION

wet

I $/$ Vn $~$ SPECIAL

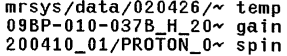

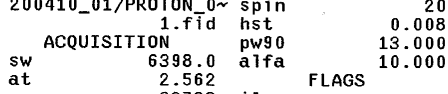

$\mathrm{fp}$
$\mathrm{fb}$
$\mathrm{bs}$

bs

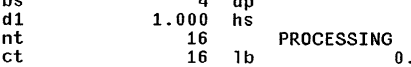

tn TRANSMITTER H1 fn PISPAY 131072

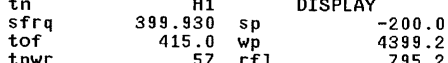

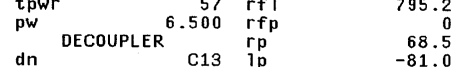

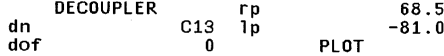

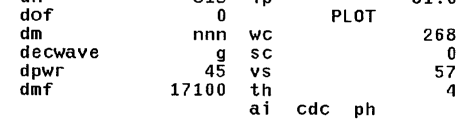

\section{$\stackrel{\substack{c \\ i}}{i}$}

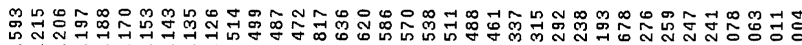

$\stackrel{\circ}{\vdots}$

ai cdc ph
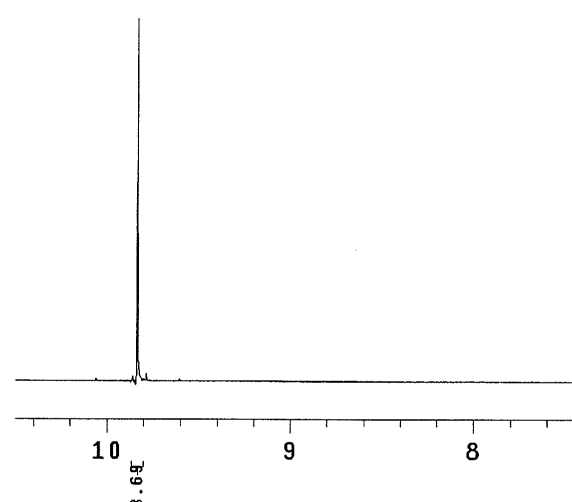

$10 \underset{\substack{b \\ \dot{m}}}{0}$

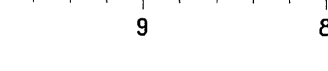

$7+1,190$

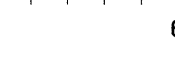

6

5

桨

${ }^{1} \mathrm{H}$ NMR spectrum for compound $\mathbf{1 3}$ 


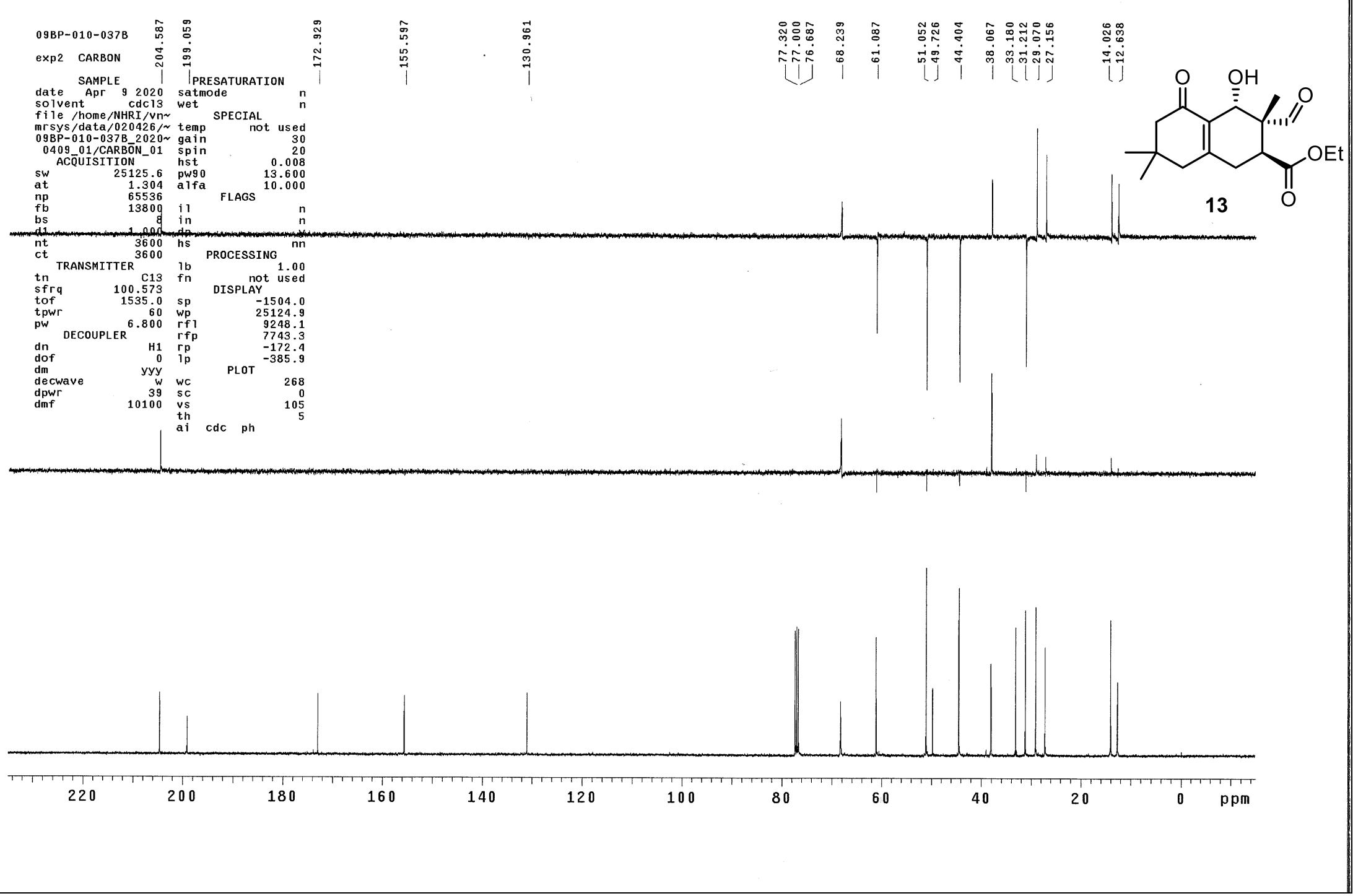

${ }^{13} \mathrm{C}$ NMR + DEPT spectra for compound $\mathbf{1 3}$ 


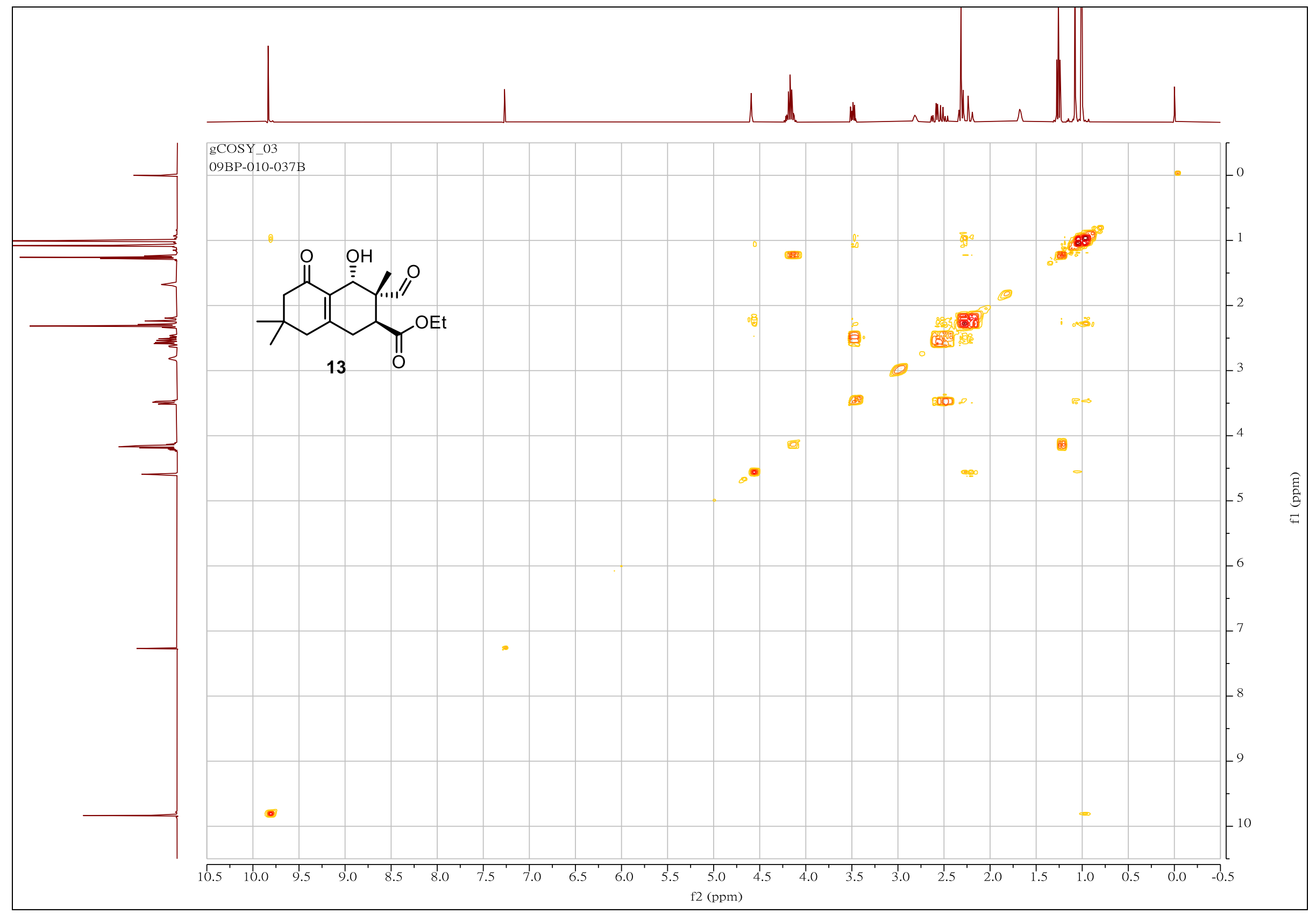

${ }^{1} \mathrm{H}-{ }^{1} \mathrm{H}$ COSY spectrum for compound $\mathbf{1 3}$ 


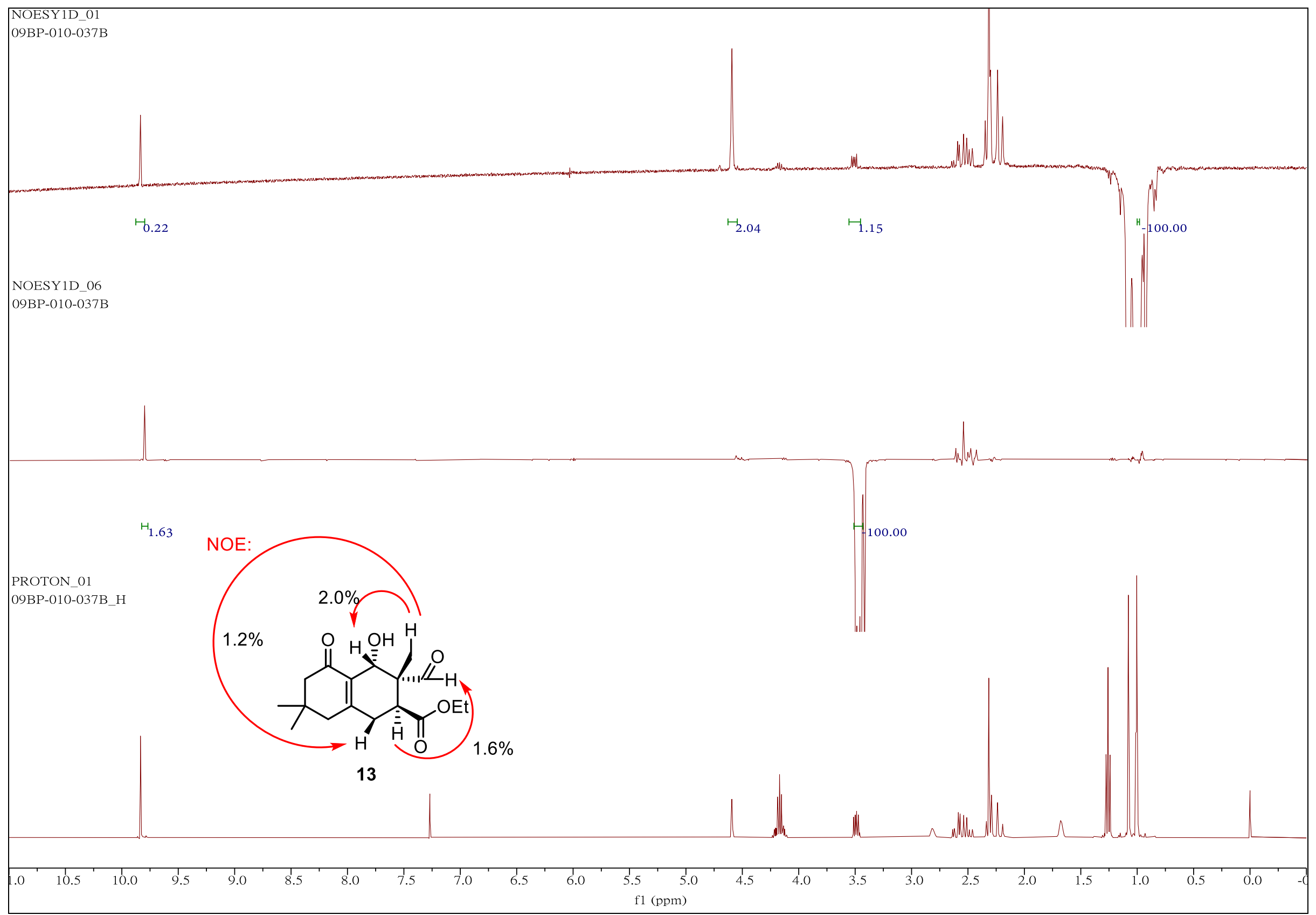

1D NOESY spectra for compound $\mathbf{1 3}$ 
$09 \mathrm{BP}-0 \stackrel{0}{50} 0-029+\mathrm{H}$

exp2 Pिं0ToN

date MAMPLE $242020 \begin{gathered}\text { PRESATURATION } \\ \text { satmode }\end{gathered}$

date
solvent 24 dCl3 wet

mrsys/data/020426/ $\sim$ temp SPECIAL not used

mo9BP-010-029_H_202 gain

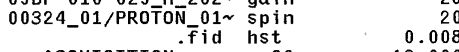

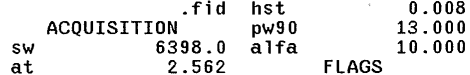

$\begin{array}{lrl}\text { at } & 2.562 & \\ \mathrm{np} & 32788 & \mathrm{il}\end{array}$

$\begin{array}{lrl}\mathrm{fb} & \text { not used } & \text { in } \\ \text { bs } & \text { dp } \\ \text { d1 } & 1.000 & \text { h }\end{array}$

1.0070

ct TRANSMITTER $\begin{array}{lll}16 & \text { lb } & 0.20 \\ \text { fn } & 131072\end{array}$

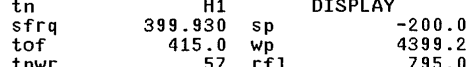

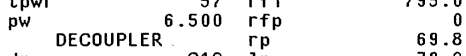

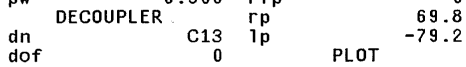

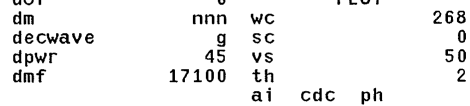

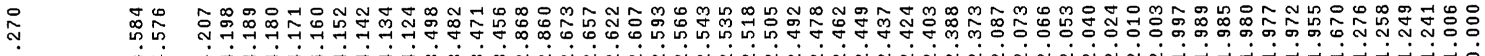
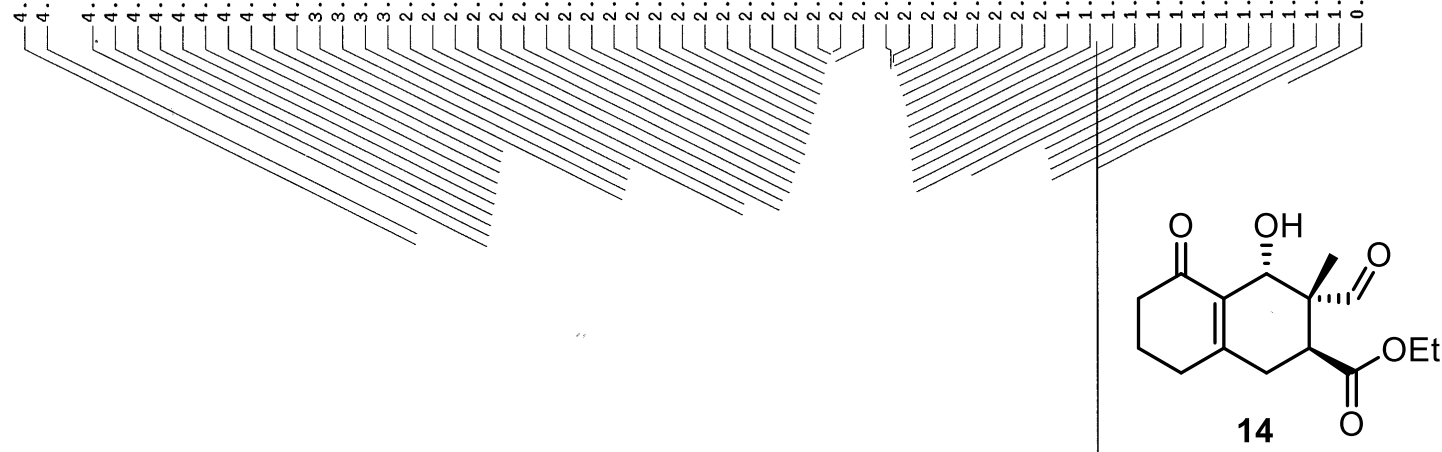

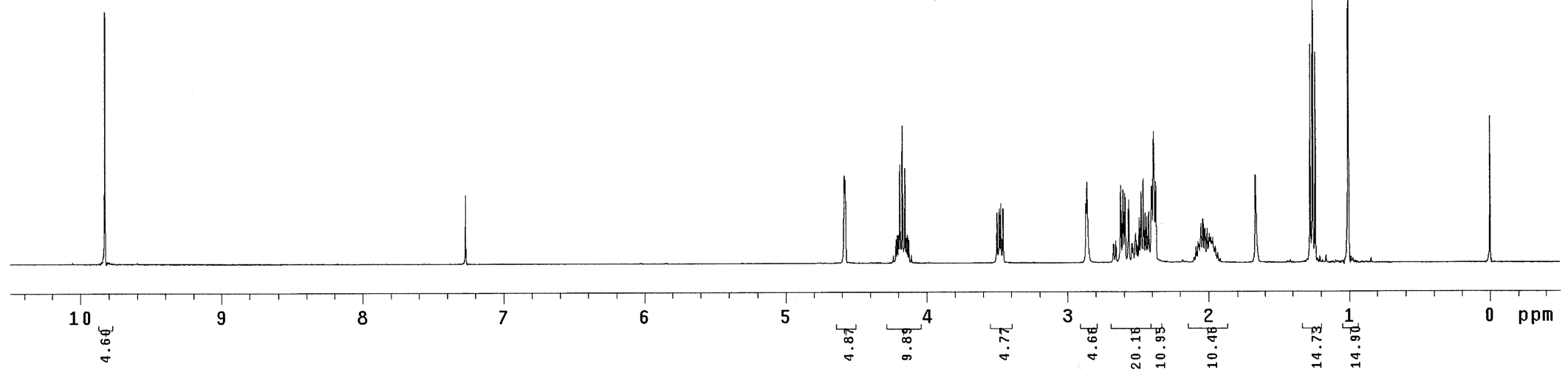

${ }^{1} \mathrm{H}$ NMR spectrum for compound $\mathbf{1 4}$ 


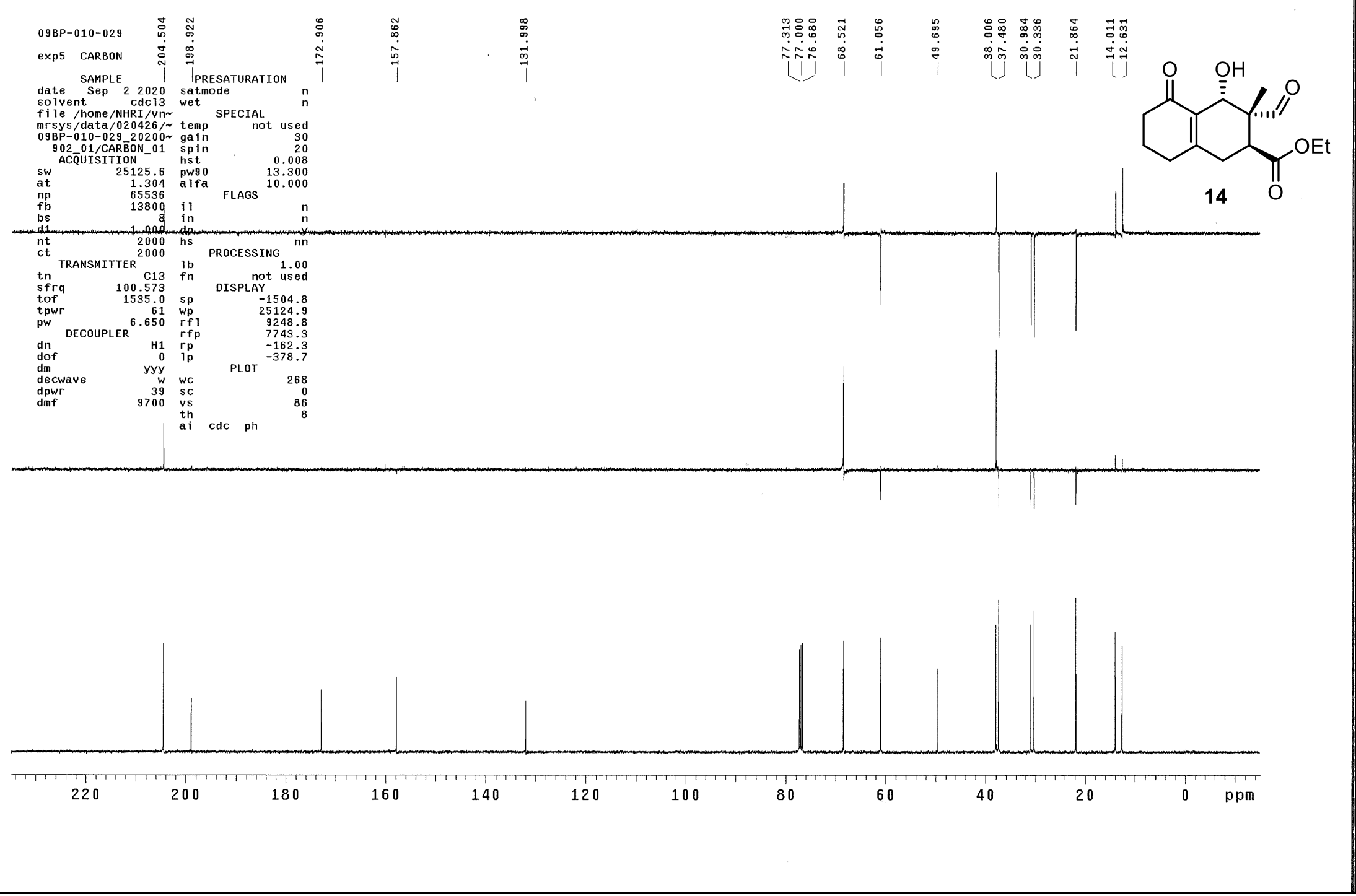

${ }^{13} \mathrm{C}$ NMR + DEPT spectra for compound $\mathbf{1 4}$ 


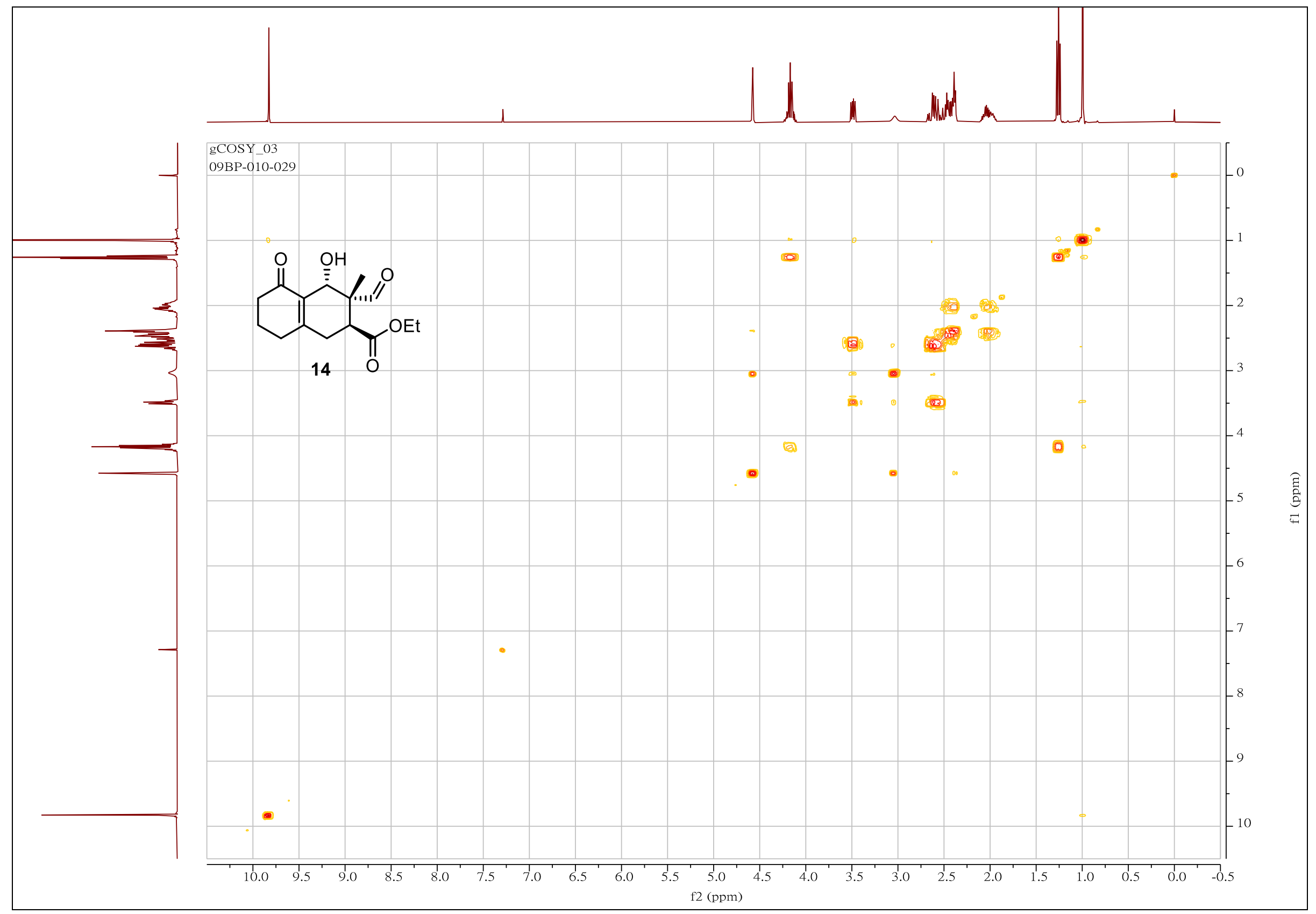

${ }^{1} \mathrm{H}-{ }^{1} \mathrm{H}$ COSY spectrum for compound $\mathbf{1 4}$ 


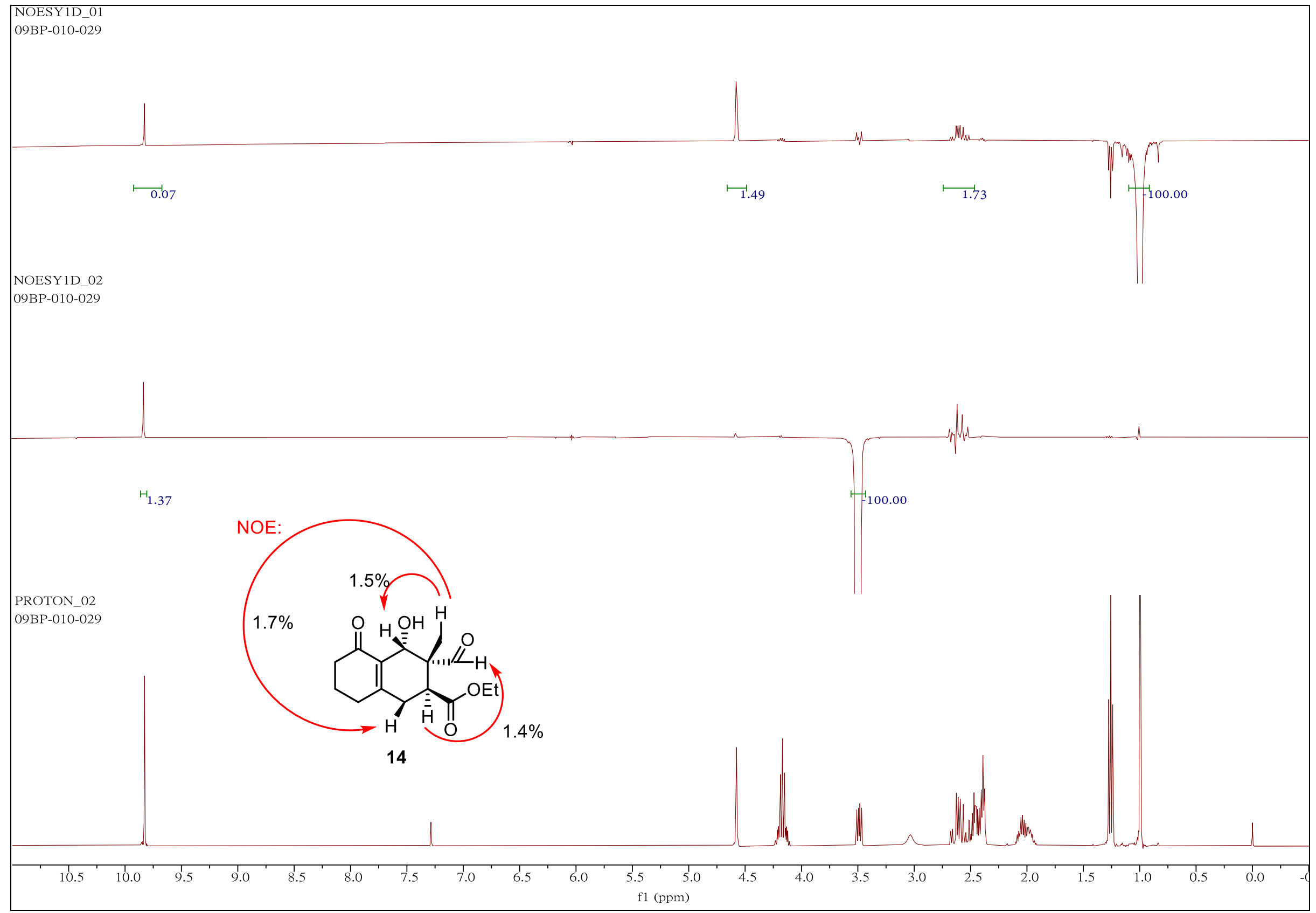

1D NOESY spectra for compound $\mathbf{1 4}$ 


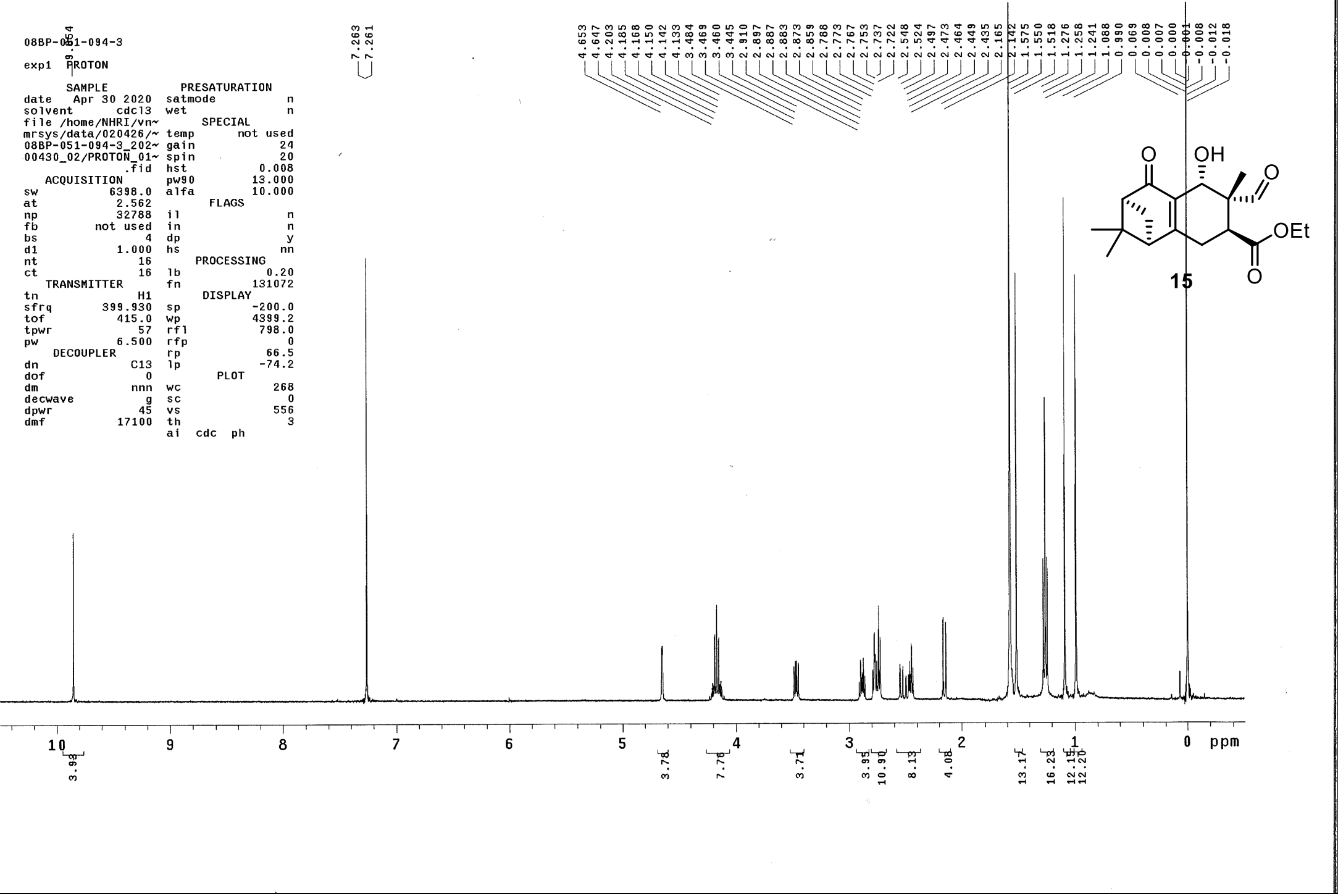

${ }^{1} \mathrm{H}$ NMR spectrum for compound $\mathbf{1 5}$ 


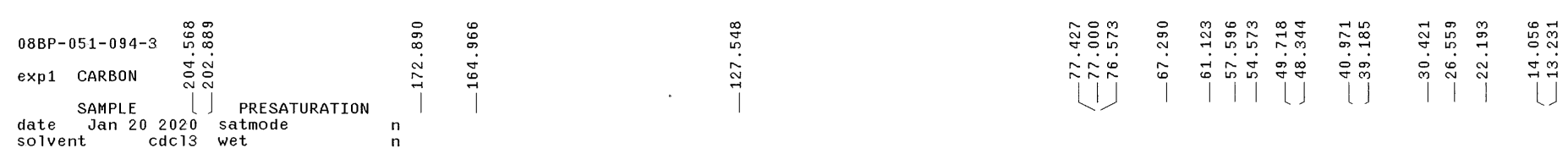

file /home/NHRI/Vn $\sim$ Wet SPECIAL

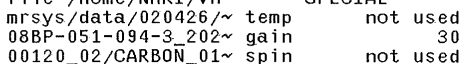

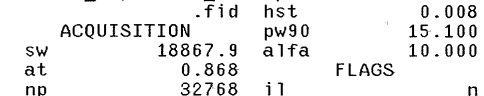

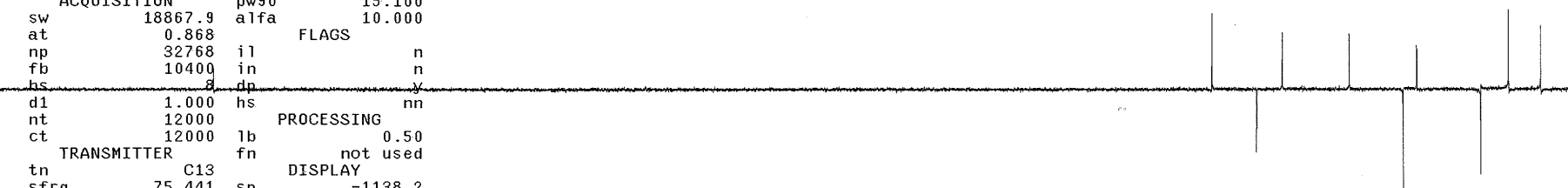

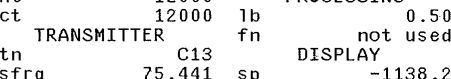

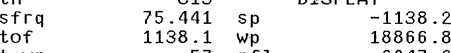

$\begin{array}{lrlr}\text { tpwr } & 57 & \mathrm{rf} & 6 \\ \mathrm{pw} & 7.550 & \mathrm{rfp} & 6947.6 \\ & & 5808.3\end{array}$

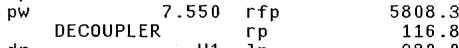

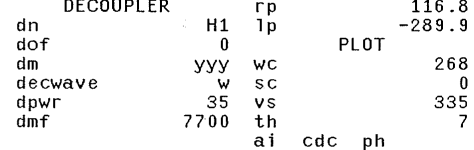

$\underset{\substack{268 \\ 305}}{309}$

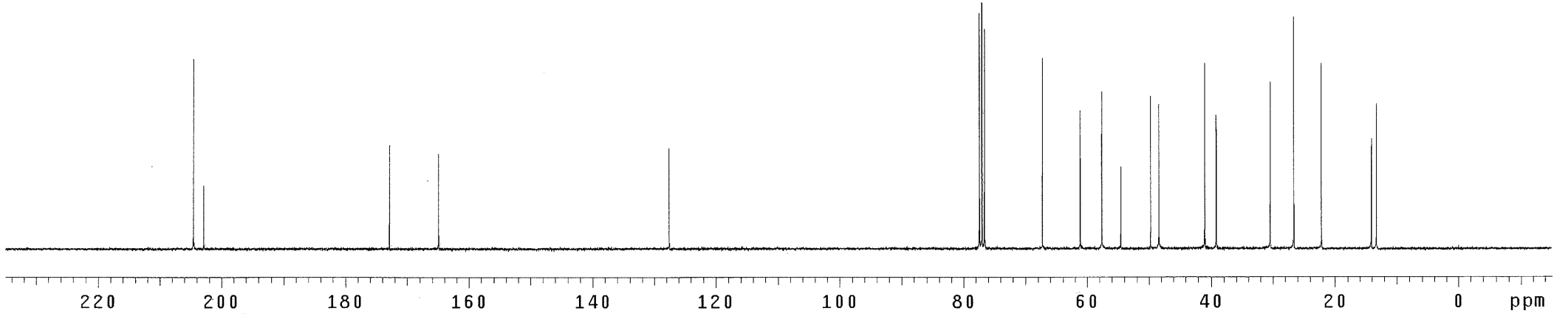

${ }^{13} \mathrm{C}$ NMR + DEPT spectra for compound $\mathbf{1 5}$ 
09BP-0 $\stackrel{m}{\stackrel{\varpi}{\leftrightarrows} 0-061 \mathrm{~B}}$

exp1 PROTON

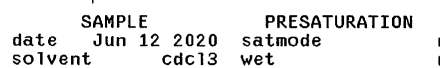

file /home /NHRI/Vn $\sim$ SPECIAL

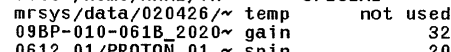

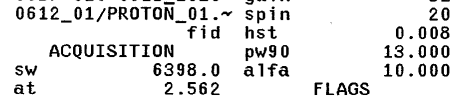

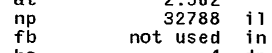

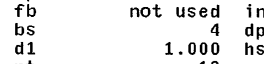

$\begin{array}{llll}10 & 16 & \text { hrocessing } \\ \mathrm{ct} & 16 & \text { ib }\end{array}$

tn TRANSMITER H1 fn DISPLAY 131072

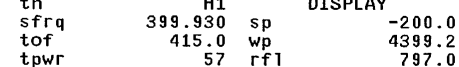

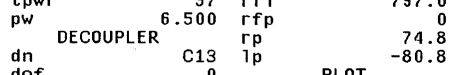

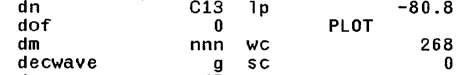

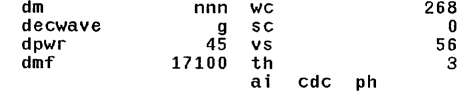

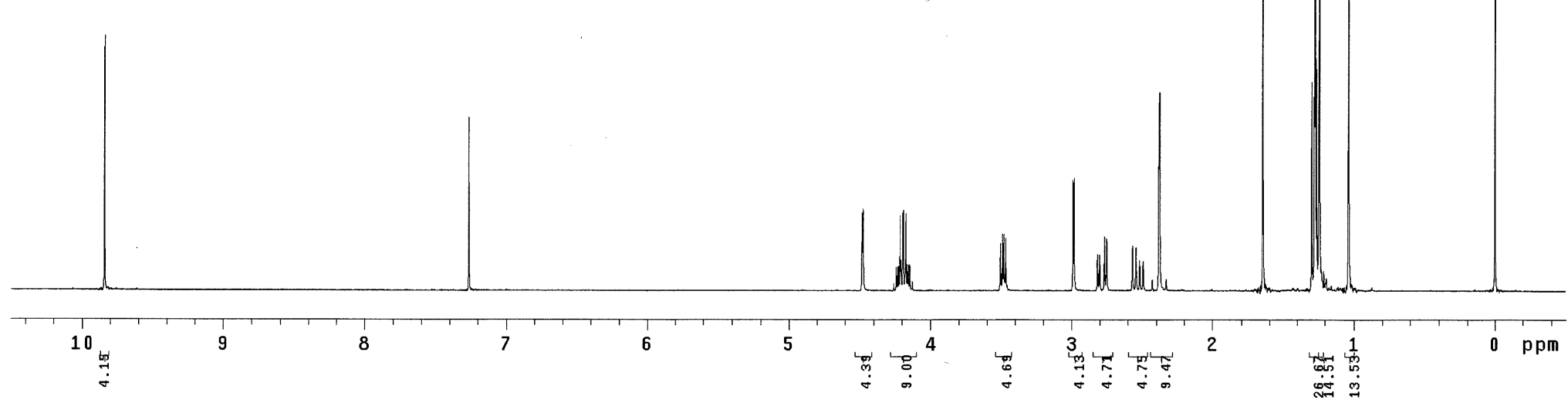

0
0
0

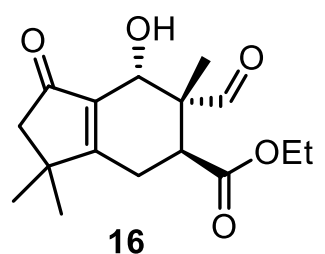

${ }^{1} \mathrm{H}$ NMR spectrum for compound $\mathbf{1 6}$ 


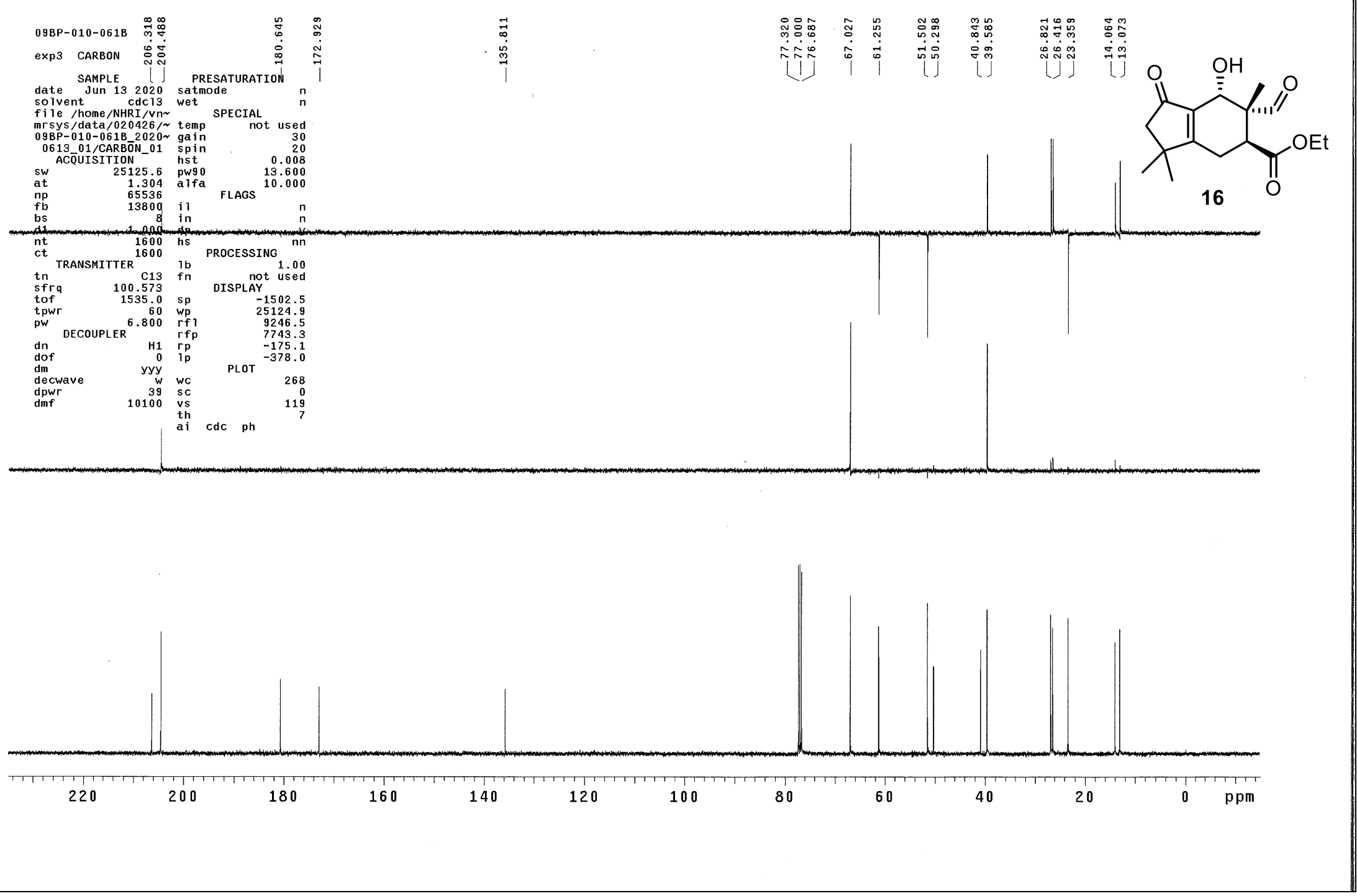

${ }^{13} \mathrm{C}$ NMR + DEPT spectra for compound 16 


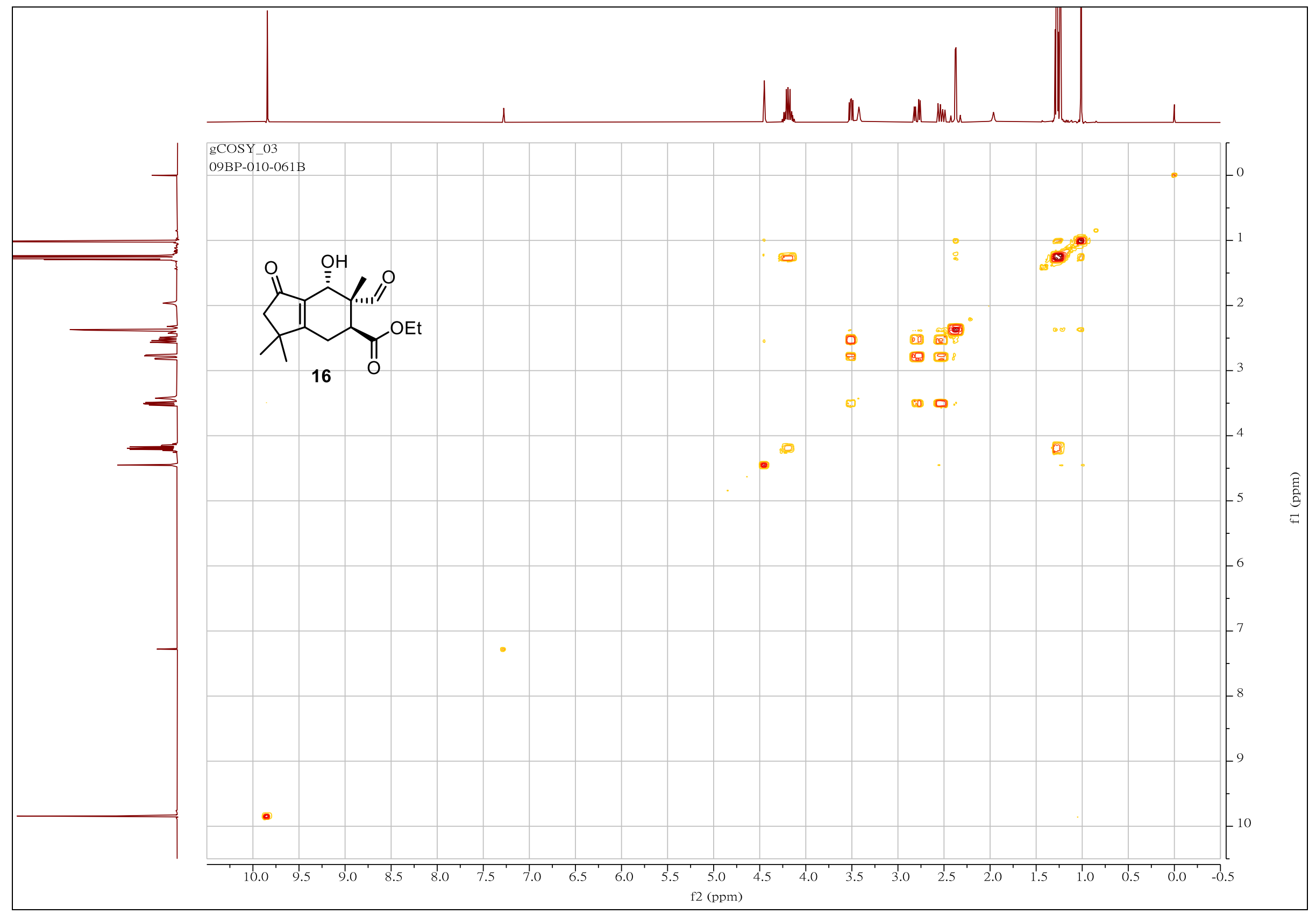

${ }^{1} \mathrm{H}-{ }^{1} \mathrm{H}$ COSY spectrum for compound $\mathbf{1 6}$ 


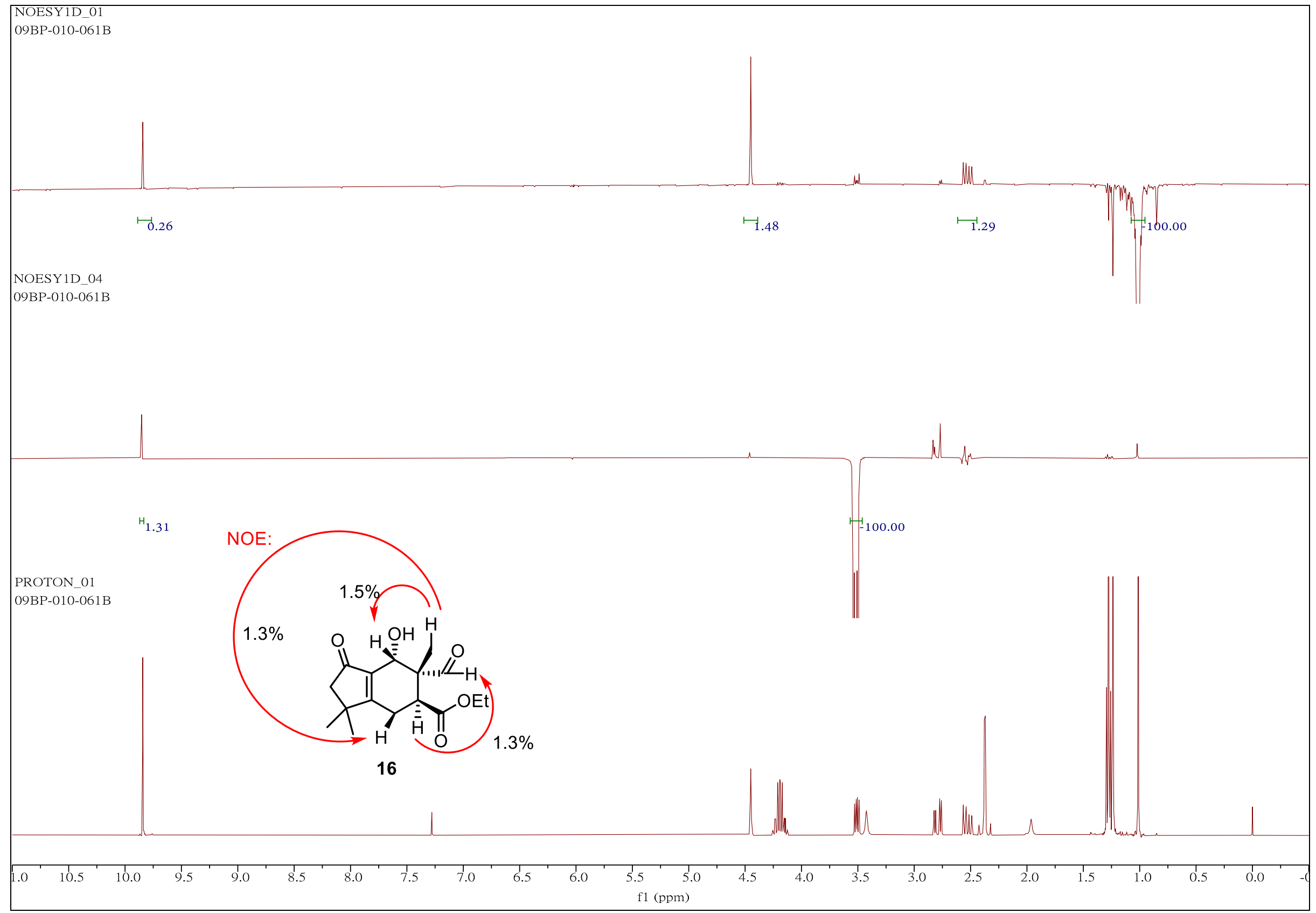

1D NOESY spectra for compound $\mathbf{1 6}$ 


\section{$09 B P-0$ 藏- 0478 \\ exp3 i⿱乛龰 PिоTоN}

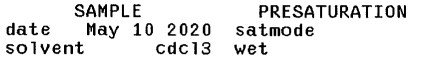

file /home/NHRI/Vn SPECIAL

not used

0510_01/PROTON ${ }_{-02 . \sim} 22 \sim \mathrm{gpin}$

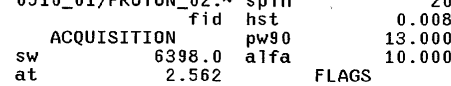

$\begin{array}{lllll}2.562 & & \text { FLAGS } \\ \text { np } & 32788 & \text { il } & & \text { FLA }\end{array}$

$\mathrm{fb}$
$\mathrm{bs}$
$\mathrm{d}$

$\begin{array}{llll}\mathrm{nt} & 1.000 & \text { hs } & \\ \mathrm{ct} & 16 & & \\ \mathrm{ct} & \mathrm{1} & \mathrm{y}\end{array}$

tn TRANSMITTER ${ }_{\text {H1 }}$ fn ${ }^{131072}$

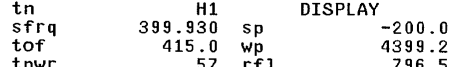

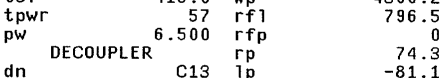

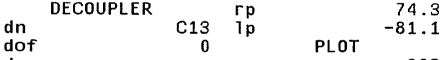

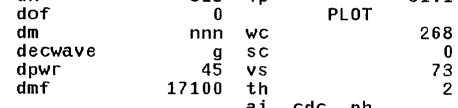

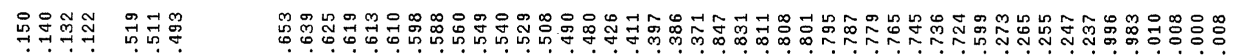

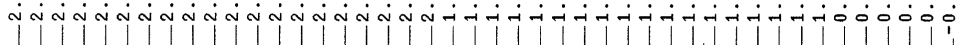
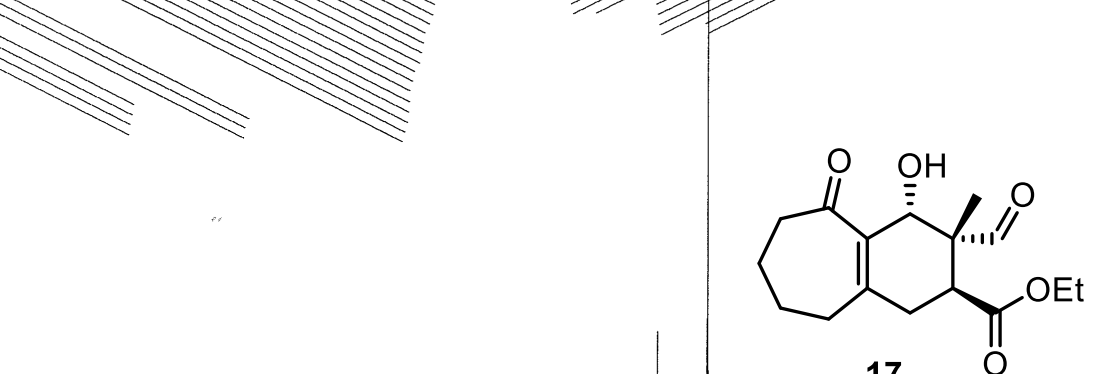

17

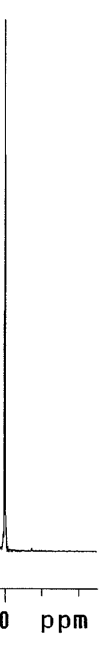

10

4,07

5

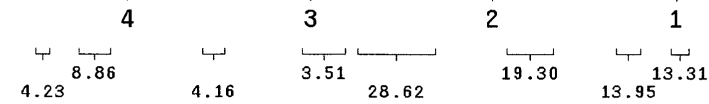

${ }^{1} \mathrm{H}$ NMR spectrum for compound $\mathbf{1 7}$ 


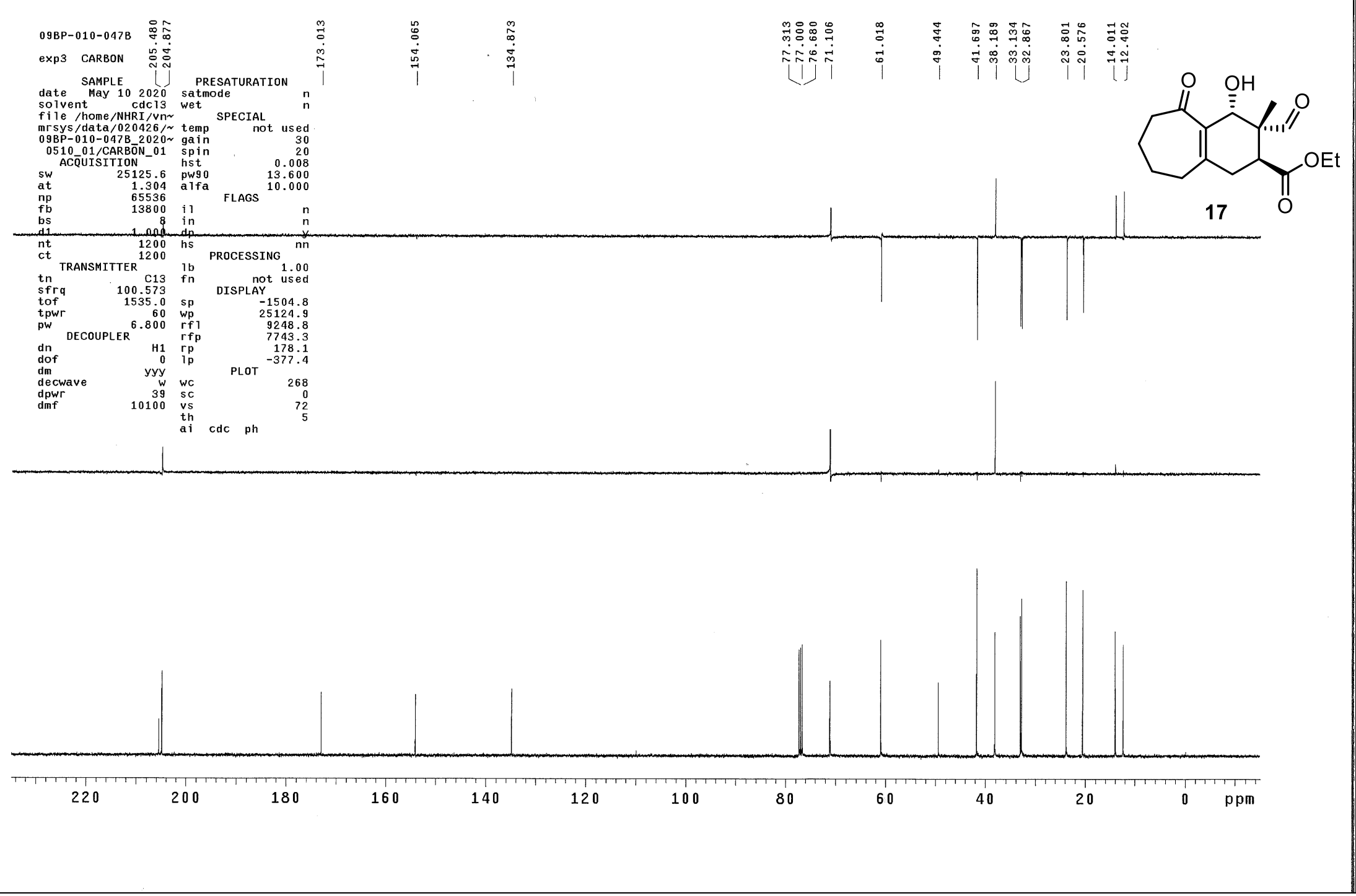

${ }^{13} \mathrm{C}$ NMR + DEPT spectra for compound $\mathbf{1 7}$ 


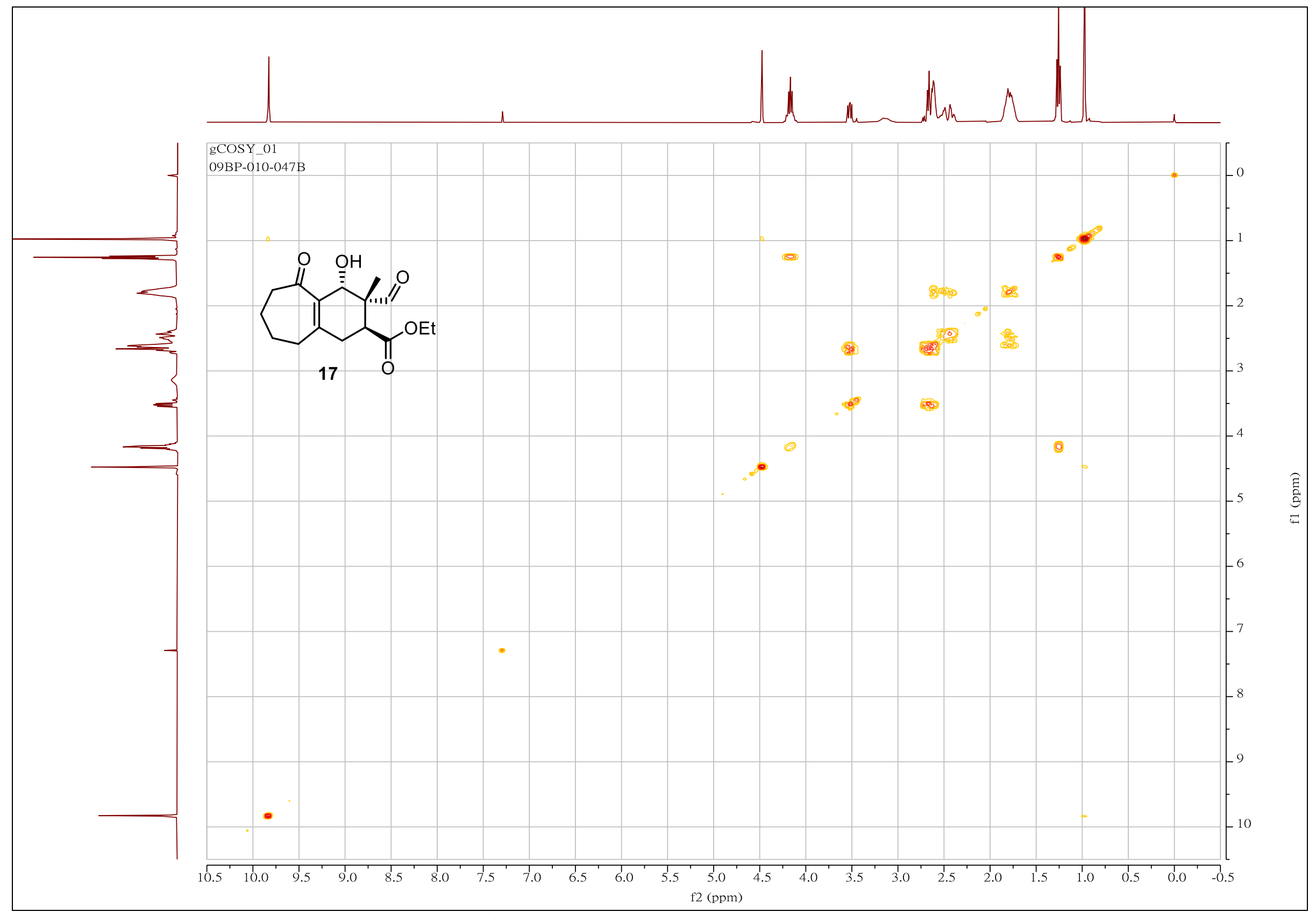

${ }^{1} \mathrm{H}-{ }^{1} \mathrm{H}$ COSY spectrum for compound $\mathbf{1 7}$ 


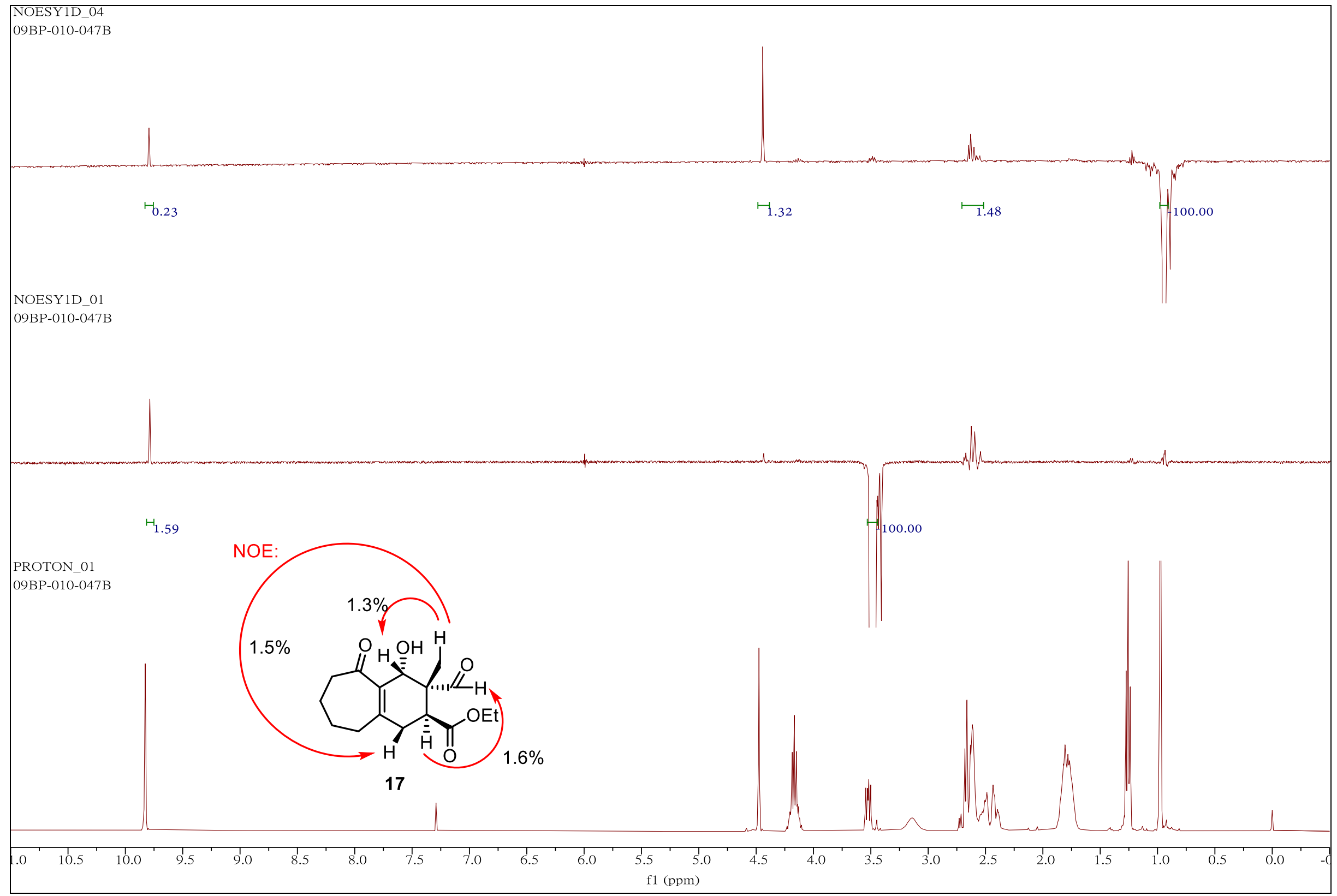

1D NOESY spectra for compound $\mathbf{1 7}$ 
09BP- 0 若- 076

exp1
PROTON
PRoton

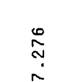

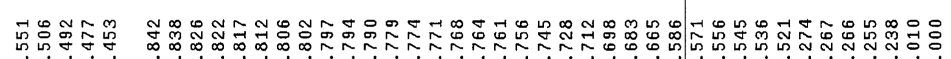

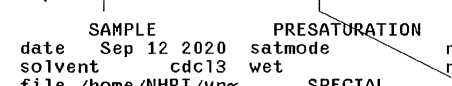

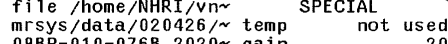

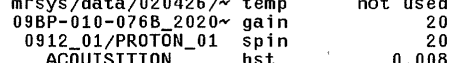

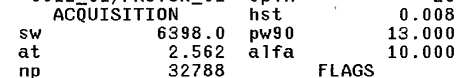

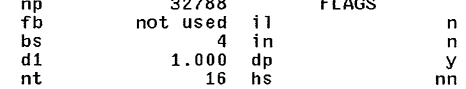

$\begin{array}{lrrrr}\mathrm{d} & 1.000 & \mathrm{dp} & \\ \mathrm{nt} & 16 & \mathrm{hs} \\ \mathrm{ct} & 16 & \begin{array}{c}\mathrm{y} \\ \text { ct }\end{array}\end{array}$

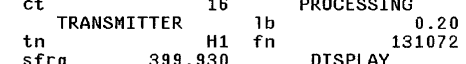

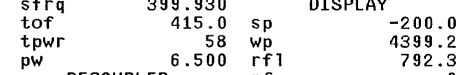

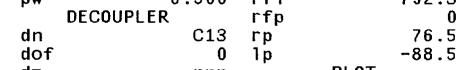

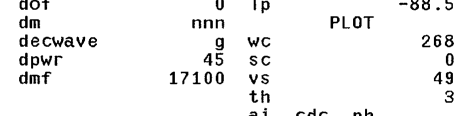

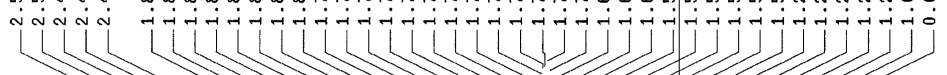

ai cdc ph
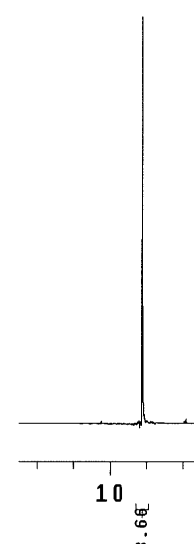

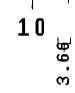

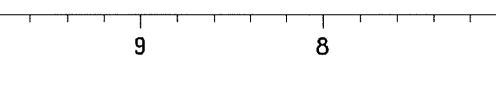

$7+11$

6

5

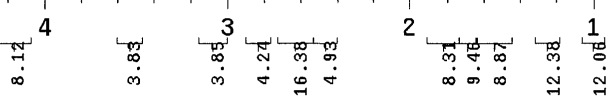

${ }^{1} \mathrm{H}$ NMR spectrum for compound $\mathbf{1 8}$ 


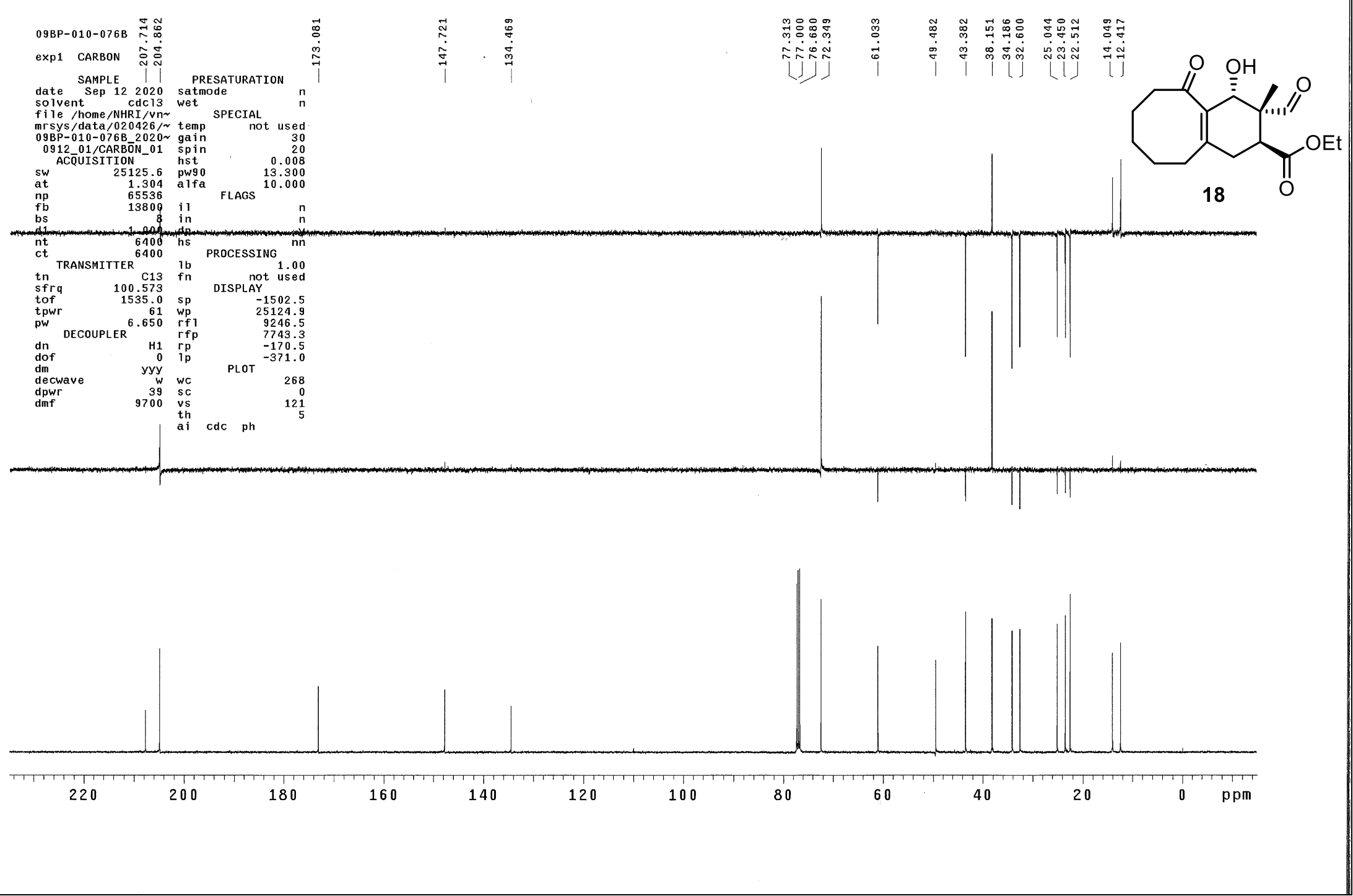

${ }^{13} \mathrm{C}$ NMR + DEPT spectra for compound $\mathbf{1 8}$ 


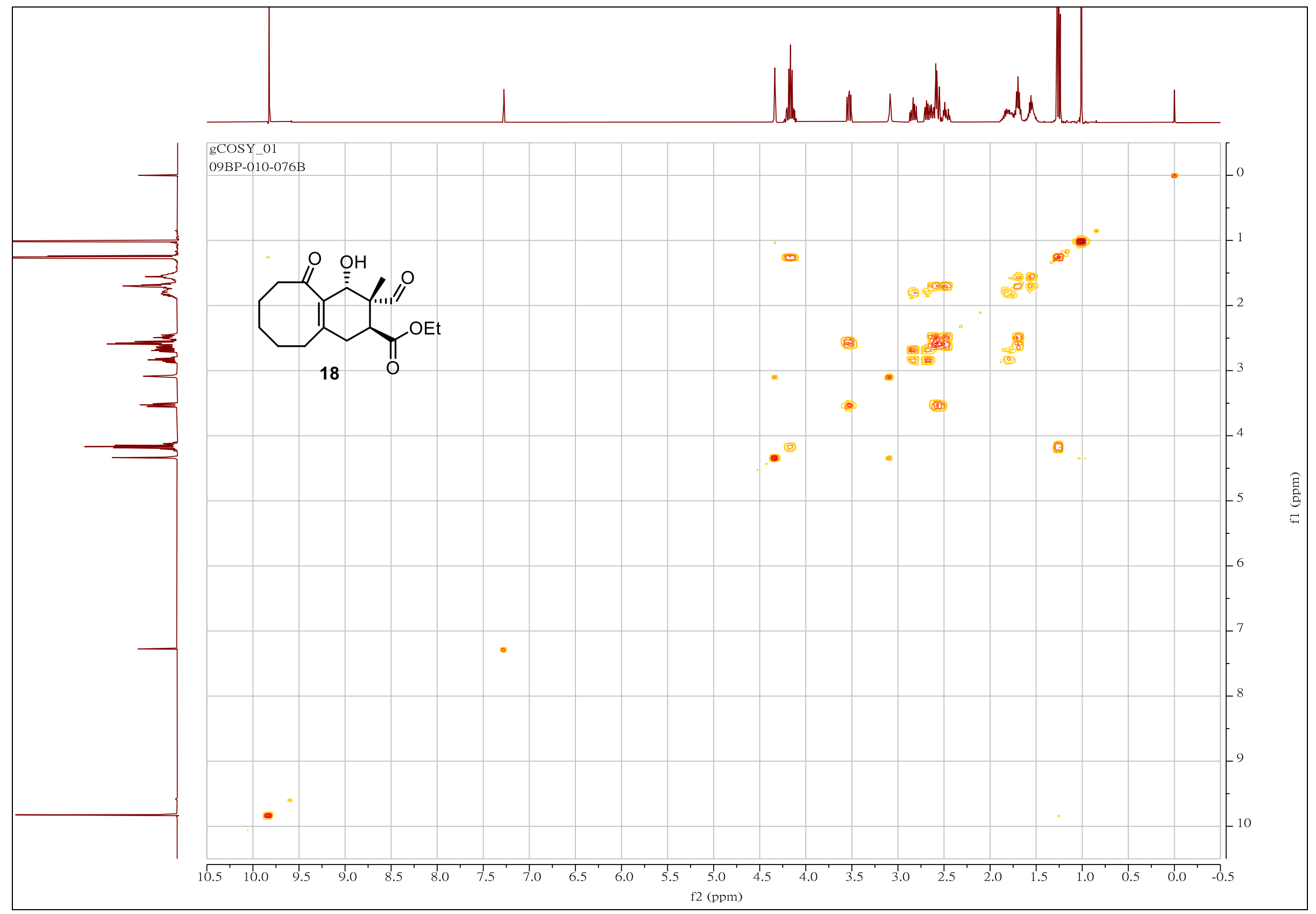

${ }^{1} \mathrm{H}-{ }^{1} \mathrm{H}$ COSY spectrum for compound $\mathbf{1 8}$ 


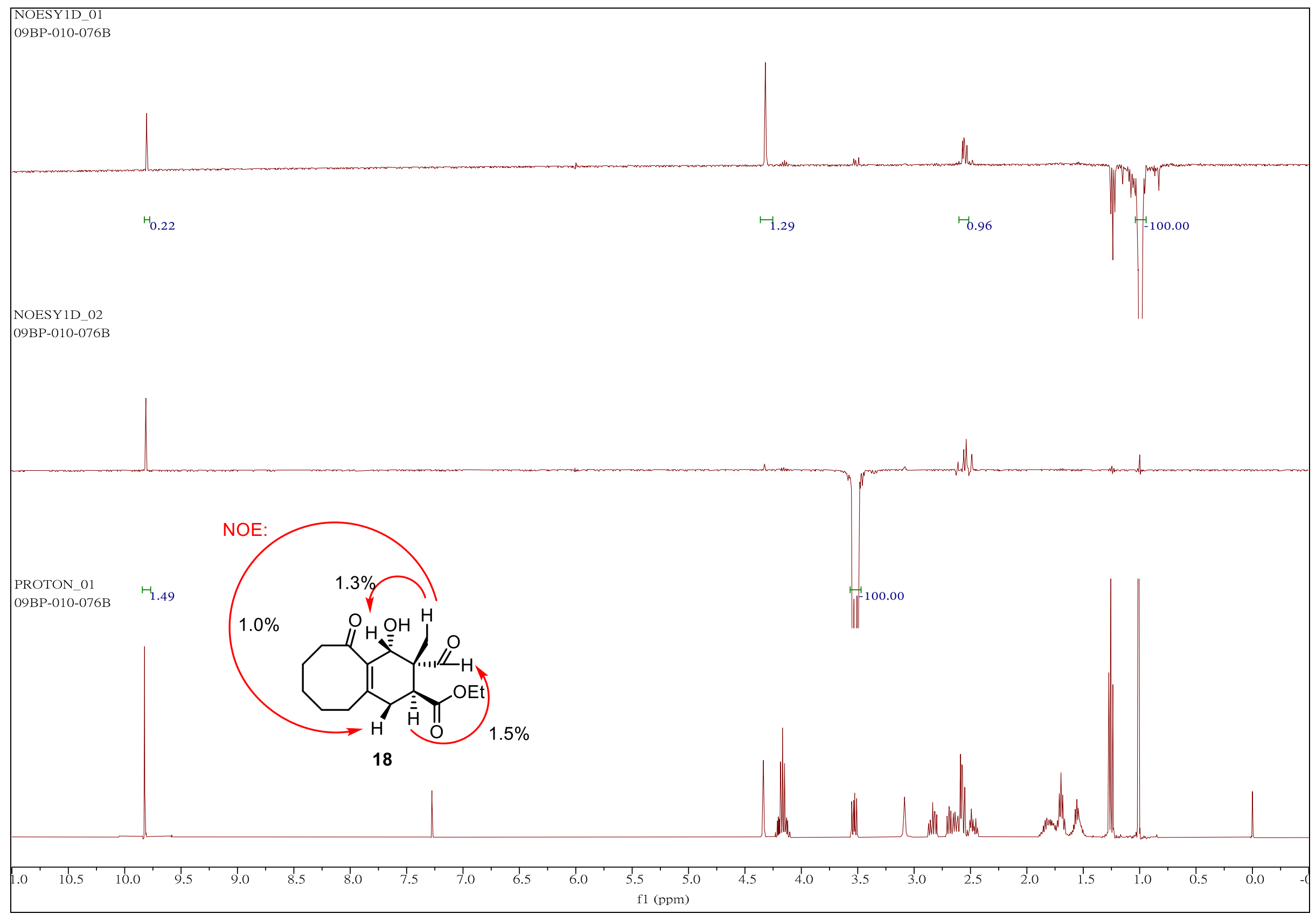

1D NOESY spectra for compound $\mathbf{1 8}$ 


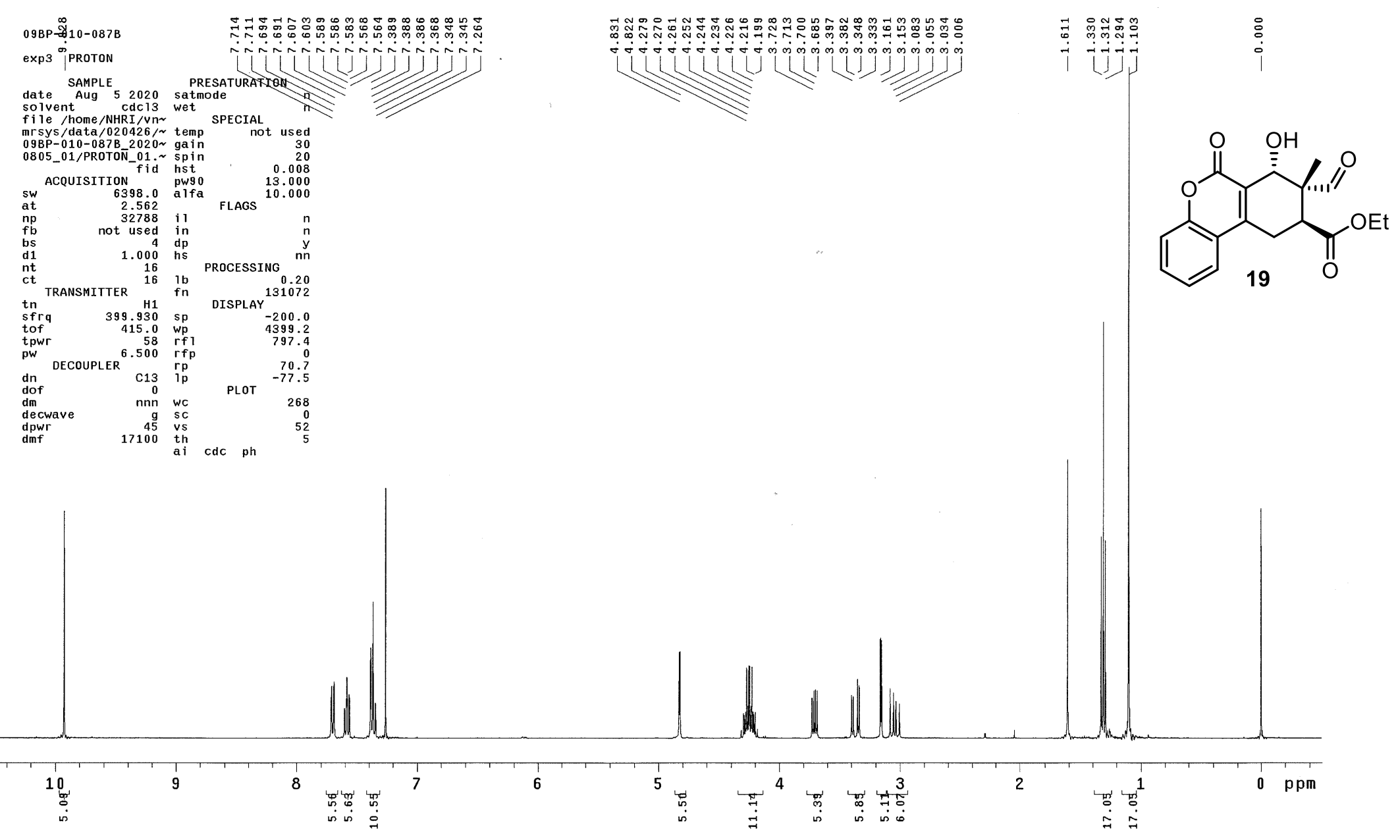

${ }^{1} \mathrm{H}$ NMR spectrum for compound 19 


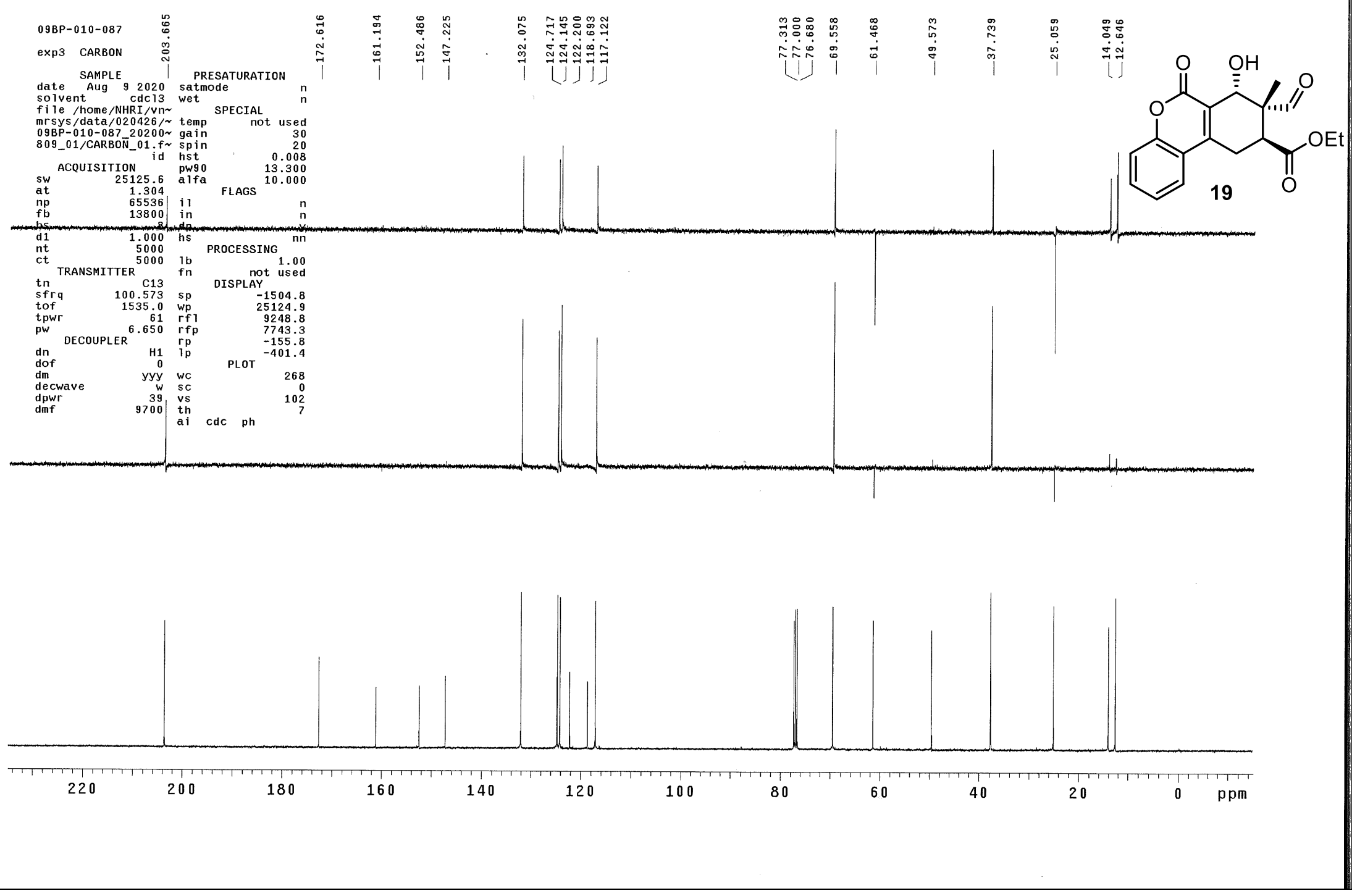

${ }^{13}$ C NMR + DEPT spectra for compound 19 


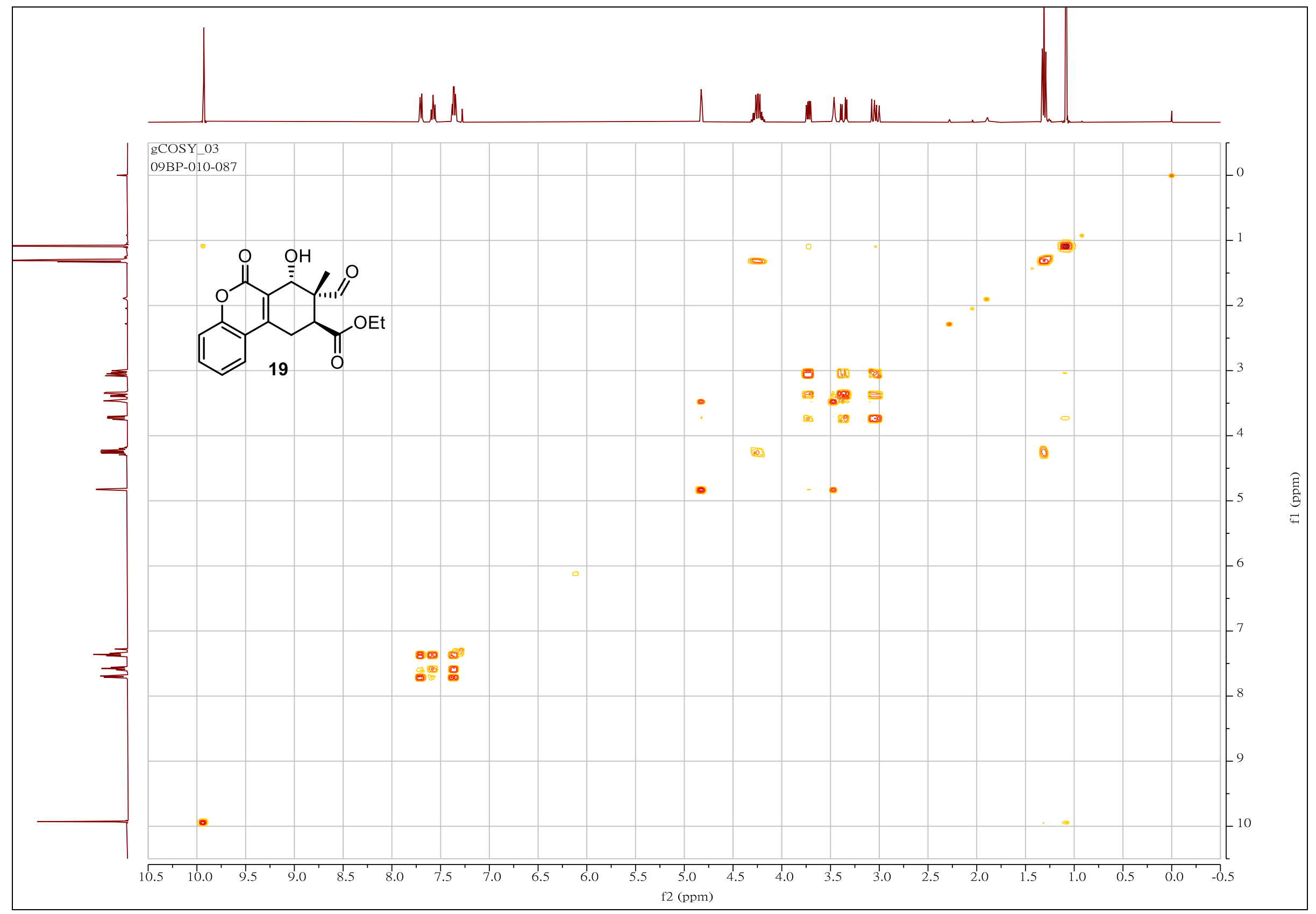

${ }^{1} \mathrm{H}-{ }^{1} \mathrm{H}$ COSY spectrum for compound 19 


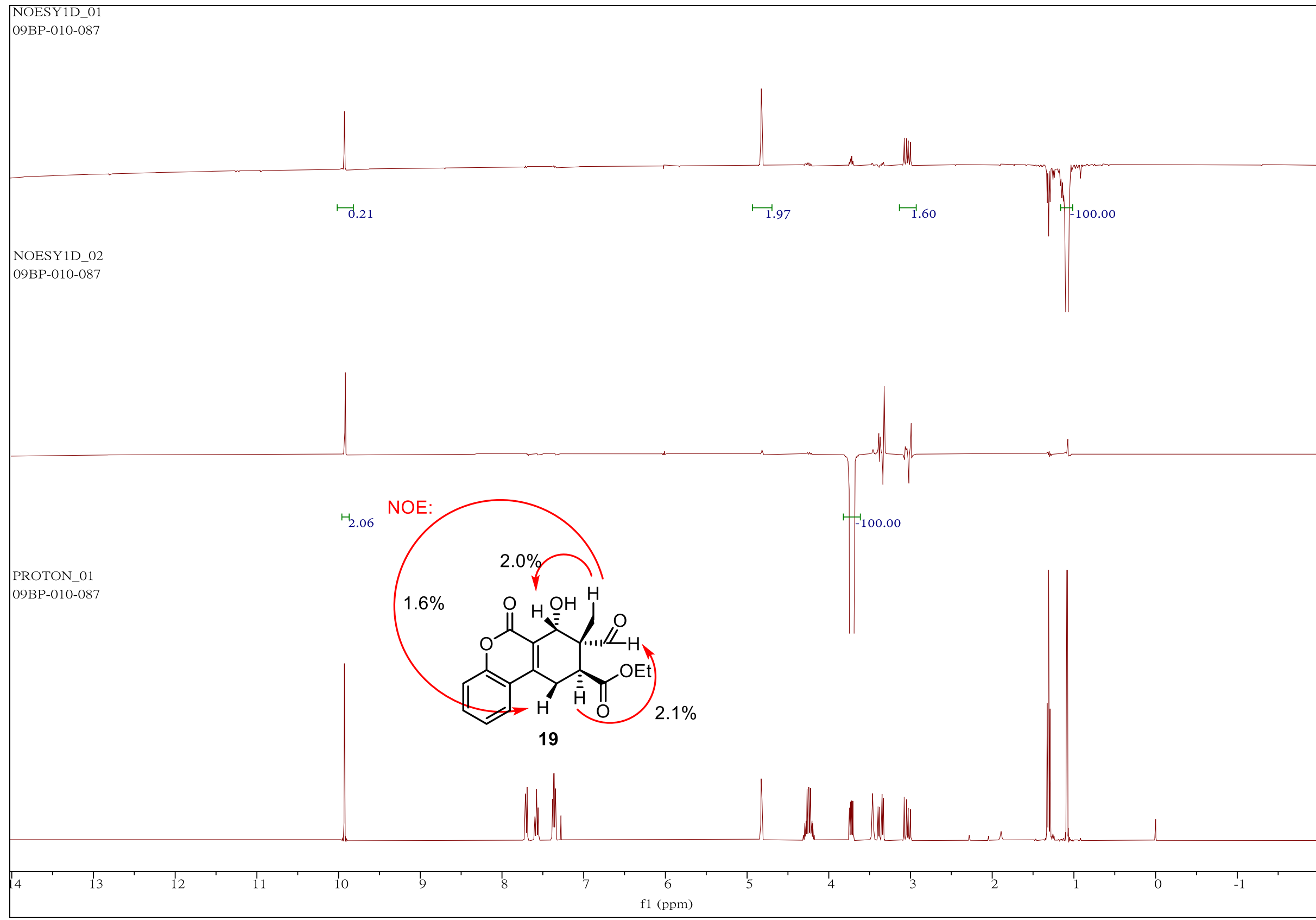

1D NOESY spectra for compound $\mathbf{1 9}$ 


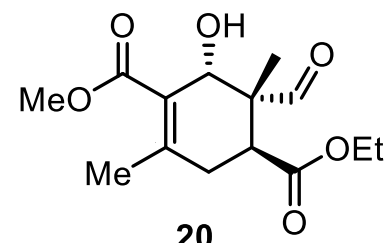

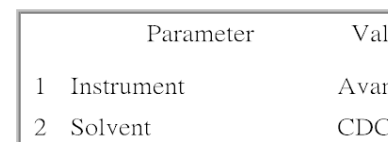

3 Temperature

4 Number of Scans

5 Receiver Gain

6 Relaxation Delay $\mathrm{CDCl3}$ 298.0

7 Pulse Width 90.5

1.0000

8 Presaturation Frequency

9 Spectrometer Frequency 600.14

10 Spectral Width

11 Lowest Frequency

12 Nucleus

13 Acquired Size

14 Spectral Size

$-2246.5$

$1 \mathrm{H}$

15 Digita Resolution

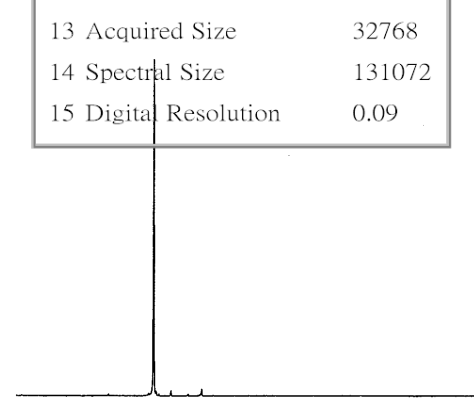

\begin{tabular}{|c|c|c|c|c|c|c|c|c|c|c|c|c|c|c|c|c|c|c|c|c|c|c|c|}
\hline \begin{tabular}{l}
$1+$ \\
\hdashline \\
8 \\
0
\end{tabular} & & & & & & & & & & & $\begin{array}{l}T \\
\text { T } \\
5 \\
0\end{array}$ & 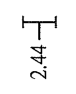 & $\begin{array}{l}T^{1} \\
\text { ले }\end{array}$ & $\begin{array}{l}+1 \\
8 \\
8 \\
-\end{array}$ & & 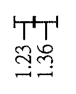 & $\begin{array}{l}\uparrow \\
\stackrel{1}{2} \\
\dot{m}\end{array}$ & & & $\begin{array}{l}T \\
T \\
\delta \\
\dot{m}\end{array}$ & & & \\
\hline 10.0 & 9.5 & 9.0 & 8.5 & 8.0 & 7.5 & 7.0 & 6.5 & 6.0 & 5.5 & $\begin{array}{c}5.0 \\
\mathrm{fl}(\mathrm{ppm})\end{array}$ & 4.5 & 4. & & 3.5 & 3.0 & 2.5 & 2.0 & 1.5 & & 1.0 & 0.5 & 0.0 & -0 \\
\hline
\end{tabular}

${ }^{1} \mathrm{H}$ NMR spectrum for compound $\mathbf{2 0}$ 


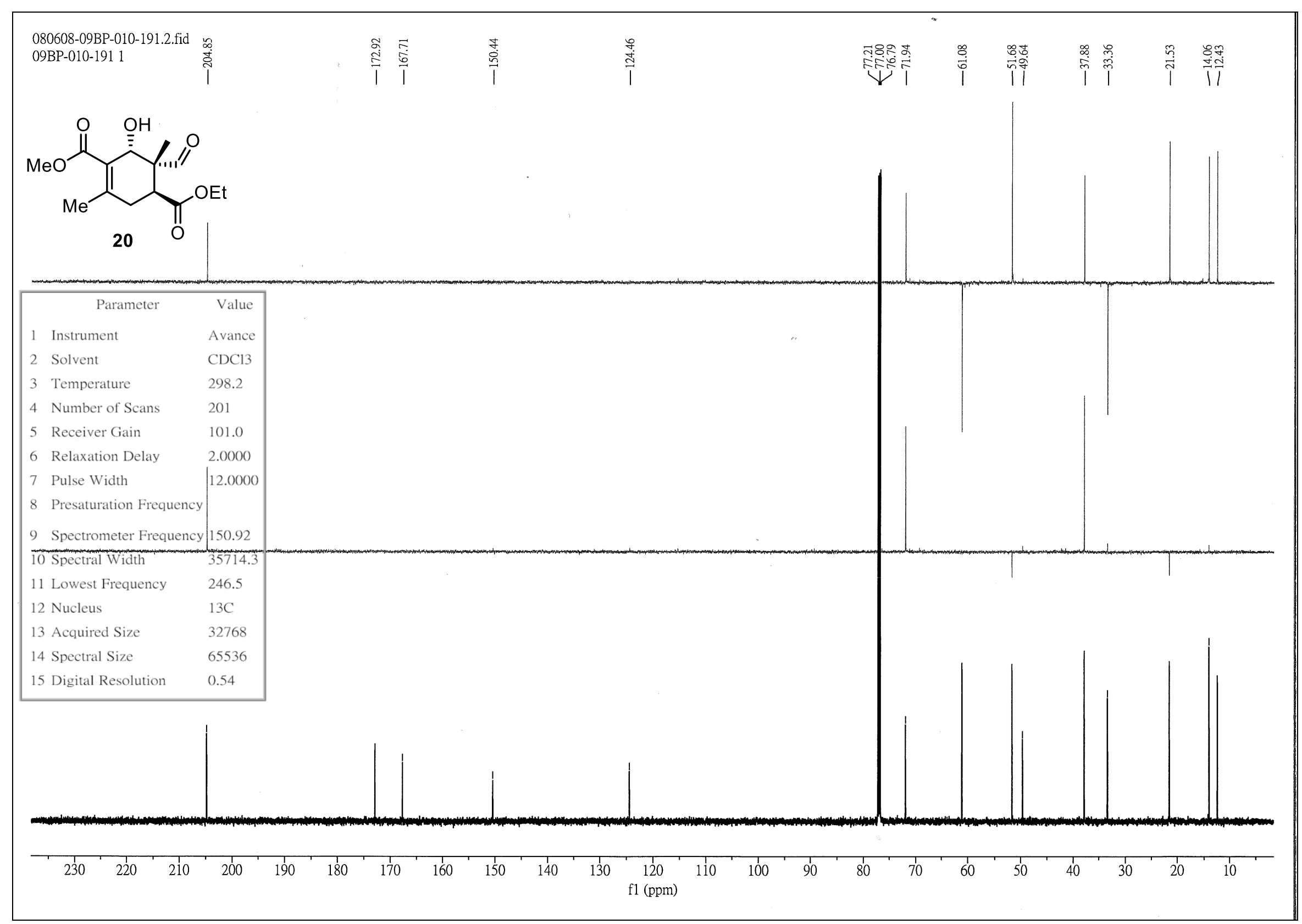

${ }^{13} \mathrm{C}$ NMR + DEPT spectra for compound $\mathbf{2 0}$ 


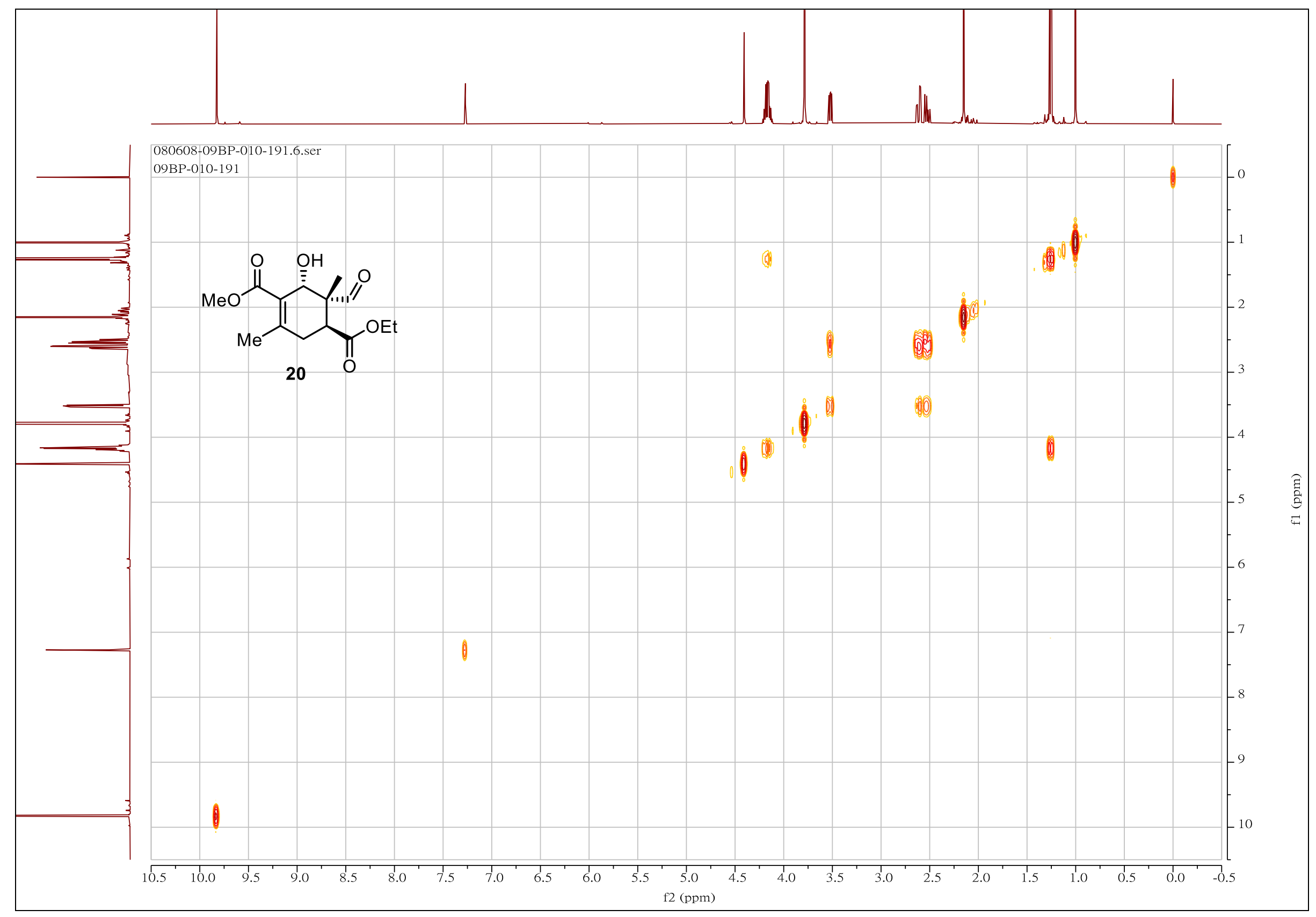

${ }^{1} \mathrm{H}-{ }^{1} \mathrm{H}$ COSY spectrum for compound 20 


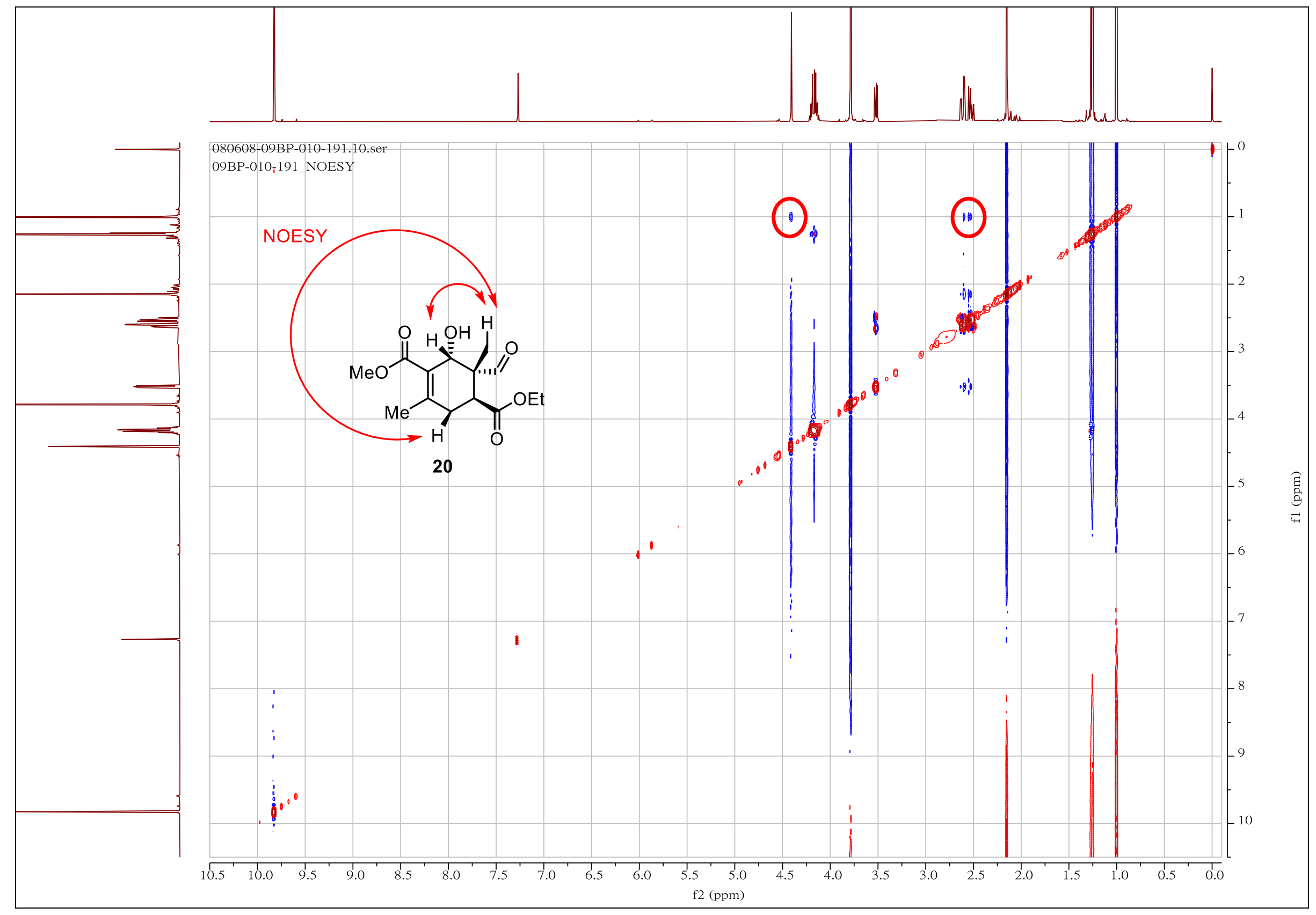

2D NOESY spectrum for compound $\mathbf{2 0}$ 


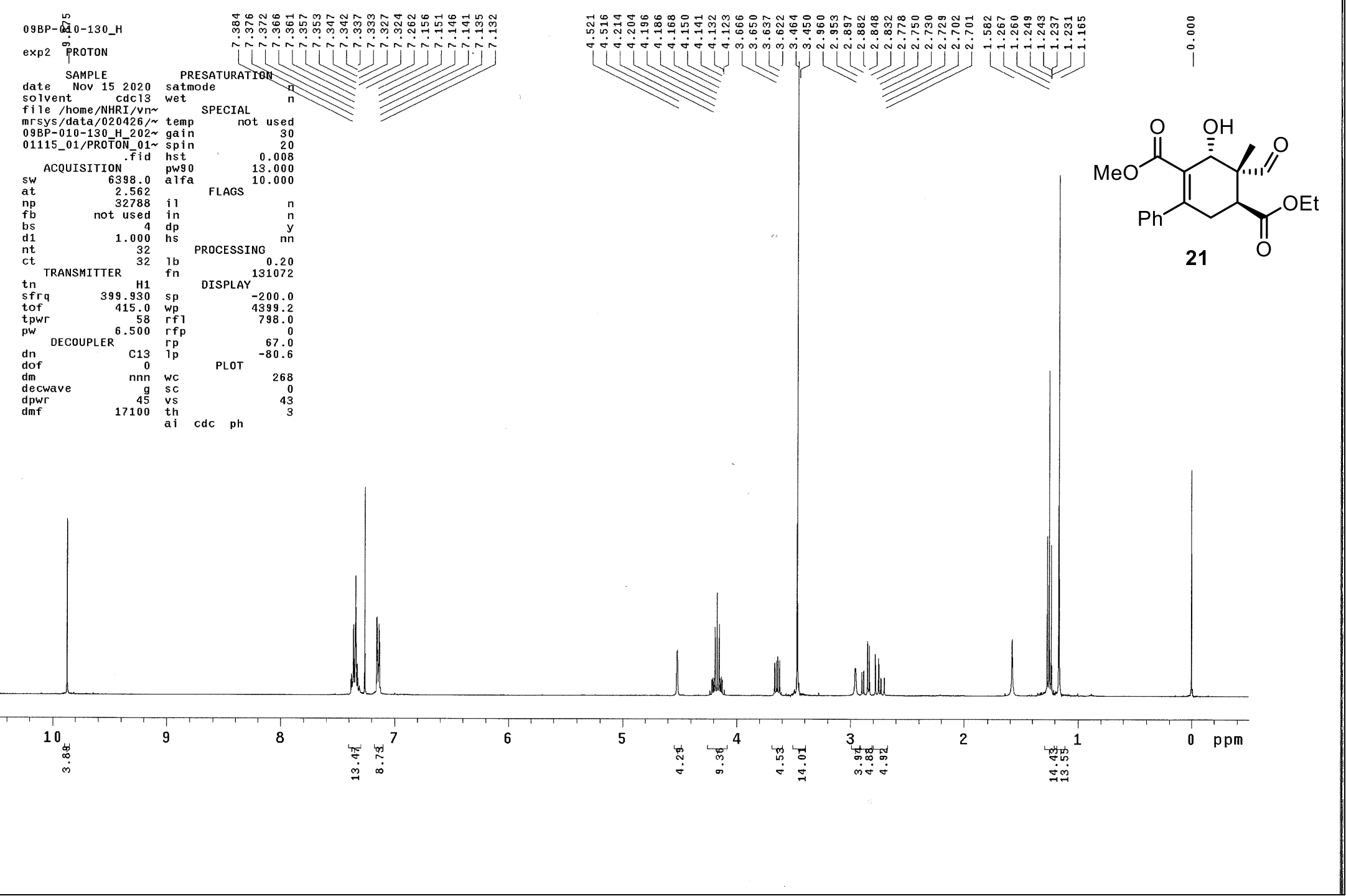

${ }^{1} \mathrm{H}$ NMR spectrum for compound $\mathbf{2 1}$ 


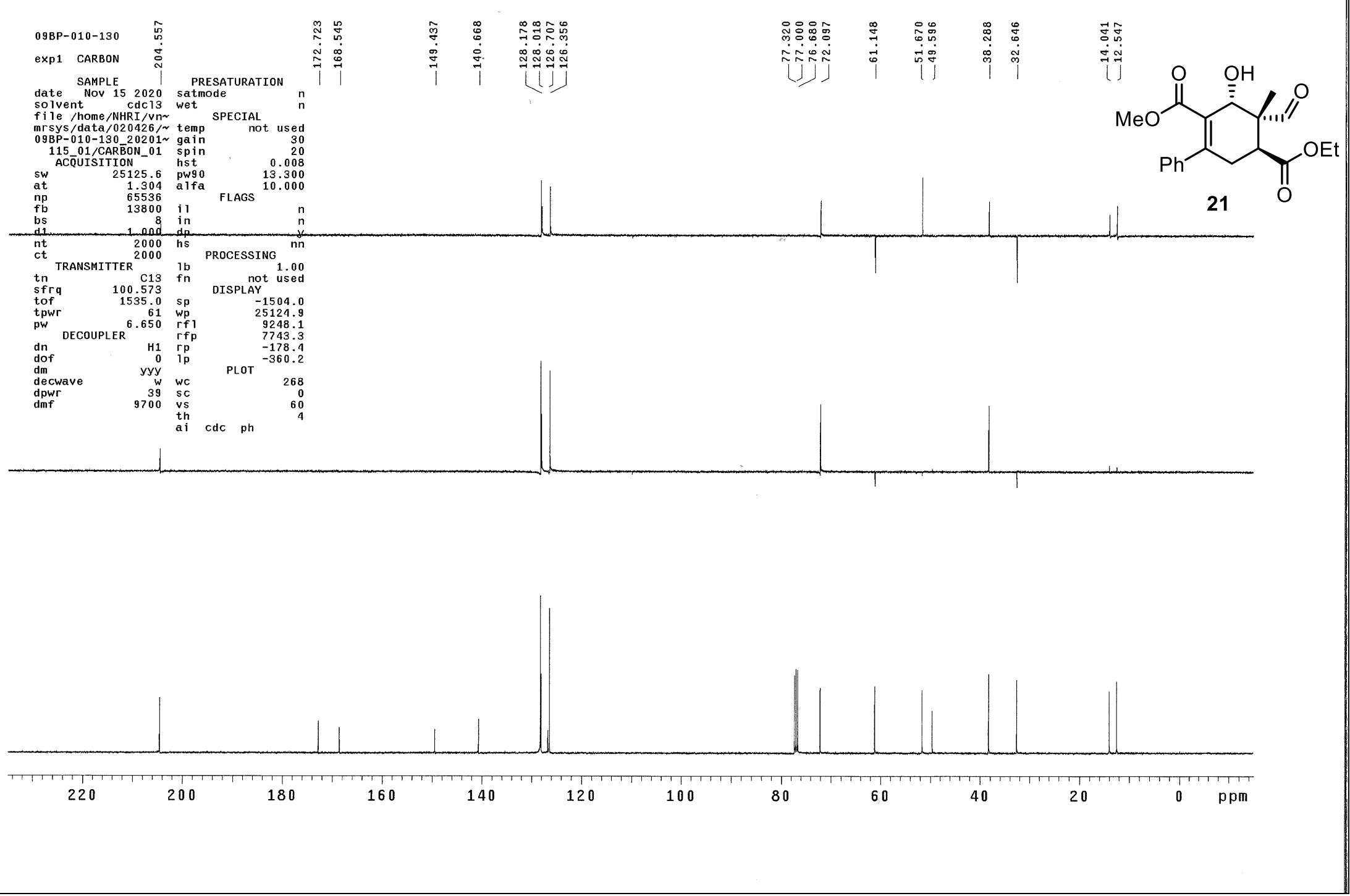

${ }^{13} \mathrm{C}$ NMR + DEPT spectra for compound 21 


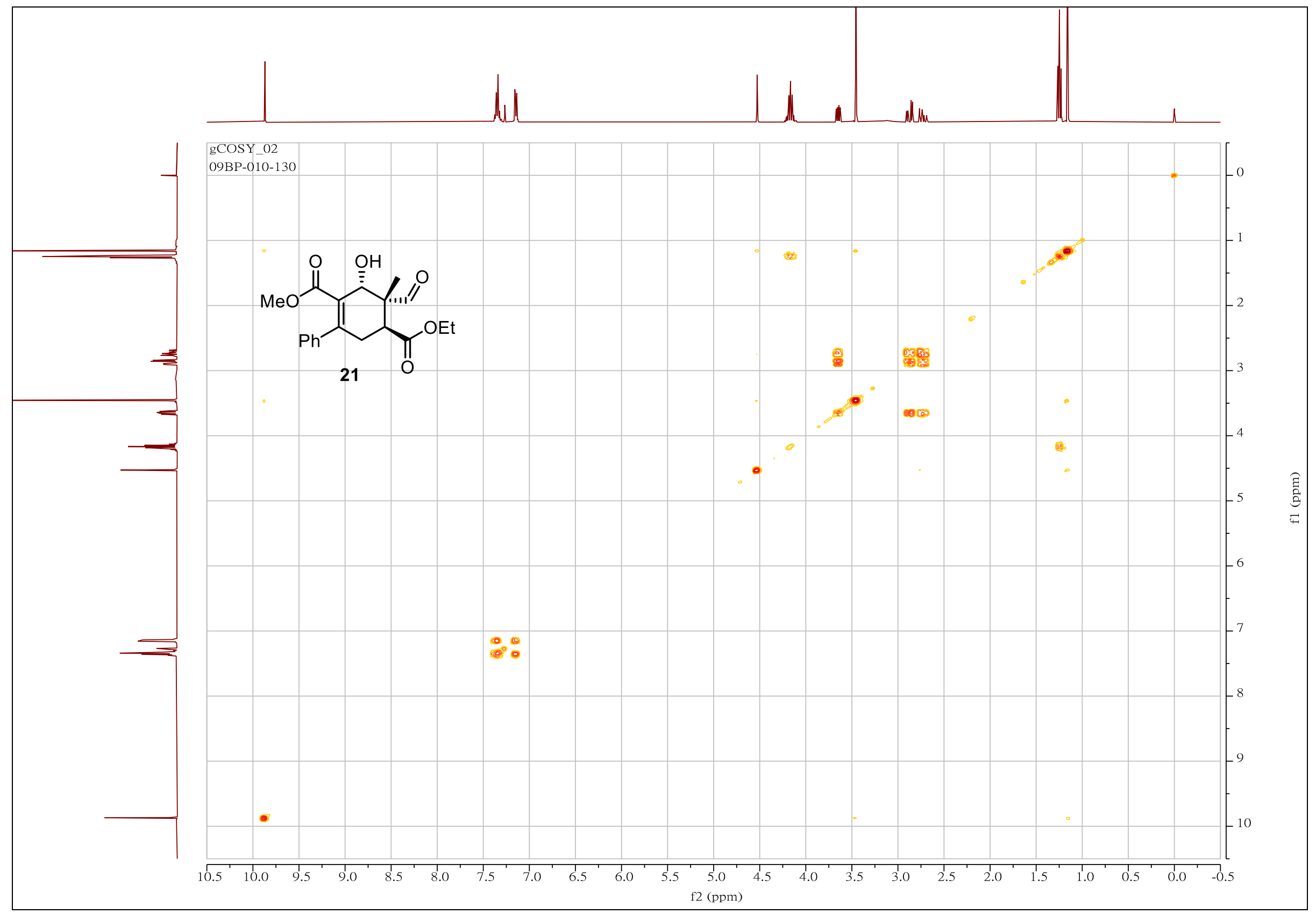

${ }^{1} \mathrm{H}-{ }^{1} \mathrm{H}$ COSY spectrum for compound 21 


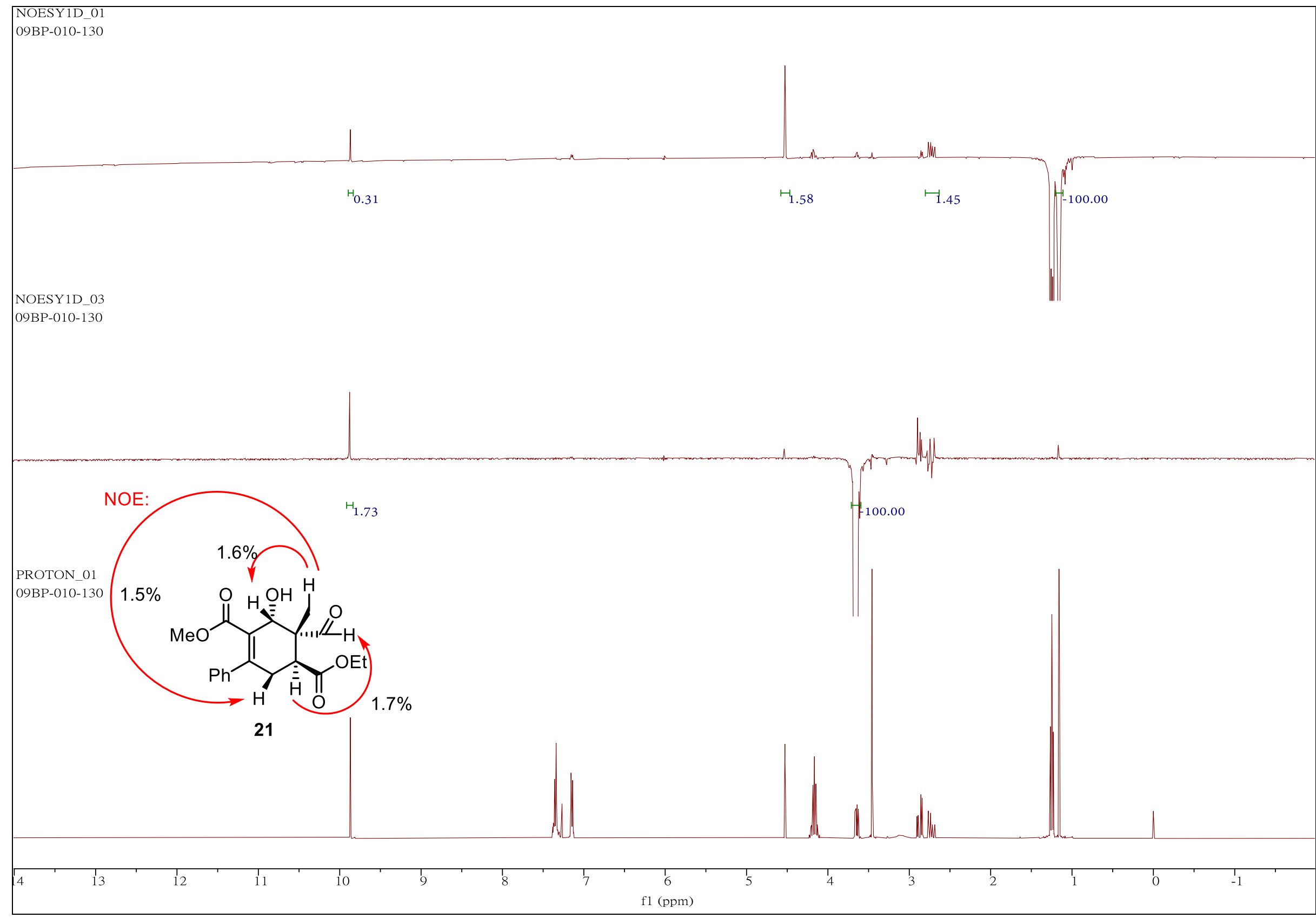

1D NOESY spectra for compound $\mathbf{2 1}$ 


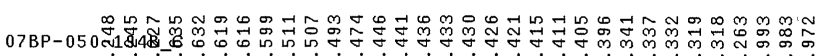

exp2 PRómon

SAMPLE PRESATURAY IOA

date Jan 820

file /home/NHRI/un wet

mrsys/data/020426/ temp SPECIAL not

$07 \mathrm{BP}-050-194 \mathrm{~B}-\mathrm{C}_{2} 20 \sim$ ga in
$200107-01 / \mathrm{PROT} O \mathrm{~N}_{0}$

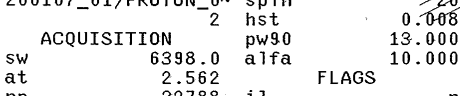

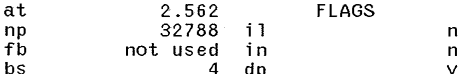

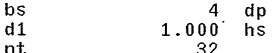

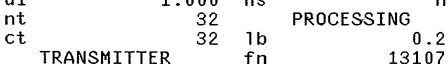

tn ${ }^{2}$ H1 fn DISPLAY ${ }^{131072}$

$\begin{array}{lrlr}\text { tn } & 399.930 & \text { sp } & \text { DISPLAY } \\ \text { sfra } & 399.900 .0 \\ \text { tof } & 415.0 & \text { wp } & 3999.2 \\ \text { top } & 5.57 & \text { ff } & 397.7\end{array}$

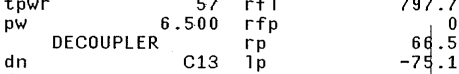

$\begin{array}{lll}d \text { dof } & \text { PLOT } & \\ \mathrm{dm} & -75.1\end{array}$

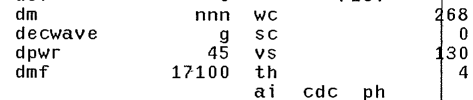

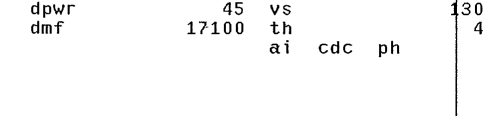

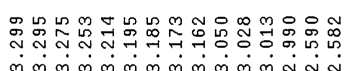

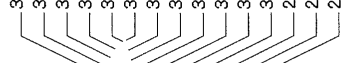

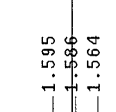

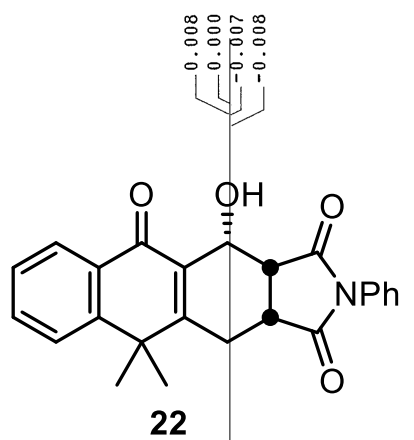

22

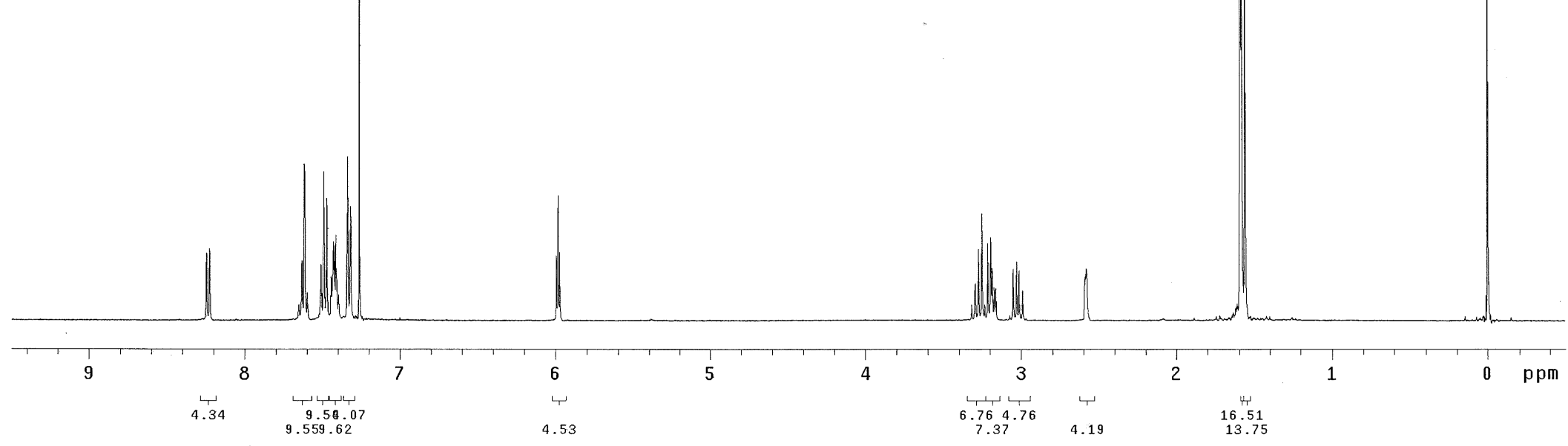

${ }^{1} \mathrm{H}$ NMR spectrum for compound 22 


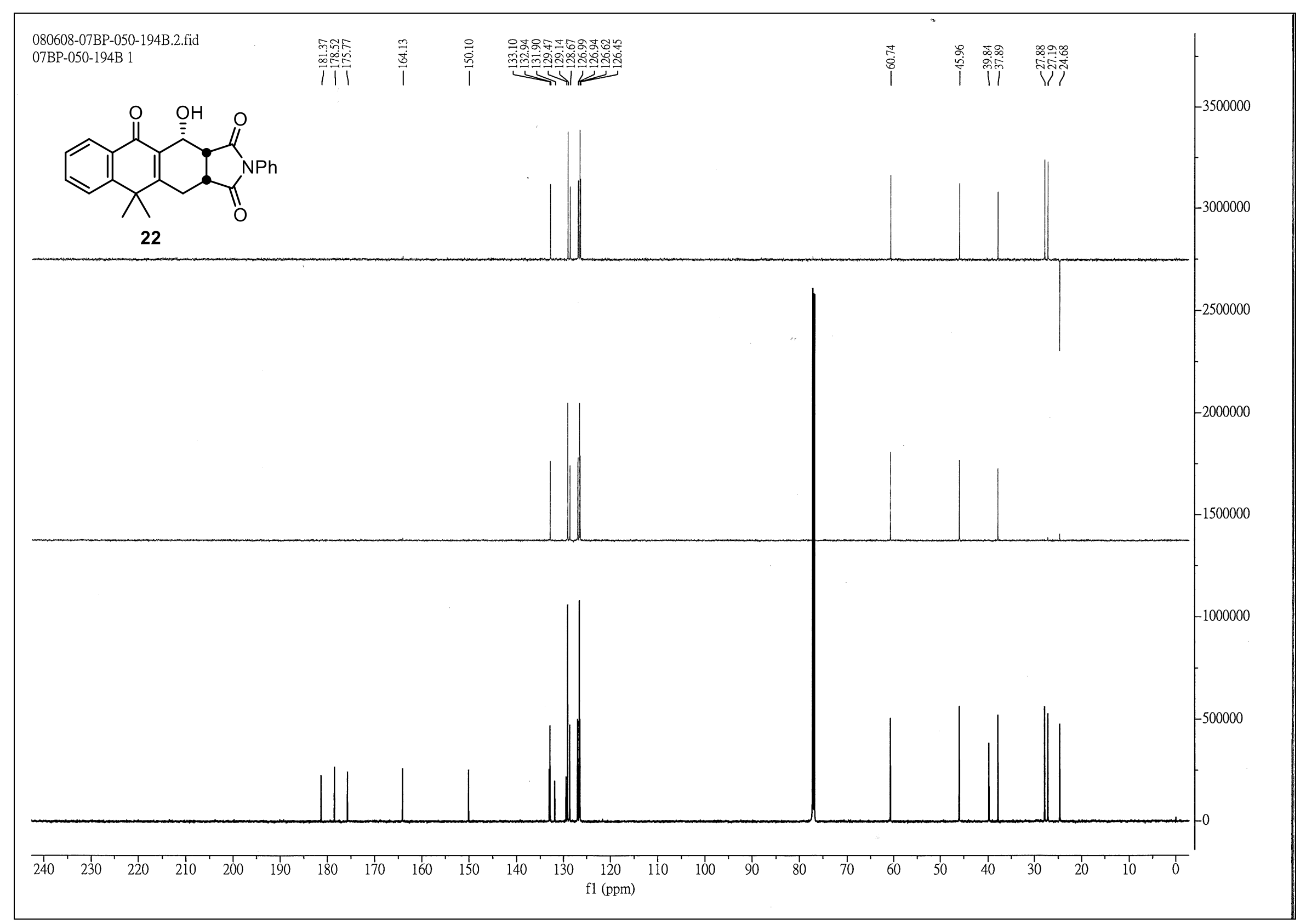

${ }^{13} \mathrm{C}$ NMR + DEPT spectra for compound 22 


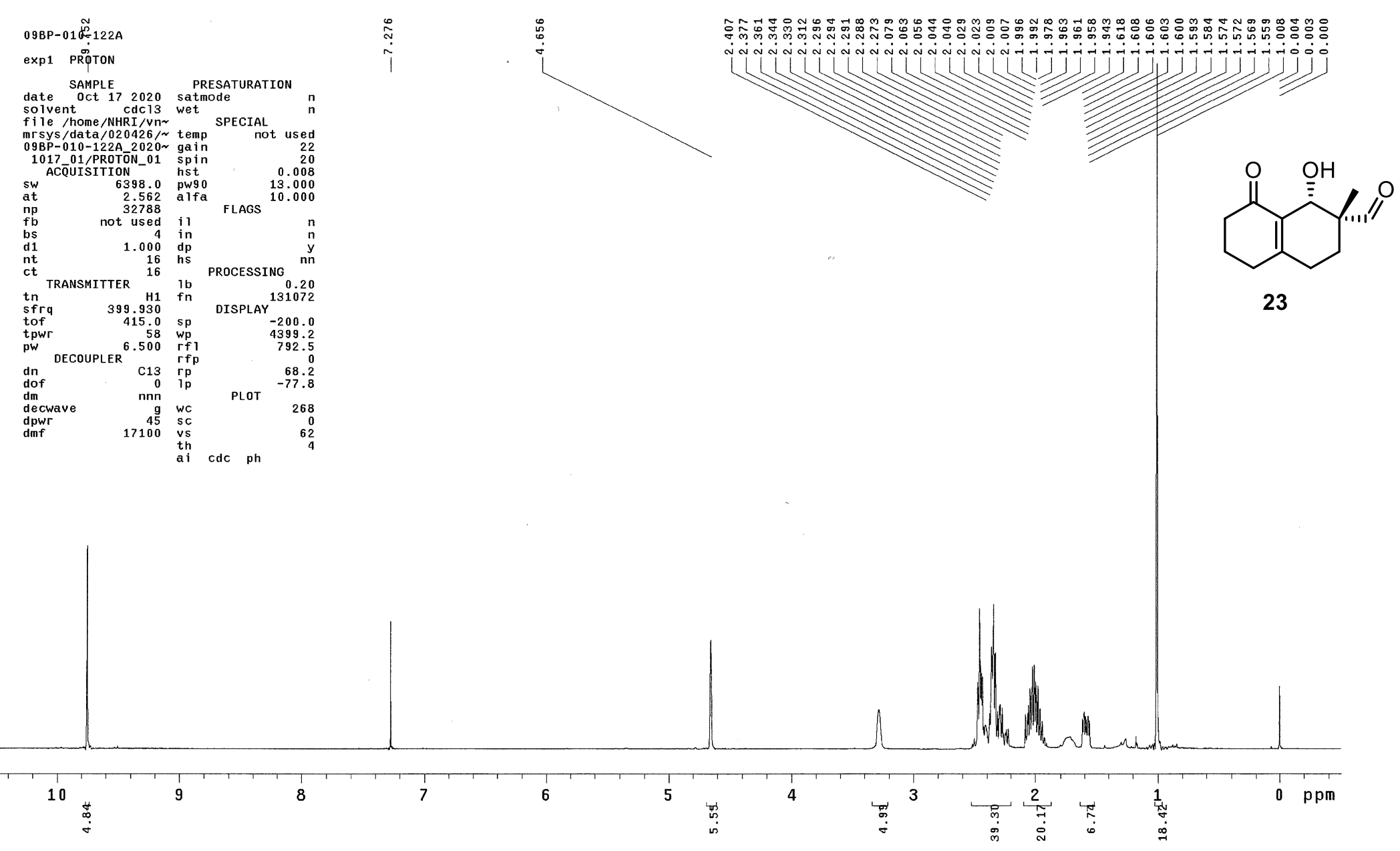

${ }^{1} \mathrm{H}$ NMR spectrum for compound $\mathbf{2 3}$ 


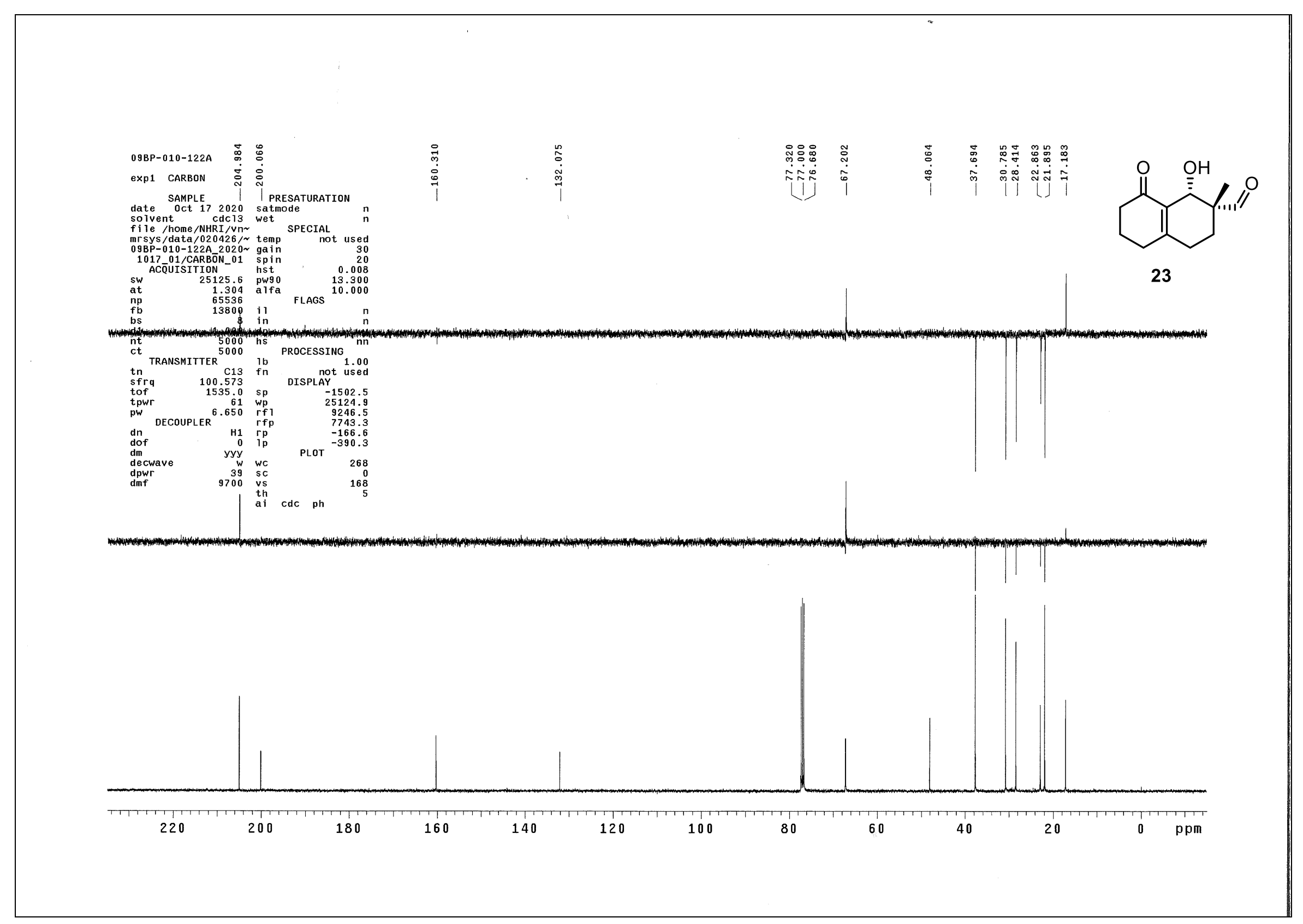

${ }^{13}$ C NMR + DEPT spectra for compound $\mathbf{2 3}$ 


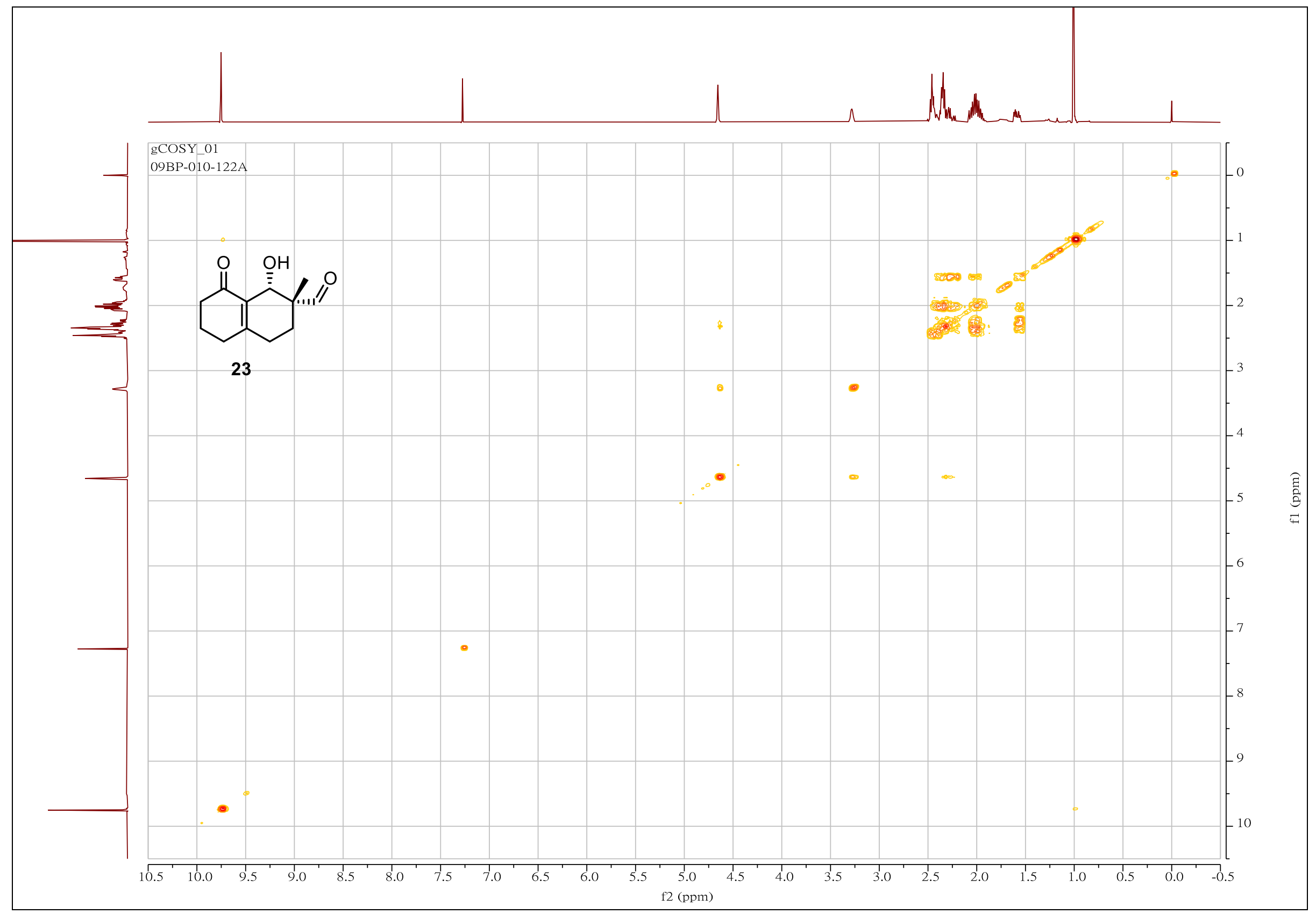

${ }^{1} \mathrm{H}-{ }^{1} \mathrm{H}$ COSY spectrum for compound 23 


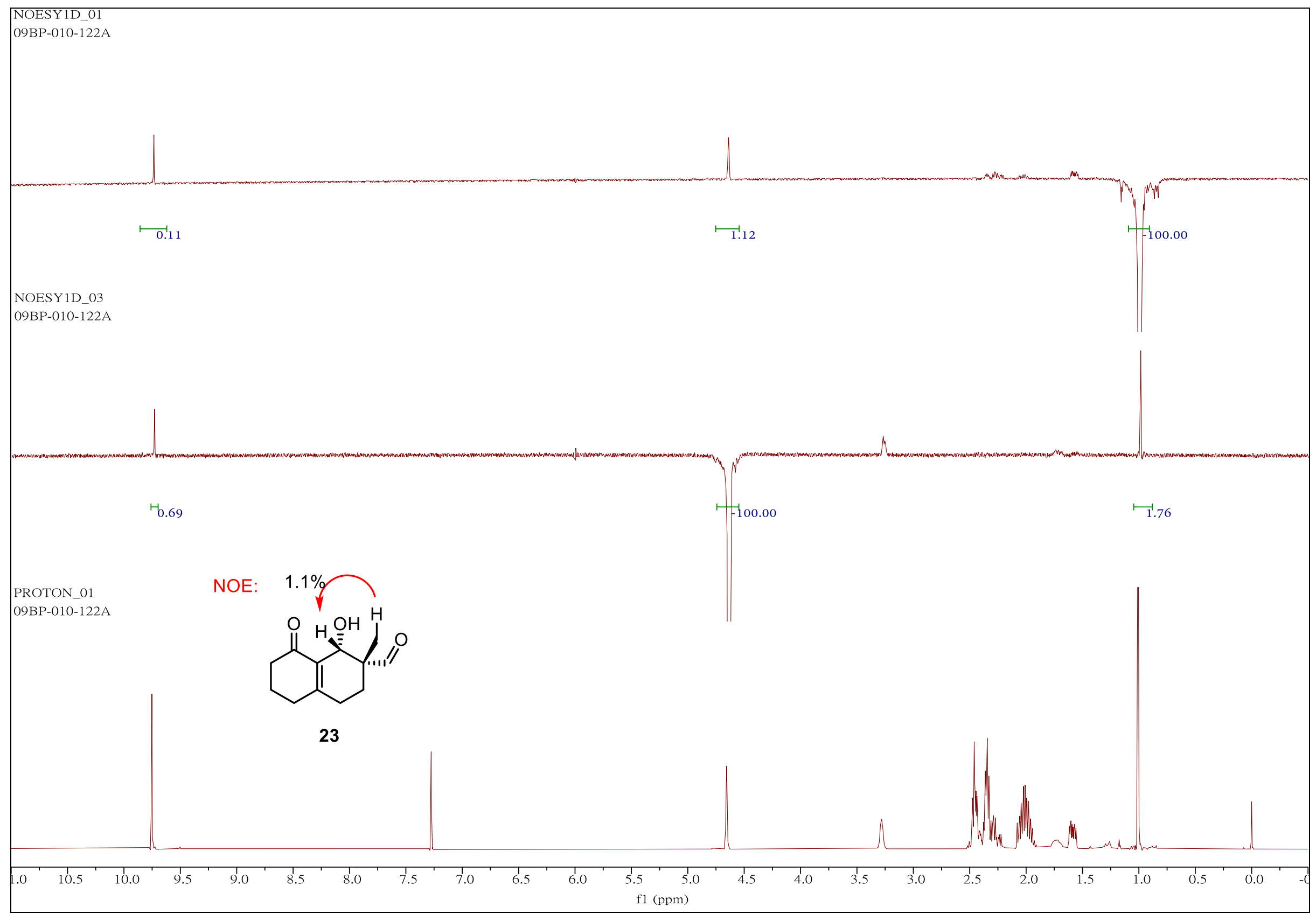

1D NOESY spectra for compound $\mathbf{2 3}$ 


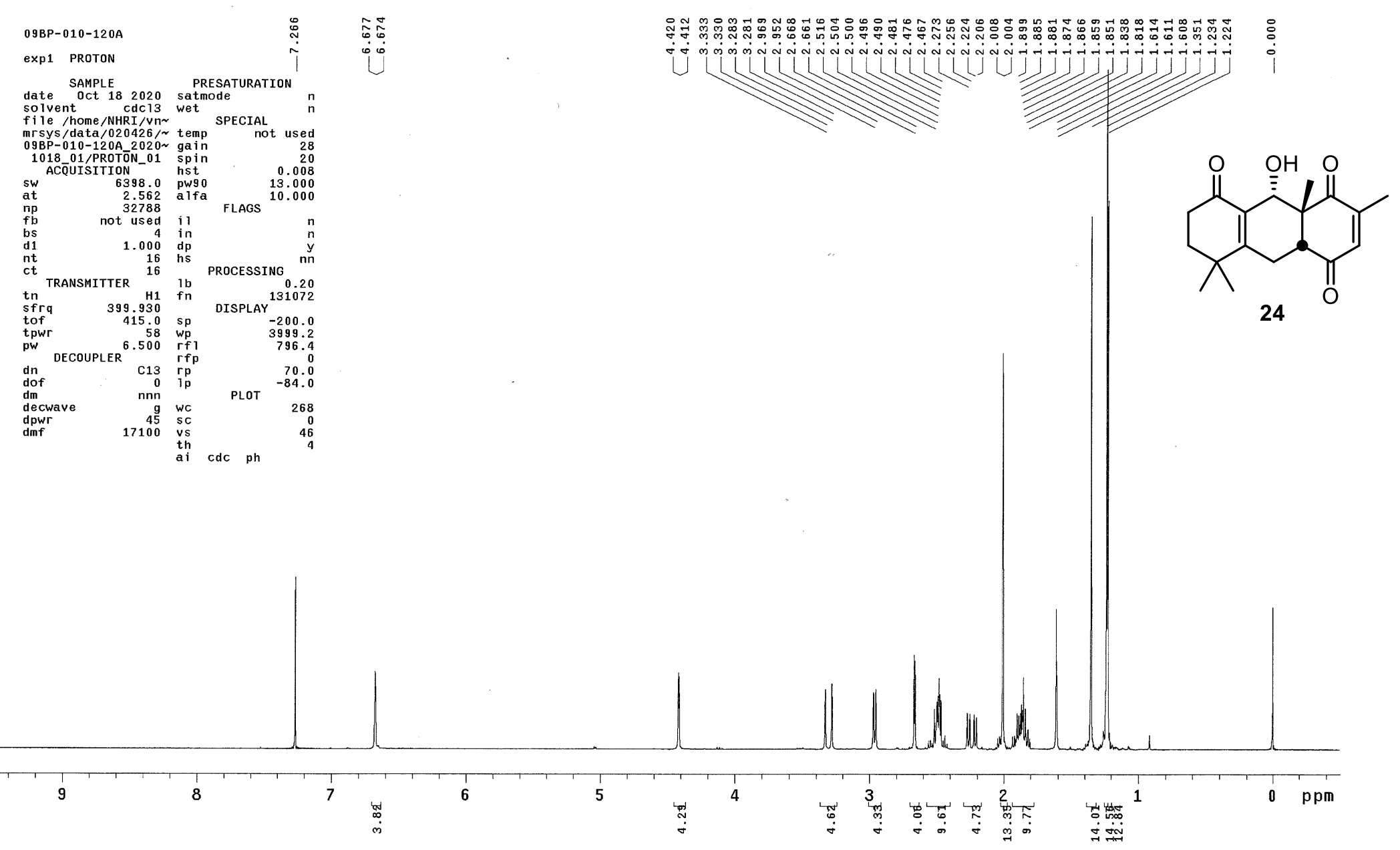

${ }^{1} \mathrm{H}$ NMR spectrum for compound 24 


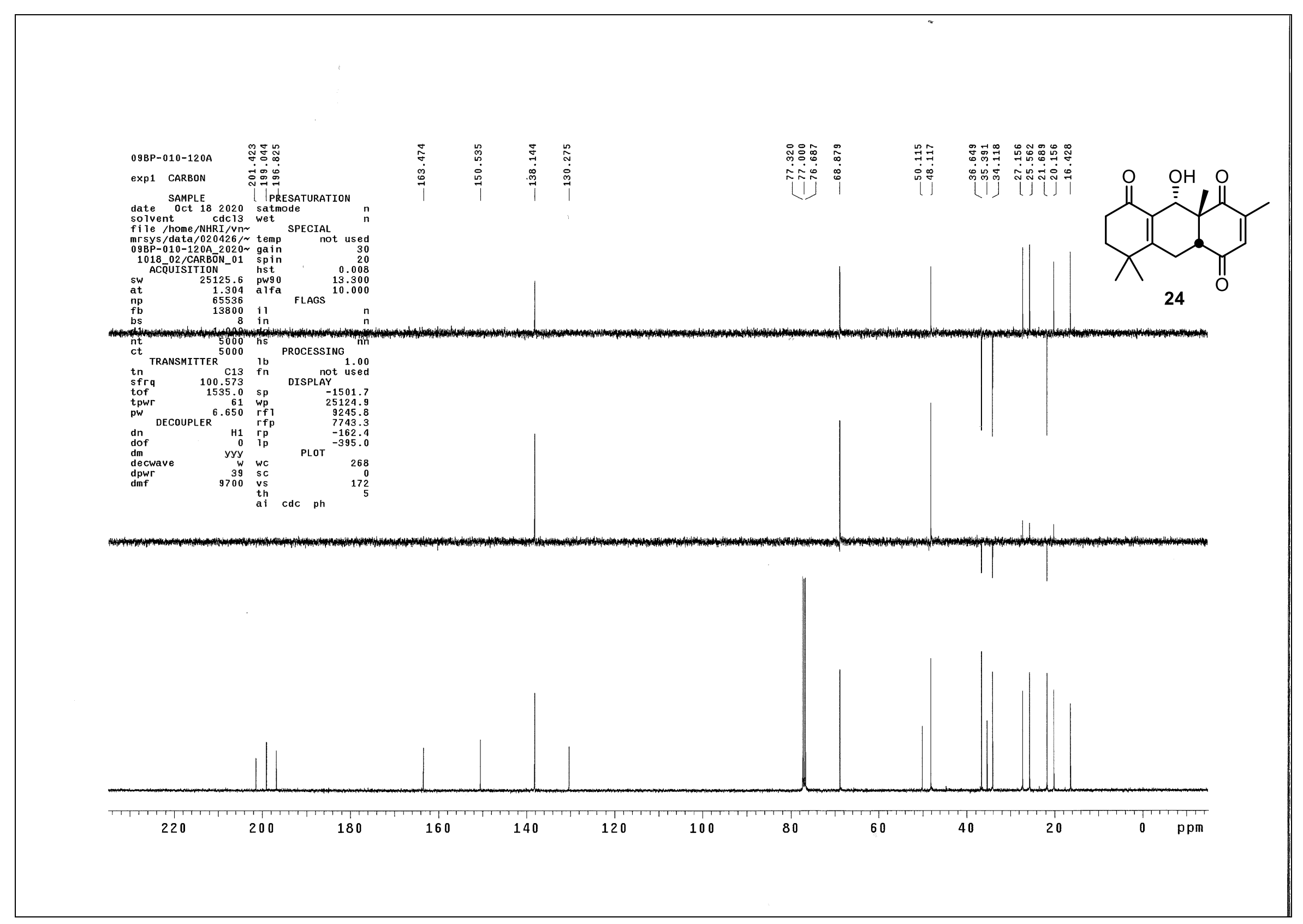

${ }^{13}$ C NMR + DEPT spectra for compound 24 


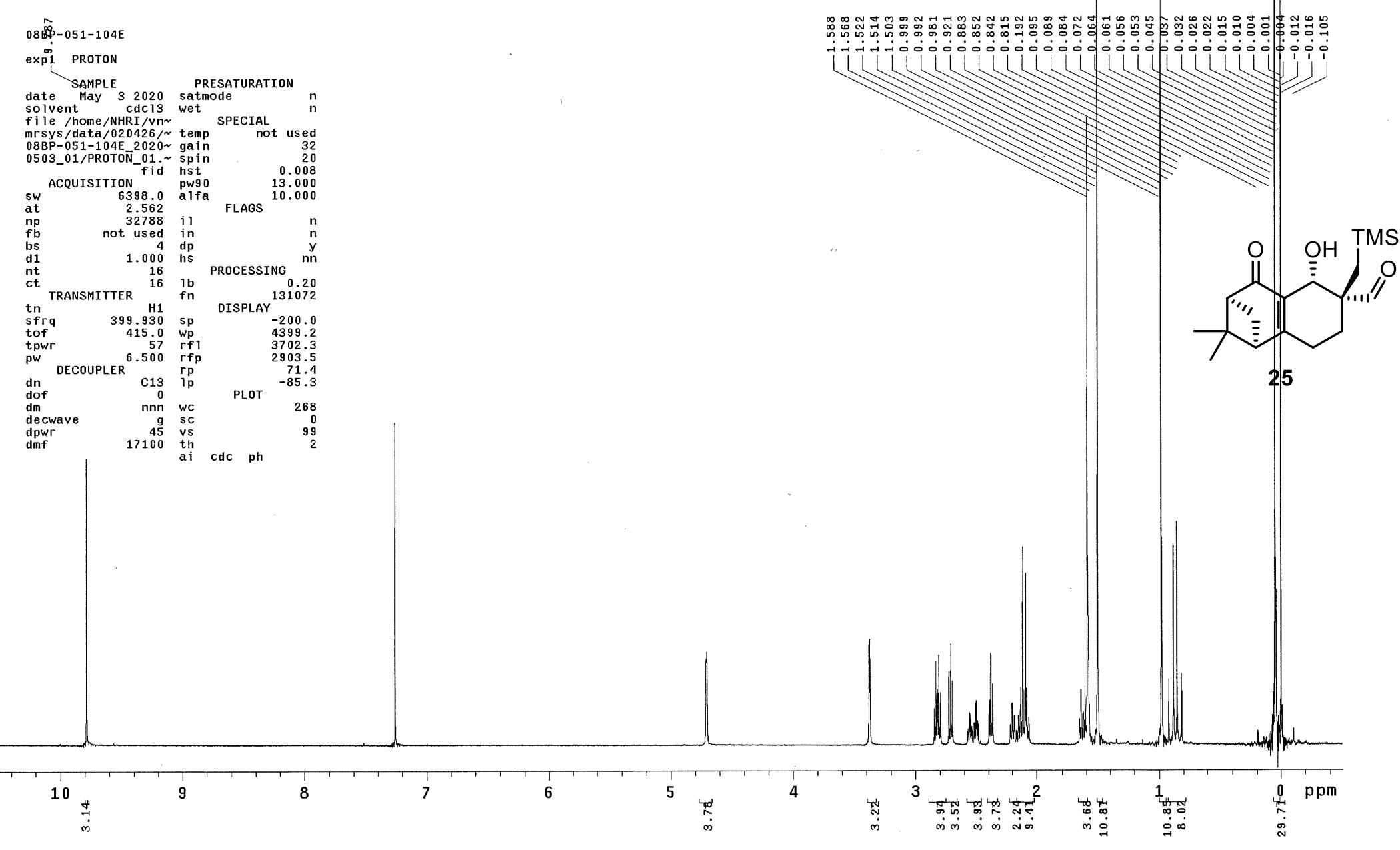

${ }^{1} \mathrm{H}$ NMR spectrum for compound 25 


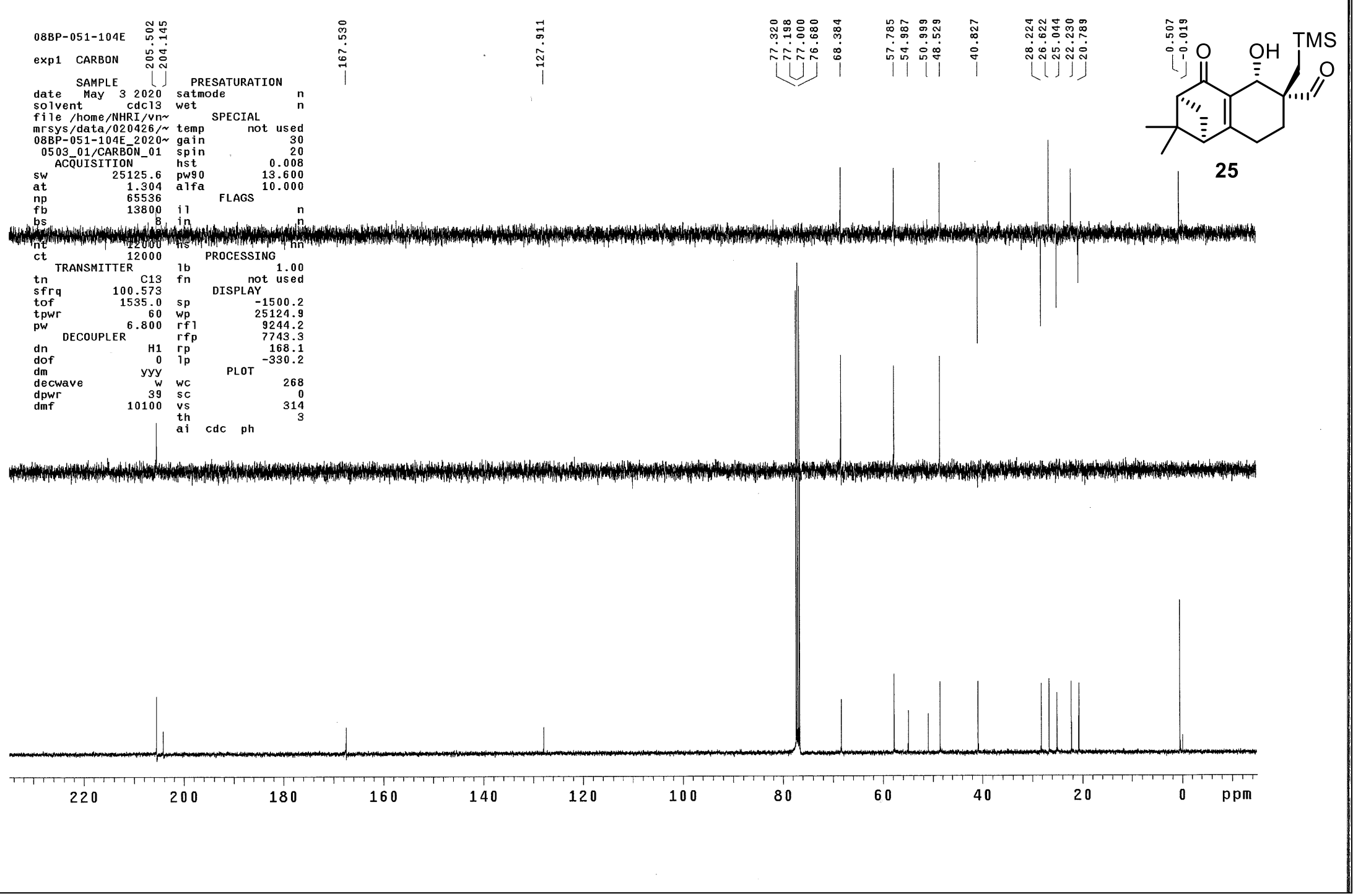

${ }^{13} \mathrm{C}$ NMR + DEPT spectra for compound 25 


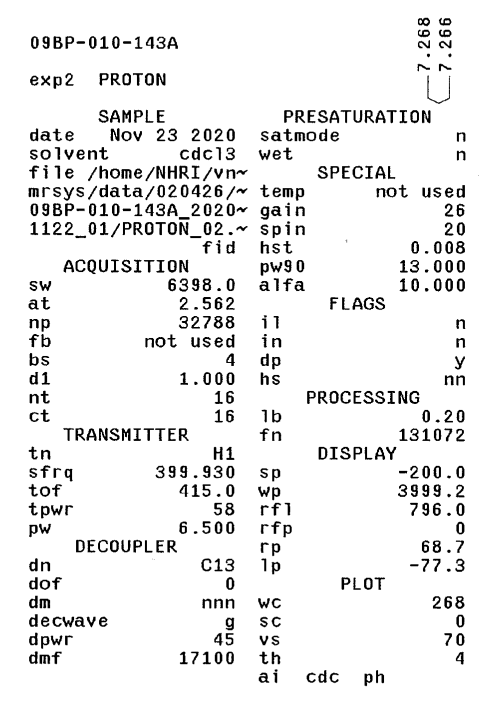

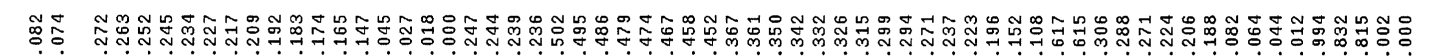

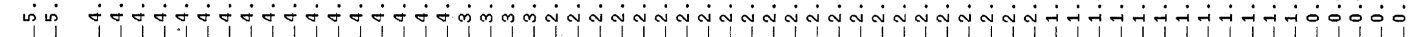

date SAMPLE 2020 PRESATURATION mosys data $02024 \sim 2$ temp net

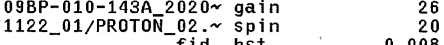

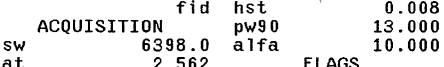

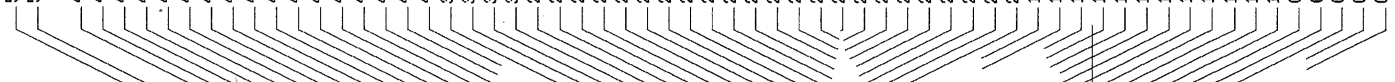

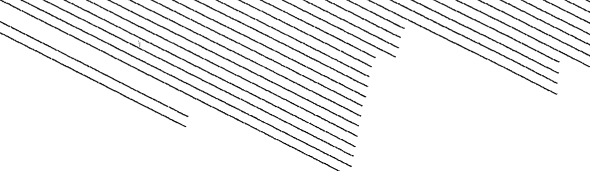




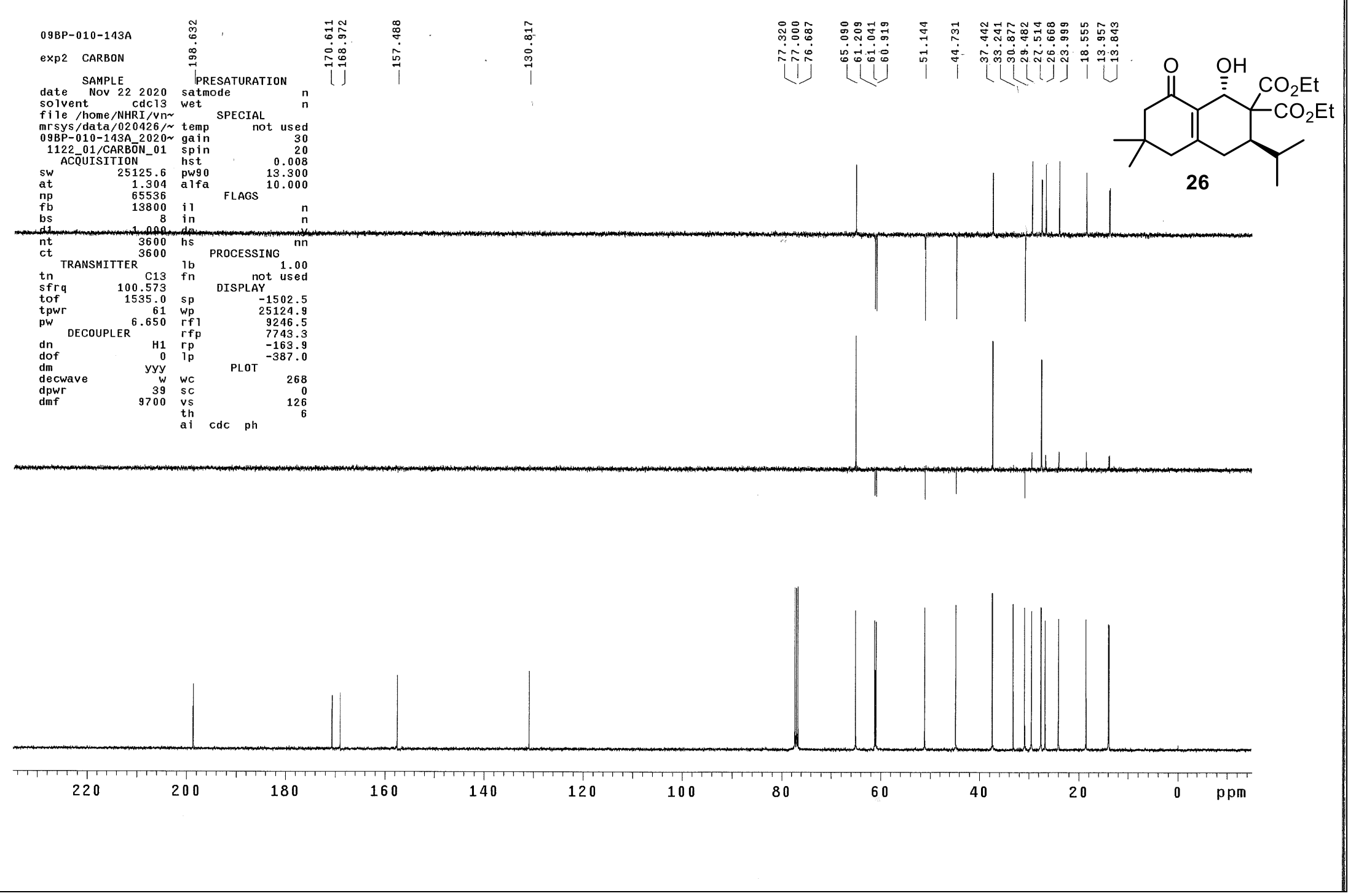

${ }^{13} \mathrm{C}$ NMR + DEPT spectra for compound $\mathbf{2 6}$ 


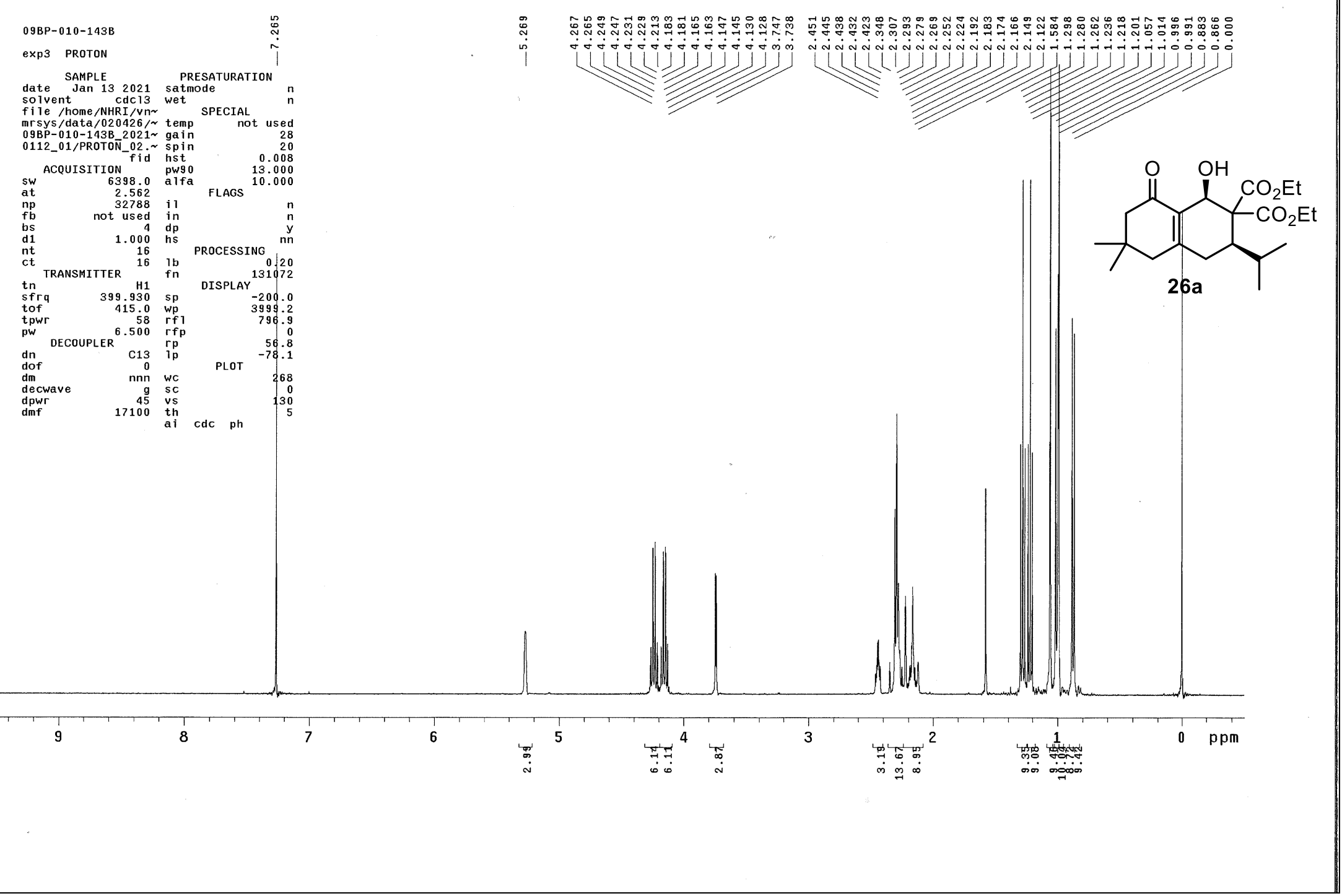

${ }^{1}$ H NMR spectrum for compound $26 a$ 


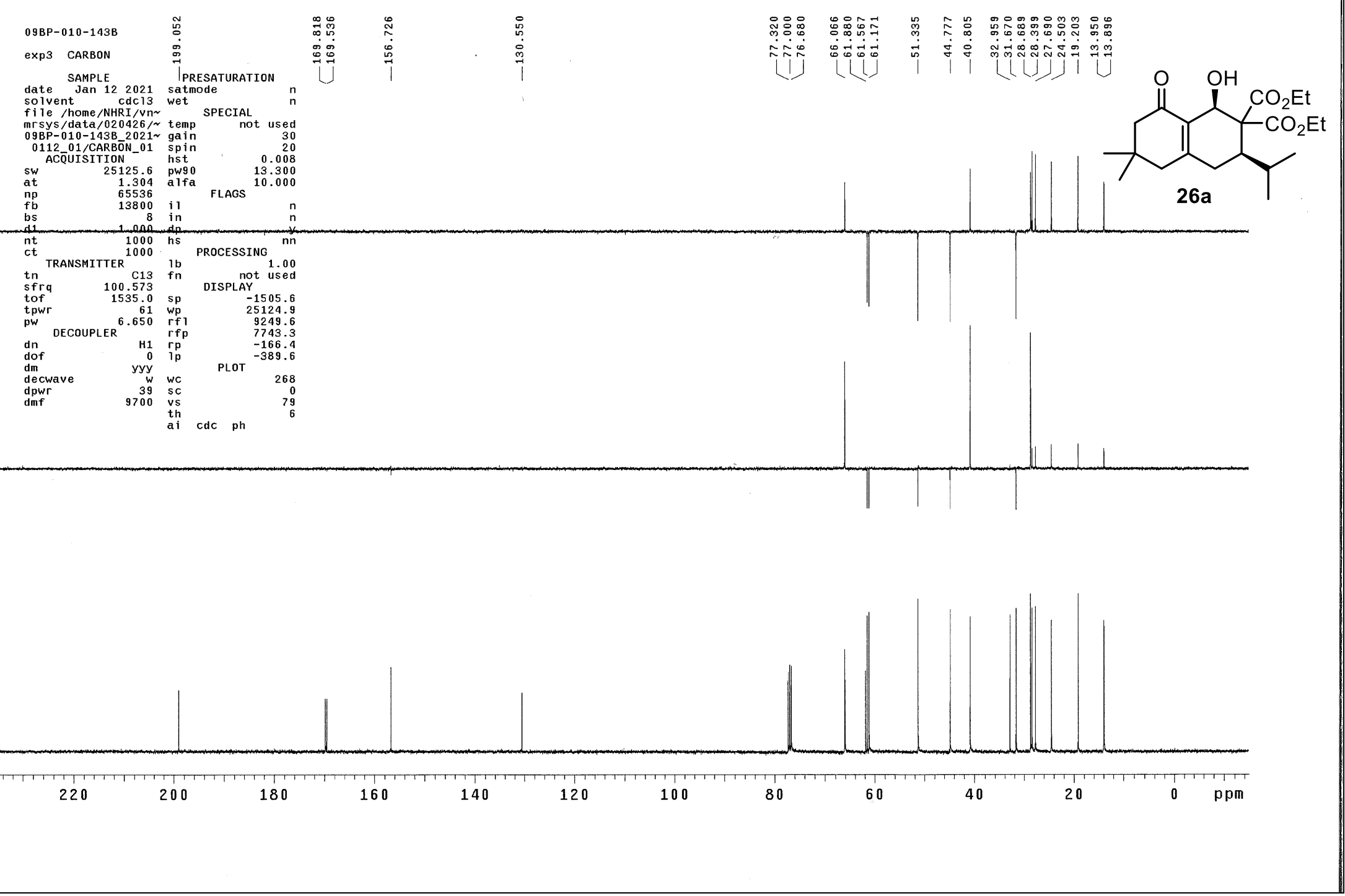

${ }^{13} \mathrm{C}$ NMR + DEPT spectra for compound 26a 


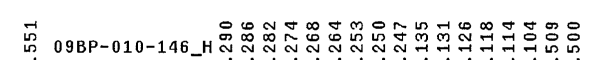

oxp2 PROTON

SAMPLE

date Nov 252020 Satminate URATIOA

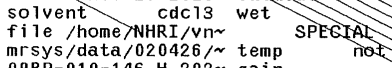

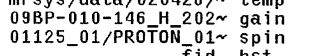

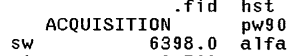

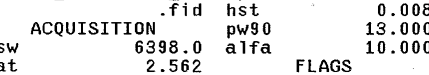

$\begin{aligned} \text { np } & 32788 \text { in } \\ \text { fb } & \text { not used in }\end{aligned}$

bs
d1
nt

PROCESSING nn

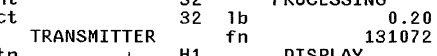

tn
sfrq
tof

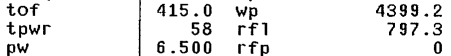

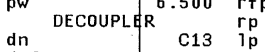

dm
dec
dpw

$\underset{\substack{d p w \\ d m f}}{\substack{d \\ d}}$

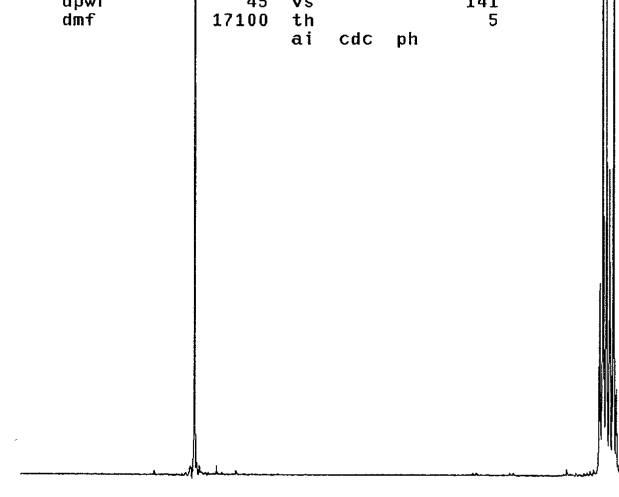

$\stackrel{+}{\stackrel{\alpha}{\infty}}$

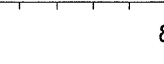

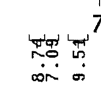

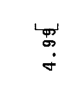

5

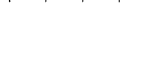

4

4

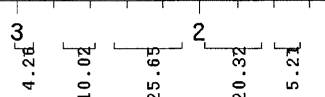

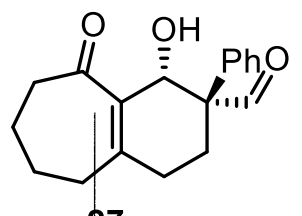

27

${ }^{1} \mathrm{H}$ NMR spectrum for compound 27 

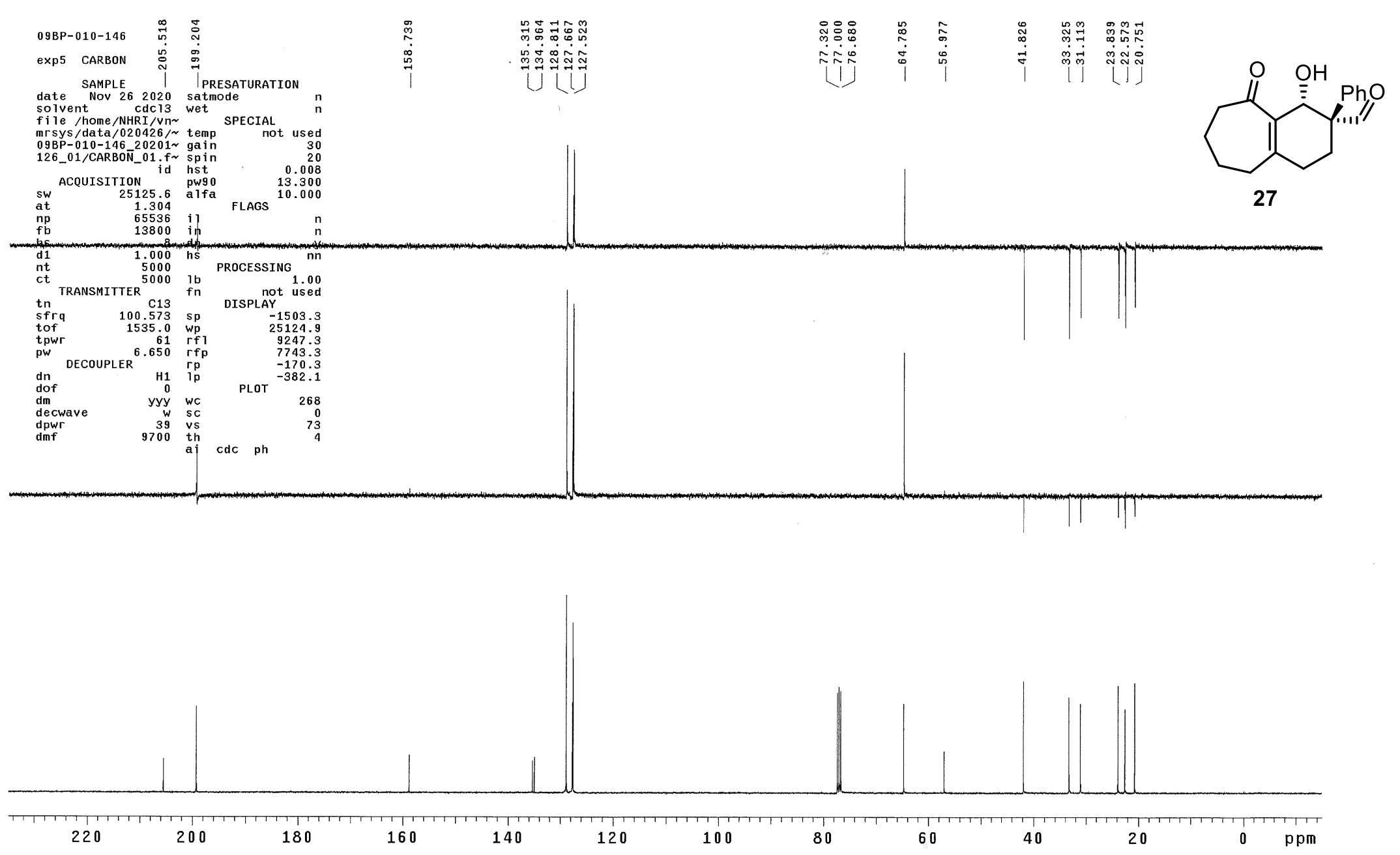

${ }^{13} \mathrm{C}$ NMR + DEPT spectra for compound 27 


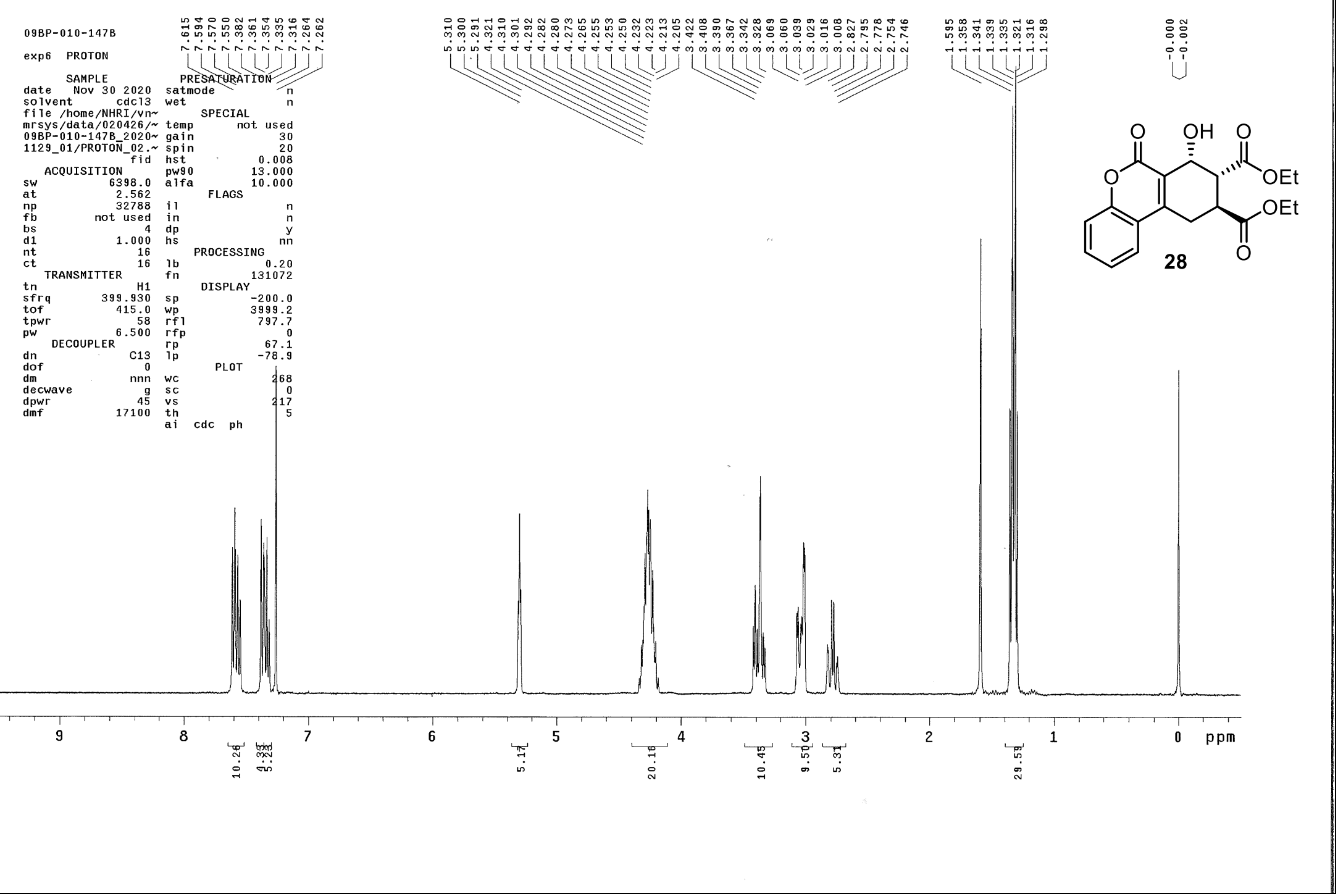

${ }^{1}$ H NMR spectrum for compound 28 


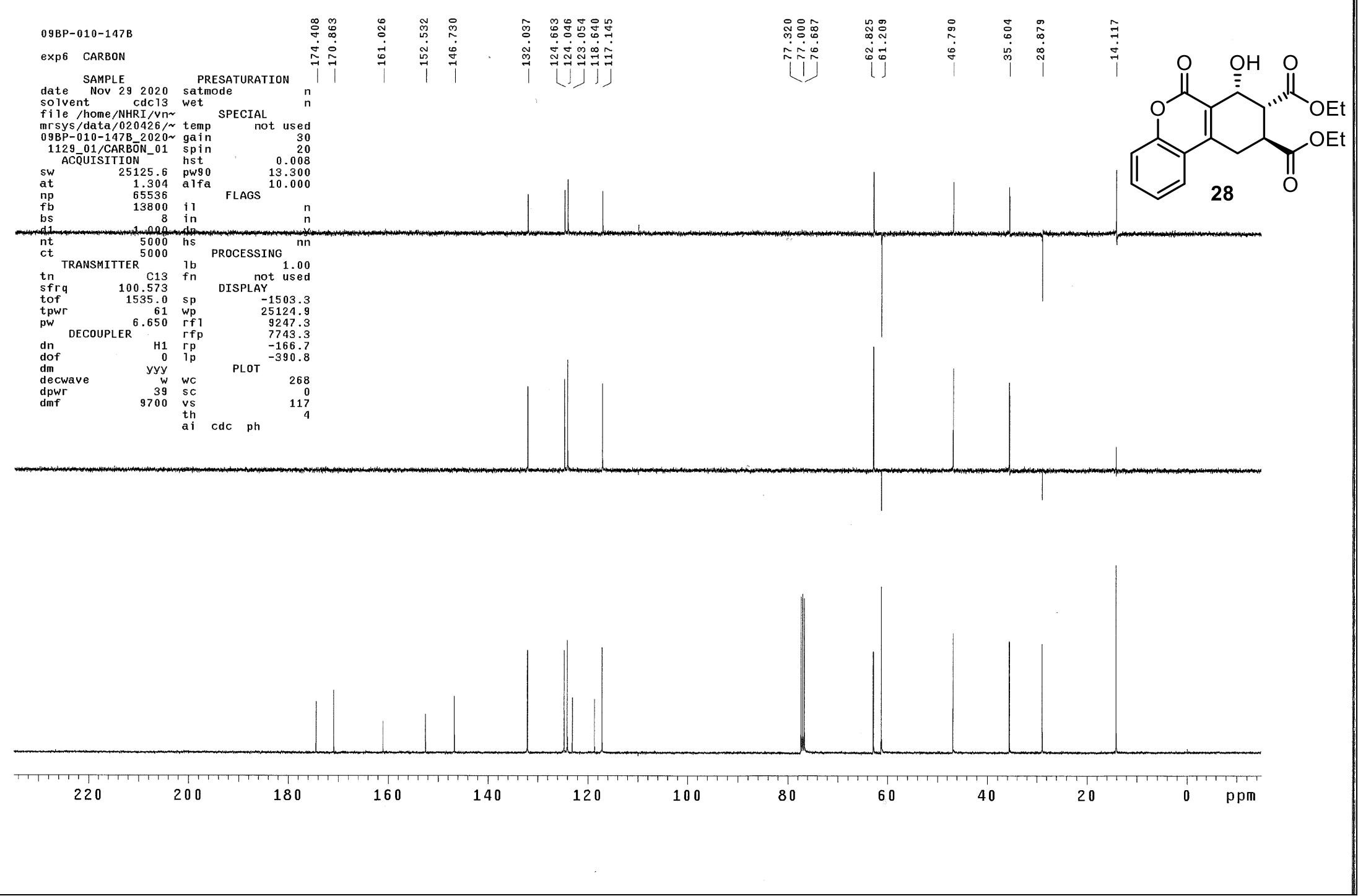

${ }^{13} \mathrm{C}$ NMR + DEPT spectra for compound $\mathbf{2 8}$ 


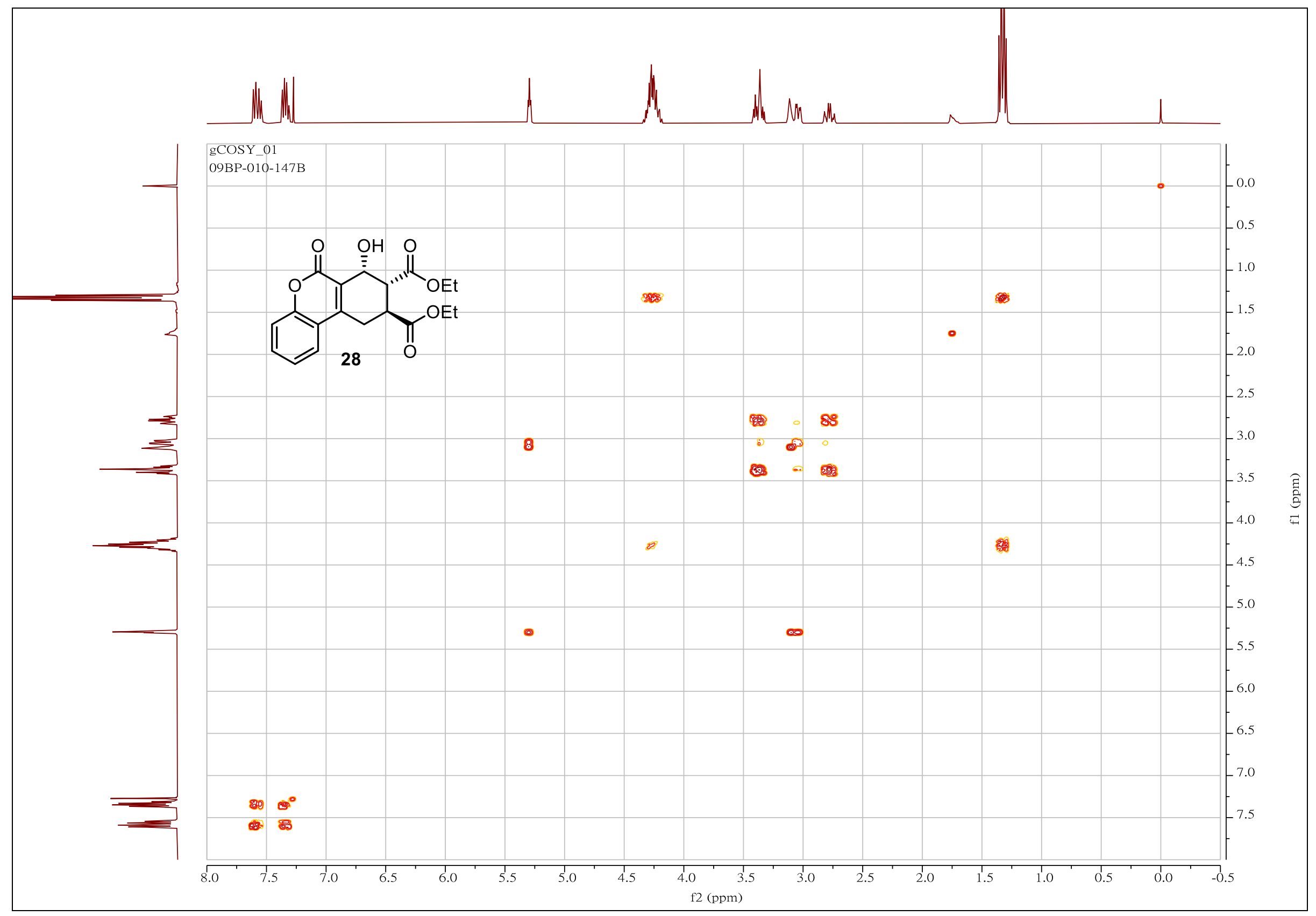

${ }^{1} \mathrm{H}-{ }^{1} \mathrm{H}$ COSY spectrum for compound 28 


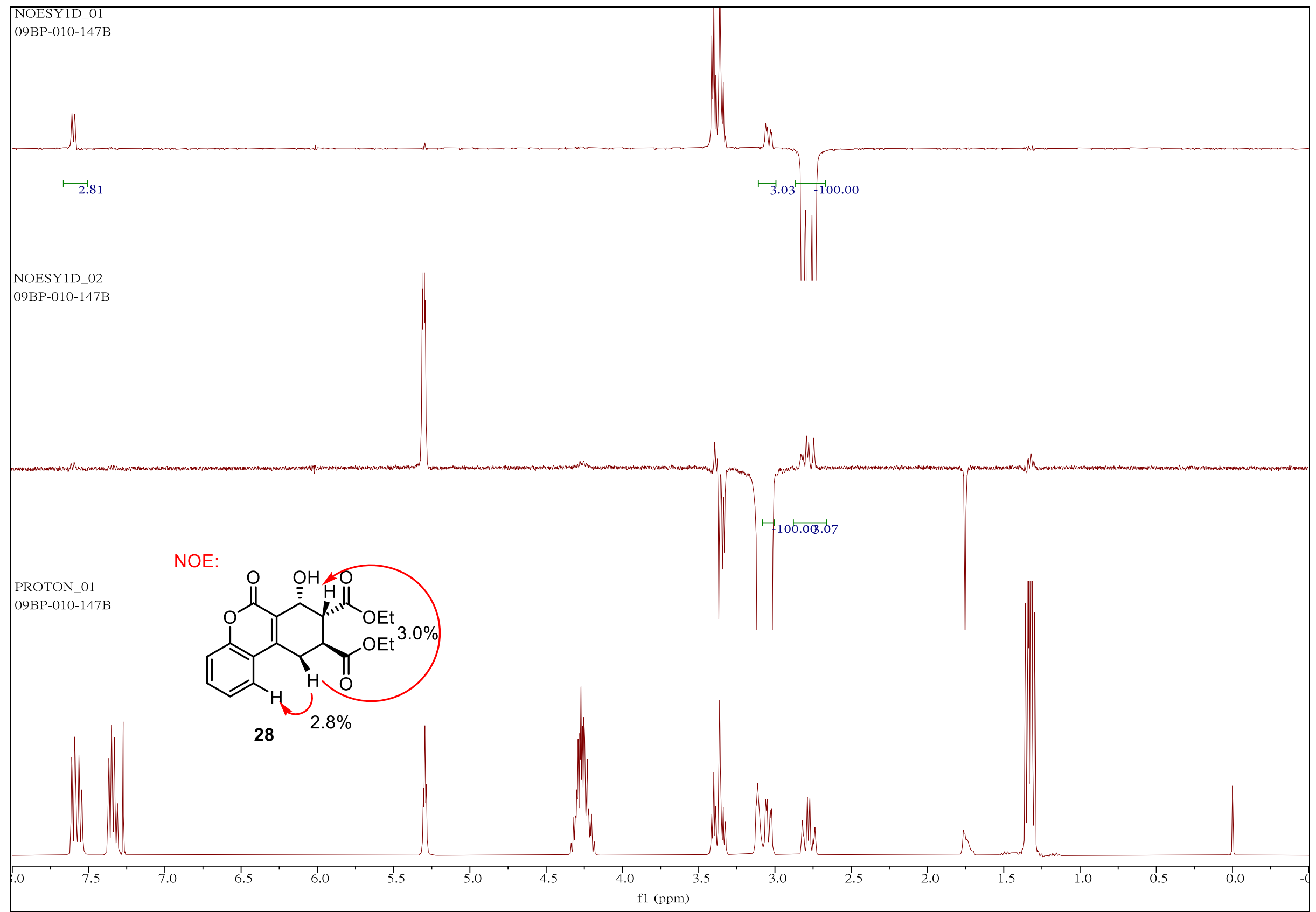

1D NOESY spectra for compound $\mathbf{2 8}$ 


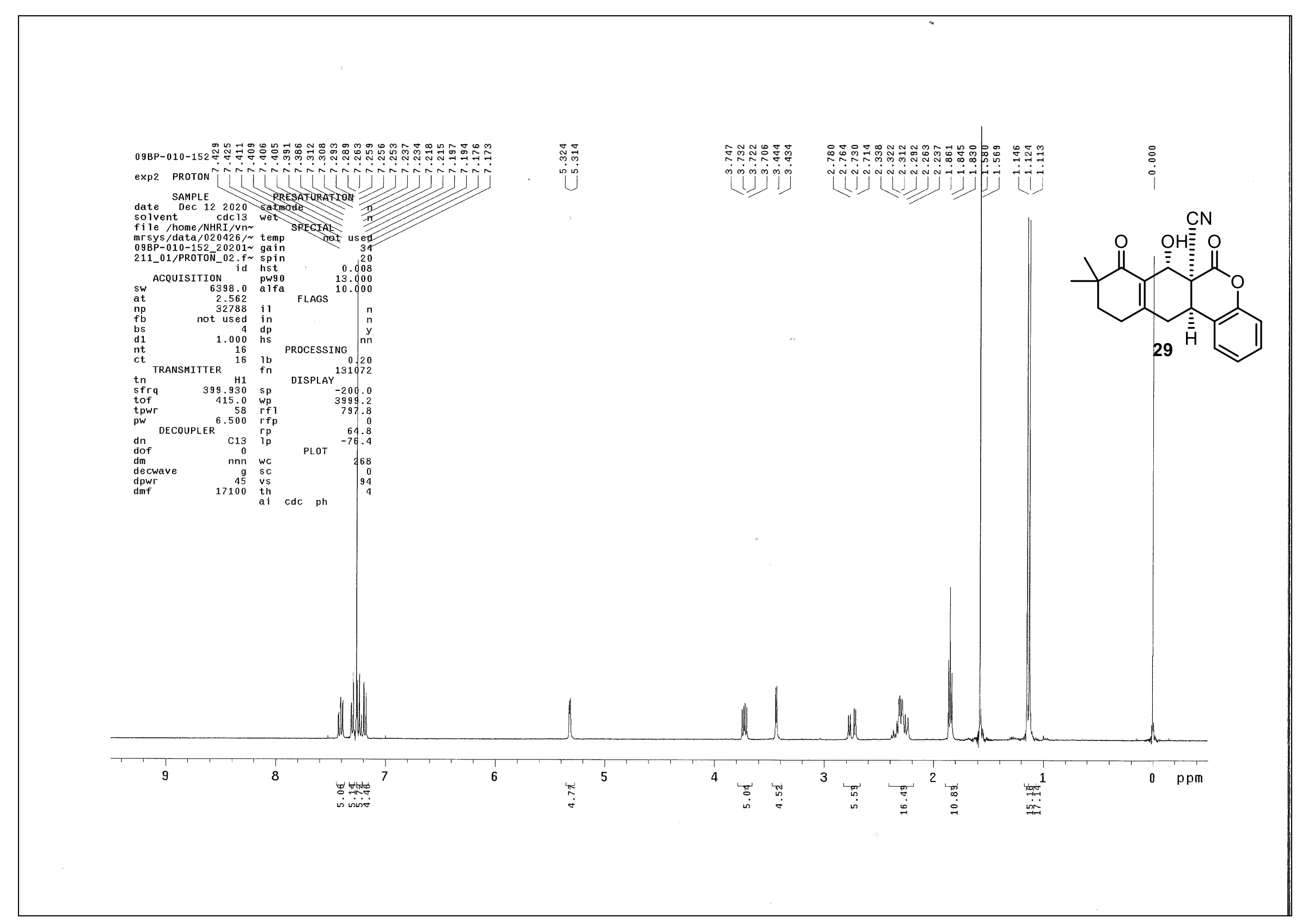

${ }^{1} \mathrm{H}$ NMR spectrum for compound 29 


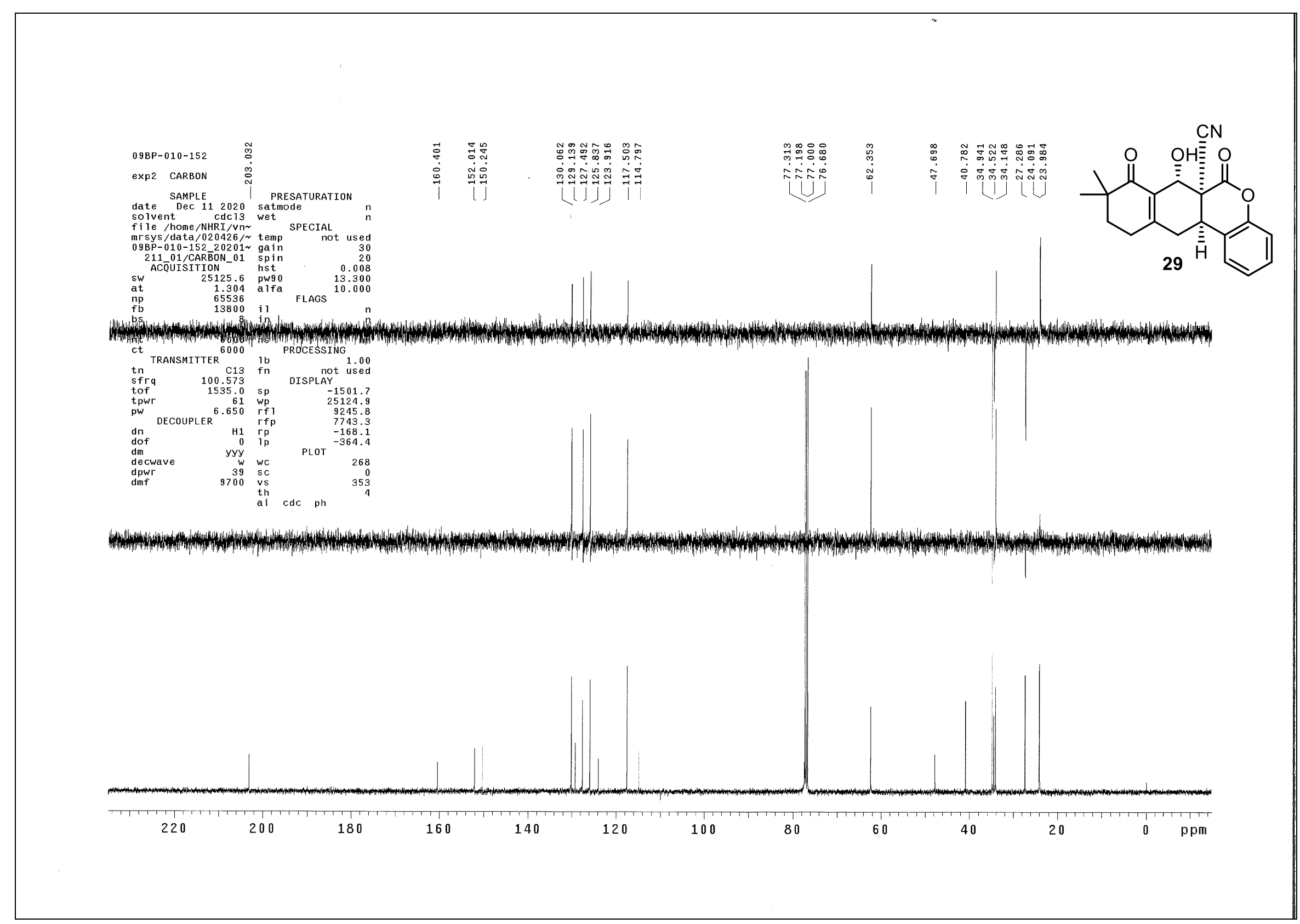

${ }^{13} \mathrm{C}$ NMR + DEPT spectra for compound $\mathbf{2 9}$ 

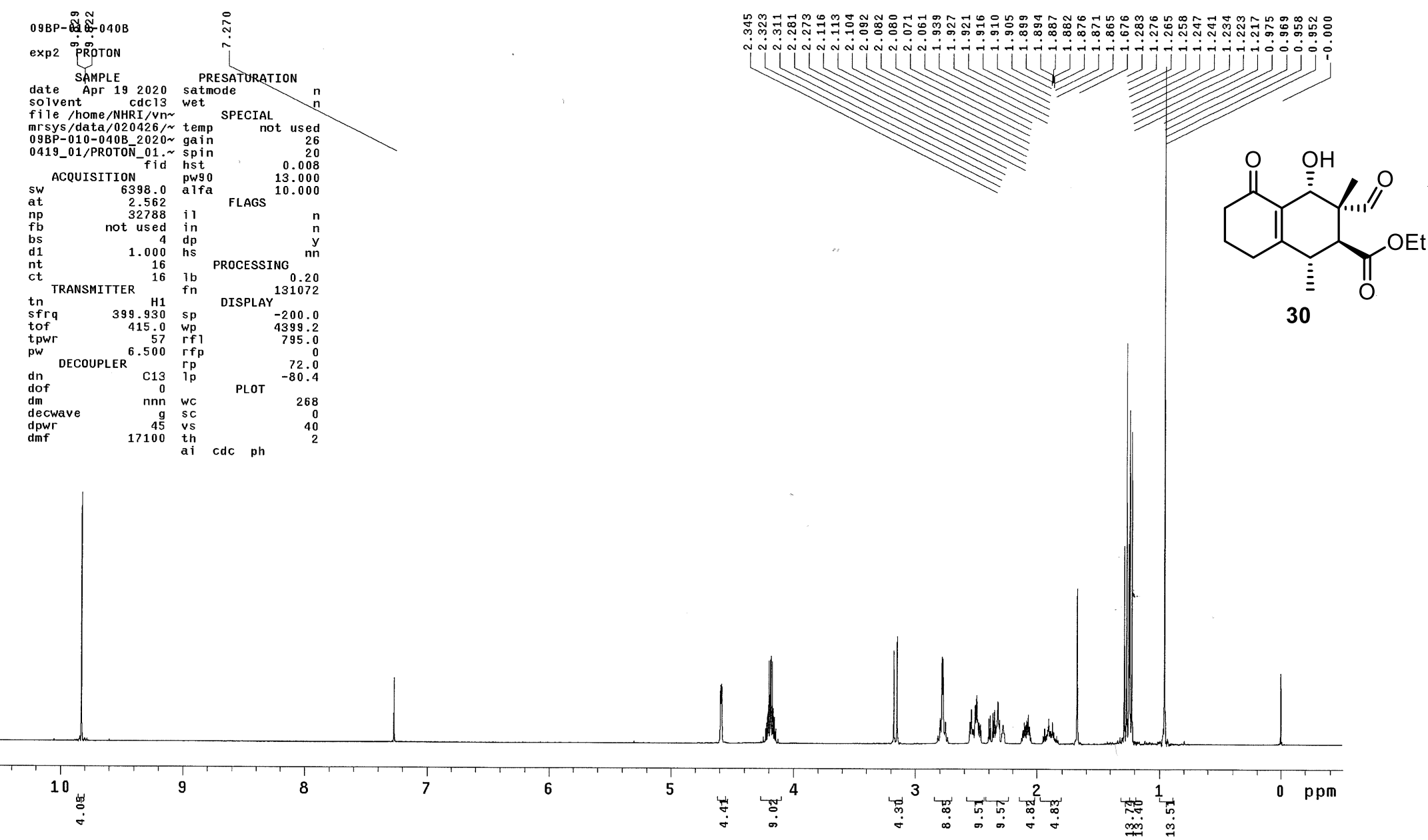

${ }^{1} \mathrm{H}$ NMR spectrum for compound $\mathbf{3 0}$ 


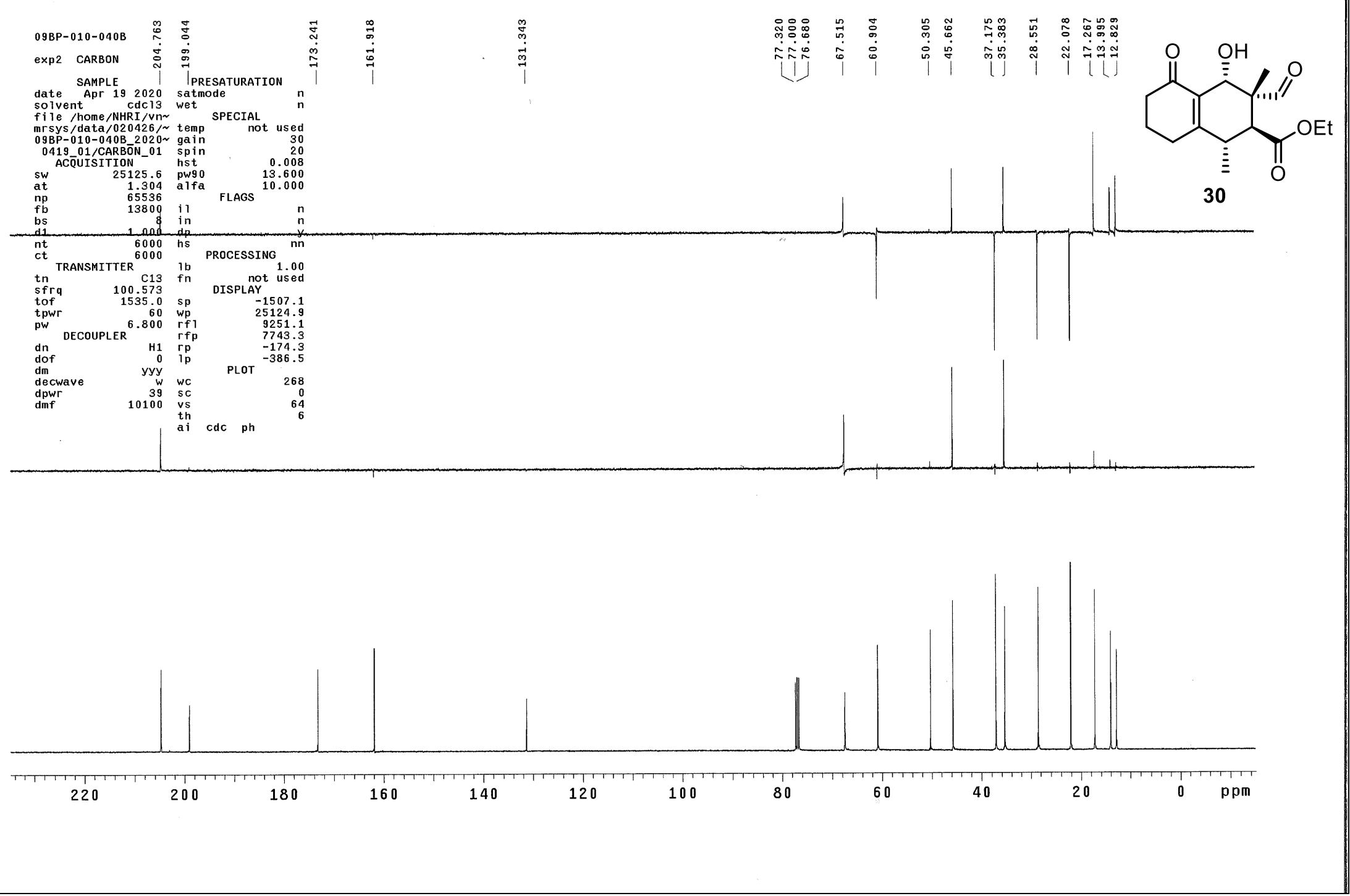

${ }^{13} \mathrm{C}$ NMR + DEPT spectra for compound $\mathbf{3 0}$ 


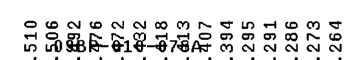

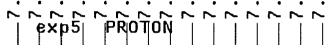

SAMPIE PRESATURATION

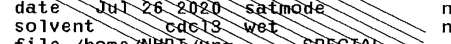

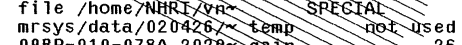

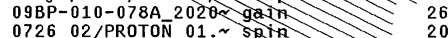

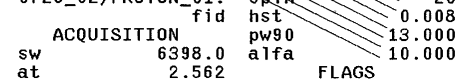

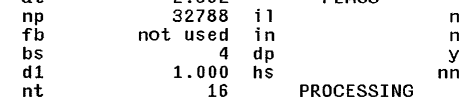

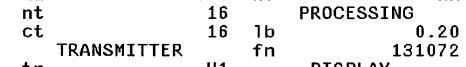

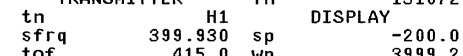

$\begin{array}{lrlr}\text { sfrq } & 399.930 & \mathrm{sp} & -200.0 \\ \text { tof } & 415.0 & \text { wp } & 3999.2 \\ \text { tpwr } & 5.580 & \text { rf } & 797.4 \\ \text { pw } & 6.500 & \text { rfp } & 74.0\end{array}$

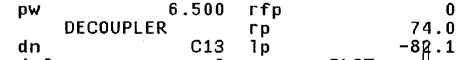

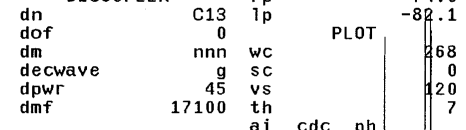

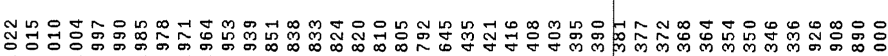

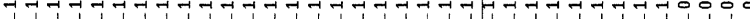

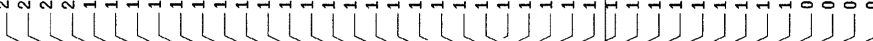

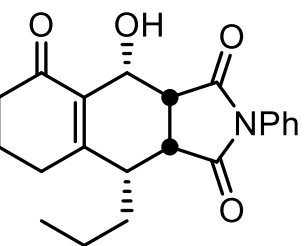

31

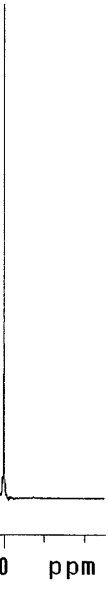

${ }^{1} \mathrm{H}$ NMR spectrum for compound $\mathbf{3 1}$ 

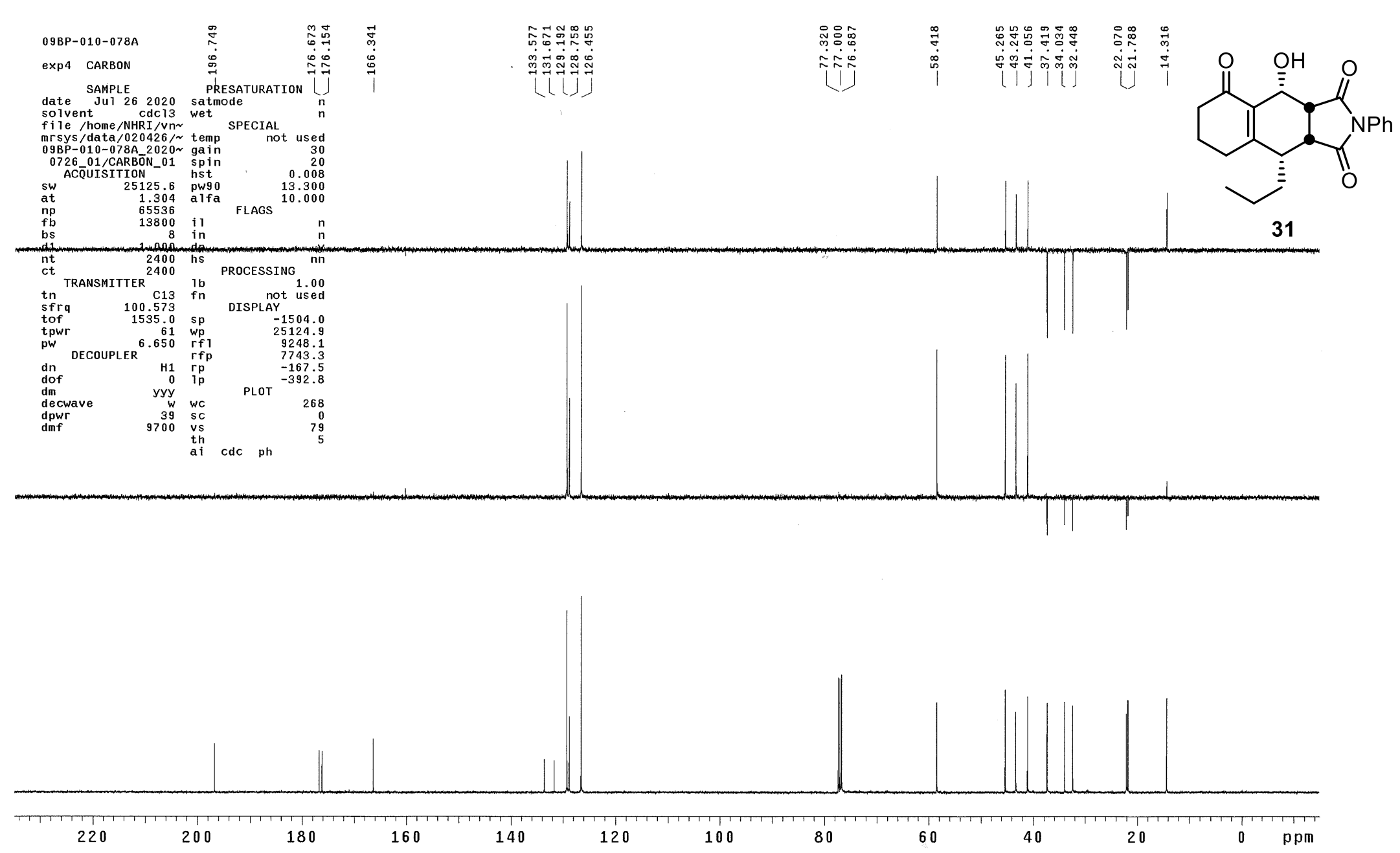

${ }^{13} \mathrm{C}$ NMR + DEPT spectra for compound $\mathbf{3 1}$ 


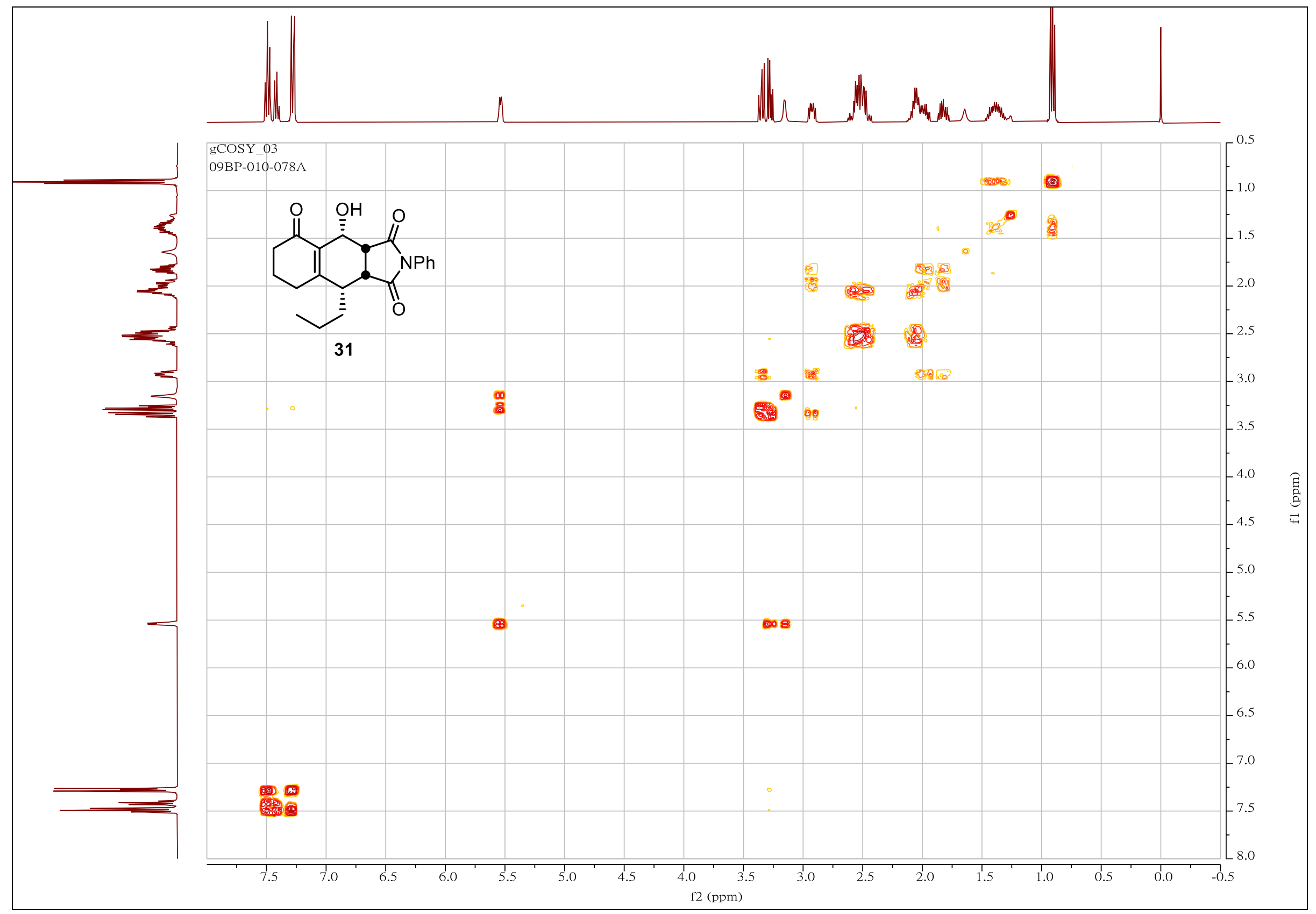

${ }^{1} \mathrm{H}-{ }^{1} \mathrm{H}$ COSY spectrum for compound 31 


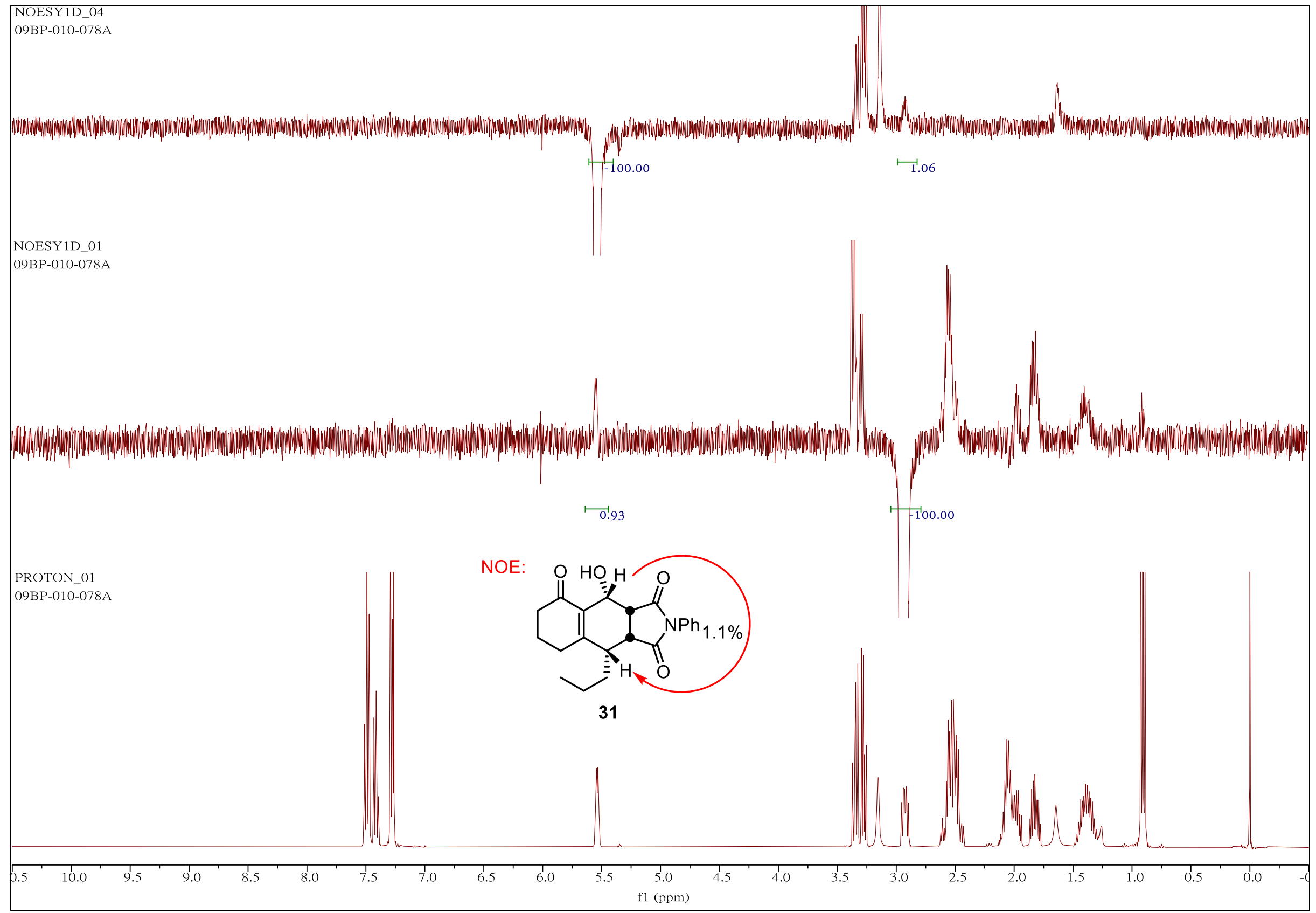

1D NOESY spectra for compound $\mathbf{3 1}$ 


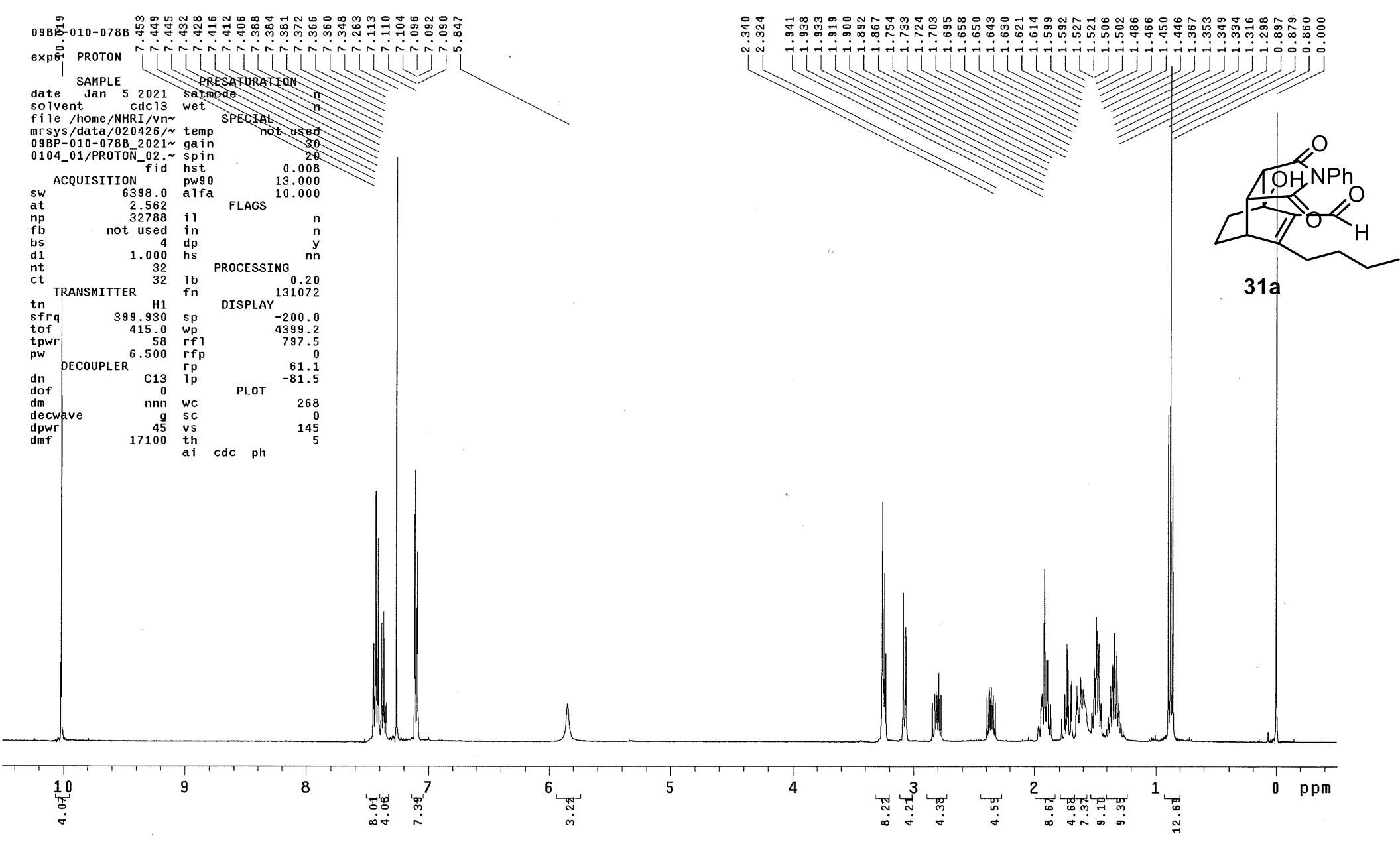

${ }^{1} \mathrm{H}$ NMR spectrum for compound 31a 


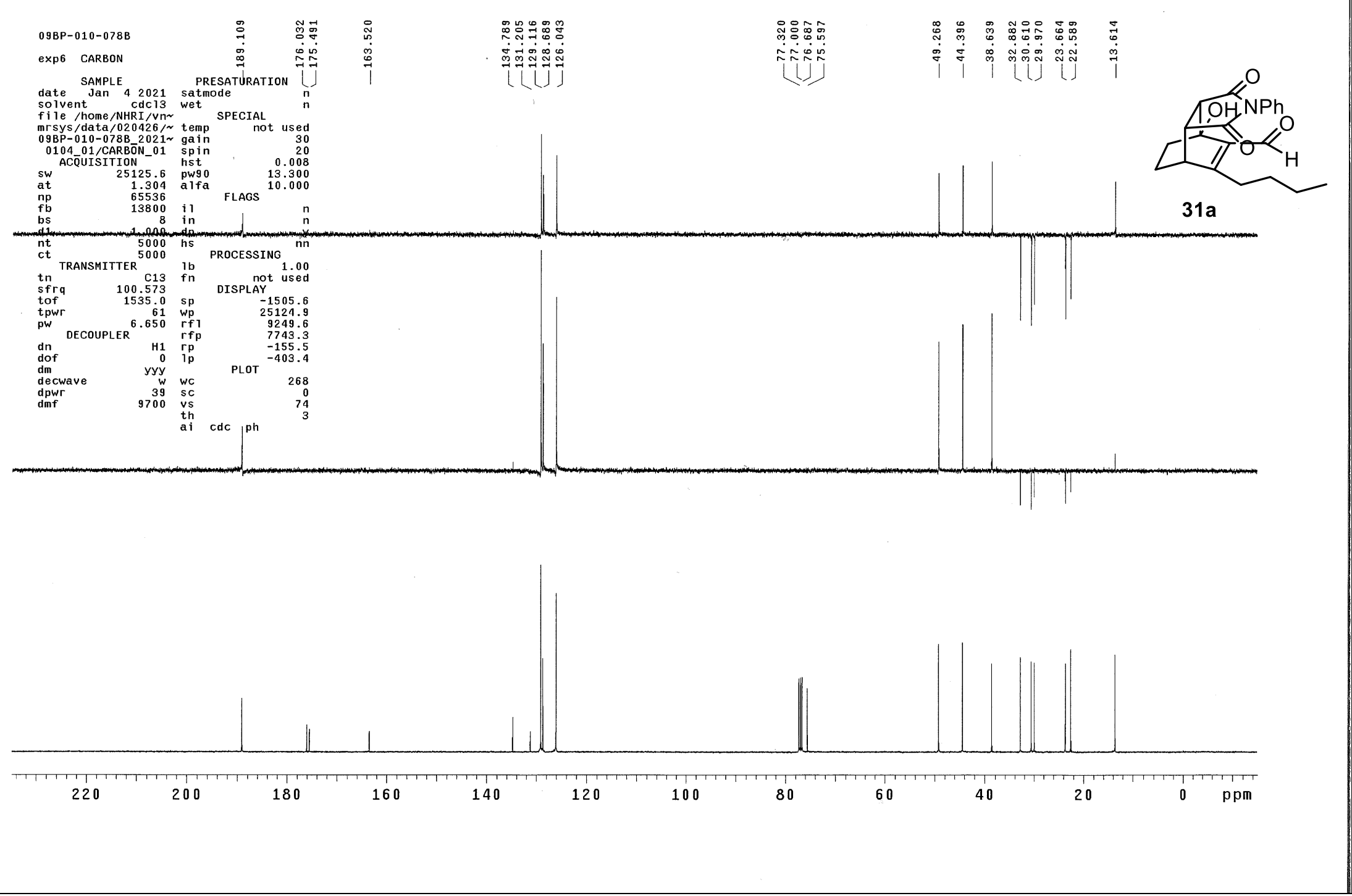

${ }^{13} \mathrm{C}$ NMR + DEPT spectra for compound 31a 


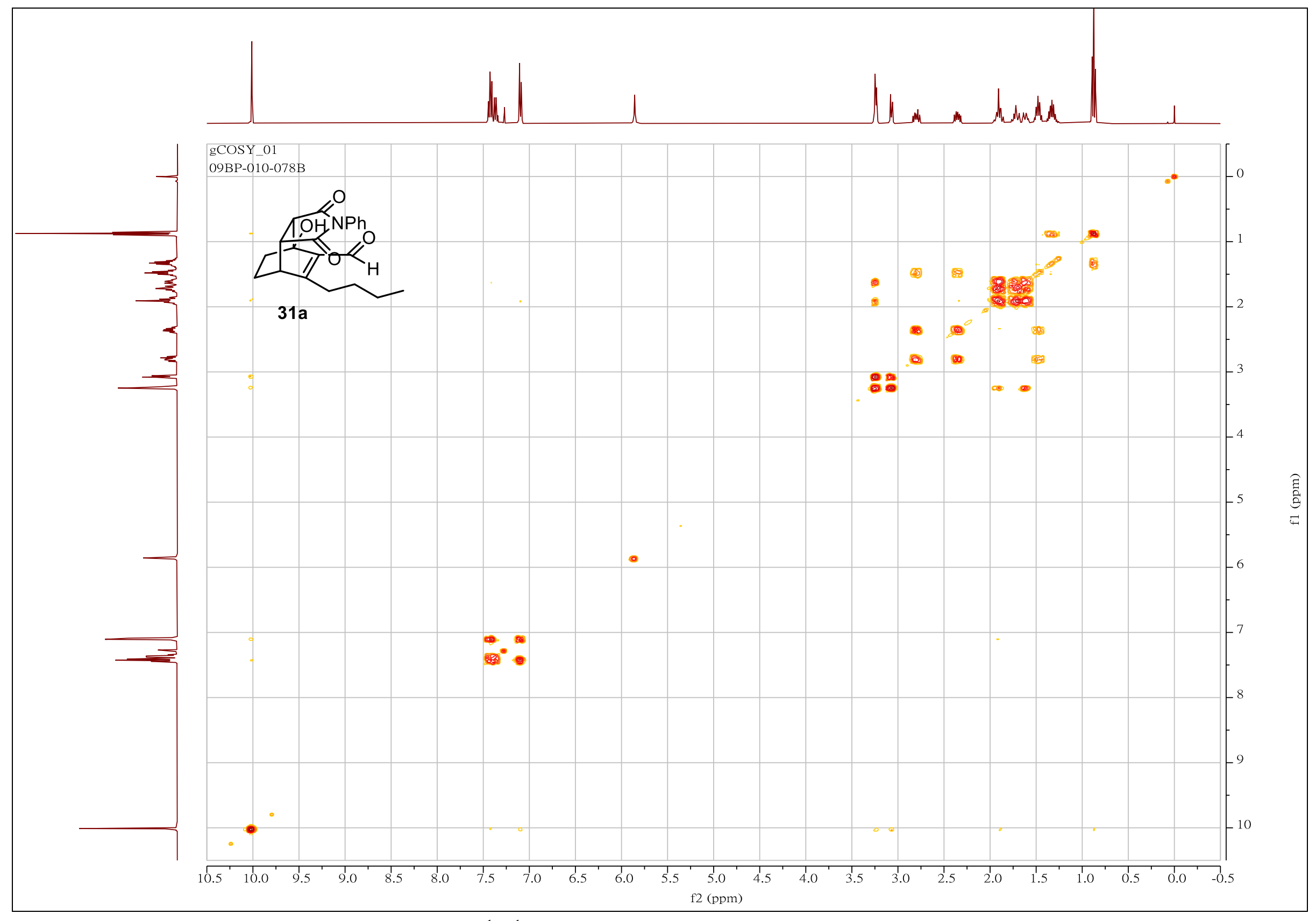

${ }^{1} \mathrm{H}-{ }^{1} \mathrm{H}$ COSY spectrum for compound $\mathbf{3 1 a}$ 


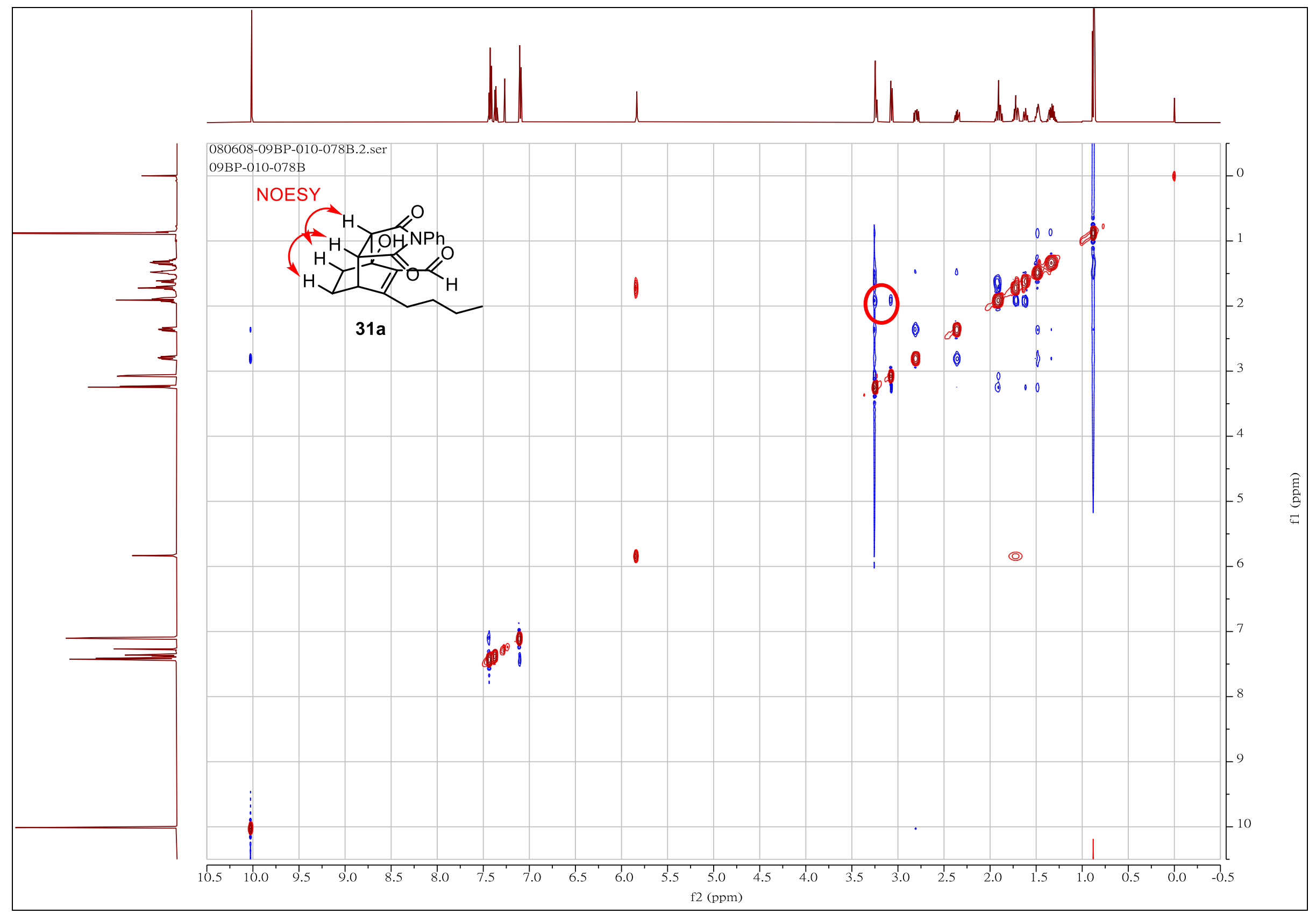

2D NOESY spectrum for compound 31a 


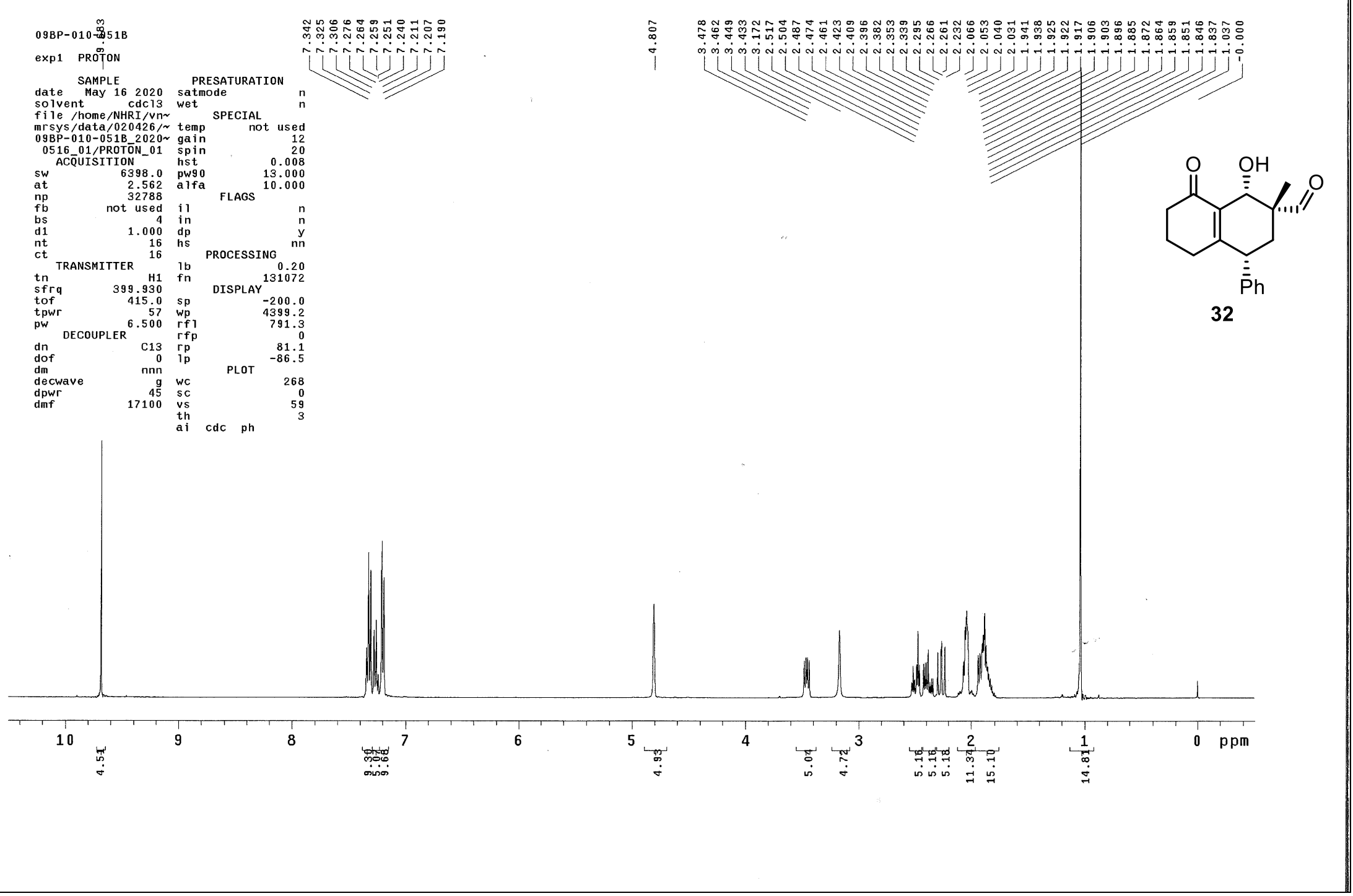

${ }^{1} \mathrm{H}$ NMR spectrum for compound $\mathbf{3 2}$ 


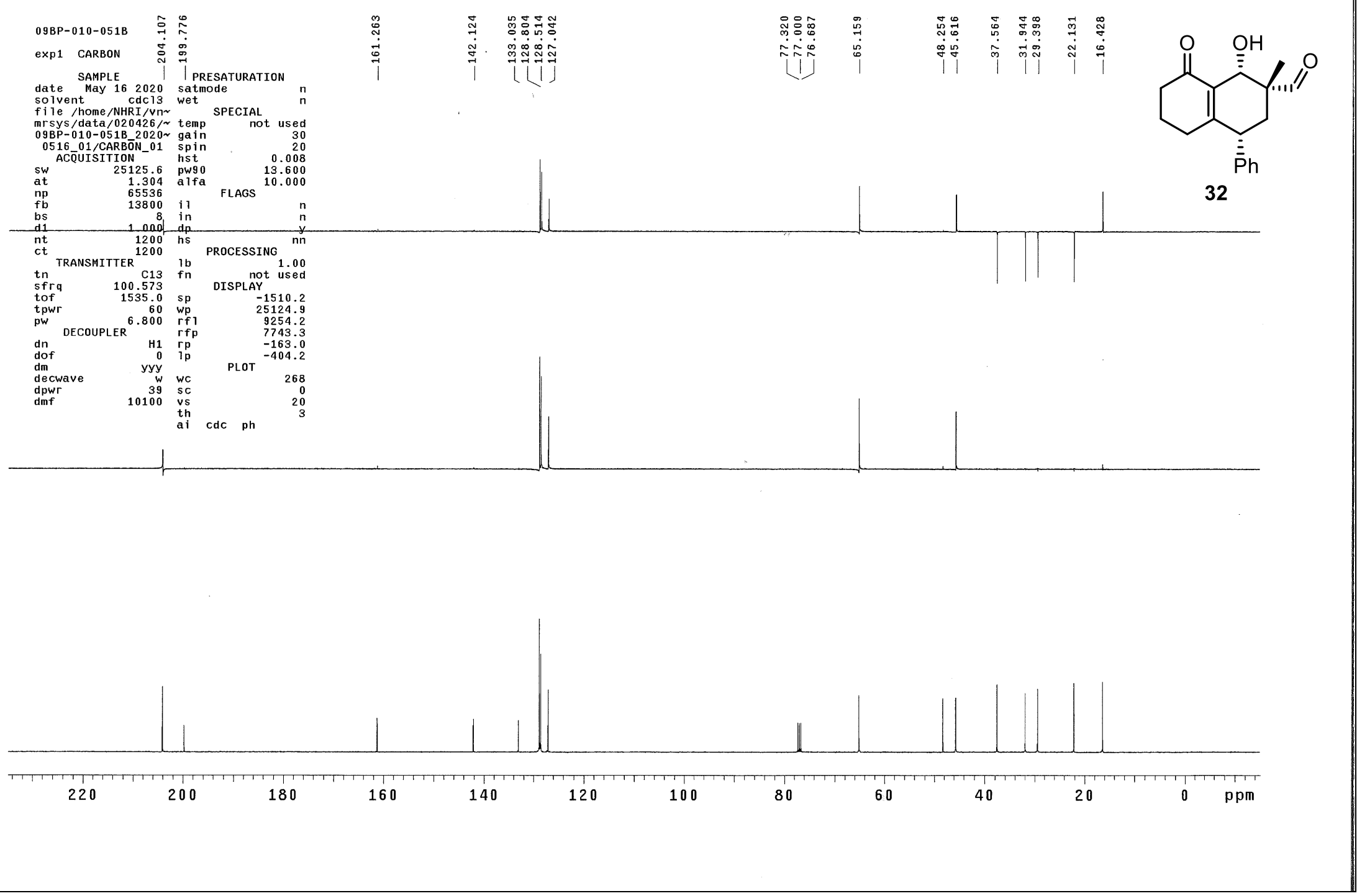

${ }^{13} \mathrm{C}$ NMR + DEPT spectra for compound 32 


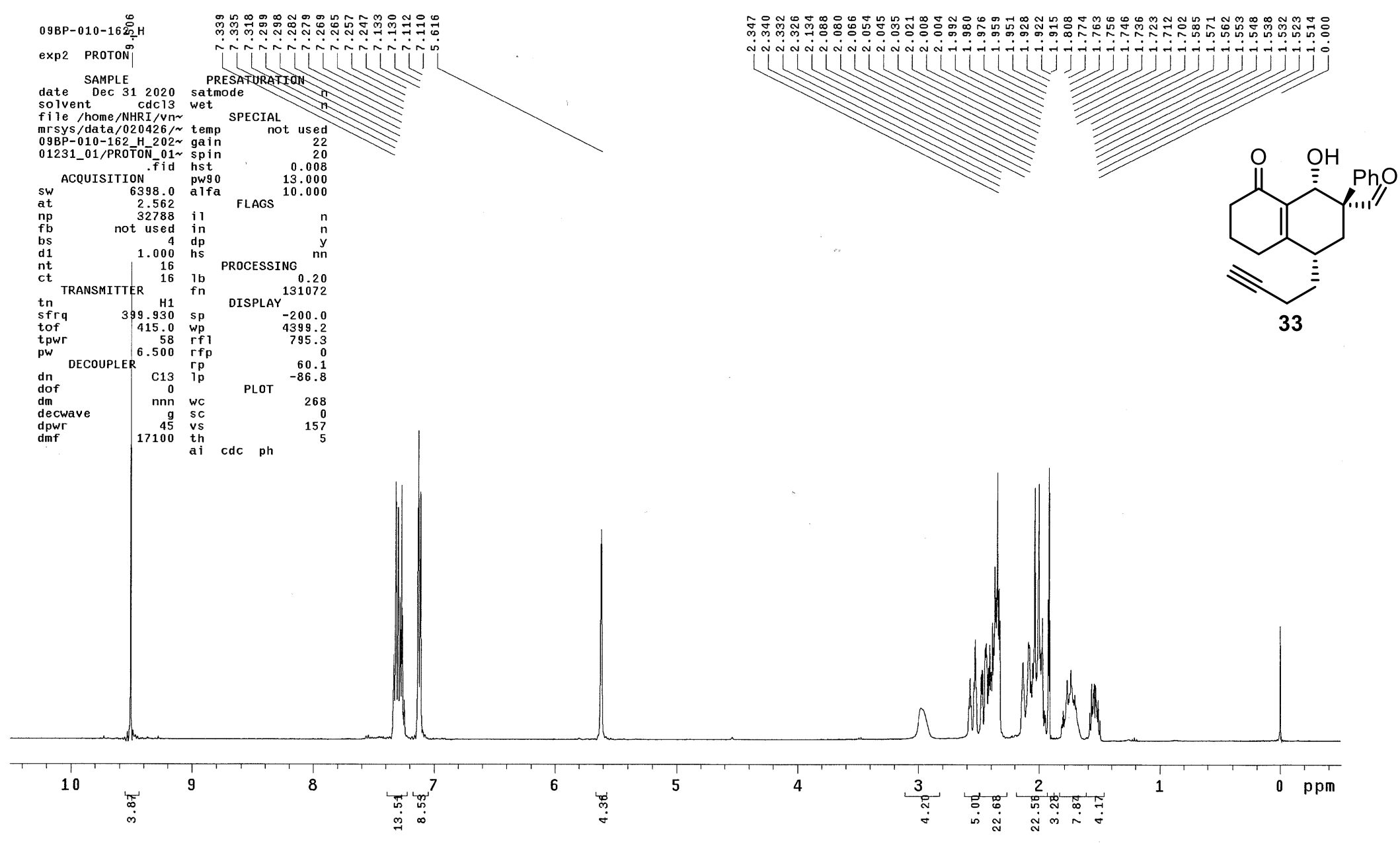

${ }^{1} \mathrm{H}$ NMR spectrum for compound $\mathbf{3 3}$ 

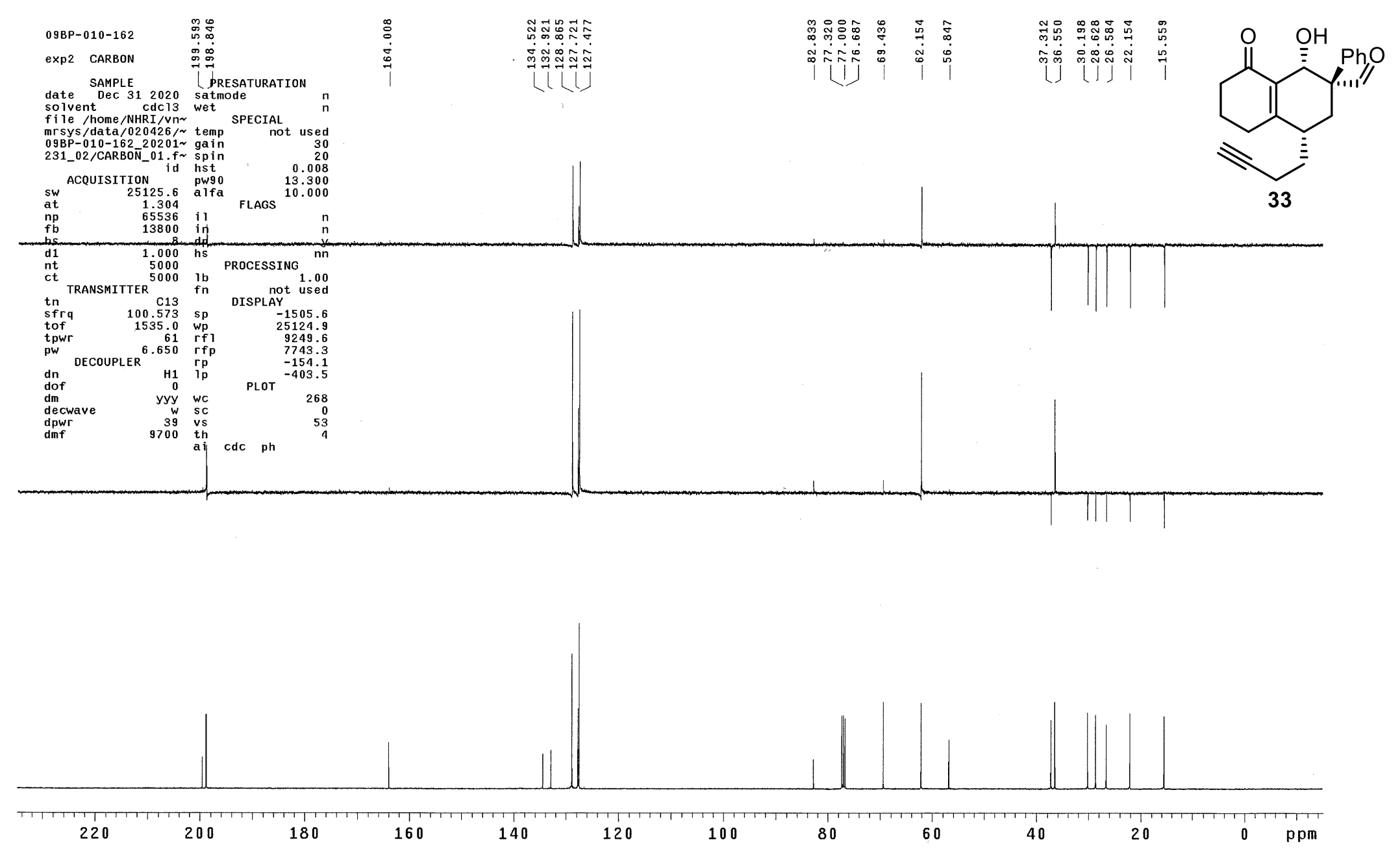

${ }^{13} \mathrm{C}$ NMR + DEPT spectra for compound 33 


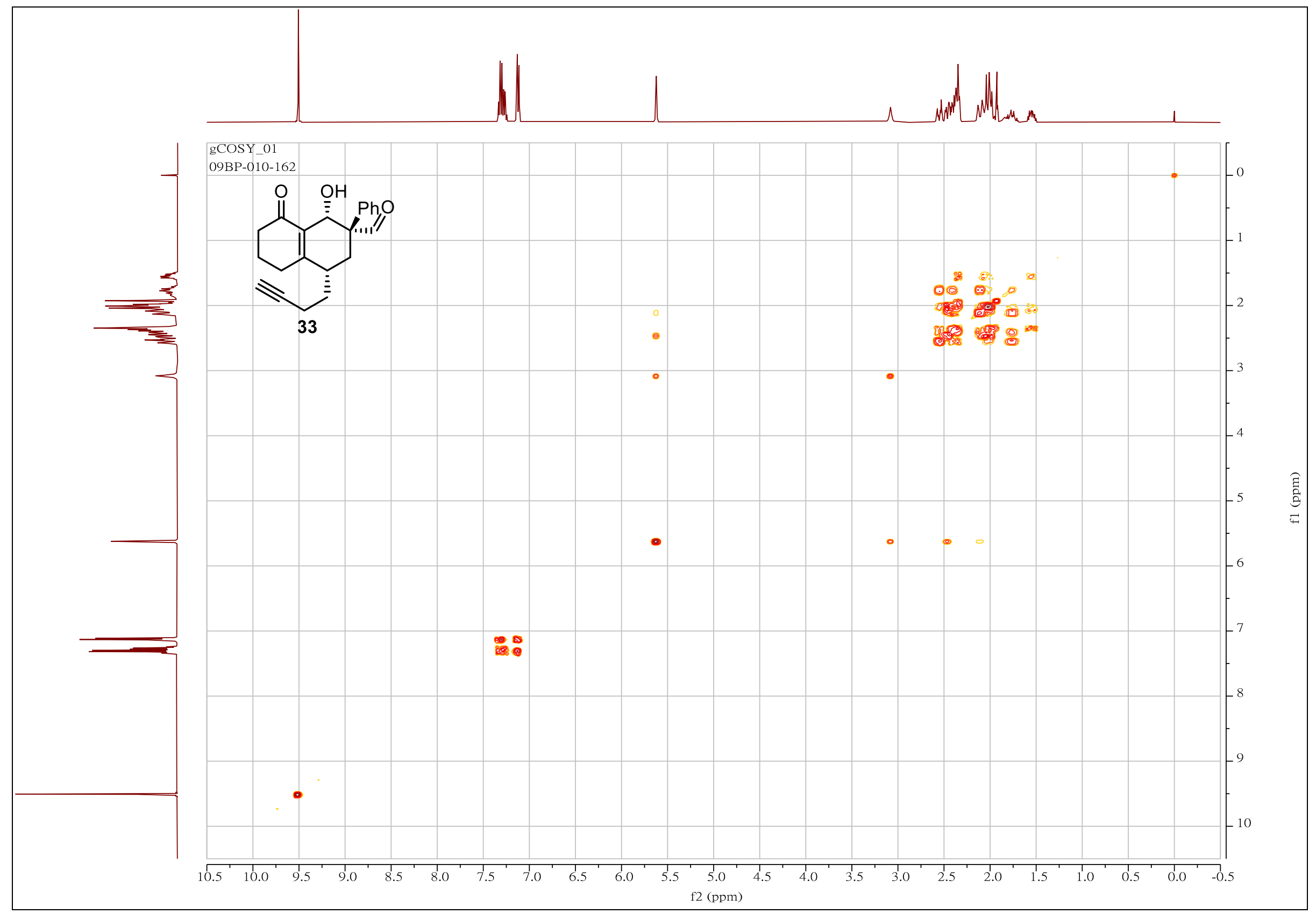

${ }^{1} \mathrm{H}-{ }^{1} \mathrm{H}$ COSY spectrum for compound 33 


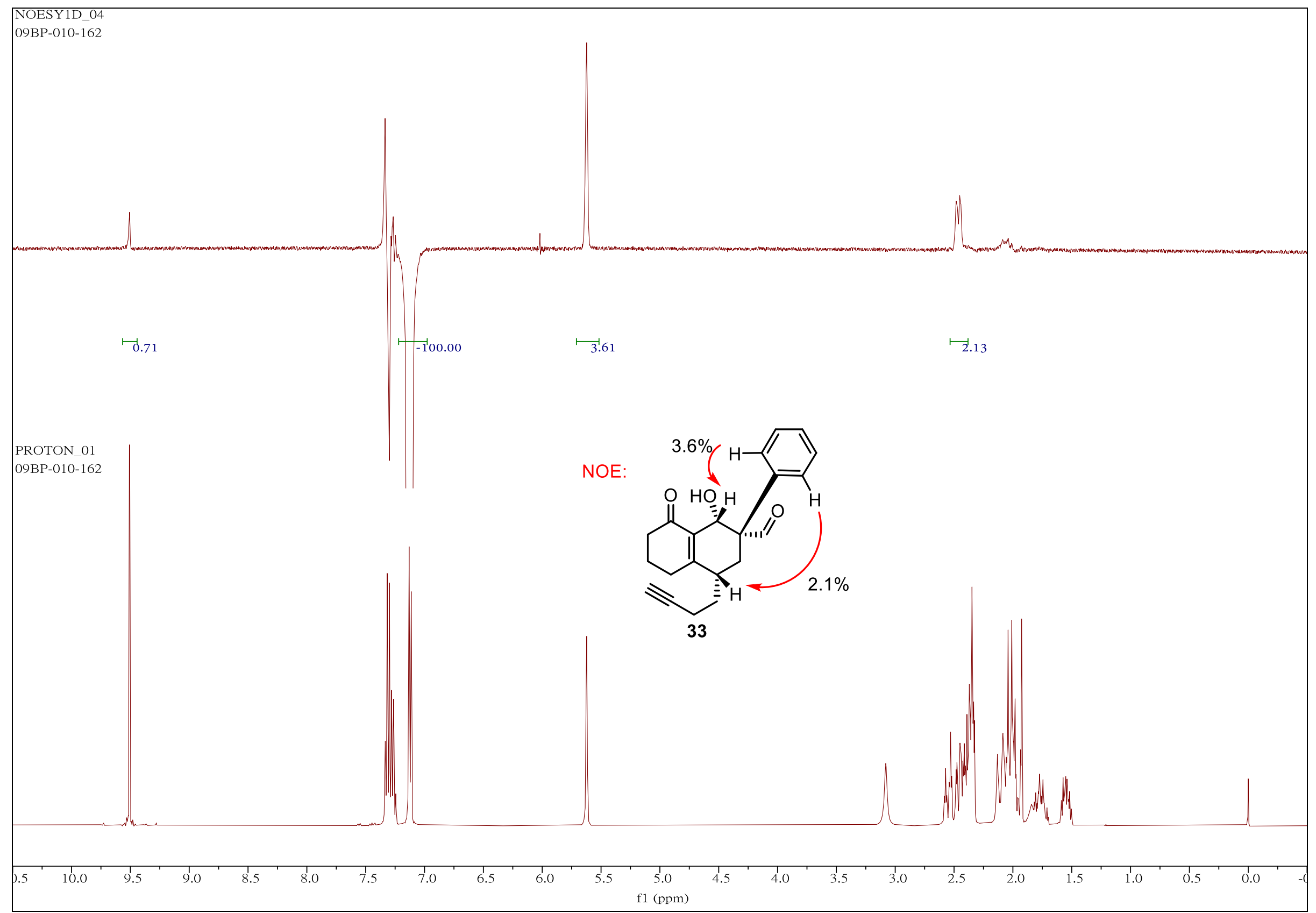

1D NOESY spectra for compound $\mathbf{3 3}$ 


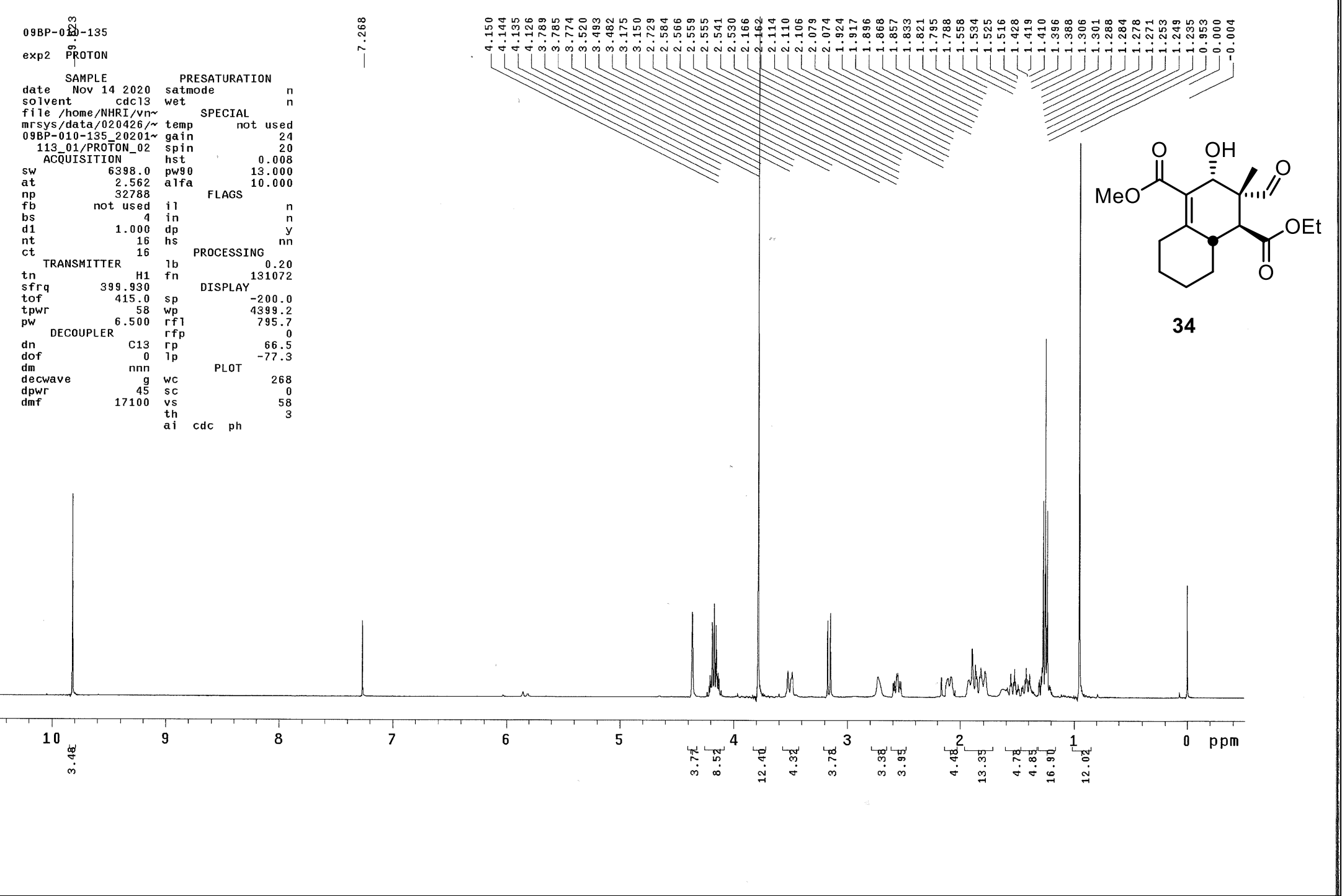

${ }^{1} \mathrm{H}$ NMR spectrum for compound 34 


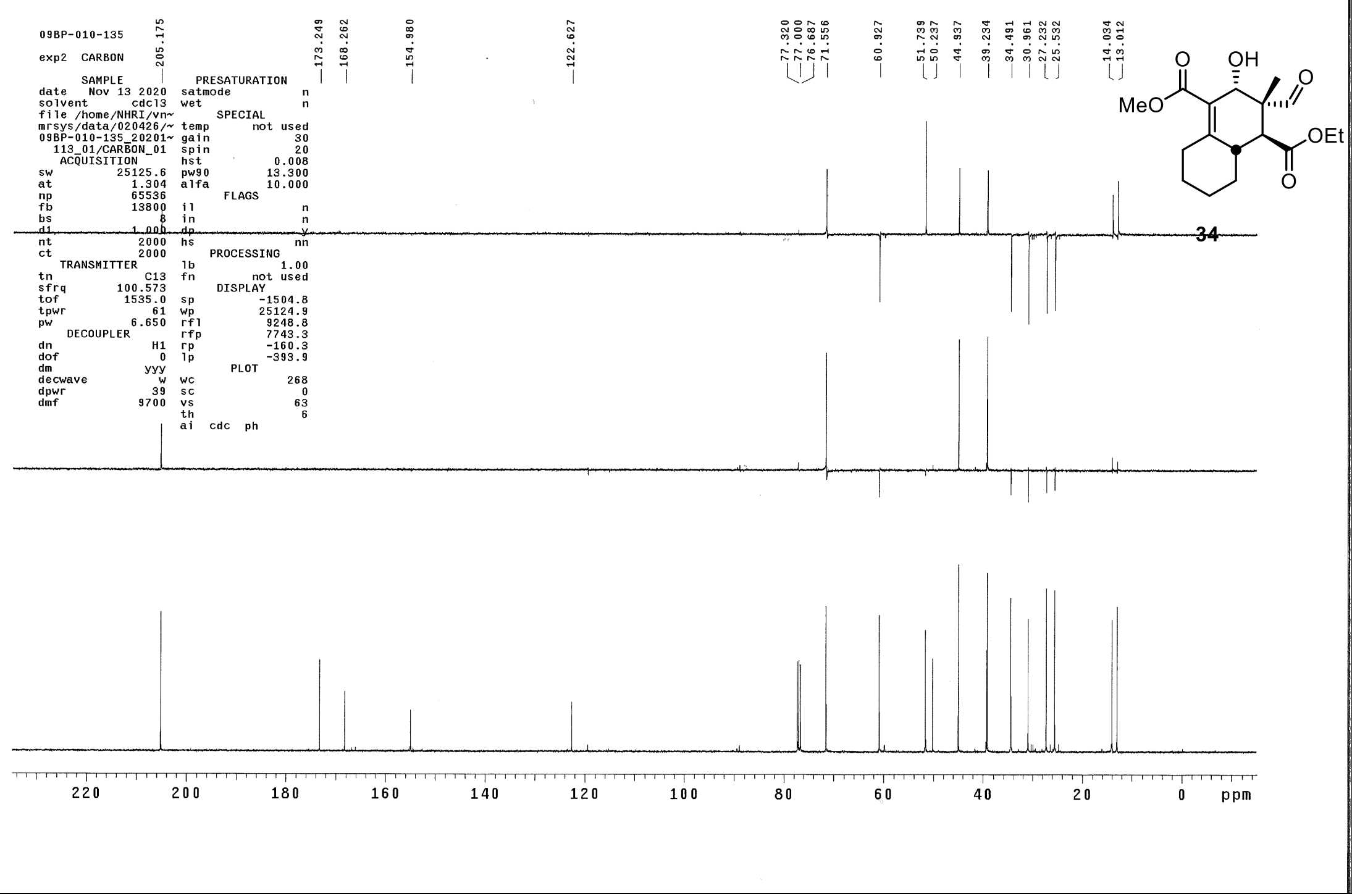

${ }^{13} \mathrm{C}$ NMR + DEPT spectra for compound $\mathbf{3 4}$ 


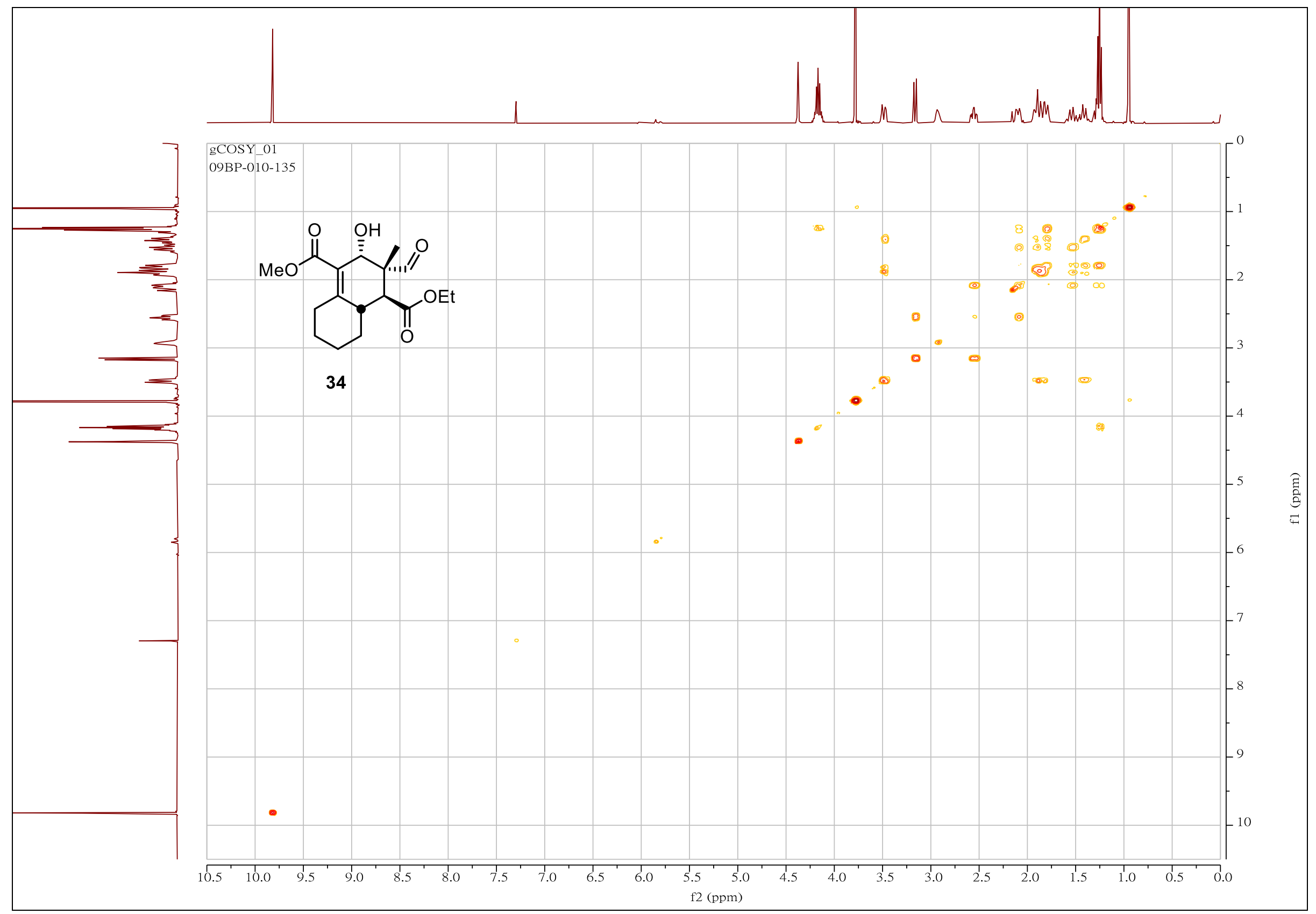

${ }^{1} \mathrm{H}-{ }^{1} \mathrm{H}$ COSY spectrum for compound 34 


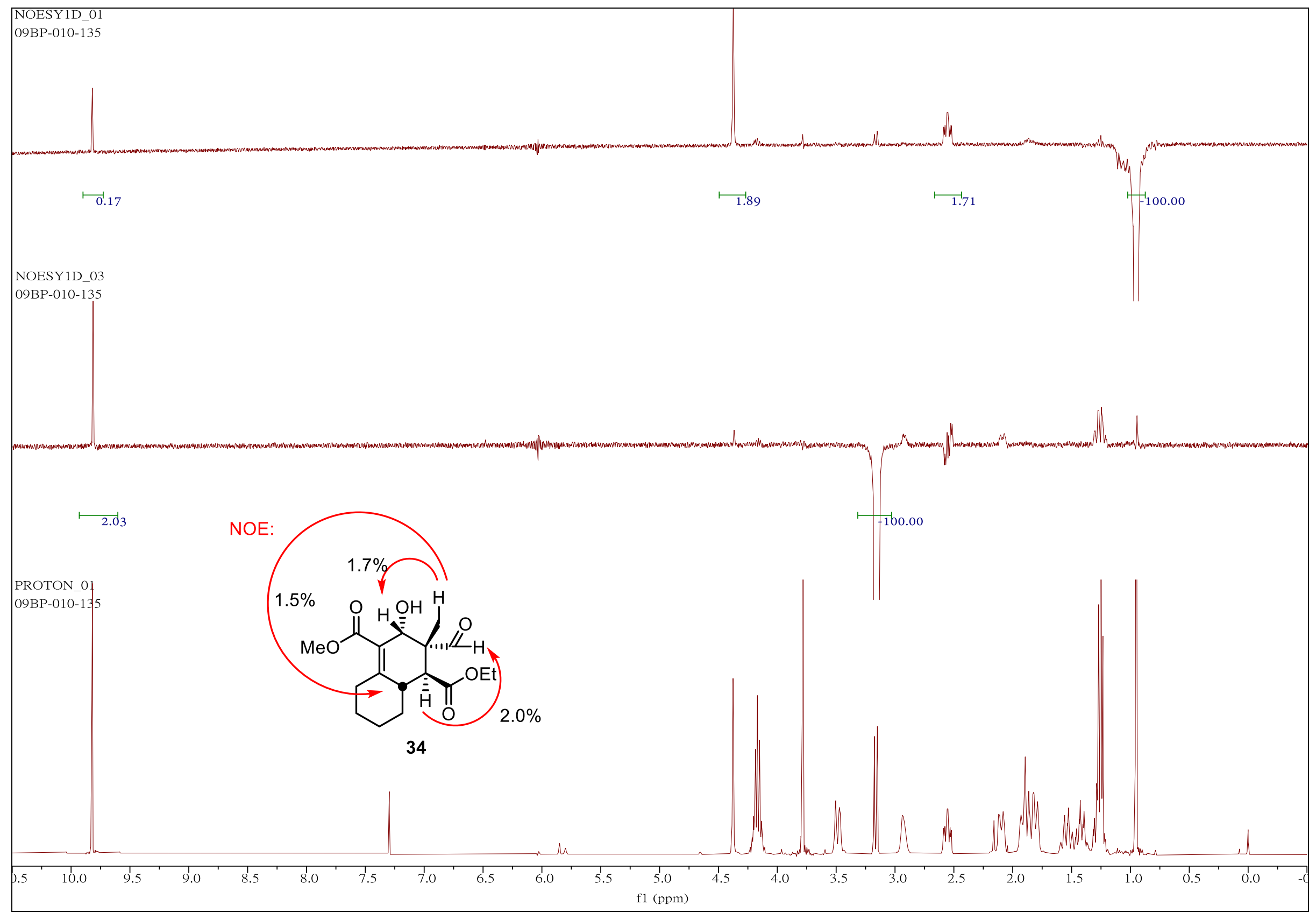

1D NOESY spectra for compound $\mathbf{3 4}$ 


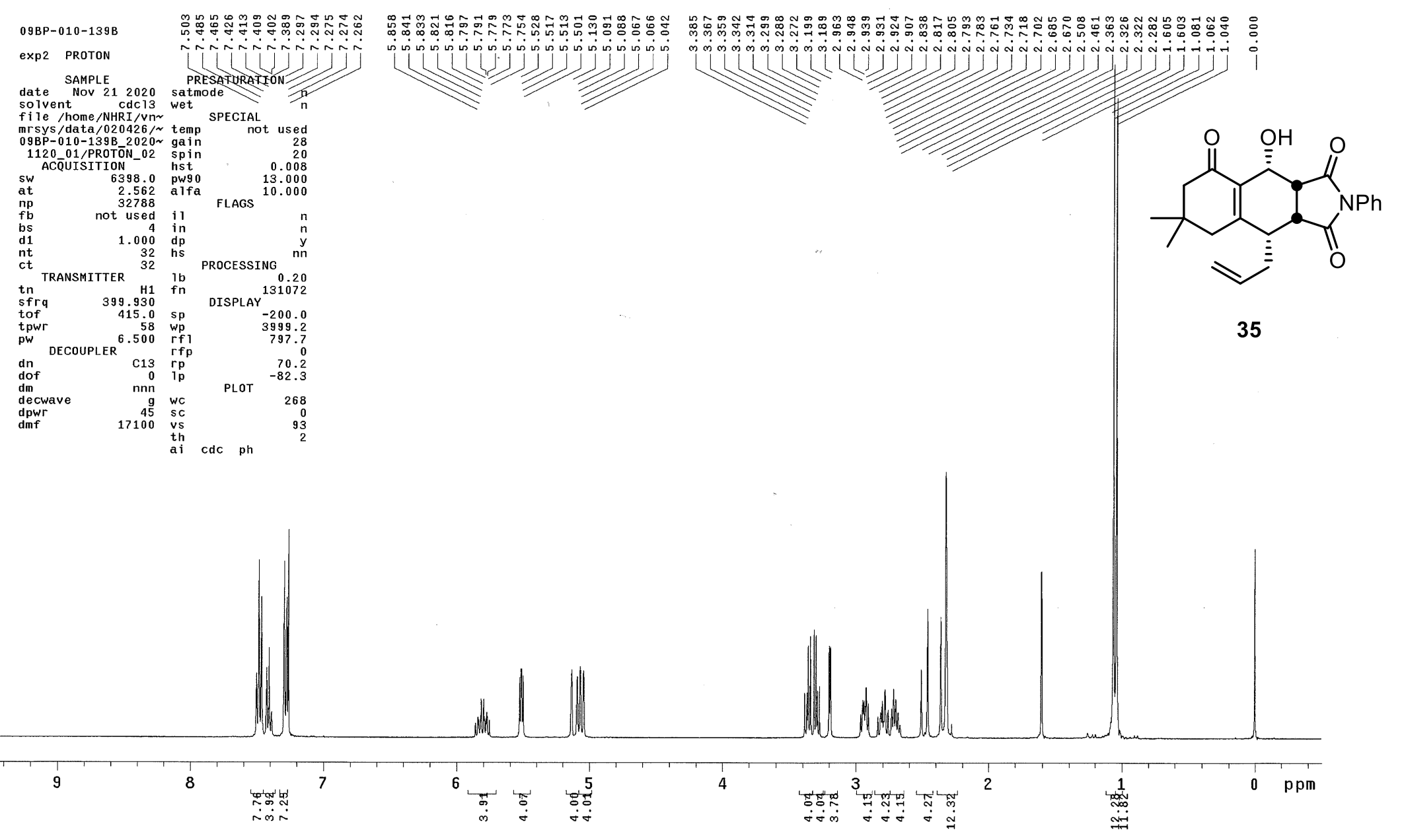

${ }^{1} \mathrm{H}$ NMR spectrum for compound $\mathbf{3 5}$ 


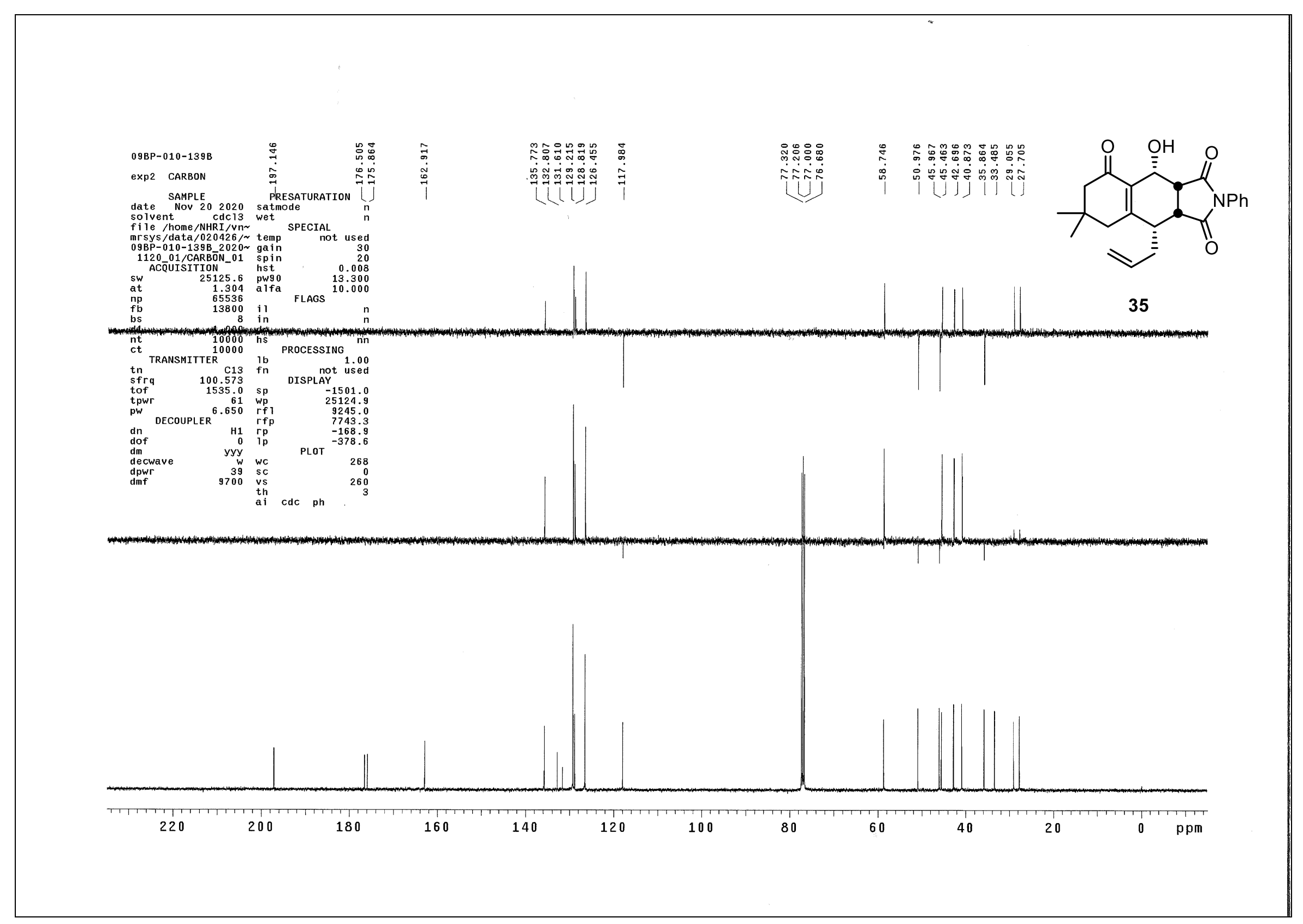

${ }^{13} \mathrm{C}$ NMR + DEPT spectra for compound 35 


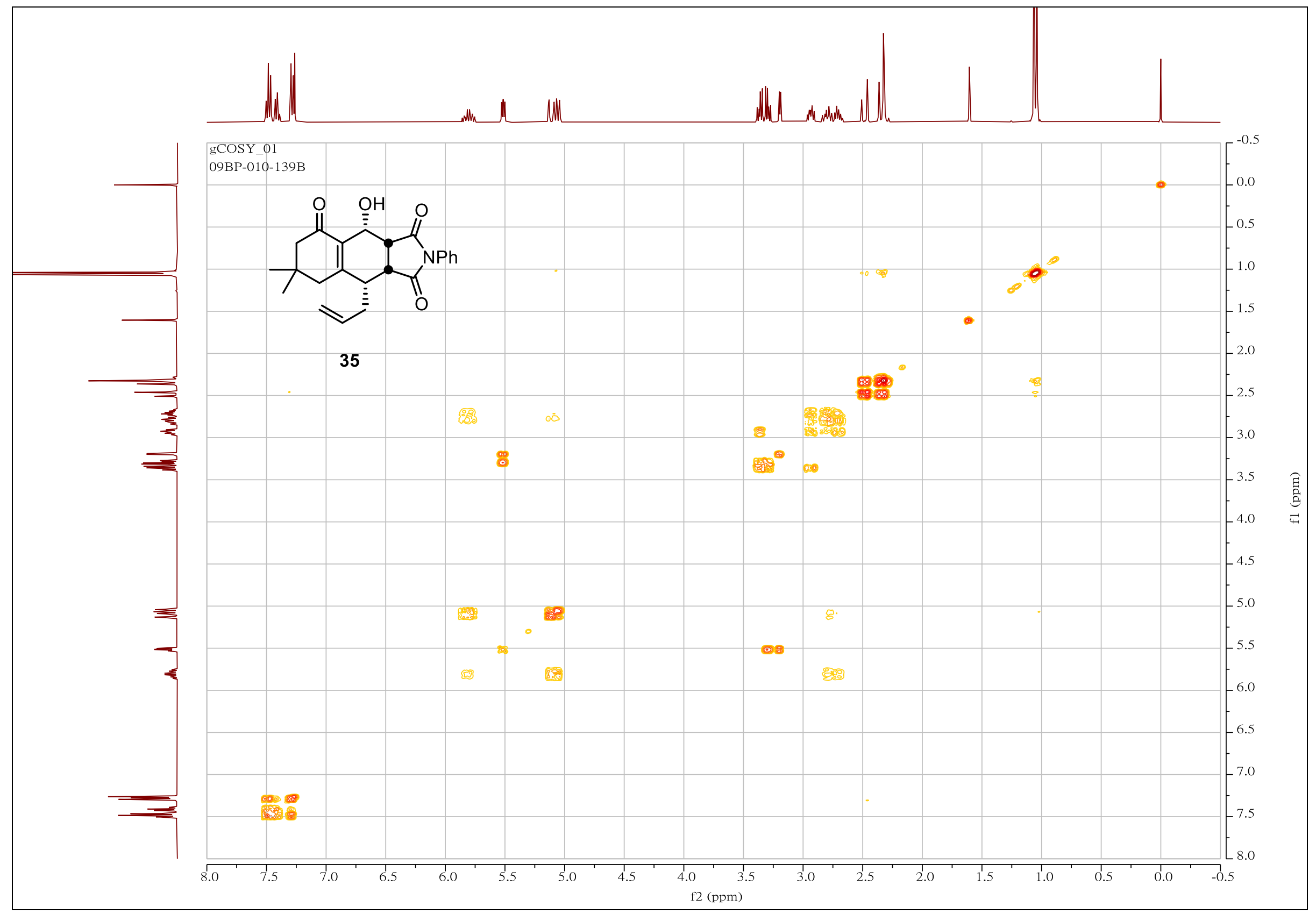

${ }^{1} \mathrm{H}-{ }^{1} \mathrm{H}$ COSY spectrum for compound 35 


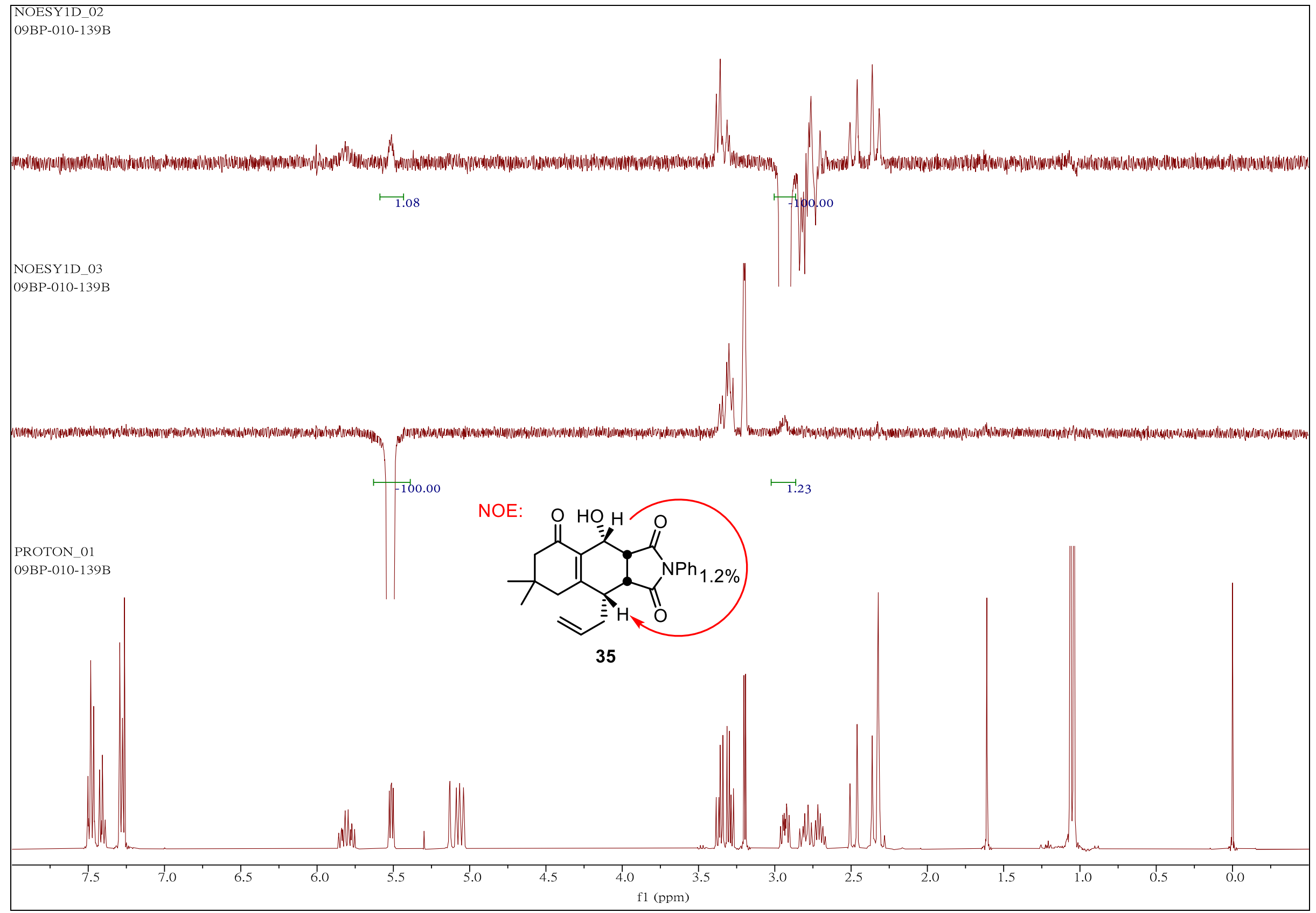

1D NOESY spectra for compound $\mathbf{3 5}$ 


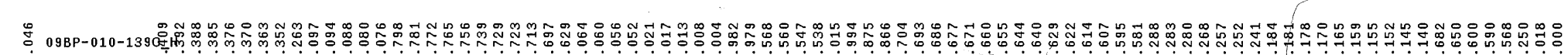

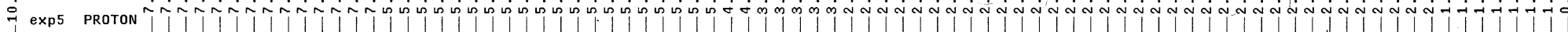

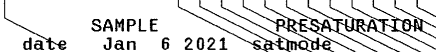

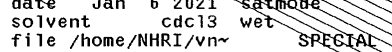

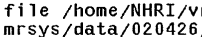

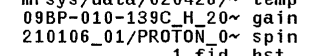

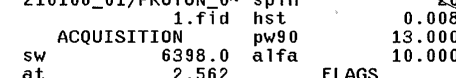

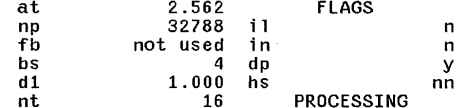

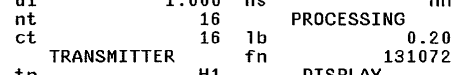

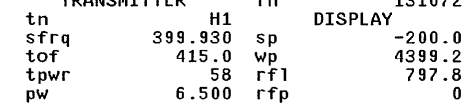

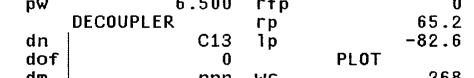

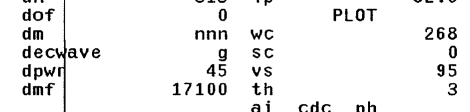

$\begin{array}{llll}17100 & \text { th } \\ \text { ai } & \text { cdc ph } & & \\ & & & \end{array}$
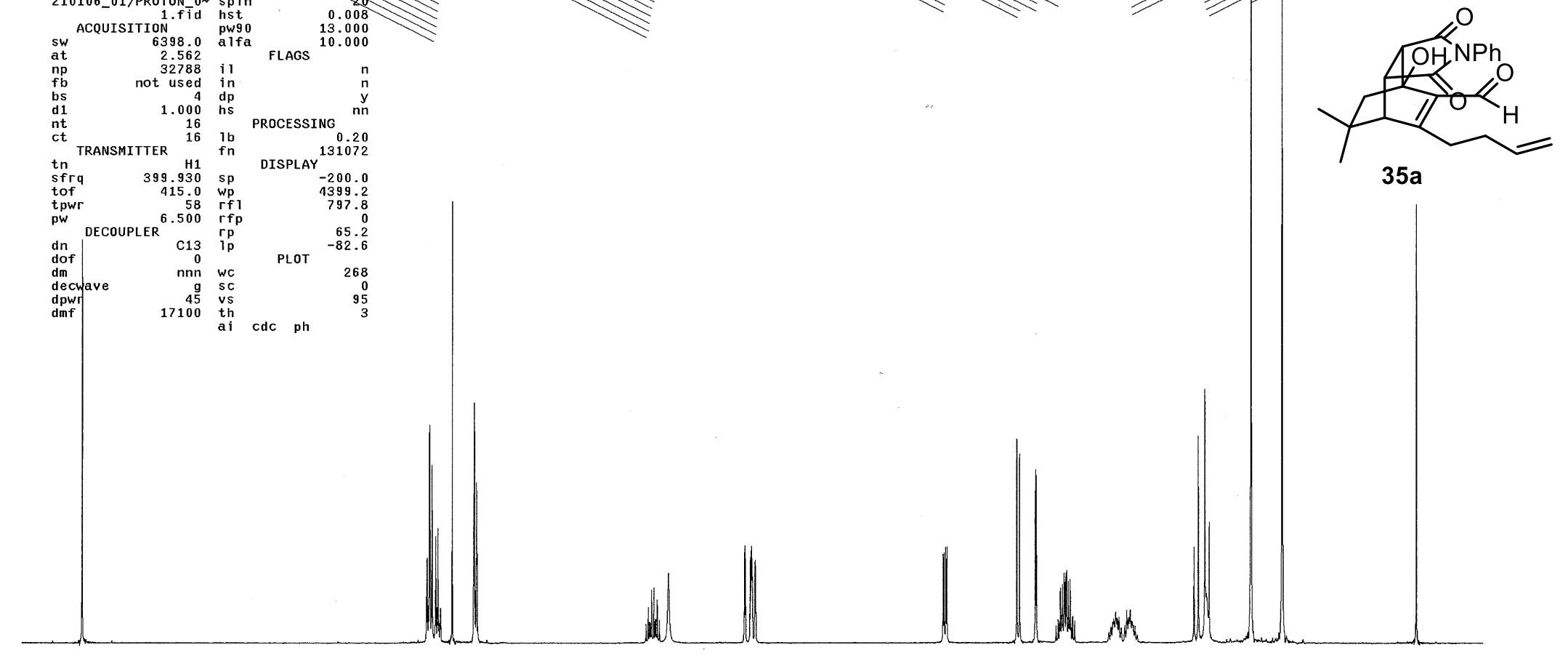

$35 a$

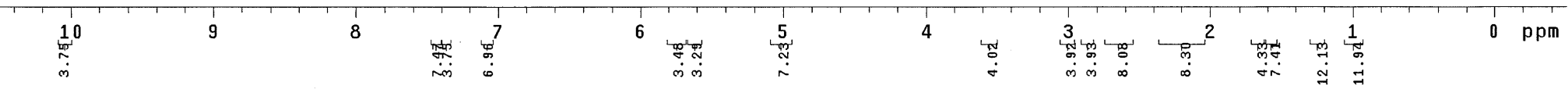

${ }^{1} \mathrm{H}$ NMR spectrum for compound $\mathbf{3 5 a}$ 

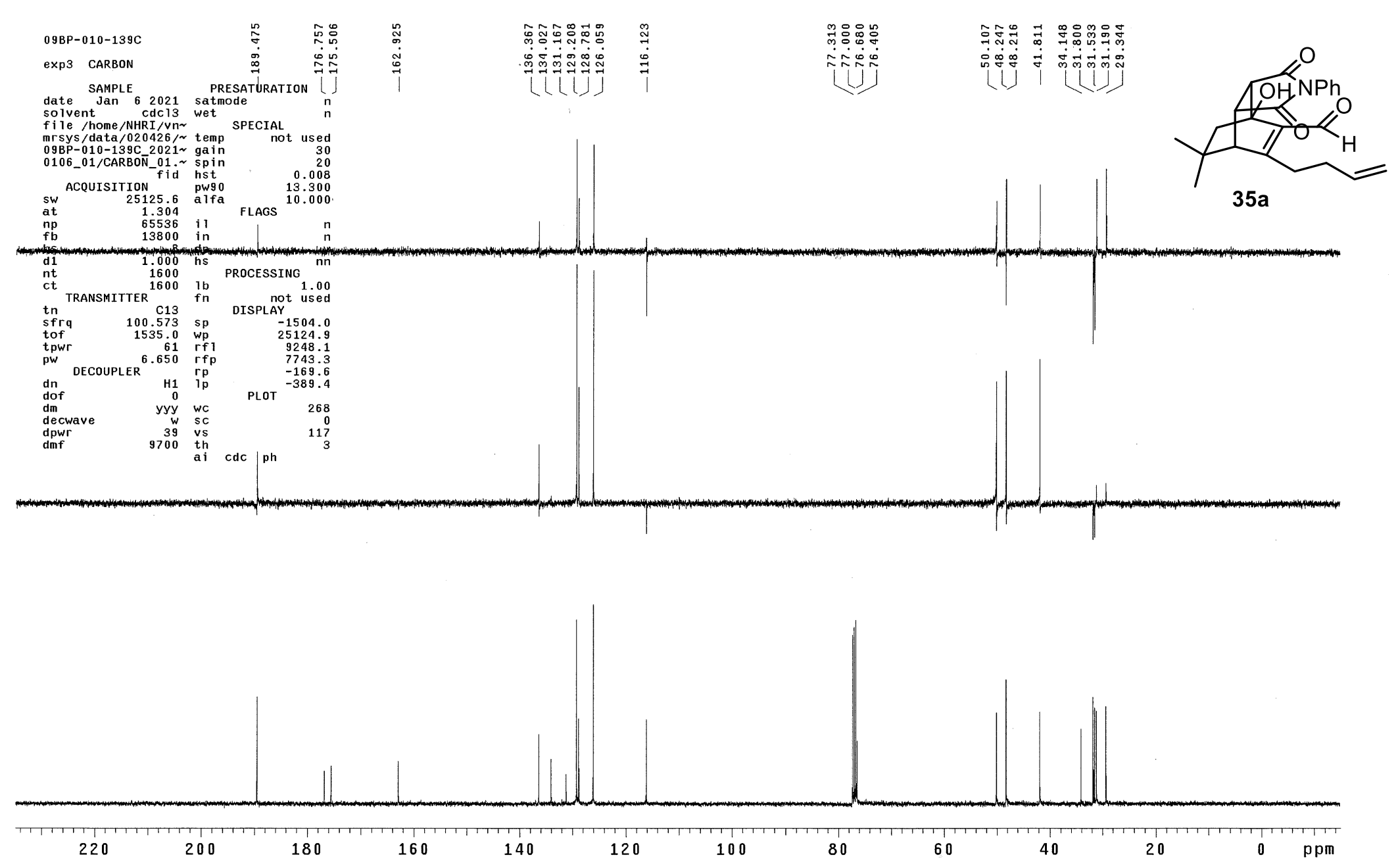

${ }^{13} \mathrm{C}$ NMR + DEPT spectra for compound $\mathbf{3 5 a}$ 


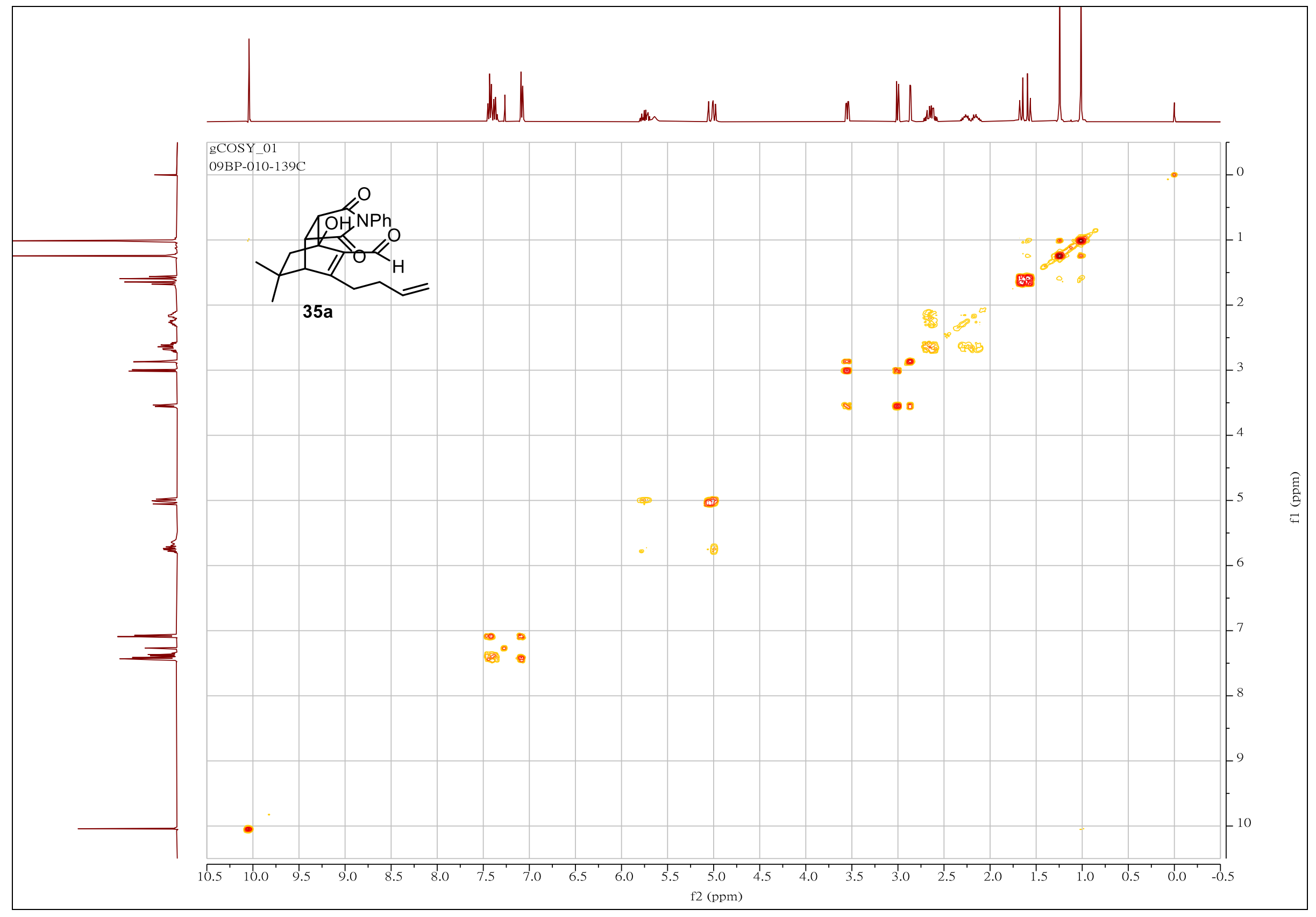

${ }^{1} \mathrm{H}-{ }^{1} \mathrm{H}$ COSY spectrum for compound $\mathbf{3 5 a}$ 


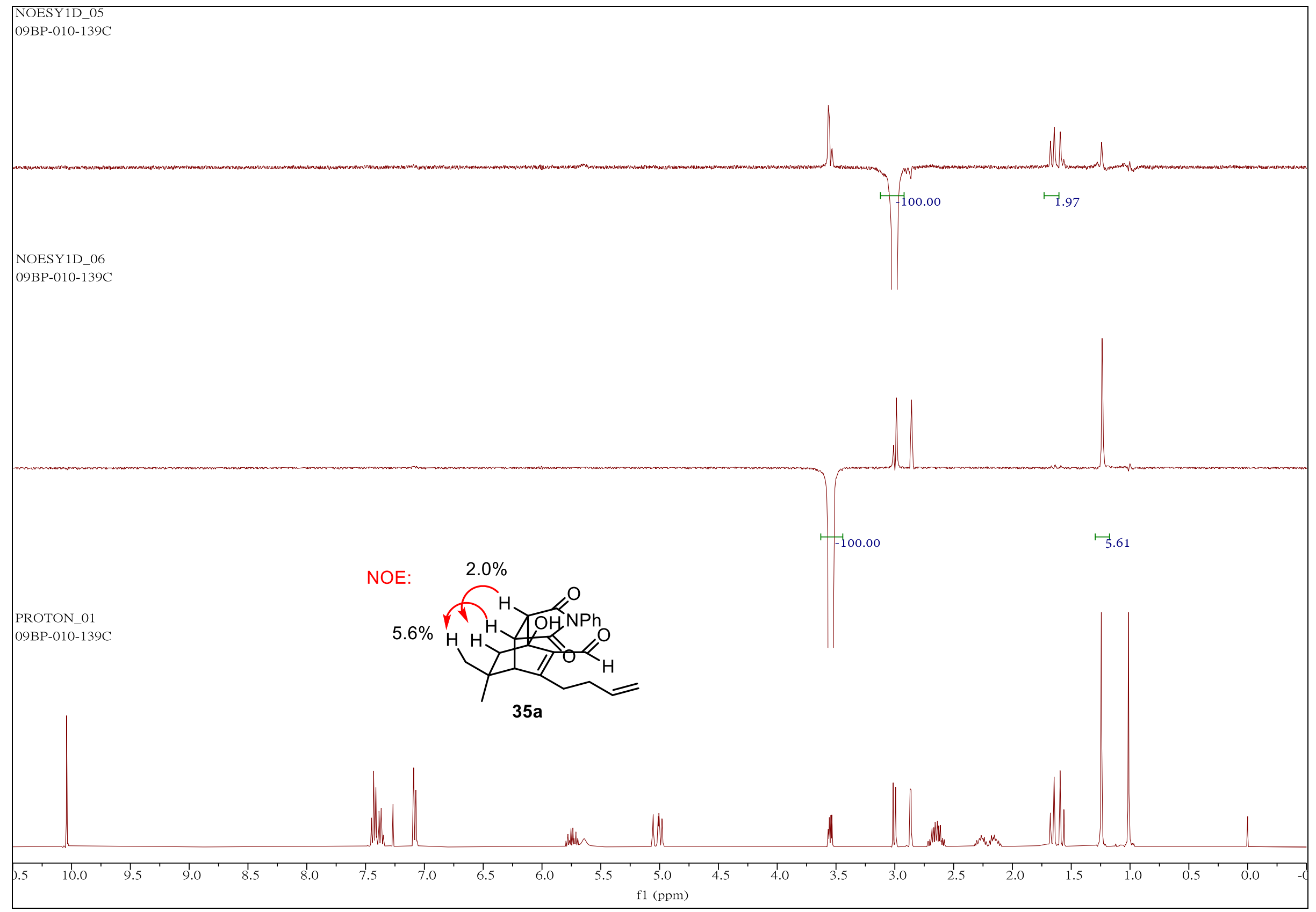

1D NOESY spectra for compound $\mathbf{3 5 a}$ 


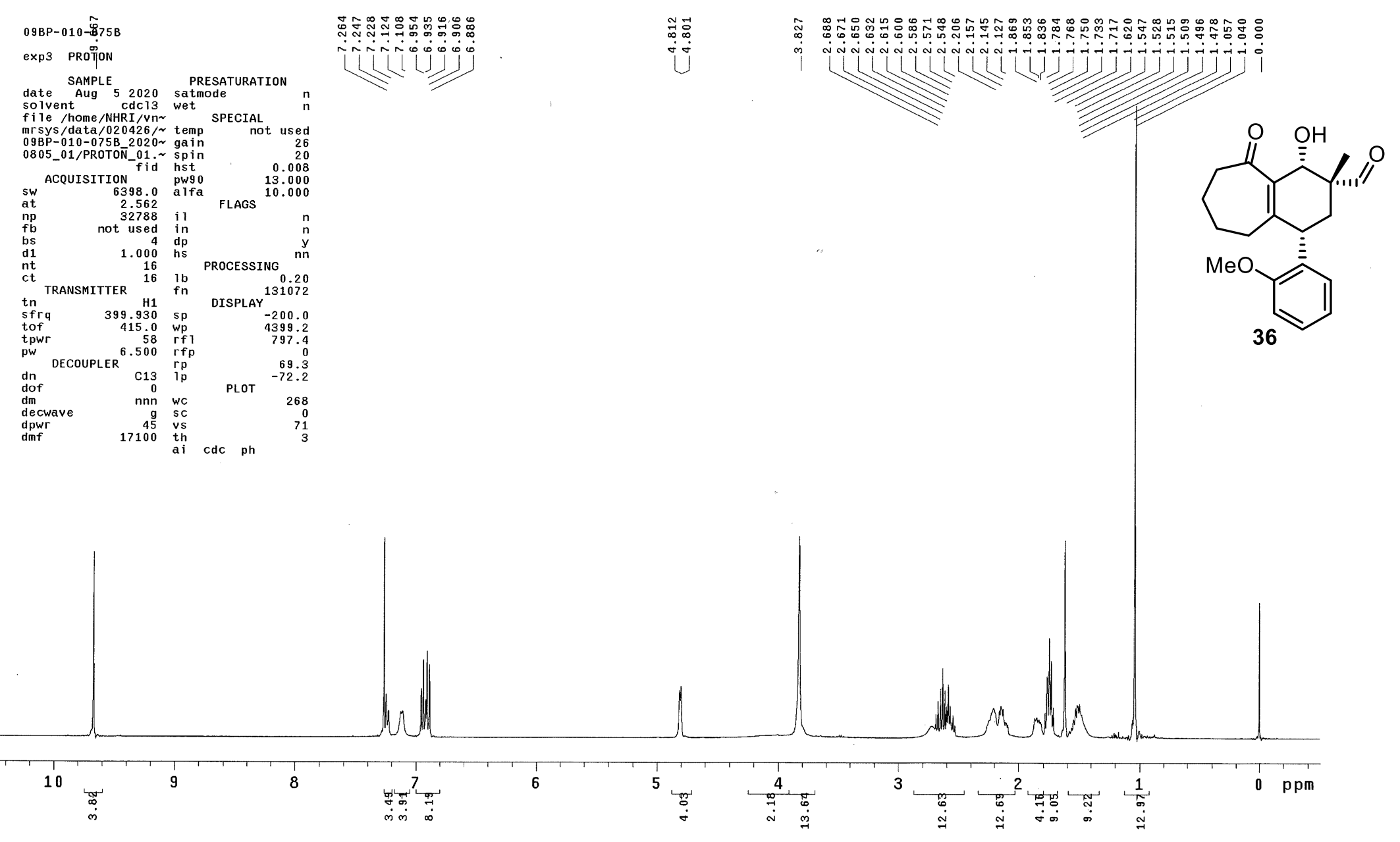

${ }^{1} \mathrm{H}$ NMR spectrum for compound $\mathbf{3 6}$ 


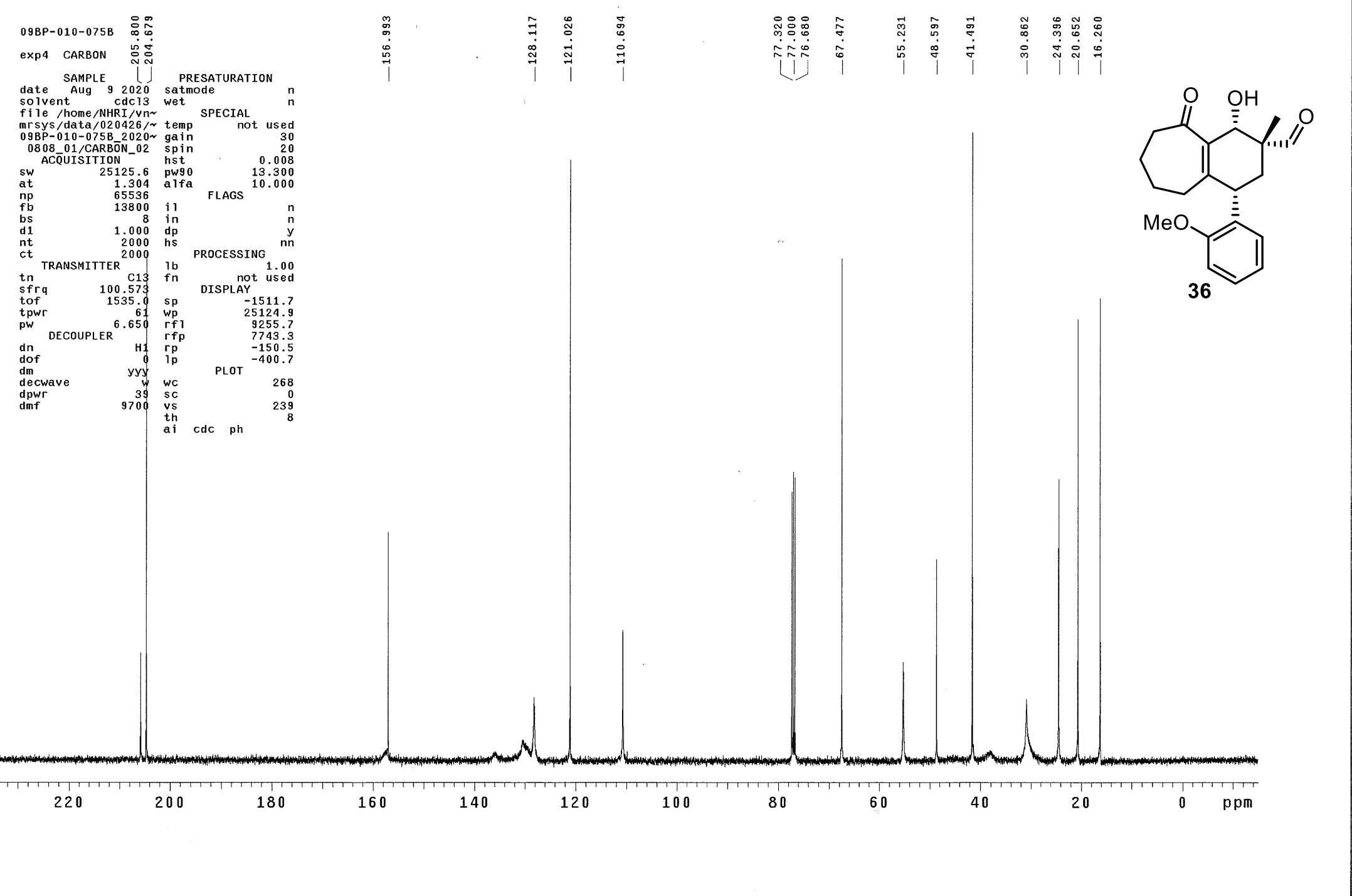

${ }^{13} \mathrm{C}$ NMR spectrum for compound $\mathbf{3 6}$ 


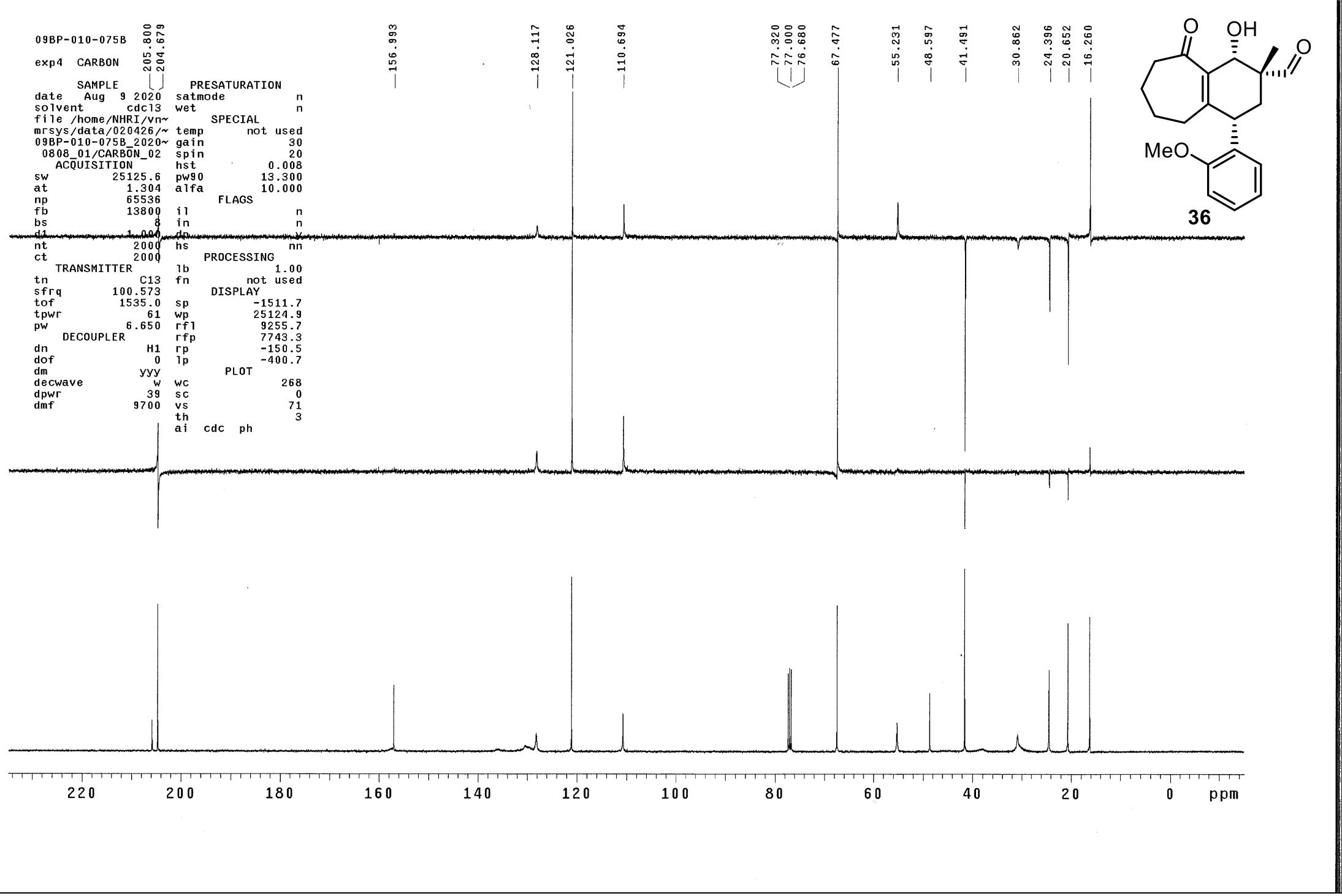

${ }^{13} \mathrm{C}$ NMR + DEPT spectra for compound $\mathbf{3 6}$ 


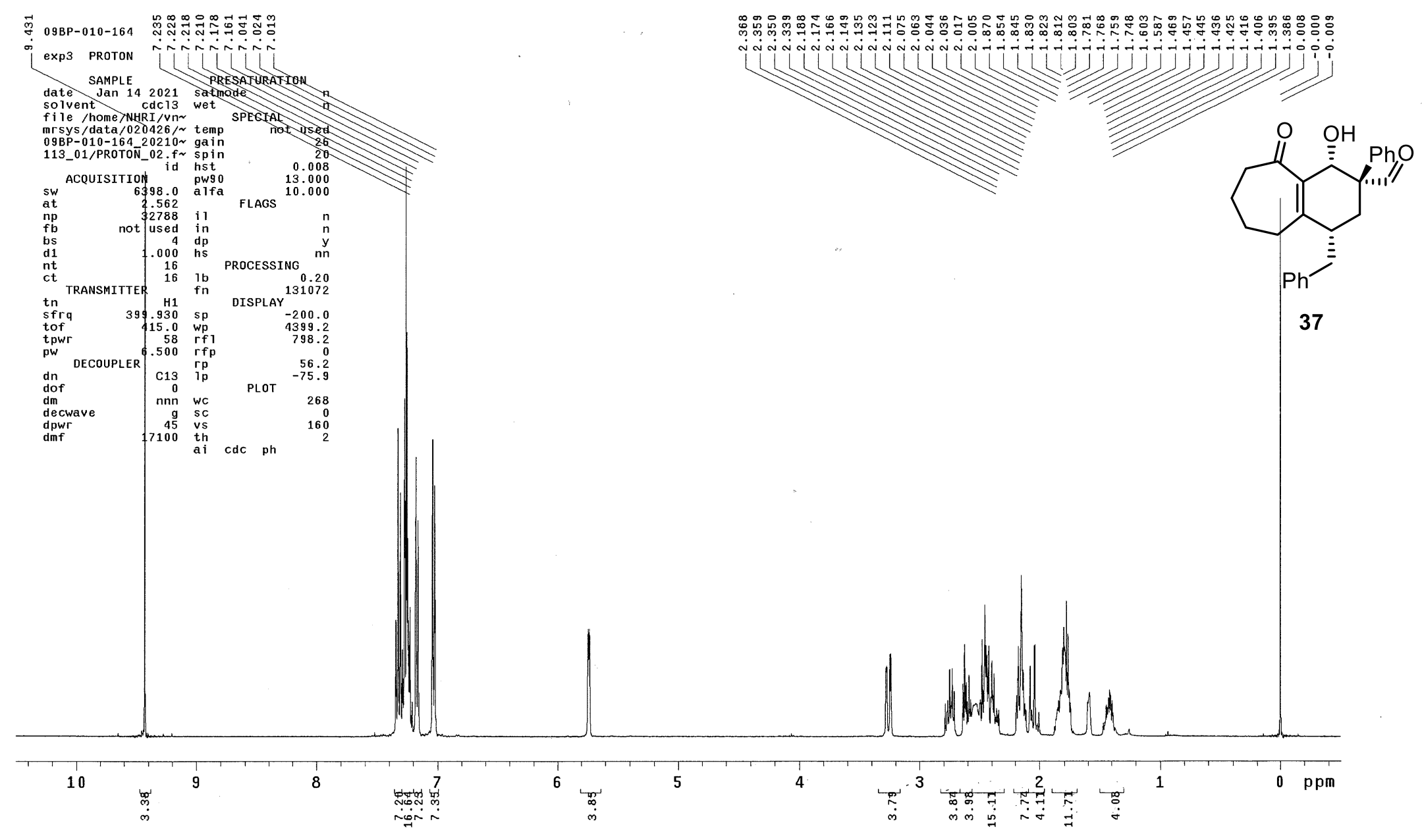

${ }^{1} \mathrm{H}$ NMR spectrum for compound $\mathbf{3 7}$ 


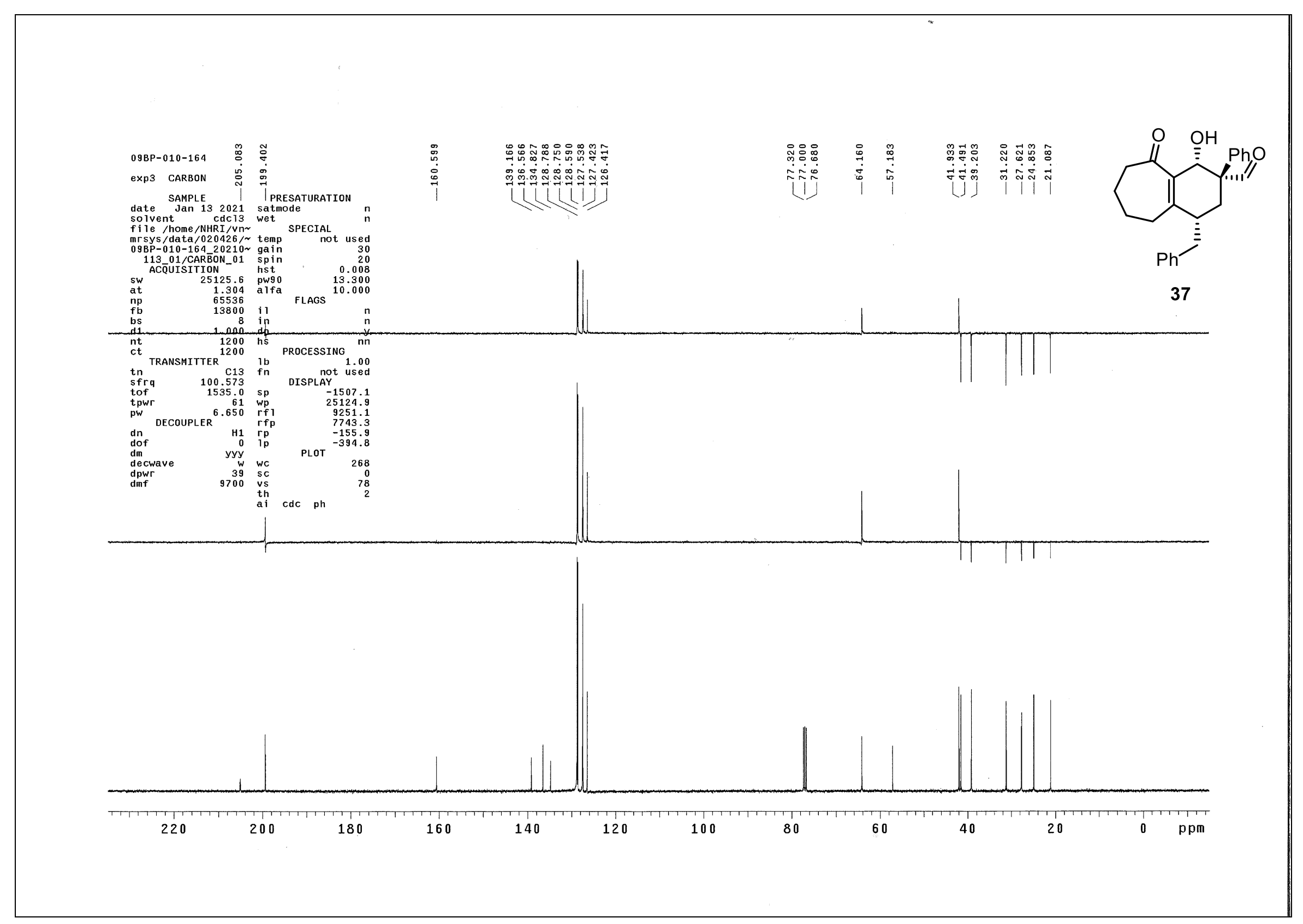

${ }^{13} \mathrm{C}$ NMR + DEPT spectra for compound $\mathbf{3 7}$ 


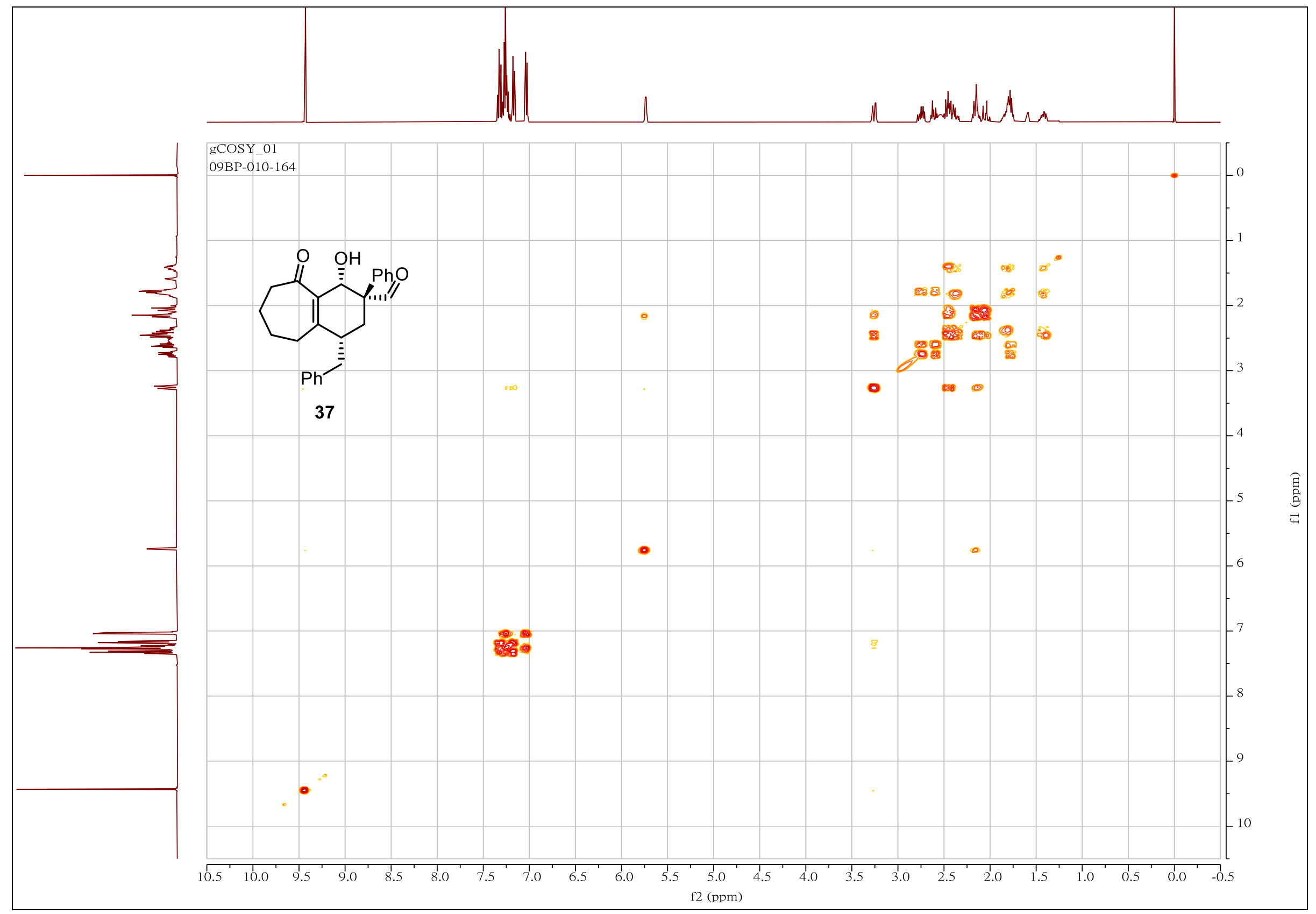

${ }^{1} \mathrm{H}-{ }^{1} \mathrm{H}$ COSY spectrum for compound 37 


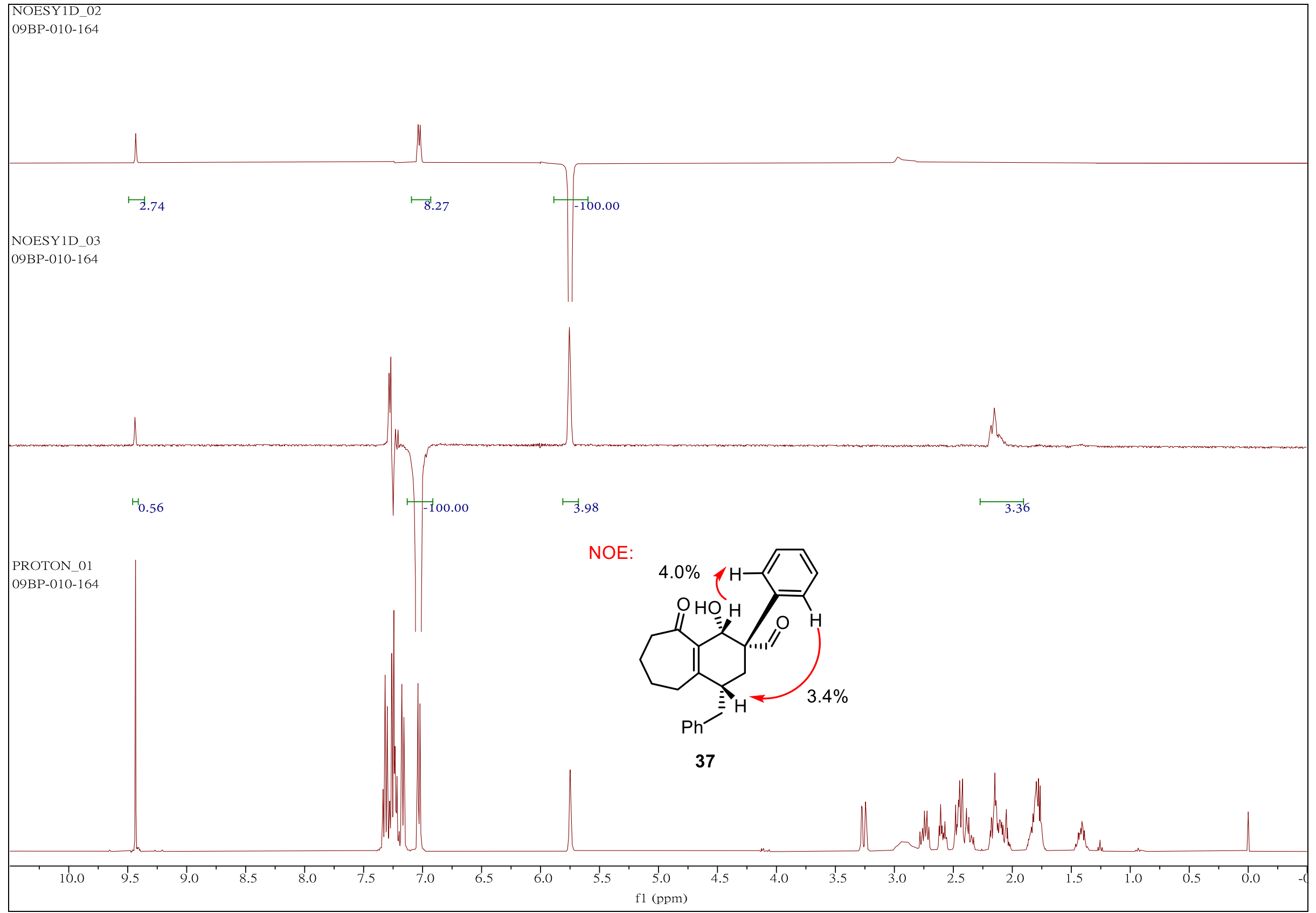

1D NOESY spectra for compound 37 
080608-09BP-010-186.1.fid 09BP-010-186

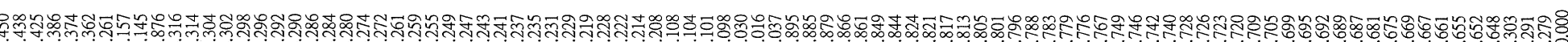

\begin{tabular}{|lll|}
\hline & \multicolumn{1}{|c|}{ Parameter } & \multicolumn{1}{c|}{ Value } \\
1 & Instrument & Avance \\
2 & Solvent & CDCl3 \\
3 & Temperature & 298.0 \\
4 & Number of Scans & 16 \\
5 & Receiver Gain & 101.0 \\
6 & Relaxation Delay & 1.0000 \\
7 & Pulse Width & 10.0000 \\
8 & Presaturation Frequency \\
9 & Spectrometer Frequency 600.14 \\
10 & Spectral Width & 11904.8 \\
11 & Lowest Frequency & -2259.5 \\
12 & Nucleus & $1 \mathrm{H}$ \\
13 & Acquired Size & 32768 \\
14 & Spectral Size & 131072. \\
15 & Digital Resolution & 0.09 \\
\hline
\end{tabular}
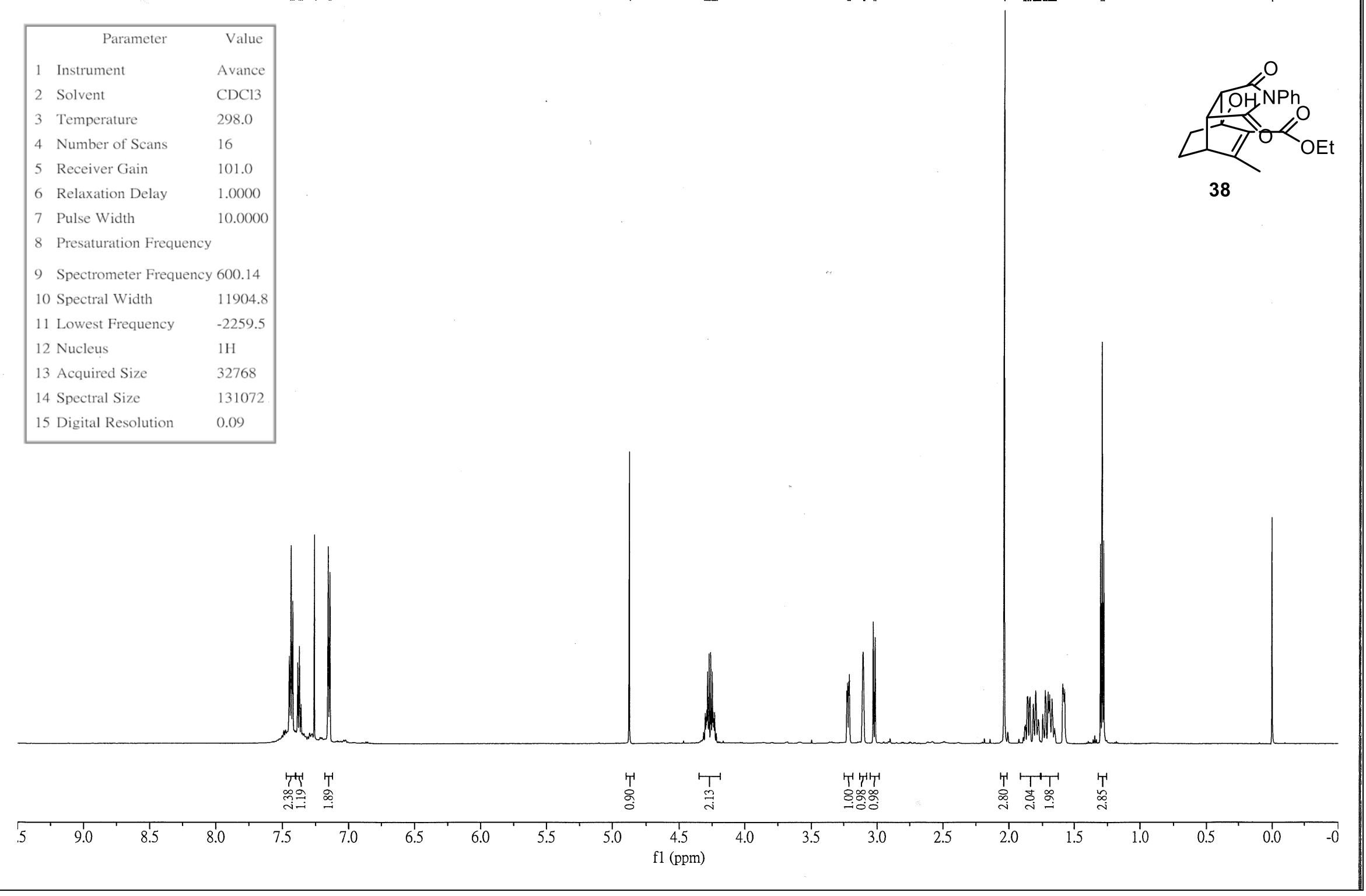

${ }^{1} \mathrm{H}$ NMR spectrum for compound $\mathbf{3 8}$ 


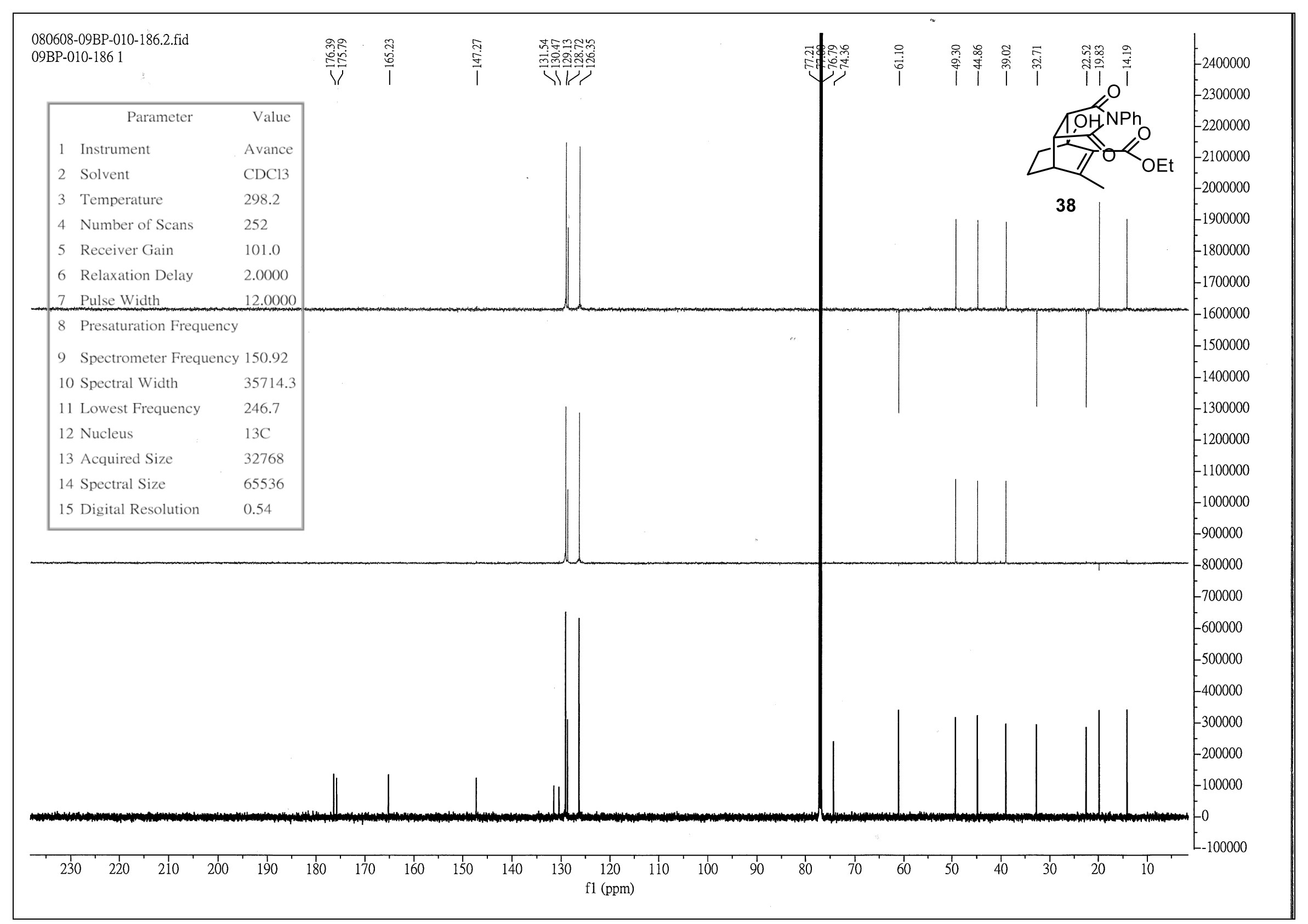

${ }^{13} \mathrm{C}$ NMR + DEPT spectra for compound $\mathbf{3 8}$ 


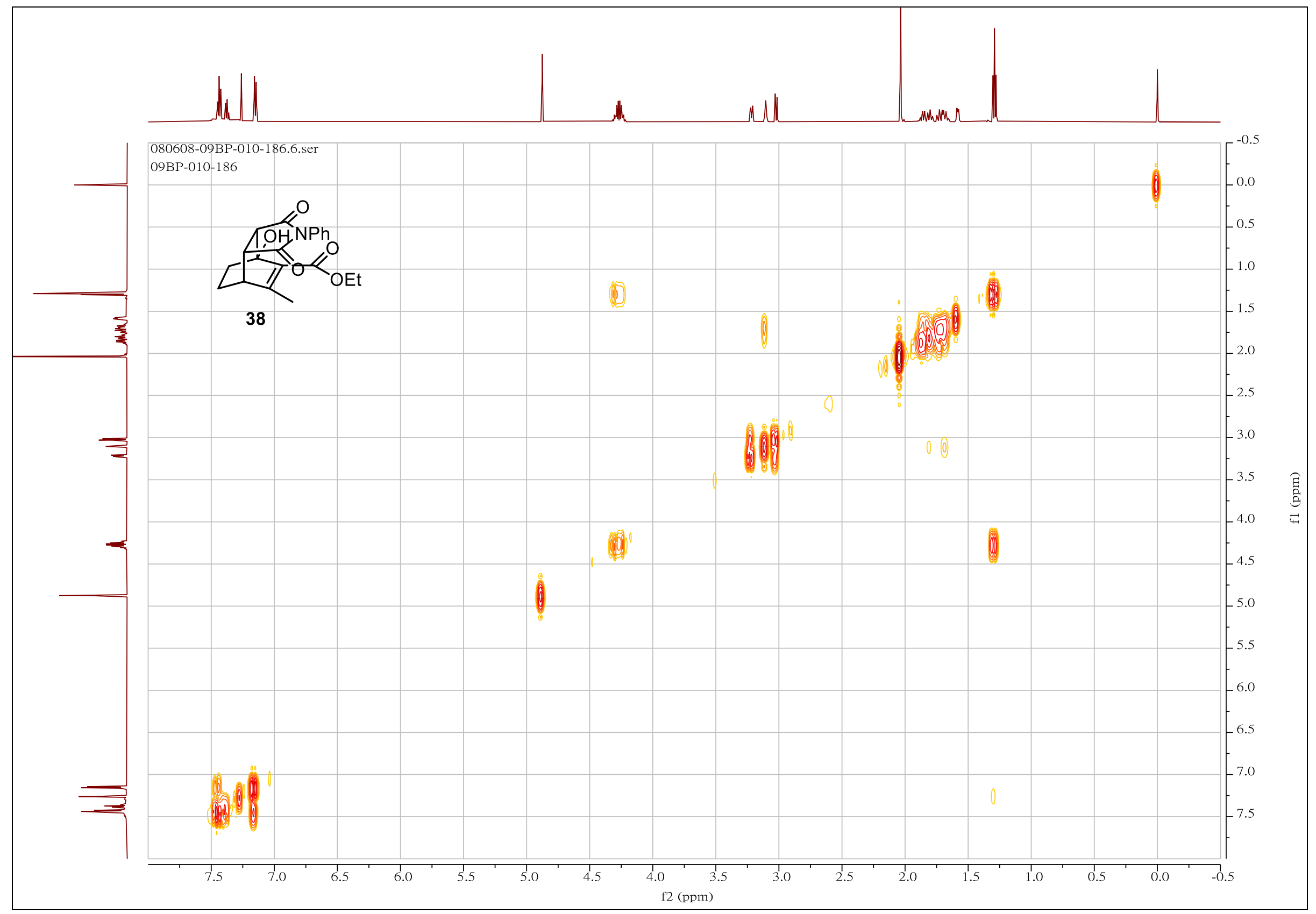

${ }^{1} \mathrm{H}-{ }^{1} \mathrm{H}$ COSY spectrum for compound $\mathbf{3 8}$ 


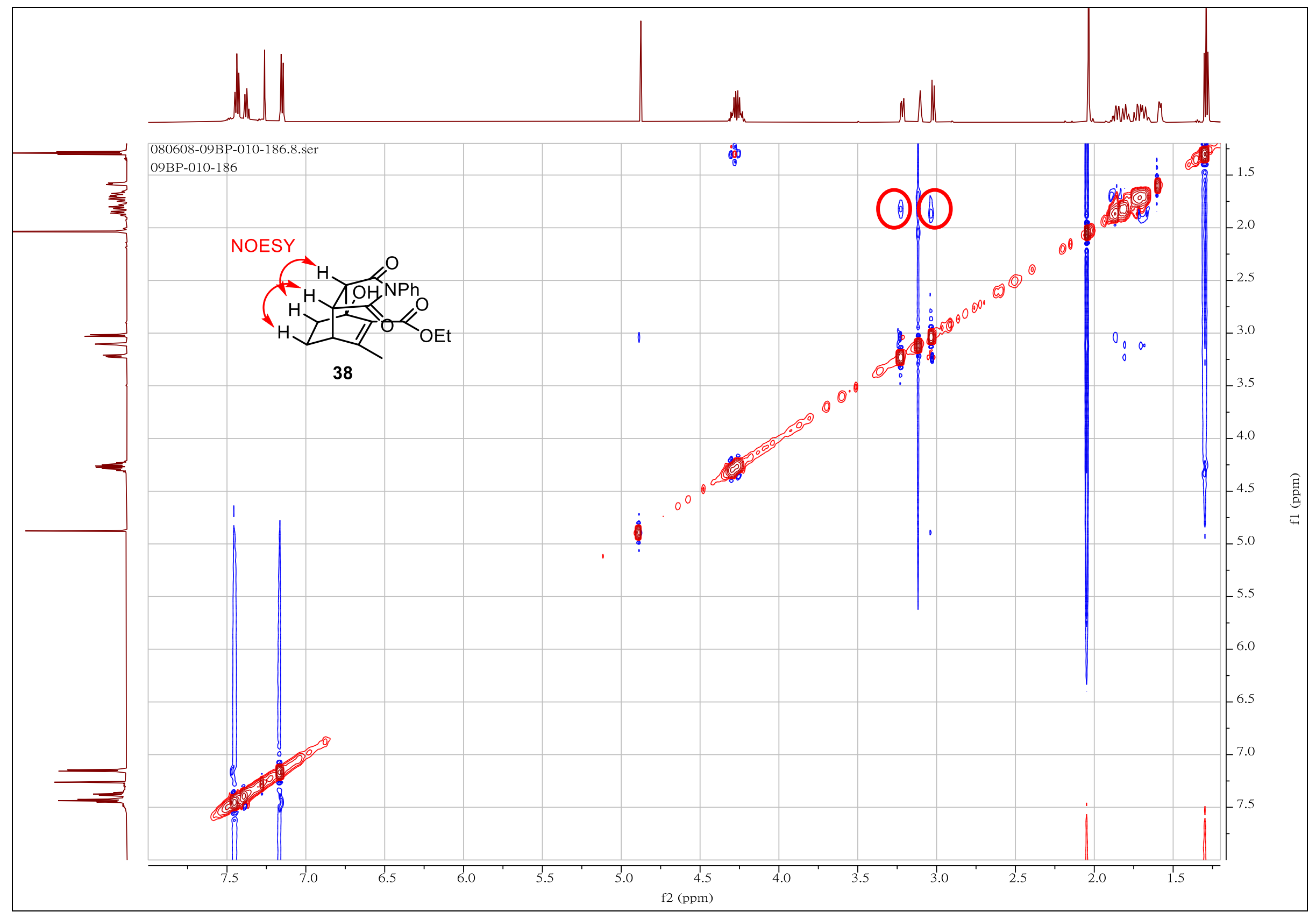

2D NOESY spectrum for compound $\mathbf{3 8}$ 


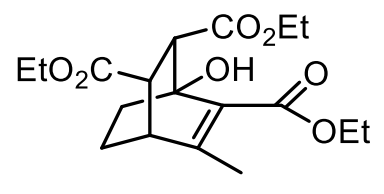

39

\begin{tabular}{|lll|}
\hline & \multicolumn{1}{|c}{ Parameter } & \multicolumn{1}{c|}{ Value } \\
1 & Instrument & Avance \\
2 & Solvent & CDCl3 \\
3 & Temperature & 294.7 \\
4 & Number of Scans & 16 \\
5 & Receiver Gain & 101.0 \\
6 & Relaxation Delay & 1.0000 \\
7 & Pulse Width & 8.0000 \\
8 & Presaturation Frequency \\
9 & Spectrometer Frequency 400.17 \\
10 & Spectral Width & 7812.5 \\
11 & Lowest Frequency & -1442.4 \\
12 & Nucleus & $1 \mathrm{H}$ \\
13 & Acquired Size & 32768 \\
14 & Spectral Size & 65536 \\
15 & Digital Resolution & 0.12 \\
\hline
\end{tabular}
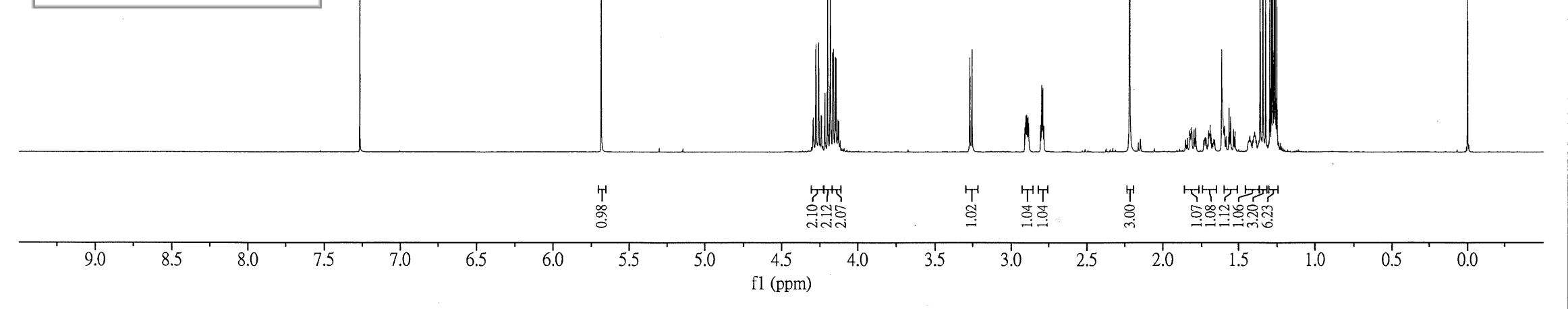

${ }^{1} \mathrm{H}$ NMR spectrum for compound 39 
080608-09BP-010-198.2.fid

09BP-010-198 1

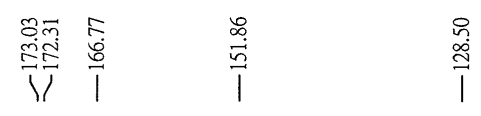

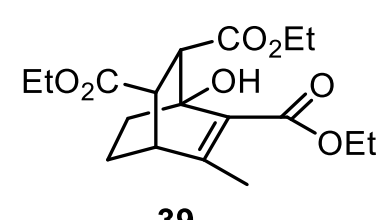

39

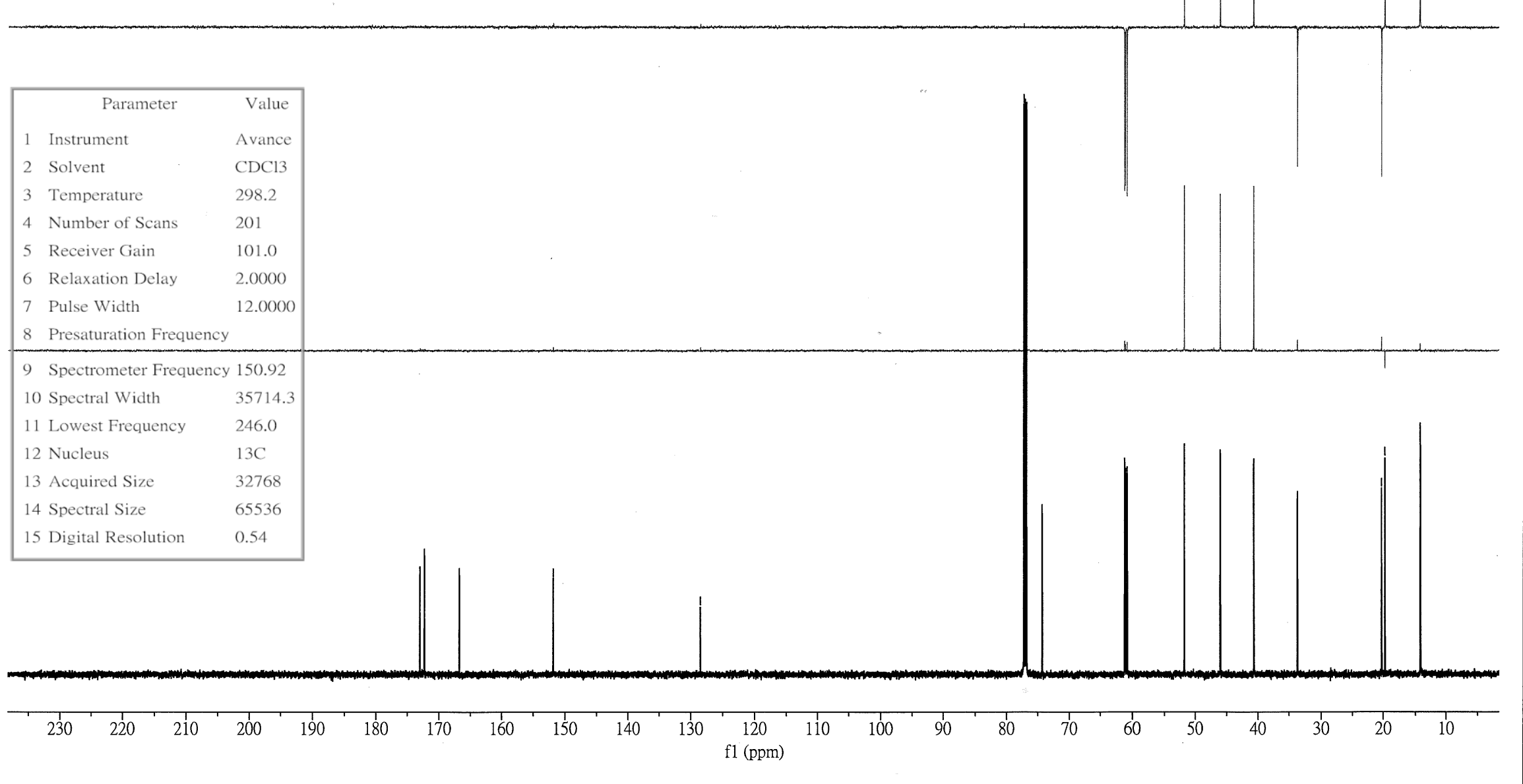

${ }^{13} \mathrm{C}$ NMR + DEPT spectra for compound 39 


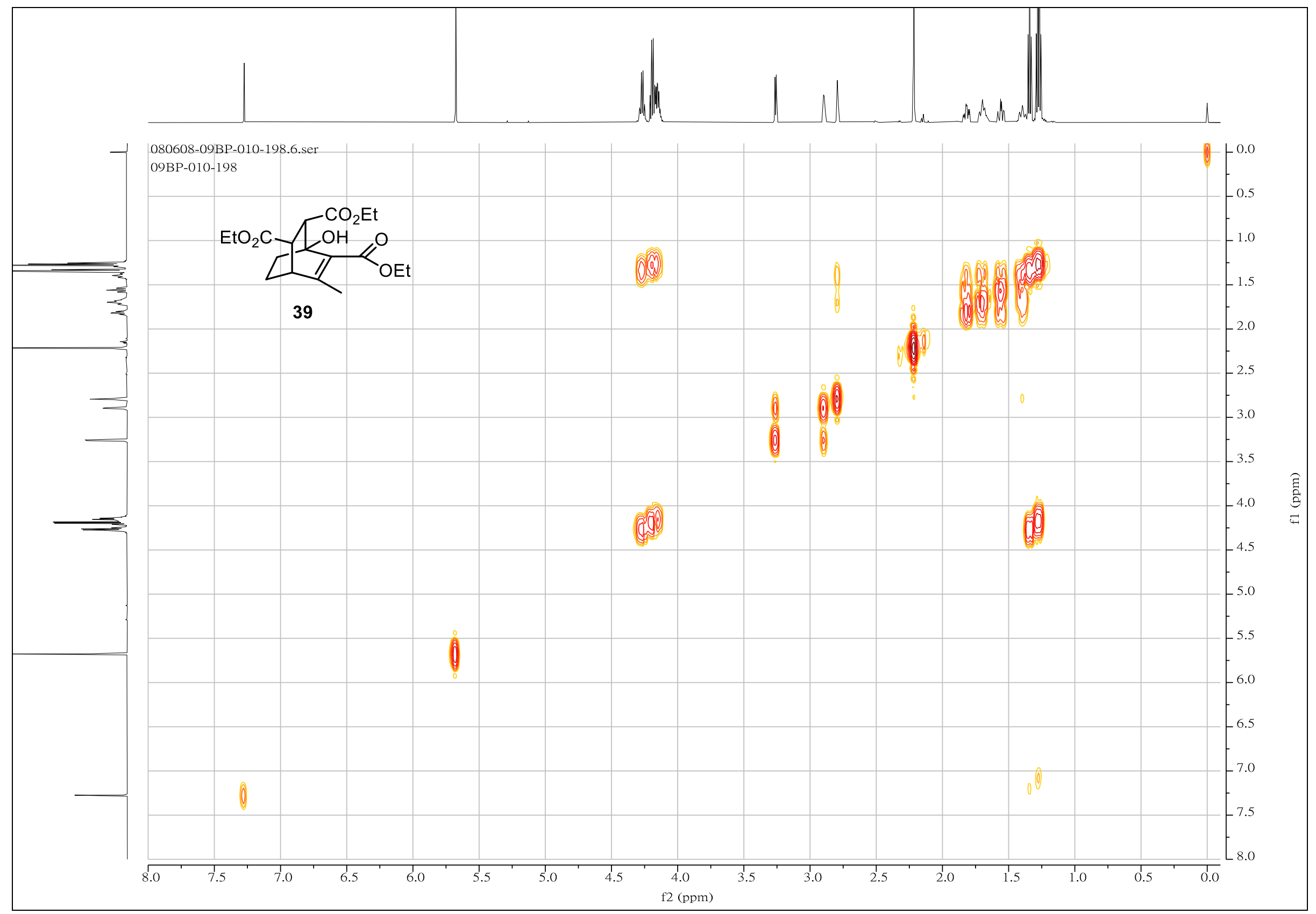

${ }^{1} \mathrm{H}-{ }^{1} \mathrm{H}$ COSY spectrum for compound 39 


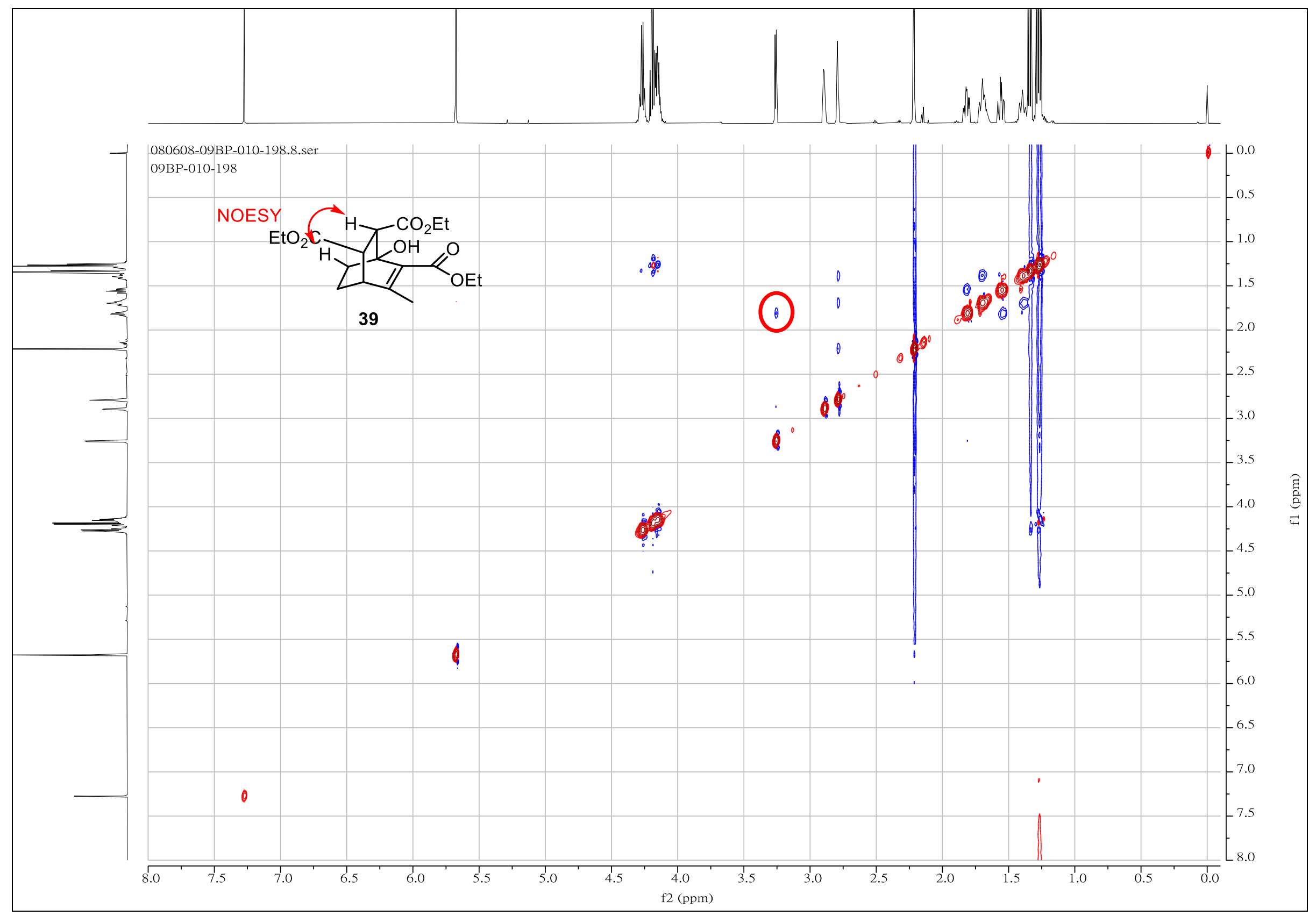

2D NOESY spectrum for compound 39 


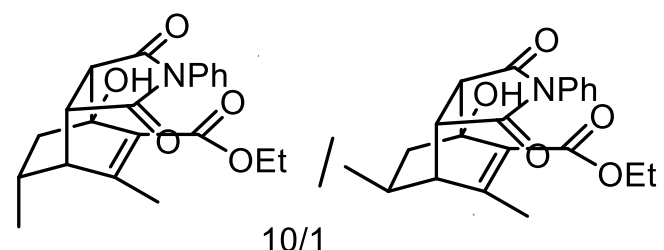

40

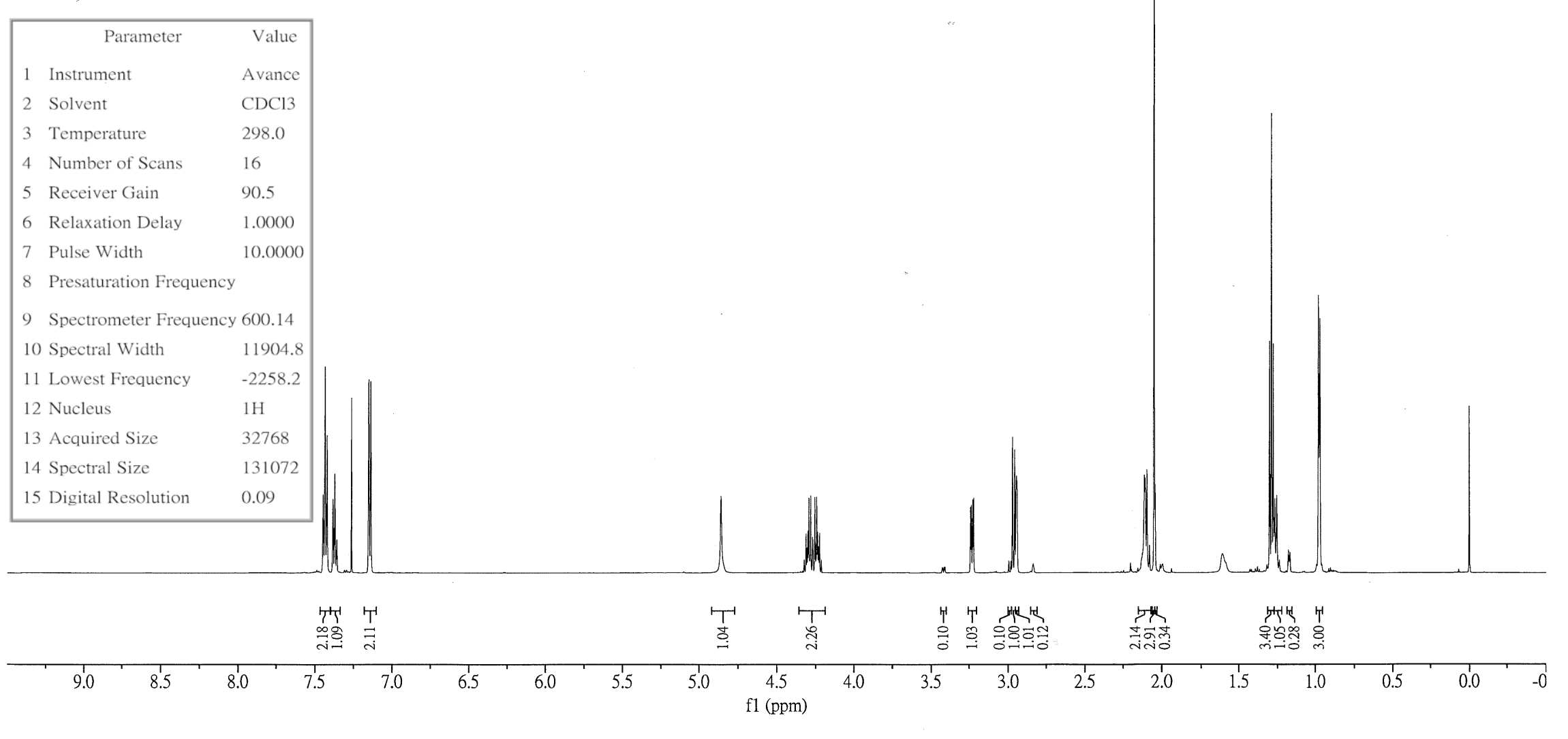

${ }^{1} \mathrm{H}$ NMR spectrum for compound 40 


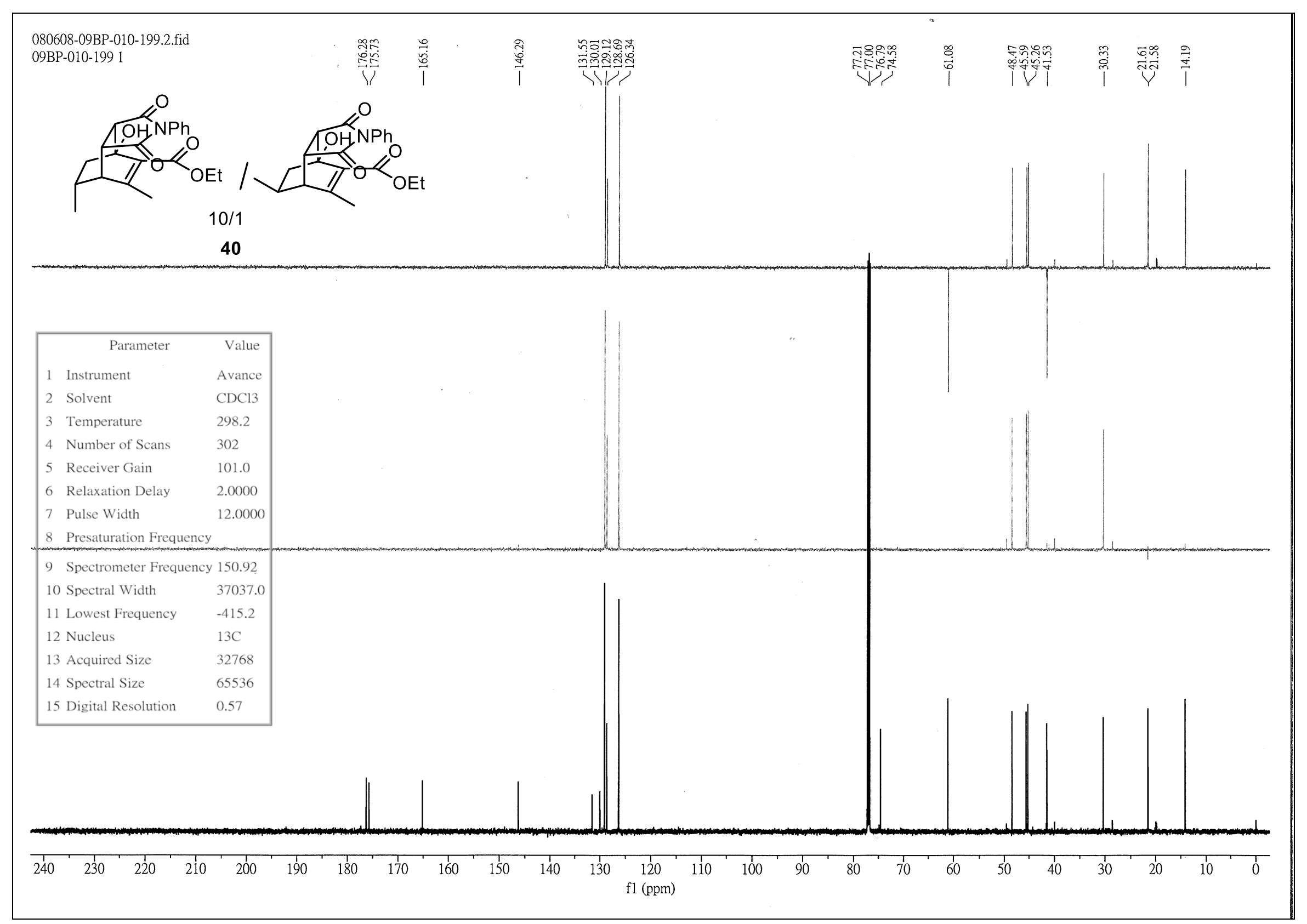

${ }^{13} \mathrm{C}$ NMR + DEPT spectra for compound $\mathbf{4 0}$ 


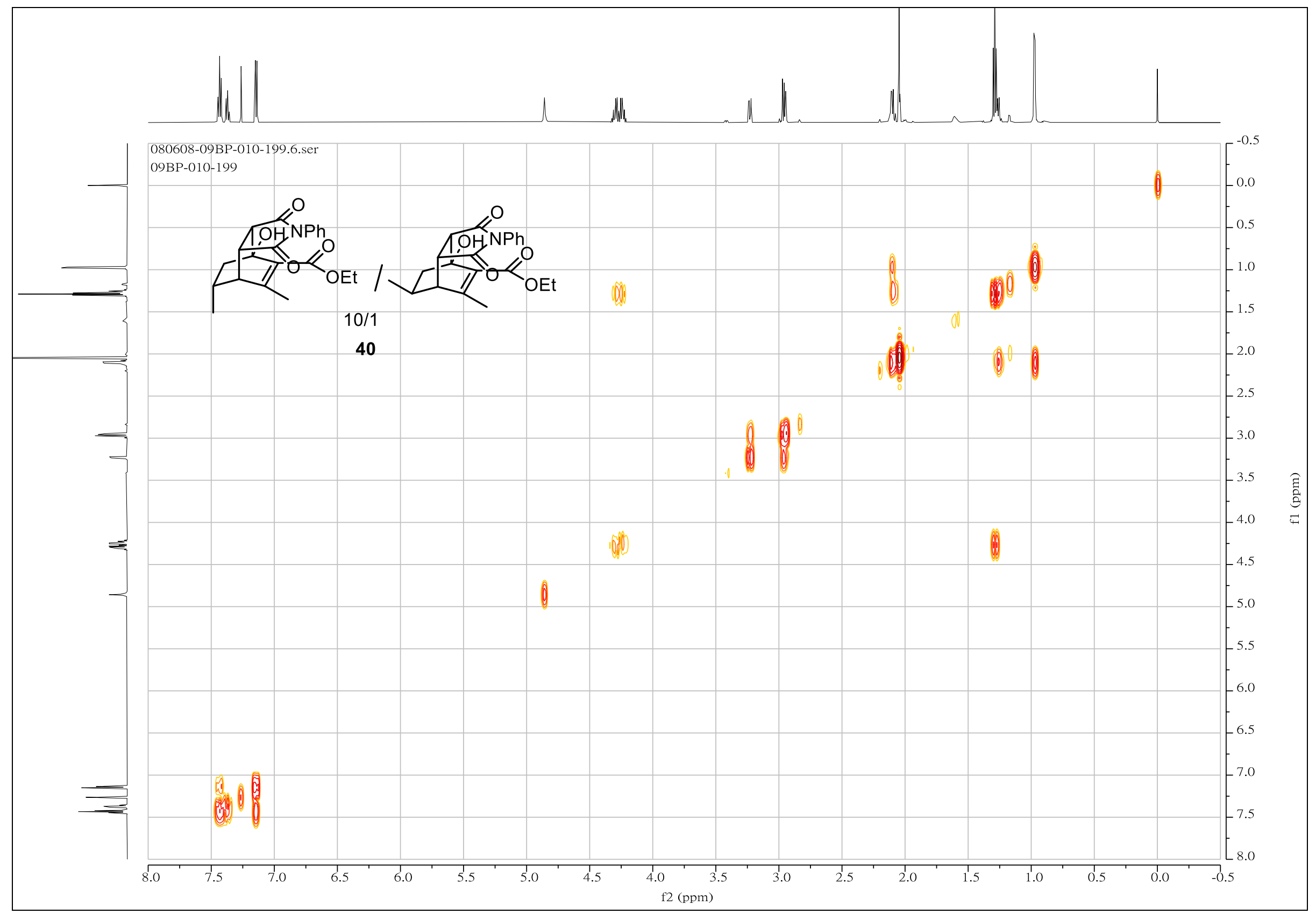

${ }^{1} \mathrm{H}-{ }^{1} \mathrm{H}$ COSY spectrum for compound $\mathbf{4 0}$ 


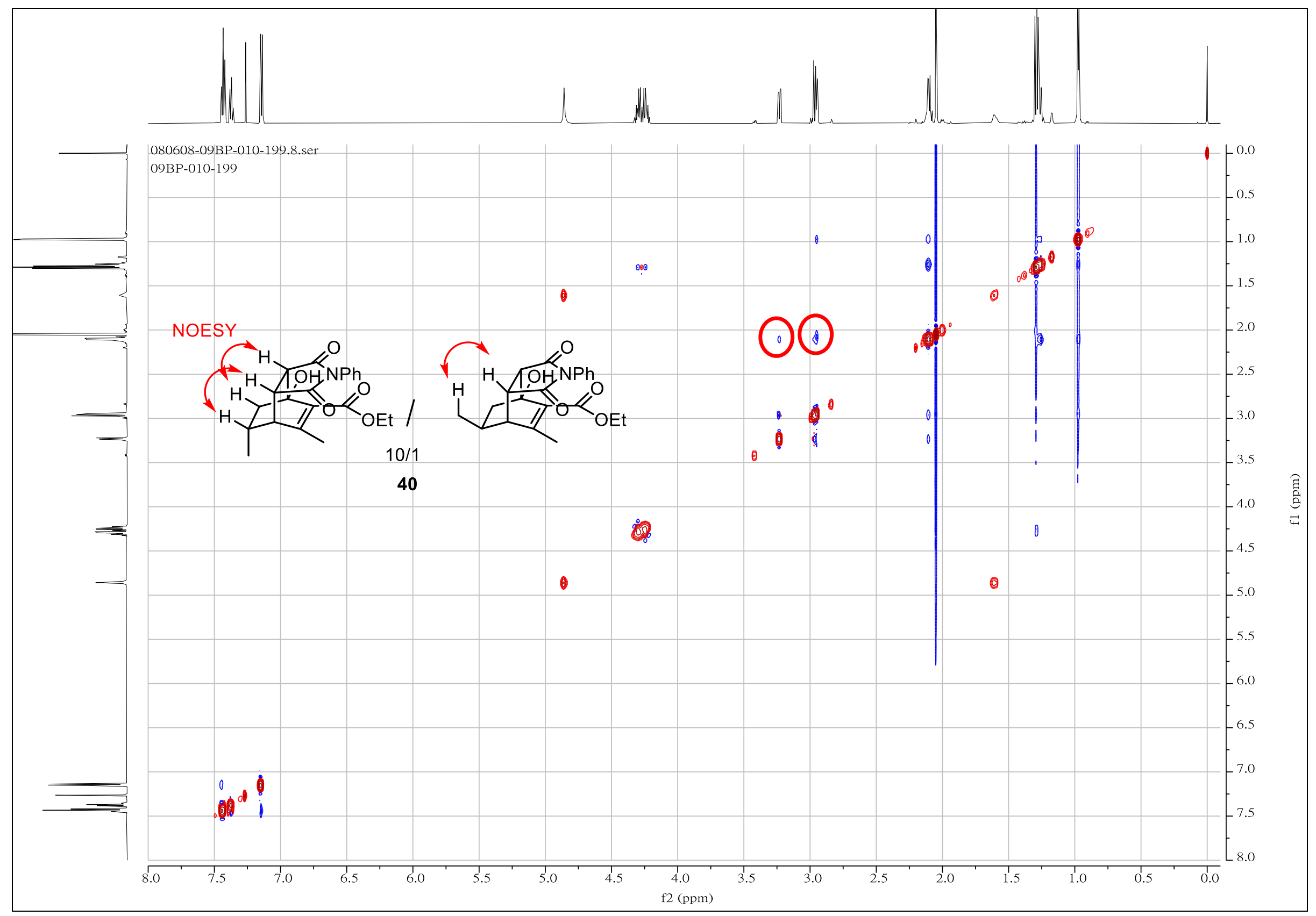

2D NOESY spectrum for compound $\mathbf{4 0}$ 


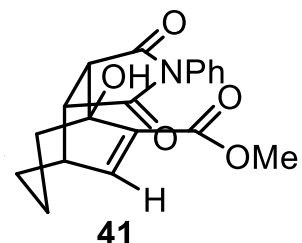

\begin{tabular}{|lll|}
\hline & \multicolumn{1}{c}{ Parameter } & \multicolumn{1}{c}{ Value } \\
1 & Instrument & Avance \\
2 & Solvent & CDCl3 \\
3 & Temperature & 294.7 \\
4 & Number of Scans & 16 \\
5 & Receiver Gain & 101.0 \\
6 & Relaxation Delay & 1.0000 \\
7 & Pulse Width & 8.0000 \\
8 & Presaturation Frequency \\
9 & Spectrometer Frequency & 400.17 \\
10 & Spectral Width & 7812.5 \\
11 & Lowest Frequency & -1444.2 \\
12 & Nucleus & $1 \mathrm{H}$ \\
13 & Acquired Size & 32768 \\
14 & Spectral Size & 65536 \\
15 & Digital Resolution & 0.12
\end{tabular}

15 Digitat Resolution 0.12
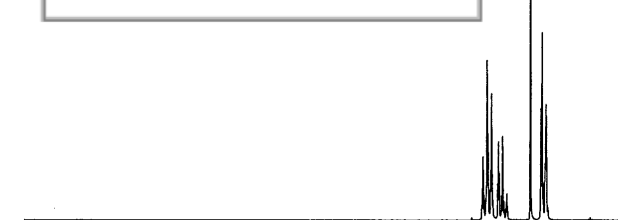
Nhathen
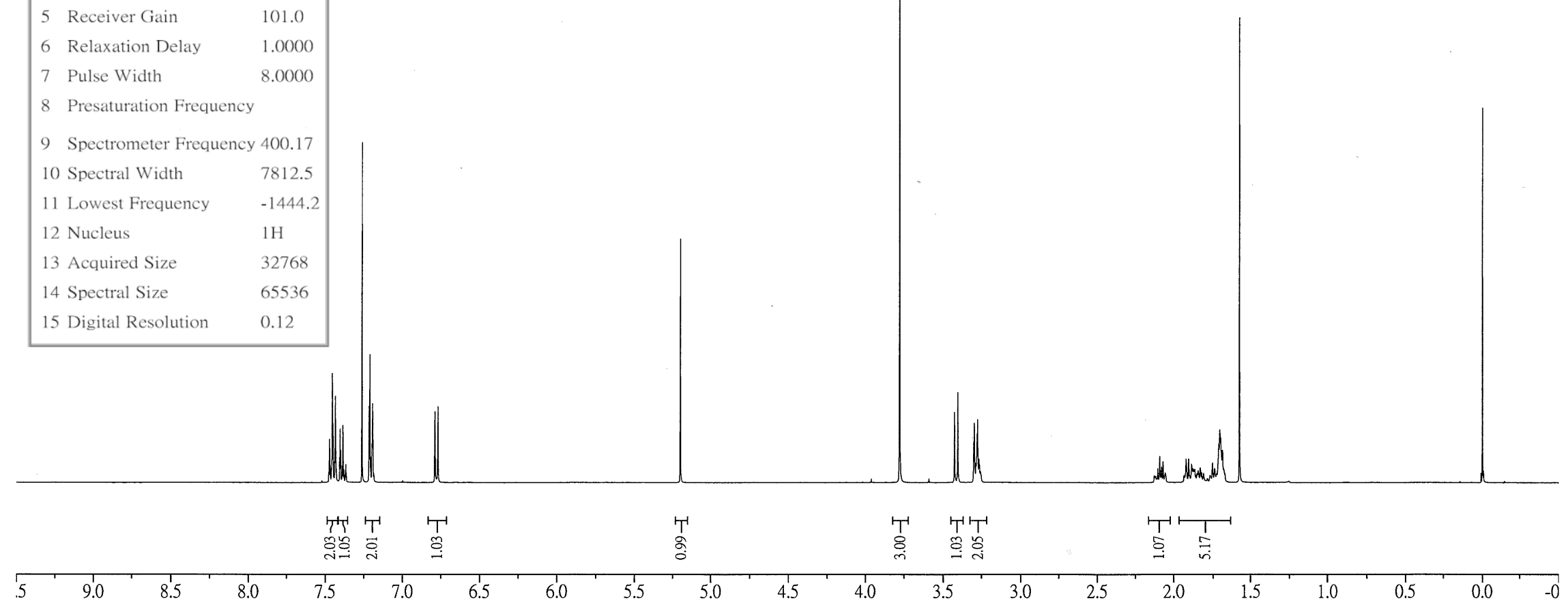

4.5
$\mathrm{f} 1(\mathrm{ppm})$

${ }^{1} \mathrm{H}$ NMR spectrum for compound $\mathbf{4 1}$ 

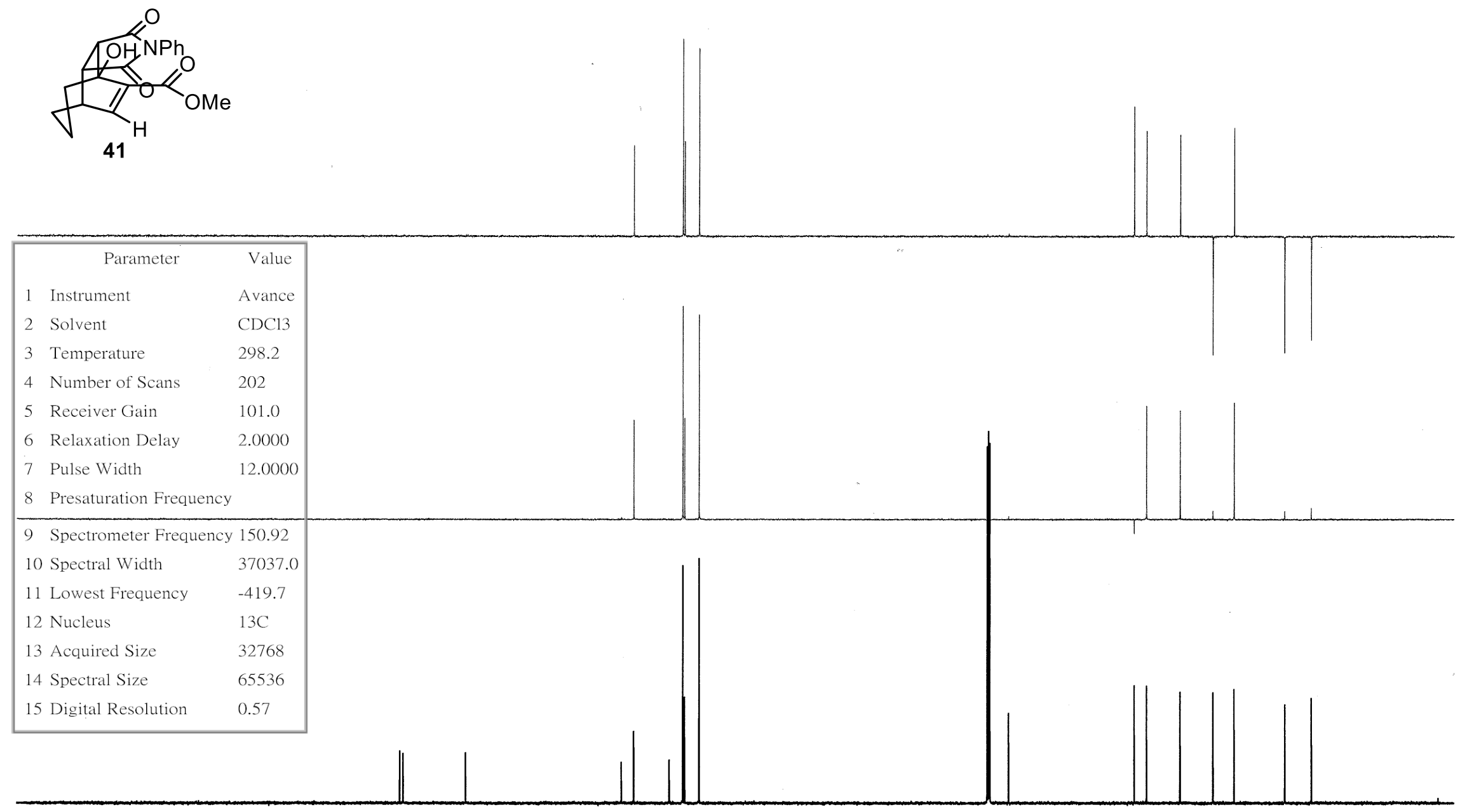

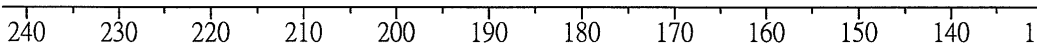

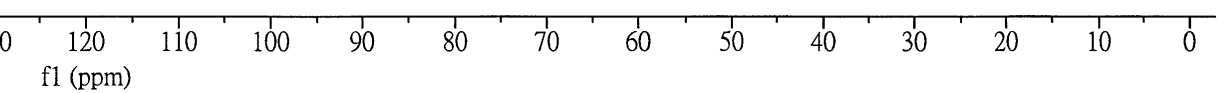

${ }^{13} \mathrm{C}$ NMR + DEPT spectra for compound 41 


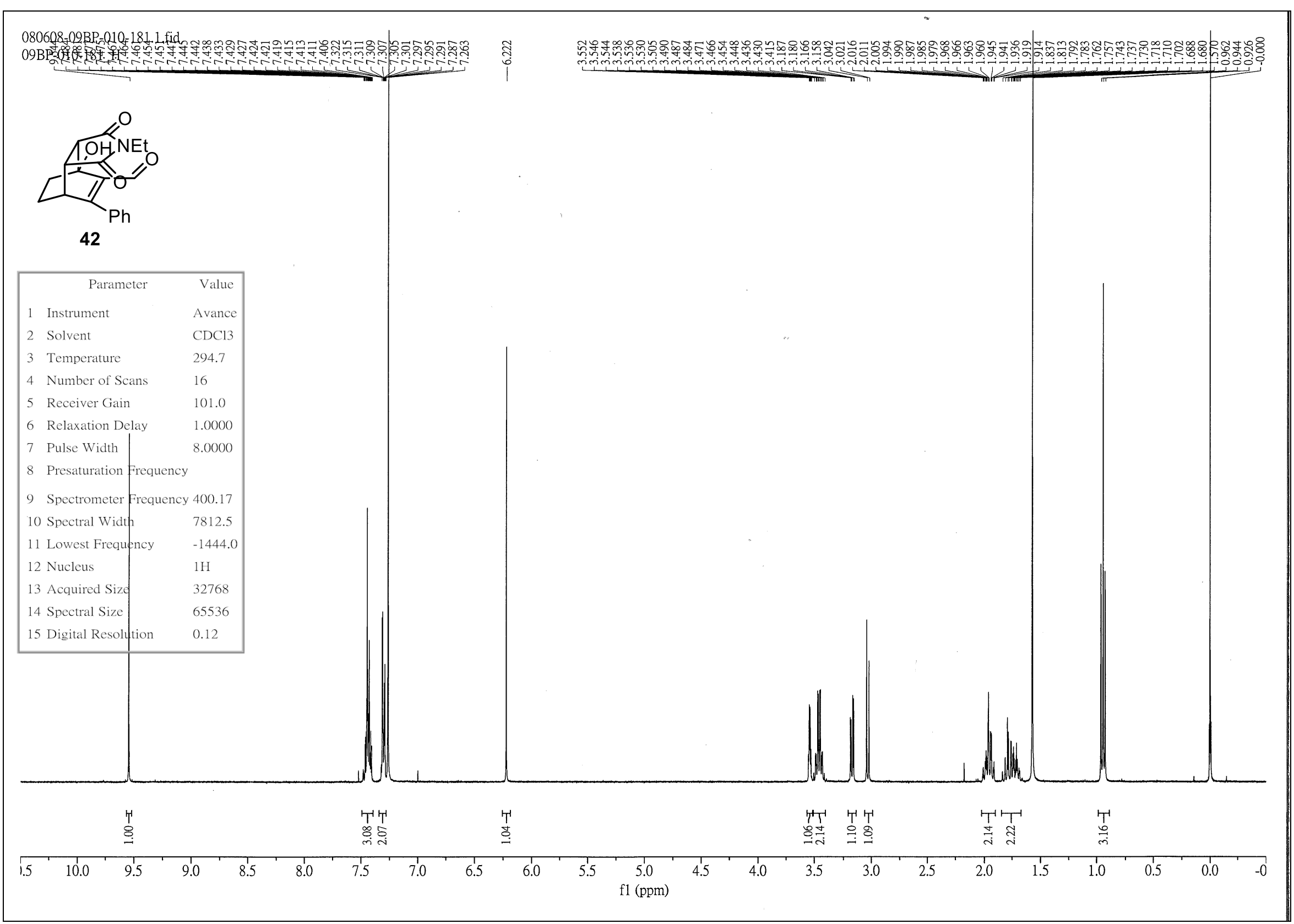

${ }^{1} \mathrm{H}$ NMR spectrum for compound $\mathbf{4 2}$ 


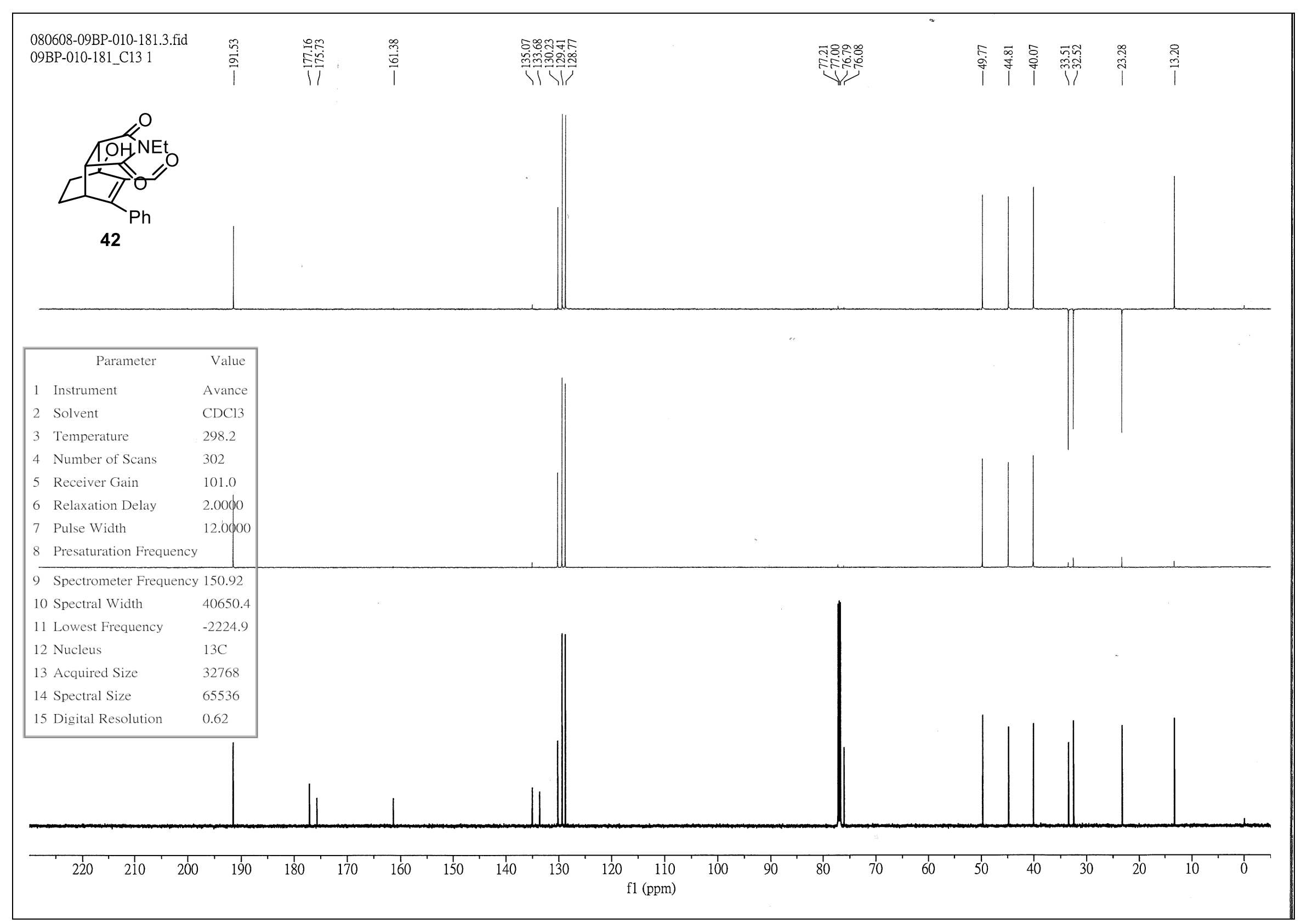

${ }^{13} \mathrm{C}$ NMR + DEPT spectra for compound 42 


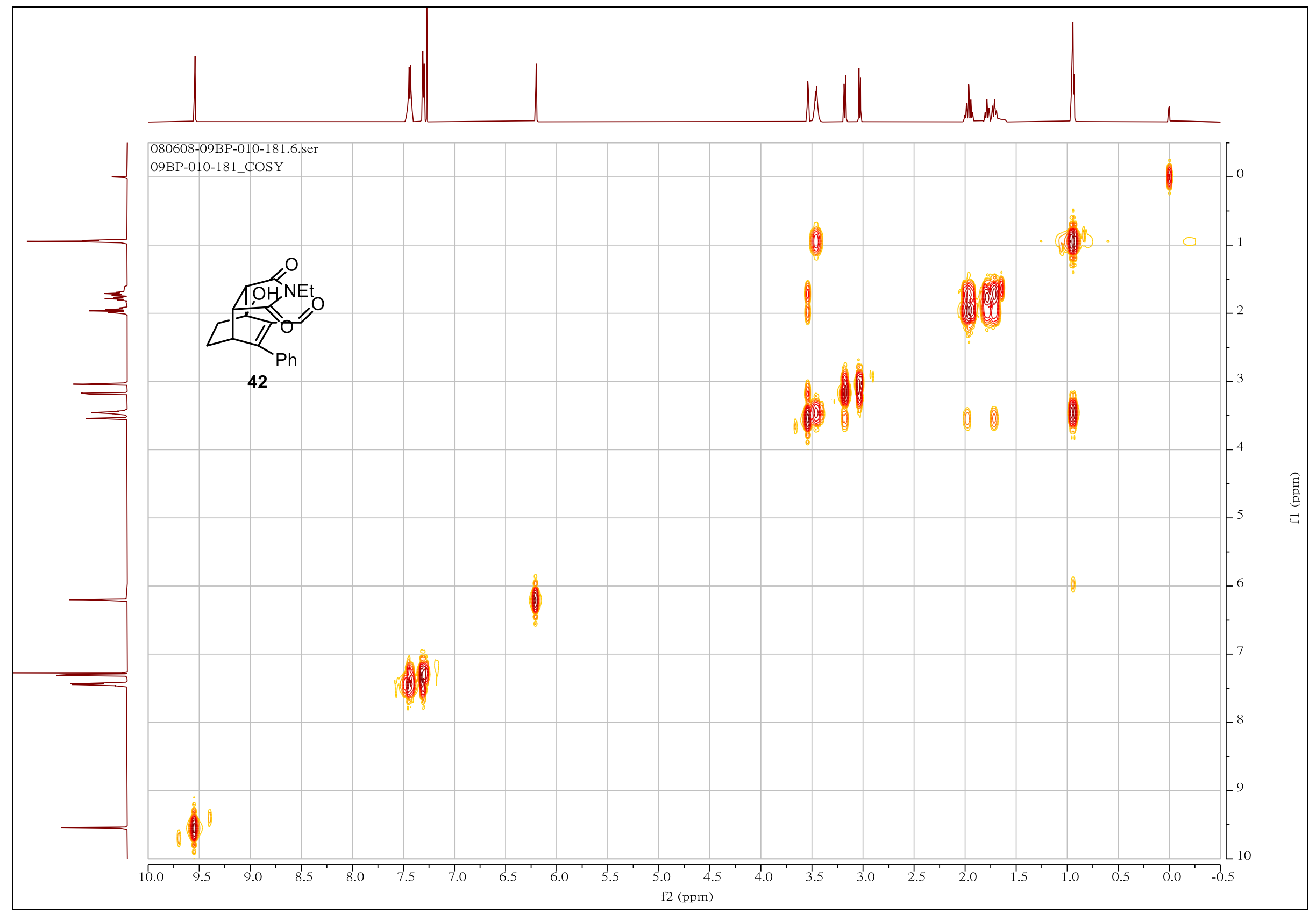

${ }^{1} \mathrm{H}-{ }^{1} \mathrm{H}$ COSY spectrum for compound 42 


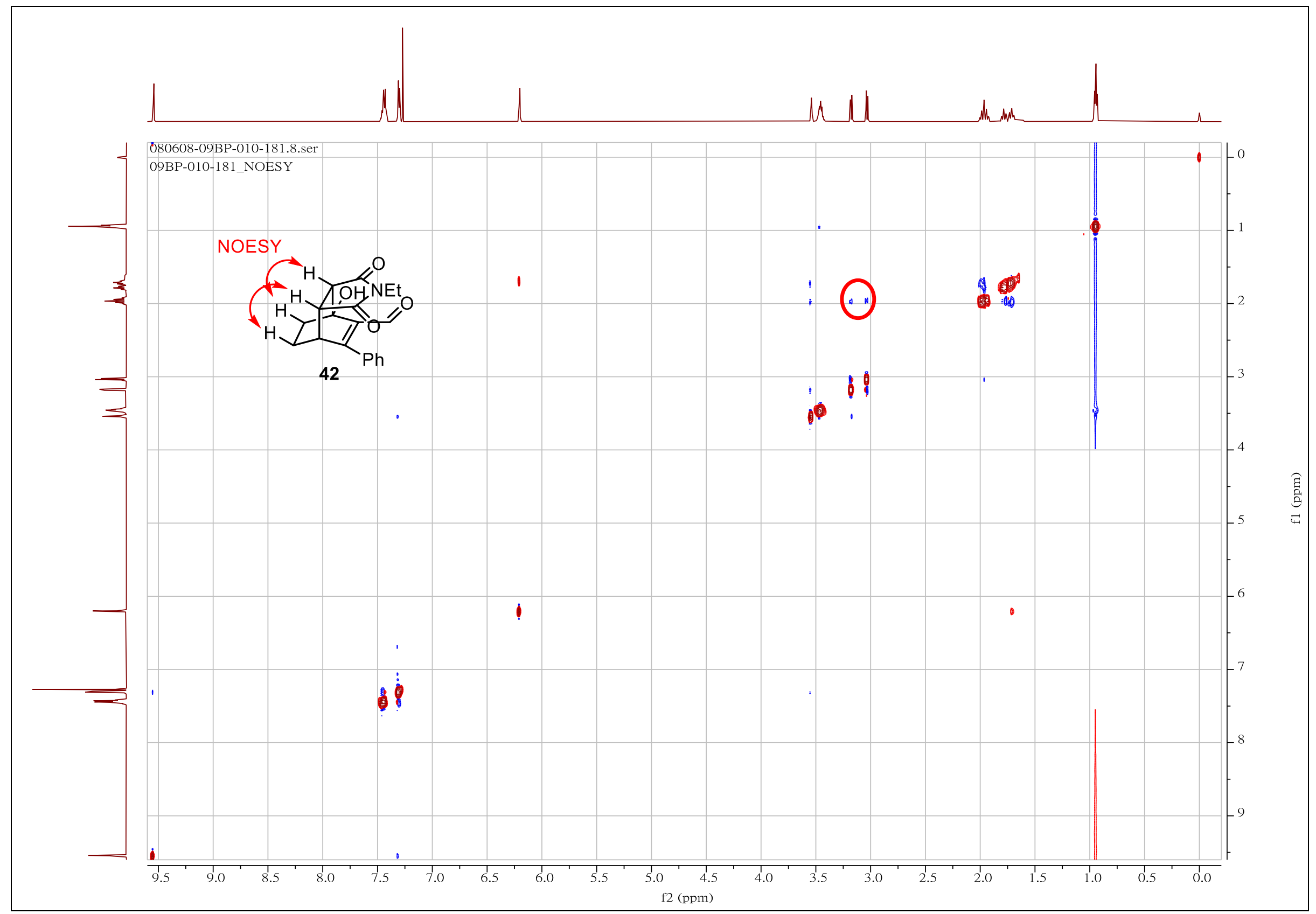

2D NOESY spectrum for compound $\mathbf{4 2}$ 


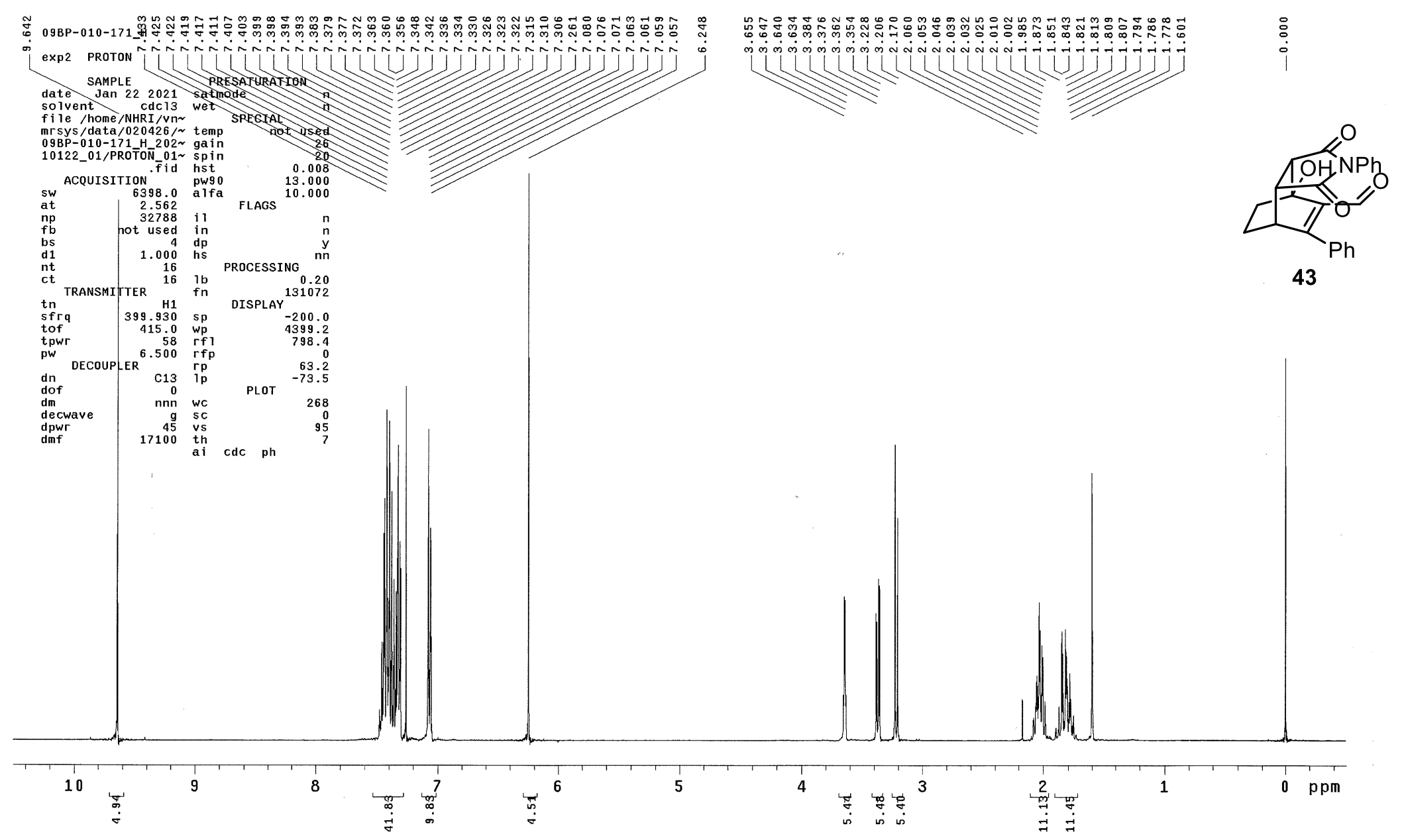

${ }^{1} \mathrm{H}$ NMR spectrum for compound $\mathbf{4 3}$ 


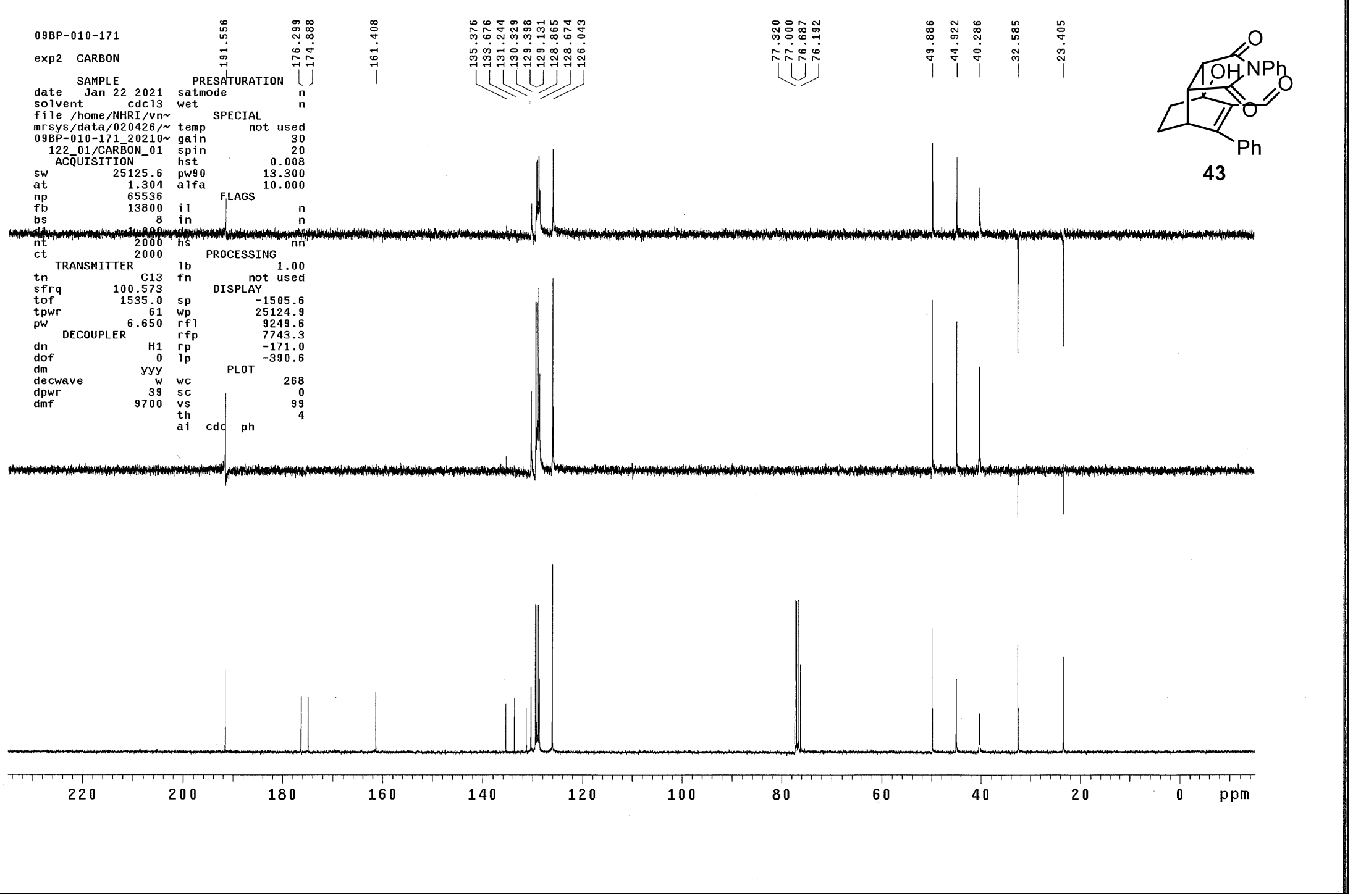

${ }^{13} \mathrm{C}$ NMR + DEPT spectra for compound $\mathbf{4 3}$ 


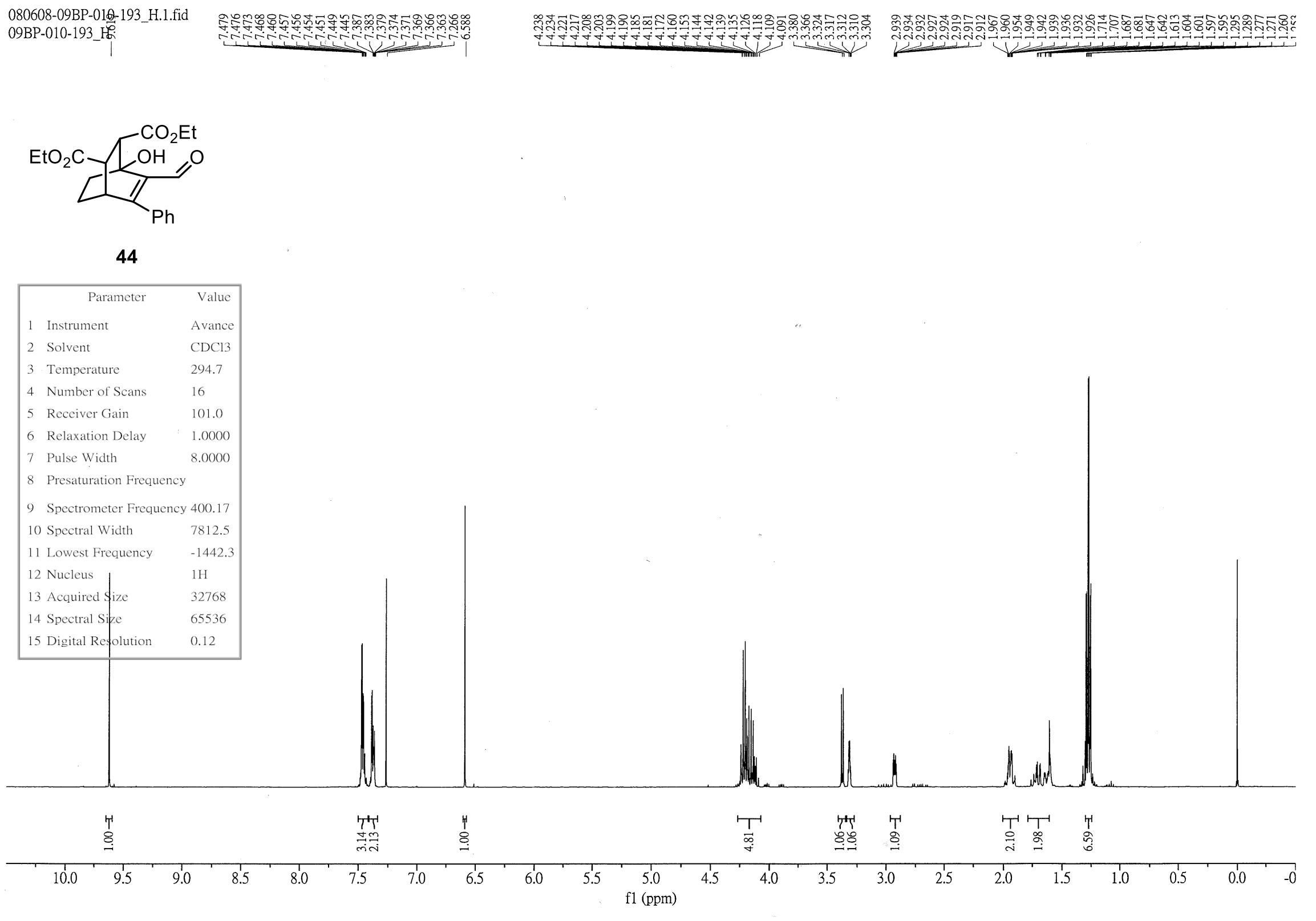

${ }^{1} \mathrm{H}$ NMR spectrum for compound 44 


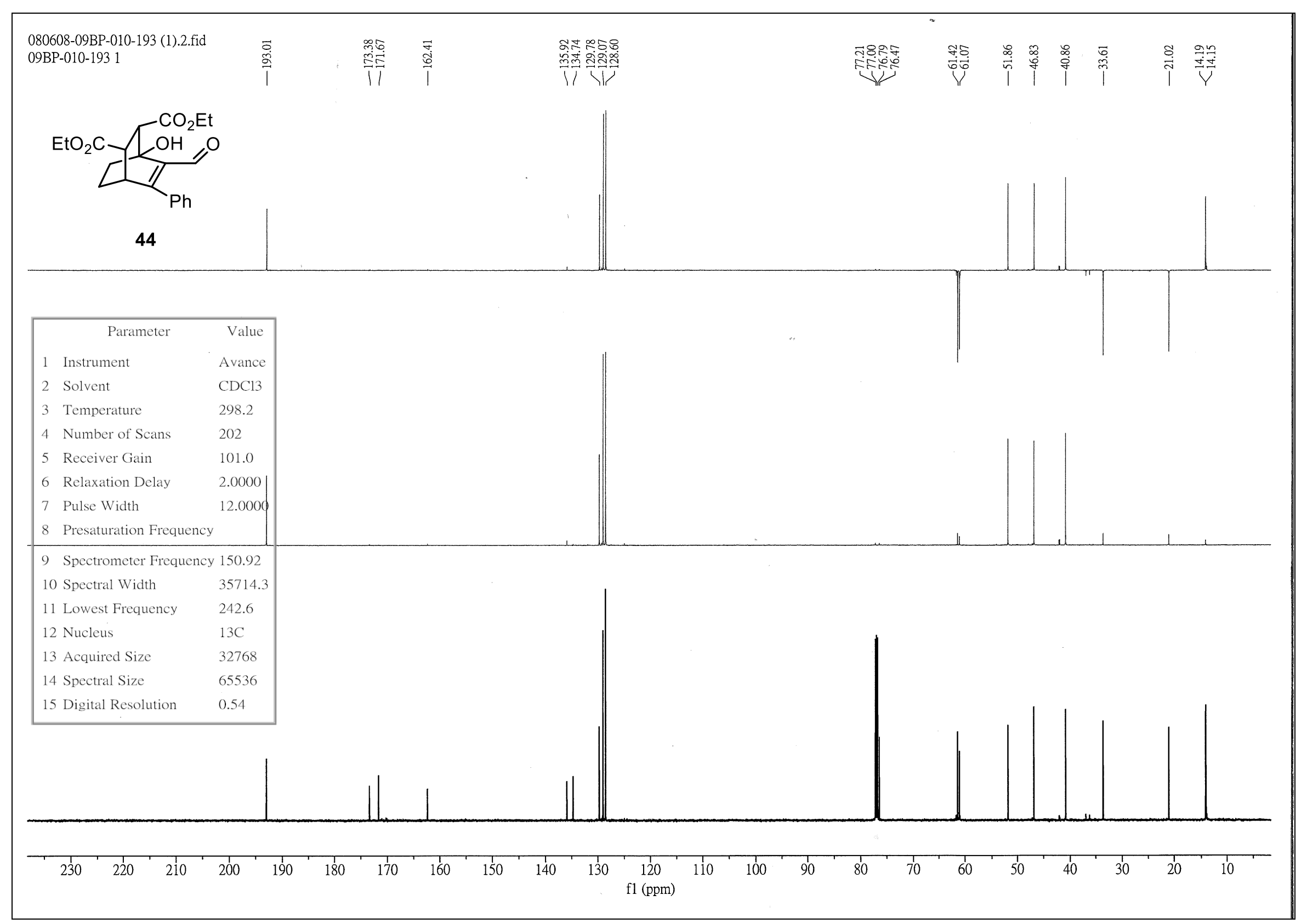

${ }^{13} \mathrm{C}$ NMR + DEPT spectra for compound 44 


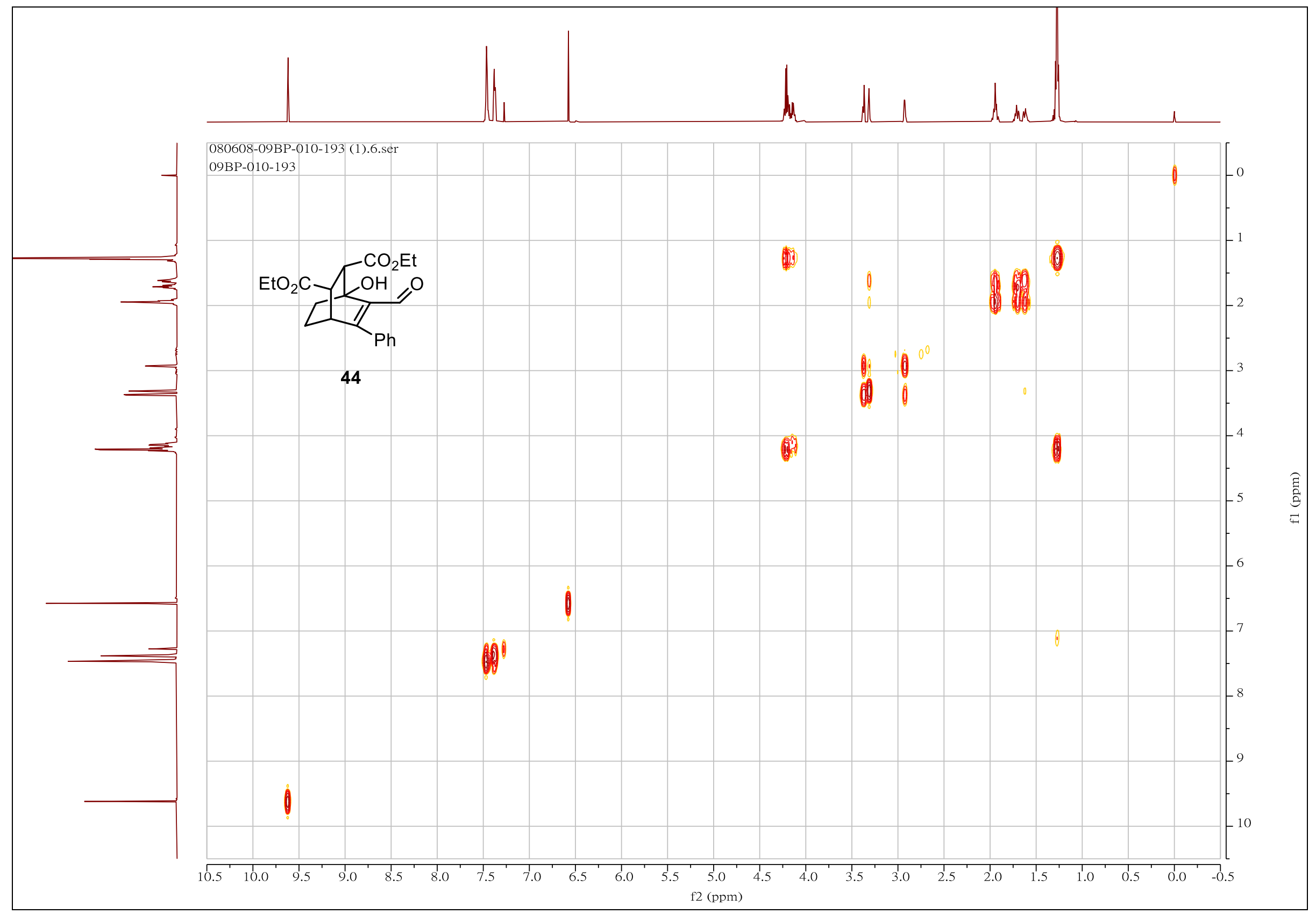

${ }^{1} \mathrm{H}-{ }^{1} \mathrm{H}$ COSY spectrum for compound 44 


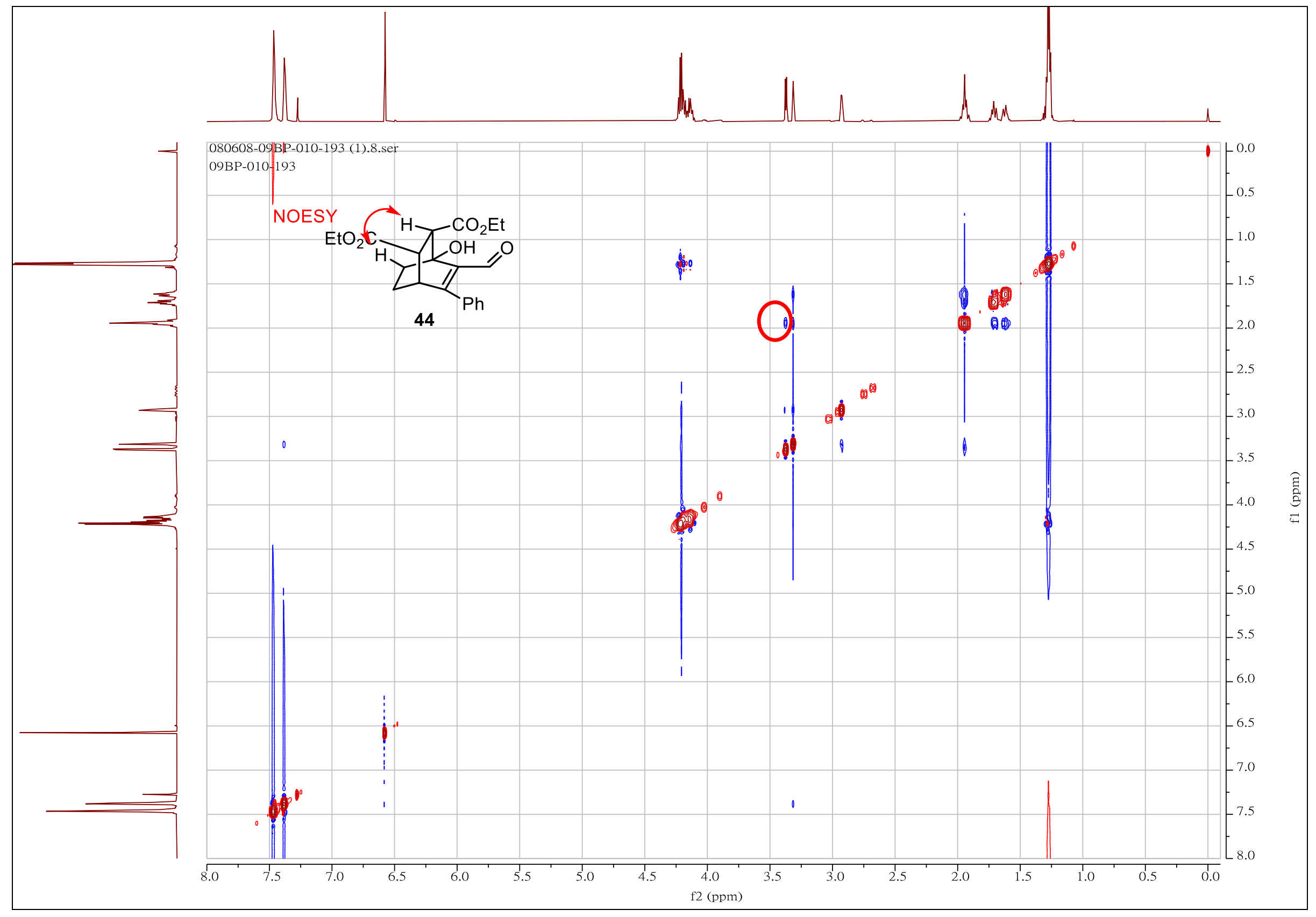

2D NOESY spectrum for compound $\mathbf{4 4}$ 


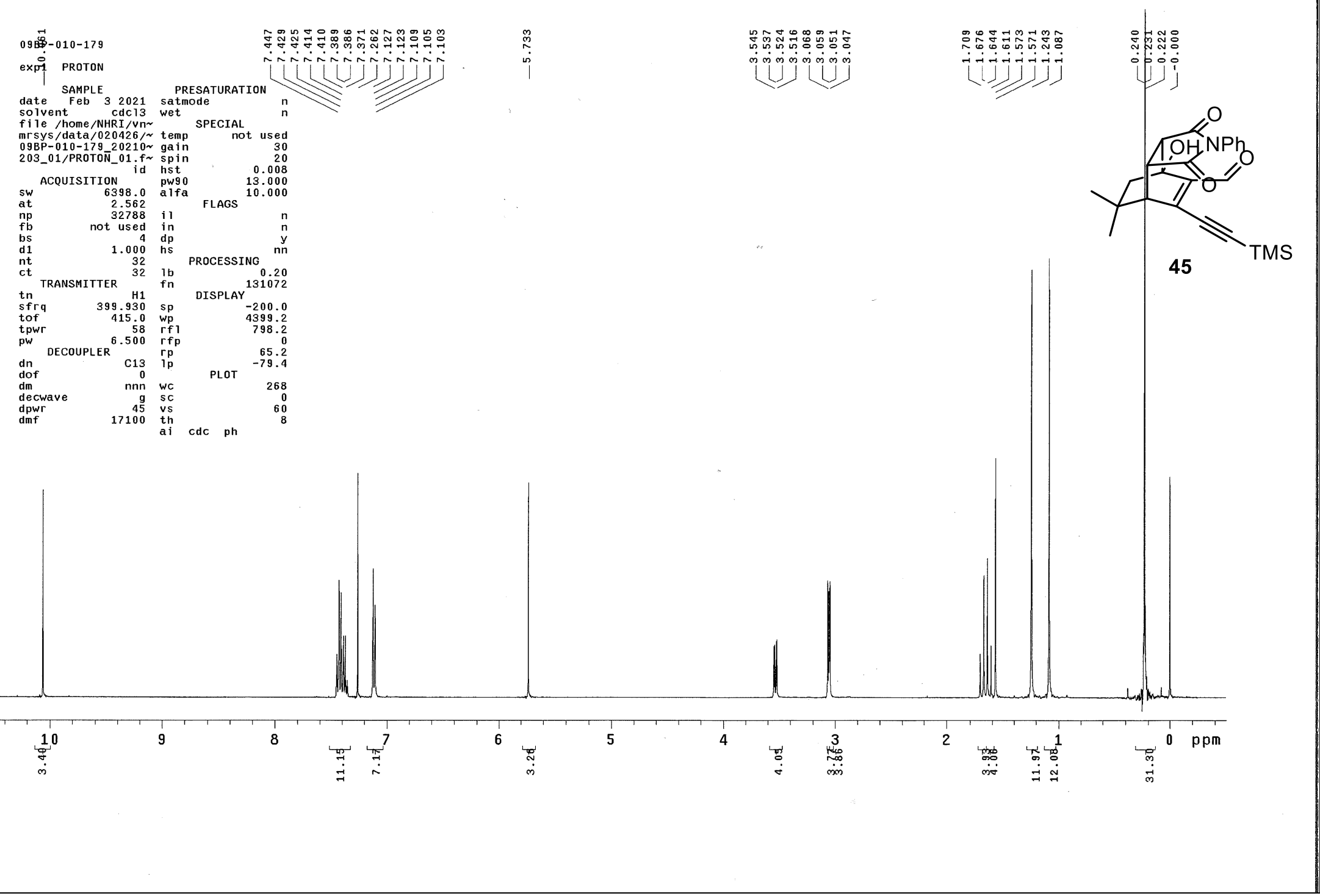

${ }^{1} \mathrm{H}$ NMR spectrum for compound 45 


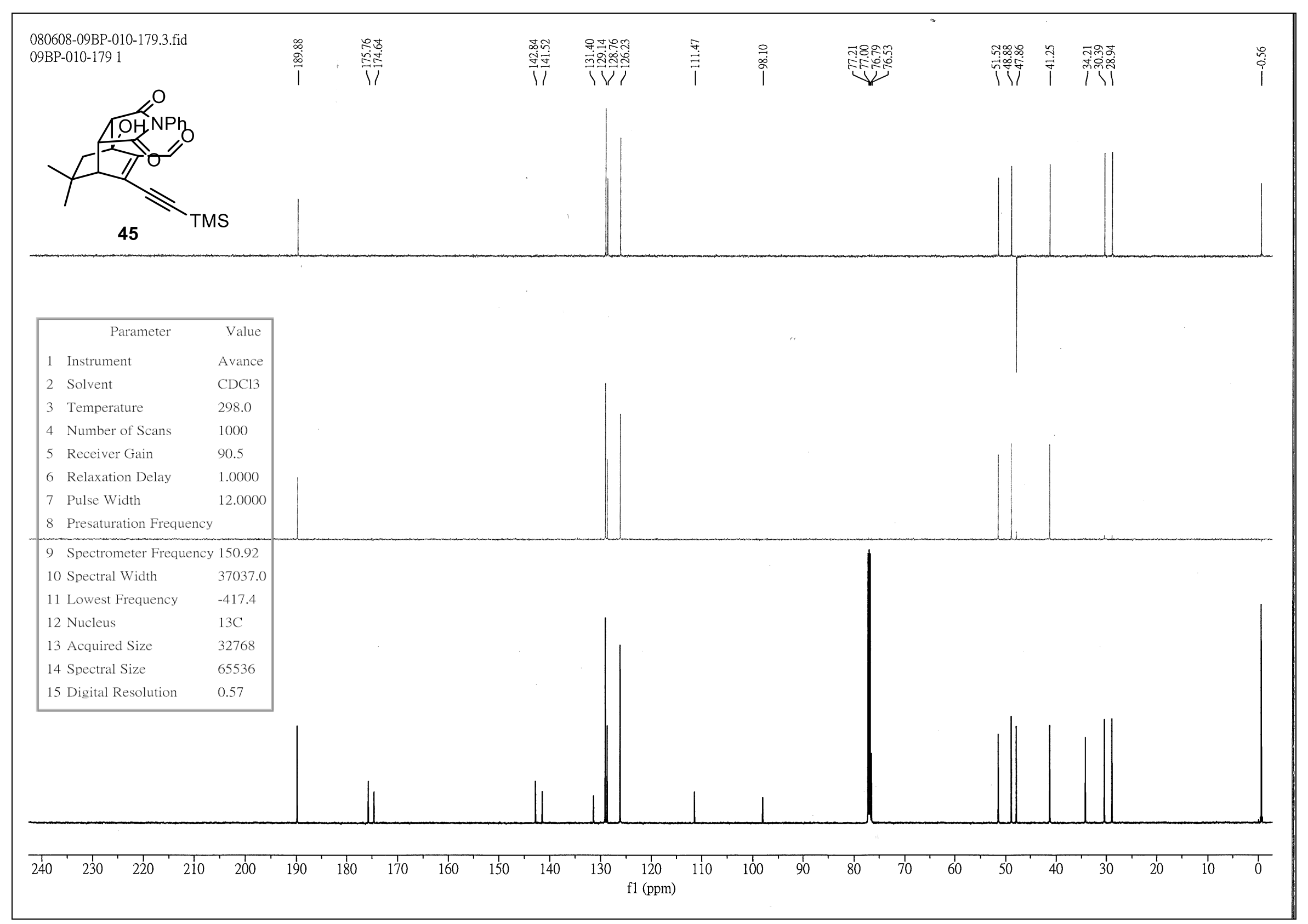

${ }^{13} \mathrm{C}$ NMR + DEPT spectra for compound 45 


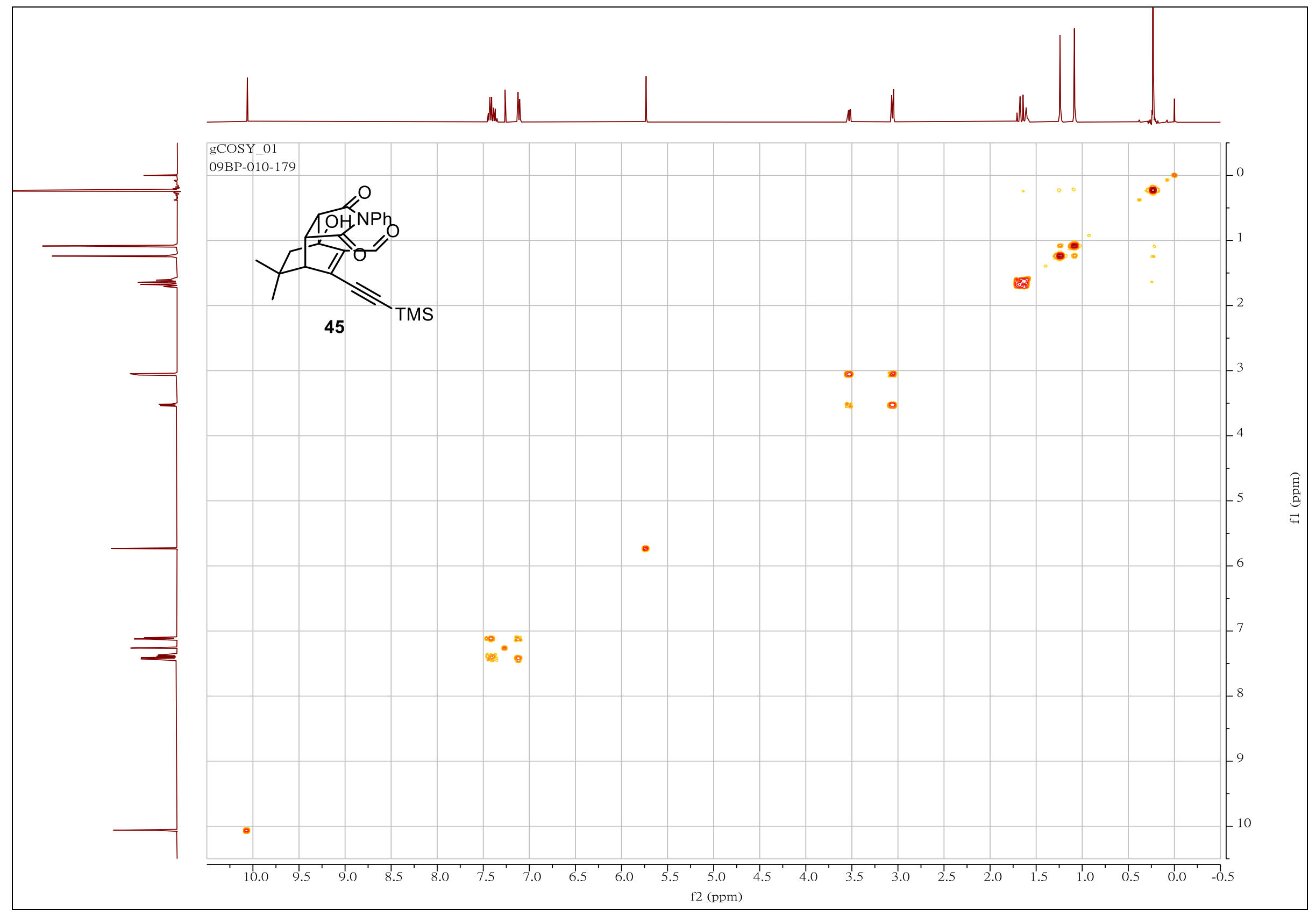

${ }^{1} \mathrm{H}-{ }^{1} \mathrm{H}$ COSY spectrum for compound 45 


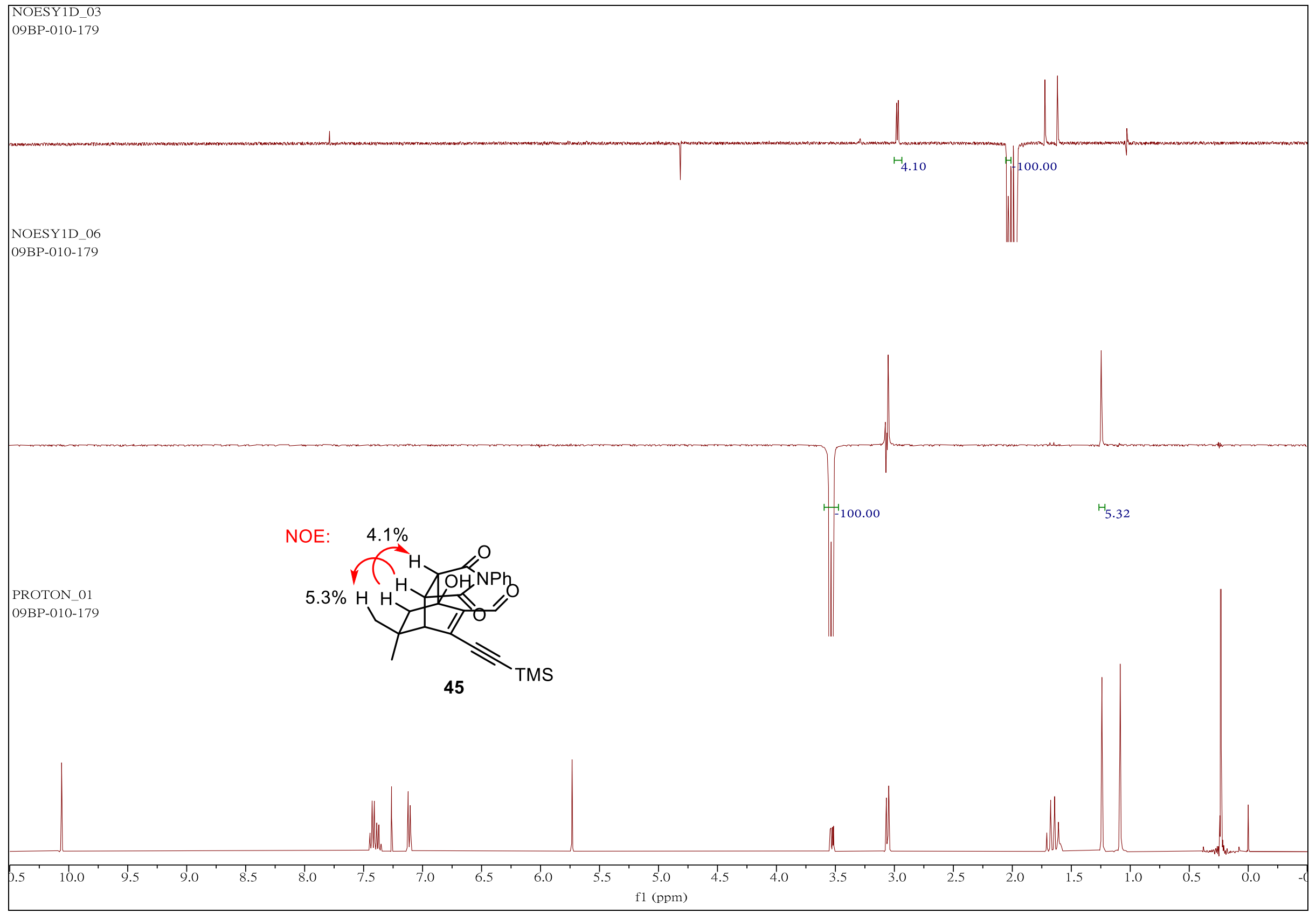

1D NOESY spectra for compound $\mathbf{4 5}$ 


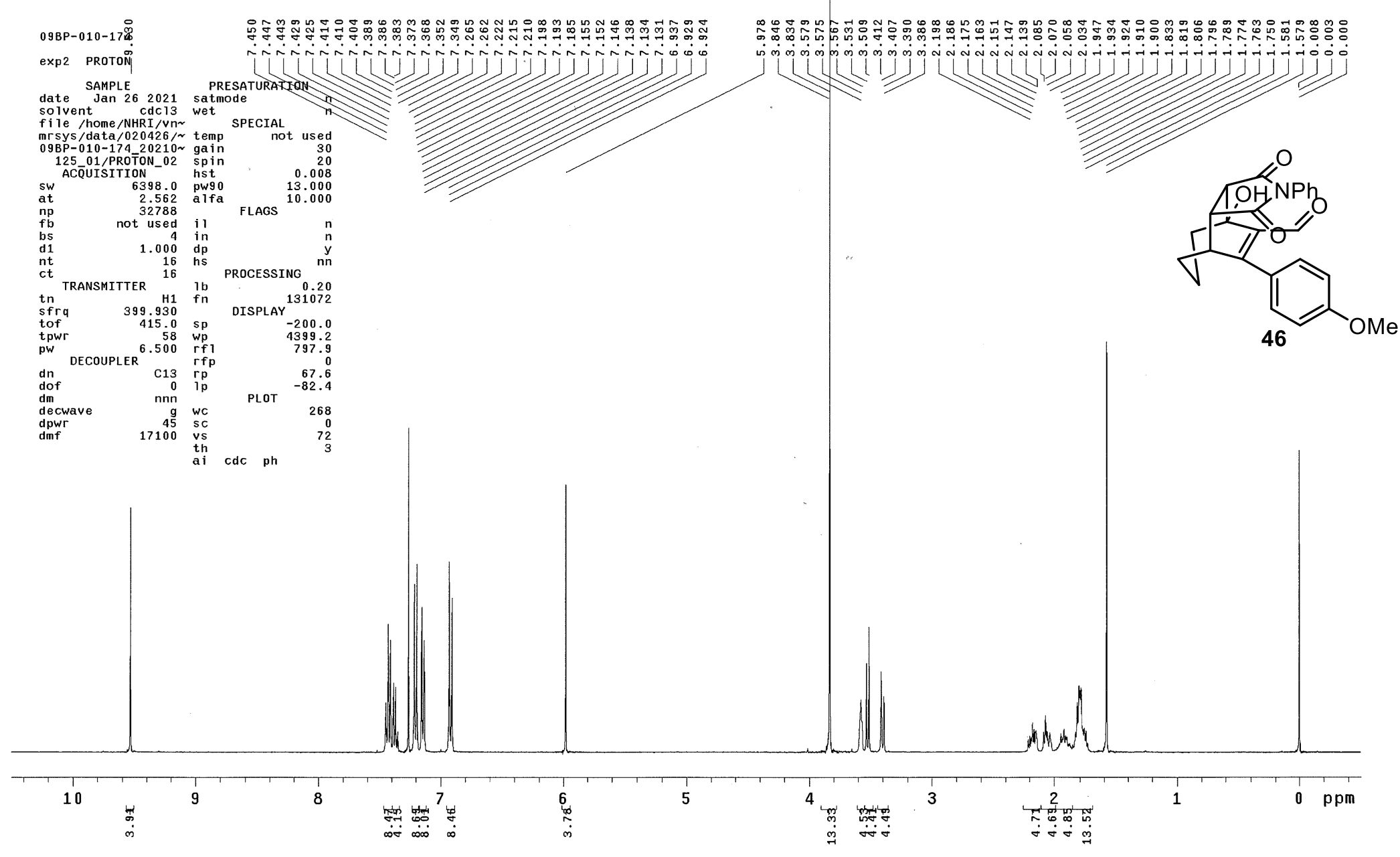

${ }^{1} \mathrm{H}$ NMR spectrum for compound 46 


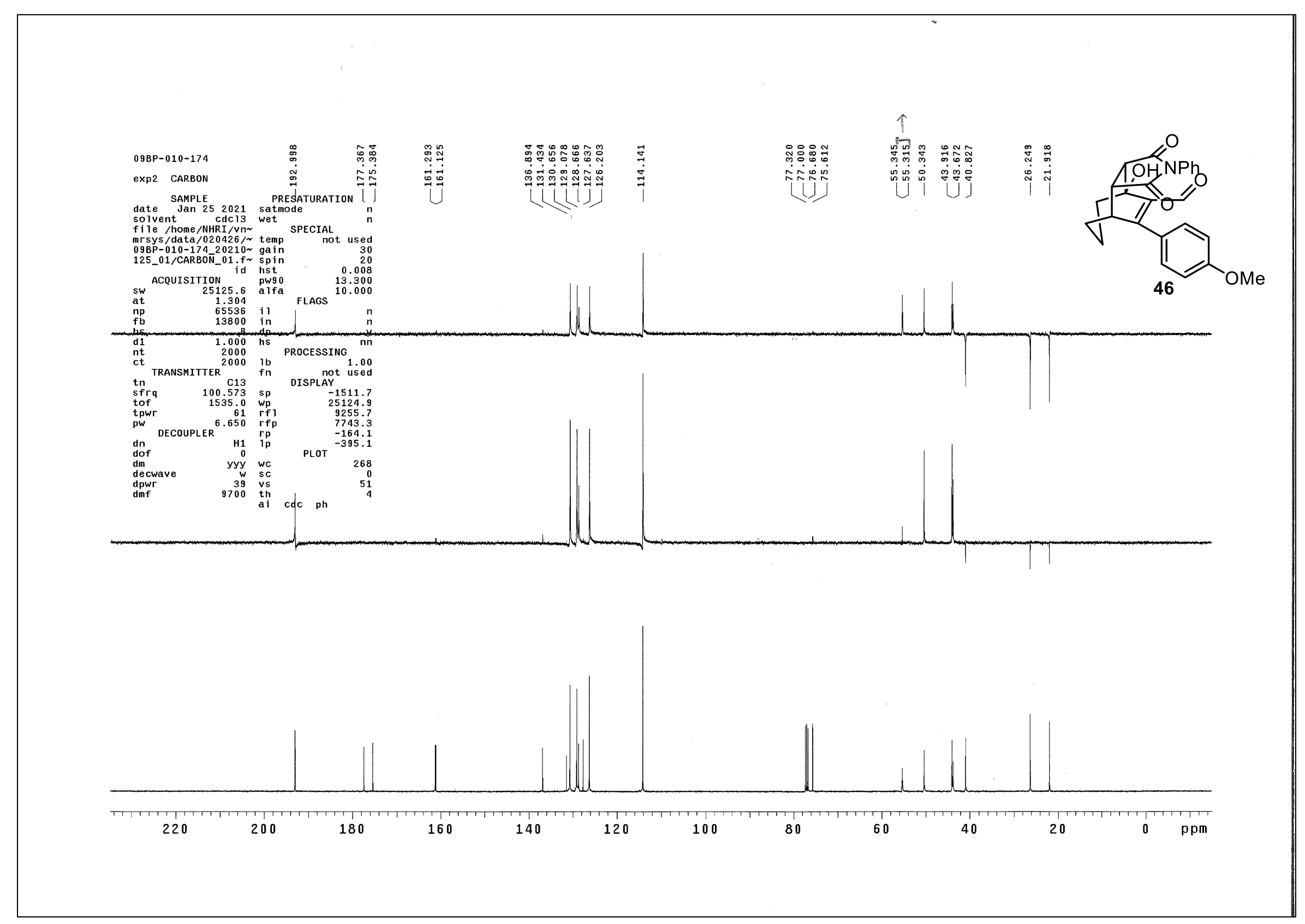

${ }^{13} \mathrm{C}$ NMR + DEPT spectra for compound 46 


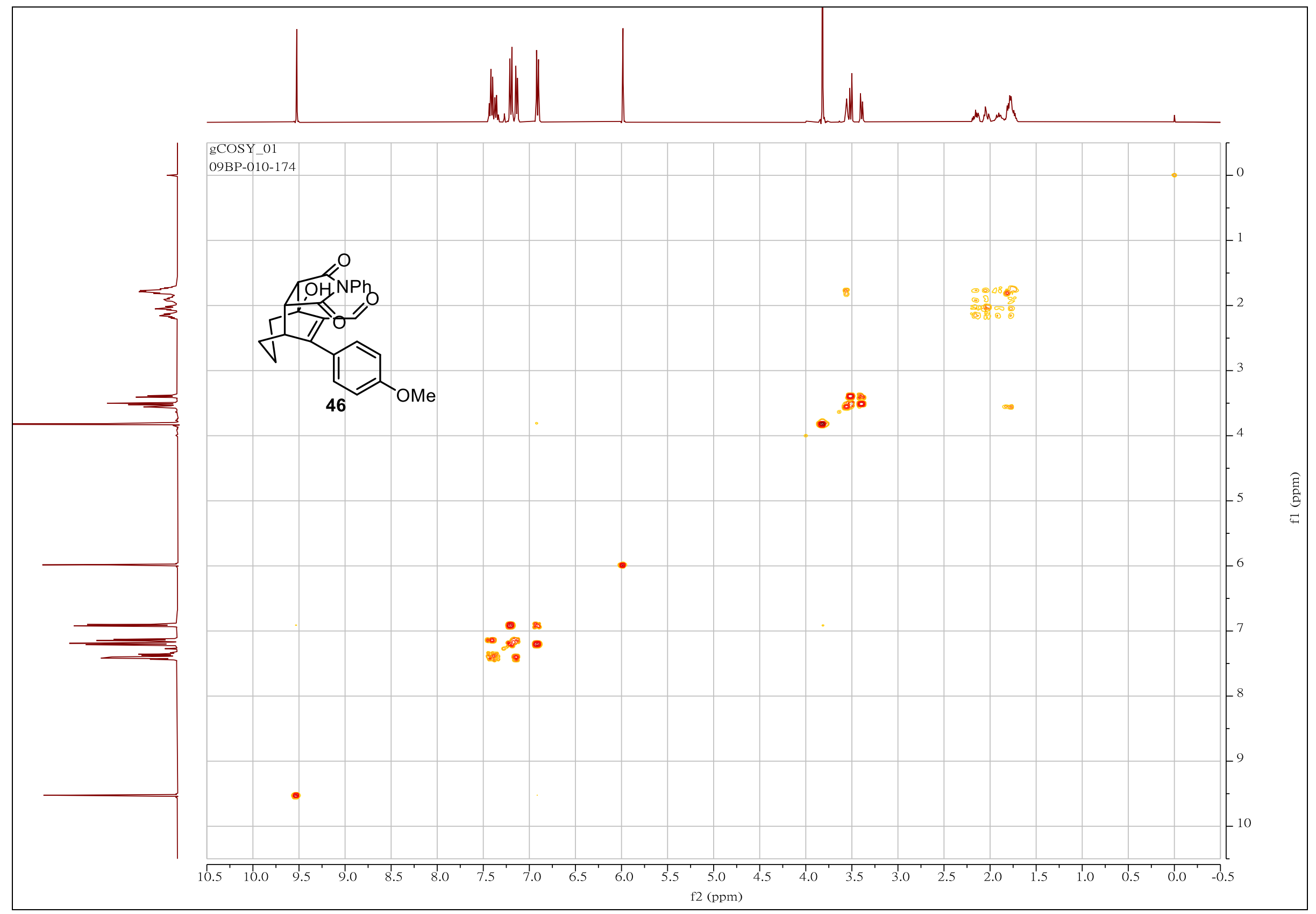

${ }^{1} \mathrm{H}-{ }^{1} \mathrm{H}$ COSY spectrum for compound 46 


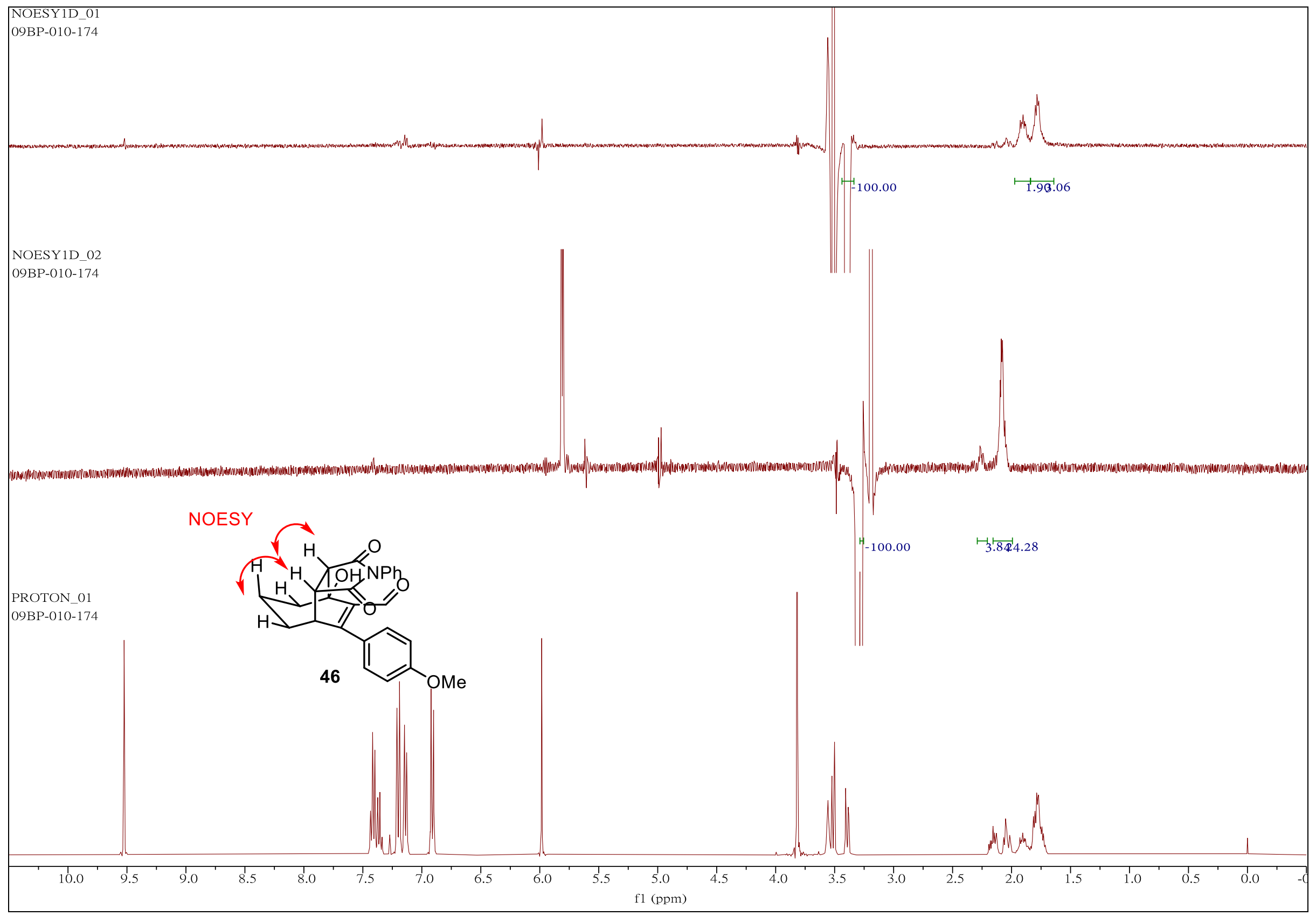

1D NOESY spectra for compound $\mathbf{4 6}$ 


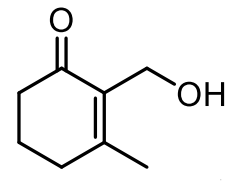

s1

\begin{tabular}{|lll|}
\hline \multicolumn{2}{|c|}{ Parameter } & \multicolumn{1}{c|}{ Value } \\
1 & Instrument & Avance \\
2 & Solvent & CDC13 \\
3 & Temperature & 294.7 \\
4 & Number of Scans & 16 \\
5 & Receiver Gain & 101.0 \\
6 & Relaxation Delay & 1.0000 \\
7 & Pulse Width & 8.0000 \\
8 & Presaturation Frequency \\
9 & Spectrometer Frequency 400.17 \\
10 & Spectral Width & 7812.5 \\
11 & Lowest Frequency & -1437.4 \\
12 & Nucleus & $1 \mathrm{H}$ \\
13 & Acquired Size & 32768 \\
14 & Spectral Size & 65536 \\
15 & Digital Resolution & 0.12 \\
\hline
\end{tabular}
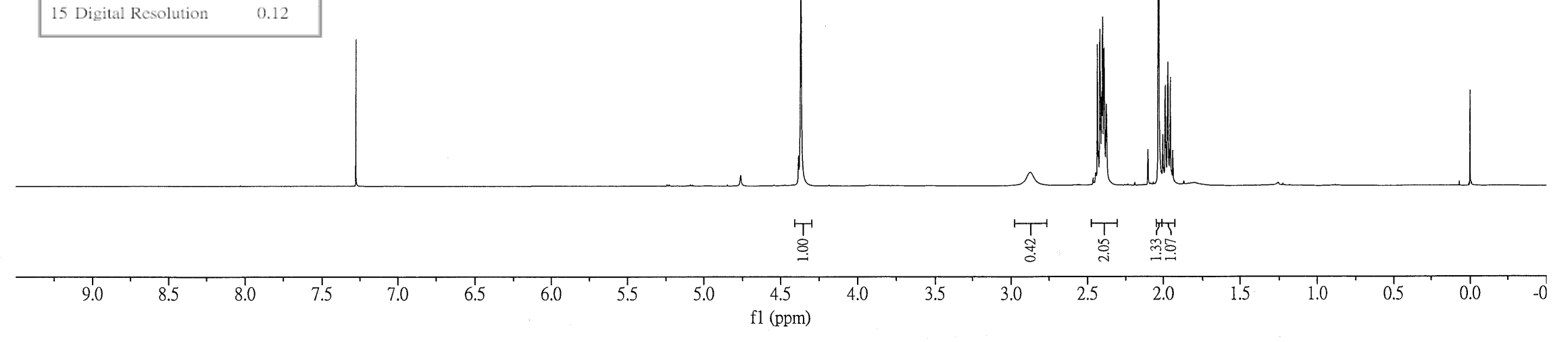

${ }^{1}$ H NMR spectrum for compound $\mathbf{S 1}$ 


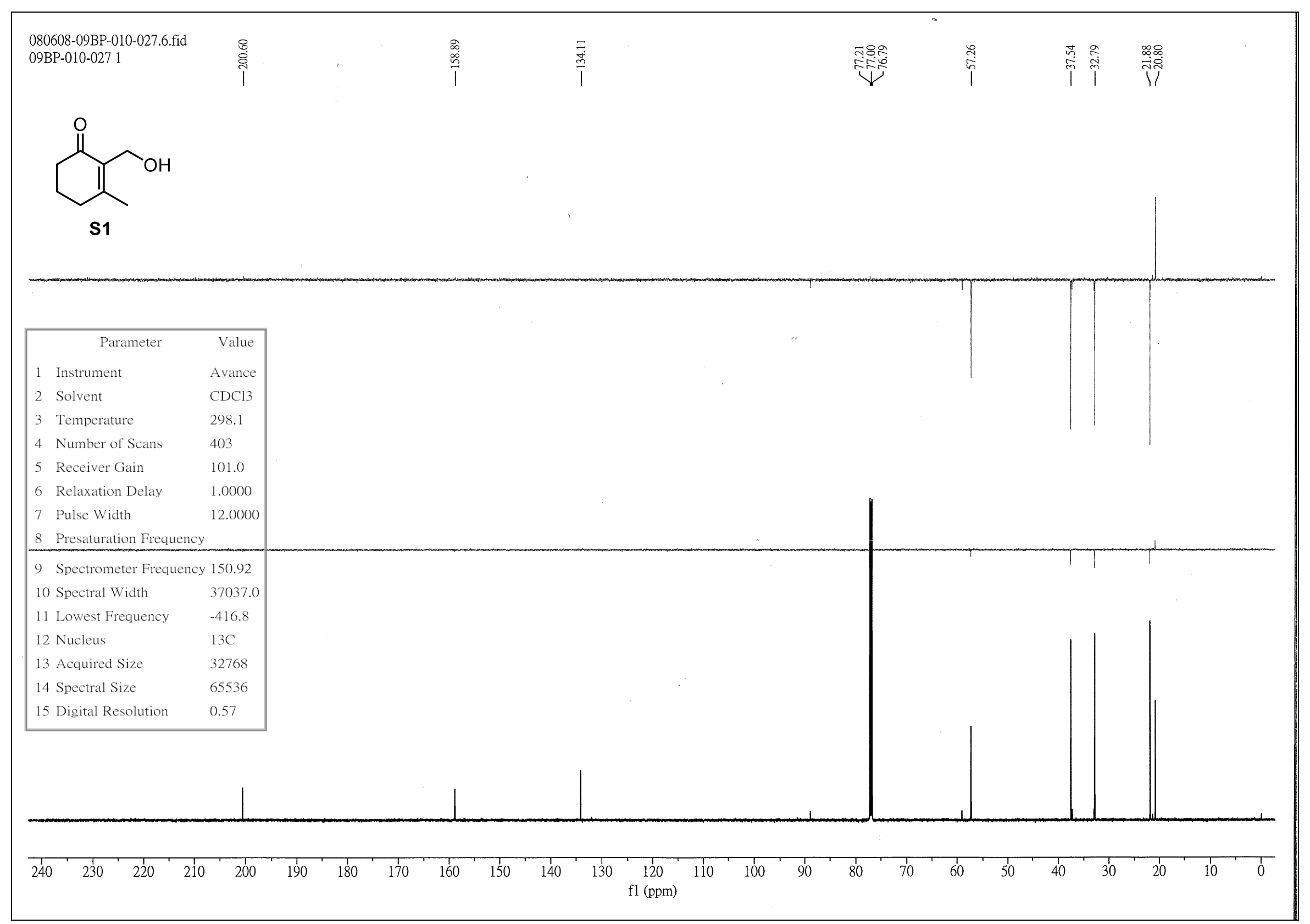

${ }^{13} \mathrm{C}$ NMR + DEPT spectra for compound S1 


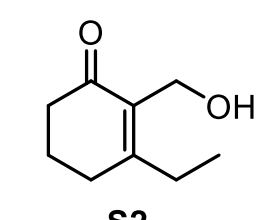

\section{S2}

\begin{tabular}{|lll|}
\hline & \multicolumn{1}{|c}{ Parameter } & \multicolumn{1}{c|}{ Value } \\
1 & Instrument & Avance \\
2 & Solvent & $\mathrm{CDCl} 3$ \\
3 & Temperature & 294.7 \\
4 & Number of Scans & 16 \\
5 & Receiver Gain & 101.0 \\
6 & Relaxation Delay & 1.0000 \\
7 & Pulse Width & 8.0000 \\
8 & Presaturation Frequency \\
9 & Spectrometer Frequency \\
10 & Spectral Width & 7812.5 \\
11 & Lowest Frequency & -1439.8 \\
12 & Nucleus & $1 \mathrm{H}$ \\
13 & Acquired Size & 32768 \\
14 & Spectral Size & 65536 \\
15 & Digital Resolution & 0.12 \\
\hline \multicolumn{2}{l}{} \\
\hline
\end{tabular}
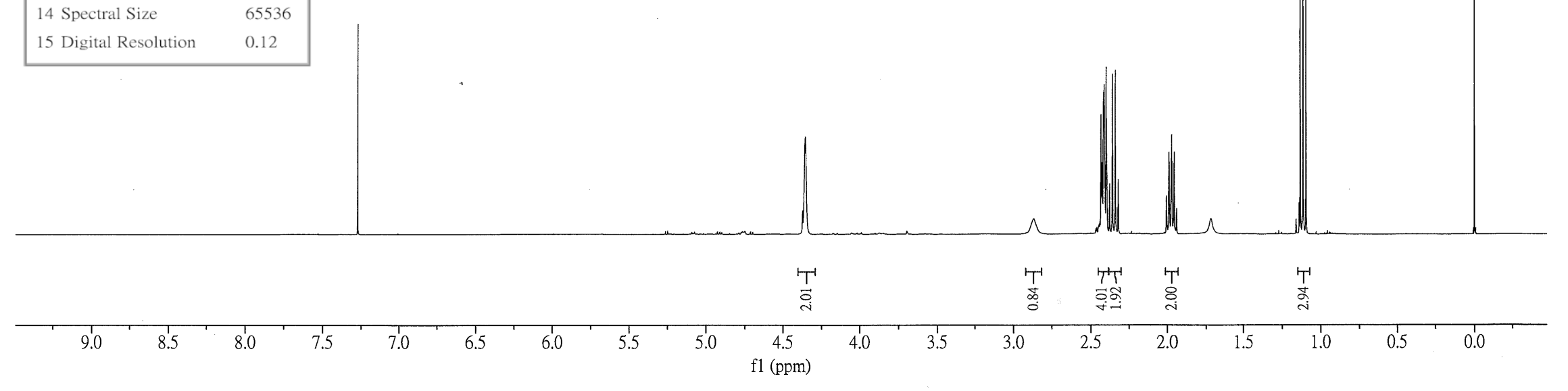

${ }^{1} \mathrm{H}$ NMR spectrum for compound $\mathbf{S 2}$ 


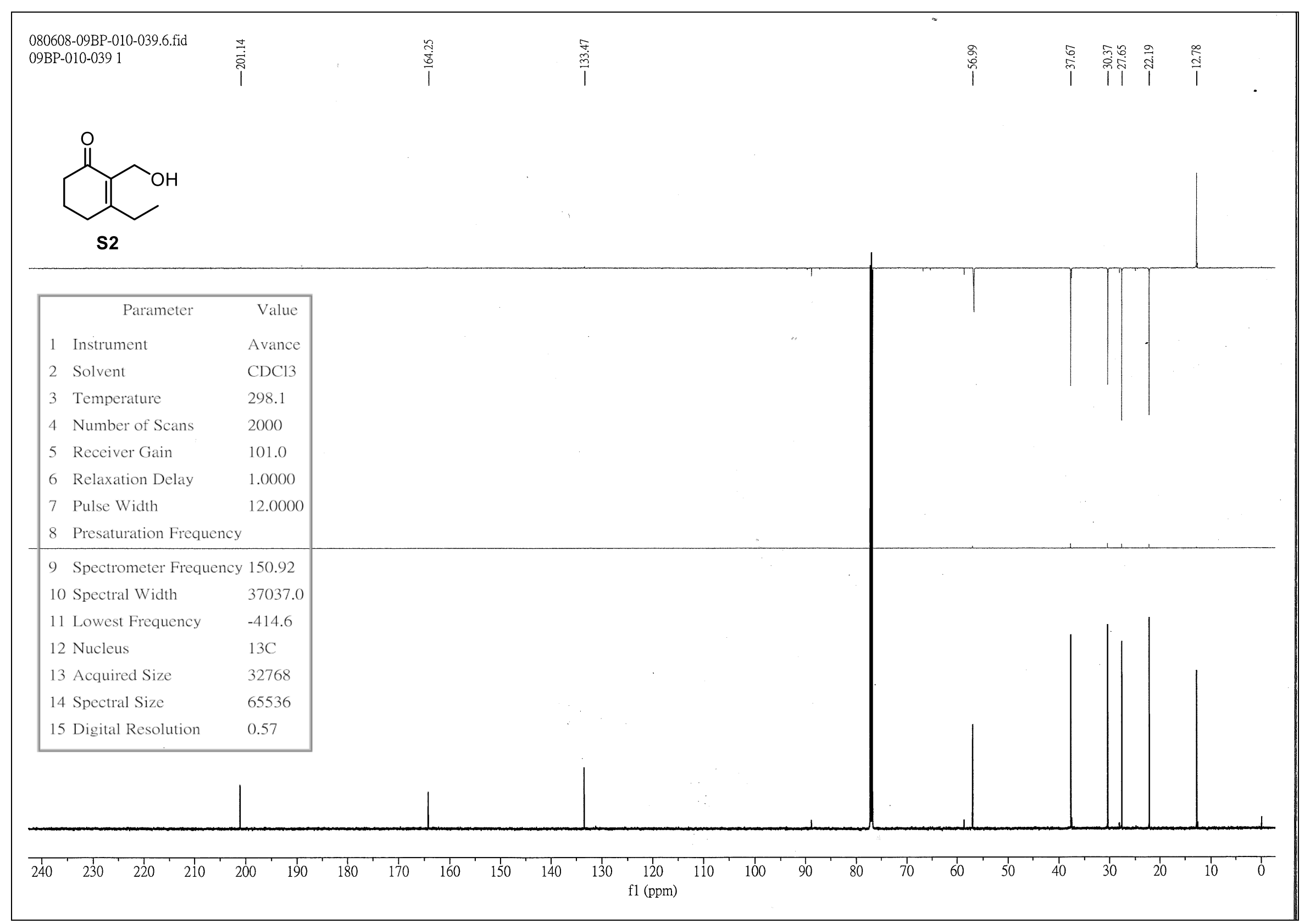

${ }^{13} \mathrm{C}$ NMR + DEPT spectra for compound $\mathbf{S 2}$ 


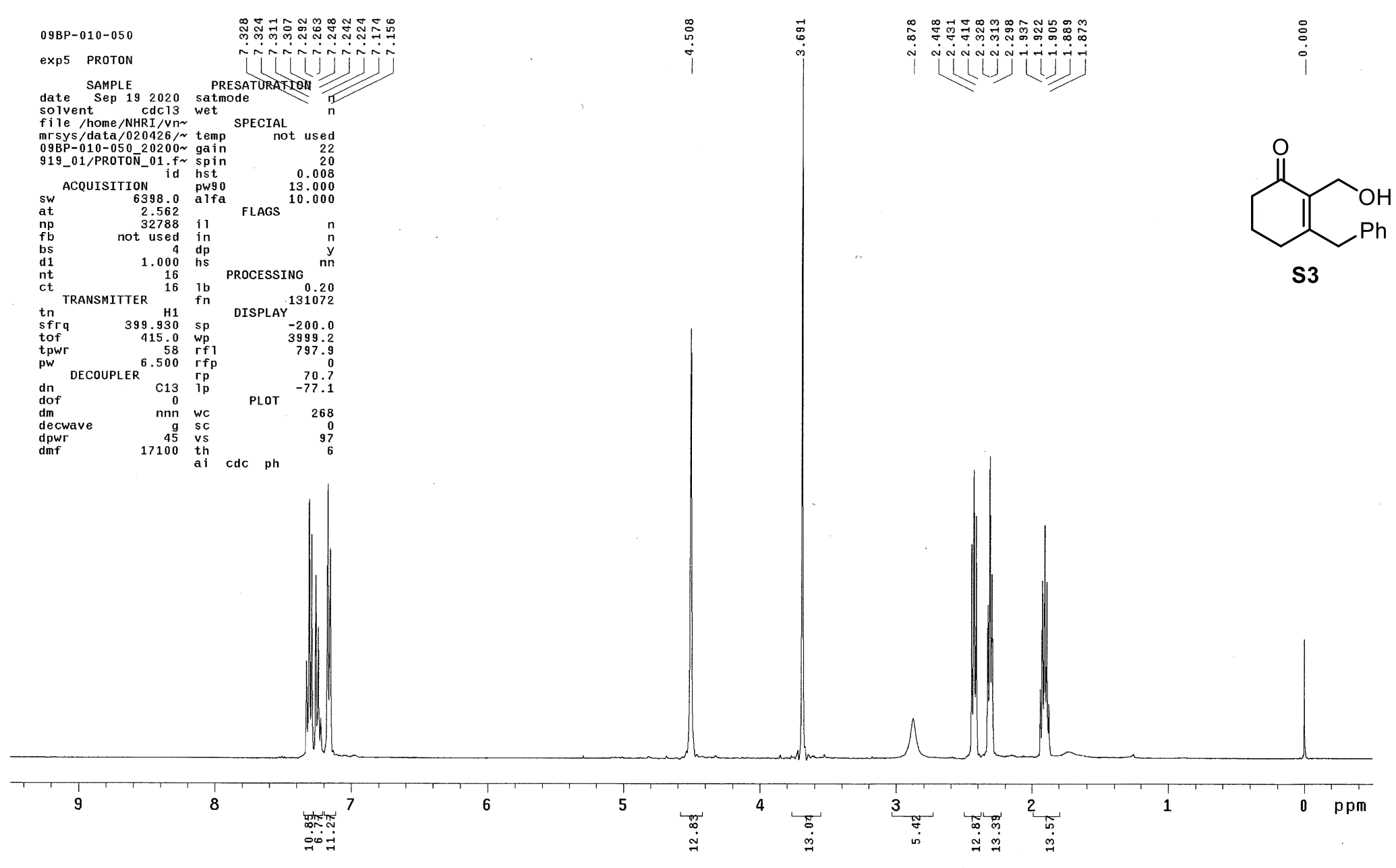

${ }^{1}$ H NMR spectrum for compound $\mathbf{S 3}$ 

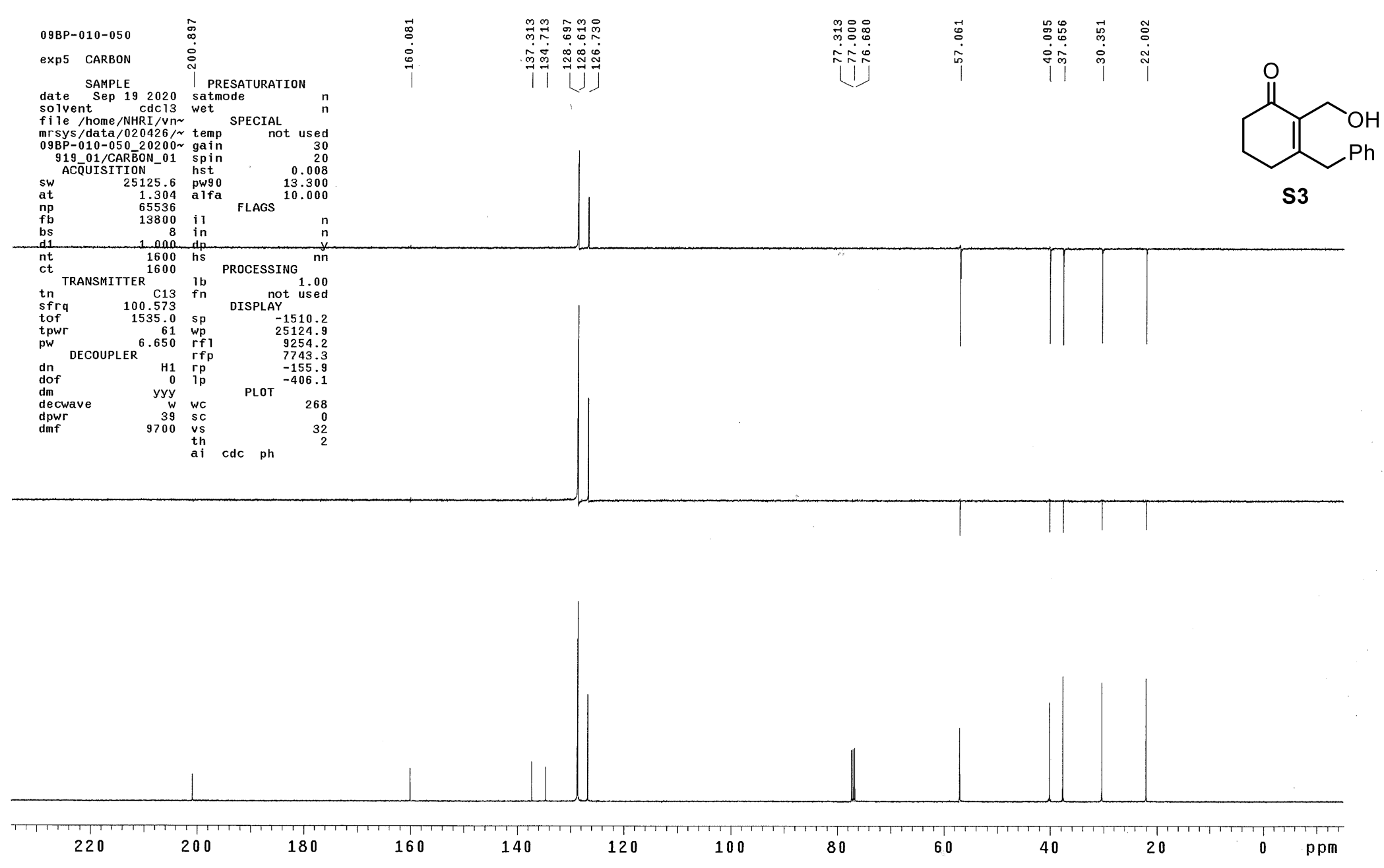

${ }^{13} \mathrm{C}$ NMR + DEPT spectra for compound $\mathbf{S 3}$ 


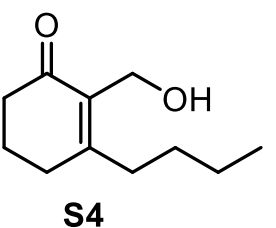

S4

\begin{tabular}{|lll|}
\hline & \multicolumn{1}{|c}{ Parameter } & \multicolumn{1}{c|}{ Value } \\
1 & Instrument & Avance \\
2 & Solvent & CDCl3 \\
3 & Temperature & 294.7 \\
4 & Number of Scans & 16 \\
5 & Receiver Gain & 53.1 \\
6 & Relaxation Delay & 1.0000 \\
7 & Pulse Width & 8.0000 \\
8 & Presaturation Frequency \\
9 & Spectrometer Frequency & 400.17 \\
10 & Spectral Width & 7812.5 \\
11 & Lowest Frequency & -1434.0 \\
12 & Nucleus & $1 \mathrm{H}$ \\
13 & Acquired Size & 32768 \\
14 & Spectral Size & 65536 \\
15 & Digital Resolution & 0.12 \\
\hline
\end{tabular}
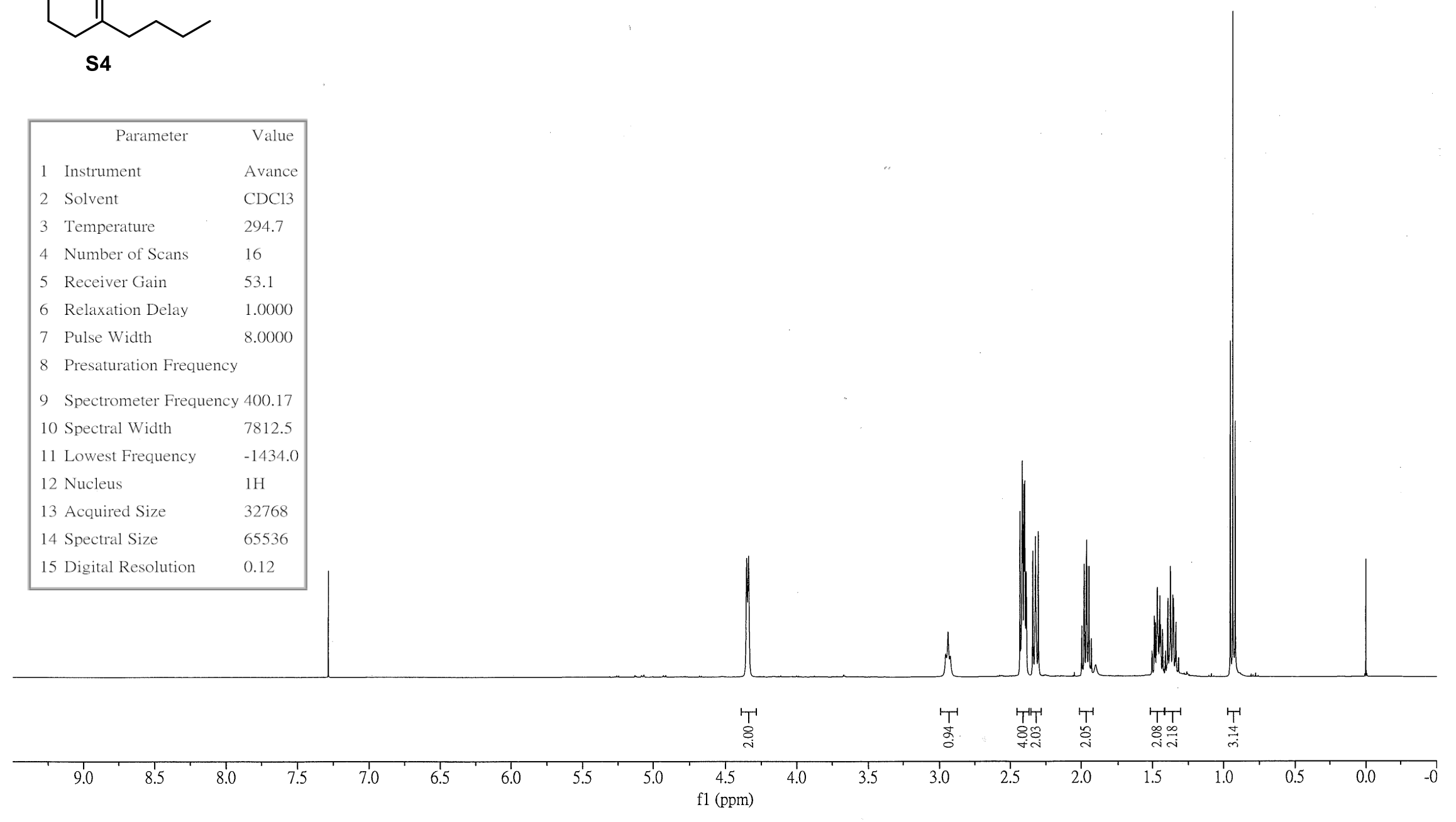

${ }^{1}$ H NMR spectrum for compound $\mathbf{S} 4$ 


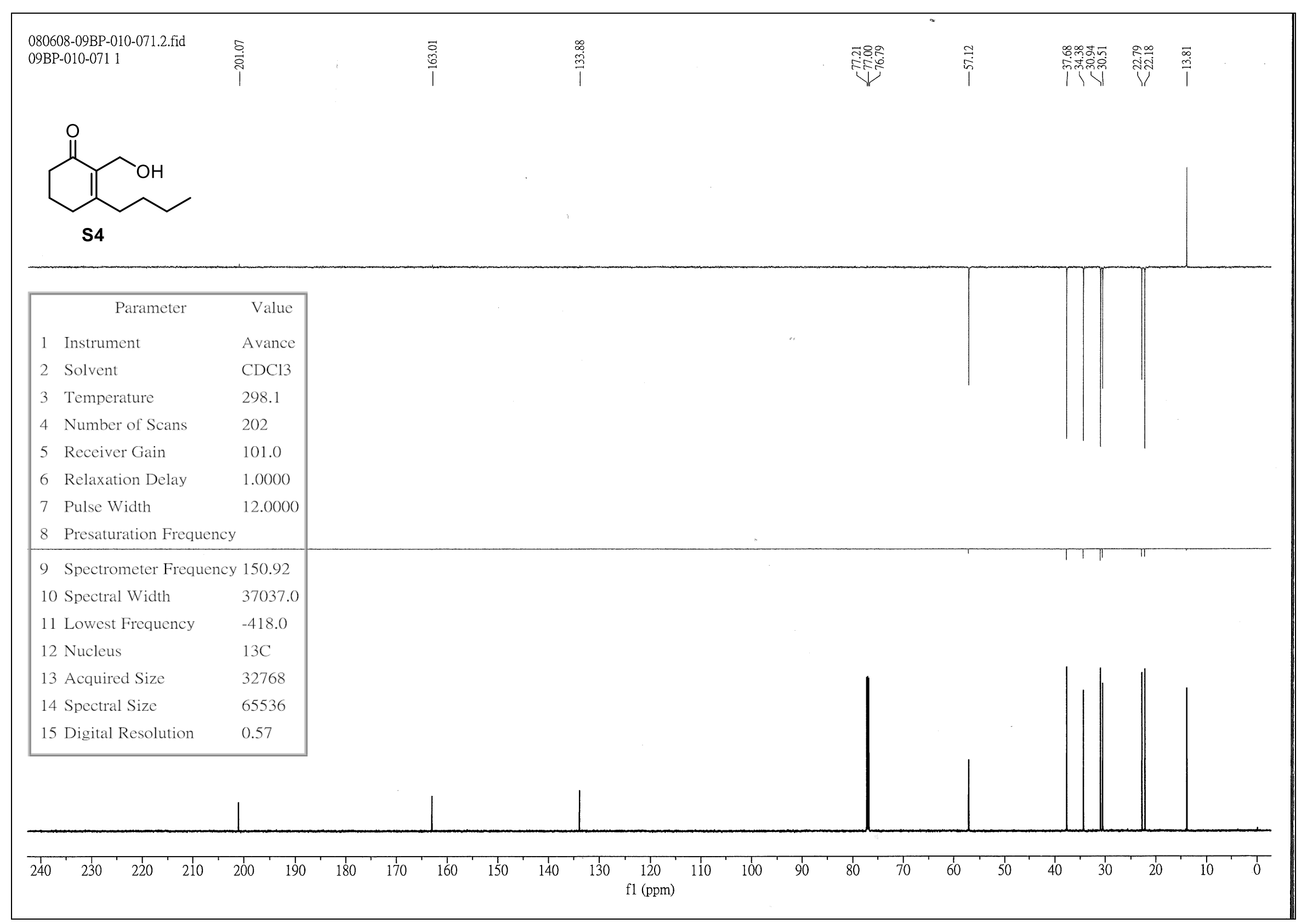

${ }^{13} \mathrm{C}$ NMR + DEPT spectra for compound S4 


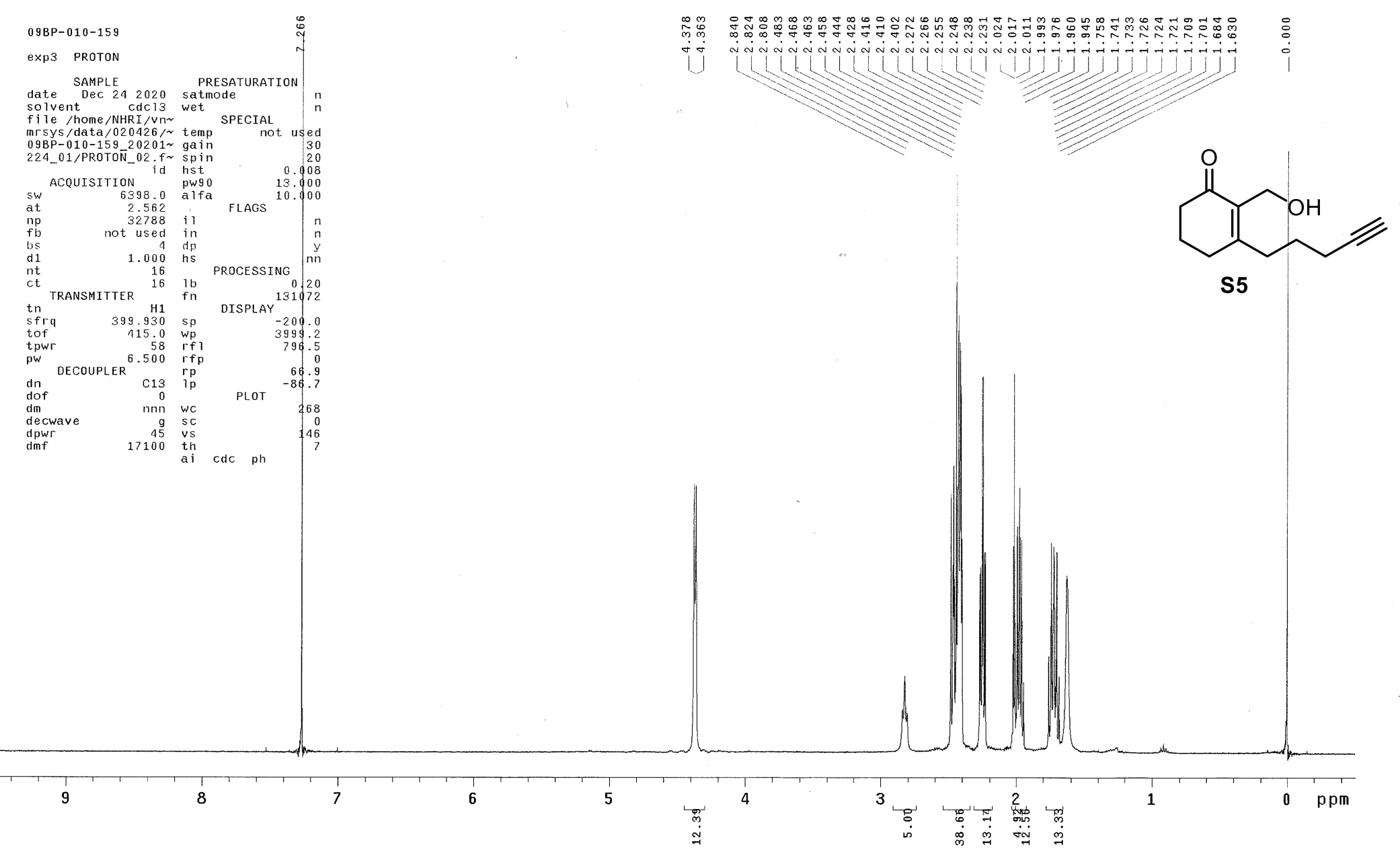

${ }^{1}$ H NMR spectrum for compound S5 

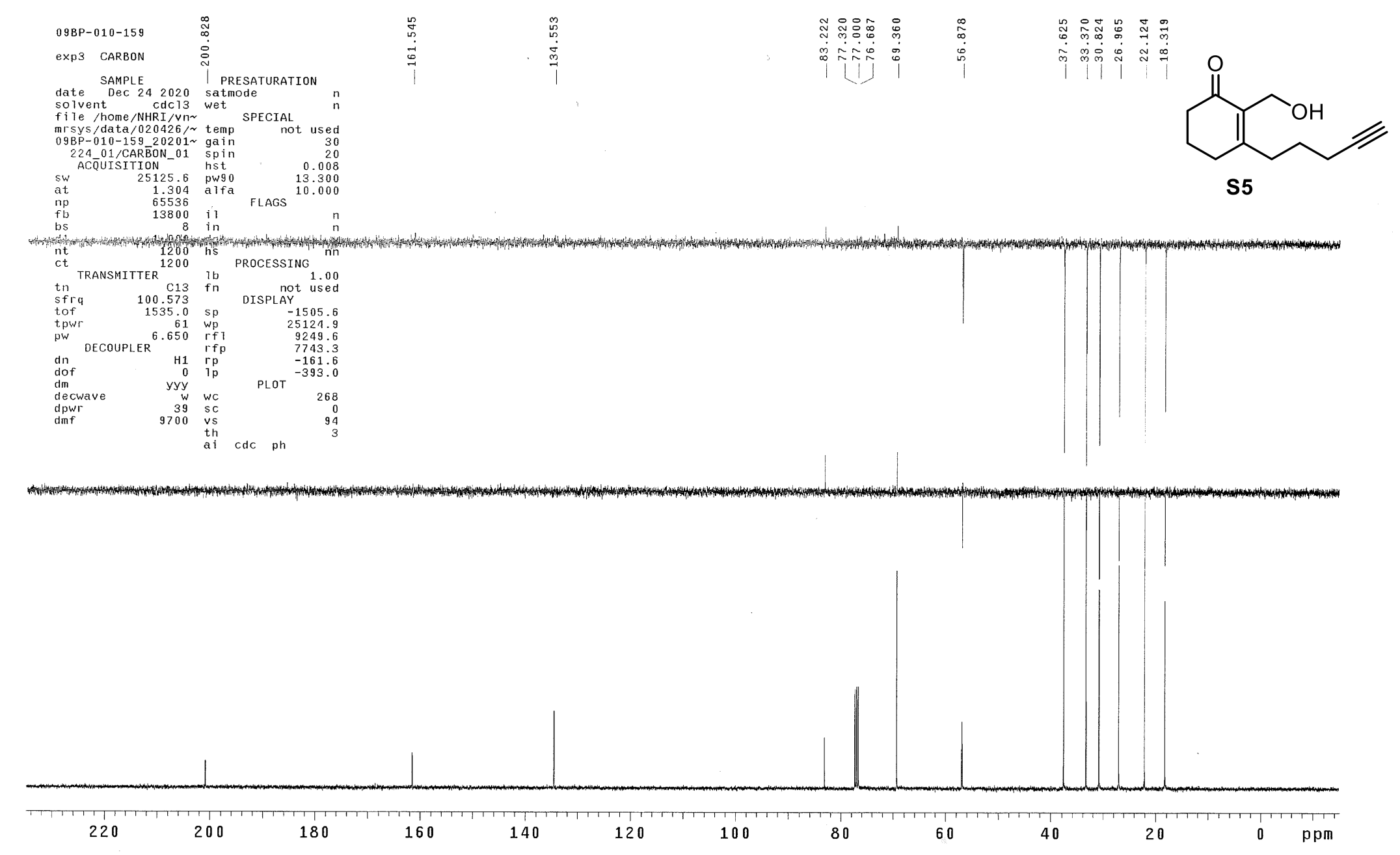

${ }^{13} \mathrm{C}$ NMR + DEPT spectra for compound S5 


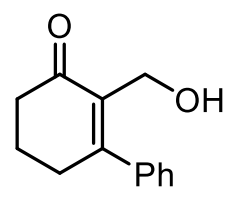

s6

\begin{tabular}{|lll|}
\hline \multicolumn{1}{|c}{ Parameter } & \multicolumn{1}{c}{ Value } \\
1 & Instrument & Avance \\
2 & Solvent & $\mathrm{CDCl} 3$ \\
3 & Temperature & 294.7 \\
4 & Number of Scans & 16 \\
5 & Receiver Gain & 101.0 \\
6 & Relaxation Delay & 1.0000 \\
7 & Pulse Width & 8.0000 \\
8 & Presaturation Frequency \\
9 & Spectrometer Frequency 400.17 \\
10 & Spectral Width & 7812.5 \\
11 & Lowest Frequency & -1441.4 \\
12 & Nucleus & $1 \mathrm{H}$ \\
13 & Acquired Size & 32768 \\
14 & Spectral Size & 65536 \\
15 & Digital Resolution & 0.12 \\
\hline
\end{tabular}
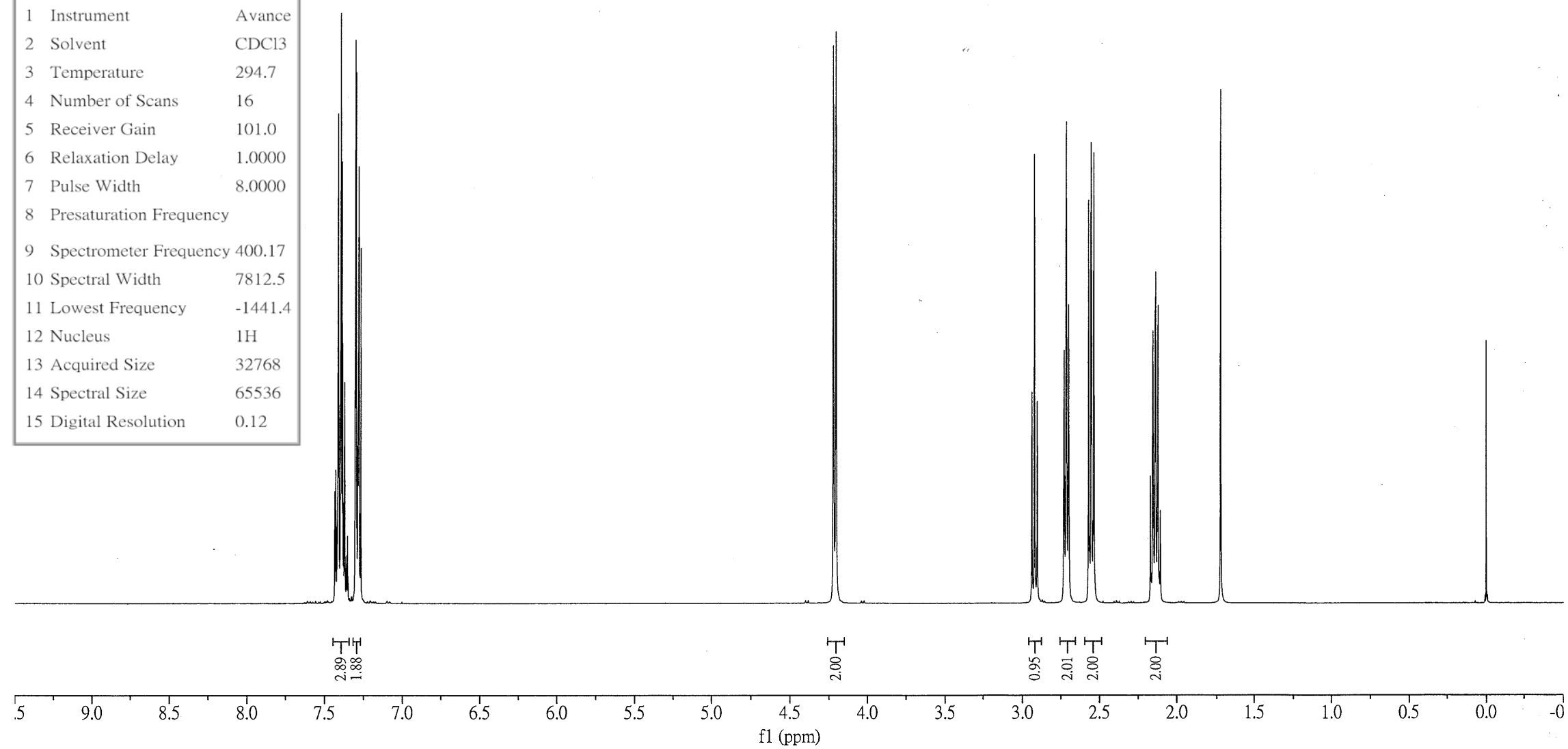

${ }^{1} \mathrm{H}$ NMR spectrum for compound S6 


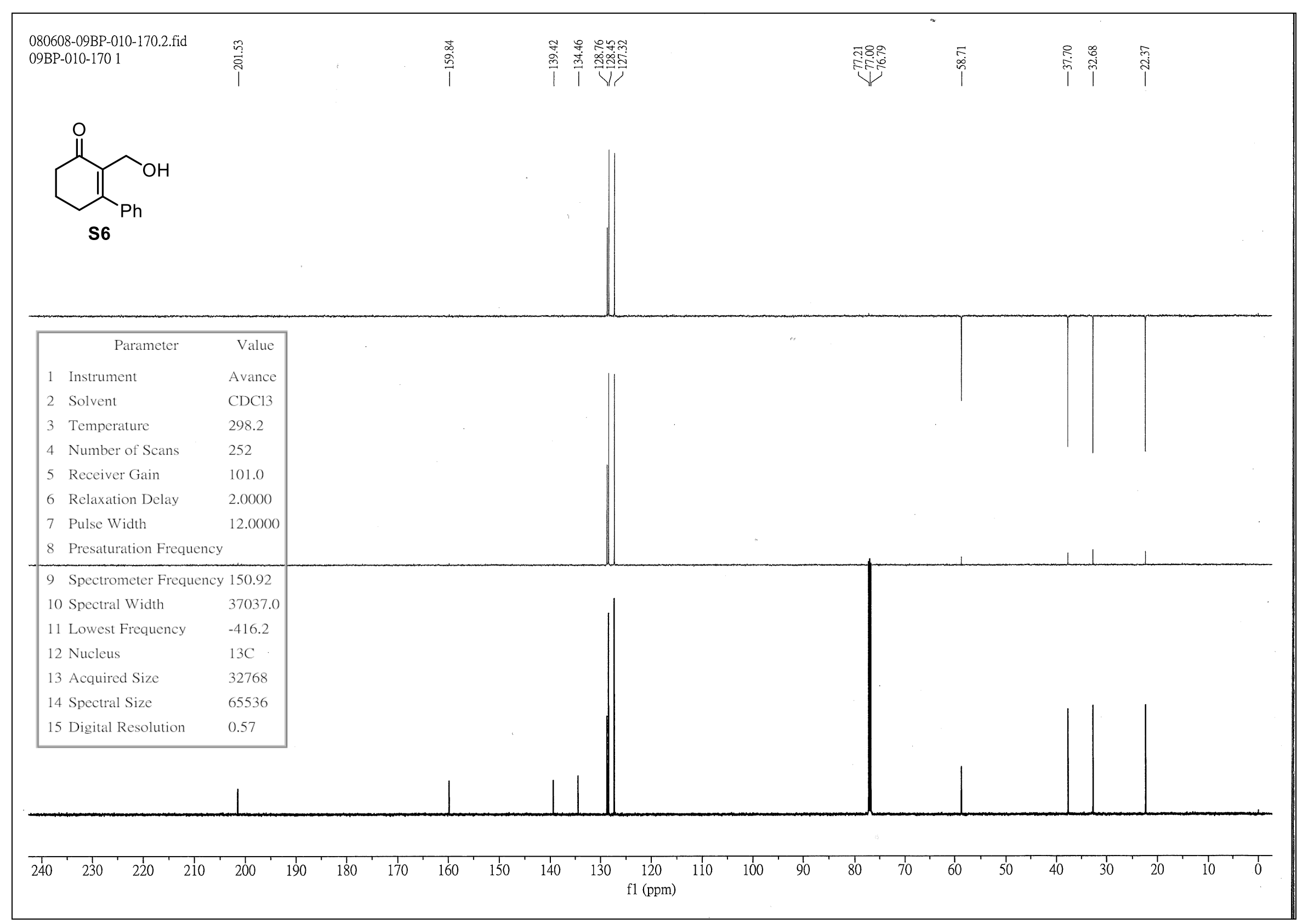

${ }^{13} \mathrm{C}$ NMR + DEPT spectra for compound S6 


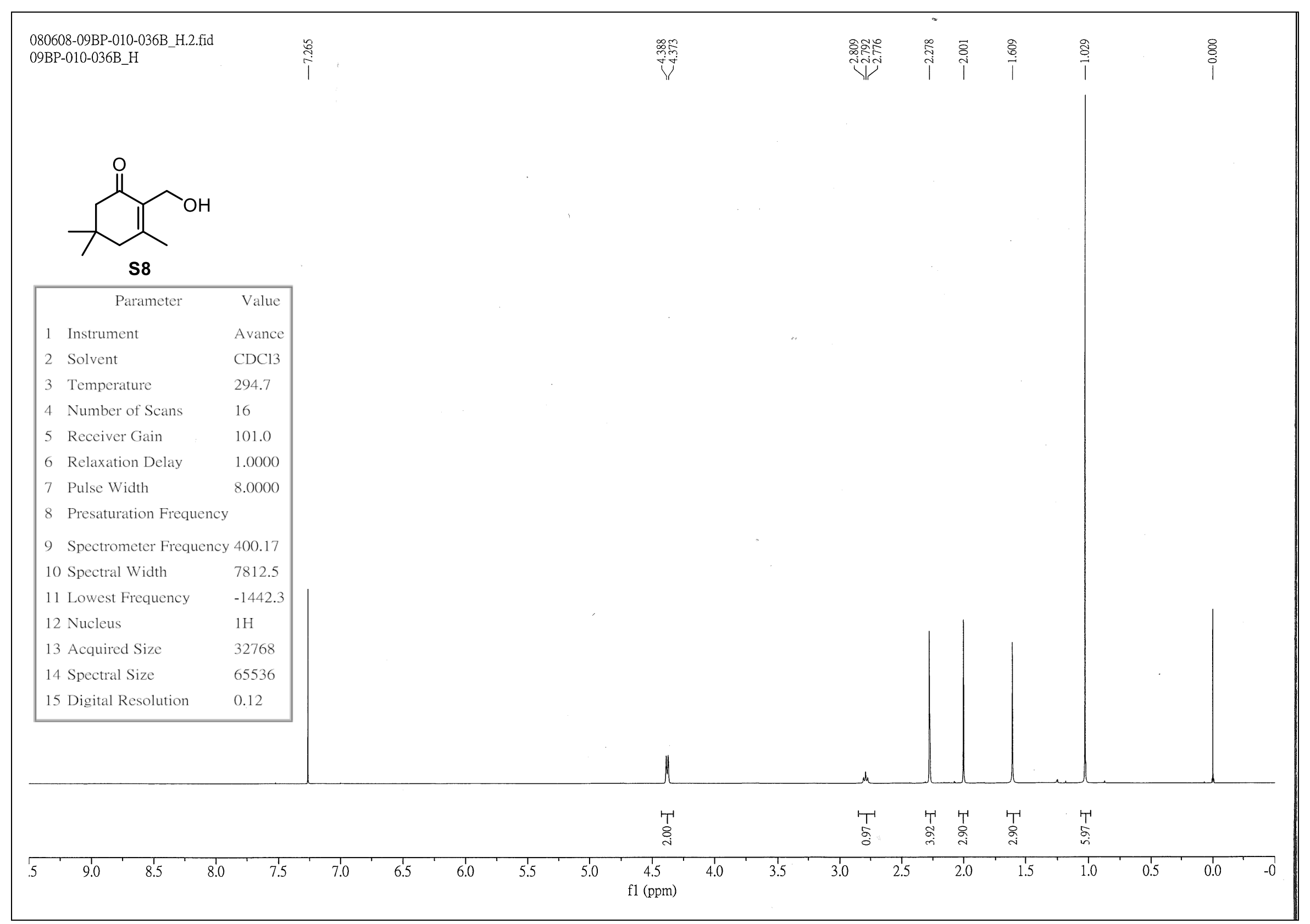

${ }^{1}$ H NMR spectrum for compound $\mathbf{S 8}$ 


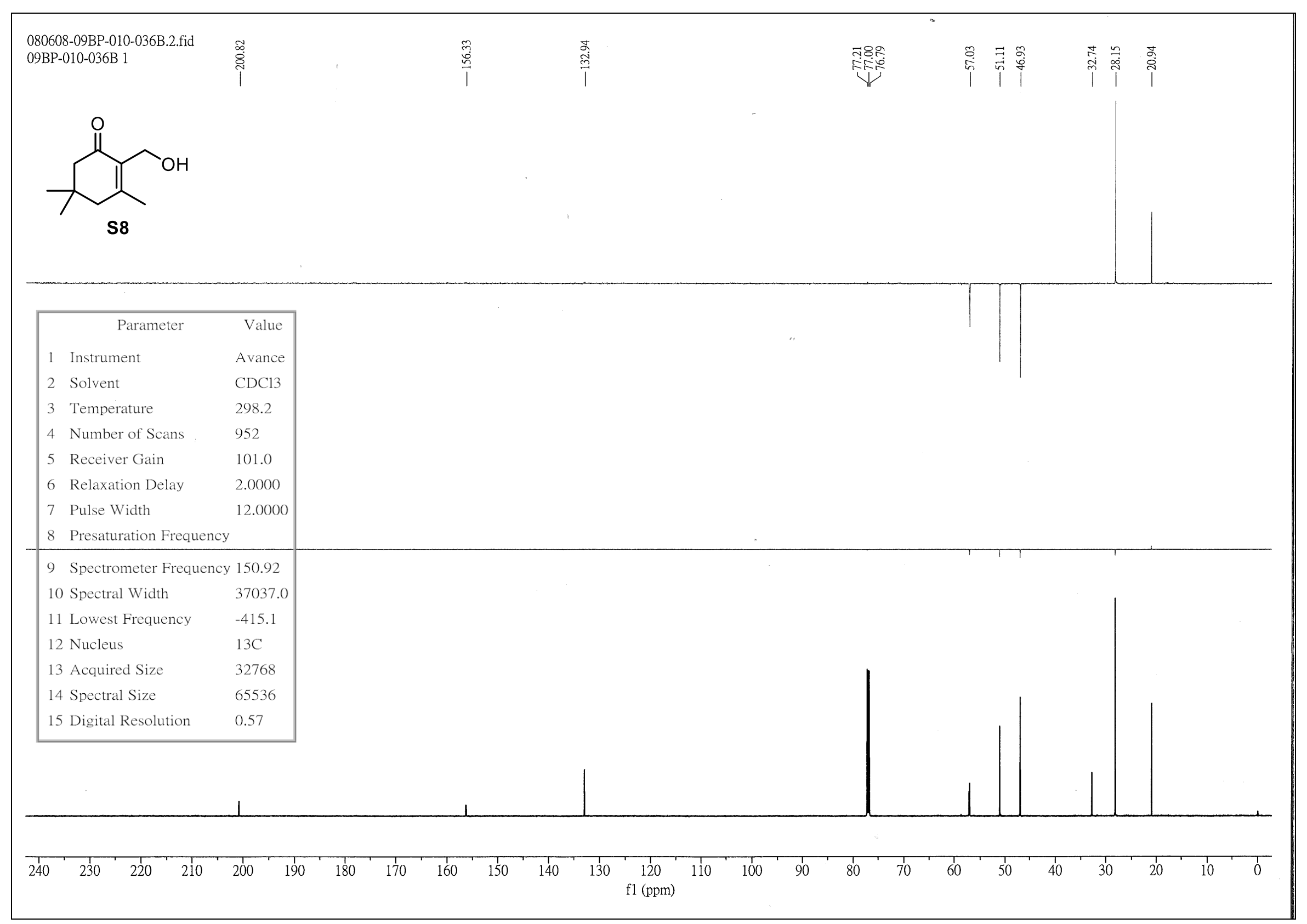

${ }^{13} \mathrm{C}$ NMR + DEPT spectra for compound S8 


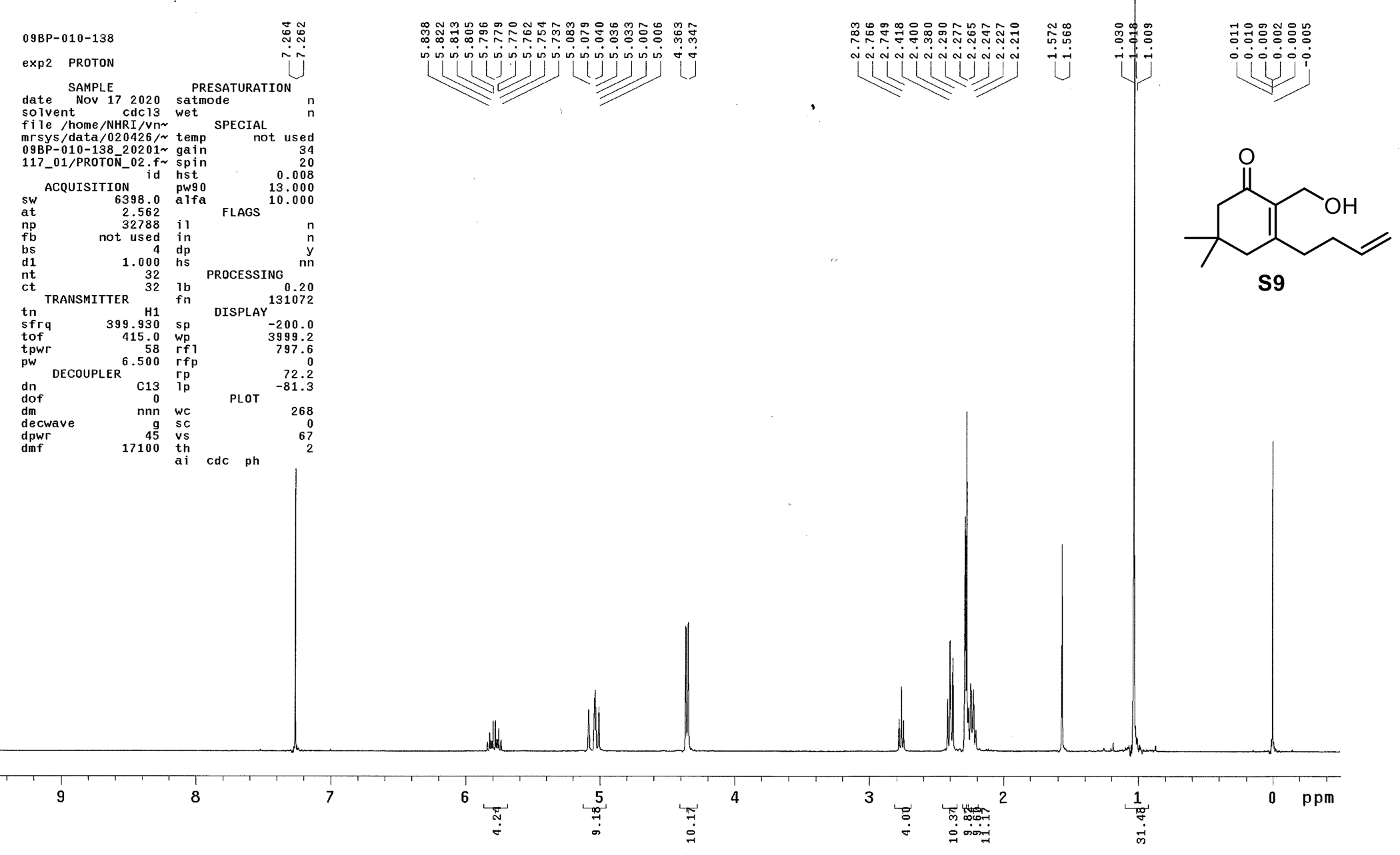

${ }^{1}$ H NMR spectrum for compound $\mathbf{S 9}$ 


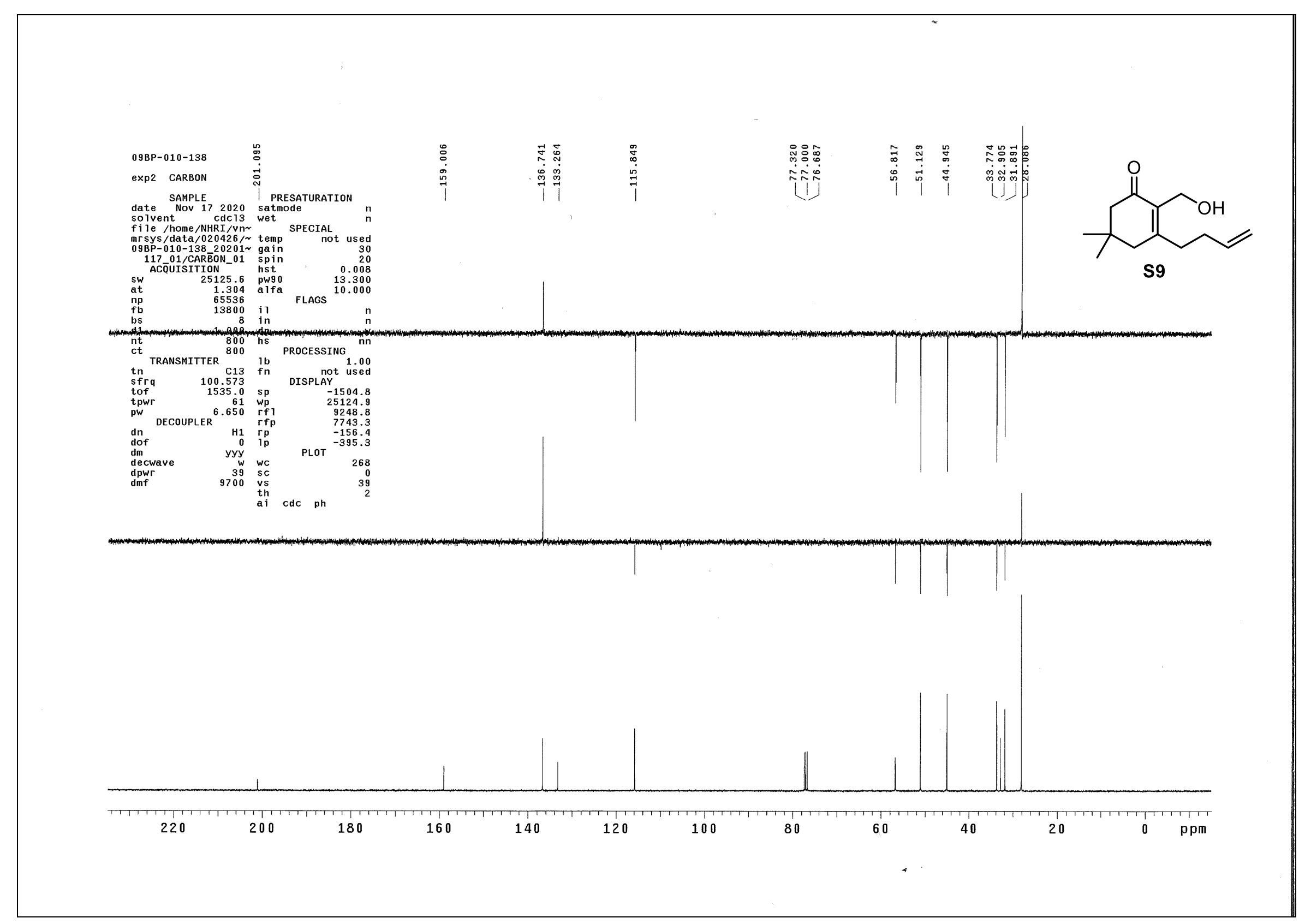

${ }^{13} \mathrm{C}$ NMR + DEPT spectra for compound S9 


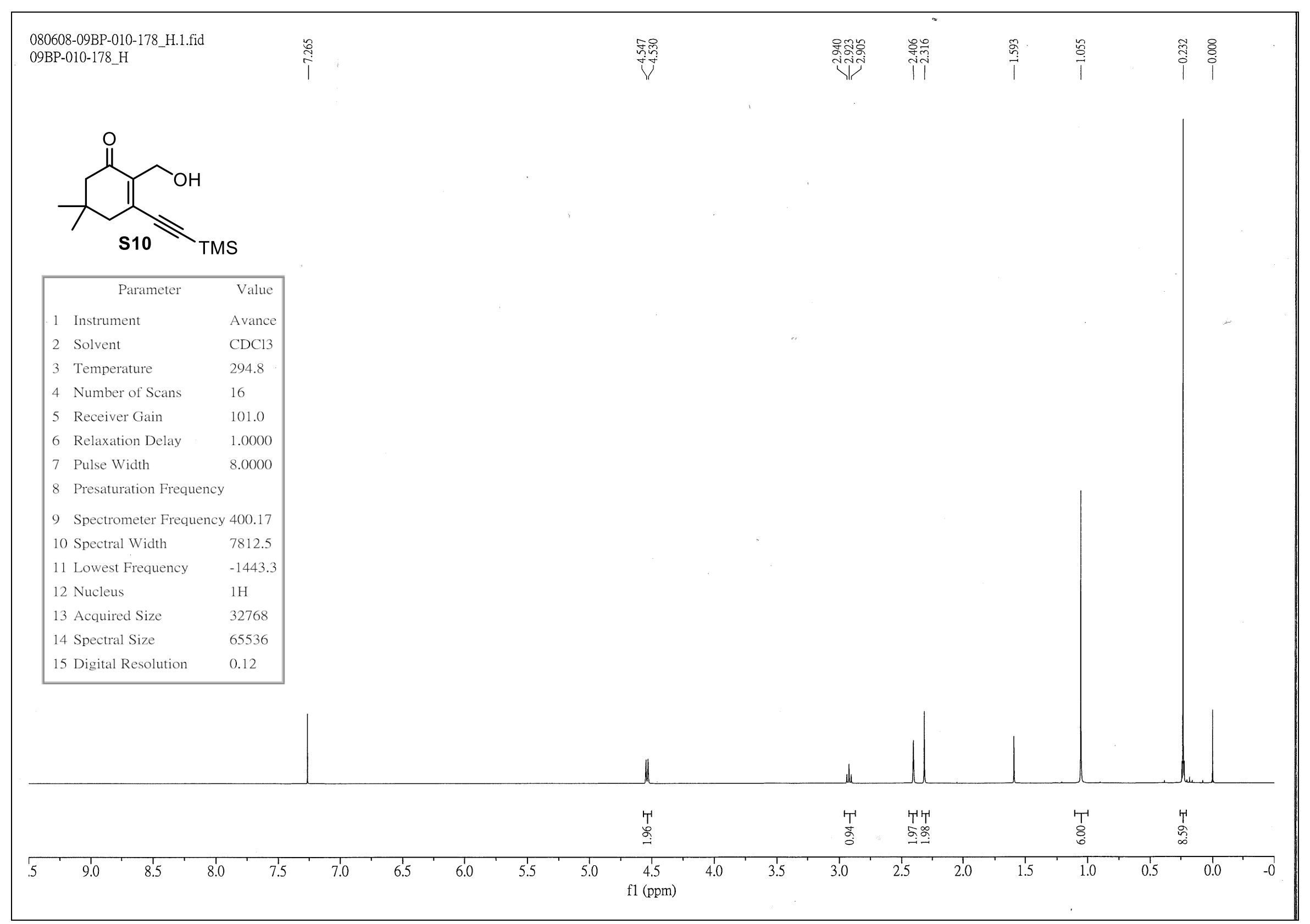

${ }^{1} \mathrm{H}$ NMR spectrum for compound S10 


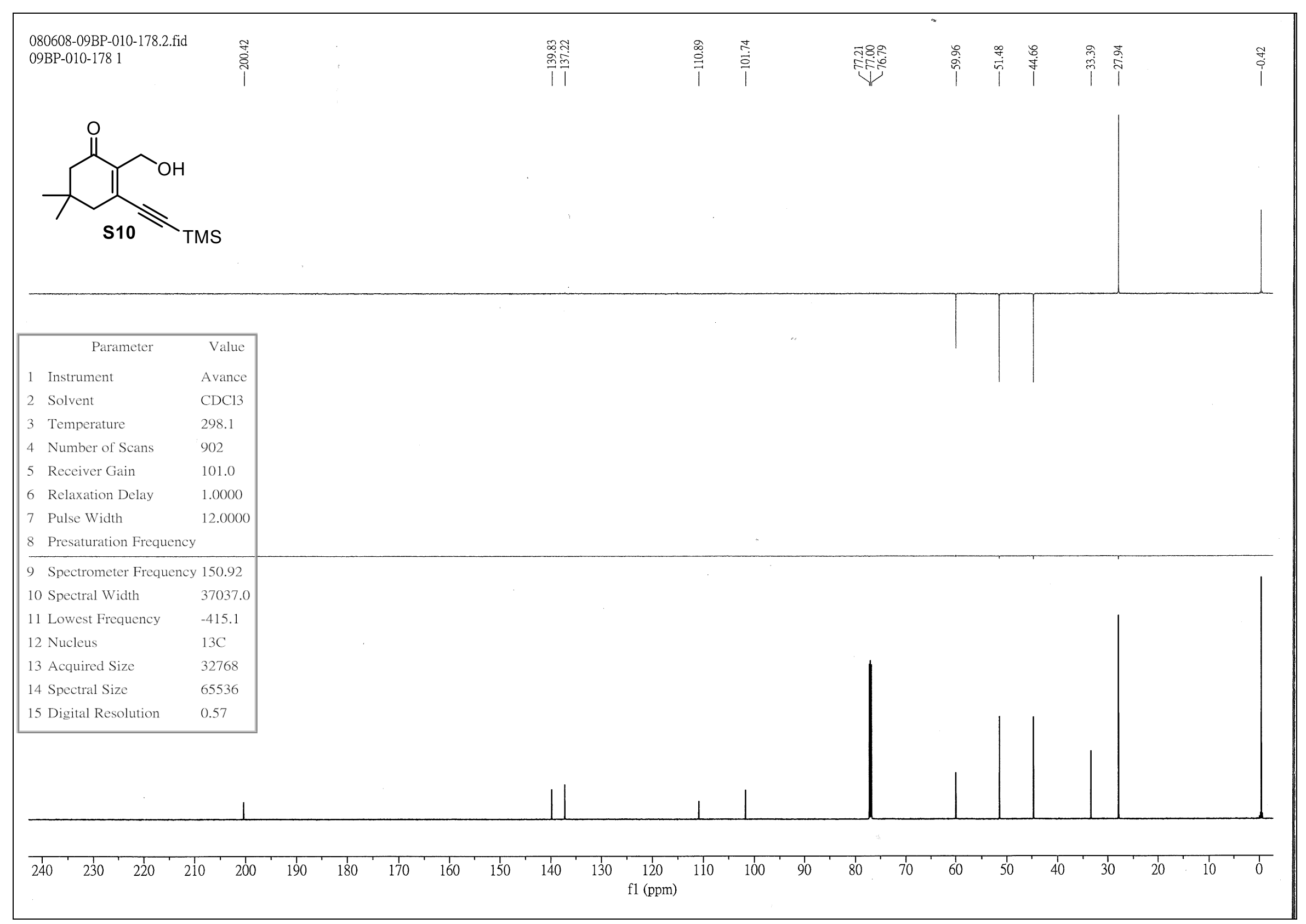

${ }^{13} \mathrm{C}$ NMR + DEPT spectra for compound $\mathbf{S 1 0}$ 


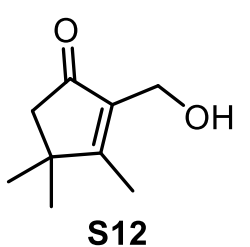

s12

\begin{tabular}{|lll|}
\hline \multicolumn{1}{|c}{ Parameter } & \multicolumn{1}{c|}{ Value } \\
1 & Instrument & Avance \\
2 & Solvent & $\mathrm{CDCl} 3$ \\
3 & Temperature & 294.7 \\
4 & Number of Scans & 16 \\
5 & Receiver Gain & 101.0 \\
6 & Relaxation Delay & 1.0000 \\
7 & Pulse Width & 8.0000 \\
8 & Presaturation Frequency \\
9 & Spectrometer Frequency & 400.17 \\
10 & Spectral Width & 7812.5 \\
11 & Lowest Frequency & -1440.3 \\
12 & Nucleus & $1 \mathrm{H}$ \\
13 & Acquired Size & 32768 \\
14 & Spectral Size & 65536 \\
15 & Digital Resolution & 0.12 \\
\hline
\end{tabular}

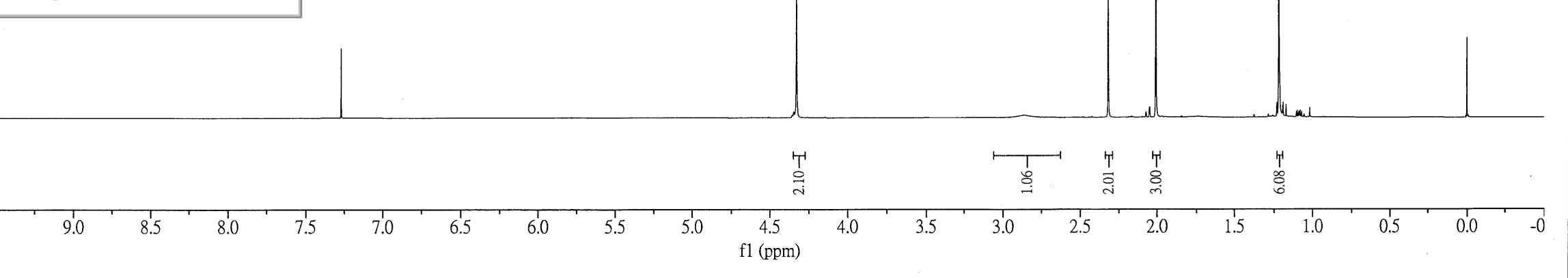

${ }^{1} \mathrm{H}$ NMR spectrum for compound $\mathbf{S 1 2}$ 


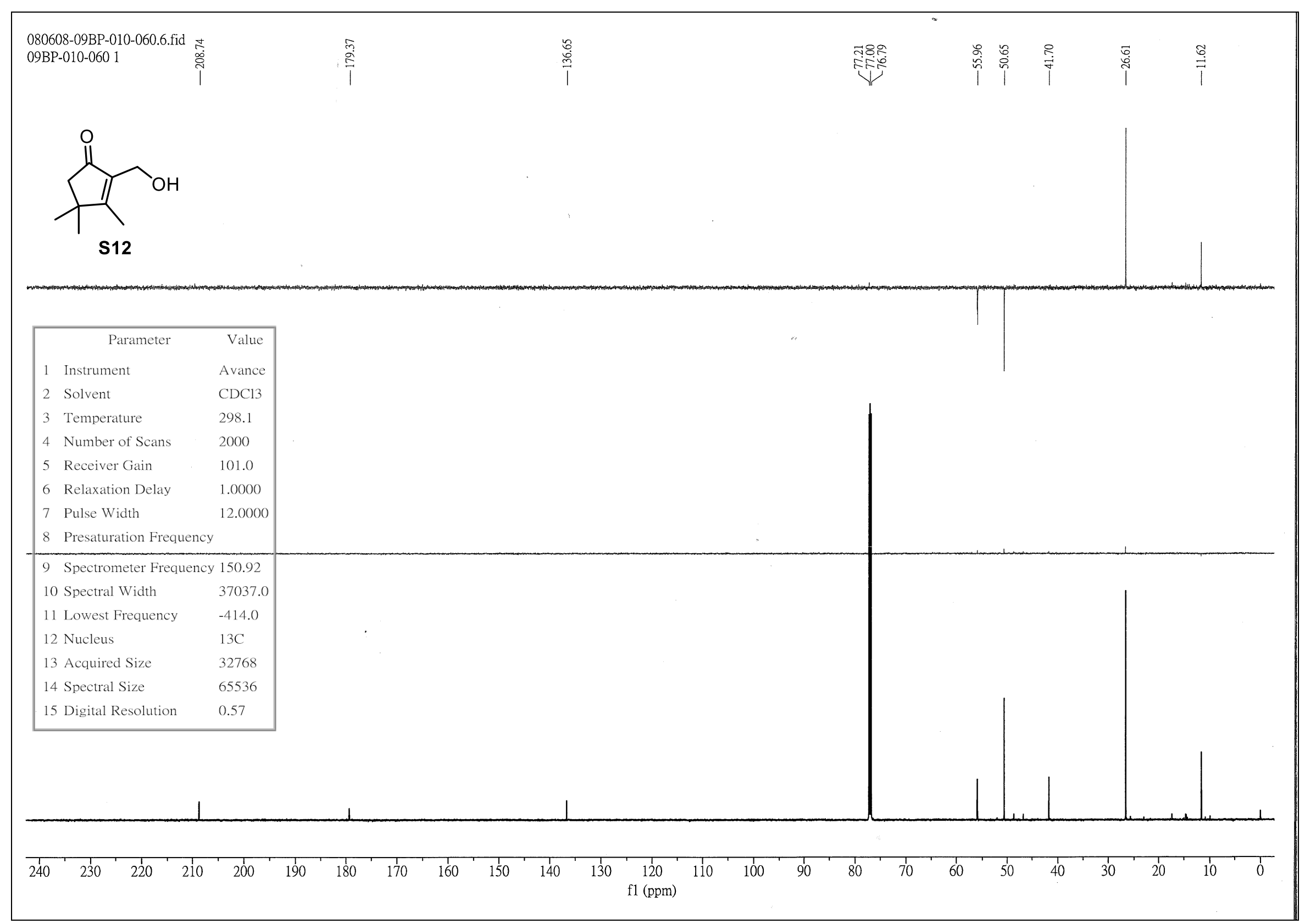

${ }^{13} \mathrm{C}$ NMR + DEPT spectra for compound $\mathbf{S 1 2}$ 


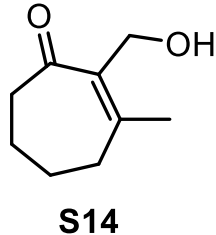

\begin{tabular}{|lll|}
\hline \multicolumn{1}{|c|}{ Parameter } & \multicolumn{1}{c|}{ Value } \\
1 & Instrument & Avance \\
2 & Solvent & $\mathrm{CDCl3}$ \\
3 & Temperature & 294.7 \\
4 & Number of Scans & 16 \\
5 & Receiver Gain & 101.0 \\
6 & Relaxation Delay & 1.0000 \\
7 & Pulse Width & 8.0000 \\
8 & Presaturation Frequency \\
9 & Spectrometer Frequency 400.17 \\
10 & Spectral Width & 7812.5 \\
11 & Lowest Frequency & -1443.5 \\
12 & Nucleus & $1 \mathrm{H}$ \\
13 & Acquired Size & 32768 \\
14 & Spectral Size & 65536 \\
15 & Digital Resolution & 0.12 \\
\hline
\end{tabular}

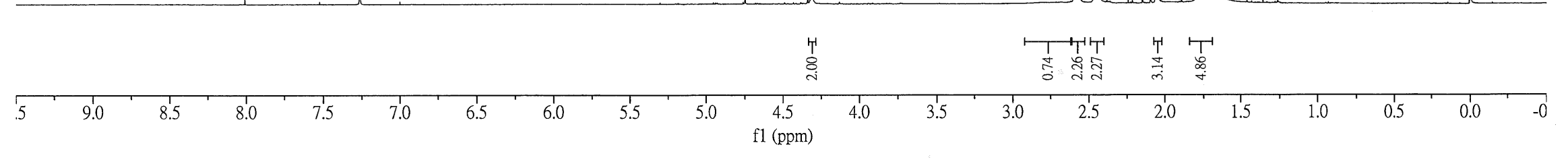

${ }^{1} \mathrm{H}$ NMR spectrum for compound $\mathbf{S 1 4}$ 


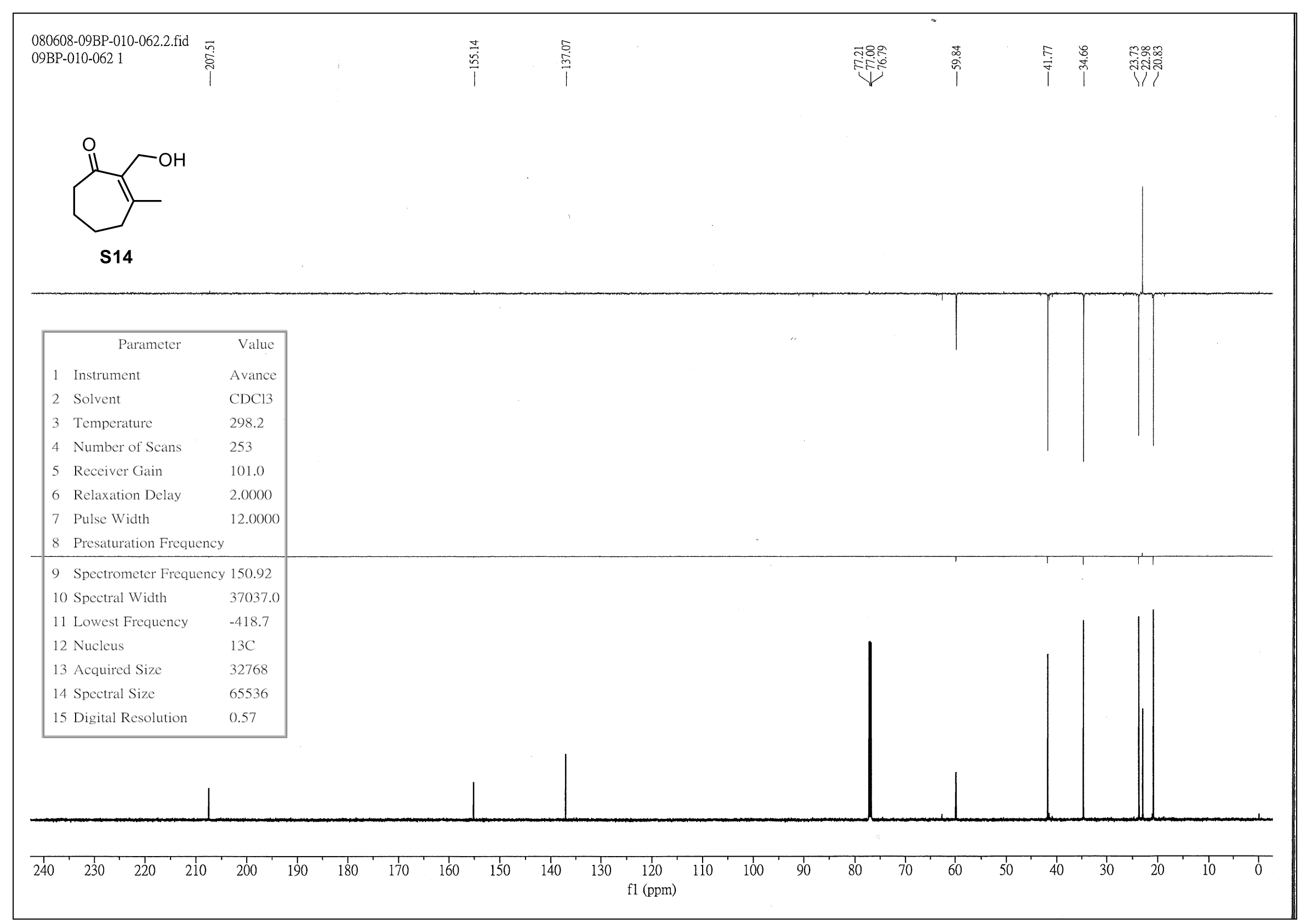

${ }^{13} \mathrm{C}$ NMR + DEPT spectra for compound S14 


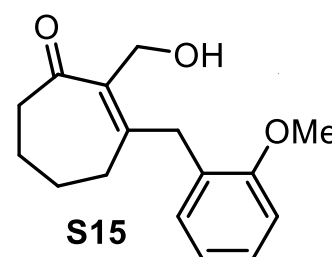

\begin{tabular}{|lll|}
\hline & \multicolumn{1}{|c}{ Parameter } & \multicolumn{1}{c|}{ Value } \\
1 & Instrument & Avance \\
2 & Solvent & CDCl3 \\
3 & Temperature & 294.7 \\
4 & Number of Scans & 16 \\
5 & Receiver Gain & 101.0 \\
6 & Relaxation Delay & 1.0000 \\
7 & Pulse Width & 8.0000 \\
8 & Presaturation Frequency \\
9 & Spectrometer Frequency & 400.17 \\
10 & Spectral Width & 7812.5 \\
11 & Lowest Frequency & -1442.9 \\
12 & Nucleus & $1 \mathrm{H}$ \\
13 & Acquired Size & 32768 \\
14 & Spectral Size & 65536 \\
15 & Digital Resolution & 0.12 \\
\hline
\end{tabular}
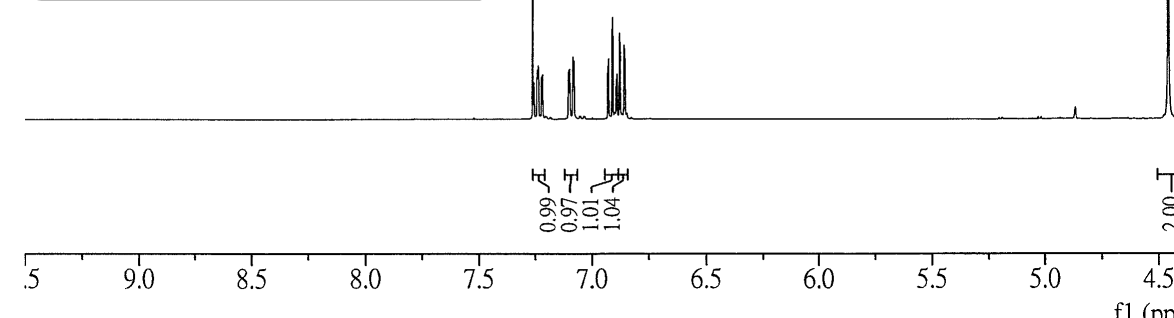

${ }^{1} \mathrm{H}$ NMR spectrum for compound $\mathbf{S 1 5}$ 


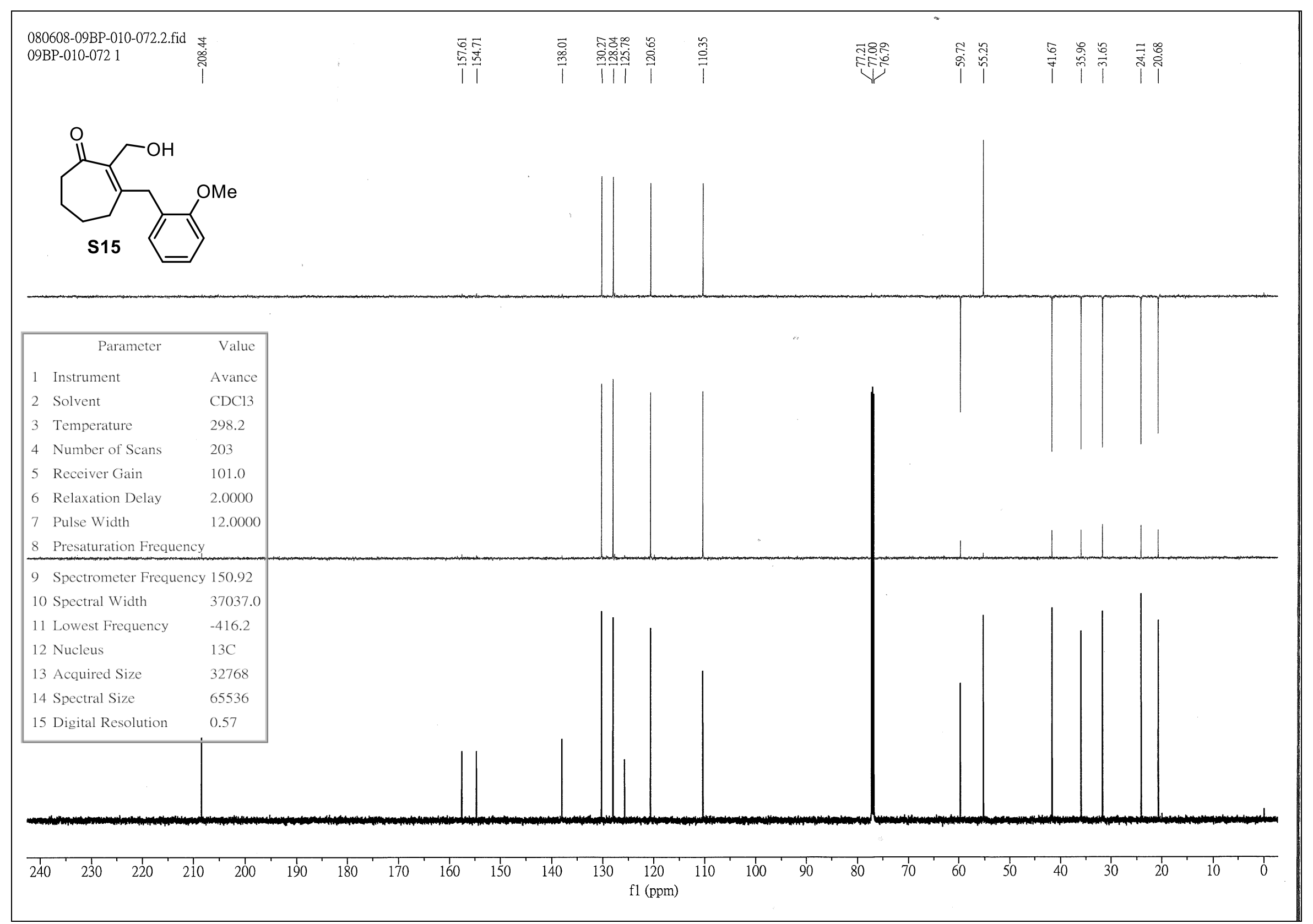

${ }^{13} \mathrm{C}$ NMR + DEPT spectra for compound $\mathbf{S 1 5}$ 


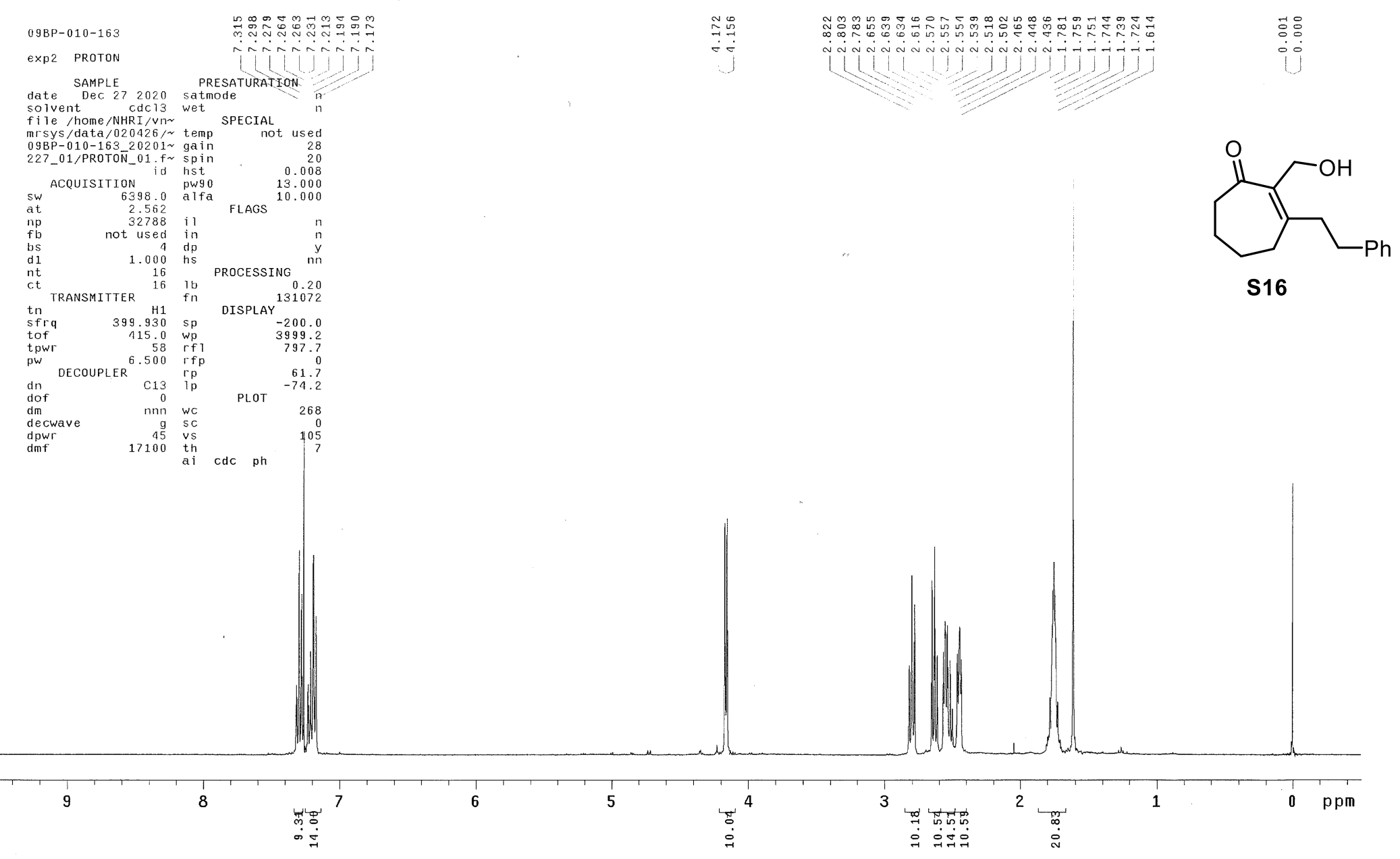

${ }^{1} \mathrm{H}$ NMR spectrum for compound $\mathbf{S 1 6}$ 

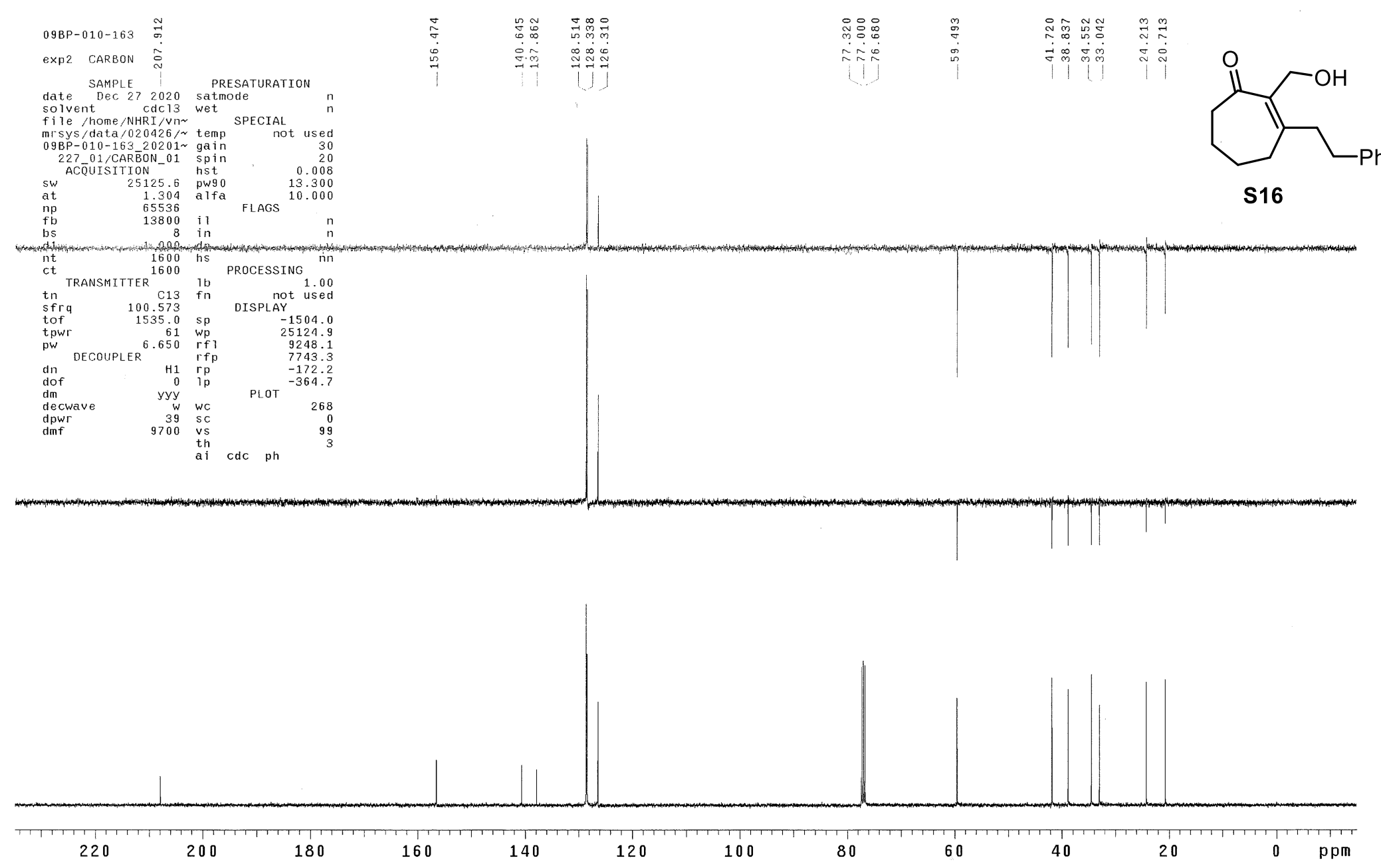

${ }^{13} \mathrm{C}$ NMR + DEPT spectra for compound S16 


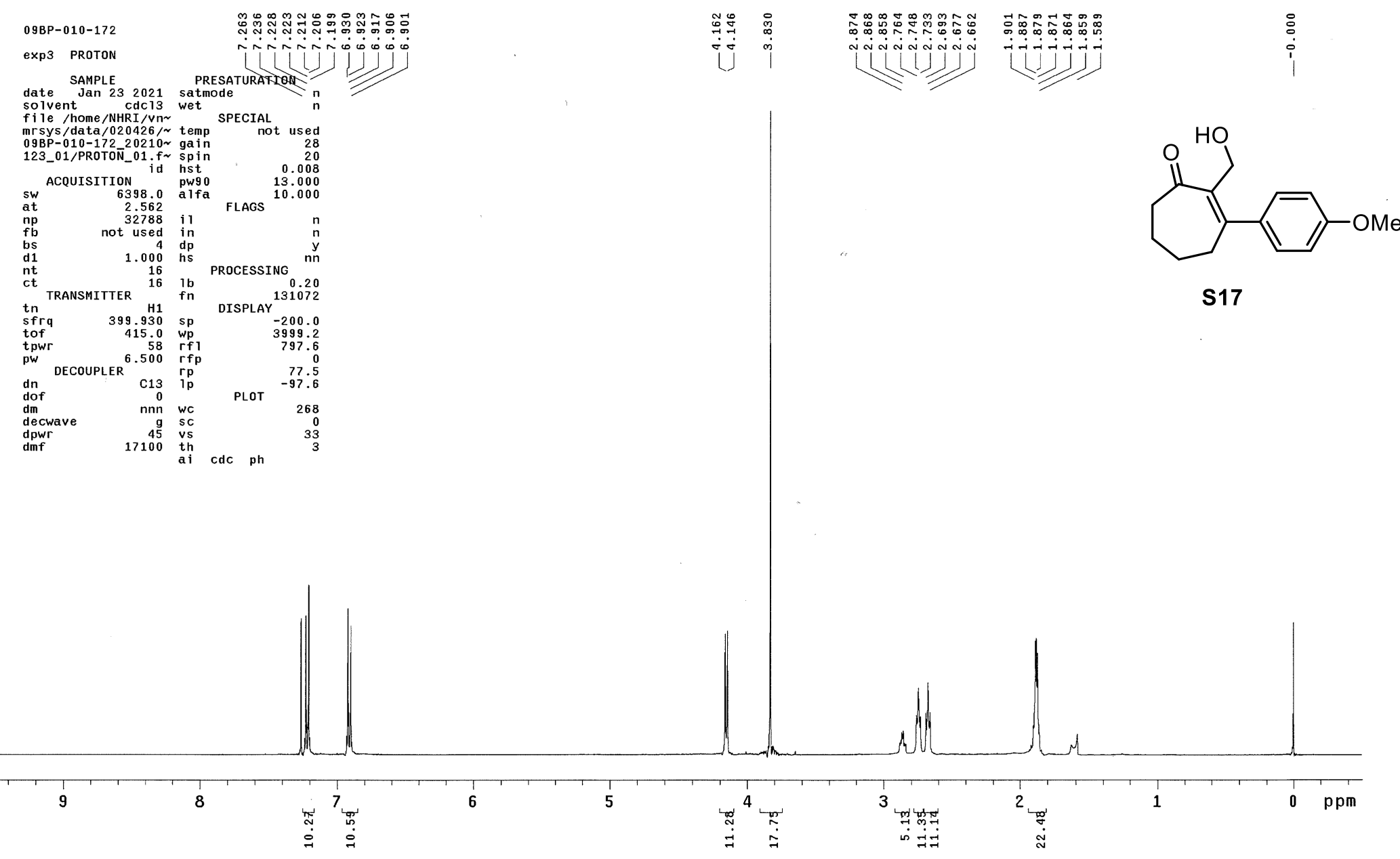

${ }^{1} \mathrm{H}$ NMR spectrum for compound $\mathbf{S 1 7}$ 


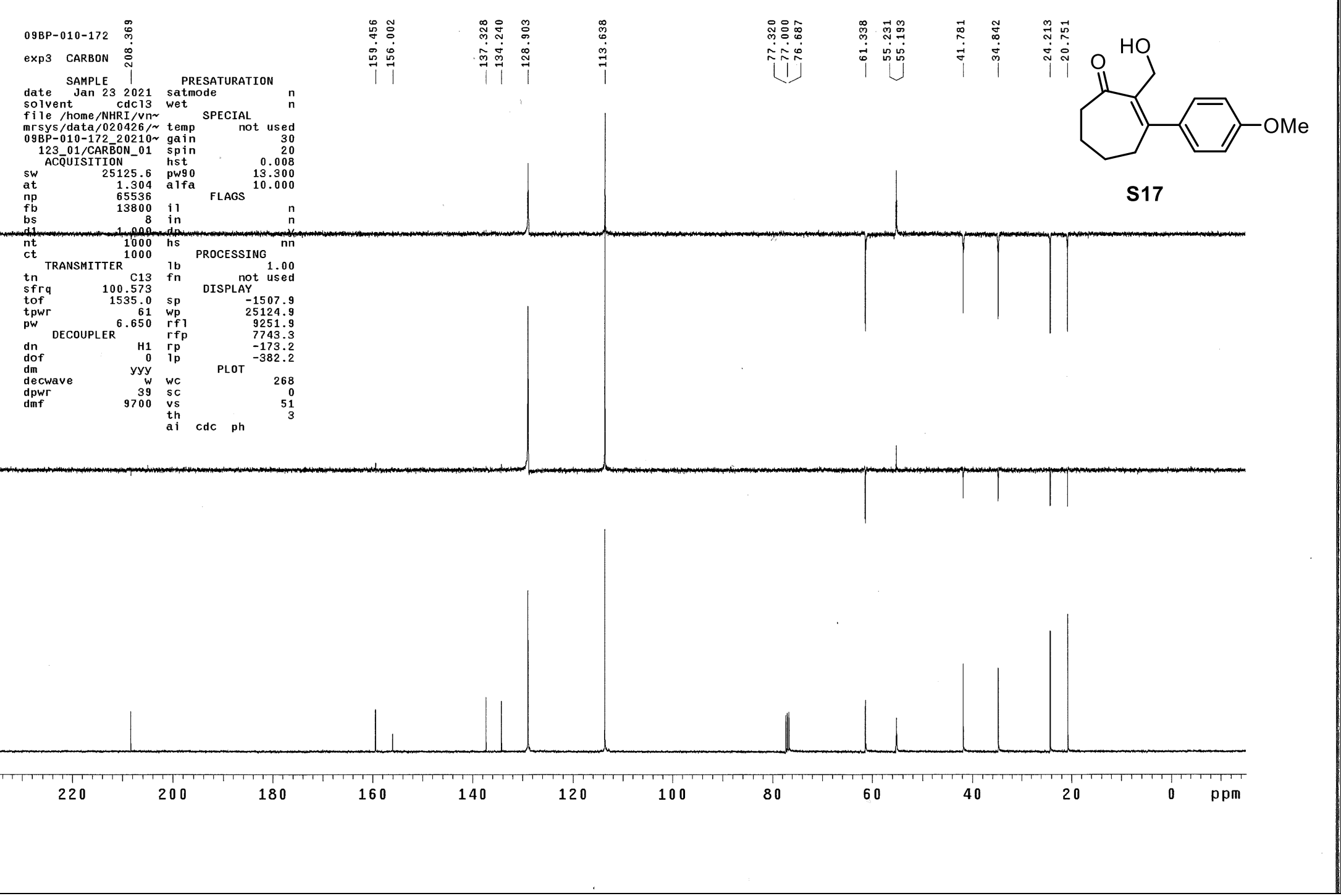

${ }^{13} \mathrm{C}$ NMR + DEPT spectra for compound $\mathbf{S 1 7}$ 


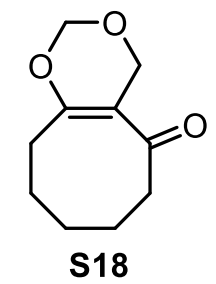

\begin{tabular}{|lll|}
\hline & \multicolumn{1}{|c}{ Parameter } & \multicolumn{1}{c|}{ Value } \\
1 & Instrument & Avance \\
2 & Solvent & CDCl3 \\
3 & Temperature & 294.7 \\
4 & Number of Scans & 16 \\
5 & Receiver Gain & 101.0 \\
6 & Relaxation Delay & 1.0000 \\
7 & Pulse Width & 8.0000 \\
8 & Presaturation Frequency \\
9 & Spectrometer Frequency 400.17 \\
10 & Spectral Width & 7812.5 \\
11 & Lowest Frequency & -1442.0 \\
12 & Nucleus & $1 \mathrm{H}$ \\
13 & Acquired Size & 32768 \\
14 & Spectral Size & 65536 \\
15 & Digital Resolution & 0.12 \\
\hline
\end{tabular}
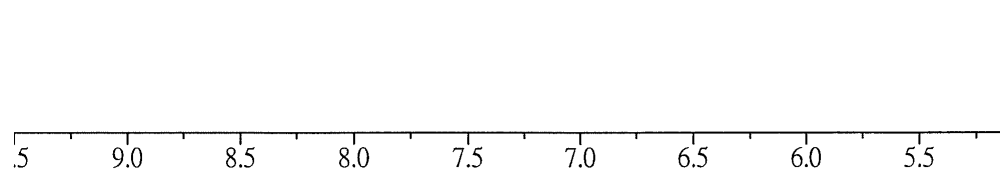

$5.5 \quad 5.0$

\begin{tabular}{|c|}
\hline 4.5 \\
\hline
\end{tabular}

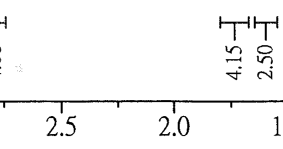
员

${ }^{1}$ H NMR spectrum for compound $\mathbf{S 1 8}$ 


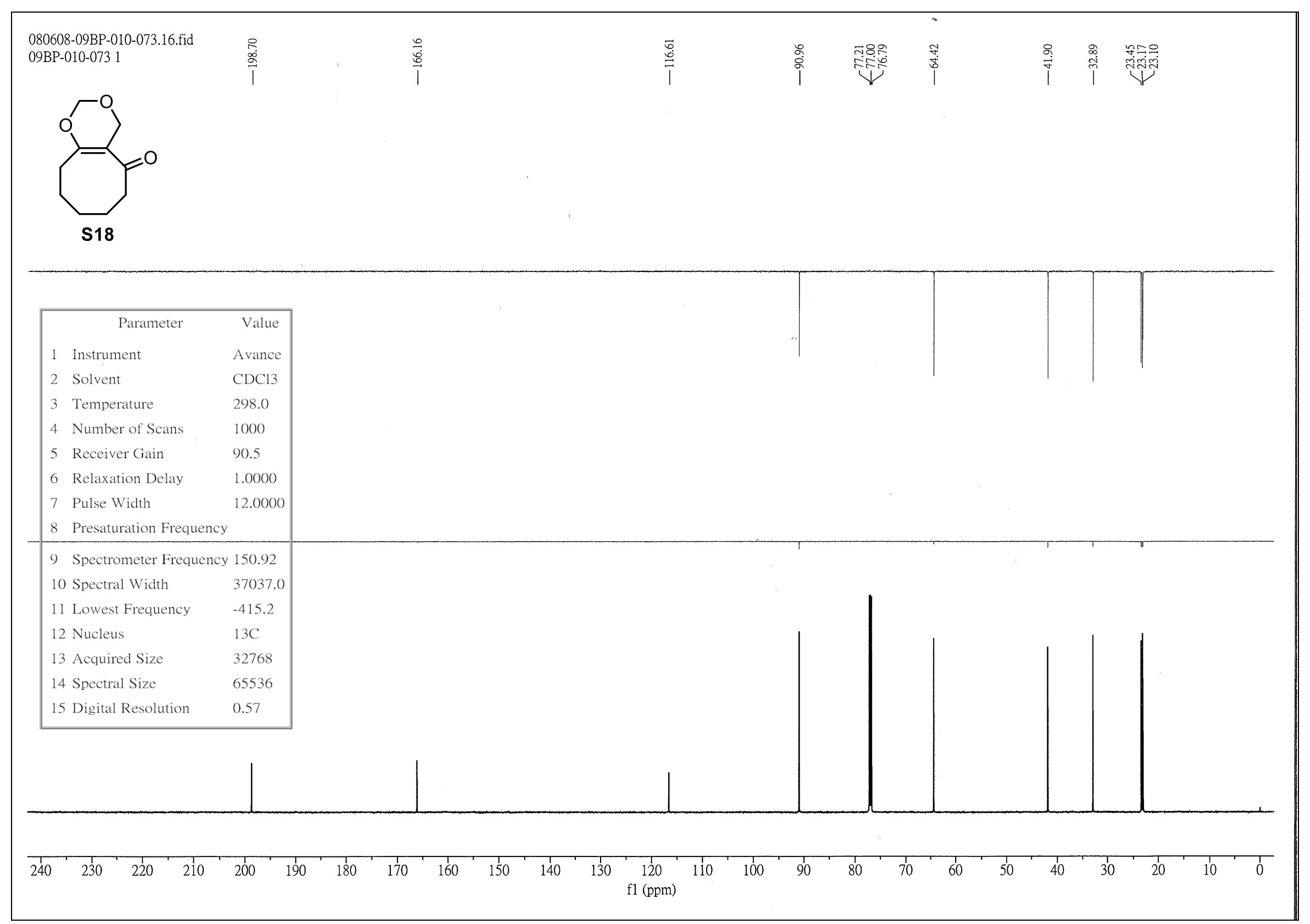

${ }^{13} \mathrm{C}$ NMR + DEPT spectra for compound $\mathbf{S 1 8}$ 


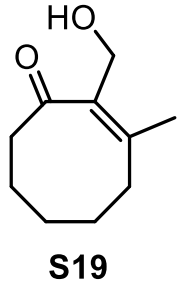

\begin{tabular}{|lll|}
\hline & \multicolumn{1}{|c}{ Parameter } & \multicolumn{1}{c|}{ Value } \\
1 & Instrument & Avance \\
2 & Solvent & CDCl3 \\
3 & Temperature & 298.0 \\
4 & Number of Scans & 16 \\
5 & Receiver Gain & 90.5 \\
6 & Relaxation Delay & 1.0000 \\
7 & Pulse Width & 10.0000 \\
8 & Presaturation Frequency \\
9 & Spectrometer Frequency 600.14 \\
10 & Spectral Width & 11904.8 \\
11 & Lowest Frequency & -2249.7 \\
12 & Nucleus & $1 \mathrm{H}$ \\
13 & Acquired Size & 32768 \\
14 & Spectral Size & 131072 \\
15 & Digital Resolution & 0.09 \\
\hline
\end{tabular}

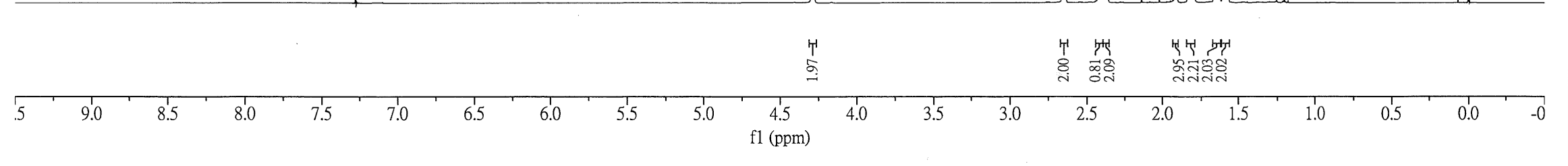

${ }^{1}$ H NMR spectrum for compound S19 


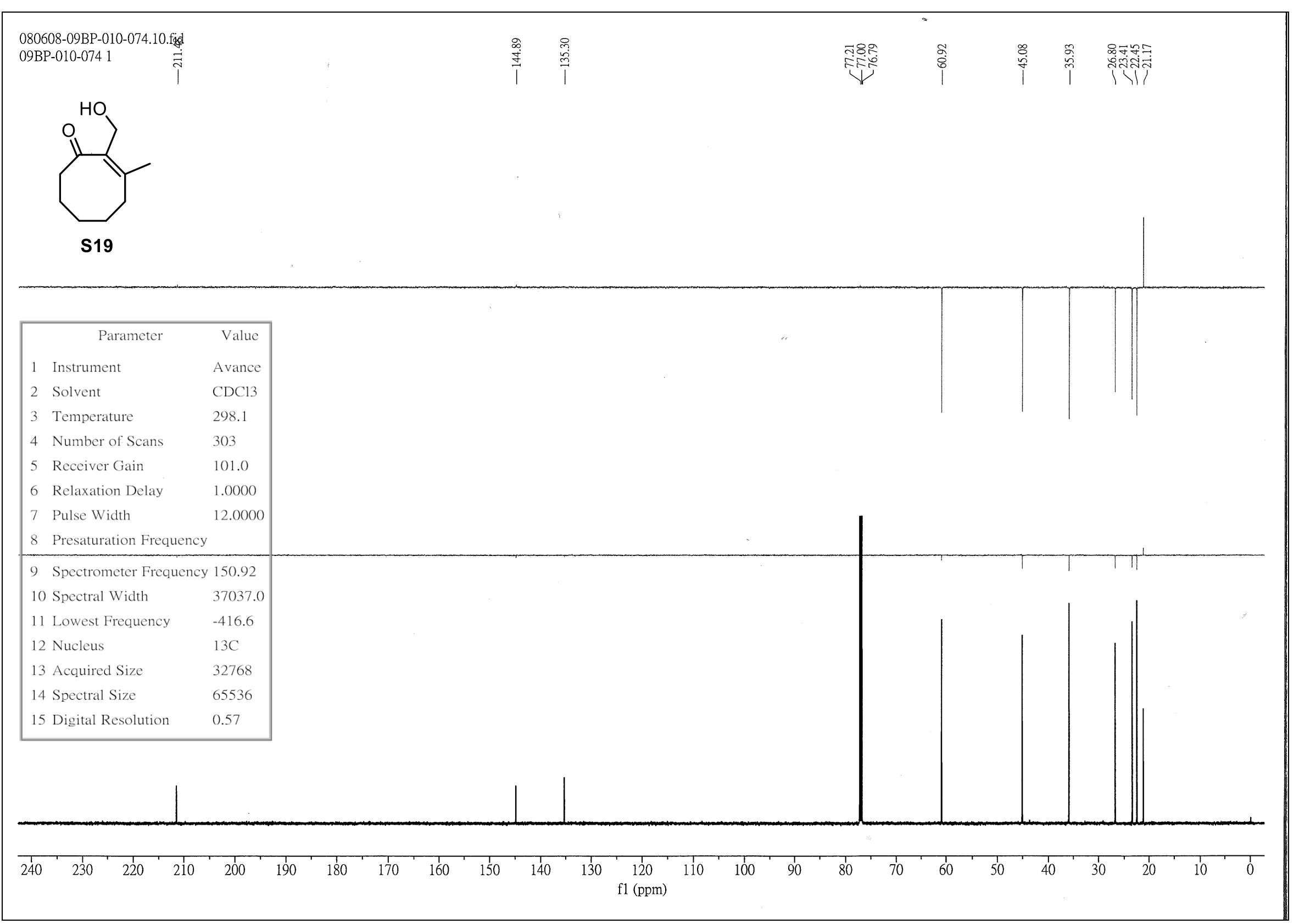

${ }^{13} \mathrm{C}$ NMR + DEPT spectra for compound S19 


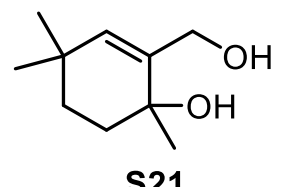

S21

\begin{tabular}{|lll}
\hline \multicolumn{1}{|c}{ Parameter } & \multicolumn{1}{c}{ Value } \\
1 & Instrument & Avance \\
2 & Solvent & CDCl3 \\
3 & Temperature & 294.7 \\
4 & Number of Scans & 16 \\
5 & Receiver Gain & 101.0 \\
6 & Relaxation Delay & 1.0000 \\
7 & Pulse Width & 8.0000 \\
8 & Presaturation Frequency \\
9 & Spectrometer Frequency 400.17 \\
10 & Spectral Width & 7812.5 \\
11 & Lowest Frequency & -1441.8 \\
12 & Nucleus & $1 \mathrm{H}$ \\
13 & Acquired Size & 32768 \\
14 & Spectral Size & 65536 \\
15 & Digital Resolution & 0.12 \\
\hline
\end{tabular}
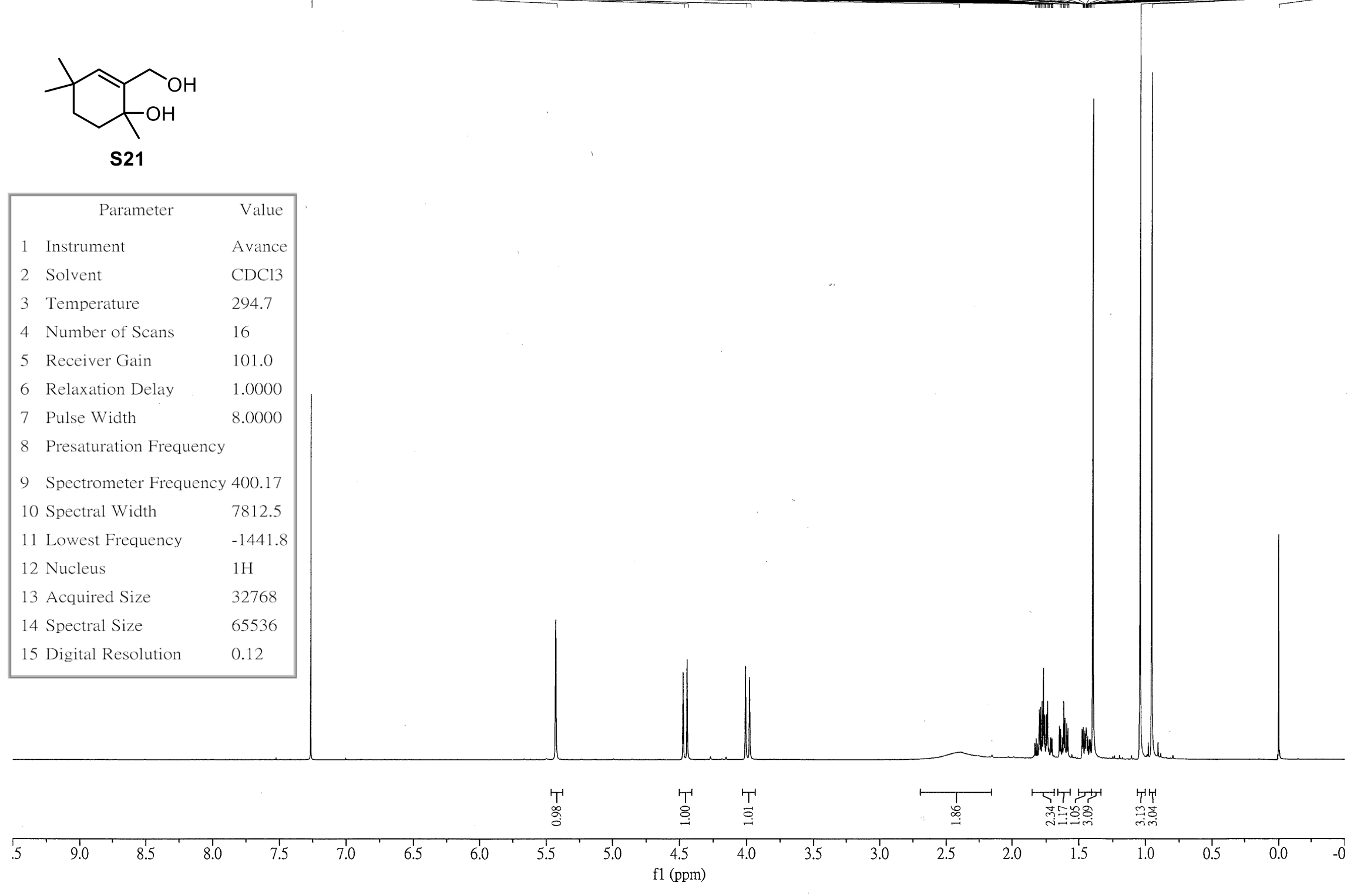

${ }^{1}$ H NMR spectrum for compound $\mathbf{S 2 1}$ 


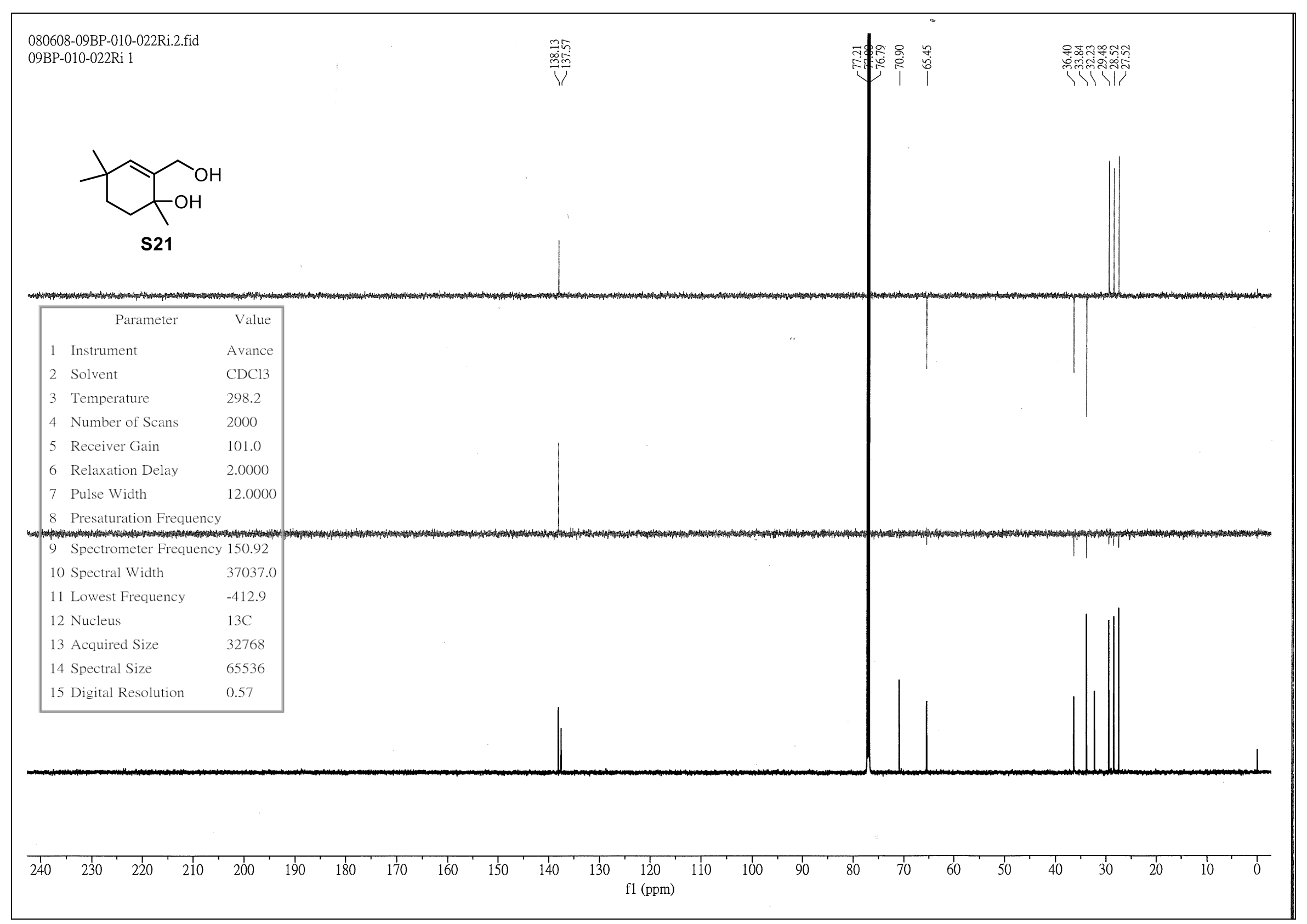

${ }^{13} \mathrm{C}$ NMR + DEPT spectra for compound S21 


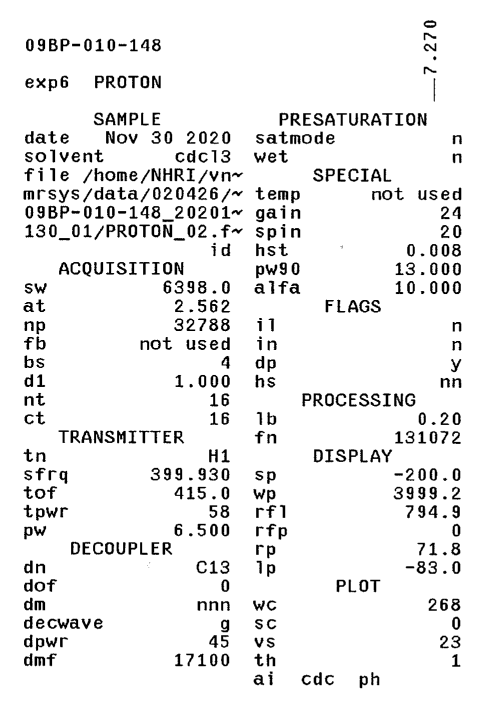

satmode

Solvent $\begin{gathered}\text { cdcl } 13 \\ \text { file wet }\end{gathered}$

not used
2ad

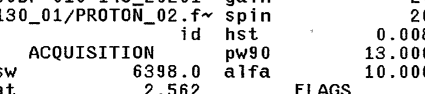

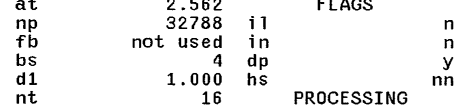

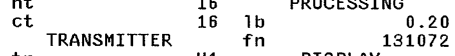

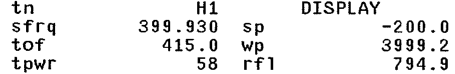

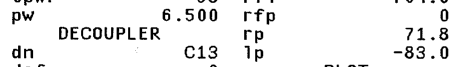

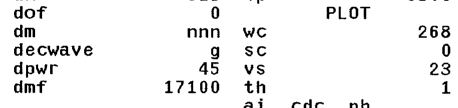

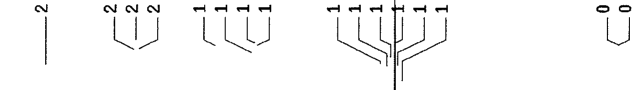

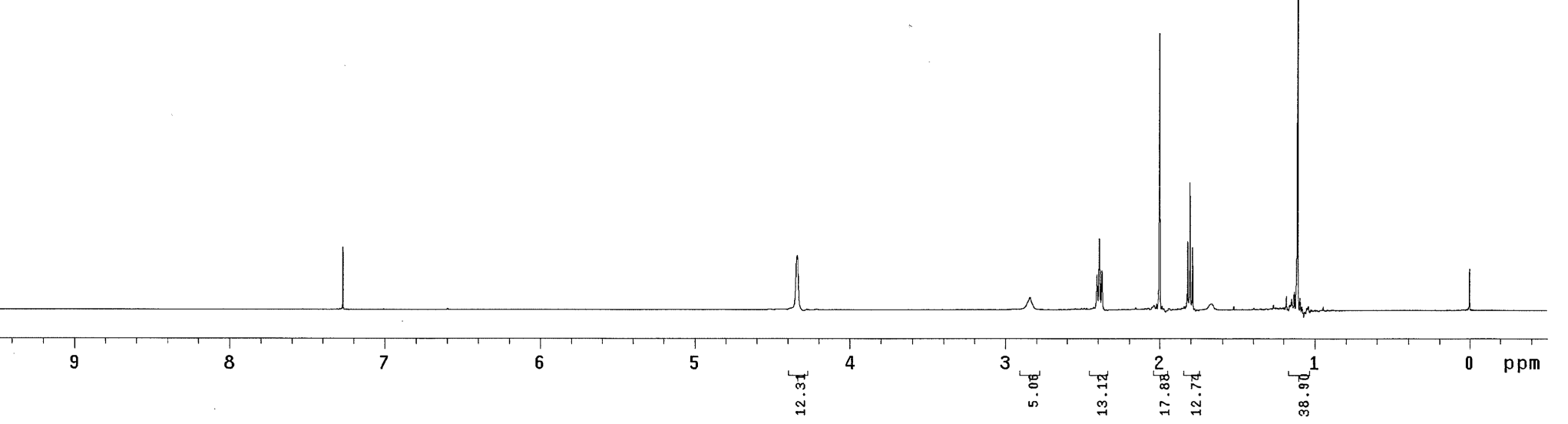



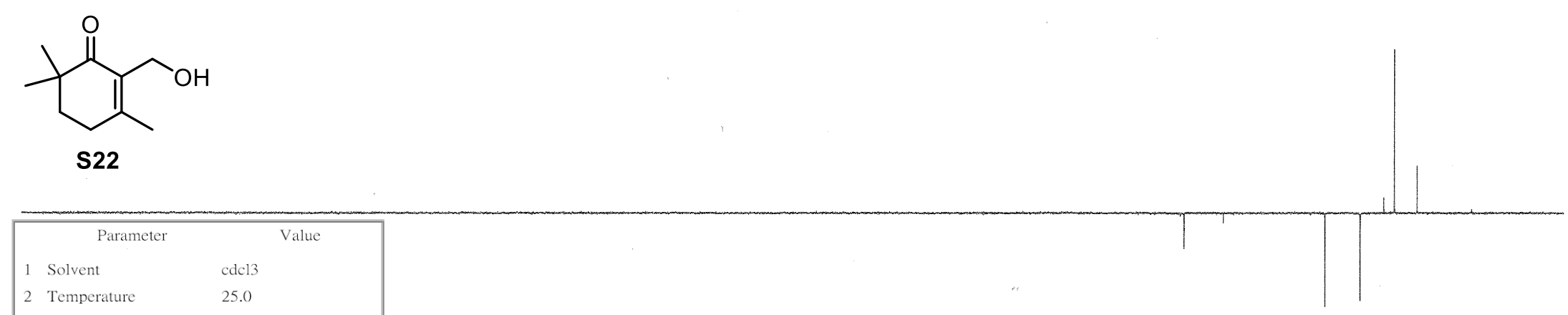

3 Pulse Sequence s2p

4 Experiment

5 Number of Scans 1200

6 Receiver Gain $\quad 30$

7 Relaxation Delay $\quad 1.0000$

8 Pulse Width 6.6500

9 Presaturation Frequency

10 Modification Date 2020-11-30T13:46:58

11 Class

12 Spectrometer Frequency 100.57

13 Spectral Width 25125.6

14 Lowest Frequency $\quad-1507.6$

15 Nucleus

16 Acquired Size

17 Spectral Size

18 Digital Resolution

0.38

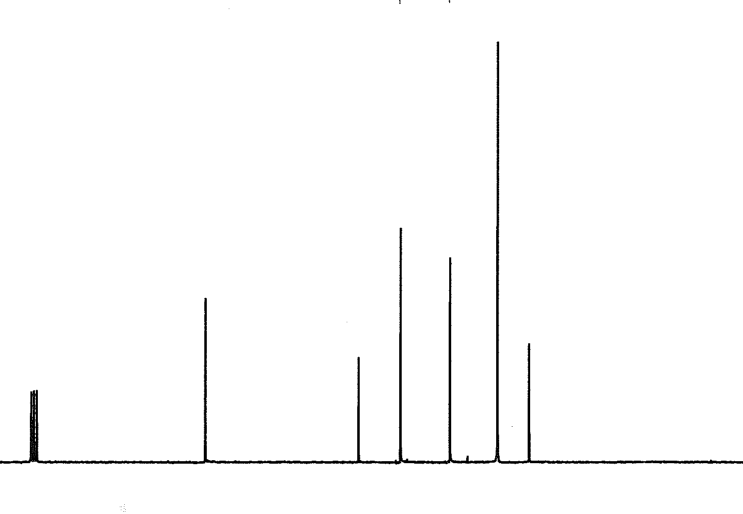

\begin{tabular}{llllll}
\hline 140 & 230 & 220 & 210 & 200 & 190
\end{tabular}

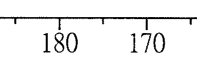

160150

$\begin{array}{cccc}140 & 130 & 120 & 110 \\ \text { f1 } & 1 \mathrm{ppm})\end{array}$

${ }^{3} \mathrm{C}$ NMR + DEPT spectra for compound $\mathbf{S 2 2}$ 

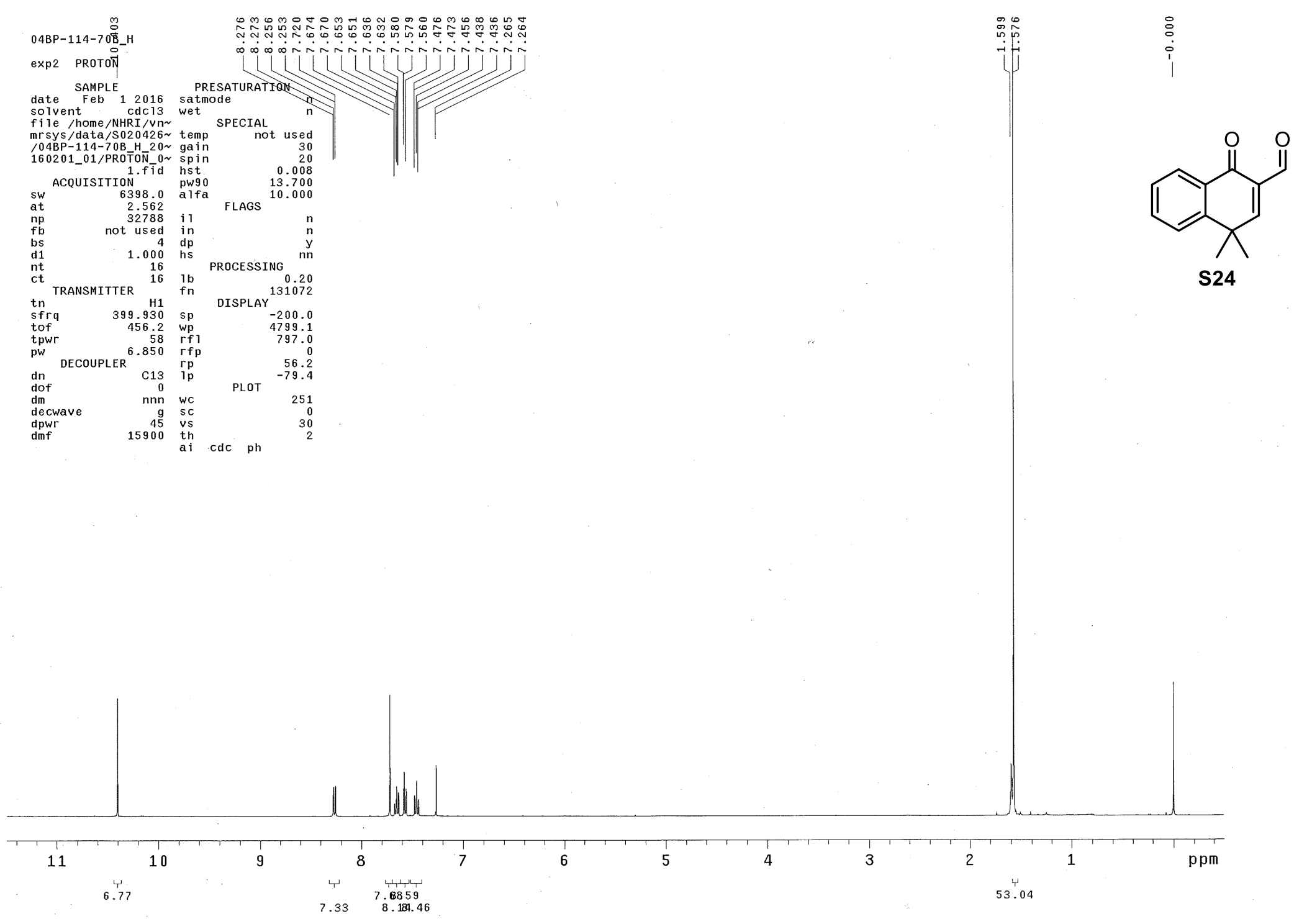

${ }^{1}$ H NMR spectrum for compound S24 


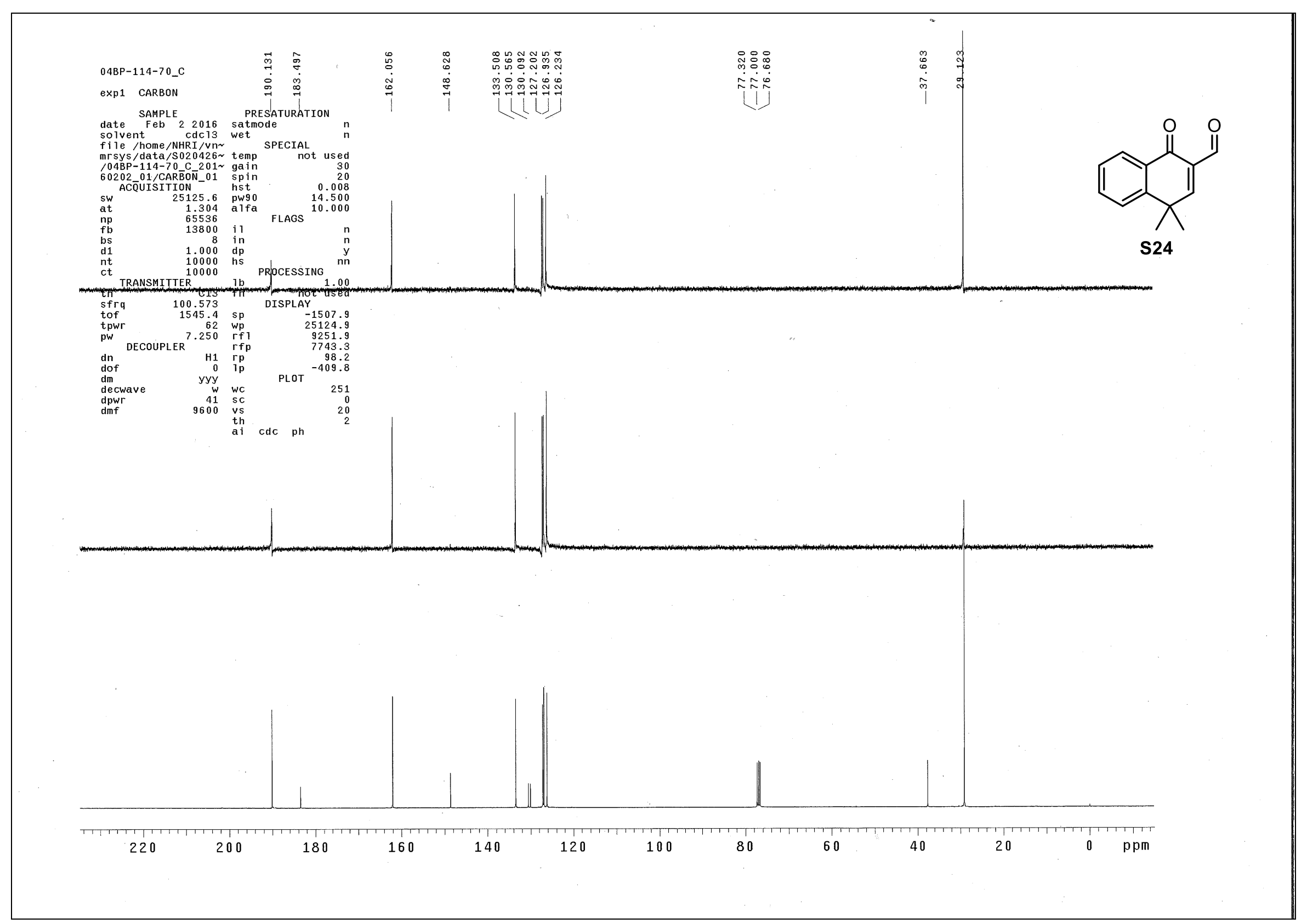

${ }^{13} \mathrm{C}$ NMR + DEPT spectra for compound S24 


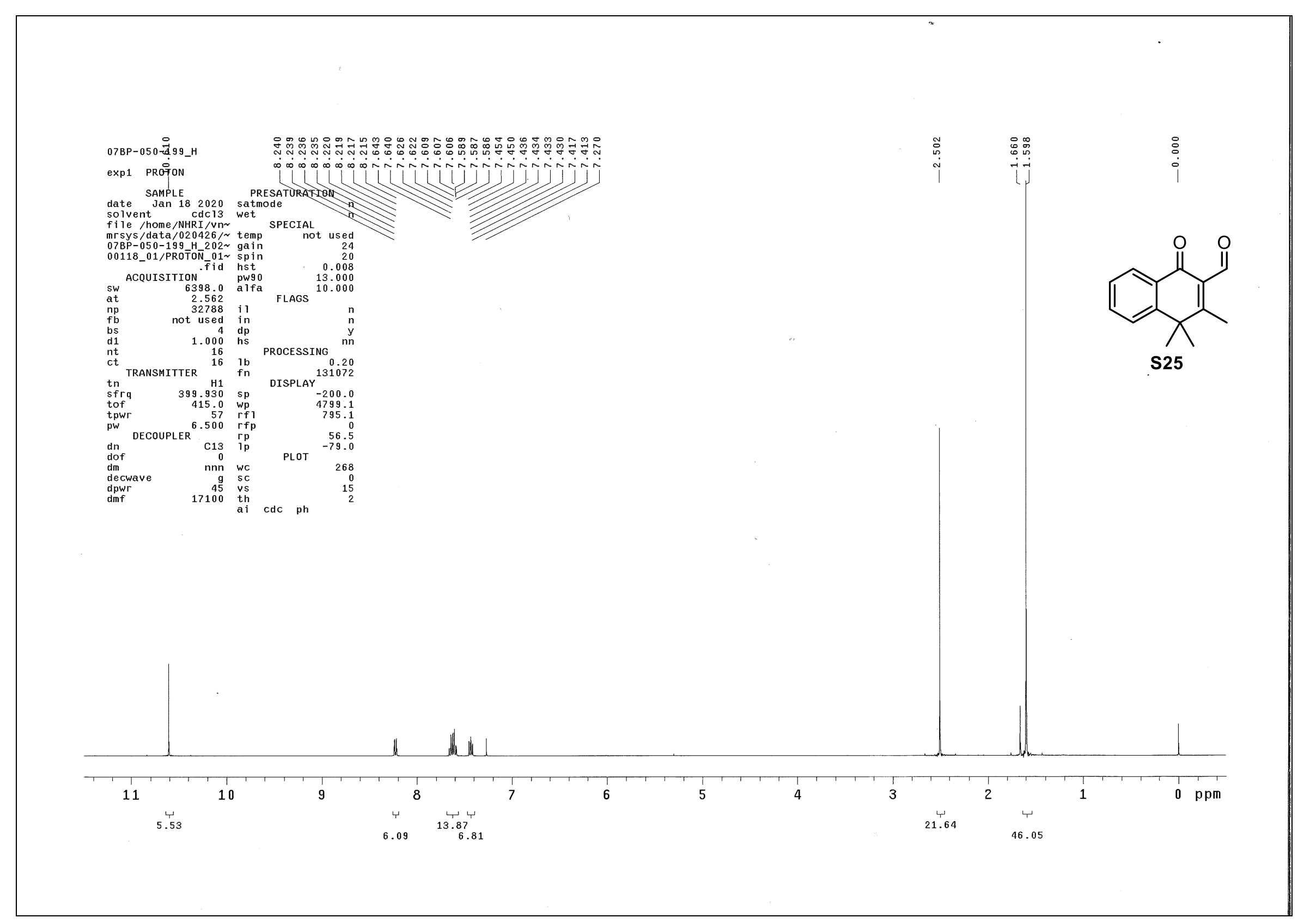

${ }^{1}$ H NMR spectrum for compound S25 


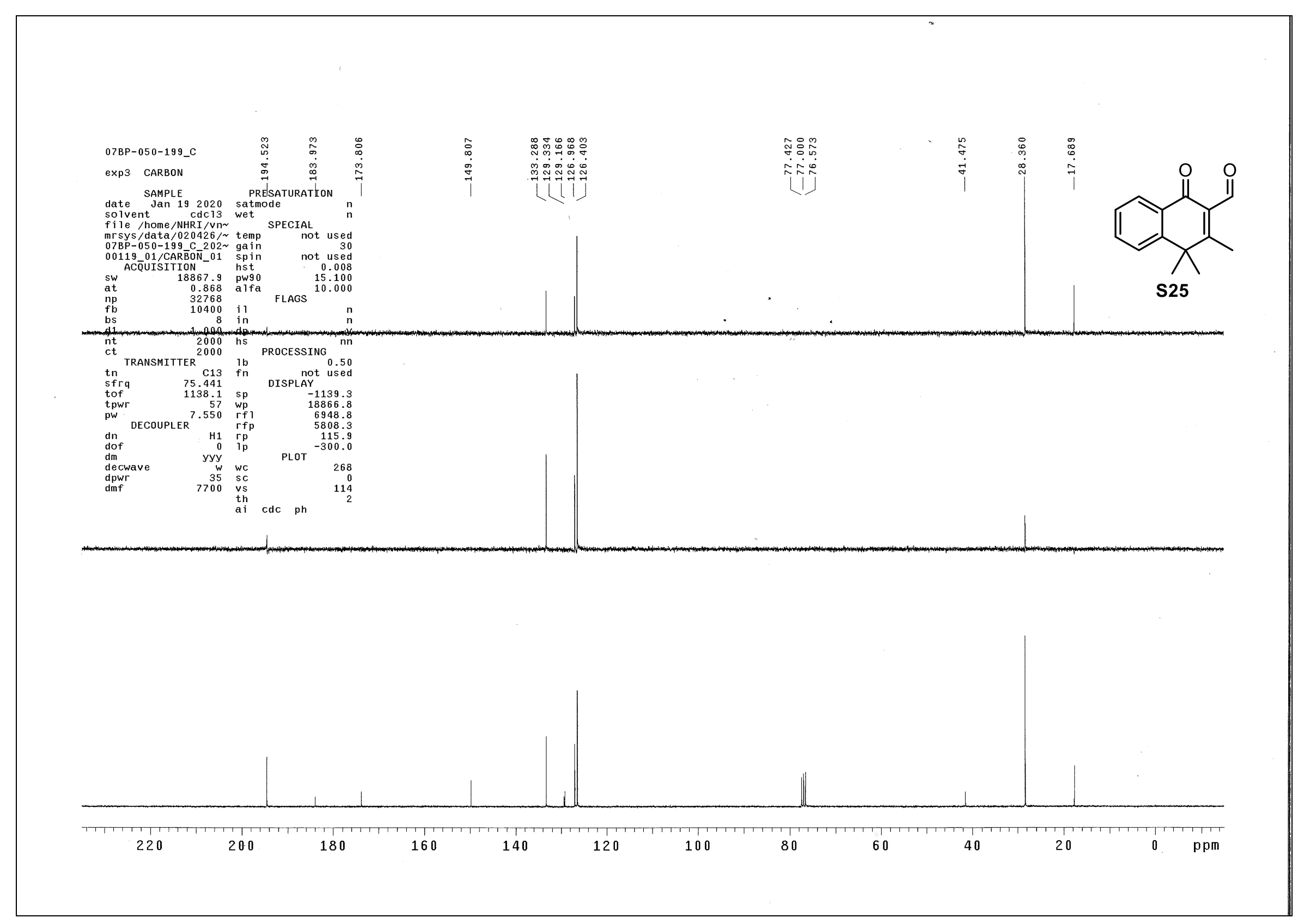

${ }^{13} \mathrm{C}$ NMR + DEPT spectra for compound $\mathbf{S 2 5}$ 


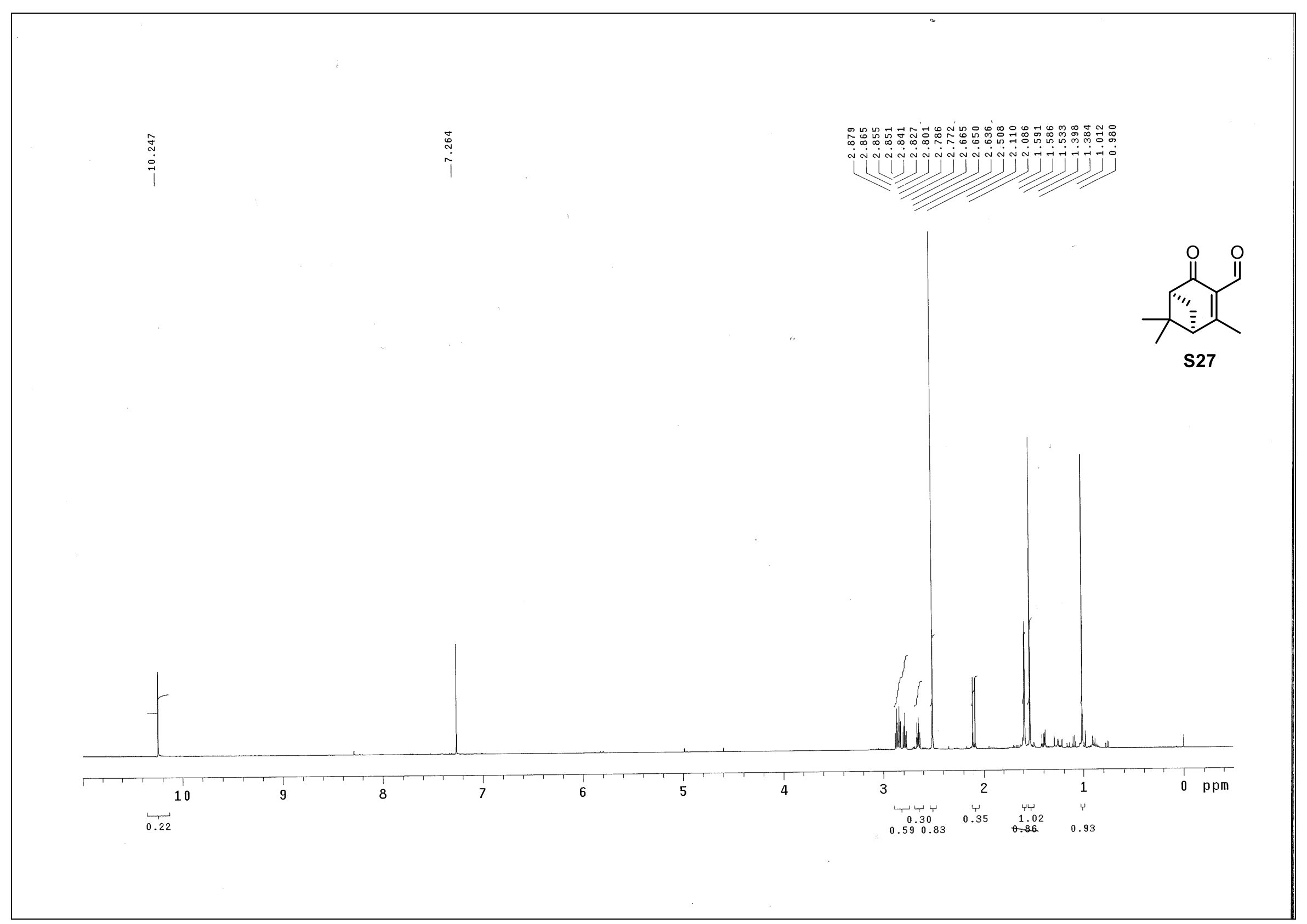

${ }^{1}$ H NMR spectrum for compound S27 

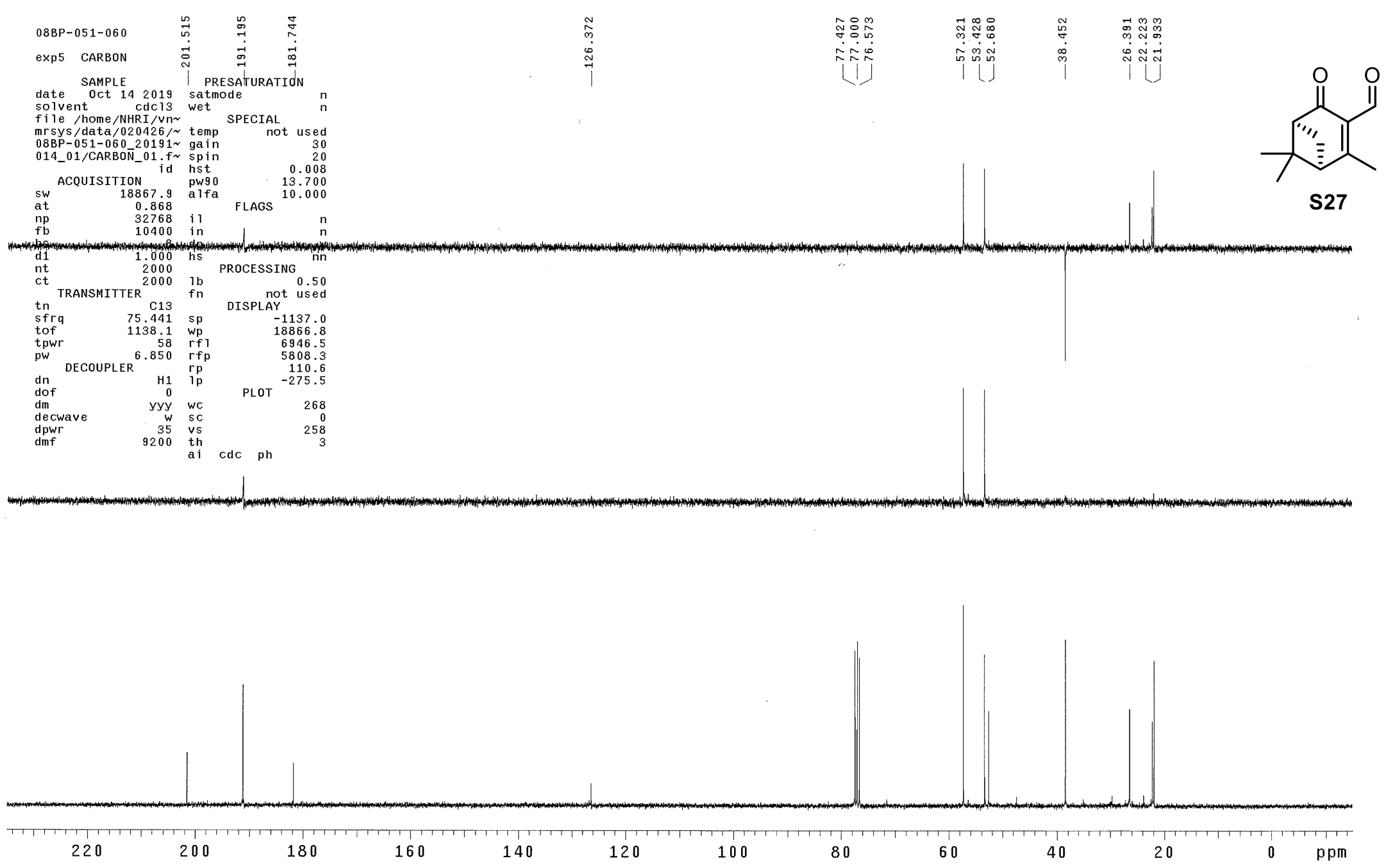

${ }^{13} \mathrm{C}$ NMR + DEPT spectra for compound S27 


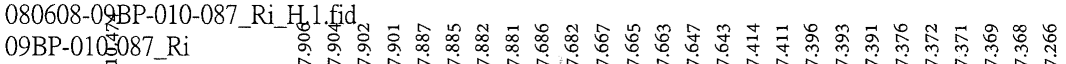

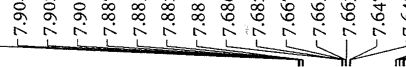
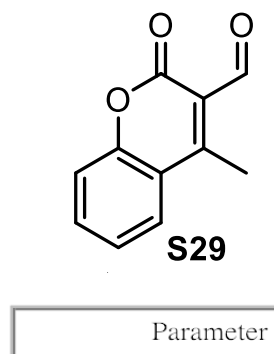

1 Instrument

2 Solvent

3 Temperature

4 Number of Scans

5 Receiver Gain

6 Relaxation Delay

7 Pulke Width

8 Prepaturation Frequency

9 Spectrometer Frequency 400.17

10 Spectral Width

11 Lowest Frequency

2 Nupleus

13 Acquired Size

14 Spectral Size

\begin{tabular}{l|l|l}
15 Di & ital Resolution $\quad 0.12$ \\
\hline &
\end{tabular}

$1 \mathrm{H}$

32768

65536
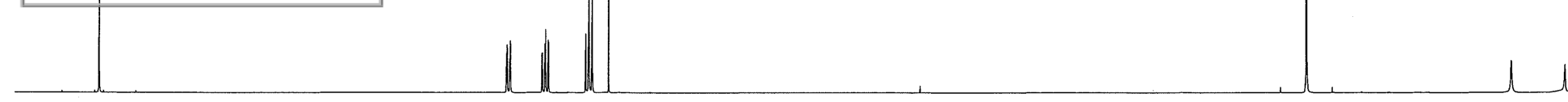

(a)

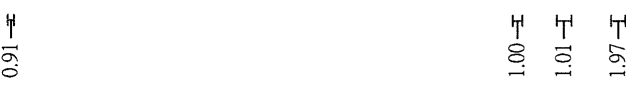

案

$9.5 \cdot 9.0$

$8.5 \quad 8.0 \quad 7.5$

$6.5 \cdot 6.0$

$5.5,5.0$

${ }^{1} \mathrm{H}$ NMR spectrum for compound $\mathbf{S 2 9}$ 


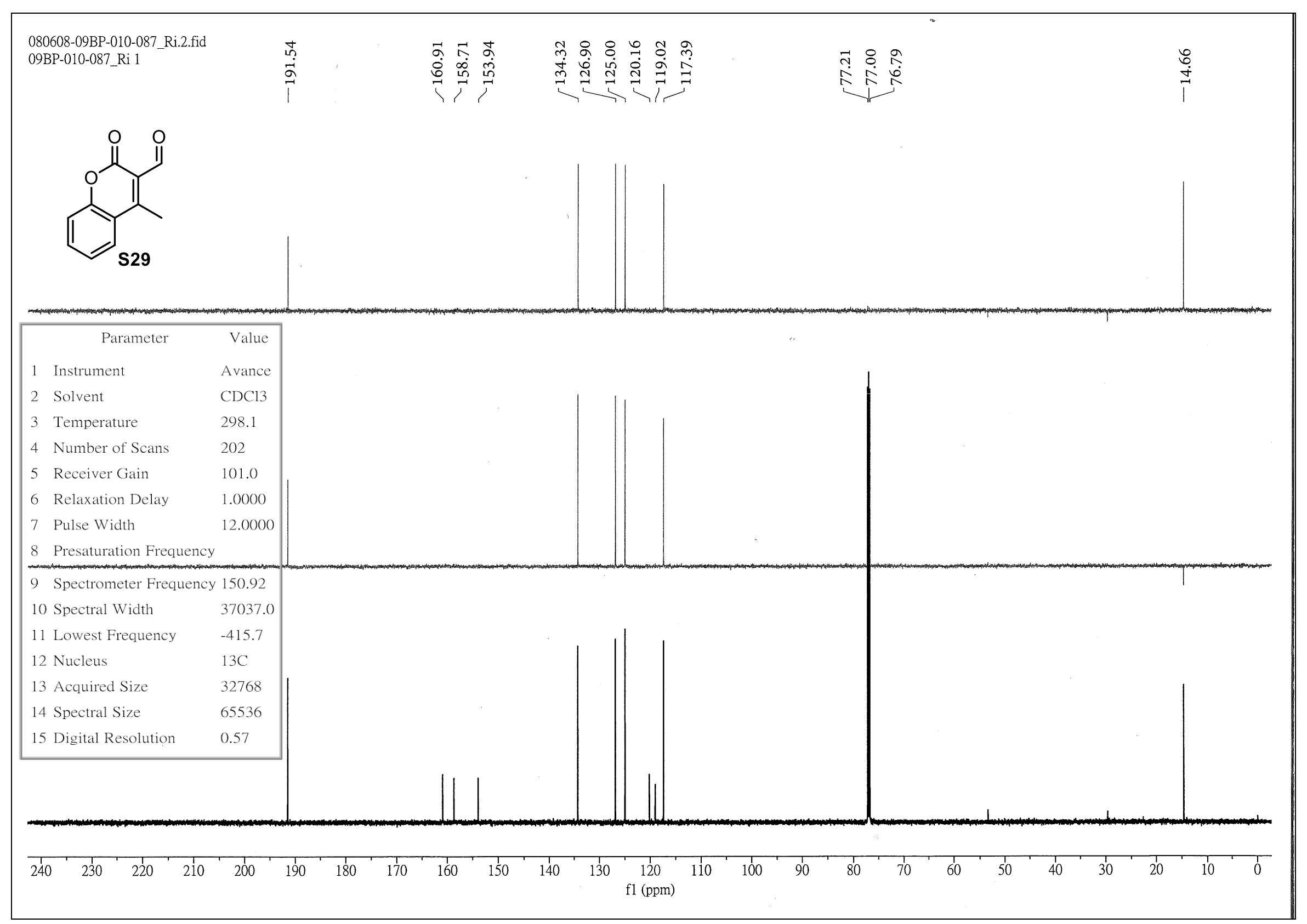

${ }^{13}$ C NMR + DEPT spectra for compound S29 


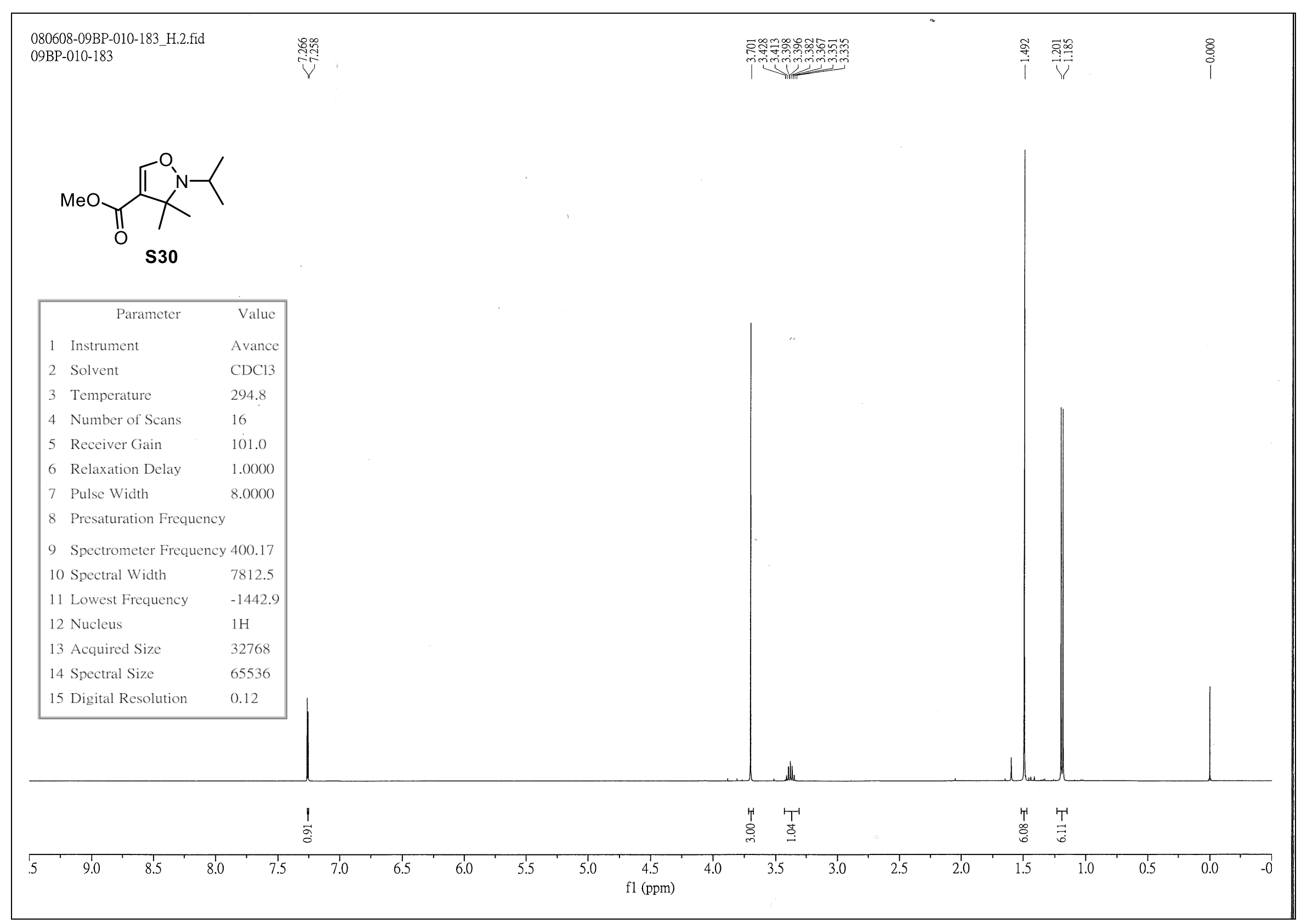

${ }^{1} \mathrm{H}$ NMR spectrum for compound $\mathbf{S 3 0}$ 


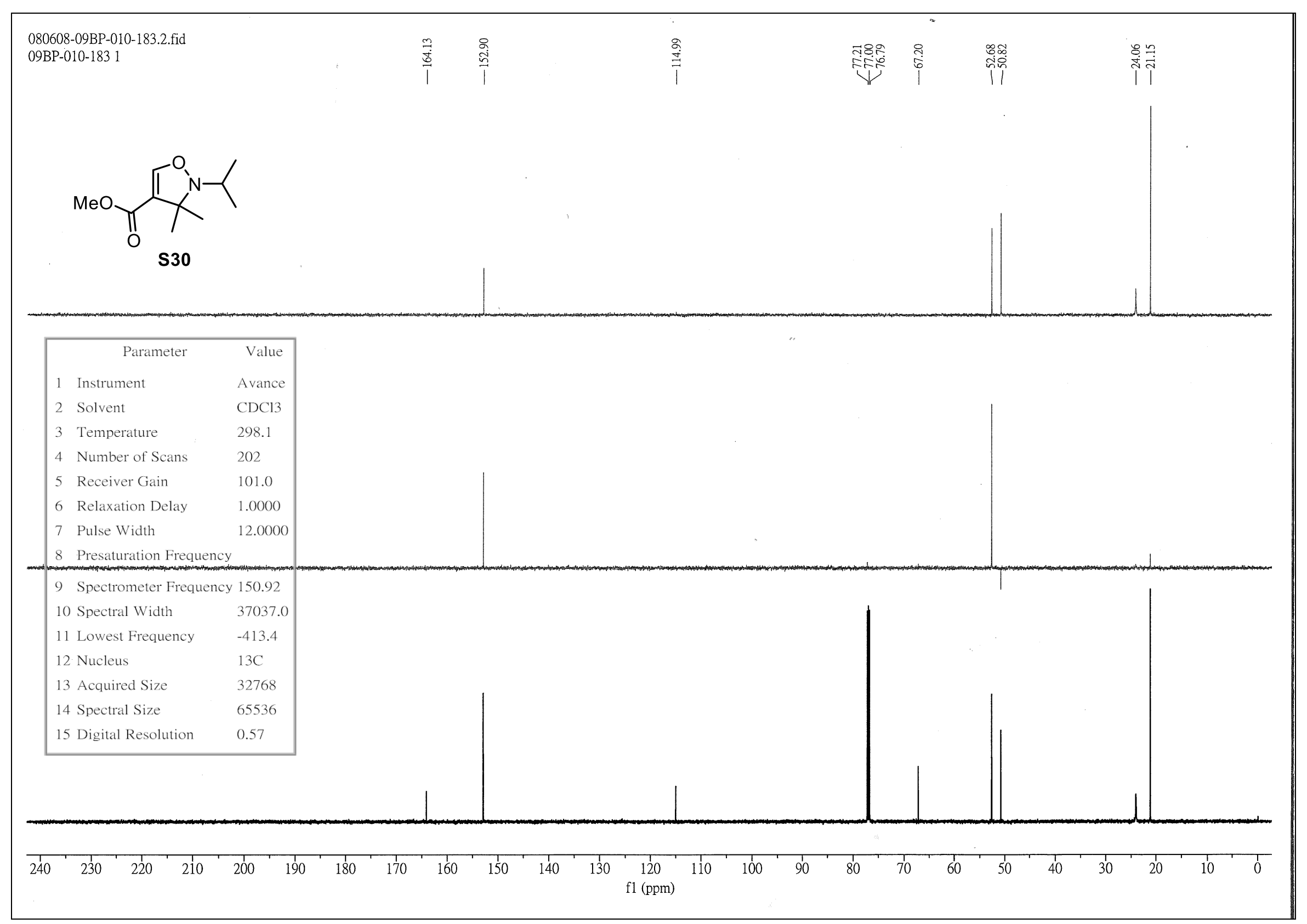

${ }^{13} \mathrm{C}$ NMR + DEPT spectra for compound $\mathbf{S 3 0}$ 


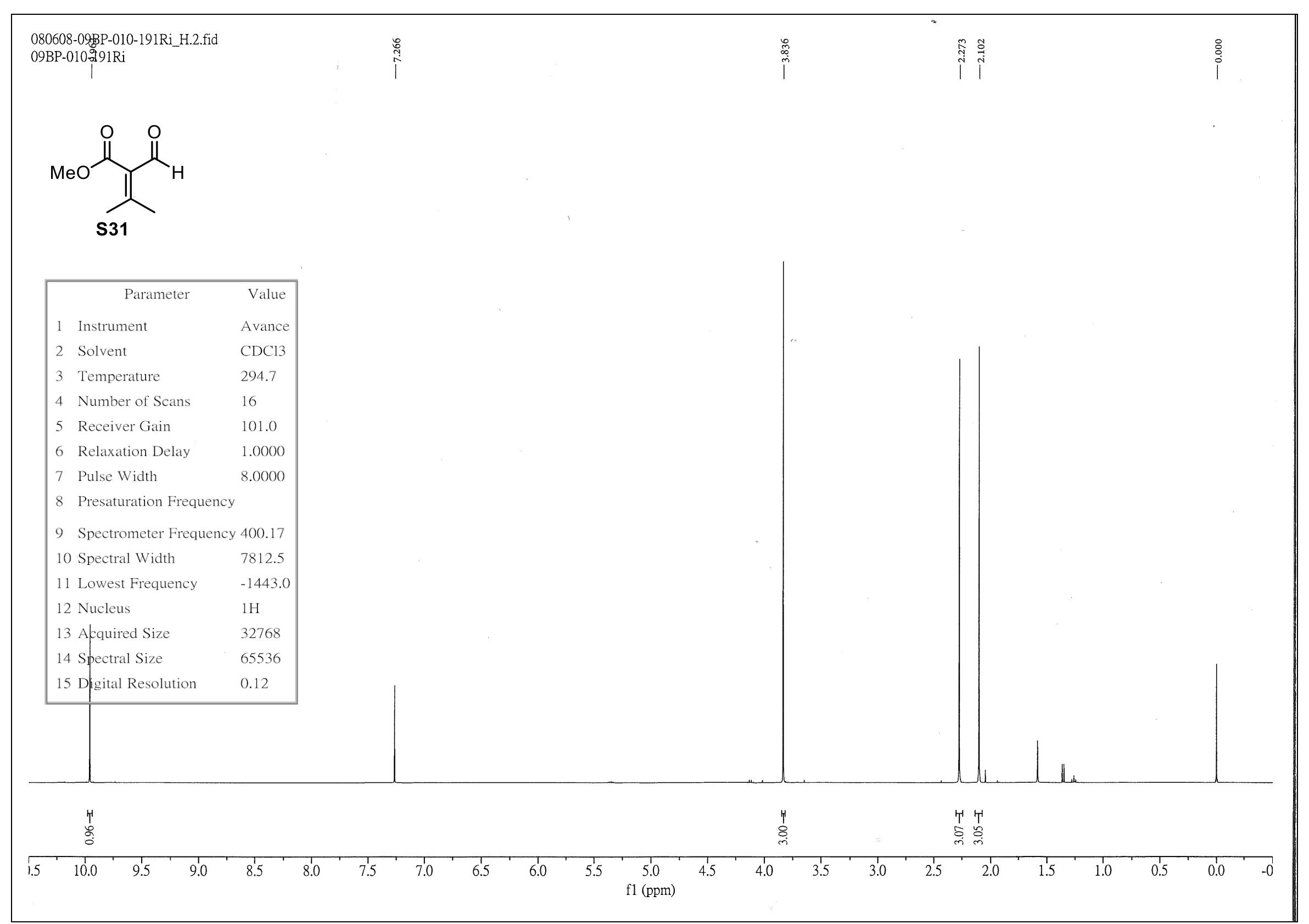

${ }^{1}$ H NMR spectrum for compound $\mathbf{S 3 1}$ 


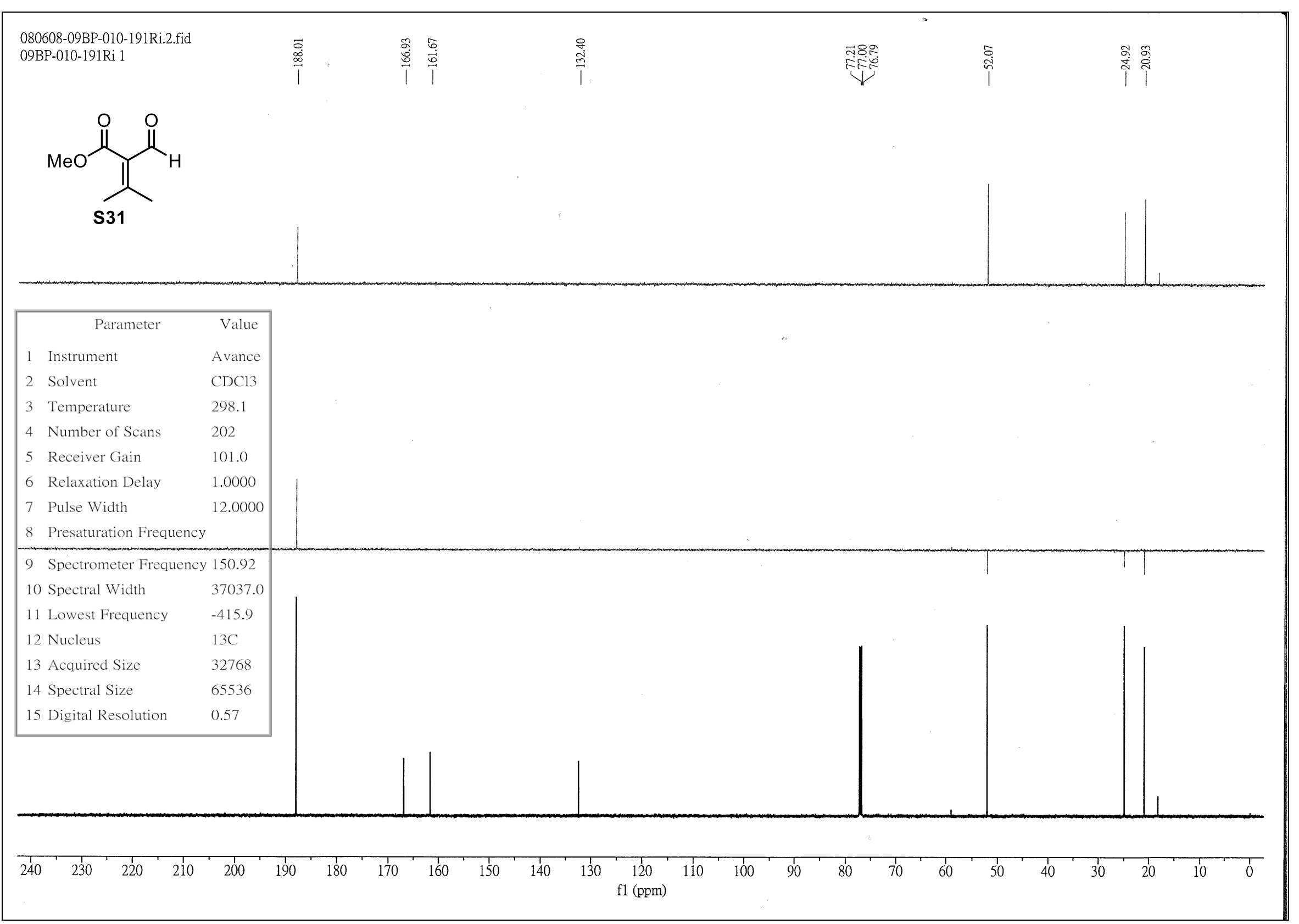

${ }^{13} \mathrm{C}$ NMR + DEPT spectra for compound $\mathbf{S 3 1}$ 


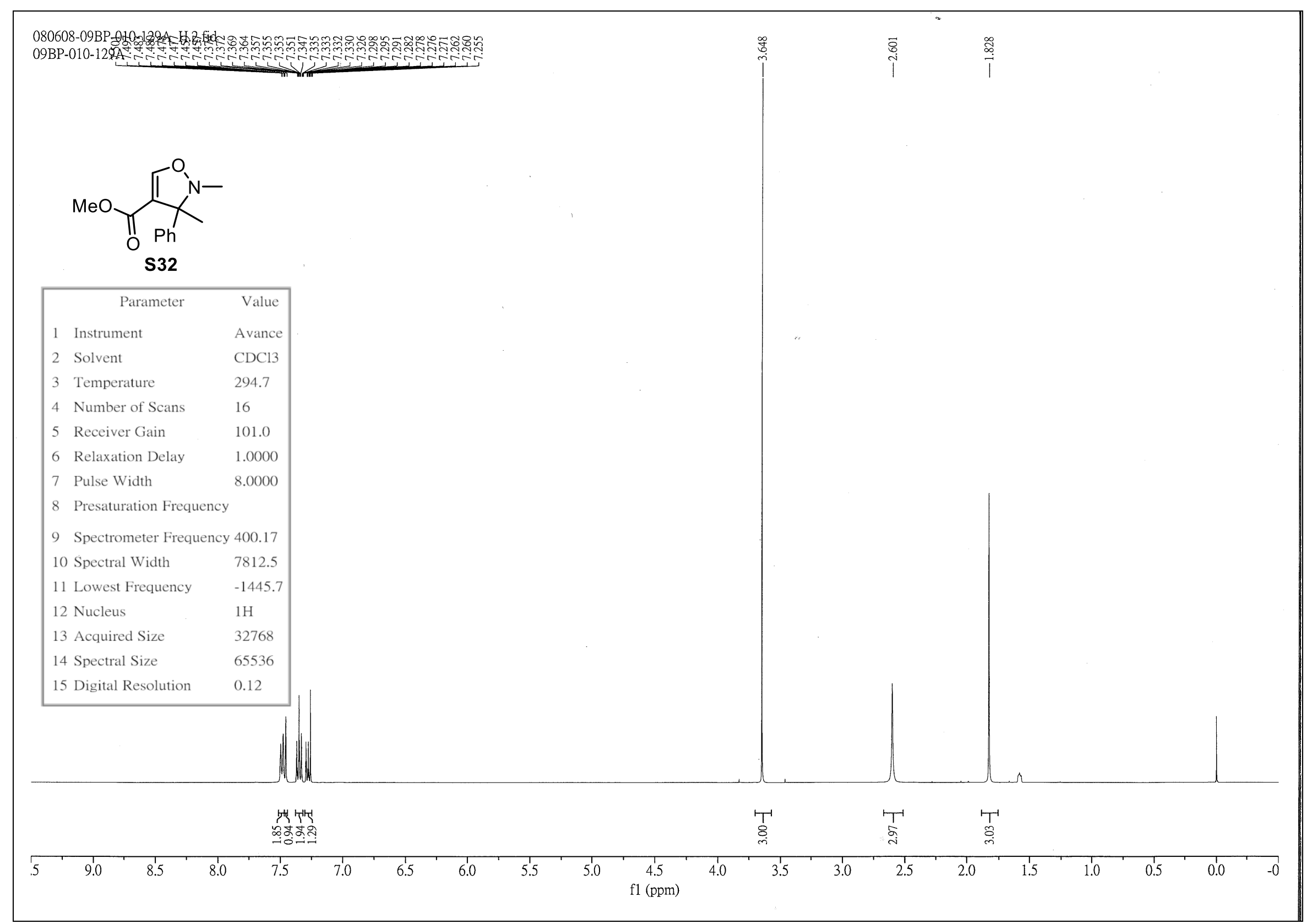

${ }^{1} \mathrm{H}$ NMR spectrum for compound $\mathbf{S 3 2}$ 


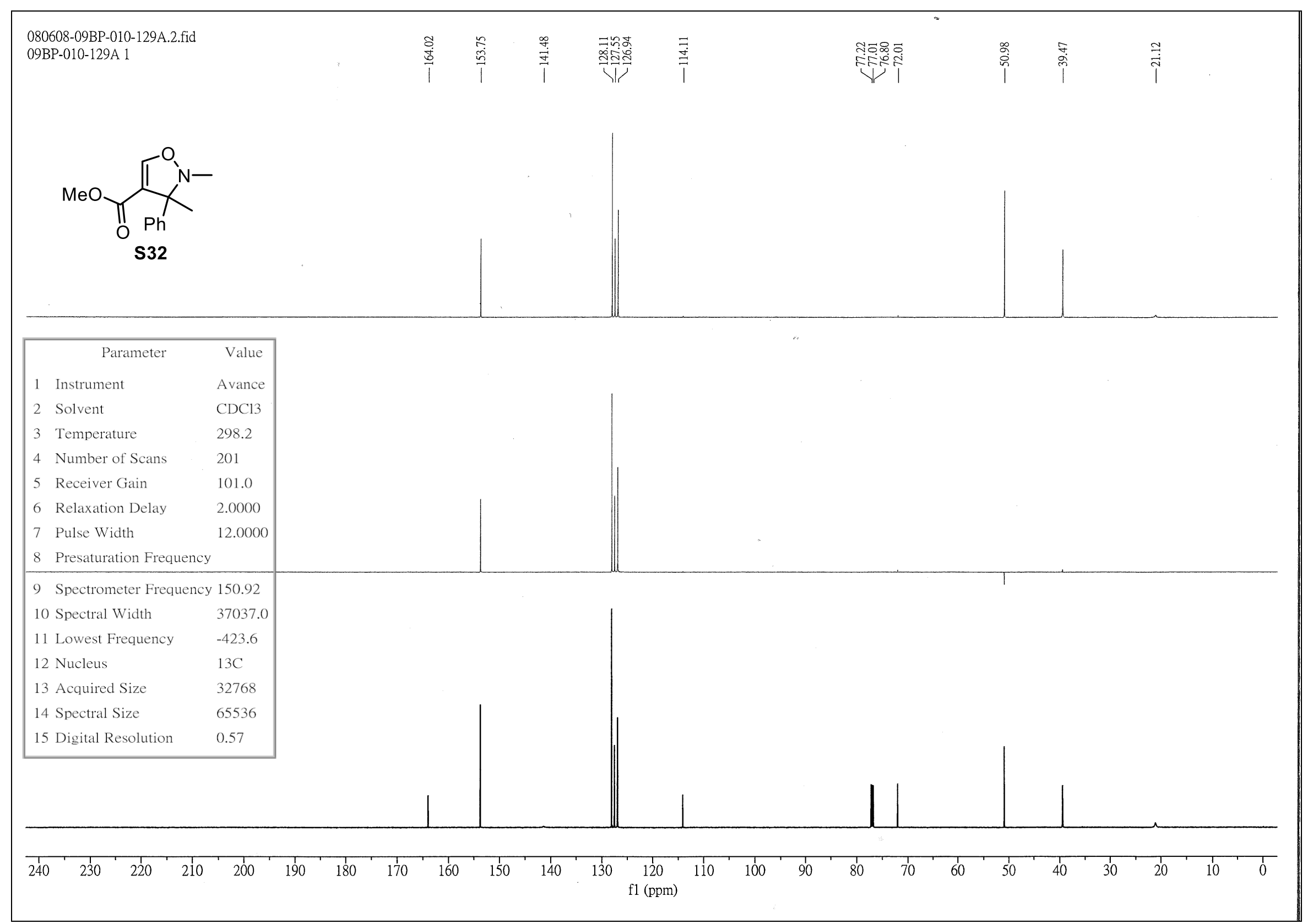

${ }^{13} \mathrm{C}$ NMR + DEPT spectra for compound $\mathbf{S 3 2}$ 


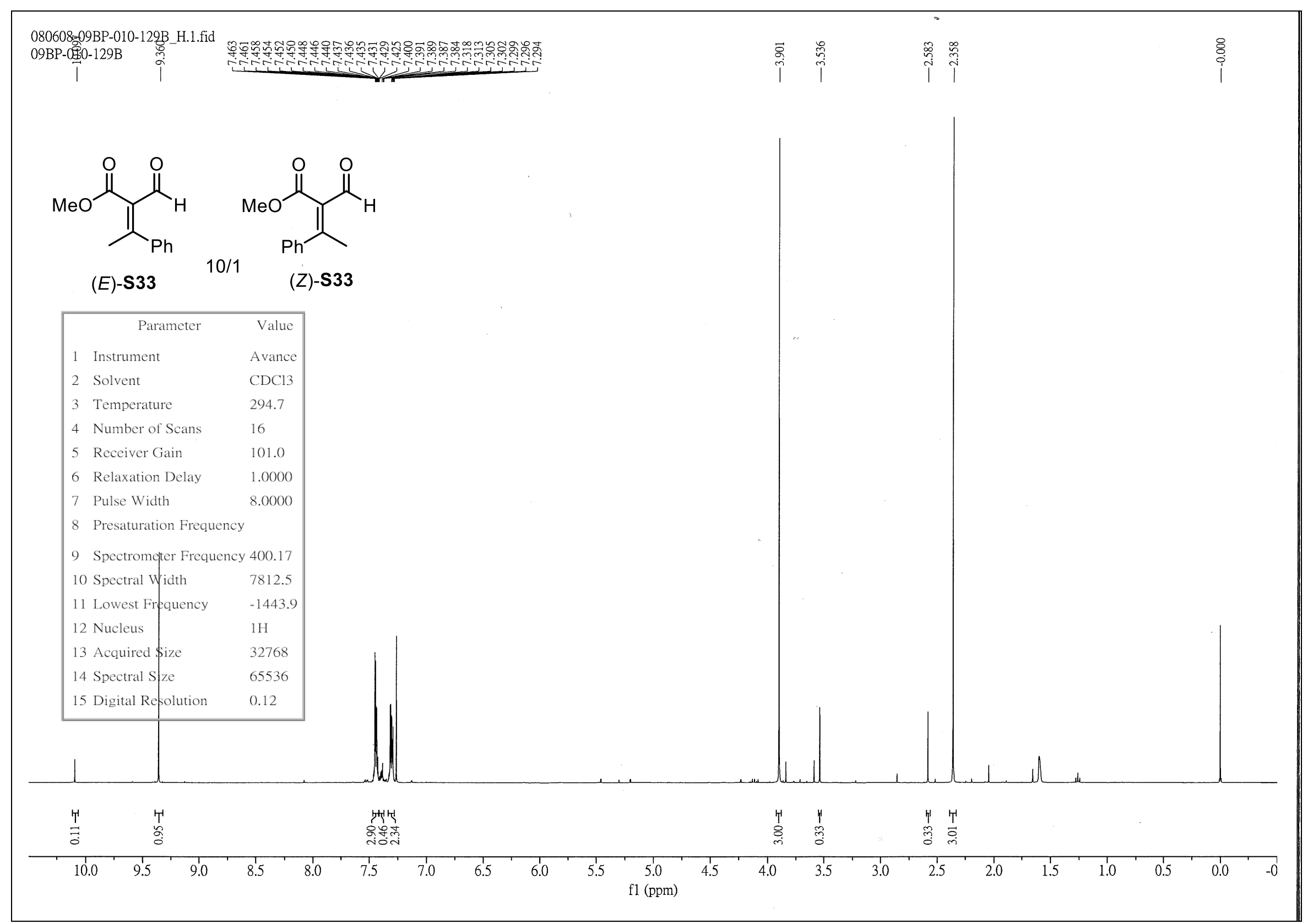

${ }^{1} \mathrm{H}$ NMR spectrum for compounds (E)-S33 and (Z)-S33 


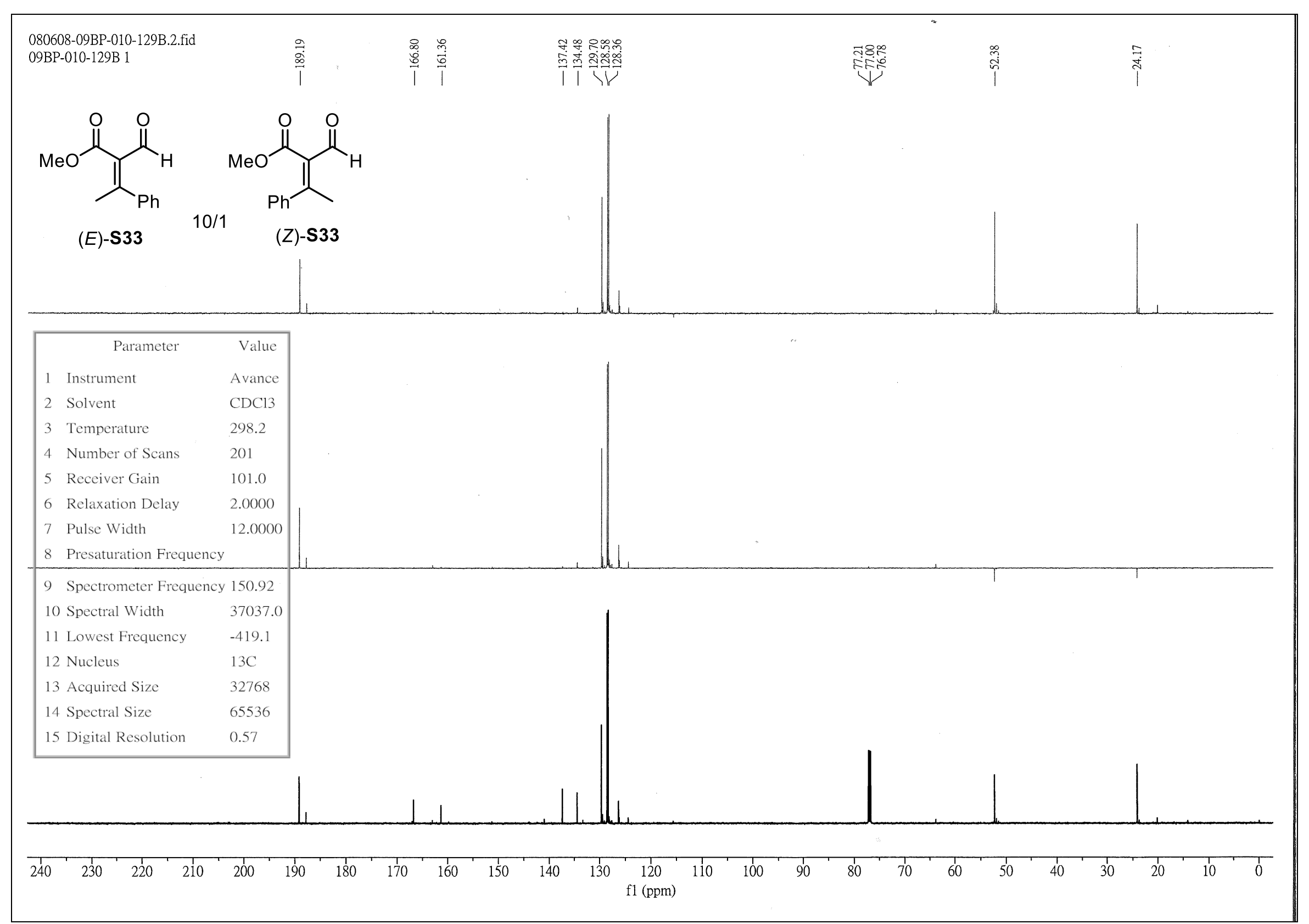

${ }^{13} \mathrm{C}$ NMR + DEPT spectra for compounds (E)-S33 and (Z)-S33 


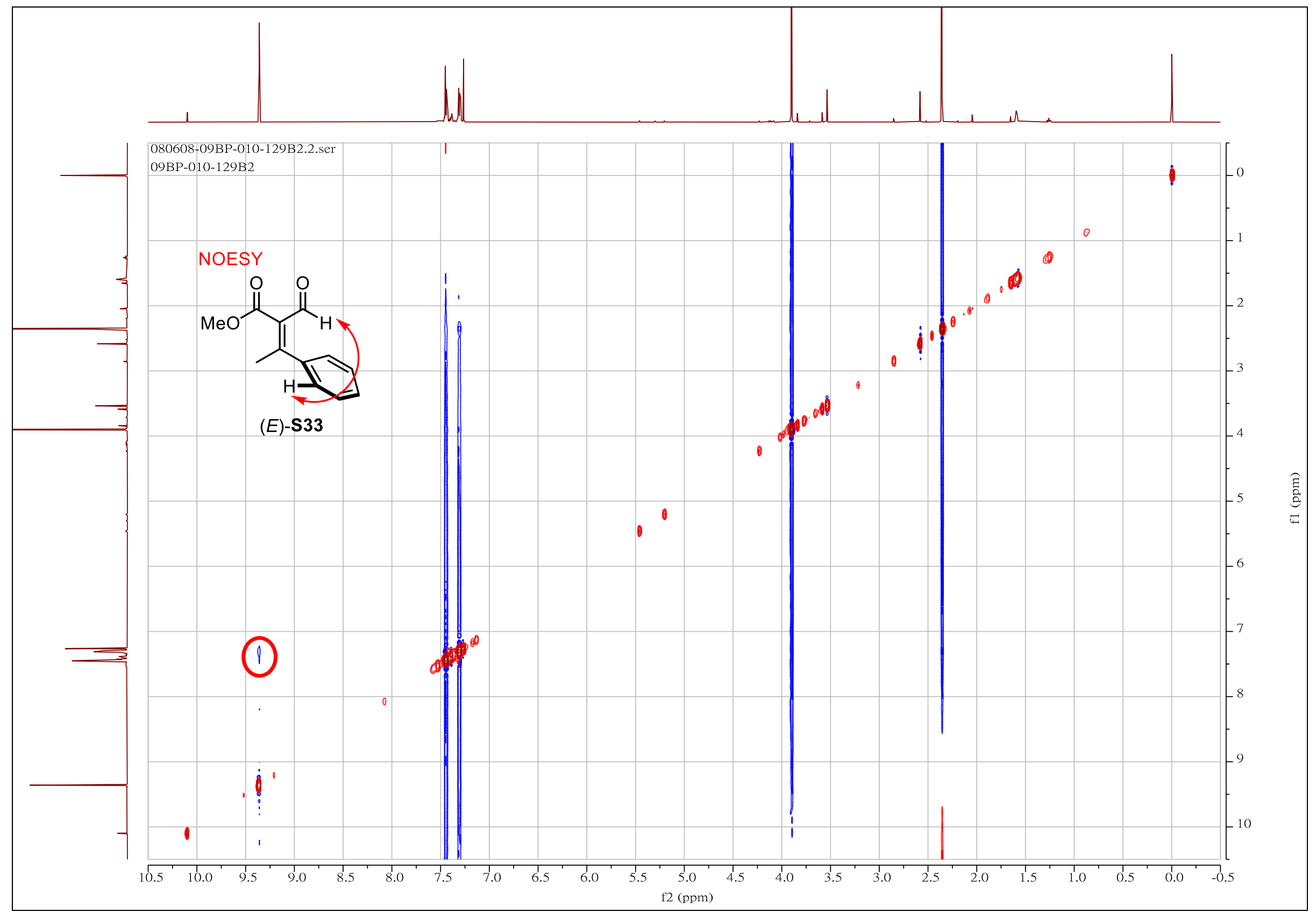

2D NOESY spectrum for compound $\mathbf{S 3 3}$ 


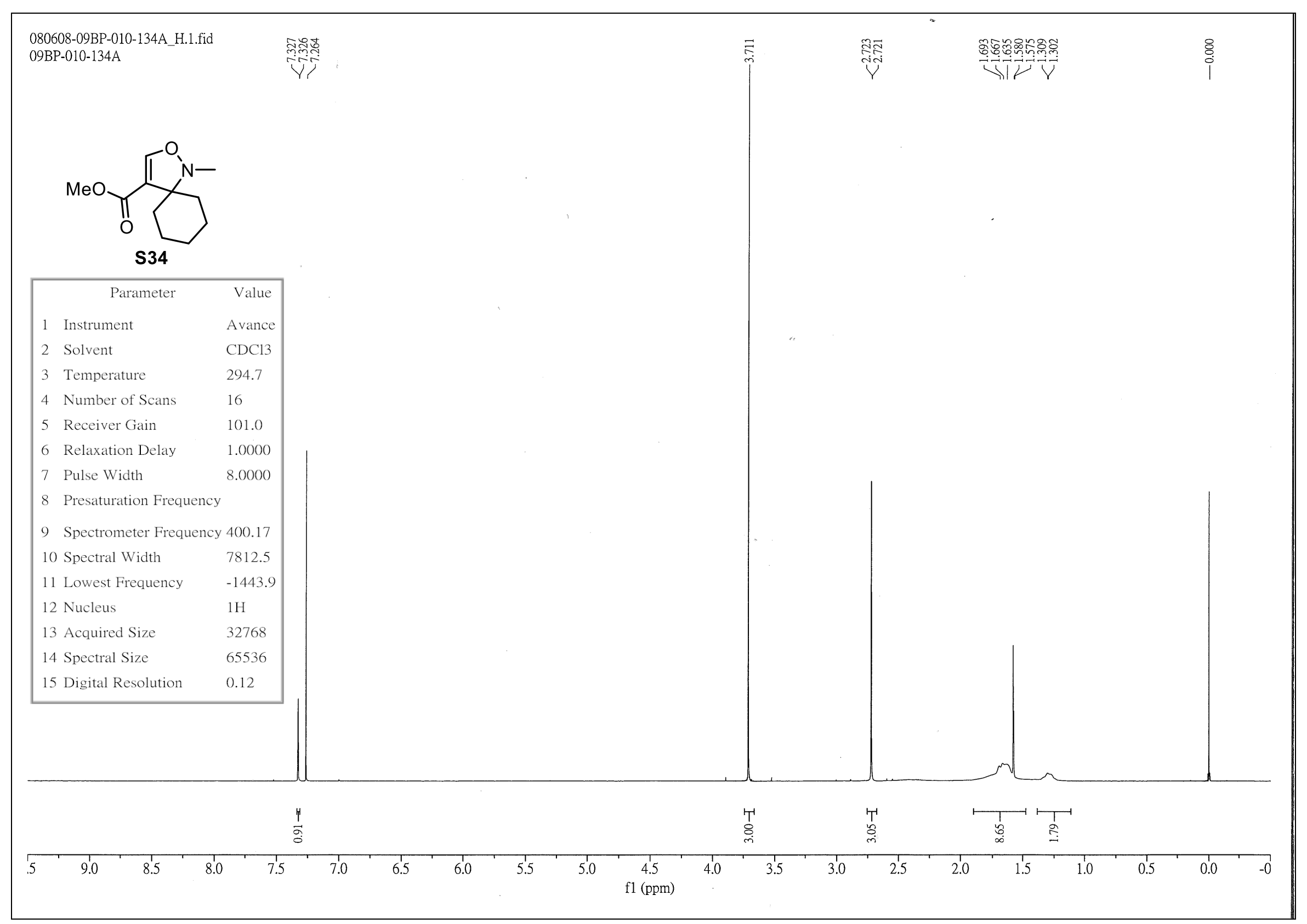

${ }^{1}$ H NMR spectrum for compound S34 


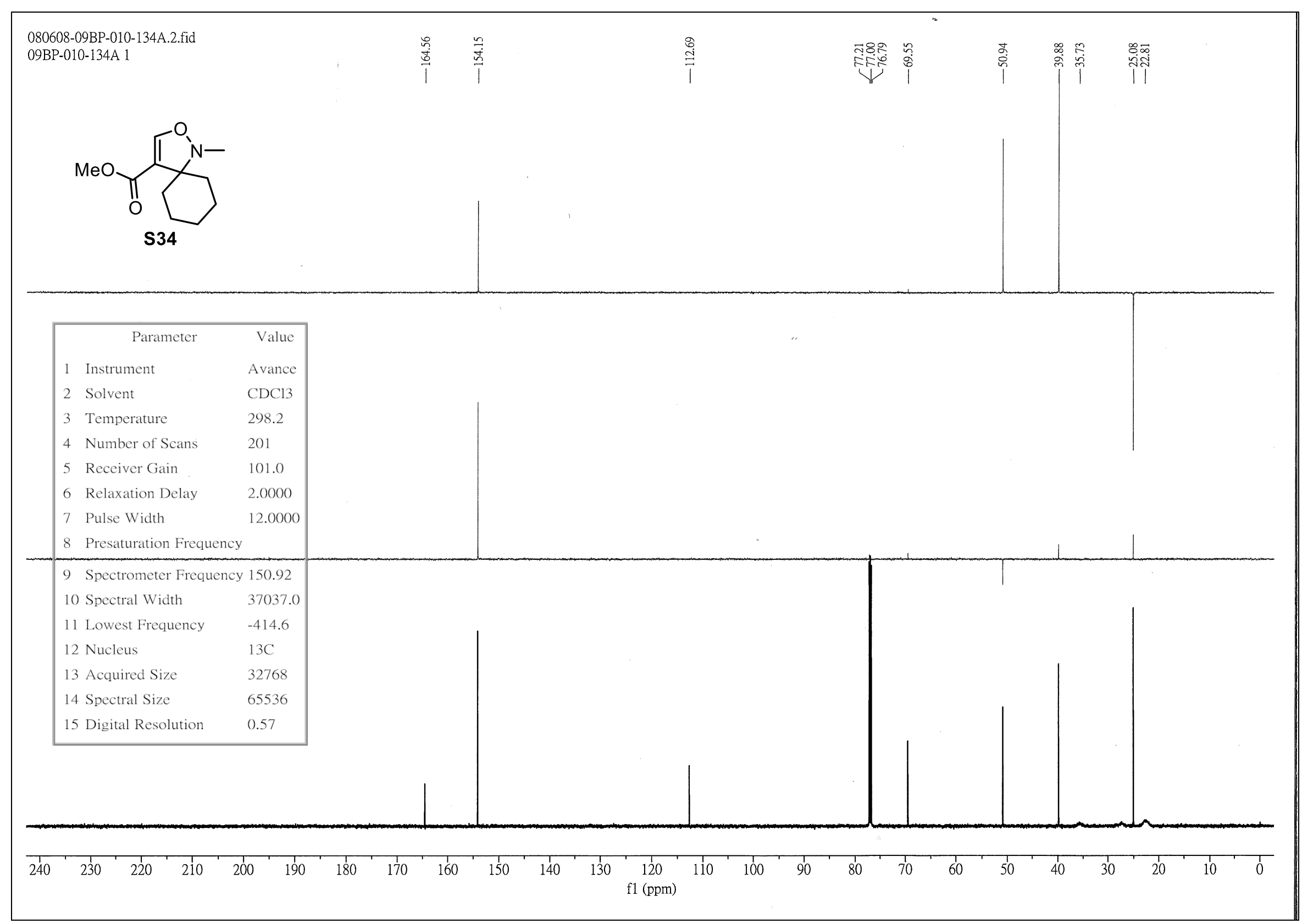

${ }^{13} \mathrm{C}$ NMR + DEPT spectra for compound S34 


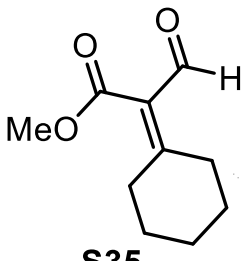

S35

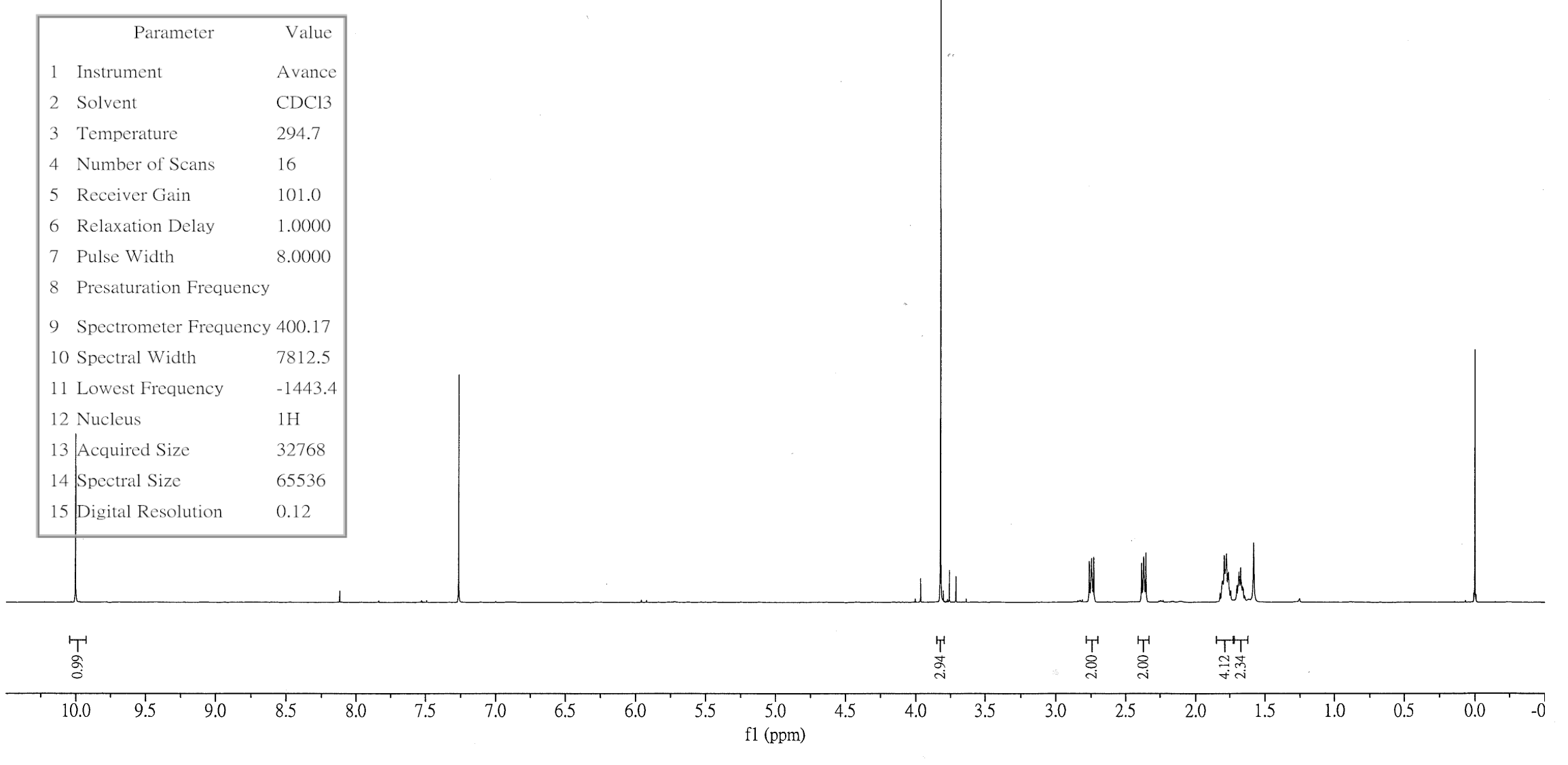

${ }^{1}$ H NMR spectrum for compound $\mathbf{S 3 5}$ 


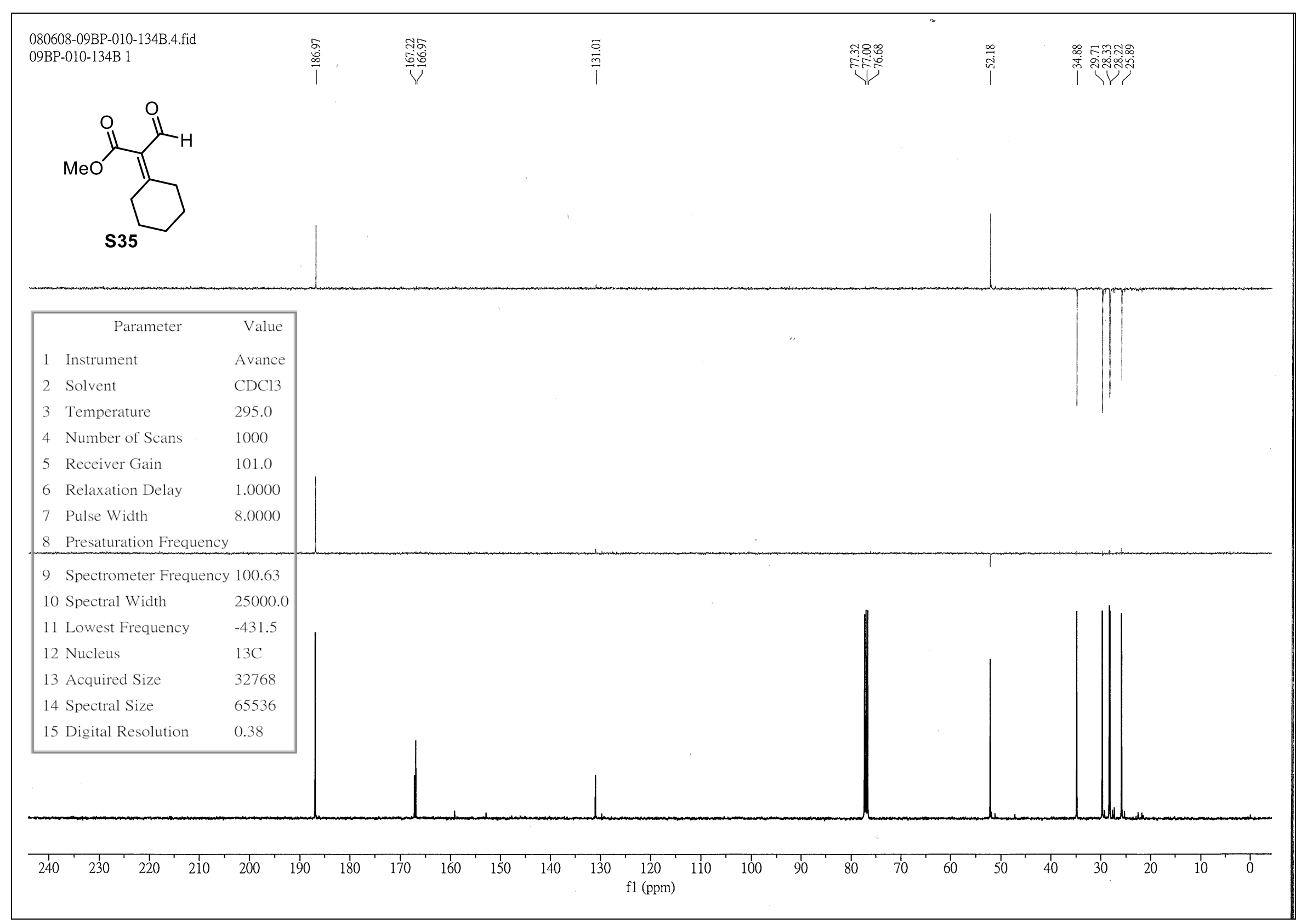

${ }^{13} \mathrm{C}$ NMR + DEPT spectra for compound $\mathbf{S 3 5}$ 